\title{
Preorganized Bimetallic Nickel Complexes of Pyrazolate-Bridged Ligands for Cooperative Substrate Transformation
}

\author{
Dissertation \\ zur Erlangung des mathematisch-naturwissenschaftlichen Doktorgrades \\ "Doctor rerum naturalium" \\ der Georg-August-Universität Göttingen \\ im Promotionsprogramm Chemie \\ der Georg-August University School of Science (GAUSS)
}

vorgelegt von

Dennis-Helmut Manz

aus Northeim

Göttingen 2016 


\section{Betreuungsausschuss}

Prof. Dr. Franc Meyer Institut für Anorganische Chemie, Universität Göttingen

Prof. Dr. Sven Schneider Institut für Anorganische Chemie, Universität Göttingen

\section{Referent}

Prof. Dr. Franc Meyer Institut für Anorganische Chemie, Universität Göttingen

\section{Korreferent}

Prof. Dr. Sven Schneider Institut für Anorganische Chemie, Universität Göttingen

\section{Weitere Mitglieder der Prüfungskommission}

Prof. Dr. Ricardo Mata Institut für Physikalische Chemie, Universität Göttingen

Jun.-Prof. Dr. Selvan Demir Institut für Anorganische Chemie, Universität Göttingen

Dr. Inke Siewert Institut für Anorganische Chemie, Universität Göttingen

Dr. Michael John Institut für Organische und Biomolekulare Chemie, Universität Göttingen

Tag der mündlichen Prüfung: 19.10.2016 


\section{Contents}

I. Extremely Slow Proton and Water Exchange of a Metal-Bridging Hydroxide 1

1. Extremely Slow Proton and Water Exchange of a Metal-Bridging Hydroxide 3

1.1. Introduction . . . . . . . . . . . . . . . 4

1.2. Objective .......................... 5

1.3. Preparation of the Pvrrole-Pyrazole Based Ligand Svstem . . . . . . . 6

1.4. Preparation and Characterization of the Bimetallic Complex . . . . . . 7

1.5. Exchange with Water. . . . . . . . . . . . . . . . 9

1.6. Determination of Kinetic and Thermodvnamic Parameters of the Exchange 12

1.6.1. Pseudo-First Order Reaction Conditions and Determination of $k$. . 14

1.6.2. Determination of the Rate Constants $k_{1}$ and $k_{2}$. . . . . . . . 16

1.6.3. Determination of Thermodvnamic Parameters . . . . . . . . . 17

1.6.4. DFT Calculations and Mechanistic Considerations . . . . . . . . 19

1.7. Summary . . . . . . . . . . . . . . . . . . . 21

II. Complexes of a Versatile Pyrazolate-based Bis( $\beta$-diketiminate) Ligand and Their Applications

2. A Bimetallic Nickel(II) Complex of a Pyrazolate-linked "Nacnac" Ligand as a Versatile Scaffold

2.1. Introduction . . . . . . . . . . . . . . . . . 26

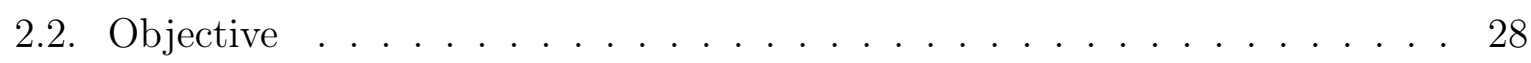

2.3. Ligand Svnthesis and Characterization . . . . . . . . . . . . . 29

2.4. Complex Synthesis . . . . . . . . . . . . . . . . . . 32

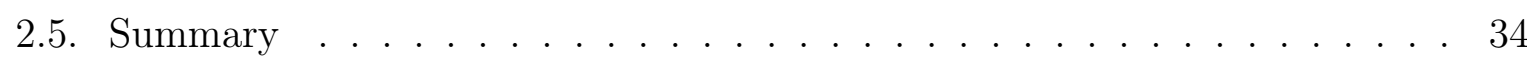

3. A $\beta$-Diketiminato Based $\mu$-Hydroxo Nickel Complex 35

3.1. Preparation of a Bimetallic $\beta$-Diketiminato Based Nickel $\mu$-Hydroxo Com-

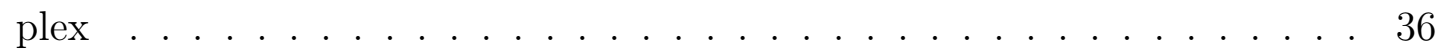

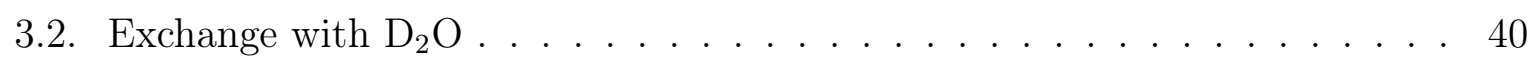




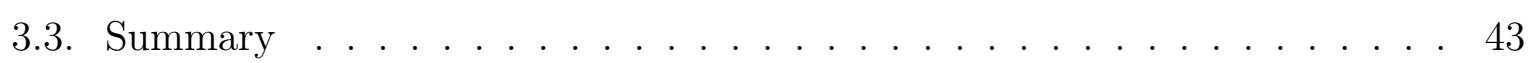

4. A Dinuclear Nickel Dihydride Complex $\quad 45$

4.1. State of Knowledge . . . . . . . . . . . . . . . . . . . 46

4.2. Motivation . . . . . . . . . . . . . . . . . . . . . 48

4.3. Preparation of $\mathrm{K}\left[\mathrm{L}^{\text {bisnac }} \mathrm{Ni}_{2}(\mathrm{H})_{2}\right]$. . . . . . . . . . . . . . . . . . . . . . . . . . . . 49

4.4. Exchange with $\mathrm{D}_{2}$. . . . . . . . . . . . . . . . . . 53

4.5. Reaction with Water . . . . . . . . . . . . . . . 65

4.6. Summary and Outlook . . . . . . . . . . . . . . . . . . 74

5. A Phenvlvinul-Bridged Dinuclear Nickel(II) Complex 75

5.1. State of Knowledge . . . . . . . . . . . . . . . . . . 76

5.2. Motivation . . . . . . . . . . . . . . . 78

5.3. Preparation and Characterization of the Complex . . . . . . . . 79

5.3.1. Synthesis and Molecular Structure . . . . . . . . . . . . . 79

5.3.2. Characterization by NMR Spectroscopv . . . . . . . . . . . 82

5.3.3. Rotational Barrier of the Phenvl Substituent . . . . . . . . . 88

5.4. Studies on the Reaction ... . . . . . . . . . . . . . 94

5.4.1. Formation of Stvrene . . . . . . . . . . . . . . 94

5.4.2. Deuterium Labeling. . . . . . . . . . . . . . . . . . . . 96

5.4.3. Para Hydrogen Induced Polarization Experiments . . . . . . . . . 102

5.4.4. Mechanistic Considerations . . . . . . . . . . . . . 110

5.4.5. Summary . . . . . . . . . . . . . . . . . . . . 111

5.5. Investigation of Phenvlacetvlene Semihvdrogenation . . . . . . . . . 113

5.5.1. Mechanism of Phenvlacetvlene Semihvdrogenation . . . . . . . . 117

5.5.2. Influences on the Catalytic Semihydrogenation Process . . . . . . 120

5.5.2.1. High Pressure . . . . . . . . . . . . . . . 120

$5.5 .2 .2 . \quad$ Mixing Issues $\ldots \ldots \ldots \ldots$

5.5.2.3. Conclusion . . . . . . . . . . . . . . . . 128

5.5.3. Investigation of Species X . . . . . . . . . . . . . 129

5.5.3.1. The Formation . . . . . . . . . . . . . . . . . 129

5.5.3.2. Characterization . . . . . . . . . . . . . . 132

5.5.3.3. Unusual Dvnamic Effects . . . . . . . . . . . . . 135

5.5.3.4. Intramolecular $\mathrm{C}-\mathrm{H}$ Activation . . . . . . . . . 136

5.5.3.5. Conclusion . . . . . . . . . . . . . . . 139

5.6. Summary and Outlook . . . . . . . . . . . . . . . . . . . . 140

6. New Model Complexes of Intermediates in the Process of Nitrogen Fixation 143

6.1. Introduction . . . . . . . . . . . . . . . . . . . . 144

6.2. Motivation . . . . . . . . . . . . . . . . . . . . 146

6.3. Preparation of an end-on Bridged Hydrazido Complex . . . . . . . . 147 
6.4. Deprotonation of the Hvdrazido Bridge . . . . . . . . . . . . . . . . 151

6.5. Protonation of the Hvdrazido Bridge . . . . . . . . . . . . . . . . 156

6.6. Thermal Conversion to an Amido-Bridged Complex . . . . . . . . . . . . . 159

6.7. Oxidation of the Hydrazido Ligand . . . . . . . . . . . . . . . . . . 167

6.8. $\mathrm{K}\left[\mathrm{L}^{\text {bisnac }} \mathrm{Ni}_{2}(\mathrm{H})_{2}\right]$ - a Potential Scaffold for $\mathrm{N}_{2}$ Fixation . . . . . . . . . . . 171

6.9. Summary and Outlook . . . . . . . . . . . . . . . . 176

$\begin{array}{ll}\text { 7. Experimental Section } & 181\end{array}$

7.1. General Considerations . . . . . . . . . . . . . . . . . . . . . 181

7.2. Svnthesis of Ligands and Ligand Precursors . . . . . . . . . . . . . . . . 183

7.3. Svnthesis of Complexes . . . . . . . . . . . . . . . . . . . . . 187

7.4. DFT Calculations . . . . . . . . . . . . . . . . . 196

\begin{tabular}{ll}
\hline Appendices & 197
\end{tabular}

A. Structures of Important Compounds 199

B. Crystallographic Data 201

$\begin{array}{ll}\text { C. Part One } & 207\end{array}$

C.1. Analysis of Isotopically Labeled $\left[\mathrm{L}^{\mathrm{pyr}} \mathrm{Ni}_{2}(\mu-\mathrm{OH})\right] \ldots$. . . . . . . . . . 207

C.2. Additional Plots . . . . . . . . . . . . . . . . . . . . . . . . 210

$\begin{array}{ll}\text { D. Part Two } & 217\end{array}$

D.1. Gas Drving Procedure . . . . . . . . . . . . . . . . . . . 218

D.2. Investigation of the Exchange of $\left.\mathrm{K}_{\mathrm{L}} \mathrm{L}^{\text {bisnac }} \mathrm{Ni}_{2}(\mathrm{H})_{2}\right]$ with Parahydrogen $\ldots 219$

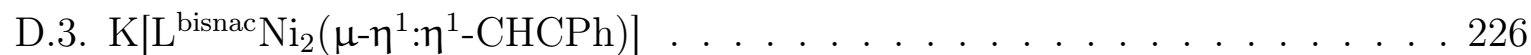

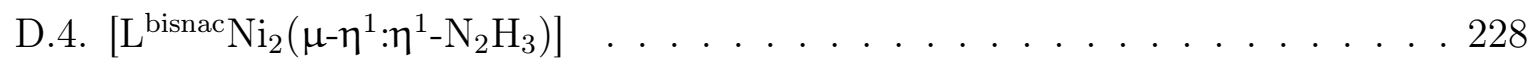

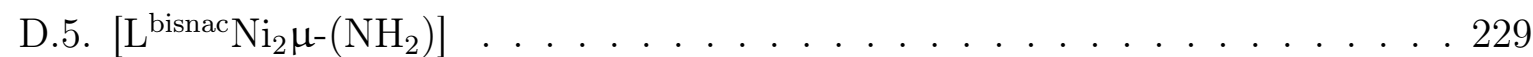

D.6. $\left[\mathrm{L}^{\text {bisnac }} \mathrm{Ni}_{2}\left(\mu-\eta^{1}: \eta^{1}-\mathrm{N}_{2} \mathrm{H}\right)\right]$. . . . . . . . . . . . . . . . 231

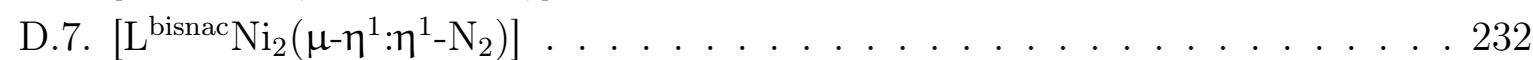

$\begin{array}{ll}\text { References } & 234\end{array}$

\begin{tabular}{ll}
\hline List of Abbreviations & 253
\end{tabular} 



\section{Part I.}

Extremely Slow Proton and Water Exchange of a Metal-Bridging Hydroxide 



\section{Extremely Slow Proton and Water Exchange of a Metal-Bridging Hydroxide}

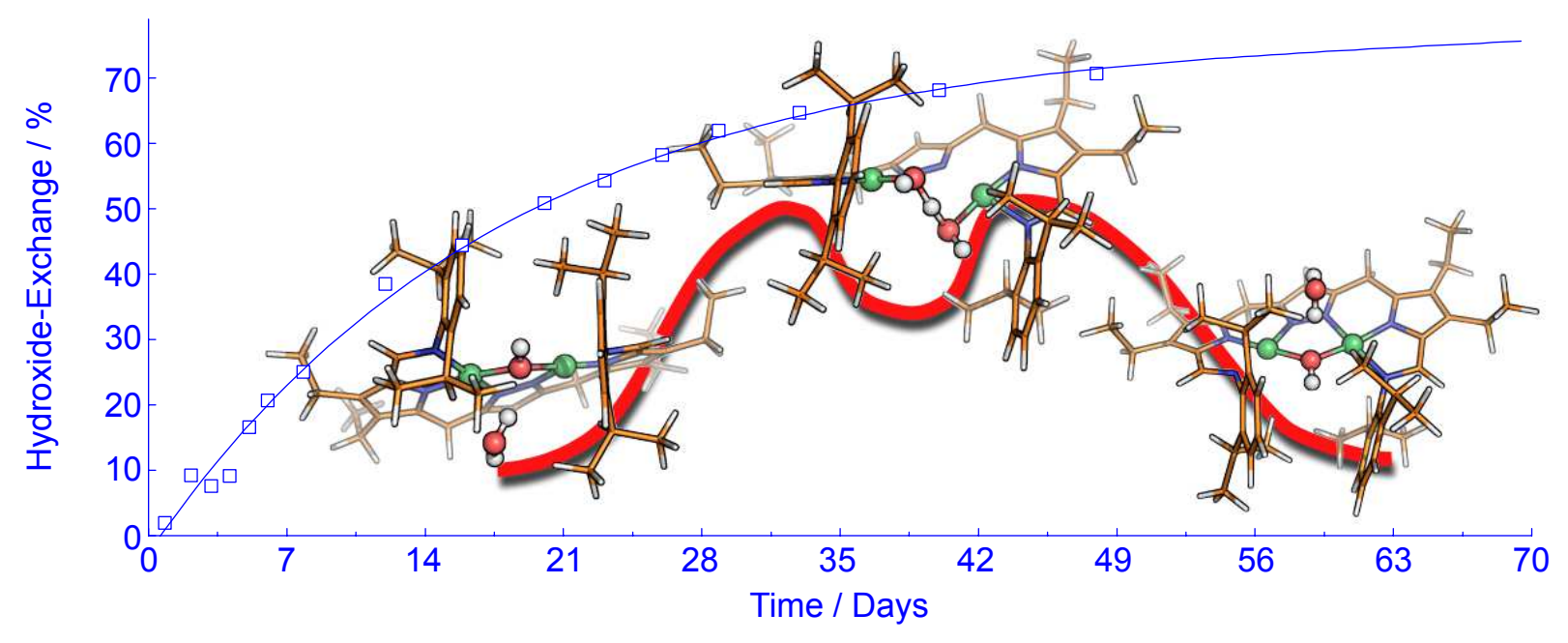

Abstract A pyrrole-pyrazolate-based ligand was prepared to give rise to a bimetallic complex comprising a bridging hydroxide. This diamagnetic complex was characterized by NMR spectroscopy, showing a strongly upfield shifted resonance at $-6.7 \mathrm{ppm}$ for the bridging hydroxide proton. Furthermore, water exchange experiments with $\mathrm{H}_{2}{ }^{17} \mathrm{O}$ and $\mathrm{D}_{2} \mathrm{O}$ have been conducted to reveal an extremely slow hydroxide exchange with a half-life of around five weeks at ambient conditions. Exchange kinetics have been analyzed in detail by means of NMR spectroscopy and a mechanistic picture could be derived from obtained kinetic and thermodynamic parameters, which was further supported by density functional theory (DFT) calculations. 


\subsection{Introduction}

\section{Exchange Reactions}

Understanding the reactivity of metal ions in biology and in solutions is continually of great interest. In this context exchange reactions are of major importance. ${ }^{[\underline{\underline{1}]}}$ In particular, the exchange of water ligands is regarded as the most essential exchange reaction. ${ }^{[\underline{1}-\underline{3}]}$ Although exchange processes in the aqueous phase are well investigated, studies of water exchange in media other than water are relatively rare. Nonetheless, these reactions are important, since the interpretation of complex enzymatic mechanisms is highly correlated to exchange reactions in enzymes. While in aqueous solution the charge of the metal complex can be stabilized by the high dielectric constant, in biological systems metal centers are often isolated by low dielectric proteins. Therefore, metal centers of high charges are unlikely in these systems, which makes neutral compounds suitable candidates for model systems to investigate water exchange processes. ${ }^{[\underline{1}]}$

\section{A Bimetallic Bridged Pyrazolate-Pyrrole-Based Complex}

Acyclic pyrrole-based diimine ligands were first synthesized in 1967 by WEBER et al. and form complexes with various metals $\left(\mathrm{M}=\mathrm{Pd}^{\mathrm{II}}, \mathrm{Cu}^{\mathrm{II}}, \mathrm{Ni}^{\mathrm{II}}, \mathrm{Zn}^{\mathrm{II}}\right) .^{\left[{ }^{[4]}\right]}$ During the last decade, various new systems with remarkable properties were synthesized. ${ }^{[\underline{\underline{5}-9]}}$

Dinuclear systems are of great interest due to their capability to mediate cooperativity between the metal cores. ${ }^{[10]}$ In particular, 3,5-disubstituted pyrazolate ligands have shown to be effective chelates for the preorganization of two metal centers. ${ }^{[9.11]}$ The dinuclear pyrazolate-pyrrole-based complexes shown in Scheme 1.1 feature bridging ligands such as $\mathrm{Cl}^{-}$or $\mathrm{OH}^{-} . \underline{\underline{\underline{9,11}]}}$

Since hydroxo-bridged bimetallic cores in metalloenzymes are suitable to catalyze various hydrolytic or oxidation processes, the dinuclear nickel(II) complex in particular is of interest. This kind of complex features two coordinatively unsaturated nickel(II) ions in a nitrogen-rich environment, which are bridged by a hydroxide, similar to the metalloenzyme urease. Furthermore, it was found that the hydroxo-bridged compound features an unusually slow exchange behaviour with water in a THF solution. ${ }^{[11]}$ 


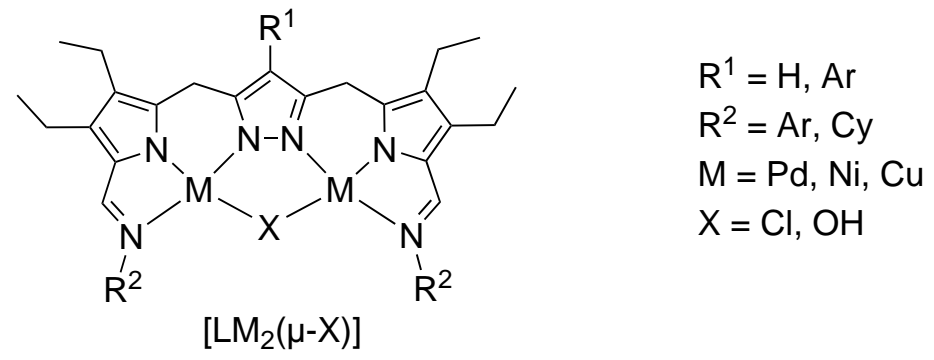

Scheme 1.1: Bimetallic bridged pyrazolate-pyrrole-based complexes.

\subsection{Objective}

The bimetallic hydroxo-bridged complex $\left[\mathrm{L}^{\mathbf{p y r}} \mathbf{N i}_{\mathbf{2}}(\mu-\mathrm{OH})\right]$ (Scheme 1.2) exhibits an upfield shifted proton resonance $\left(-6.7 \mathrm{ppm}\right.$ in $\mathrm{THF}^{\left.-\mathrm{D}_{8}\right)}$ of the hydroxo group in the ${ }^{1} \mathrm{H}$ NMR spectrum, which lies within the range of reported nickel-hydroxides $(-0.49-$ $-8.4 \mathrm{ppm}) . \stackrel{[12-20]}{\underline{2}}$ In the presence of water, $\left[\mathbf{L}^{\mathrm{pyr}} \mathrm{Ni}_{\mathbf{2}}(\mu-\mathrm{OH})\right]$ showed an extremely slow exchange of the bridging ligand, which was proven by isotopic labeling experiments and monitoring by NMR spectroscopy.

This work focuses on the in-depth investigation of the exchange, including the establishment of kinetic and thermodynamic parameters. With additional support from DFT calculations, a mechanistic picture is to be derived.
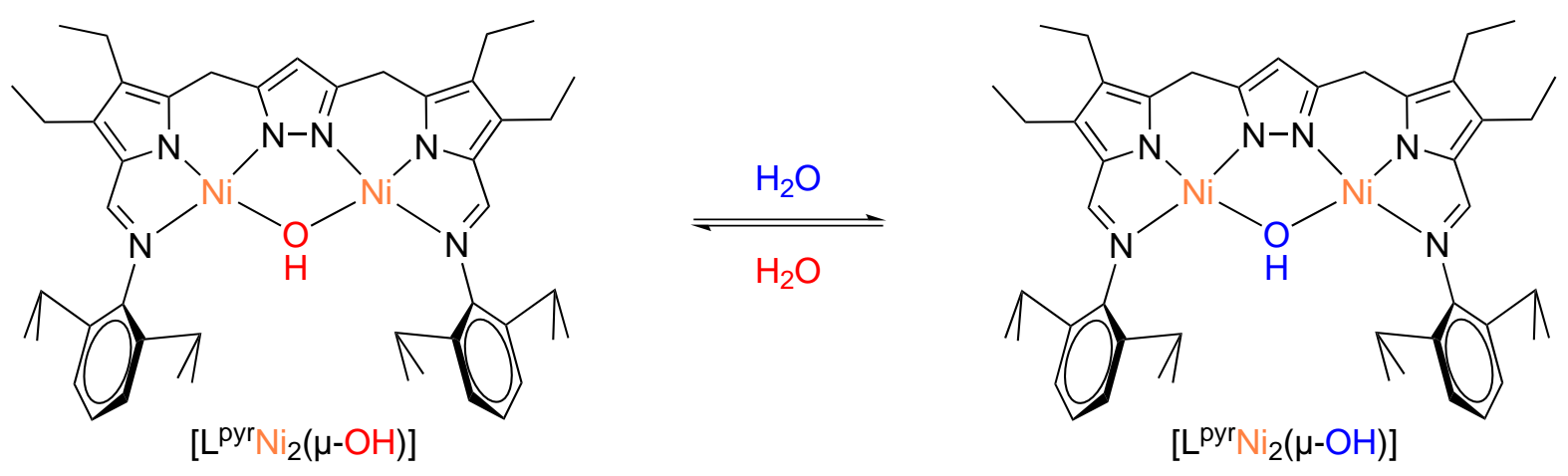

Scheme 1.2: Hydroxyl-exchange in $\left[\mathrm{L}^{\mathrm{pyr}} \mathrm{Ni}_{2}(\mu-\mathrm{OH})\right]$ with water in a THF solution. 


\subsection{Preparation of the Pyrrole-Pyrazole Based Ligand System}

The main building block of the acyclic diimine-ligand system is the dialdehyde (5), which may be reacted with primary amines. This compound was synthesized by starting with the pyrazole compound 3,5-Bis(chloromethyl)-pyrazole hydrochloride (1), which was pre-

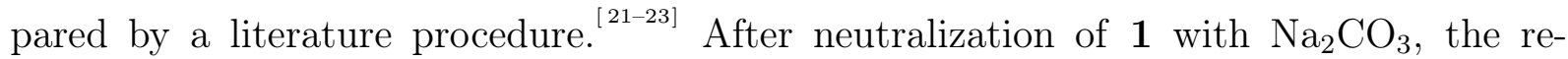
sulting 3,5-Bis(chloromethyl)-pyrazole (2) was substituted with 3,4-diethylpyrrole $\stackrel{[24,25]}{\underline{20}}$ (3) to form the pyrazole-pyrrole compound (4). In the next step a formylation via Vilsmeier-Haack procedure ${ }^{\underline{[26]}}$ yielded the aldeyde (5). Finally, condensation with 2,6-diisopropylaniline gives the ligand $\mathbf{H}_{\mathbf{3}} \mathbf{L}^{\mathbf{p y r}}$. ${ }^{[9,11]}$<smiles>ClCc1cc(CCl)[nH]n1</smiles>

1

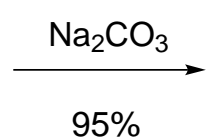

$95 \%$<smiles>ClCc1cc(CCl)[nH]n1</smiles>

2

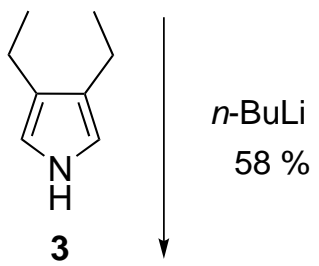<smiles>CCc1c(C=O)[nH]c(Cc2cc(Cc3[nH]c(C=O)c(CC)c3CC)[nH]n2)c1CC</smiles>

5

1) $\mathrm{DMF},(\mathrm{COCl})_{2}$

2) $\mathrm{NaOAc}$

$59 \%$<smiles>CCc1c[nH]c(Cc2cc(Cc3[nH]cc(CC)c3CC)[nH]n2)c1CC</smiles>

4<smiles>C=Cc1cccc(C=C)c1N=Cc1[nH]c(Cc2cc(Cc3[nH]c(C=Nc4c(C=C)cccc4C(C)C)c(CC)c3CC)n[nH]2)c(CC)c1CC</smiles>

$H_{3} L^{p y r}$

Figure 1.1.: Synthesis of the ligand $\mathbf{H}_{3} \mathbf{L}^{\mathrm{pyr}}$. [9,11] 


\subsection{Preparation and Characterization of the Bimetallic Complex}

Addition of a solution of $\mathrm{Ni}(\mathrm{OAc})_{2} \cdot 4 \mathrm{H}_{2} \mathrm{O}$ (2 eq.) in $\mathrm{MeOH}$ to a stirred yellow solution of $\mathbf{H}_{3} \mathbf{L}^{\mathbf{p y r}}$ and triethylamine (1 eq.) in THF results in the formation of the deep red $\left[\mathbf{L}^{\mathbf{p y r}} \mathbf{N i}_{\mathbf{2}}(\mu-\mathrm{OH})\right]$. The ORTEP diagram of the molecular structure of $\left[\mathbf{L}^{\mathbf{p y r}} \mathbf{N i}_{\mathbf{2}}(\mu-\mathrm{OH})\right]$ is shown in Figure 1.2. The complex crystallizes in a triclinic crystal space group $P \overline{1}$ with two molecules in the unit cell.
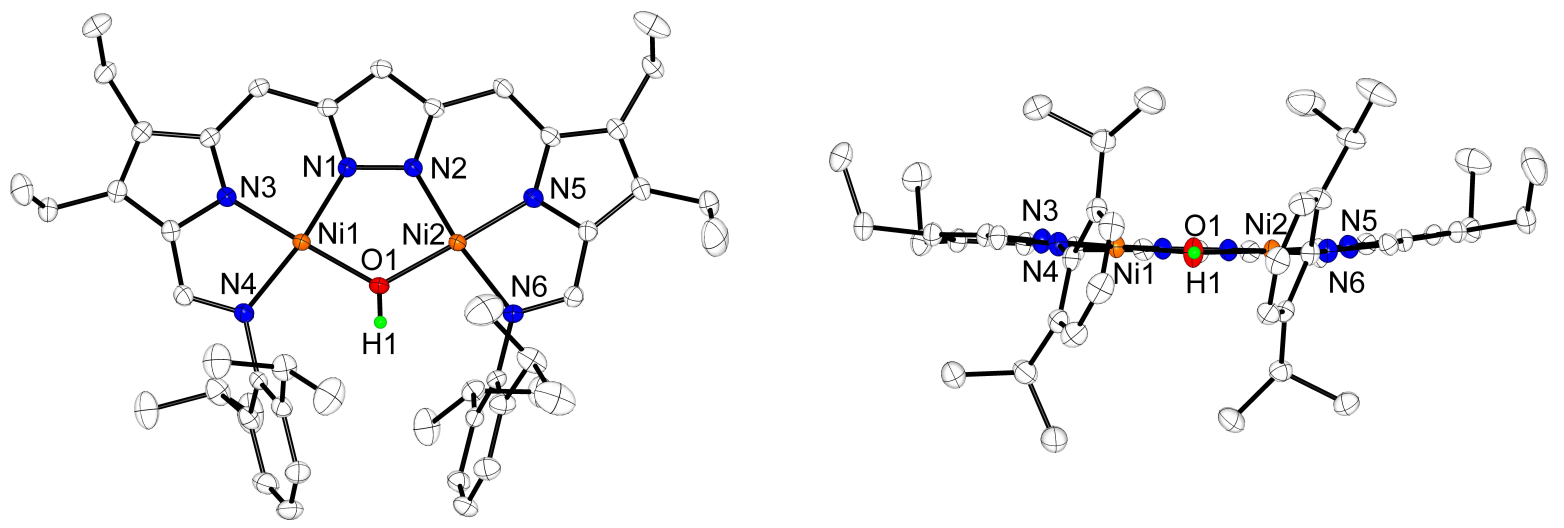

Figure 1.2.: Thermal displacement ellipsoids (shown at $50 \%$ probability) of the molecular structure of [ $\left.{ }^{\text {pyr }} \mathrm{Ni}_{2}(\mu-\mathrm{OH})\right]$ from the top (left) and the front side (right). All hydrogen atoms except the hydroxohydrogen atom have been omitted for the sake of clarity (the hydrogen atom is displayed as ball-and-stick representation with a fixed radius of $0.135 \AA$ ).

The angles between the nickel atoms and the coordinated nitrogen or oxygen atoms provide insight into the coordination environment. For instance, for Ni1 of $\left[\mathbf{L}^{\mathbf{p y r}} \mathbf{N i}_{\mathbf{2}}(\boldsymbol{\mu}-\right.$ $\mathrm{OH})$ ], the two largest angles to neighboring atoms are $\Varangle \mathrm{N} 3-\mathrm{Ni1}-\mathrm{O} 1=178^{\circ}$ and $\Varangle \mathrm{N} 1-\mathrm{Ni} 1-\mathrm{N} 4=173^{\circ}$ and the other four are nearly $90^{\circ}$. This indicates a square-planar coordination of Ni1 (Figure 1.2). A similar environment was determined for Ni2. The $\mathrm{Ni} \cdot \cdots \mathrm{Ni}$ distance is $3.25 \AA$ and the angle $\Varangle \mathrm{Ni} 2-\mathrm{O} 1-\mathrm{Ni1}=121.24^{\circ}$. Further selected bond lengths and angles are shown in Table 1.1.

Figure 1.3 shows the ${ }^{1} \mathrm{H}$ NMR spectrum of $\left[\mathbf{L}^{\mathbf{p y r}} \mathbf{N i}_{\mathbf{2}}(\mu-\mathrm{OH})\right]$ measured in THF-D 8 . The proton resonance of the hydroxo group is shifted to higher fields of the spectrum $\left(\delta\left\{{ }^{1} \mathrm{H}\right\}_{\mathrm{OH}}\right.$ $=-6.7 \mathrm{ppm}$ ). To investigate the effect of ring current from the DIPP moieties, the ligand $\mathbf{H}_{3} \mathbf{L}^{\mathbf{p y r}}$ was modified (the DIPP moieties were replaced by cyclohexyl substituents). ${ }^{[11]}$ With this ligand a hydroxo-bridged bimetallic nickel complex was obtained, which revealed a proton resonance at $-4.1 \mathrm{ppm}$ for the hydroxo group. ${ }^{[11]}$ This indicated that the effect of an aryl ring current on the electronic shielding of the hydroxo-hydrogen was marginal. Strong electronic shielding likely originates from the relatively electron rich (trianionic) ligand system itself, accounting for the upfield chemical shift of $\left[\mathbf{L}^{\mathbf{p y r}} \mathbf{N i}_{\mathbf{2}}(\mu-\right.$ $\mathrm{OH})$ ]. This is substantiated by proton resonances of hydroxo groups of literature known 
Ni-OH complexes (-6.2 ppm, $-7.8 \mathrm{ppm}$ and $-8.4 \mathrm{ppm})$, featuring comparable electron rich ligands. $\stackrel{[14,17]}{ }$

Table 1.1.: Selected bond lengths, distances and angles of [ $\left.\mathrm{L}^{\mathrm{pyr}} \mathrm{Ni}_{2}(\mu-\mathrm{OH})\right]$.

\begin{tabular}{|c|c|c|c|c|c|}
\hline \multicolumn{2}{|c|}{ Bond lengths / $\AA$} & \multicolumn{4}{|c|}{ Angle $/^{\circ}$} \\
\hline Ni1-N3 & $1.8346(2)$ & N3-Ni1-N1 & $89.79(6)$ & N5-Ni2-O1 & $178.26(6)$ \\
\hline $\mathrm{Ni1}-\mathrm{N} 1$ & $1.8603(1)$ & N3-Ni1-O1 & $177.94(7)$ & N2-Ni2-O1 & $89.05(6)$ \\
\hline $\mathrm{Ni1-O1}$ & $1.8646(1)$ & $\mathrm{N} 1-\mathrm{Ni1}-\mathrm{O} 1$ & $88.87(6)$ & N5-Ni2-N6 & $83.36(6)$ \\
\hline Ni1-N4 & $1.9275(1)$ & N3-Ni1-N4 & $82.97(6)$ & $\mathrm{Ni2}-\mathrm{O} 1-\mathrm{Ni1}$ & $121.24(7)$ \\
\hline Ni2-N5 & $1.8326(1)$ & $\mathrm{N} 1-\mathrm{Ni1}-\mathrm{N} 4$ & $172.76(6)$ & N2-N1-Ni1 & $120.50(1)$ \\
\hline $\mathrm{Ni2}-\mathrm{N} 2$ & $1.8609(1)$ & O1-Ni1-N4 & $98.36(6)$ & N1-N2-Ni2 & $120.12(1)$ \\
\hline $\mathrm{Ni2}-\mathrm{O} 1$ & $1.8619(1)$ & N5-Ni2-N2 & $89.20(6)$ & $\mathrm{Ni2}-\mathrm{O} 1-\mathrm{Ni1}$ & $121.24(7)$ \\
\hline $\mathrm{Ni2}-\mathrm{N} 6$ & $1.9343(1)$ & & & & \\
\hline $\mathrm{N} 1-\mathrm{N} 2$ & $1.369(2)$ & & & & \\
\hline $\mathrm{O} 1-\mathrm{H} 1$ & $0.7804(3)$ & & & & \\
\hline $\mathrm{Ni} 1 \ldots \mathrm{Ni} 2$ & $3.2472(4)$ & & & & \\
\hline
\end{tabular}

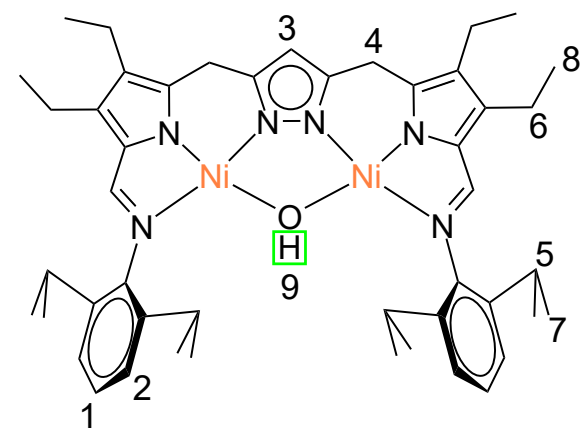
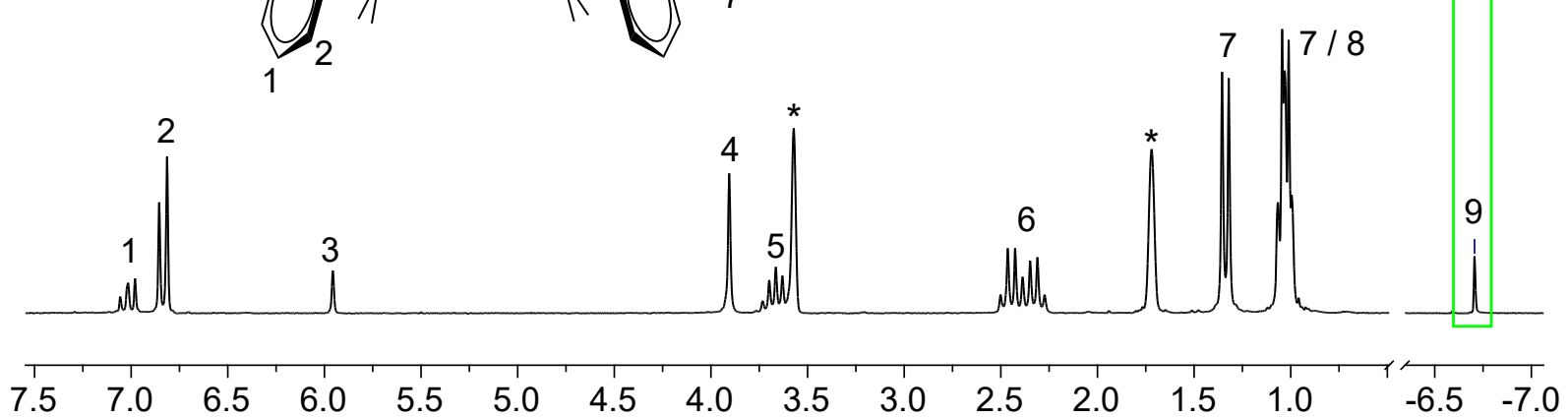

$\delta / \mathrm{ppm}$

Figure 1.3.: ${ }^{1} \mathrm{H}$ NMR spectrum $(200 \mathrm{MHz})$ of $\left[\mathrm{L}^{\mathrm{pyr}} \mathrm{Ni}_{2}(\mu-\mathrm{OH})\right]$ measured in THF- $\mathrm{D}_{8}$. The pronounced upfield shift of the $\mu-\mathrm{OH}$ ligand is highlighted. Residal solvents are marked $\left({ }^{*}\right)$. 


\subsection{Exchange with Water}

\section{Experimental Setup}

For detailed studies on the exchange mechanisms, several NMR samples (in NMR tubes with a J. Young PTFE valve) with $10 \mathrm{mg}(12 \mu \mathrm{mol})$ of $\left[\mathbf{L}^{\mathbf{p y r}} \mathbf{N i}_{\mathbf{2}}(\mu-\mathbf{O H})\right]$ in dry THF- $\mathrm{d}_{8}$ $(0.5 \mathrm{~mL})$ were prepared. At different constant temperatures from $30-60{ }^{\circ} \mathrm{C}$, the exchange reaction was initiated by addition of an excess of water $\left(\mathrm{D}_{2} \mathrm{O}\right.$ or $\mathrm{H}_{2}{ }^{17} \mathrm{O}, 10-100$ eq. $)$ to the sample tubes. The exchange was determined by the integral ratio $\mathrm{H}^{\mathrm{OH}} / \mathrm{H}^{\mathrm{Pz}}$ which was derived from ${ }^{1} \mathrm{H}$ NMR spectra. Measurements were recorded using a $200 \mathrm{MHz}$ NMR spectrometer periodically until the saturation region was reached (after 6 weeks at room temperature). The exchange reactions with $\mathrm{D}_{2} \mathrm{O}$ were conducted at constant temperatures $^{\dagger}$ : all the prepared NMR sample tubes were kept in a self prepared heating device over the course of the reaction. To investigate exchange of the complete hydroxo group, ${ }^{17}$ O-labeled water $(90 \%)$ was used.

\section{Exchange with $\mathrm{D}_{2} \mathrm{O}$}

Exchange with $\mathrm{D}_{2} \mathrm{O}$ was probed by means of ${ }^{1} \mathrm{H}$ NMR and IR spectroscopy (shown in the appendix, C.1). An example of the slow exchange at $30{ }^{\circ} \mathrm{C}$ is illustrated in Figure 1.5. The conversion to the deuterium labeled product was determined from monitoring the decreasing hydroxo-hydrogen signal by ${ }^{1} \mathrm{H}$ NMR . At higher amounts of $\mathrm{D}_{2} \mathrm{O}$ the exchange rate increases. Further experiments performed at higher temperatures are depicted in the appendix (Figure C.3).

\section{Exchange with $\mathrm{H}_{2}{ }^{17} \mathrm{O}$}

Exchange of the hydroxyl group in $\left[\mathrm{L}^{\mathrm{pyr}} \mathrm{Ni}_{\mathbf{2}}\left(\mu^{-17} \mathrm{OH}\right)\right]$ can be observed via mass spectrometry (Figure C.2) and ${ }^{17} \mathrm{O}$ NMR spectroscopy. Figure 1.4 illustrates the increase of the ${ }^{17} \mathrm{O}$ labeled $\mu-\mathrm{OH}$ group in the complex over 28 days. The ${ }^{17} \mathrm{O}$ peak of the hydroxo ligand features pronounced line broadening $(\approx 100 \mathrm{ppm})$ due to the fast relaxation of the ${ }^{17} \mathrm{O}$ nucleus. ${ }^{[27-29]}$

\footnotetext{
${ }^{\dagger}$ Since it was not possible to heat the $200 \mathrm{MHz}$ NMR spectrometer during the time of measurement the samples cooled off to ambient temperature during the ${ }^{1} \mathrm{H}$ NMR measurement. The systematic error, which results by that working procedure was considered to be marginal due to the timescale of the overall measuring session and was therefore ignored.
} 


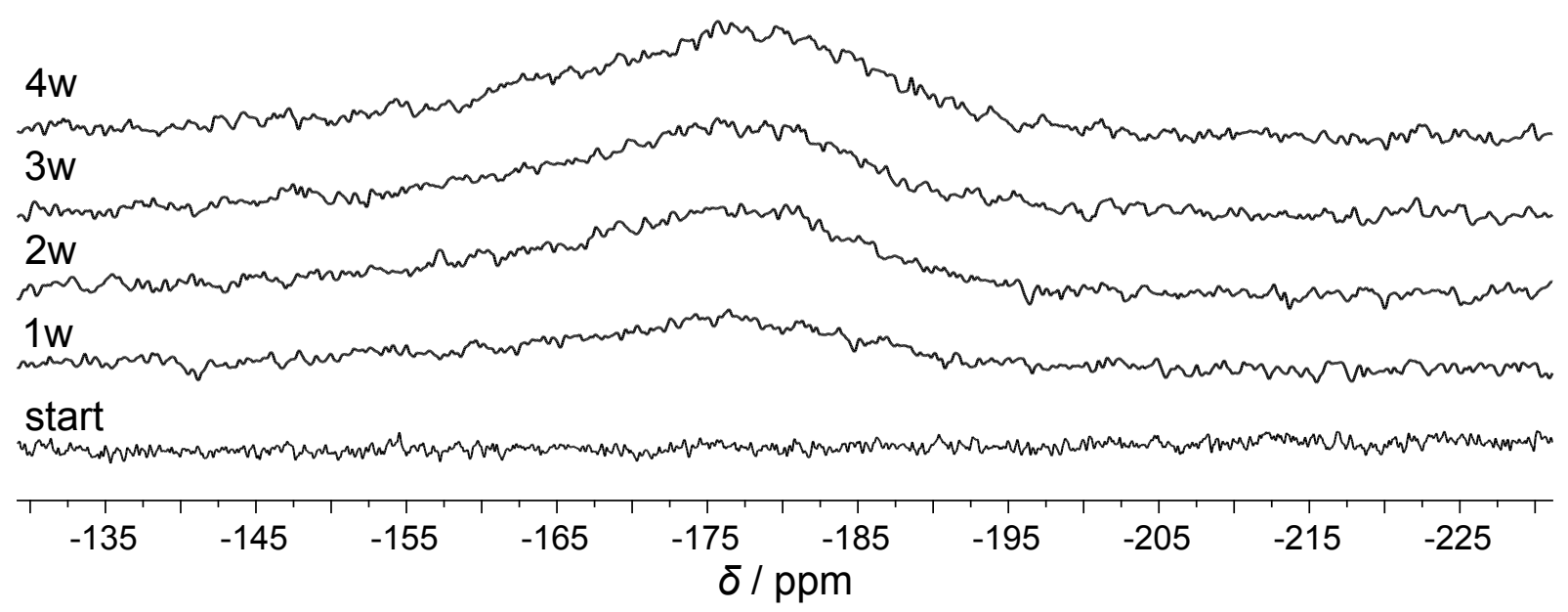

Figure 1.4.: ${ }^{16} \mathrm{OH} /{ }^{17} \mathrm{OH}$ exchange of $\left[\mathrm{L}^{\text {pyr }} \mathrm{Ni}_{2}\left(\mu-{ }^{17} \mathrm{OH}\right)\right]$ with $\mathrm{H}_{2}{ }^{17} \mathrm{O}$ (100 eq.) in THF- $\mathrm{D}_{8}$ followed by ${ }^{17} \mathrm{O}$ NMR $(68 \mathrm{MHz})$ over one month.

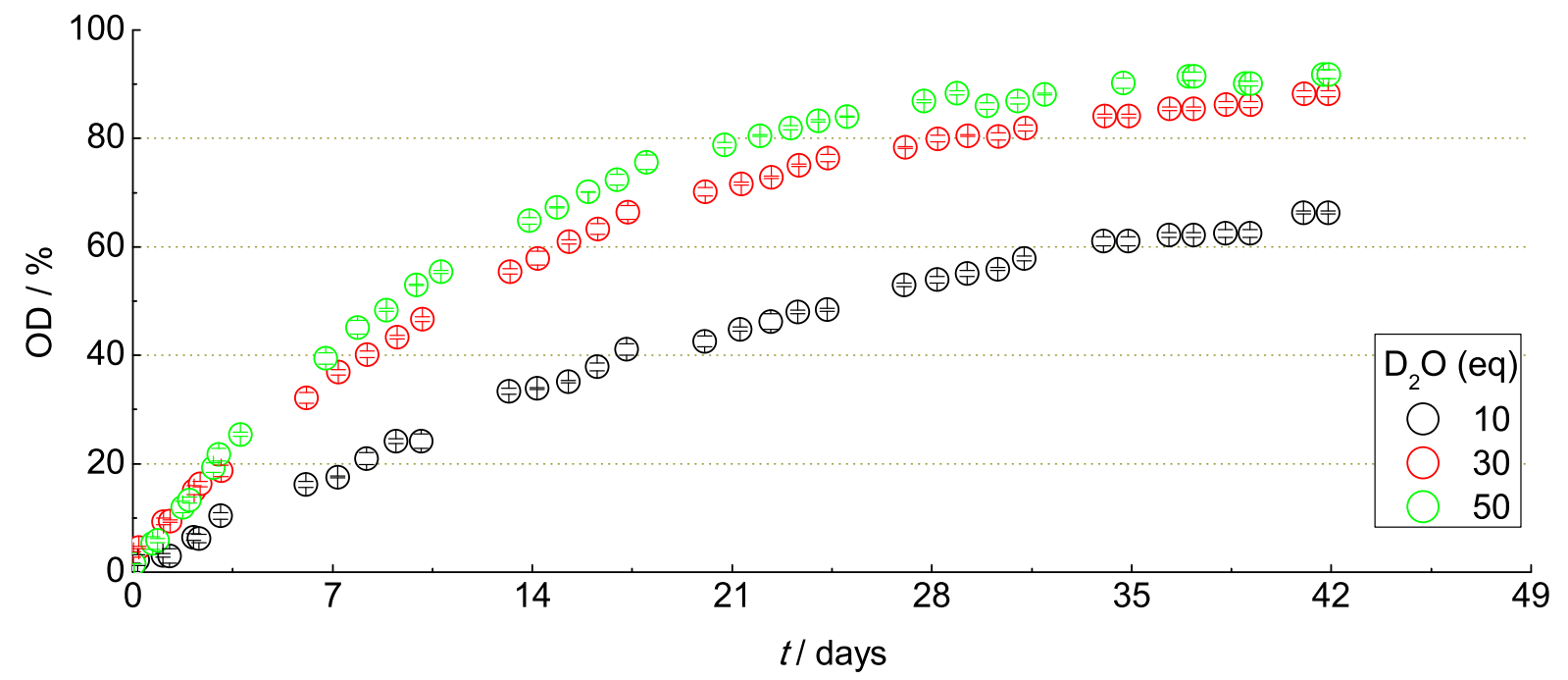

Figure 1.5.: $\mathrm{H}-\mathrm{D}$ exchange of $\left[\mathrm{L}^{\text {pyr }} \mathrm{Ni}_{2}(\mu-\mathrm{OH})\right]$ with $\mathrm{D}_{2} \mathrm{O}\left(10-50\right.$ eq.) in THF- $\mathrm{D}_{8}$ at $30{ }^{\circ} \mathrm{C}$. 
The dynamic ${ }^{16} \mathrm{OH} /{ }^{17} \mathrm{OH}$ exchange was monitored by ${ }^{1} \mathrm{H}$ NMR and quantified by means of the decreasing ${ }^{16} \mathrm{OH}$ signal via line-fitting. ${ }^{\ddagger}$ Figure 1.6 shows the ${ }^{1} \mathrm{H}$ NMR spectrum of a sample containing $\left[\mathbf{L}^{\mathbf{p y r}} \mathbf{N i}_{\mathbf{2}}\left(\mu \mathbf{-}^{\mathbf{1 6}} \mathrm{OH}\right)\right]$ and an excess of $\mathrm{H}_{2}{ }^{17} \mathrm{O}$, measured after several days. The ${ }^{16} \mathrm{O}-\mathrm{H}$ hydrogen resonance decreased while the ${ }^{17} \mathrm{O}-\mathrm{H}$ signal emerged. Similar to the ${ }^{17} \mathrm{O}$ NMR experiment, the hydrogen resonance of the ${ }^{17} \mathrm{O}$ labeled $\mu$-hydroxo ligand exhibits pronounced line broadening. The strong quadrupolar moment $(I=5 / 2)$ of the ${ }^{17} \mathrm{O}$ nucleus exhibits a short relaxation time, which in turn affects the bound hydrogen atom. Consequently, relaxation of the hydroxo-hydrogen is dramatically increased.

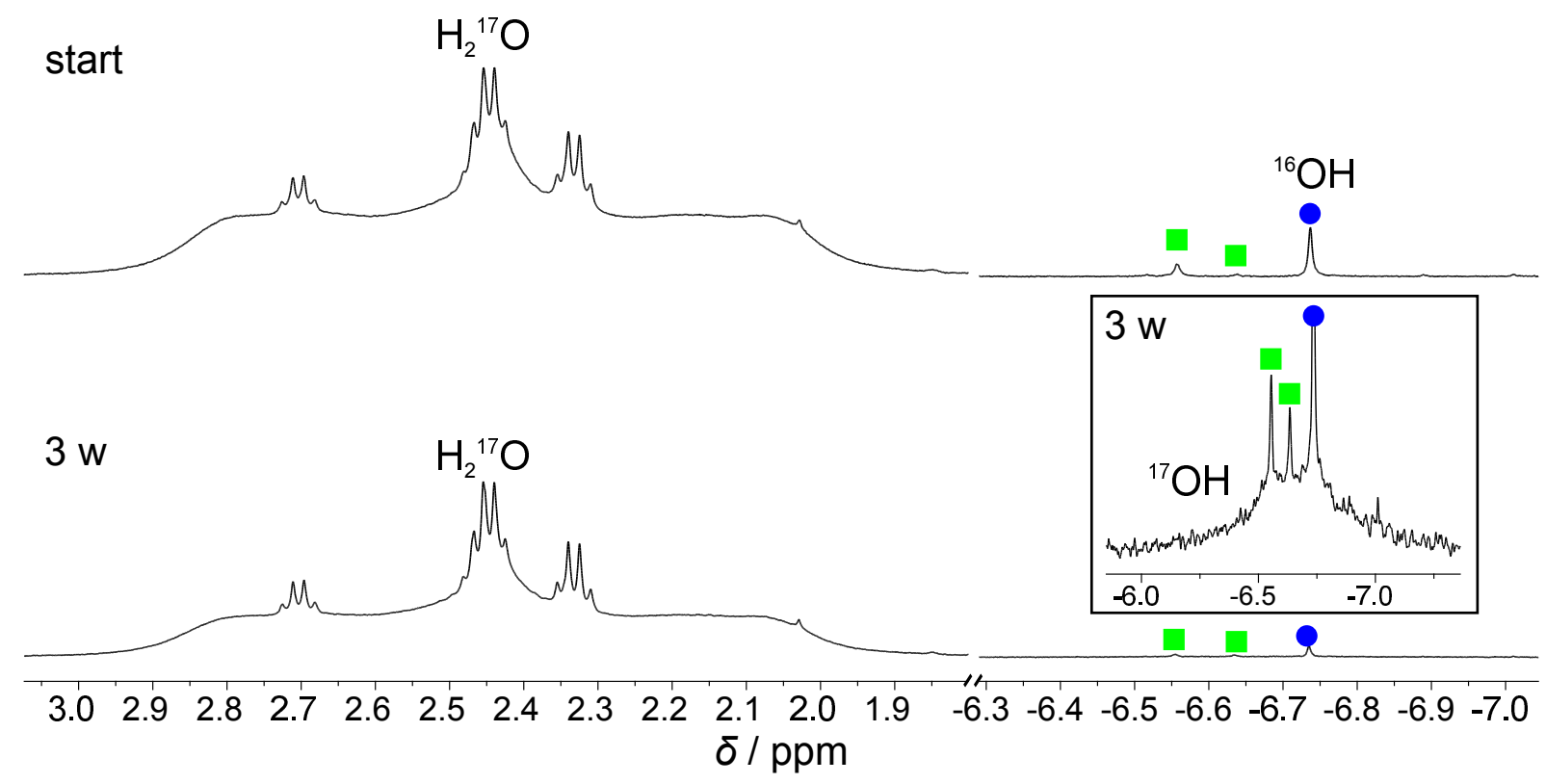

Figure 1.6.: ${ }^{16} \mathrm{OH} /{ }^{17} \mathrm{OH}$ exchange of $\left[\mathrm{L}^{\text {pyr }} \mathrm{Ni}_{2}(\mu-\mathrm{OH})\right]$ with $\mathrm{H}_{2}{ }^{17} \mathrm{O}$ (30 eq.) in THF- $\mathrm{D}_{8}$ monitored by ${ }^{1} \mathrm{H}$ NMR $(200 \mathrm{MHz})$ at $30{ }^{\circ} \mathrm{C}$. The ${ }^{17} \mathrm{O}$-water causes pronounced line broadening. Three weeks (bottom) after addition of the labeled water, the sharp ${ }^{16} \mathrm{OH}$ resonance $\bullet$ of the complex decreased while the broad ${ }^{17} \mathrm{OH}$ peak emerged (illustrated in the box). Further resonances at -6.55 and -6.63 ppm may be attributed to the presence of an intermediate $(\square)$.

Figure 1.7 illustrates the exchange of $\left[\mathbf{L}^{\mathbf{p y r}} \mathbf{N i}_{\mathbf{2}}(\mu-\mathrm{OH})\right]$ with different amounts of $\mathrm{H}_{2}{ }^{17} \mathrm{O}$ at $30{ }^{\circ} \mathrm{C}$. The exchange was quantified by means of integration of the decreasing ${ }^{16} \mathrm{OH}$ hydrogen signal in ${ }^{1} \mathrm{H}$ NMR. Increasing the equivalents of labelled water led to an increase of the exchange rate. Further experiments performed at higher temperatures are presented in the appendix (Figure C.4.

${ }^{\ddagger}$ The exchange determination via ${ }^{17} \mathrm{O}$ NMR was difficult and unprecise due to line broadening $(\approx$ $100 \mathrm{ppm}$ ) and an unfavorable signal-to-noise ratio. 


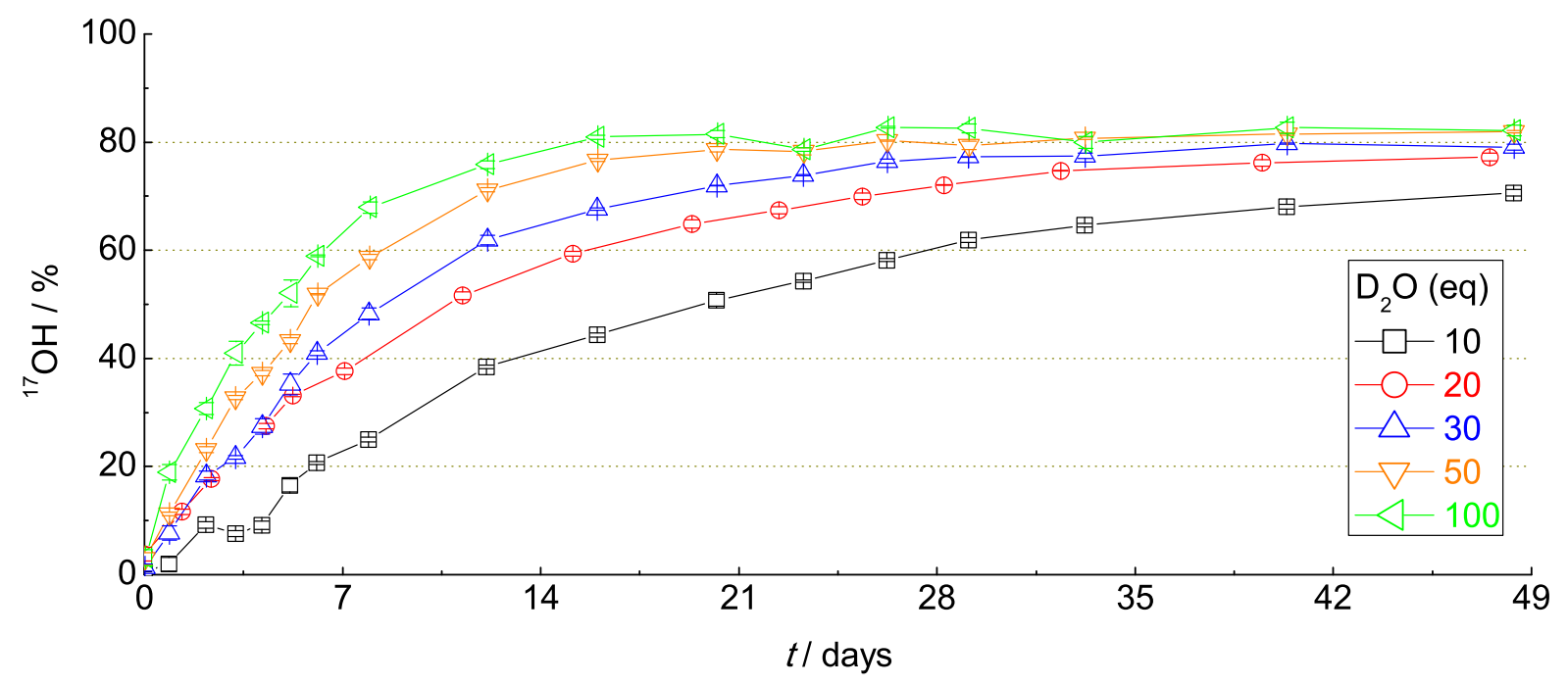

Figure 1.7.: Exchange of [ $\left.\mathrm{L}^{\text {pyr }} \mathrm{Ni}_{2}(\mu-\mathrm{OH})\right]$ with $\mathrm{H}_{2}{ }^{17} \mathrm{O}\left(10-100\right.$ eq.) in $\mathrm{THF}-\mathrm{D}_{8}$ at $30{ }^{\circ} \mathrm{C}$.

\subsection{Determination of Kinetic and Thermodynamic Parameters of the Exchange}

Considering the reaction $\mathrm{R}_{3} \mathrm{MX}+\mathrm{Y} \rightarrow \mathrm{R}_{3} \mathrm{MY}+\mathrm{X}$, substitution and exchange for squareplanar complexes is described by a rate law that consists of one first-order term in the metal complex MX and a second term, which is first-order both in MX and the substituting reagent $\mathrm{Y}$ :

$$
v=-\frac{\left[\mathrm{R}_{3} \mathrm{MX}\right]}{d t}=\left(k_{1}+k_{2}[\mathrm{Y}]\right)\left[\mathrm{R}_{3} \mathrm{MX}\right]
$$

Substitution or exchange reactions for square-planar complexes can take place via two different pathways (Scheme 1.3 and Scheme 1.4). As shown in Scheme 1.3 the overall reaction can follow either a dissociative or an associative substitution / exchange mechanism. Usually for square-planar complexes, exchange takes place via an associative mechanism where a higher ordered (five-coordinate) intermediate is formed. Hence the activation entropy is negative. On the other hand, entropy is positive for the dissociative mechanism due to a less ordered intermediate. 


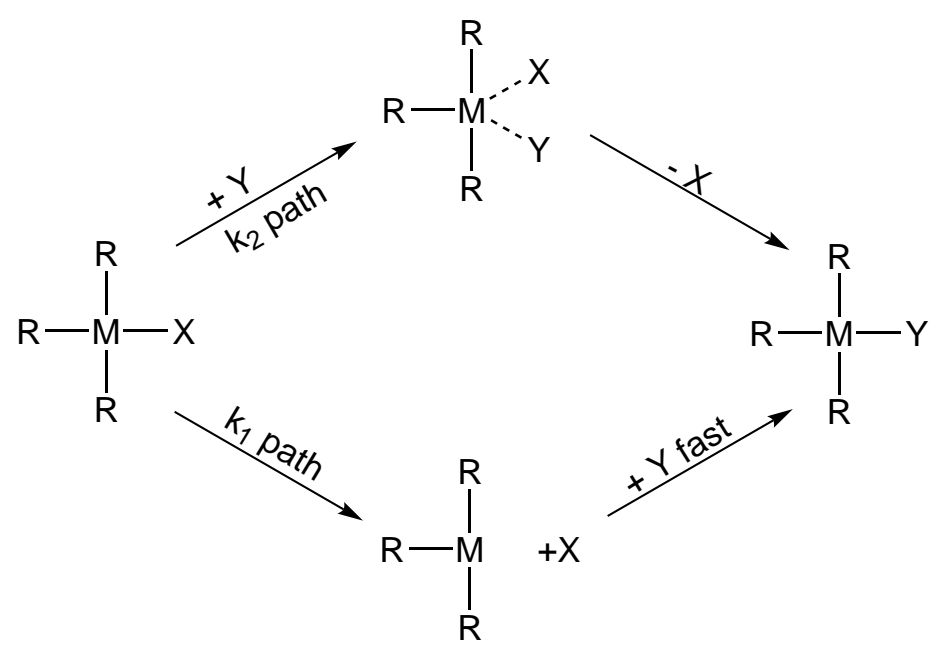

3-coordinated intermediate

Scheme 1.3: Parallel associative and dissociative mechanisms. ${ }^{[30]}$

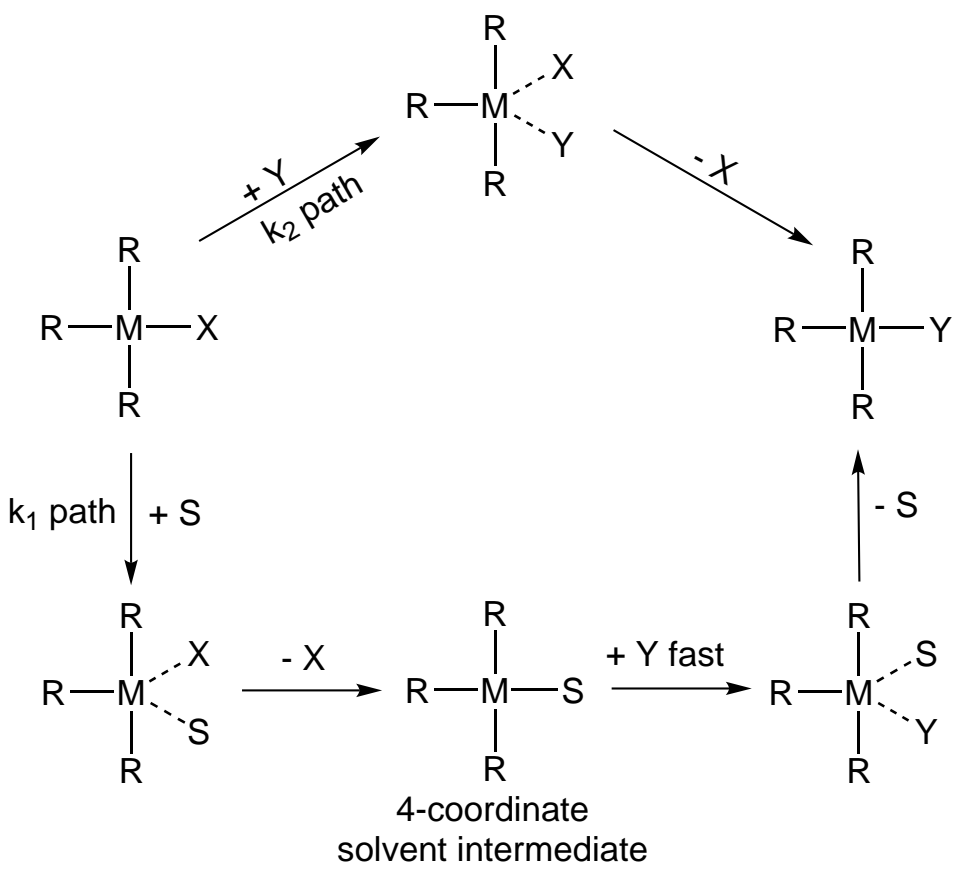

Scheme 1.4: Associative solvolysis and direct substitution. ${ }^{[30]}$

If the solvent is participating in the exchange reaction in an associative solvolysis as described in Scheme 1.4, a four-coordinate solvent intermediate will be formed. In the next step the solvent is substituted by the exchanging group. In this associative solvolysis, the $k_{1}$ term has the most pronounced impact since the rate determining step is coordination and substitution with the solvent molecule, while the last substitution step with the exchange reagent will be very fast in comparison.

If, like in this case, the rate of reaction is independent from the concentration of the 
exchanging / substituting reagent $\mathrm{Y}$, the $k_{2}$ term will be very small and therefore negligible, resulting in an overall first order rate behaviour (eq. 1.1). On the other hand, $k_{1}$ typically is very small for reactions in non-coordinating solvents and the rate of the exchange reaction is of second order overall. ${ }^{[30]}$

\subsubsection{Pseudo-First Order Reaction Conditions and Determination of $k$}

Usually substitution or exchange experiments are carried out using an excess (at least 10 equivalents with respect to the other reactant) of one reagent, which in this case is exchanging substance $Y$. The overall concentration of that reagent can then be considered constant over the whole reaction time. Hence the rate constant $k_{2}$ and the concentration [Y] (considered as constant) can be included in a new experimental rate constant $k$ which yields a new simplified rate law of pseudo-first order:

$$
v=-\frac{d\left[\mathrm{R}_{3} \mathrm{MX}\right]}{d t}=k\left[\mathrm{R}_{3} \mathrm{MX}\right]
$$

with

$$
k=k_{1}+k_{2}[\mathrm{Y}]
$$

By rearranging 1.1 the following equation will be obtained:

$$
\frac{d\left[\mathrm{R}_{3} \mathrm{MX}\right]}{\left[\mathrm{R}_{3} \mathrm{MX}\right]}=-k d t
$$

that can afterwards be integrated between $\left[\mathrm{R}_{3} \mathrm{MX}\right]_{0}$ and $\left[\mathrm{R}_{3} \mathrm{MX}\right]$ and $t=0$ and $t$ :

$$
\int_{\left[\mathrm{R}_{3} \mathrm{MX}\right]_{0}}^{\left[\mathrm{R}_{3} \mathrm{MX}\right]} \frac{d\left[\mathrm{R}_{3} \mathrm{MX}\right]}{\left[\mathrm{R}_{3} \mathrm{MX}\right]}=-k \int_{0}^{t} d t
$$

which gives:

$$
\ln \left[\mathrm{R}_{3} \mathrm{MX}\right]=\ln \left[\mathrm{R}_{3} \mathrm{MX}\right]_{0}-k t
$$


To determine this experimental rate constant the natural logarithmic plot of the decreasing complex concentration $\mathrm{c}\left(\left[\mathbf{L}^{\mathbf{p y r}} \mathbf{N i}_{\mathbf{2}}(\mu-\mathbf{O H})\right]\right)$ as a function of time was established. An expample describing the linear fits for different $\mathrm{D}_{2} \mathrm{O}$ concentrations at $30{ }^{\circ} \mathrm{C}$ is given in Figure 1.8. Further plots associated with measurements using $\mathrm{D}_{2} \mathrm{O}$ and $\mathrm{H}_{2}{ }^{17} \mathrm{O}$ in the temperature range $30-50^{\circ} \mathrm{C}$ are shown in the appendix (Figure C.5 and Figure C.6). The slope of the linear fit of the data yields the negative rate constant of pseudo-first order where $m=-k$. Limiting the selection of data points to the initial rate region minimizes the influence of side-reactions. ${ }^{[31]}$

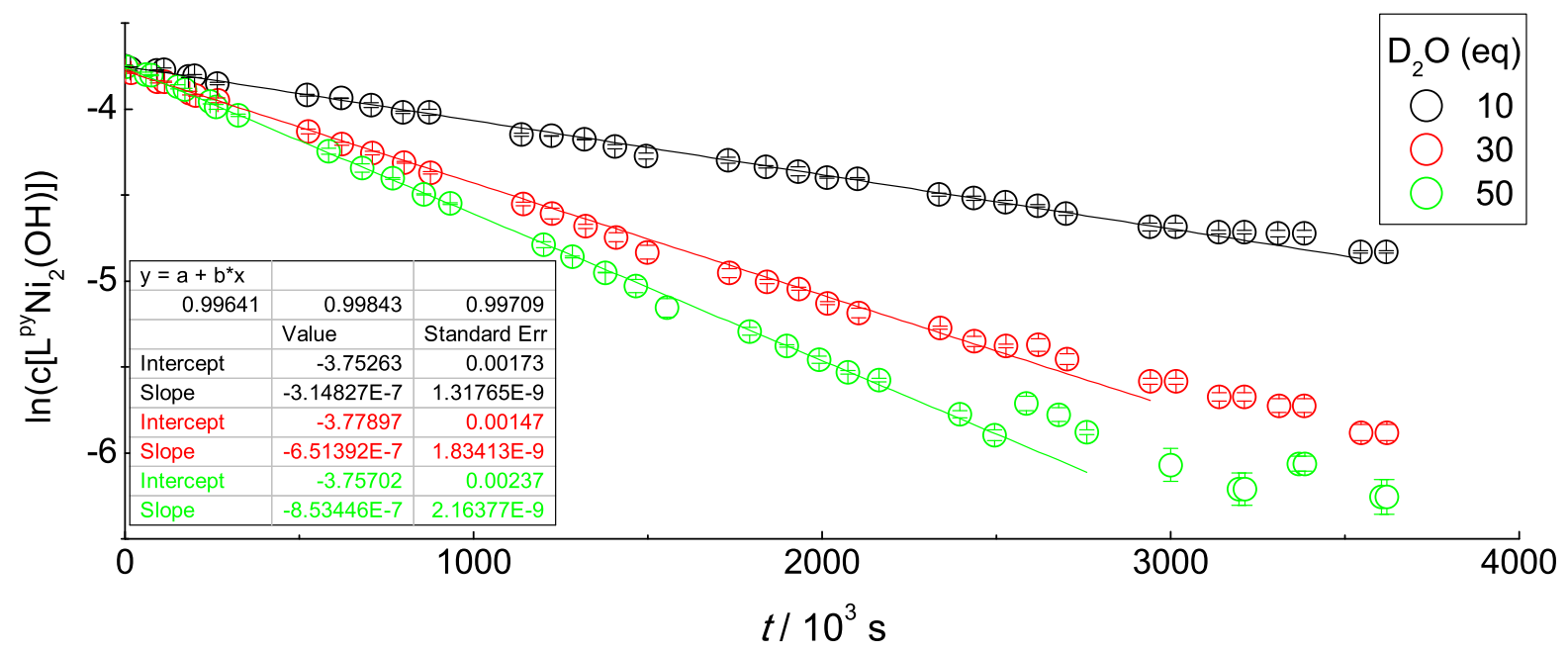

Figure 1.8.: Natural-log plot of the concentration of $\left[\mathrm{L}^{\mathrm{pyr}} \mathrm{Ni}_{2}(\mu-\mathrm{OH})\right]$ vs time for measurements with different equivalents of $\mathrm{D}_{2} \mathrm{O}$ performed at $30^{\circ} \mathrm{C}$. Line-fitting of initial data points is indicated. 


\subsubsection{Determination of the Rate Constants $k_{1}$ and $k_{2}$}

To determine the rate constant, a plot of the previously determined experimental rate constant $k$ as a function of excess concentration of the exchanging reagent $\left(\mathrm{D}_{2} \mathrm{O}, \mathrm{H}_{2}{ }^{17} \mathrm{O}\right)$ was established. An example of the exchange experiment with $\mathrm{D}_{2} \mathrm{O}$ performed at $40{ }^{\circ} \mathrm{C}$ is illustrated in Figure 1.9. Further results are depicted in the appendix (C.2). The linear plots confirm the dependence of reaction rate on water concentration. According to Equation 1.3 the slope of the linear fit yields $k_{2}$ and the intercept gives $k_{1}$.

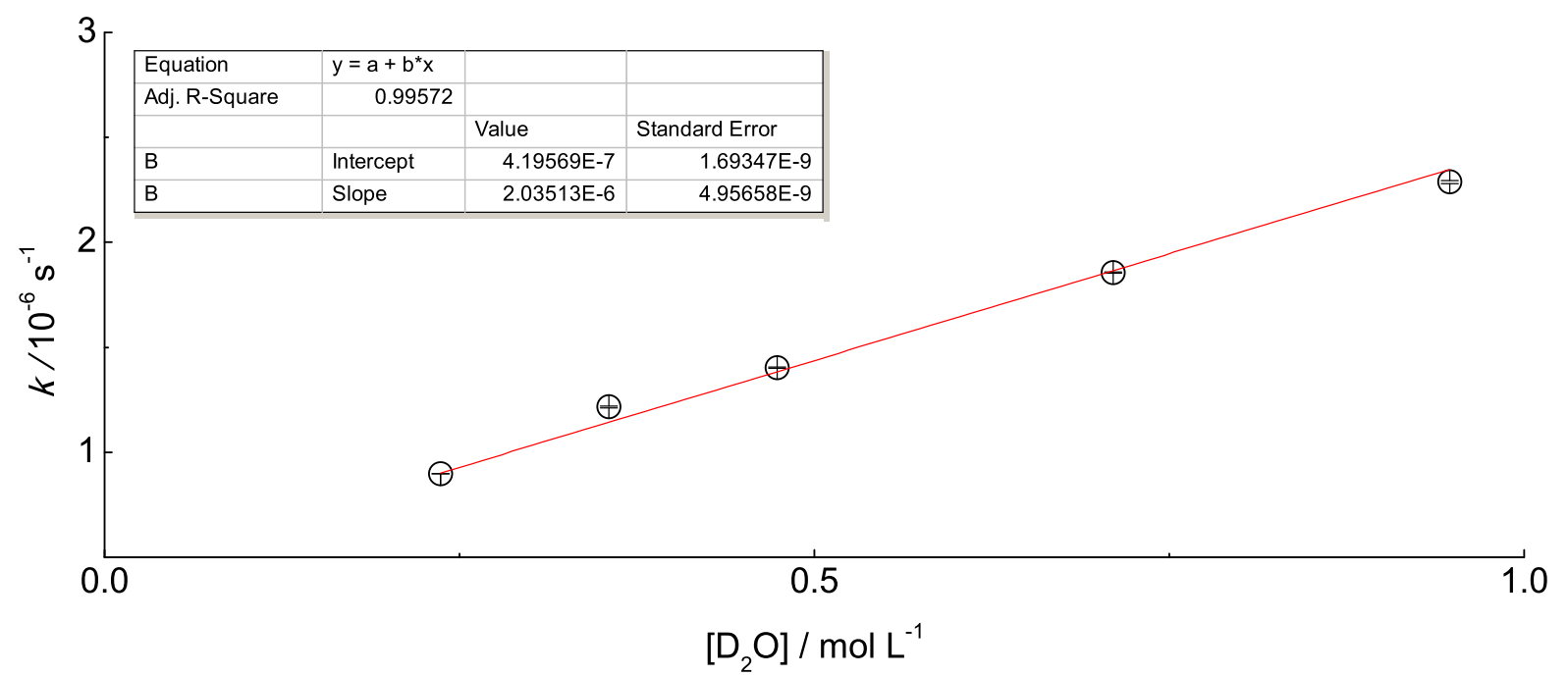

Figure 1.9.: Plot of experimental rate constant $k$ versus concentration of $\mathrm{D}_{2} \mathrm{O}$ at $40{ }^{\circ} \mathrm{C}$. The linear fit is indicated.

The obtained rate constants $k_{1}$ and $k_{2}$ for the exchange reactions with $\mathrm{D}_{2} \mathrm{O}$ and $\mathrm{H}_{2}{ }^{17} \mathrm{O}$ performed at different temperatures $\left(30-60{ }^{\circ} \mathrm{C}\right)$ are summarized in Table 1.2.

Table 1.2.: Graphically determined values for the rate constants $k_{1}$ and $k_{2}$ for the exchange of [ $\mathrm{L}^{\text {pyr }} \mathrm{Ni}_{2}(\mu-\mathrm{OH})$ ] with $\mathrm{D}_{2} \mathrm{O}$ and $\mathrm{H}_{2}{ }^{17} \mathrm{O}$ at different temperatures.

\begin{tabular}{cccccc}
\hline & \multicolumn{2}{c}{$\mathrm{H} / \mathrm{D}$ exchange with $\mathrm{D}_{2} \mathrm{O}$} & & \multicolumn{2}{c}{$\mathrm{OH}$ exchange with $\mathrm{H}_{2}{ }^{17} \mathrm{O}$} \\
\cline { 2 - 3 } \cline { 5 - 6 } $\mathrm{T} /{ }^{\circ} \mathrm{C}$ & $k_{1}{ }^{[\mathrm{a}]} / 10^{-7} \mathrm{~s}^{-1}$ & $k_{2}{ }^{[\mathrm{a}]} / 10^{-7} \mathrm{~L} \mathrm{~mol}^{-1} \mathrm{~s}^{-1}$ & & $k_{1}{ }^{[\mathrm{a}]} / 10^{-7} \mathrm{~s}^{-1}$ & $k_{2}{ }^{[\mathrm{a}]} / 10^{-7} \mathrm{~L} \mathrm{~mol}^{-1} \mathrm{~s}^{-1}$ \\
\hline 30 & $1.87 \pm 0.02$ & $5.90 \pm 0.03$ & & $1.68 \pm 0.03$ & $9.73 \pm 0.08$ \\
40 & $4.20 \pm 0.02$ & $20.35 \pm 0.05$ & & $4.67 \pm 0.08$ & $22.3 \pm 0.2$ \\
50 & $8.0 \pm 0.2$ & $64.4 \pm 0.4$ & & $8.5 \pm 0.4$ & $85.3 \pm 0.8$ \\
60 & $30 \pm 1$ & $134 \pm 1$ & & $96 \pm 30$ & $222 \pm 55$ \\
\hline
\end{tabular}

${ }^{[a]}$ Confidence intervals from graphical determination.

The values of $k_{1}$ and $k_{2}$ exhibit a larger relative error at higher temperatures, which is due to a smaller initial rate region with fewer data points. Nevertheless, a comparison of $k_{1}$ and $k_{2}$ shows that the values of $k_{1}$ are much smaller than for $k_{2}$. This is consistent 
with the dependence of exchange on the $\mathrm{D}_{2} \mathrm{O}$ and $\mathrm{H}_{2}{ }^{17} \mathrm{O}$ concentration. Although it is difficult to determine accurate values for $k_{1}$ using a graphical method, it is assumed that participation of the solvent THF- $\mathrm{D}_{8}$ in the exchange is marginal or does not even occur. If a solvolytic mechanism can be excluded, the first order term $k_{1}$ becomes small and can be ignored in the rate law (1.1) leading to:

$$
v=-\frac{\left[\mathrm{R}_{3} \mathrm{MX}\right]}{d t}=k_{2}[\mathrm{Y}]\left[\mathrm{R}_{3} \mathrm{MX}\right]
$$

The exchange reaction would then be first order in both $[\mathrm{Y}]$ and $\left[\mathrm{R}_{3} \mathrm{MX}\right]$ and second order overall.

\subsubsection{Determination of Thermodynamic Parameters}

With the obtained rate constants (Table 1.2), thermodynamic parameters were determined. The activation energy $E_{A}$ can be obtained graphically by use of the Arrhenius equation:

$$
\ln (k)=-\frac{E_{A}}{R T}+\ln (A)
$$

A natural-log plot of $\ln k_{2}$ against the reciprocal temperature yields the negative activation energy divided by the gas constant $R$ as slope of the linear fit with the pre-exponential factor $\mathrm{A}$ as the intercept.

The Eyring Equation [1.9 can be used to determine the activation enthalpy $\Delta H^{\ddagger}$ and activation entropy $\Delta S^{\ddagger}$ :

$$
\ln \left(\frac{k}{T}\right)=-\frac{\Delta H^{\ddagger}}{R T}+\ln \left(\frac{k_{B}}{h}\right)+\frac{\Delta S^{\ddagger}}{R} .
$$

A linear fit of $\ln \left(\frac{k_{2}}{T}\right)$ plotted against the reciprocal temperature yields the activation enthalpy $\Delta H^{\ddagger}$ determined from the slope of the fit $\left(-\frac{\Delta H^{\ddagger}}{R}\right)$. The intercept of the linear fit equals $\ln \left(\frac{k_{B}}{h}\right)+\frac{\Delta S^{\ddagger}}{R}$, which yields the activation entropy $\Delta S^{\ddagger}$. Furthermore, the plots for exchange with $\mathrm{D}_{2} \mathrm{O}$ and $\mathrm{H}_{2}{ }^{17} \mathrm{O}$ are nearly identical, indicating exclusive hydroxide exchange.

Thermodynamic parameters are summarized in Table 1.3. It can be seen that the activation entropy is strongly negative for both $\mathrm{H} / \mathrm{D}$ and $\mathrm{OH}$ exchange. This indicates that the exchange takes place via an associative mechanism, which is common for a low-spin $\mathrm{d}^{8}$ complex. ${ }^{[30]}$ In addition the activation energy $E_{A}$ and the activation enthalpy $\Delta H^{\ddagger}$ are quite high due to the fact that the exchange is very slow in both cases. 

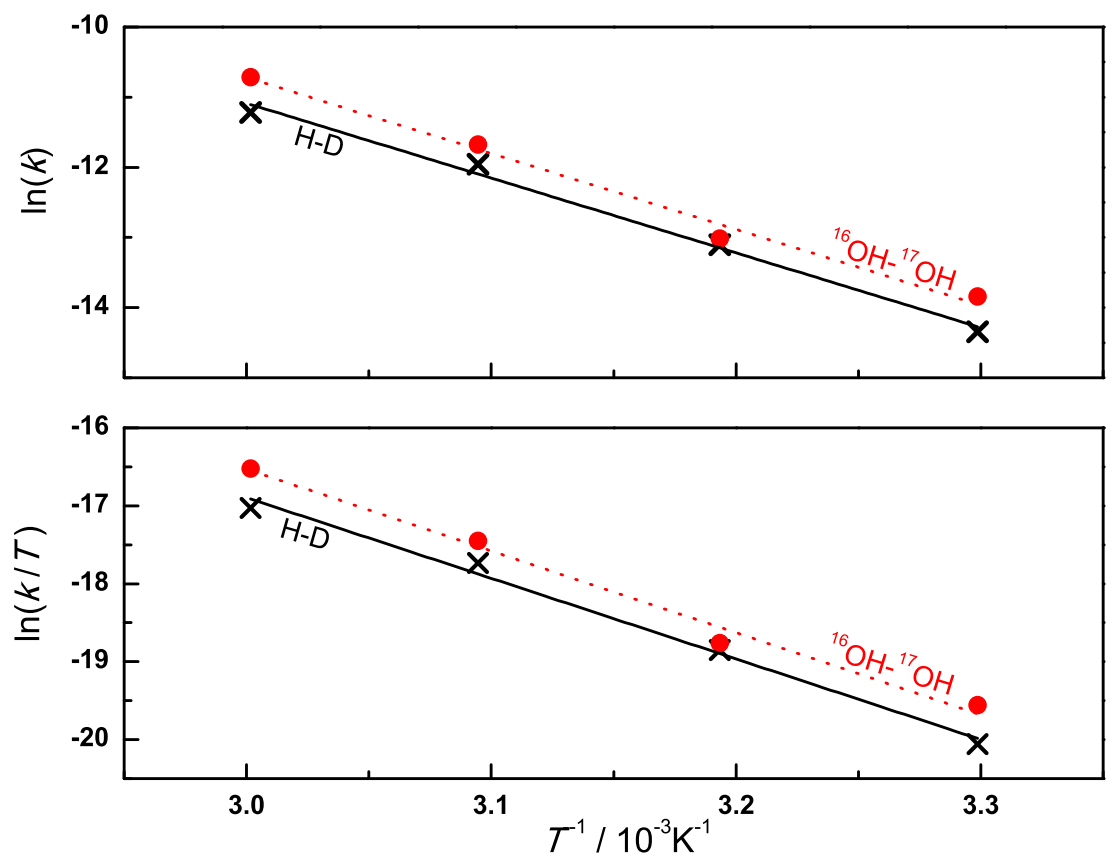

Figure 1.10.: Arrhenius plot (top) and Eyring plot (bottom) for the $\mathrm{H} / \mathrm{D}$ exchange (-) and $\mathrm{OH}$ exchange $(\cdots)$ for $\left[\mathrm{L}^{\mathrm{pyr}} \mathrm{Ni}_{2}(\mu-\mathrm{OH})\right]$.

Table 1.3.: Graphically determined values of the thermodynamic parameters $E_{A}, \Delta H^{\ddagger}, \Delta S^{\ddagger}$ and the calculated value of $\Delta G^{\ddagger} 298 \mathrm{~K}$ for the exchange of [ $\left.\mathrm{L}^{\text {pyr }} \mathrm{Ni}_{2}(\mu-\mathrm{OH})\right]$ with $\mathrm{D}_{2} \mathrm{O}$ and $\mathrm{H}_{2}{ }^{17} \mathrm{O}$.

\begin{tabular}{ccccc}
\hline used water & $E_{A}^{[\mathrm{a}]} / \mathrm{kJ} \mathrm{mol}^{-1}$ & $\Delta H^{\ddagger[\mathrm{a}]} / \mathrm{kJ} \mathrm{mol}^{-1}$ & $\Delta S^{\ddagger[\mathrm{a}]} / \mathrm{J} \mathrm{mol}^{-1} \mathrm{~K}^{-1}$ & $\Delta G^{\ddagger} 298 \mathrm{~K}^{[\mathrm{a}]} / \mathrm{kJ} \mathrm{mol}^{-1}$ \\
\hline $\mathrm{D}_{2} \mathrm{O}$ & $89 \pm 5$ & $86 \pm 5$ & $-80 \pm 17$ & $110 \pm 5$ \\
$\mathrm{H}_{2}{ }^{17} \mathrm{O}$ & $90 \pm 6$ & $88 \pm 6$ & $-74 \pm 20$ & $109 \pm 6$ \\
\hline
\end{tabular}

${ }^{[a]}$ Confidence intervals from standard propagation of errors. 


\subsubsection{DFT Calculations and Mechanistic Considerations}

To elucidate the mechanism, extensive DFT calculations were performed in the group of Prof. MATA. The structures shown in Figure 1.11 are based on the theoretical calculations and illustrate the first step of the exchange process and formation of an intermediate.
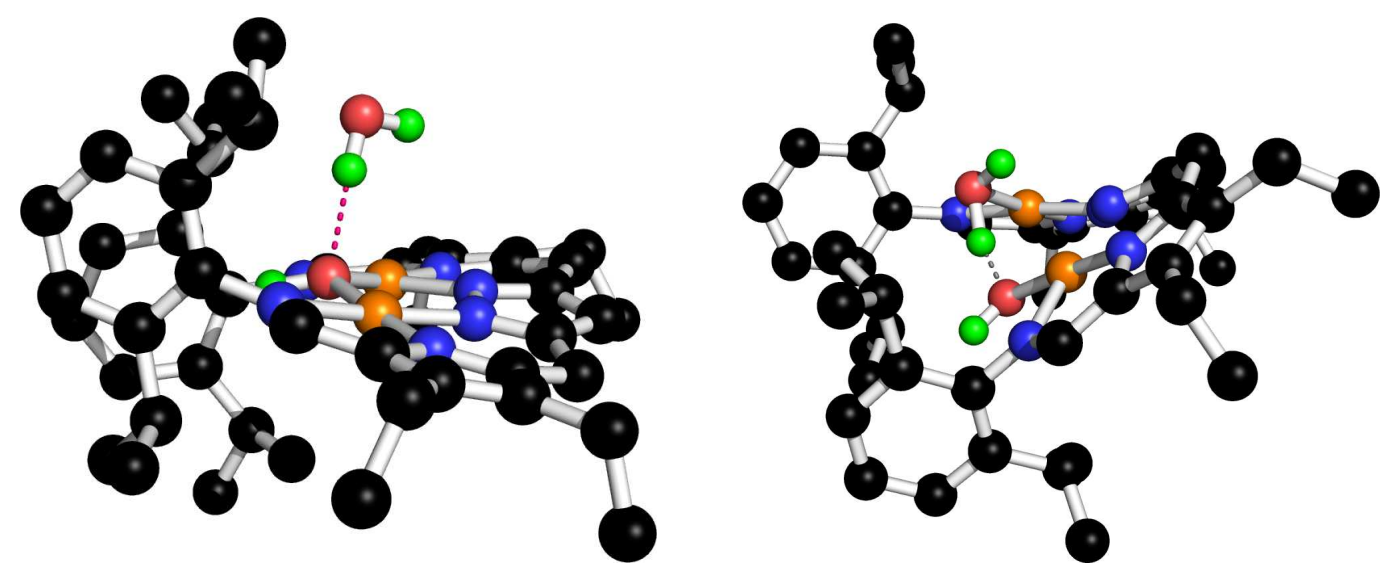

Figure 1.11.: Possible approach of the water molecule (left) and the proposed structure of the intermediate $\left[\mathrm{L}^{\mathrm{pyr}} \mathrm{Ni}_{2}\left(\mu-\mathrm{O}_{2} \mathrm{H}_{3}\right)\right]$ (right).

The water molecule approaches the complex directly at the bridging hydroxyl group leading to formation of a bridging hydrogen bond with a water hydrogen and the hydroxyl oxygen. In the next step the water molecule coordinates to one of the nickel ions and binds to the $\mathrm{OH}$ group, which leads to the intermediate $\left[\mathbf{L}^{\mathrm{pyr}} \mathbf{N i}_{\mathbf{2}}\left(\mu-\mathrm{O}_{\mathbf{2}} \mathbf{H}_{\mathbf{3}}\right)\right]$. This type of structure was also reported for a TACN (triazacyclononane)-pyrazolate-based dinuclear nickel(II) complex featuring a bridging $\left[\mathrm{H}_{3} \mathrm{O}_{2}\right]^{-}$unit. ${ }^{[32]}$ However, in this case the complex was stable due to a substantially larger $\mathrm{Ni}$. . Ni distance $(4.475 \AA)$ in comparison to $\left[\mathbf{L}^{\mathrm{pyr}} \mathrm{Ni}_{2}(\mu-\mathrm{OH})\right](3.247 \AA)$.

The associative exchange mechanism can be described according to Scheme 1.5. In the symmetric $\left[\mathbf{L}^{\text {pyr }} \mathbf{N i}_{\mathbf{2}}\left(\mu-\mathrm{O}_{\mathbf{2}} \mathbf{H}_{\mathbf{3}}\right)\right]$ the bridging ligand comprises a hydrogen atom stemming from the water molecule which lies between the oxygen atoms from the $\mathrm{OH}$ group and the water molecule. The oxygen atoms are each coordinated to one of the $\mathrm{Ni}^{\mathrm{II}}$ cations. According to computational results a large energetic barrier has to be overcome to form the intermediate (Figure 1.12). The energy of the intermediate state was calculated as $\Delta \mathrm{G}=40.79 \mathrm{~kJ} \mathrm{~mol}{ }^{-1}$. Therefore, the formation of $\left[\mathbf{L}^{\mathbf{p y r}} \mathbf{N i}_{\mathbf{2}}\left(\mu-\mathbf{O}_{\mathbf{2}} \mathbf{H}_{\mathbf{3}}\right)\right]$ is very slow compared to water elimination and is thus rate determining.

After formation of intermediate $\left[\mathbf{L}^{\mathrm{pyr}} \mathrm{Ni}_{2}\left(\mu-\mathrm{O}_{2} \mathbf{H}_{3}\right)\right]$, exchange of the former hydroxo-ligand can either proceed or revert to the original reactant. Both directions are energetically favorable, however the release of water is much faster than coordination (Figure 1.12. Small resonances at -6.55 and $-6.63 \mathrm{ppm}$ in ${ }^{1} \mathrm{H}$ NMR spectra (Figure 1.6 may be correlated to the intermediate $\left[\mathrm{L}^{\mathrm{pyr}} \mathrm{Ni}_{2}\left(\mu-\mathrm{O}_{2} \mathbf{H}_{3}\right)\right]$. 


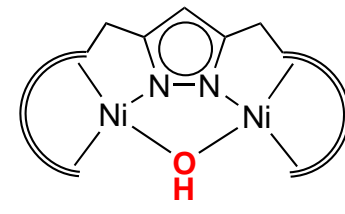

$$
\begin{gathered}
\begin{array}{c}
+\mathrm{H}_{2} \mathrm{O} \\
k_{2}
\end{array} \\
\hline \begin{array}{c}
k_{-2} \\
-\mathrm{H}_{2} \mathrm{O}
\end{array} \\
\text { slow }
\end{gathered}
$$

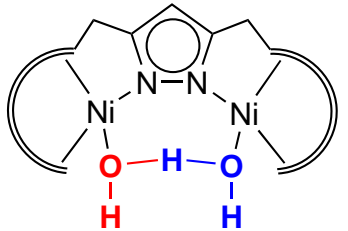

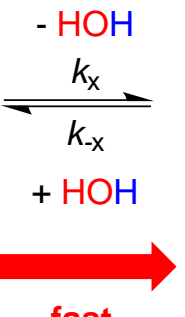

Scheme 1.5: Proposed exchange mechanism for [ $\left.\mathrm{L}^{\mathrm{pyr}} \mathrm{Ni}_{2}(\mu-\mathrm{OH})\right]$ with water. After overcoming an energetic barrier, the intermediate [ $\left.\mathrm{L}^{\mathrm{pyr}} \mathrm{Ni}_{2}(\mu-\mathrm{HOHOH})\right]$ is formed. This step is rate-deterimining and leads to a pre-equilibrium. Subsqeuently, the exchange of the entire hydroxo-group can occur giving ${ }^{\left[{ }^{p y r}\right.} \mathrm{Ni}_{2}(\mu-$ $\mathrm{OH})]$ and $\mathrm{HOH}$.

Moreover, calculations showed that exchange leads to the abstraction of the bridging hydrogen by the former hydroxyl group (Scheme 1.5). The formed water molecule is afterwards expelled from the complex. This is consistent with the experimental outcome, which also showed substitution of the entire hydroxo group.

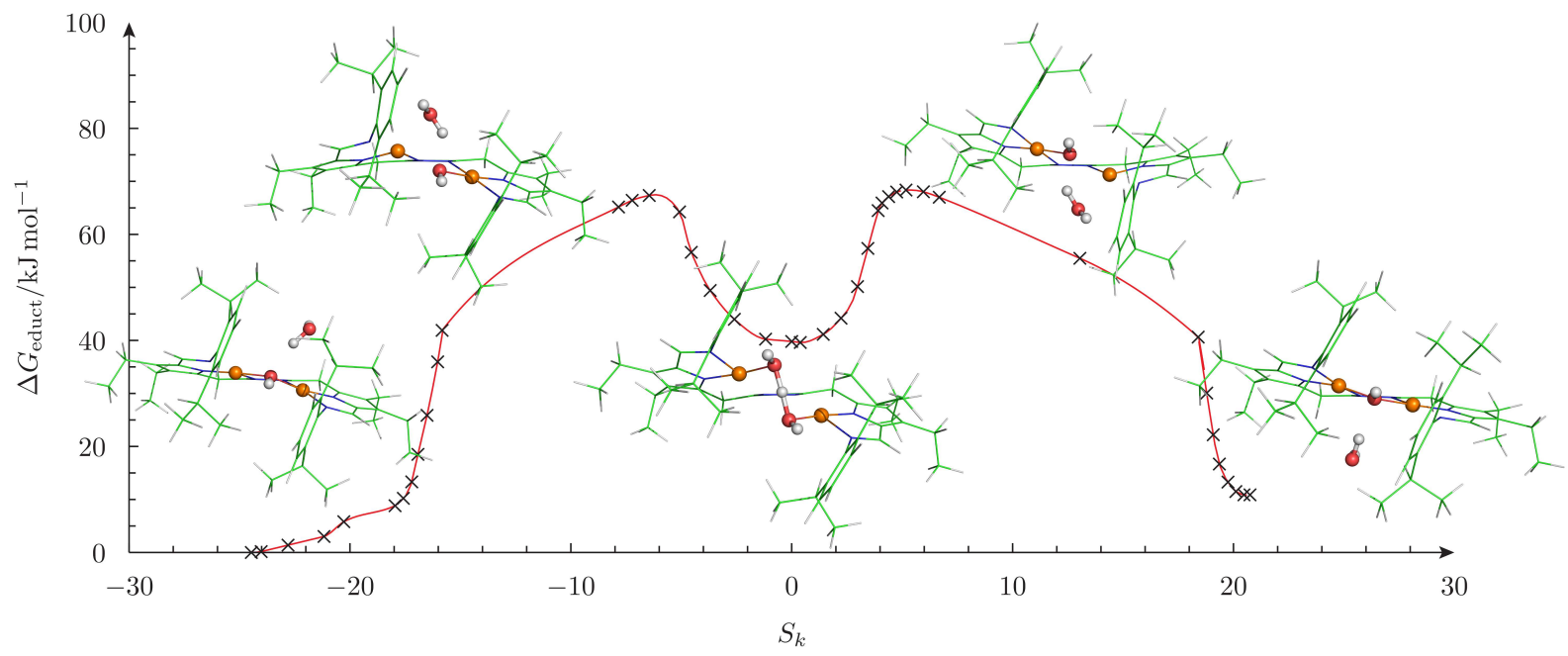

Figure 1.12.: The energy profile for the hydroxo-exchange of $\left[\mathrm{L}^{\text {pyr }} \mathrm{Ni}_{2}(\mu-\mathrm{OH})\right]$ with water based on DFT calculations (BP86) of the MATA group. 


\subsection{Summary}

The unusually slow exchange of $\left[\mathbf{L}^{\mathbf{p y r}} \mathbf{N i}_{\mathbf{2}}(\mu-\mathbf{O H})\right]$ with water was studied in depth by means of isotopic labeling and monitoring by NMR techniques. Thermodynamic and kinetic parameters for the exchange were determined from experimental data, indicating an associative exchange mechanism. The rate of the reaction is dependent on the concentration of water and is thus considered to be of second order. Furthermore, it was found that exchange involves substitution of the entire $\mu-\mathrm{OH}$ ligand of $\left[\mathbf{L}^{\mathbf{p y r}} \mathbf{N i}_{\mathbf{2}}(\mu-\mathrm{OH})\right]$ with a hydroxide originating from a water molecule. Based on two different theoretical calculation methods, which support the experimentally derived values (Table 1.4), an energy profile for the exchange was established (Figure 1.12).

Table 1.4.: Experimental and calculated values of the thermodynamic parameters $E_{A}, \Delta H^{\ddagger}, \Delta S^{\ddagger}$ and $\Delta G^{\ddagger} 303 \mathrm{~K}$ for the exchange of [ $\left.\mathrm{L}^{\mathrm{pyr}} \mathrm{Ni}_{2}(\mu-\mathrm{OH})\right]$ with water $\left(\mathrm{D}_{2} \mathrm{O}\right.$ and $\left.\mathrm{H}_{2}{ }^{17} \mathrm{O}\right)$.

\begin{tabular}{ccccc}
\hline used water & $E_{A}^{[\mathrm{a}]} / \mathrm{kJ} \mathrm{mol}^{-1}$ & $\Delta H^{\ddagger[\mathrm{a}]} / \mathrm{kJ} \mathrm{mol}^{-1}$ & $\Delta S^{\ddagger[\mathrm{a}]} / \mathrm{J} \mathrm{mol}^{-1} \mathrm{~K}^{-1}$ & $\Delta G^{\ddagger} 303 \mathrm{~K}^{[\mathrm{a}]} / \mathrm{kJ} \mathrm{mol}^{-1}$ \\
\hline $\mathrm{D}_{2} \mathrm{O}$ & $89 \pm 5$ & $86 \pm 5$ & $-80 \pm 17$ & $110 \pm 5$ \\
$\mathrm{H}_{2}{ }^{17} \mathrm{O}$ & $90 \pm 6$ & $88 \pm 6$ & $-74 \pm 20$ & $109 \pm 6$ \\
$\mathrm{H}_{2} \mathrm{O}^{[\mathrm{b}][f]}$ & - & $84^{[\mathrm{d}]} / 86^{[\mathrm{e}]}$ & - & $91^{[\mathrm{d}]} / 92^{[\mathrm{e}]}$ \\
$\mathrm{H}_{2} \mathrm{O}^{[\mathrm{c}][\mathrm{f}]}$ & - & $91^{[\mathrm{d}]} / 92^{[\mathrm{e}]}$ & - & $99^{[\mathrm{d}]} / 102^{[\mathrm{e}]}$ \\
\hline
\end{tabular}

${ }^{[a]}$ Confidence intervals from standard propagation of errors.

${ }^{[b]}$ Calculated values (B3LYP-D3).

${ }^{[c]}$ Calculated values (LMP2).

${ }^{[d]}$ Barrier for the left hand-side energyprofile.

${ }^{[e]}$ Barrier for the right hand-side energyprofile.

${ }^{[f}$ All values were computed with the def2-TZVP basis set, based on BP86/def2-SVP optimized geometries. 



\section{Part II.}

\section{Complexes of a Versatile}

Pyrazolate-based Bis( $\beta$-diketiminate)

Ligand and Their Applications 



\section{A Bimetallic Nickel(II) Complex of a Pyrazolate-linked "Nacnac" Ligand as a Versatile Scaffold}
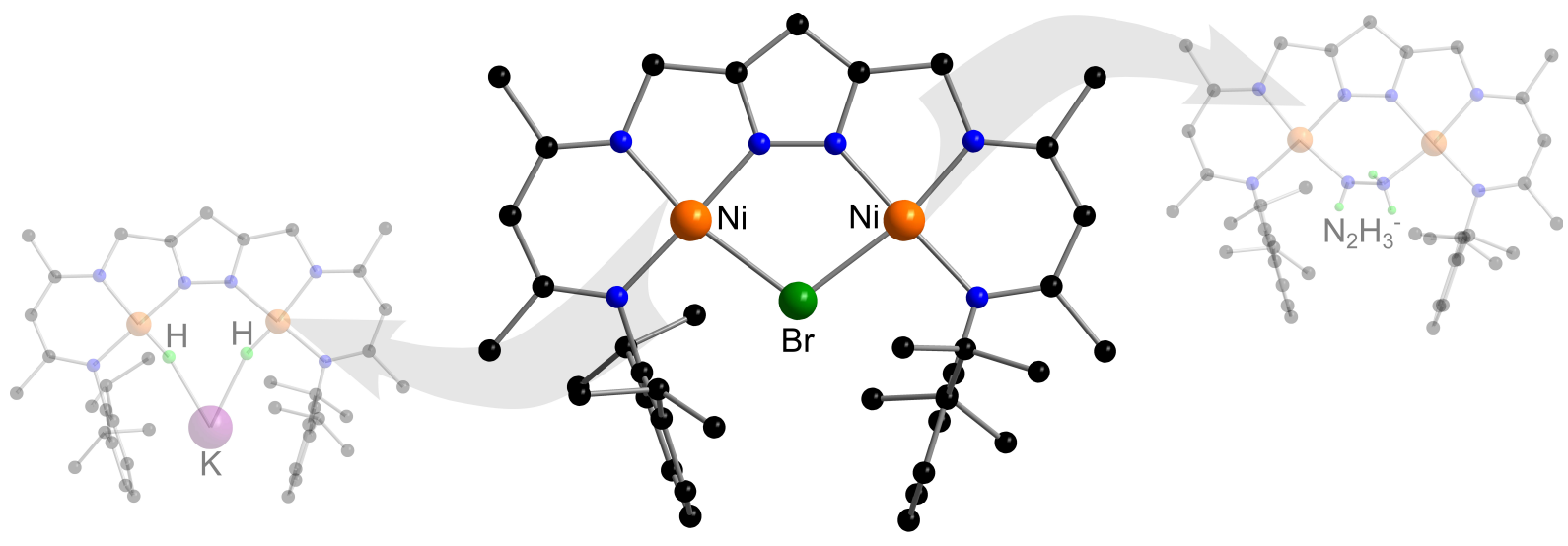

Abstract During the last decades, $\beta$-diketiminates, also referred to as "nacnac" ligands, have raised substantial interest among chemists due to their simple preparation and electronic and steric tunability. Therefore, the combination of two of these versatile moieties with pyrazolate as a linker unit was envisioned to furnish a ligand capable of preorganization upon metal complexation. This chapter deals with the preparation and characterization of the trianionic, hexadentate ligand system and the establishment of a dinuclear nickel complex as the foundation for further promising chemistry. 


\subsection{Introduction}

$N$-substituted $\beta$-diketiminato ligands, which are also known as "nacnac", are attracting more and more attention due to their monoanionic, chelating nature and their tunable steric and electronic properties. ${ }^{[33-\underline{36}]}$ They are capable of stabilizing low valent metal centers in particular. ${ }^{[33]}$ Until now, alkali and earth alkali, ${ }^{[37-43]}$ main-group, $\underline{\underline{[44}-\underline{48}]}$ transition-metal, $\stackrel{[33,49-59]}{=}$ lanthanide, ${ }^{[60]}$ and actinide ${ }^{[61,62]}$ complexes are reported, which emphazises the widespread utility of this versatile ligand.

Compared to their oxygen analogues, which are the $\beta$-diketonato ligands $\left[\mathrm{RC}\left(\mathrm{C}\left\{\mathrm{R}^{\prime}\right\} \mathrm{O}\right)_{2}\right]^{-}$, nacnac ligands $\left[\mathrm{RC}\left(\mathrm{C}\left\{\mathrm{R}^{\prime}\right\} \mathrm{NR}^{\prime \prime}\right)_{2}\right]^{-}$provide the advantage of further substitutions at the $N$-donor function (e.g. alkyl, silyl or aryl), allowing for steric protection of a chelated metal ion (Scheme 2.1) . $^{[33,35]}$<smiles></smiles><smiles>[R]c1c([R])[n+]([PH])nn1[PH]</smiles>

Scheme 2.1: A $\beta$-diketonate- and a $\beta$-diketiminate chelate.

This ligand has primarily been used to establish mononuclear complexes. Some of these complexes tend to form dimers with small molecules as metal bridging units, showing quite interesting properties in regards to potential small molecule activation. ${ }^{[54,55,59,63-68]}$ Studies including nickel complexes of $\beta$-diketiminato ligands have been reported by the

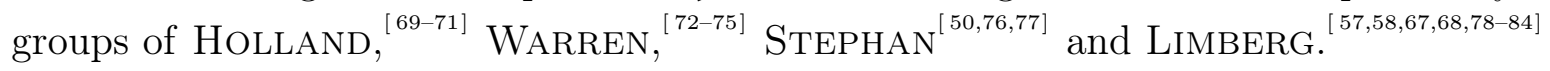

Scheme 2.2 shows an example of $\mathrm{N}_{2}$ activation based on the work of the LIMBERG group. $\stackrel{[67]}{\underline{[}}$ In this case a dimer formed under reducing conditions $\left(\mathrm{KC}_{8}\right)$ and a dinitrogen atmosphere. The dimer features $\mathrm{Ni}^{\mathrm{I}}$ metal centers and a bridged $\mathrm{N}_{2}$ in an activated state. Potassium is coordinated to this bridging unit and two of the aryl sidearms as well, caused by a cation- $\pi$-interaction. Addition of another equivalent of $\mathrm{KC}_{8}$ resulted in reduction of the $\mathrm{N}_{2}$ triple bond to a double bond.

Attempts to attach two nacnac ligands to a linking unit, yielding bis( $\beta$-diketiminato) systems are so far rarely reported in literature. ${ }^{\left[\frac{[39.85 .87]}{}\right.}$ Nonetheless, interest in these ligands appears to be constantly growing since they provide the opportunity to form preorganized dinuclear complexes with potential metal ion cooperativity. In the year 2005 HuLTzSCH et al. established several linked bis( $\beta$-diketiminato) ligands for a more effective chelation of selected metal ions, yielding complexes of higher stability (Scheme 2.3). ${ }^{[.85]}$ The use of chiral linkers, such as the cyclohexyl moiety (Scheme 2.3) provided the possibility of stereoselective control in further reactions. 


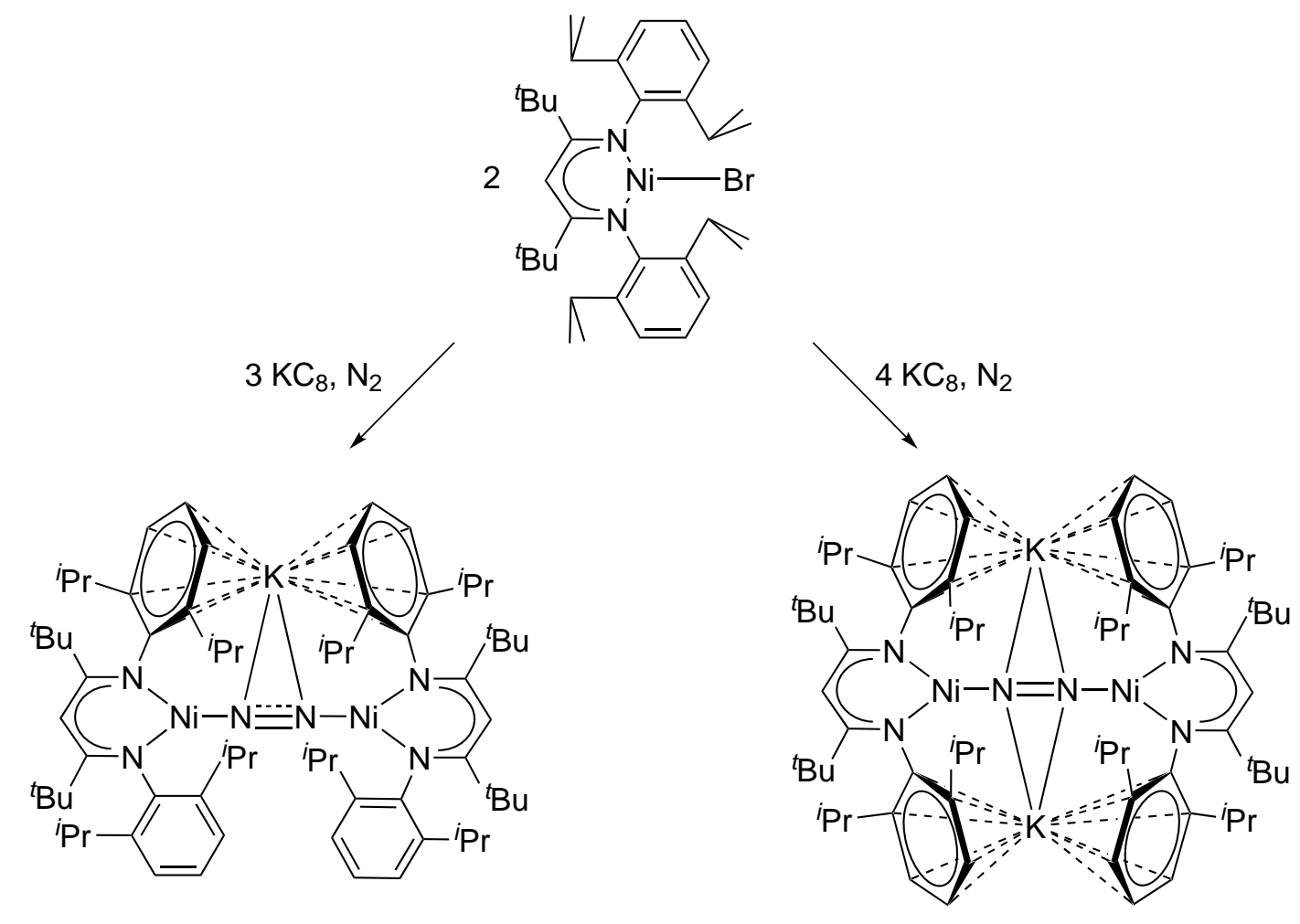

Scheme 2.2: Formation of a bridged dimer with $\mathrm{KC}_{8}$ under a nitrogen atmosphere.$^{\left[\frac{[67]}{6}\right.}$
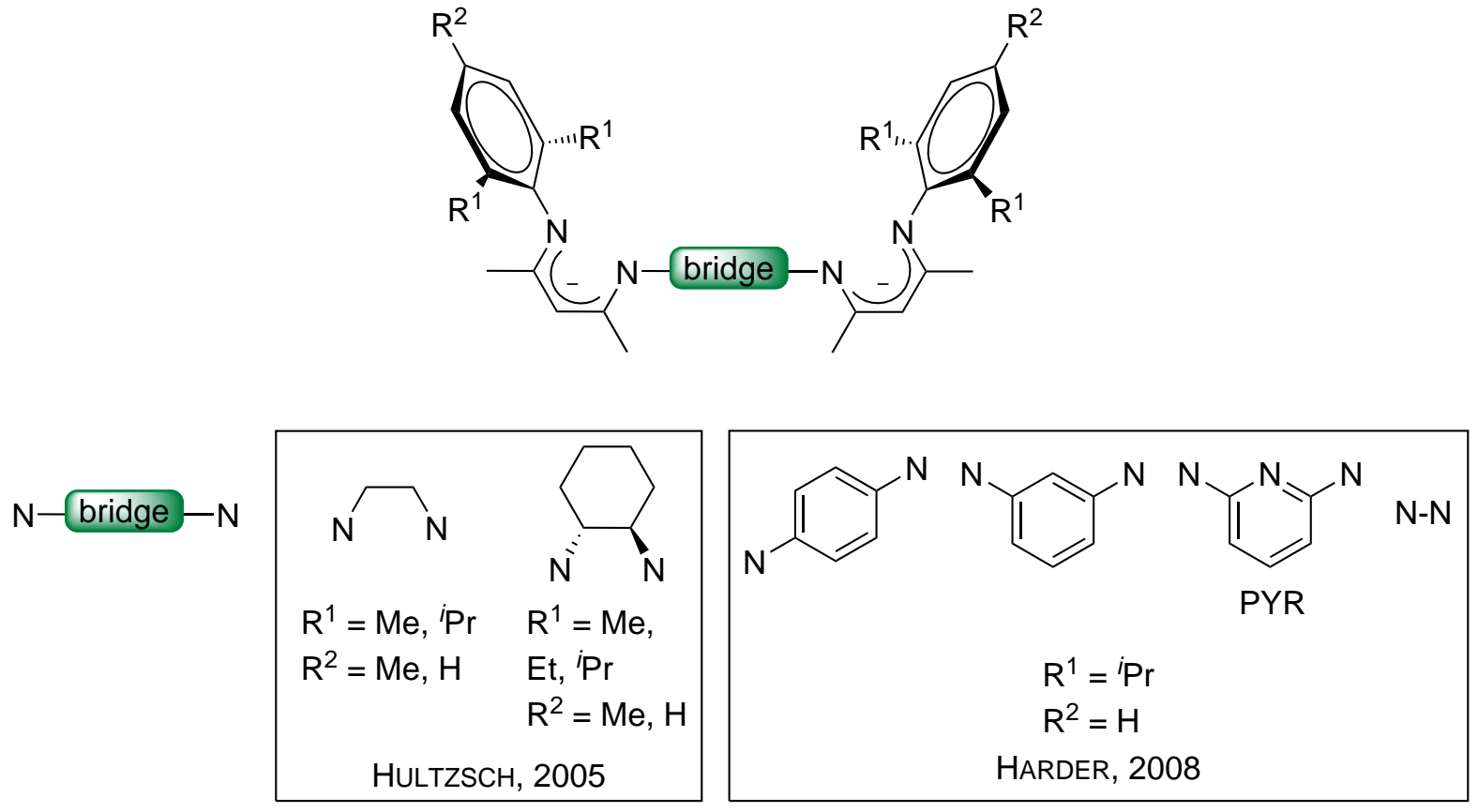

Scheme 2.3: Linked bis( $\beta$-diketiminato) ligand systems. ${ }^{[39,85,87]}$ 
Further work by HARDER et al. was reported in 2008, presenting linked sytems containing bulky DIPP (diisopropylphenyl) moieties. ${ }^{[39.87]}$ The established systems initially gave rise to dinuclear zinc complexes, which have been shown to catalyze the copolymerization of cyclohexyl oxide and $\mathrm{CO}_{2} \cdot{ }^{[39]}$ In the following years these ligand systems were also used

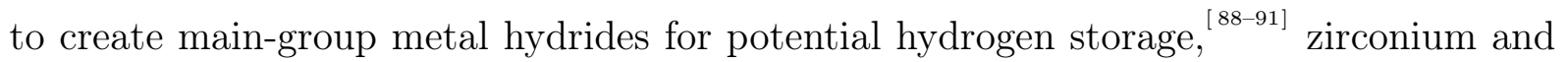
hafnium-based ethylene polymerization catalysts, ${ }^{[92]}$ and dinuclear lanthanide complexes as potential pre-catalysts in isoprene polymerization. ${ }^{[93-95]}$

These recent results, once more, show that the $\beta$-diketiminato systems are highly capable of metal center stabilization covering a large range of the periodic table. Further stabilization can be achieved by using linker units which contain donor functions like nitrogen. For instance, the pentadentate ligand system PYR (Scheme 2.3) comprises a pyridine linker unit. PYR received special interest in recent studies. ${ }^{[\underline{[9-98}]}$

This ligand system was used by LIMBERG and co-workers to establish a dinuclear pyridine-bridged bis( $\beta$-diketiminato) nickel hydride complex (Scheme 2.4) ${ }^{\left[{ }^{[98]}\right.}$ Starting from a bromide-bridged dinuclear $\mathrm{Ni}^{\mathrm{II}}$ compound, treatment with $\mathrm{KHBEt}_{3}$ eventually resulted in formation of a mixed valent $\mathrm{Ni}^{\mathrm{I}}-\mathrm{Ni}^{\mathrm{II}}$ complex featuring a hydride in the bridging position. The system was then studied in the context of reactivity and redox chemistry.
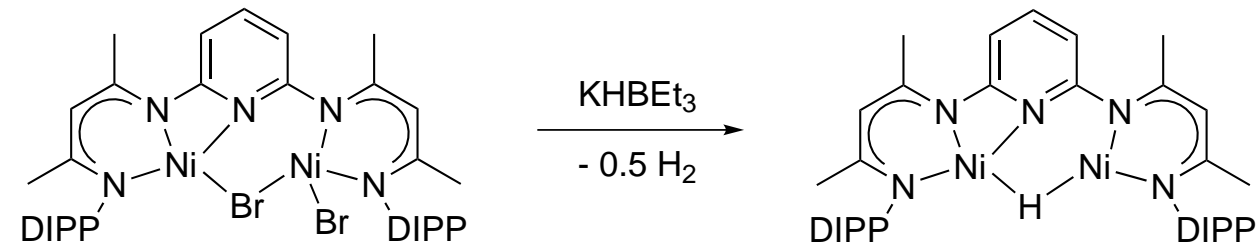

Scheme 2.4: Formation of a hydride-bridged, mixed valent $\mathrm{Ni}^{\mathrm{i}}-\mathrm{Nill}$ complex. ${ }^{\left[\frac{[98]}{}\right.}$

\subsection{Objective}

In the context of this work, establishment of a new ligand system similar to $\mathbf{H}_{\mathbf{3}} \mathbf{L}^{\mathbf{p y r}}$ was attempted. Following from ligand systems shown in Scheme 2.3, merging the DIPP substituted nacnac moieties with a bis(methylene)-pyrazolate bridging unit results in their prearrangement and yields an overall trianionic, hexadentate ligand system with two binding pockets (Scheme 2.5).

Compared to the pyridine linker unit of the literature known PYR ligand, pyrazole provides the advantage of an additional $N$-donor function. This results in a stronger chelating ligand, which can promote the formation of highly preorganized, bimetallic complexes. $\stackrel{[10]}{=}$ For example, upon coordination of $\mathrm{Ni}^{\mathrm{II}}$ cations, a dinuclear compound with an anionic bridging unit $\mathrm{X}^{-}$(Scheme 2.6) can be formed. This neutral complex is similar to $\left[\mathbf{L}^{\mathbf{p y r}} \mathbf{N i}_{\mathbf{2}}(\mu-\mathbf{X})\right]$, exhibiting five- $(-)$ and six-membered (-) ring chelates, which 

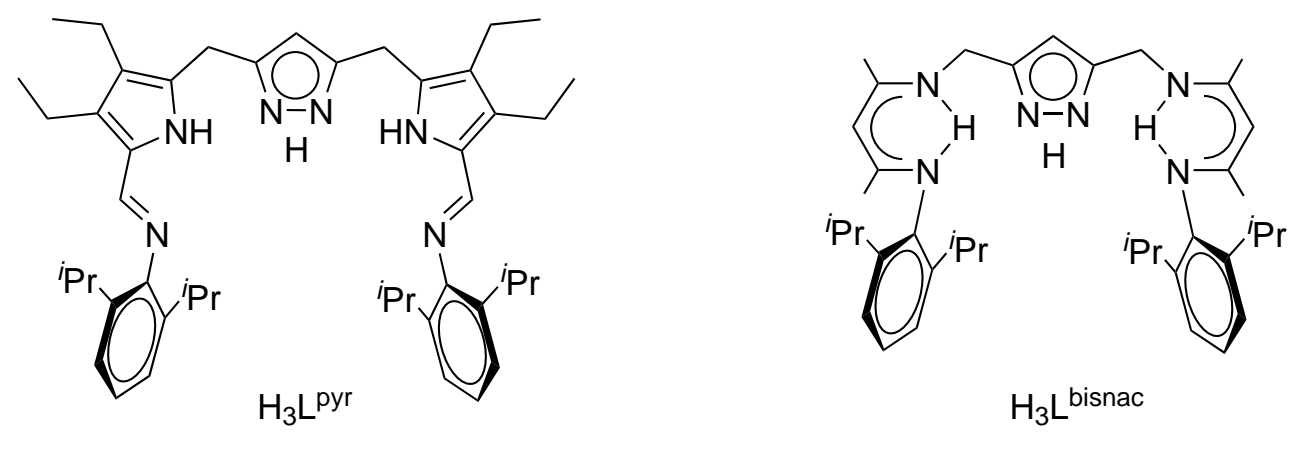

Scheme 2.5: $\mathbf{H}_{3} \mathrm{~L}^{\text {pyr }}$ and the targeted ligandsystem $\mathrm{H}_{3} \mathrm{~L}^{\text {bisnac }}$.

significantly stabilize the entire system. The bulky DIPP moieties of the ligand provide additional steric protection of the complex cavity and can potentially coordinate cations via cation- $\pi$-interactions, as was experienced in previously reported studies with similar systems. ${ }^{[67,99]}$
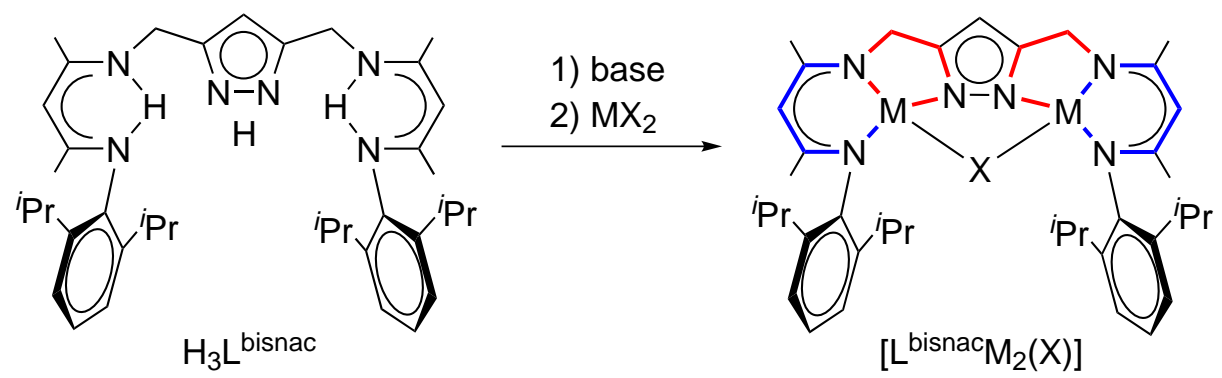

Scheme 2.6: Targeted ligand system $\mathbf{H}_{3} \mathbf{L}^{\text {bisnac }}$ and a hypothetical complex formation featuring an anionic bridging unit $\mathrm{X}^{-}$.

\subsection{Ligand Synthesis and Characterization}

Pyrazolate as the bridging unit prompted use of the compound bis(3,5-amminomethyl)pyrazole (c) as the main building block (Scheme 2.7). Starting from commercially available 3,5-dimethylpyrazole, pyrazole-3,5-carboxy methyl ester was produced in two steps according to literature procedures. ${ }^{[100-102]}$ (c) was prepared in three subsequent steps (details are found in the experimental part (7).

The $\beta$-diketiminate sidearm 2-[(2,6-diisopropylphenyl)imido]-pent-2-en-4-one (d, Scheme 2.8) was synthesized according to the literature. ${ }^{[56]}$ In the final step two parts of $(\mathbf{d})$ was attached to the linker unit (c) yielding the ligand $\mathbf{H}_{\mathbf{3}} \mathbf{L}^{\text {bisnac }}$ (Scheme 2.8, a detailed description is found in the experimental section (7).

Figure 2.1 shows the ${ }^{1} \mathrm{H}$ NMR spectrum of $\mathbf{H}_{3} \mathbf{L}^{\text {bisnac }}$. The resonance pattern indicates either a $\mathrm{C}_{2 \mathrm{v}}$ or $\mathrm{C}_{2}$ symmetry. Two separate doublet resonances (near $1.2 \mathrm{ppm}$ ) for the isopropyl $\mathrm{CH}_{3}$ groups of the DIPP sidearms indicate a structure as shown. Four of the 

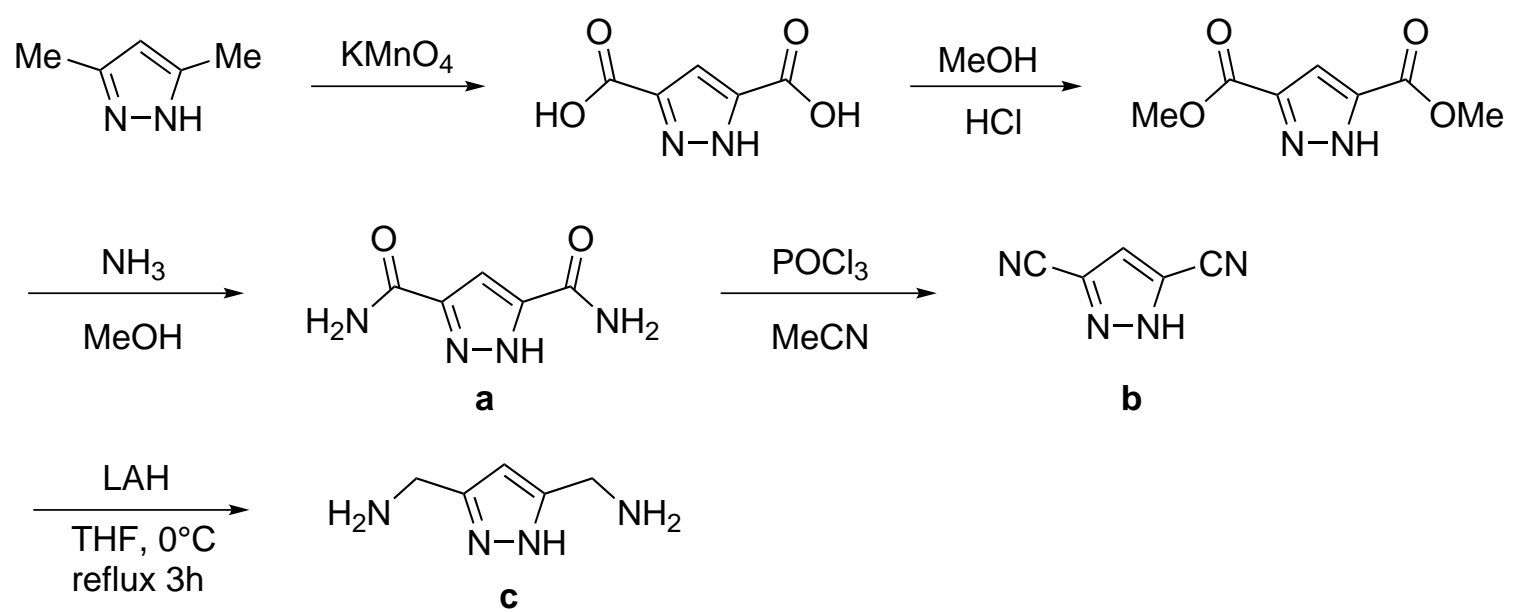

Scheme 2.7: Synthesis of the pyrazolate linker unit bis(3,5-amminomethyl)pyrazole (c).

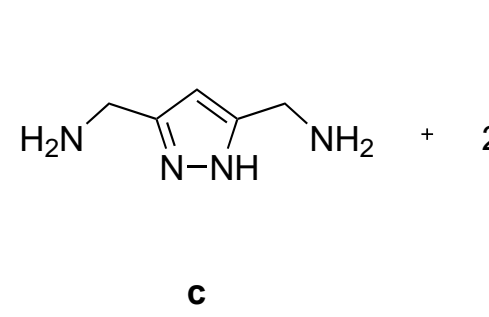

1) $\mathrm{Et}_{3} \mathrm{O}^{+} \mathrm{BF}_{4}^{-}$ $\mathrm{DCM}$ (abs.)

2) $\mathrm{Et}_{3} \mathrm{~N}$ (abs.)

$3 d$ RT

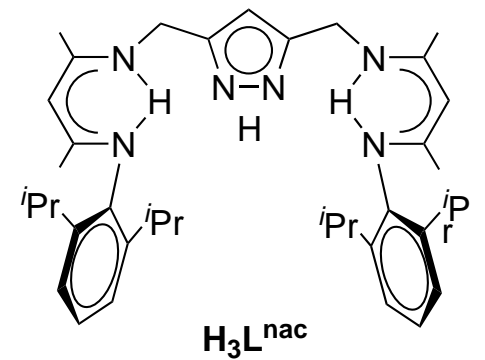

Scheme 2.8: Preparation of the bis- $\beta$-diketiminate ligand system $\mathrm{H}_{3} \mathrm{~L}^{\text {bisnac }}$.

eight isopropyl methyl groups point towards each other and the cavity of the main ligand skeleton. This allignment can cause the ring current originating from the aryl substituents to affect these particular isopropyl methyl moieties, resulting in weak electronic shielding. As a consequence, one doublet resonance is shifted to slightly higher field (๑).

At room temperature the $\mathrm{NH}$ protons can hardly be distinguished due to pronounced line broadening from rapid exchange occuring between them. Cooling of the ligand solution lead to sharpening of the NH resonances ( $b$ and $c$ in Figure 2.2). NH tautomerism of the pyrazole becomes slow on the NMR time scale which causes the molecule to lower its symmetry to $\mathrm{C}_{\mathrm{s}}$, which is reflected by a more complex ${ }^{1} \mathrm{H}$ NMR spectrum. For instance, the resonances associated with $\mathrm{CH}_{3}(\bullet)$ and $\mathrm{CH}(\boldsymbol{\square})$ of the $\beta$-diketiminato moieties separate into two distinct signals at lower temperature. 


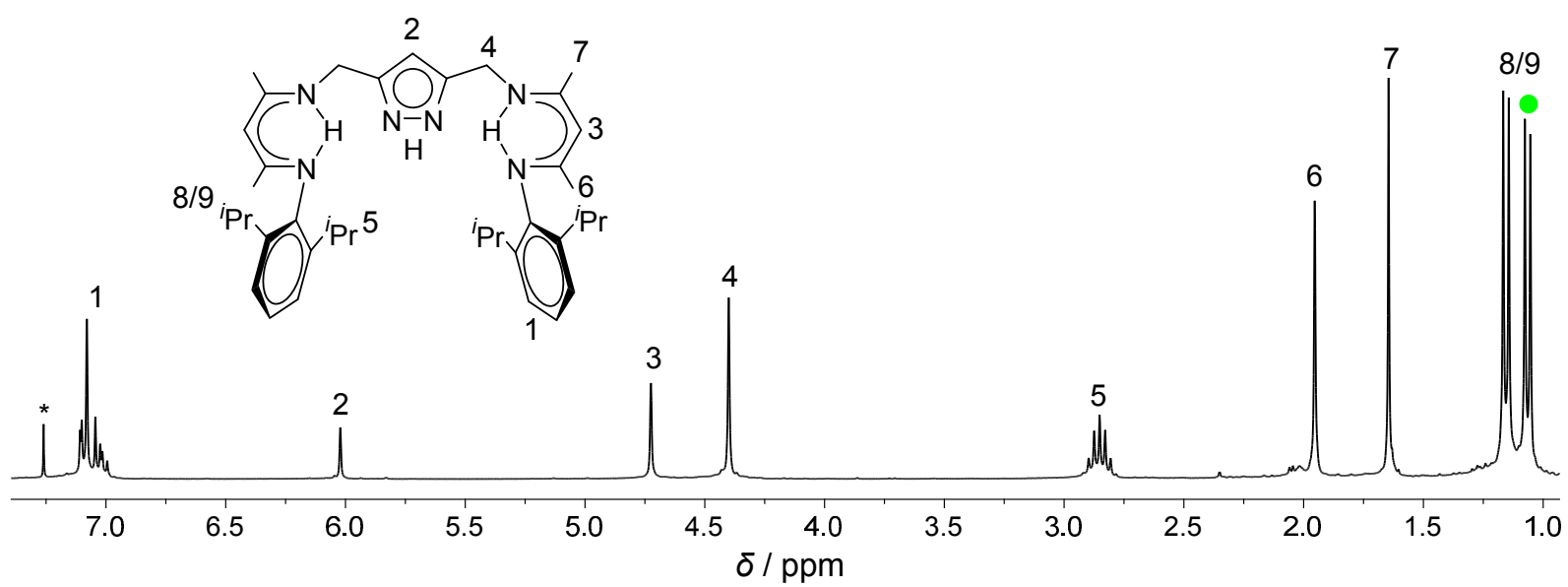

Figure 2.1.: ${ }^{1} \mathrm{H}$ NMR spectrum $(300 \mathrm{MHz})$ of $\mathbf{H}_{3} \mathrm{~L}^{\text {bisnac }}$ measured in $\mathrm{CDCl}_{3}$. Residual solvent signals are marked $\left({ }^{*}\right)$.

(a)

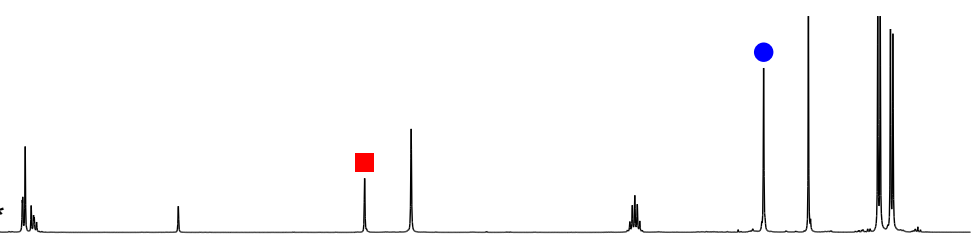

(b)

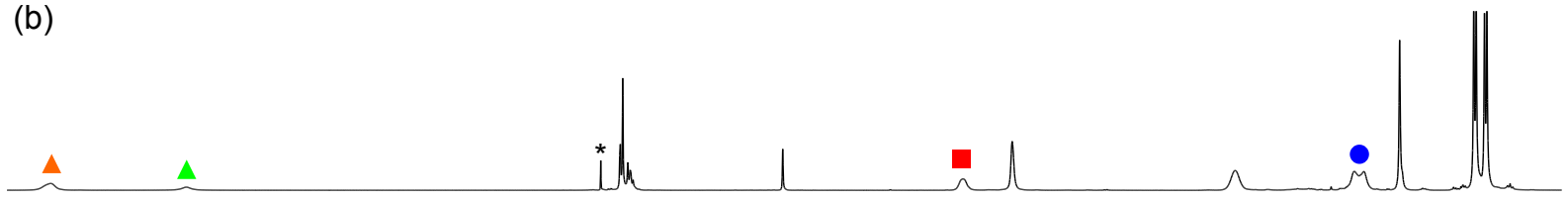

(c)

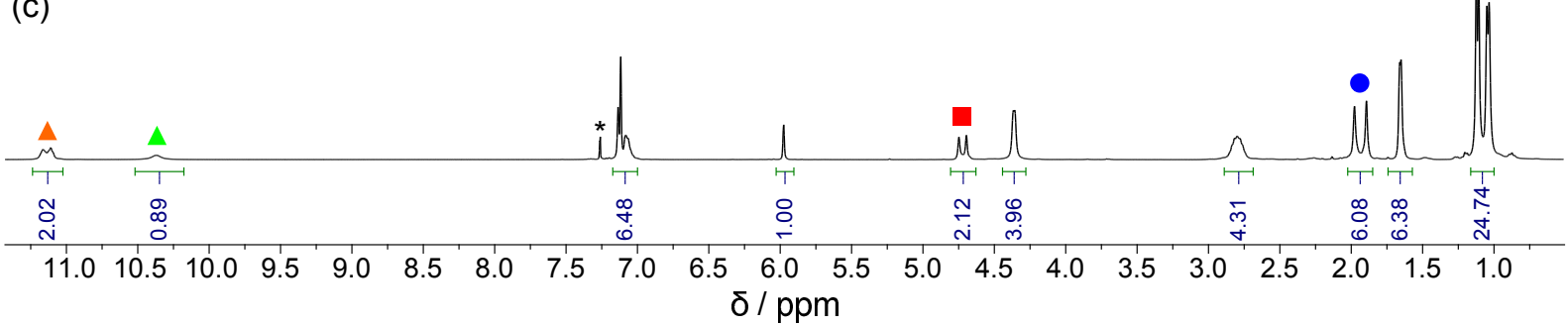

Figure 2.2.: ${ }^{1} \mathrm{H}$ NMR spectra $(400 \mathrm{MHz})$ of $\mathbf{H}_{3} \mathrm{~L}^{\text {bisnac }}$ measured at (a) room temperature, (b) $-35^{\circ} \mathrm{C}$ and (c) $-50^{\circ} \mathrm{C}$. At low temperature the proton resonances associated with $\mathrm{NH}$ of the pyrazole $\Delta$ and sidearm $\Delta$ of the ligand are resolved. Due to localization of the pyrazole $\mathrm{NH}$ proton, the molecule becomes nonsymmetric, which is reflected in particular by the splitting of the $\mathrm{CH}_{3}-(\bullet)$ and $\mathrm{CH}-(\square)$ singlet resonances belonging to the $\beta$-diketiminato sidearms to two separate signals. At $-50{ }^{\circ} \mathrm{C}$ (c) both $\mathrm{NH}$ protons of the latter $(\triangle)$ are also affected by the asymmetry and are resolved as two distinct signals. Residual solvent signals $\left(\mathrm{CHCl}_{3}\right)$ are marked $\left({ }^{*}\right)$. 


\subsection{Complex Synthesis}

$\mathbf{H}_{3} \mathbf{L}^{\text {bisnac }}$ was deprotonated with $n$-butyllithium in THF to give a deep red solution. The addition of a $\mathrm{NiBr}_{2}$ (DME) complex to this solution under inert atmosphere gave a brownish suspension, which was stirred overnight at room temperature. Eventually, a bromide bridged $\mathrm{Ni}^{\mathrm{II}}$ complex was formed, as illustrated in Scheme 2.9.

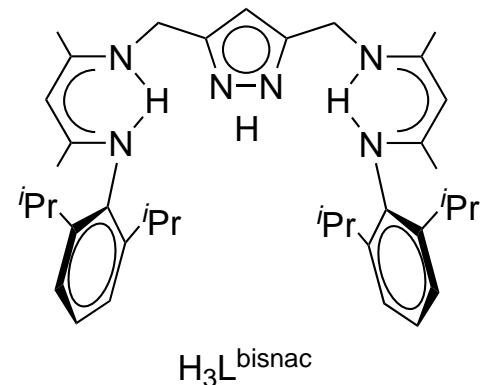

1) $n$-BuLi, $-78^{\circ} \mathrm{C}$

2) $\mathrm{NiBr}_{2}(\mathrm{DME})$, rt THF (abs.) $\mathrm{N}_{2}$

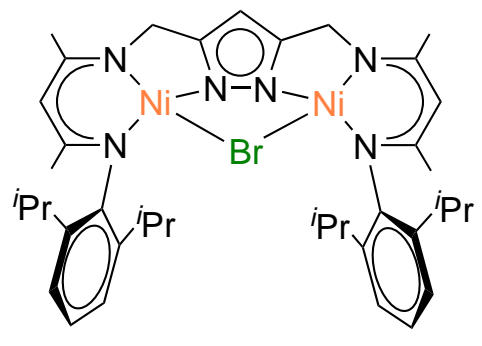

$\left[\mathrm{L}^{\text {bisnac }} \mathrm{Ni}_{2}(\mu-\mathrm{Br})\right]$

Scheme 2.9: Preparation of the bromide bridged $\mathrm{Ni}^{l l}$ complex $\left[\mathrm{L}^{\text {bisnac }} \mathrm{Ni}_{2}(\mu-\mathrm{Br})\right]$.

Workup of the reaction mixture included the use of a centrifuge to separate the precipitate ( $\left[\mathbf{L}^{\text {bisnac }} \mathbf{N i}_{\mathbf{2}}(\mu-\mathbf{B r})\right]$ ) from the mother liquor. The isolated solid was washed with a small amount of THF followed by acetone until the washing phase was colorless. After drying under vacuum the crude substance remained as a fine, beige colored powder. With this method $\left[\mathbf{L}^{\text {bisnac }} \mathbf{N i}_{\mathbf{2}}(\mu-\mathbf{B r})\right]$ was obtained in pure form and very high yield (96\%), which allowed for the facile crystallization of the complex by slow evaporation of a dichloromethane or chloroform solution. After several days dark yellow cube shaped crystals were isolated, which were suitable for X-ray diffraction. Figure 2.3 shows the ORTEP diagramm of the molecular structure. In Table 2.1 a selection of bond lengths, distances and angles is shown.

The nickel complex $\left[\mathbf{L}^{\text {bisnac }} \mathbf{N i}_{\mathbf{2}}(\boldsymbol{\mu}-\mathbf{B r})\right]$ crystallizes in the monoclinic spacegroup $P 2_{1}$ with four molecules in the unit cell.

The coordination environment of the nickel centers can be determined by the angles between one nickel atom and two adjacent atoms. For instance, the two largest angles correspond to $\Varangle \mathrm{N} 3-\mathrm{Ni1}-\mathrm{Br} 1=168^{\circ}$ and $\Varangle \mathrm{N} 1-\mathrm{Ni1}-\mathrm{N} 4=176^{\circ}$ for Ni1. The other four angles range from 83 to $93^{\circ}$. A similar geometry was found for $\mathrm{Ni} 2$ and the respective atoms coordinated to it, which indicates that the two metal centers reside in a roughly square-planar coordination environment.

[ $\left.\mathbf{L}^{\text {bisnac }} \mathbf{N i}_{\mathbf{2}}(\mu-\mathbf{B r})\right]$ exhibits a five-membered chelate (e.g. five-membered ring comprised of Ni1-N1-C2-C4-N3, marked red in Figure 2.3). The Ni $\cdots$ Ni distance of $3.81 \AA$ is within the range for nickel complexes of multifunctional pyrazolate ligands containing dinuclear five-membered chelate rings $\left(d_{\mathrm{Ni} \cdots \mathrm{Ni}}=3.476-4.661 \AA\right) . \stackrel{[10.32]}{.}$ The relatively short distance 

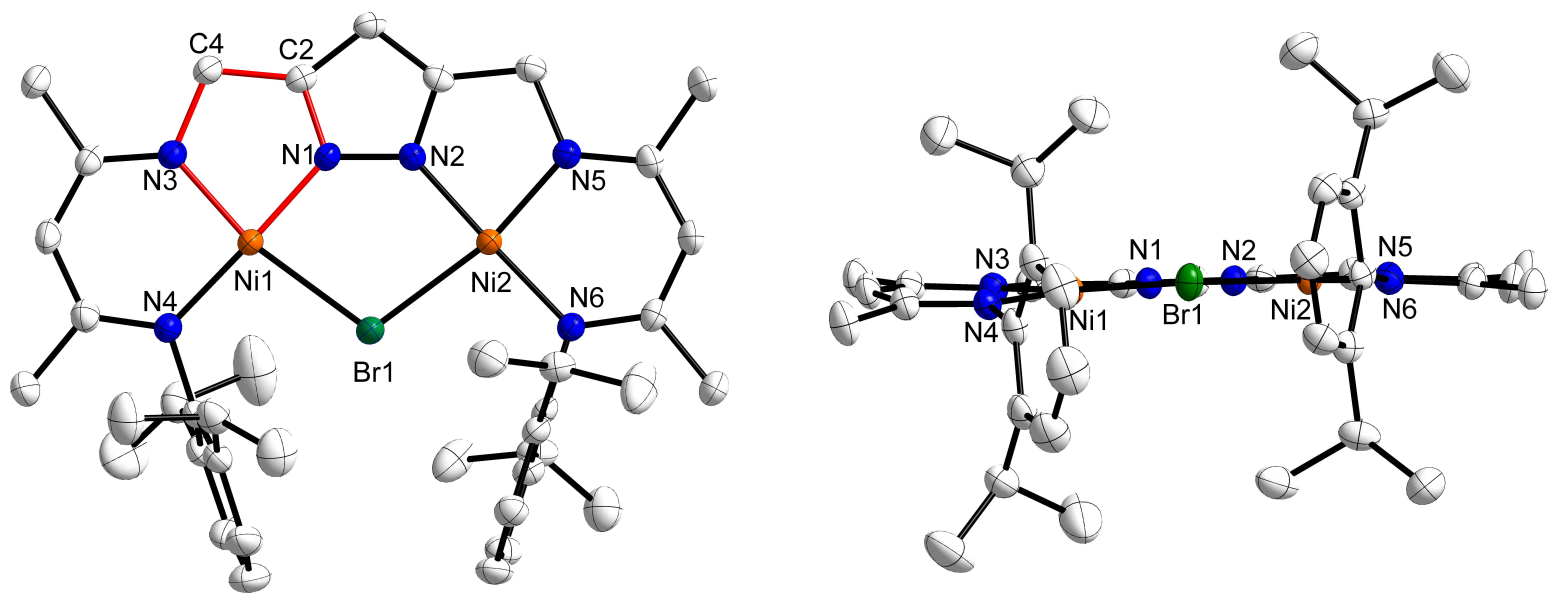

Figure 2.3.: Thermal displacement ellipsoids (shown at $50 \%$ probability) of the molecular structure of [ $\left.\mathrm{L}^{\text {bisnac }} \mathrm{Ni}_{2}(\mu-\mathrm{Br})\right]$. All hydrogen atoms have been omitted for clarity. Left: Top view of the molecular structure. The bonds marked in red illustrate a five-membered chelate. Right: side view to illustrate the square-planar coordination environment of the two metal atoms.

Table 2.1.: Selected bond lengths and angles of [ $\left.\mathrm{L}^{\text {bisnac }} \mathrm{Ni}_{2}(\mu-\mathrm{Br})\right]$.

\begin{tabular}{cccc}
\hline \multicolumn{2}{c}{ Bond lengths / } & \multicolumn{2}{c}{ Angle / } \\
\hline Ni1-N1 & $1.843(2)$ & N1-Ni1-N3 & $83.08(9)$ \\
Ni1-N3 & $1.882(2)$ & N1-Ni1-N4 & $175.98(9)$ \\
Ni1-N4 & $1.895(2)$ & N3-Ni1-N4 & $94.64(9)$ \\
Ni1-Br1 & $2.3978(4)$ & N1-Ni1-Br1 & $85.29(6)$ \\
Ni2-N2 & $1.845(2)$ & N3-Ni1-Br1 & $167.91(7)$ \\
Ni2-N5 & $1.889(2)$ & N4-Ni1-Br1 & $97.16(6)$ \\
Ni2-N6 & $1.897(2)$ & N2-Ni2-N5 & $82.56(9)$ \\
Ni2-Br1 & $2.3812(4)$ & N2-Ni2-N6 & $178.02(9)$ \\
Ni1...Ni2 & $3.8066(5)$ & N5-Ni2-N6 & $95.52(9)$ \\
Br1...Ar(right) & $3.5615(3)$ & N2-Ni2-Br1 & $85.64(6)$ \\
Br1...A Ar(left) & $3.5639(4)$ & N5-Ni2-Br1 & $168.20(6)$ \\
& & N6-Ni2-Br1 & $96.27(6)$ \\
& & Ni2-Br1-Ni1 & $105.599(13)$ \\
\hline
\end{tabular}


between the nickel centers $\left(d_{\mathrm{Ni} \cdots \mathrm{Ni}}<3.9 \AA\right)$ in $\left[\mathbf{L}^{\text {bisnac }} \mathbf{N i}_{\mathbf{2}}(\mu-\mathrm{Br})\right]$ can potentially allow the coordination of a small molecule as a bridging unit. ${ }^{[10]}$

The ${ }^{1} \mathrm{H}$ NMR spectrum of $\left[\mathbf{L}^{\text {bisnac }} \mathbf{N i}_{2}(\mu-\mathbf{B r})\right]$ is illustrated in Figure 2.4. Similar to the spectrum of the ligand (Figure 2.1), two doublet resonances are observed for the four isopropyl methyl groups belonging to the aryl sidearms. After complexation of the nickel cations, the sidearms are forced into closer proximity to each other due to the higher organization of the system. In particular, the isopropyl methyl groups are affected by the ring current originating from the aryl $\pi$-systems. As a consequence, the methyl groups pointing towards each other (10) experience a stronger electronical shielding yielding more upfield shifted signals than the outer methyl groups.

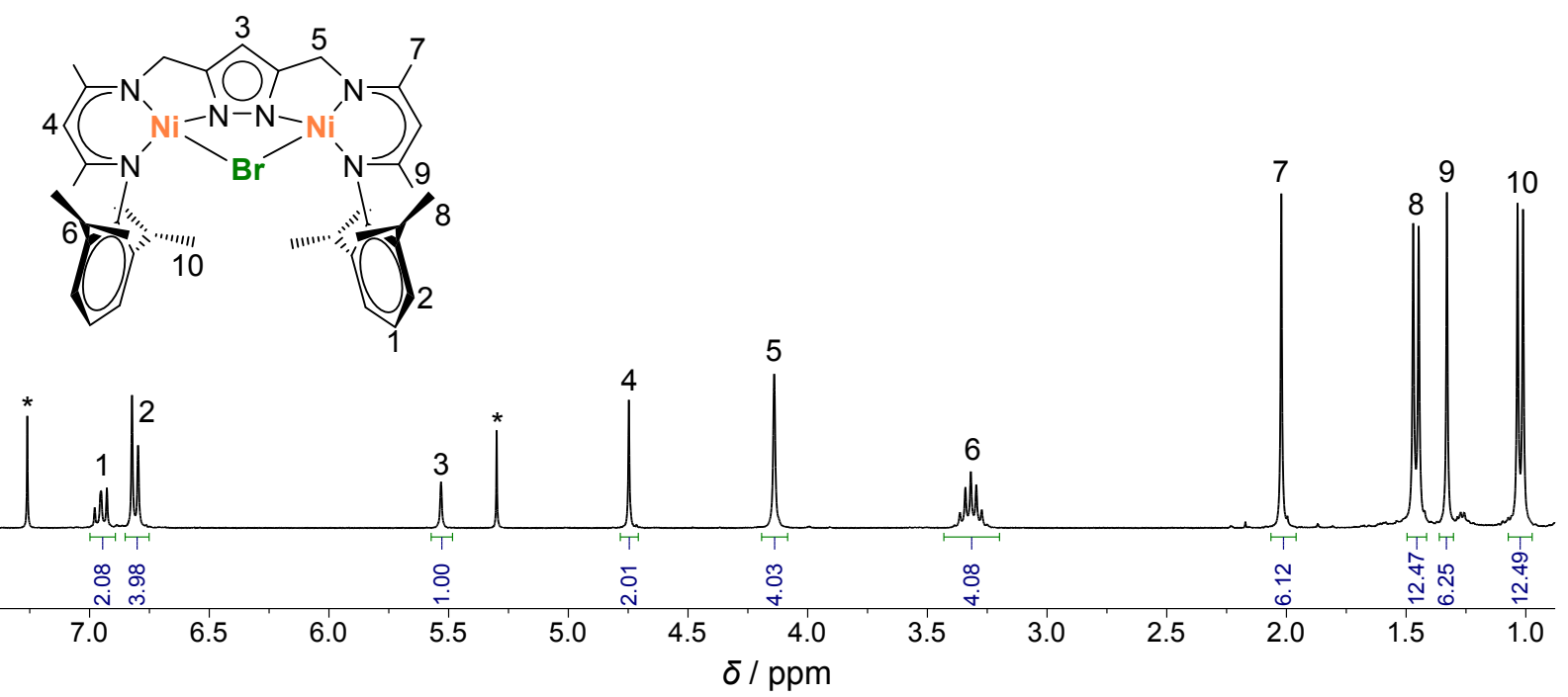

Figure 2.4.: ${ }^{1} \mathrm{H}$ NMR spectrum of [ $\left.\mathrm{L}^{\text {bisnac }} \mathrm{Ni}_{2} \mu-\mathrm{Br}\right]$ measured in $\mathrm{CDCl}_{3}$. Residual solvent signals are marked $\left({ }^{*}\right)$.

\subsection{Summary}

Combining two of the versatile $\beta$-diketiminato ligands (nacnac) with a pyrazolate-linker unit gave rise to a novel, hexadentate ligand system $\mathbf{H}_{3} \mathbf{L}^{\text {bisnac }}$. With this new ligand a dinuclear, bromido-bridged nickel complex was prepared. [ $\mathbf{L}^{\text {bisnac }} \mathbf{N i}_{\mathbf{2}}(\mu-\mathbf{B r}]$ was produced in very high yields ( $\geqslant 95 \%)$ and is stable under ambient conditions. The diamagnetic nature of the neutral $\mathrm{Ni}^{\mathrm{II}}$ complex provides the opportunity for NMR spectroscopic studies similar to the previously discussed $\left[\mathbf{L}^{\mathbf{p y r}} \mathrm{Ni}_{\mathbf{2}}(\mu-\mathrm{OH}]\right.$.

With establishment of $\left[\mathbf{L}^{\text {bisnac }} \mathbf{N i}_{2}(\mu-\mathbf{B r})\right]$, the foundation for the expanding field of novel bis( $\beta$-diketiminato)-pyrazolate-based complex chemistry was created. The following chapters present first impressions, which indicate much potential of this promising new system. 


\section{A $\beta$-Diketiminato Based $\mu$-Hydroxo Nickel Complex}

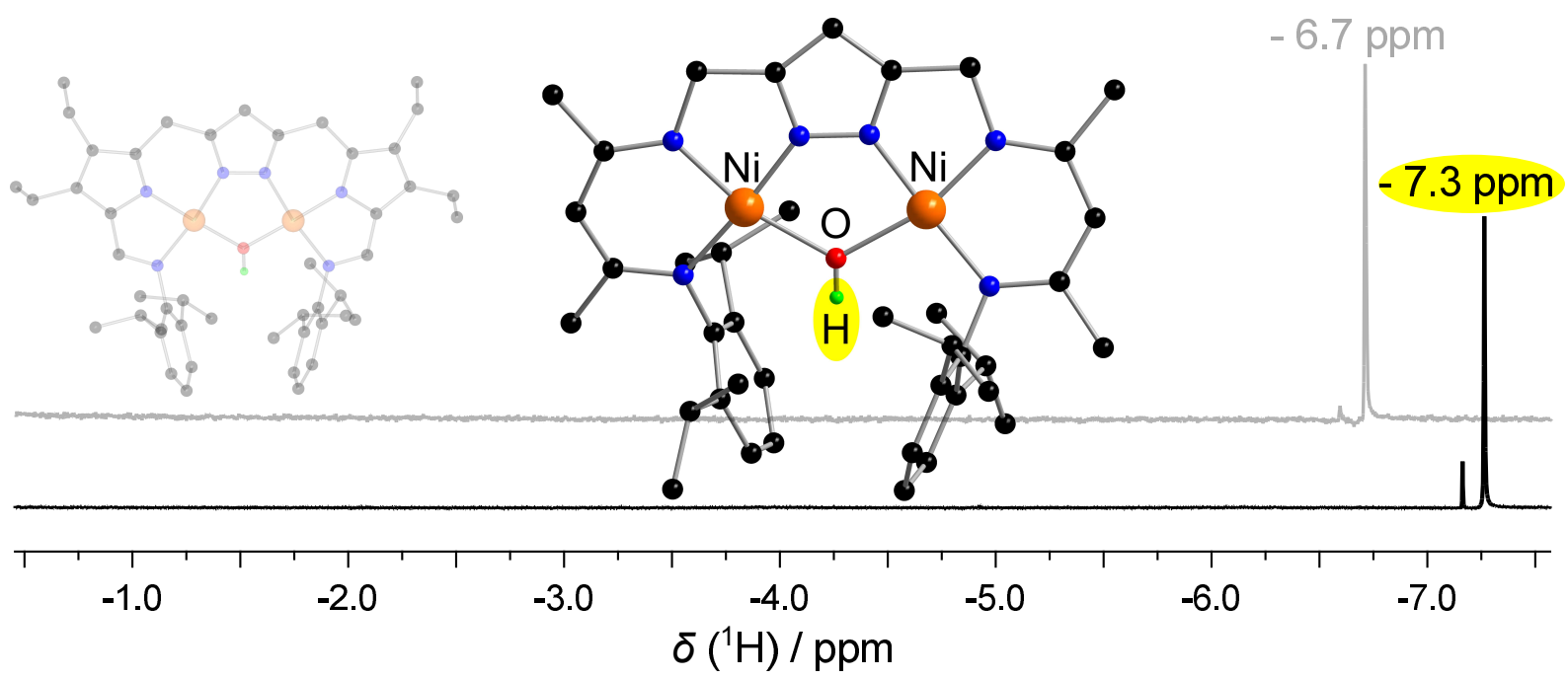

Abstract In the context of the previous part of this work, a new hydroxo bridged dinuclear nickel complex with the new ligand $\mathbf{H}_{\mathbf{3}} \mathbf{L}^{\text {bisnac }}$ was synthesized. This chapter deals with the preparation and characterization of the complex. Similar to $\left[\mathbf{L}^{\mathbf{p y r}} \mathbf{N i}_{\mathbf{2}}(\mu-\mathbf{O H})\right]$, the new $\left[\mathbf{L}^{\text {bisnac }} \mathbf{N i}_{\mathbf{2}}(\boldsymbol{\mu}-\mathbf{O H})\right]$ exhibits a hydroxide signal in the upfield of the ${ }^{1} \mathrm{H}$ NMR spectrum. Nonetheless, the exchange with $\mathrm{D}_{2} \mathrm{O}$ occurs rapidly in contrast to $\left[\mathbf{L}^{\mathbf{p y r}} \mathbf{N i}_{\mathbf{2}}(\mu-\right.$ $\mathrm{OH})]$. 


\subsection{Preparation of a Bimetallic $\beta$-Diketiminato Based Nickel $\mu$-Hydroxo Complex}

The $\mu$-hyroxo complex $\left[\mathbf{L}^{\text {bisnac }} \mathbf{N i}_{\mathbf{2}}(\boldsymbol{\mu}-\mathbf{O H})\right]$ was synthesized by means of adding an aqueous solution of $\mathrm{KOH}$ to a suspension of $\left[\mathbf{L}^{\text {bisnac }} \mathbf{N i}_{2}(\mu-\mathbf{B r})\right]$ in $\mathrm{THF}$, which eventually led to a deep green solution (Scheme 3.1).

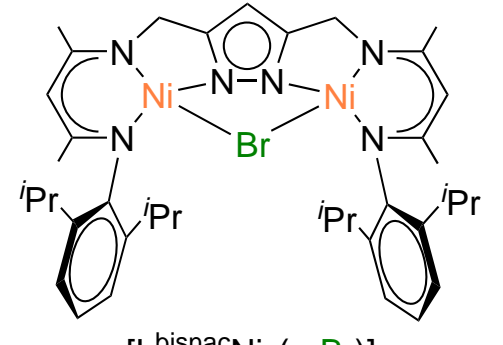

$\left[\right.$ bisnac $\left.^{\text {isi }}{ }_{2}(\mu-\mathrm{Br})\right]$

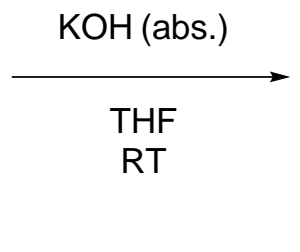

Scheme 3.1: Synthesis of [ $\left.\mathrm{L}^{\text {bisnac }} \mathrm{Ni}_{2}(\mu-\mathrm{OH})\right]$.

Slow pentane vapour diffusion into the THF solution or layering with hexanes and cooling to $-30{ }^{\circ} \mathrm{C}$ yielded green crystals suitable for X-ray diffraction measurements. The ORTEP diagram of $\left[\mathbf{L}^{\text {bisnac }} \mathbf{N i}_{\mathbf{2}}(\boldsymbol{\mu}-\mathbf{O H})\right]$ is shown in Figure 3.1 and selected bond lengths and angles are listed in Table 3.1. Both $\mathrm{Ni}^{\mathrm{II}}$ centers are coordinated in a square-planar fashion comparable to the precursor $\left[\mathbf{L}^{\text {bisnac }} \mathbf{N i}_{\mathbf{2}}(\mu-\mathbf{B r})\right]$ and the previously discussed analogue $\left[\mathbf{L}^{\mathrm{pyr}} \mathbf{N i}_{\mathbf{2}}(\mu-\mathbf{O H})\right]$. However, the complex is slightly twisted, resulting in some distortion and consequential deviation from ideal square-planar coordination (right side of Figure 3.1.
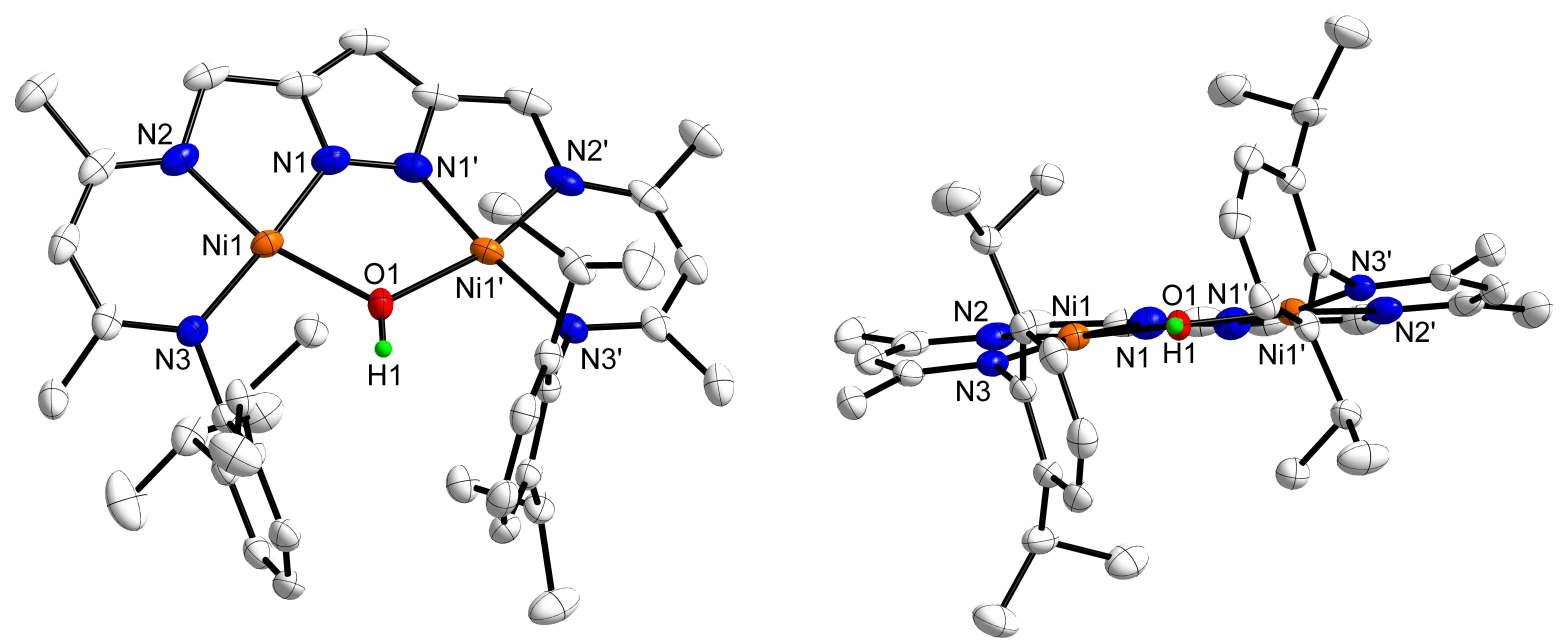

Figure 3.1.: Thermal displacement ellipsoids (shown at $50 \%$ probability) of the molecular structure of [ $\left.\mathrm{L}^{\text {bisnac }} \mathrm{Ni}_{2}(\mu-\mathrm{OH})\right]$. All hydrogen atoms (except the hydroxo hydrogen atom) have been omitted for clarity. Left: Top view of the molecular structure. Right: side view of [ $\left.\mathrm{L}^{\text {bisnac }} \mathrm{Ni}_{2}(\mu-\mathrm{OH})\right]$. 
Table 3.1.: Selected bond lengths and angles of $\left[\mathrm{L}^{\text {bisnac }} \mathrm{Ni}_{2}(\mu-\mathrm{OH})\right]$.

\begin{tabular}{cccc}
\hline \multicolumn{2}{c}{ Bond lengths $/ \AA$} & \multicolumn{2}{c}{ Angle / } \\
\hline Ni1-N1 & $1.8043(18)$ & N1-Ni1-N3 & $173.41(8)$ \\
Ni1-N3 & $1.8877(18)$ & N1-Ni1-N2 & $81.14(9)$ \\
Ni1-N2 & $1.8923(17)$ & N3-Ni1-N2 & $95.47(8)$ \\
Ni1-O & $1.9943(10)$ & N1-Ni1-O1 & $83.01(8)$ \\
Ni $\cdots \mathrm{Ni}$ & $3.4698(4)$ & N3-Ni1-O1 & $100.67(7)$ \\
O1-H1 & $0.7745(198)$ & N2-Ni1-O1 & $163.71(8)$ \\
Ar $\cdots \mathrm{H} 1$ & $3.0991(137)$ & & \\
\hline
\end{tabular}

$\left[\mathbf{L}^{\text {bisnac }} \mathbf{N i}_{\mathbf{2}}(\boldsymbol{\mu}-\mathrm{OH})\right]$ features a $\mathrm{Ni} \cdots \mathrm{Ni}$ distance of $3.47 \AA$, which is short compared to dinuclear five ring chelate nickel complexes of multifunctional pyrazolate ligands $\left(d_{\mathrm{Ni} \cdots \mathrm{Ni}}\right.$ $=3.476-4.661 \AA) . \stackrel{[10,32]}{[}$ This is obviously enforced by the small exogenous OH bridge.

Figure 3.2 shows the ${ }^{1} \mathrm{H}$ NMR spectrum of $\left[\mathbf{L}^{\text {bisnac }} \mathbf{N i}_{\mathbf{2}}(\mu-\mathrm{OH})\right]$. The hydroxo-proton signal occurs at high field $\left(\delta\left\{{ }^{1} \mathrm{H}\right\}_{\mathrm{OH}}=-7.3 \mathrm{ppm}\right)$ in THF-D $\mathrm{D}_{8}$, analogous to $\left[\mathbf{L}^{\mathbf{p y r}} \mathbf{N i}_{\mathbf{2}}(\mu-\right.$ $\mathrm{OH})]\left(\delta\left\{{ }^{1} \mathrm{H}\right\}_{\mathrm{OH}}=-6.7 \mathrm{ppm}\right)$.

Both $\mathbf{H}_{\mathbf{3}} \mathbf{L}^{\mathbf{p y r}}$ and $\mathbf{H}_{\mathbf{3}} \mathbf{L}^{\text {bisnac }}$ are trianionic, hexadentate and electron rich ligands with the same bulky diisopropylphenyl (DIPP) sidearms. These commonalities lead to a similar electronic shielding of the hydroxo group in both $\mu$-hydroxo complexes. The ${ }^{1} \mathrm{H}$ NOESY experiment (Figure 3.3) indicates the close spatial proximity of the DIPP moieties of $\left[\mathbf{L}^{\text {bisnac }} \mathbf{N i}_{2}(\mu-\mathrm{OH})\right]$ to the bridging hydroxide. As a consequence and similar to the situation in $\left[\mathbf{L}^{\mathbf{p y r}} \mathbf{N i}_{\mathbf{2}}(\mu-\mathbf{O H})\right]$, close proximity of the aryl substituents to the hydroxide hydrogen can provide enhanced electronic shielding by means of ring current effects.

Figure 3.4 illustrates the distances between the $\mu-\mathrm{OH}$ group and the DIPP moieties of $\left[\mathbf{L}^{\text {pyr }} \mathbf{N i}_{2}(\mu-\mathrm{OH})\right]$ and $\left[\mathbf{L}^{\text {bisnac }} \mathbf{N i}_{2}(\mu-\mathbf{O H})\right]$. The distance of the aryl centroids to the hydroxo-hydrogen atom (red dashed line) is smaller in $\left[\mathbf{L}^{\text {bisnac }} \mathbf{N i}_{\mathbf{2}}(\mu-\mathbf{O H})\right](3.1 \AA)$ than in $\left[\mathbf{L}^{\mathbf{p y r}} \mathbf{N i}_{\mathbf{2}}(\boldsymbol{\mu}-\mathbf{O H})\right](3.3 \AA)$ and indicates a stronger influence of the aryl ring current on the hydroxo group. Nevertheless, in the first part of this work it was revealed that ring current effects are only one cause for the high electronic shielding. Therefore, the electron rich nature of both ligand systems $\mathbf{H}_{3} \mathbf{L}^{\mathbf{p y r}}$ and $\mathbf{H}_{3} \mathbf{L}^{\text {bisnac }}$ can be considered as a main contributor to the upfield chemical shift of the $\mu-\mathrm{OH}$ group.

IR spectroscopy revealed the characteristic O-H stretching vibration at a wavenumber of $\tilde{\nu}_{\mathrm{OH}}=3608 \mathrm{~cm}^{-1}$, which is similar to the band observed in $\left[\mathbf{L}^{\mathrm{pyr}} \mathbf{N i}_{\mathbf{2}}(\mu-\mathbf{O H})\right]$ featuring an $\mathrm{O}-\mathrm{H}$ stretching vibration at $\tilde{\nu}_{\mathrm{OH}}=3602 \mathrm{~cm}^{-1}$. 


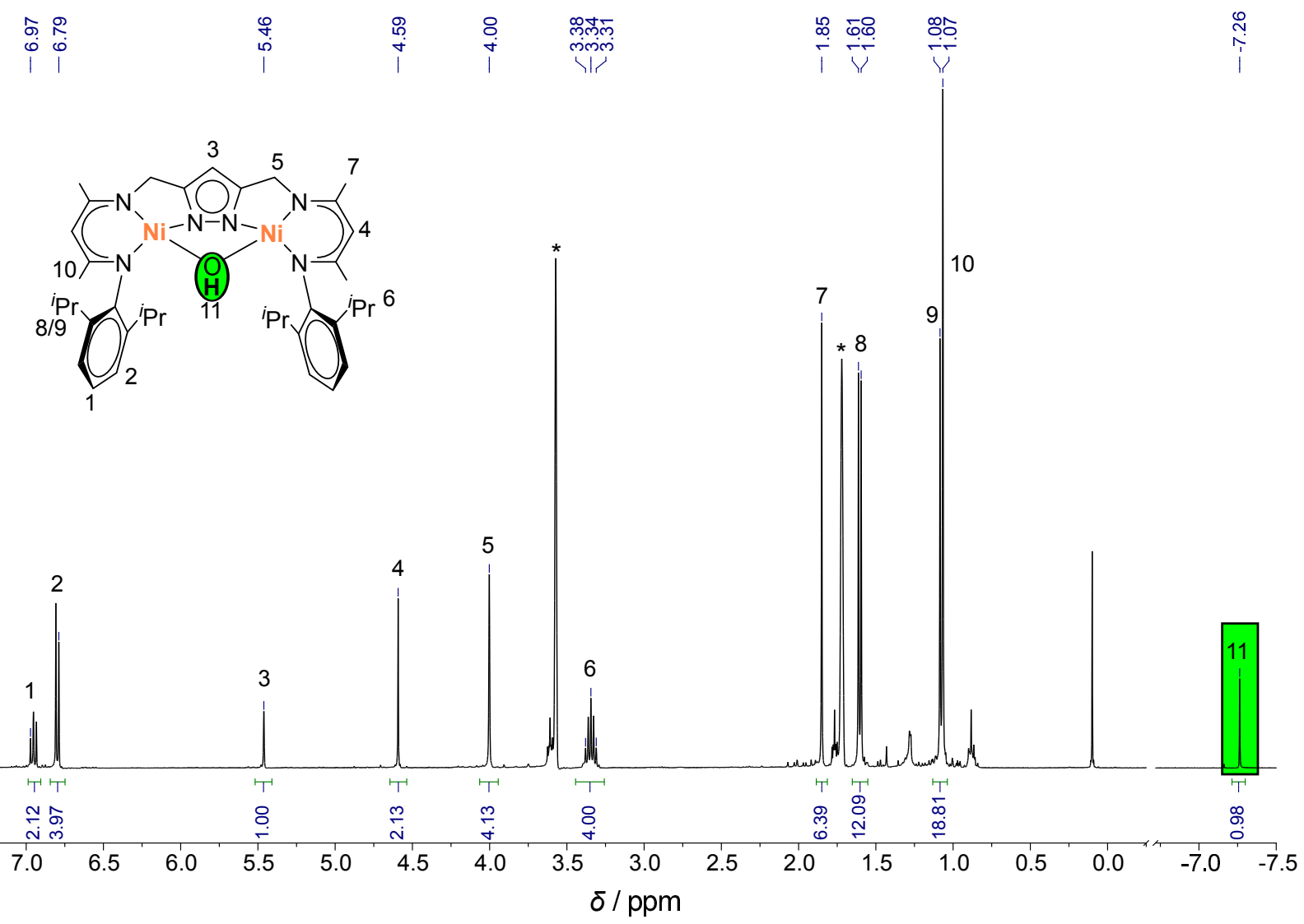

Figure 3.2.: ${ }^{1} \mathrm{H}$ NMR $(400 \mathrm{MHz})$ of $\left[\mathrm{L}^{\text {bisnac }} \mathrm{Ni}_{2}(\mu-\mathrm{OH})\right]$ measured in $T H F-\mathrm{D}_{8}$. Resonances of residual solvents are marked $\left({ }^{*}\right)$.

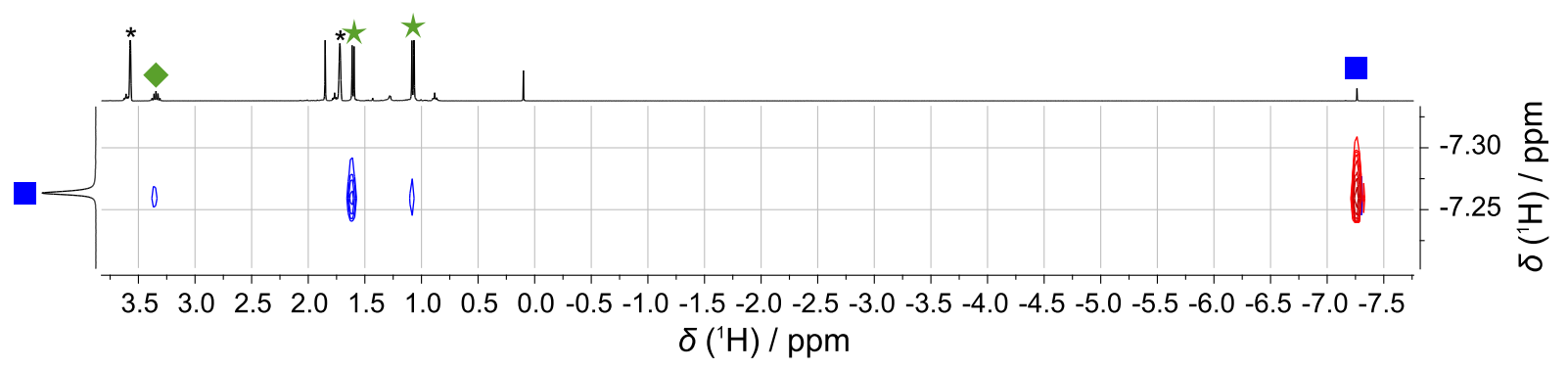

Figure 3.3.: ${ }^{1} \mathrm{H}-{ }^{1} \mathrm{H}$ NOESY $(400 \mathrm{MHz})$ of $\left[\mathrm{L}^{\text {bisnac }} \mathrm{Ni}_{2}(\mu-\mathrm{OH})\right]$ measured in THF- $\mathrm{D}_{8}$. The contour diagram illustrates the NOE correlations of the bridging $\mathrm{OH}$ proton $(\square)$ to the isopropyl $\mathrm{CH}_{3}(\star)$ and $\mathrm{CH}(\star)$ of the aromatic rings. Resonances of residual solvents $\left(\right.$ THF- $\left.\mathrm{D}_{7}\right)$ are marked $\left({ }^{*}\right)$. 


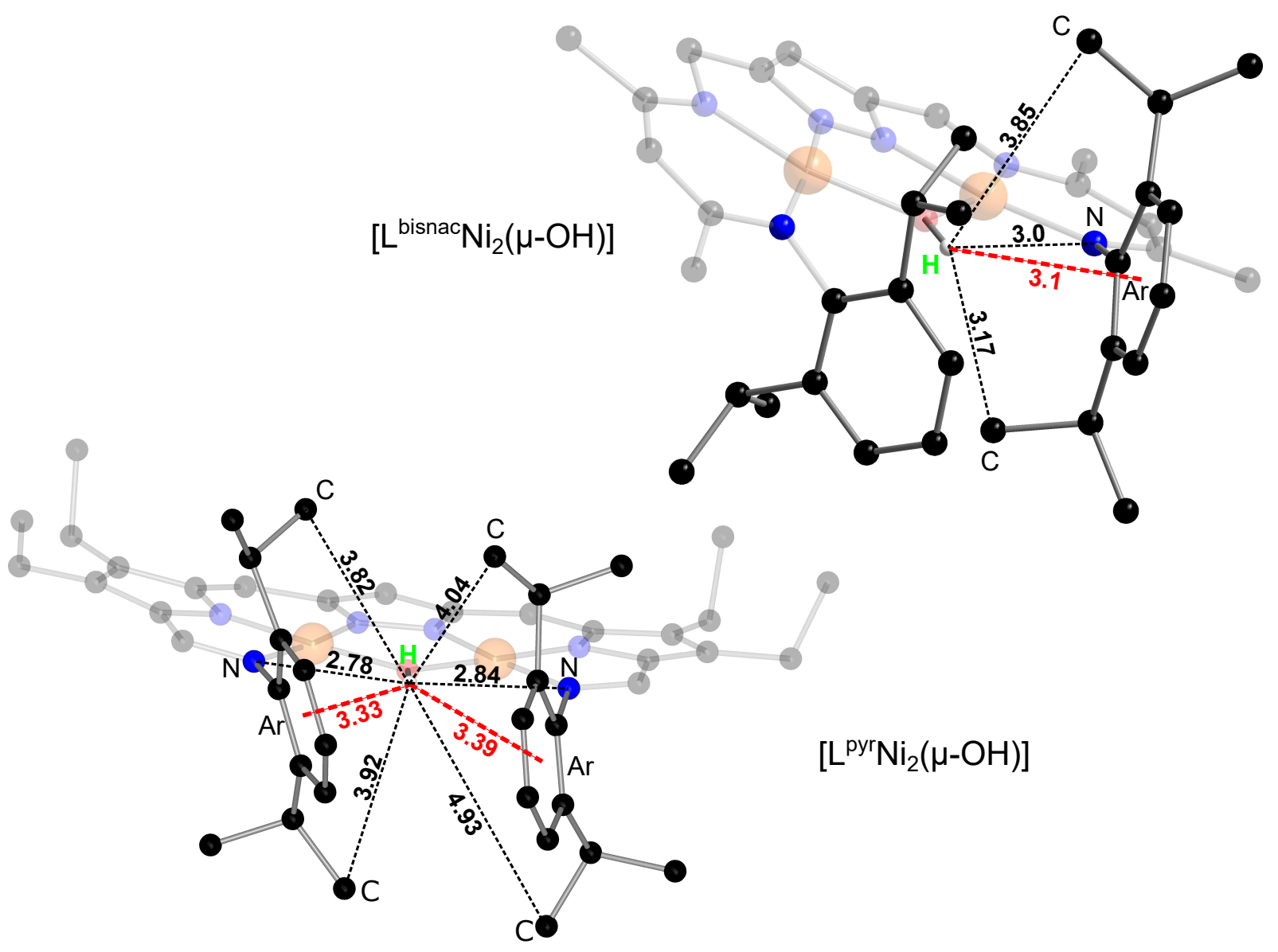

Figure 3.4.: Spatial distances (in $\AA$ ) between the hydroxo-hydrogen and the DIPP moieties in [ [ $\left.{ }^{\text {bisnac }} \mathrm{Ni}_{2}(\mu-\mathrm{OH})\right]$ (top) and [ $\left.\mathrm{L}^{\text {pyr }} \mathrm{Ni}_{2}(\mu-\mathrm{OH})\right]$ (bottom). The distances between the aryl centroids towards the hydroxo-hydrogen are marked with a red dashed line. 


\subsection{Exchange with $\mathrm{D}_{2} \mathrm{O}$}

The exchange behaviour of $\left[\mathbf{L}^{\text {bisnac }} \mathbf{N i}_{\mathbf{2}}(\boldsymbol{\mu}-\mathbf{O H})\right]$ with $\mathrm{D}_{2} \mathrm{O}$ was investigated by means of ${ }^{1} \mathrm{H}$ NMR spectroscopy. Similar to the exchange experiments performed with $\left[\mathbf{L}^{\mathbf{p y r}} \mathbf{N i}_{\mathbf{2}}(\mu-\right.$ $\mathrm{OH})$ ] a tenfold excess of $\mathrm{D}_{2} \mathrm{O}$ was added to a sample containing $10 \mathrm{mg}$ of crystalline $\left[\mathbf{L}^{\text {bisnac }} \mathbf{N i}_{2}(\mu-\mathrm{OH})\right]$ dissolved in dry $\mathrm{THF}_{-} \mathrm{D}_{8}$. After initiation of the H-D exchange reaction by mixing the substances, a very rapid exchange of $\left[\mathbf{L}^{\text {bisnac }} \mathbf{N i}_{\mathbf{2}}(\mu-\mathrm{OH})\right]$ to $\left[\mathrm{L}^{\text {bisnac }} \mathbf{N i}_{\mathbf{2}}(\mu-\mathrm{OD})\right]$ occured (Figure 3.5), contrary to the slow exchange observed for $\left[\mathrm{L}^{\mathrm{pyr}} \mathrm{Ni}_{2}(\mu-\mathrm{OH})\right]$.

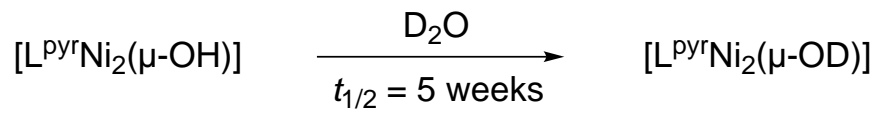

$$
\begin{aligned}
& {\left[\mathrm{L}^{\text {bisnac }} \mathrm{Ni}_{2}(\mu-\mathrm{OH})\right] \stackrel{\mathrm{D}_{2} \mathrm{O}}{\text { immediate }} \quad\left[\mathrm{L}^{\text {bisnac }} \mathrm{Ni}_{2}(\mu-\mathrm{OD})\right]}
\end{aligned}
$$

Scheme 3.2: Exchange of $\left[\mathrm{L}^{\text {pyr }} \mathrm{Ni}_{2}(\mu-\mathrm{OH})\right]$ and $\left[\mathrm{L}^{\text {bisnac }} \mathrm{Ni}_{2}(\mu-\mathrm{OH})\right]$ with $\mathrm{D}_{2} \mathrm{O}$.

(a)

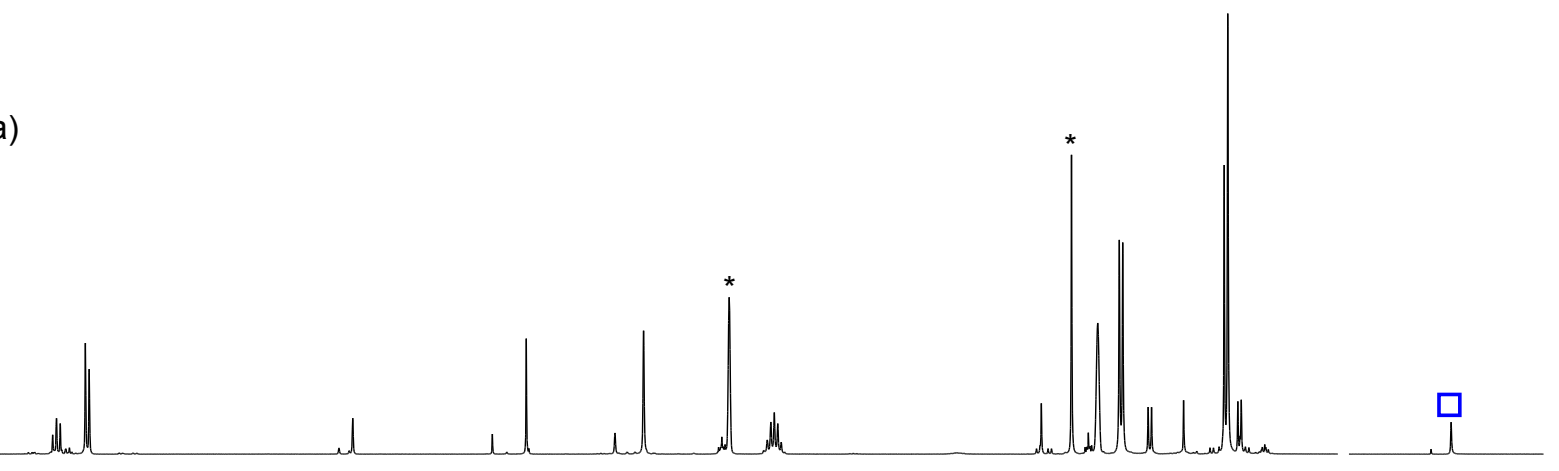

(b)

$1 \mathrm{~min}$.

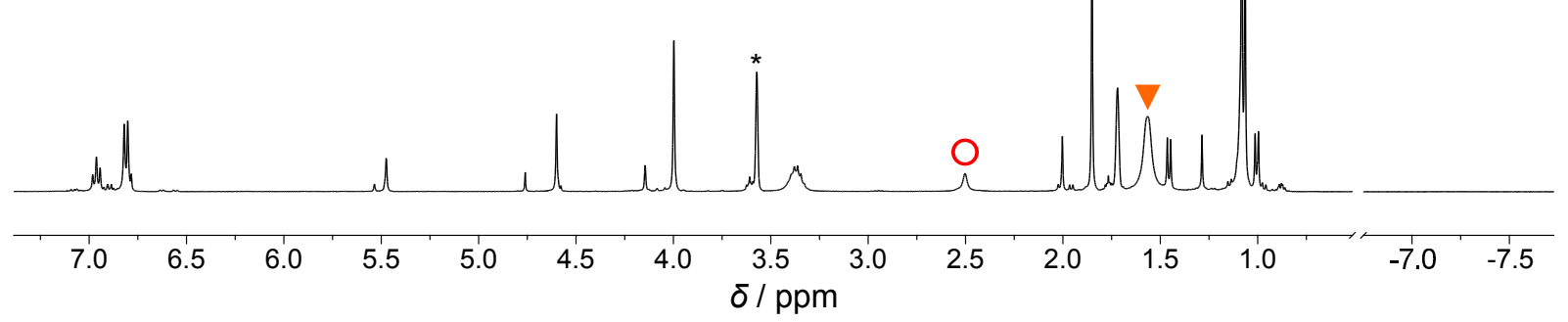

Figure 3.5.: (a) ${ }^{1} \mathrm{H}$ NMR $(400 \mathrm{MHz})$ of $\left[\mathrm{L}^{\text {bisnac }} \mathrm{Ni}_{2}(\mathrm{OH})\right]$ showing the characteristic hydroxide proton resonance $\square$ at $\delta\left\{{ }^{1} \mathrm{H}\right\}=-7.26 \mathrm{ppm}$. (b) A spectrum recorded after the treatment of the sample with $\mathrm{D}_{2} \mathrm{O}$. The hydroxo proton resonance completely vanished indicating a rapid exchange with $\mathrm{D}_{2} \mathrm{O}$. $A$ corresponding amount of $\mathrm{HDO}$ or $\mathrm{H}_{2} \mathrm{O}$ is formed (O). While all other resonances show no distinguishable changes, the signal assigned to isopropyl $\mathrm{CH}_{3}$ groups exhibits significant broadening $(\nabla)$. Resonances of residual solvents (THF-D $\left.{ }_{7}\right)$ are marked $\left(^{*}\right)$.

The extreme difference in the exchange behaviour with water can have steric reasons. As 
depicted in Scheme 3.3, $\left[\mathbf{L}^{\mathbf{p y r}} \mathbf{N i}_{\mathbf{2}}(\mu-\mathbf{O H})\right]$ is forming a six-membered chelate ring, causing the metal centers into closer proximity $(3.25 \AA)$. On the other hand, [ $\left.\mathbf{L}^{\mathbf{b i s n a c}} \mathbf{N i}_{\mathbf{2}}(\boldsymbol{\mu}-\mathbf{O H})\right]$ forms a five-membered chelate ring, which increases the metal-metal distance $(3.47 \AA)$.
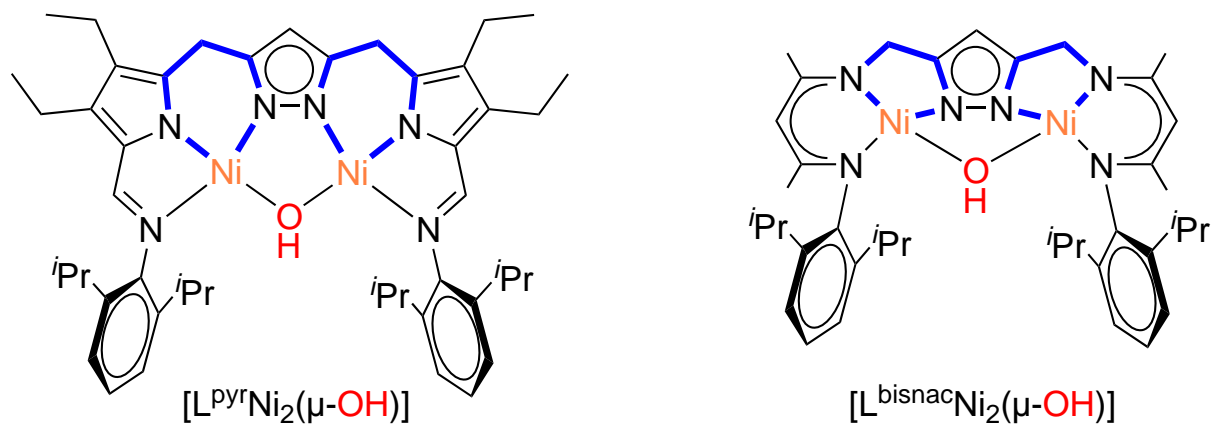

Scheme 3.3: Comparison of $\left[\mathrm{L}^{\text {pyr }} \mathrm{Ni}_{2}(\mu-\mathrm{OH})\right]$ and $\left[\mathrm{L}^{\text {bisnac }} \mathrm{Ni}_{2}(\mu-\mathrm{OH})\right]$. As indicated by a mark (-) $\left[\mathrm{L}^{\mathrm{pyr}} \mathrm{Ni}_{2}(\mu-\mathrm{OH})\right]$ is forming a six-membered chelate ring while $\left[\mathrm{L}^{\text {bisnac }} \mathrm{Ni}_{2}(\mu-\mathrm{OH})\right]$ forms a five-membered chelate ring.

Evidence for the influence of increased metal-metal distance on the exchange rate with water is provided by the palladium based analogoue of $\left[\mathbf{L}^{\mathbf{p y r}} \mathbf{N i}_{\mathbf{2}}(\mu-\mathbf{O H})\right] .{ }^{[11]}\left[\mathbf{L}^{\mathbf{p y r}} \mathbf{P d}_{\mathbf{2}}(\mu-\right.$ $\mathrm{OH})]$ features a metal-metal distance of $d_{\mathrm{Pd}-\mathrm{Pd}}=3.43 \AA$, which is very similar to the distance observed in $\left[\mathbf{L}^{\text {bisnac }} \mathbf{N i}_{\mathbf{2}}(\mu-\mathrm{OH})\right]$. Exchange experiments with $\left[\mathbf{L}^{\mathbf{p y r}} \mathbf{P d}_{\mathbf{2}}(\mu-\mathrm{OH})\right]$ and $\mathrm{D}_{2} \mathrm{O}$ also showed rapid exchange. ${ }^{[11]}$ An increase in the metal-metal distance causes a stretch of the system, which leads to an increase in the distance between the $\mu$-OH and the DIPP substituted nitrogen atoms $\left(\left[\mathbf{L}^{\mathbf{p y r}} \mathbf{N i}_{2}(\mu-\mathbf{O H})\right]: d_{\mathrm{OH} \cdots \mathrm{N}}=2.78-2.84 \AA\right.$, $\left[\mathbf{L}^{\text {bisnac }} \mathbf{N i}_{\mathbf{2}}(\mu-\mathrm{OH})\right]: d_{\mathrm{OH} \cdots \mathrm{N}}=3.00 \AA$, Figure 3.4). Although the steric shielding of $\mu-\mathrm{OH}$ at the front (Figure 3.6) is similar in both complexes, which is reflected by comparable distances between the isopropyl groups and the hydroxo-hydrogen (Figure 3.4), the apical sites in $\left[\mathbf{L}^{\text {bisnac }} \mathbf{N i}_{2}(\mu-\mathbf{O H})\right]$ are more exposed due to a longer $\mathrm{OH} \cdots \mathrm{N}$ distance. As a consequence, the bridging hydroxide in $\left[\mathbf{L}^{\text {bisnac }} \mathbf{N i}_{\mathbf{2}}(\mu-\mathrm{OH})\right]$ is more susceptible towards an approaching water molecule. The space filling models of $\left[\mathbf{L}^{\mathbf{p y r}} \mathbf{N i}_{\mathbf{2}}(\mu-\mathbf{O H})\right]$ (left) and [ $\left.\mathbf{L}^{\text {bisnac }} \mathbf{N i}_{\mathbf{2}}(\mu-\mathbf{O H})\right]$ (a, Figure 3.6) illustrate the steric shielding of the $\mu-\mathrm{OH}$ group. The distance between the nickel atoms in $\left[\mathbf{L}^{\mathbf{b i s n a c}} \mathbf{N i}_{\mathbf{2}}(\mu-\mathbf{O H})\right]$ is greater than in $\left[\mathbf{L}^{\mathbf{p y r}} \mathbf{N i}_{\mathbf{2}}(\mu-\mathbf{O H})\right]$ (a, top, Figure 3.6). The slight distortion of $\left[\mathbf{L}^{\text {bisnac }} \mathbf{N i}_{\mathbf{2}}(\mu-\mathbf{O H})\right]$ can be seen in the middle of (a). However, the DIPP moieties provide essentially the same steric protection from the front in both complexes (a, bottom, Figure 3.6).

The twist in $\left[\mathbf{L}^{\text {bisnac }} \mathbf{N i}_{\mathbf{2}}(\boldsymbol{\mu}-\mathbf{O H})\right]$ further decreases the steric protection of the $\mu$-hydroxo group, which is illustrated in (b) of Figure 3.6. Because $\left[\mathbf{L}^{\mathbf{p y r}} \mathbf{N i}_{\mathbf{2}}(\mu-\mathbf{O H})\right]$ contains additional alkyl substituents, the attack of a water molecule is sterically more hindered from one apical site of the complex. On the contrary, $\left[\mathbf{L}^{\text {bisnac }} \mathbf{N i}_{\mathbf{2}}(\mu-\mathbf{O H})\right]$ is sterically exposed at both apical sites, providing more possibilites for approach of a water molecule. Furthermore, the larger $\mathrm{Ni} \cdots \mathrm{Ni}$ distance facilitates the formation of $\mathrm{HO} \cdots \mathrm{HOH}$ intermediate which is proposed to be the rate determining step of the exchange reaction (Section 1.6.4). 
(a)

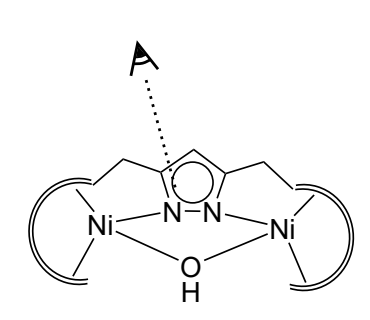

[ $\left.\mathrm{L}^{\text {pyr }} \mathrm{Ni}_{2}(\mu-\mathrm{OH})\right]$
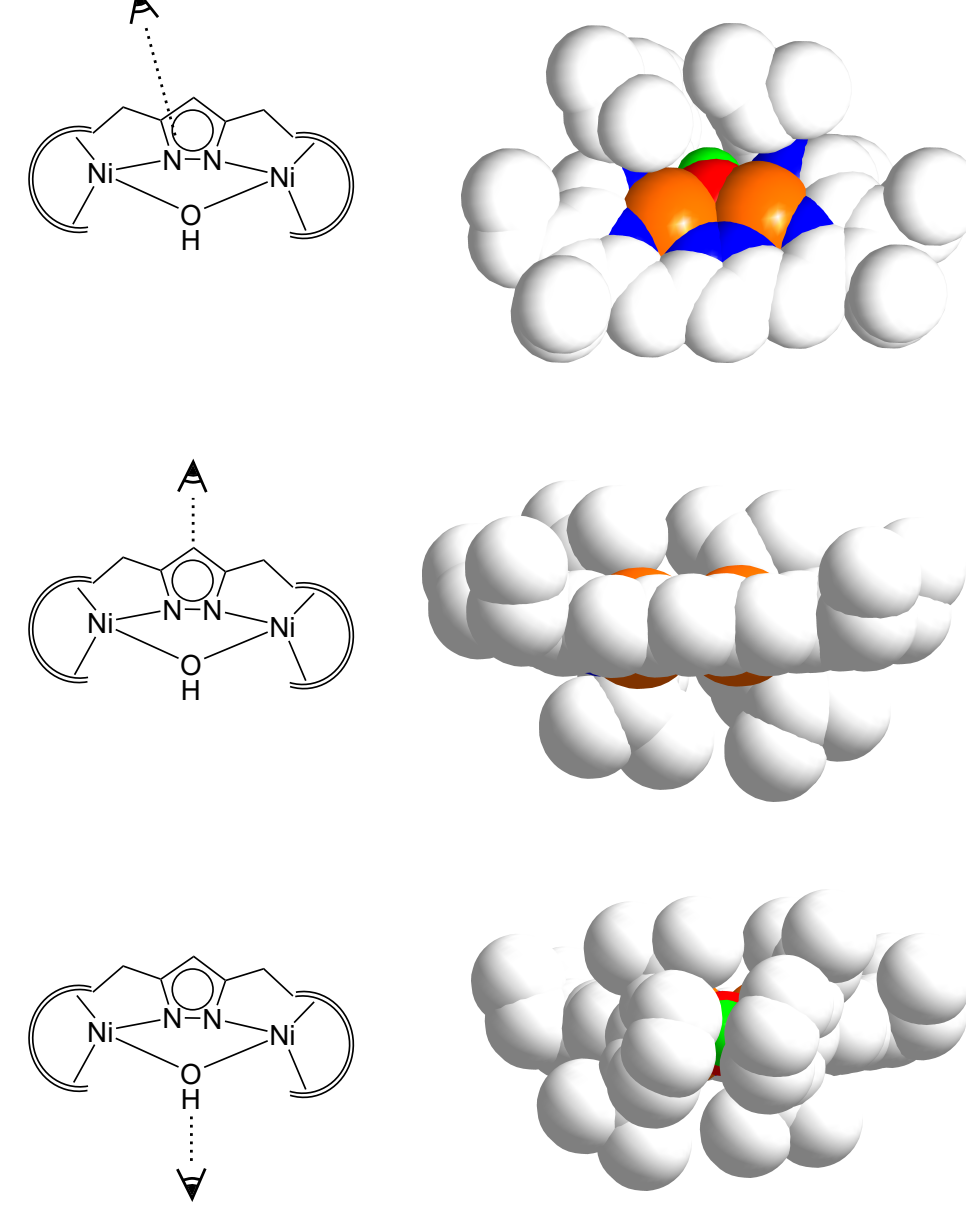

$\left[\mathrm{L}^{\text {bisnac }} \mathrm{Ni}_{2}(\mu-\mathrm{OH})\right]$
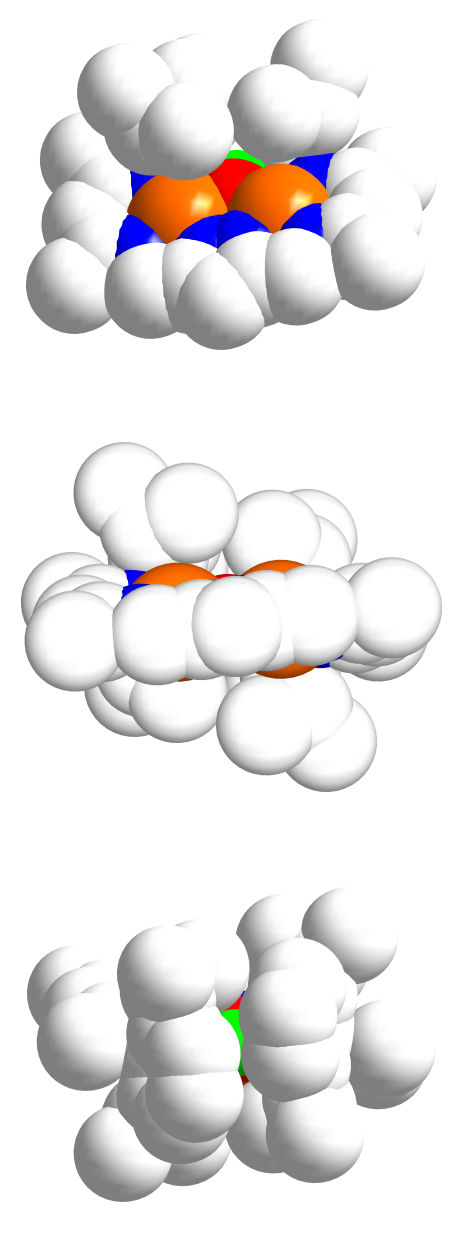

(b)

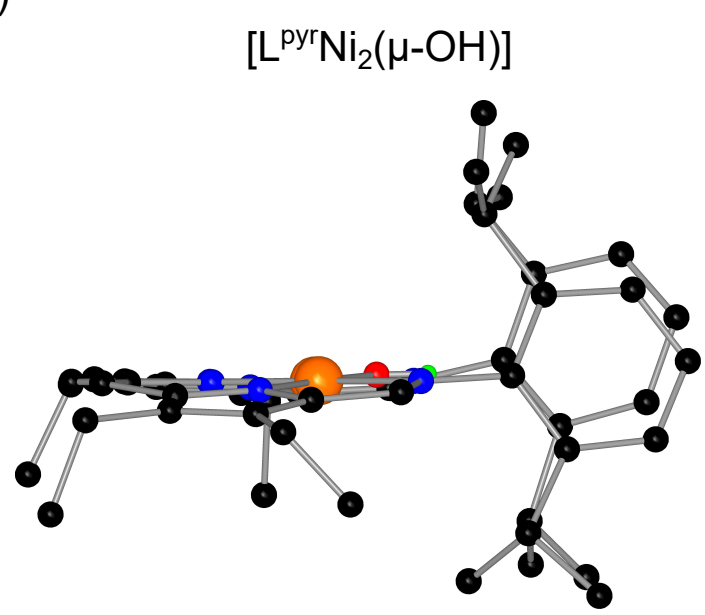

$\left[\mathrm{L}^{\text {bisnac }} \mathrm{Ni}_{2}(\mu-\mathrm{OH})\right]$

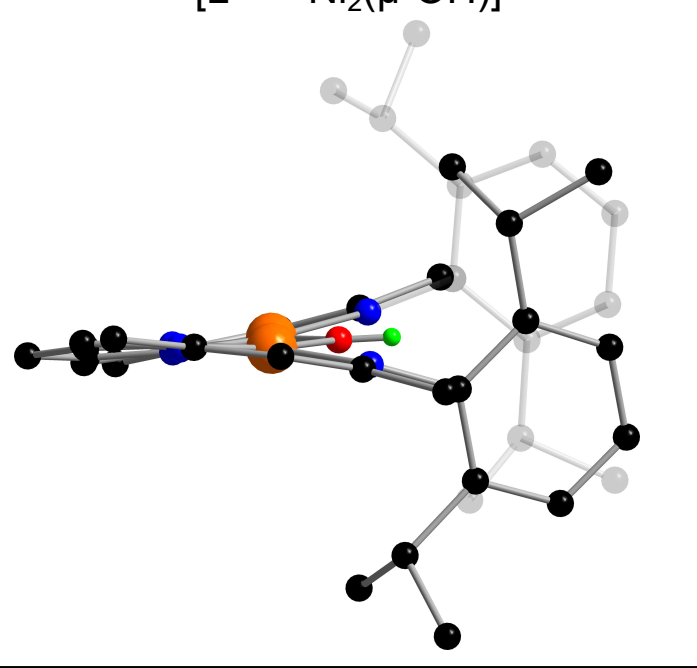

Figure 3.6.: (a) Space filling models of [ $\left.\mathrm{L}^{\text {pyr }} \mathrm{Ni}_{2}(\mu-\mathrm{OH})\right]$ (left) and [ $\left.\mathrm{L}^{\text {bisnac }} \mathrm{Ni}_{2}(\mu-\mathrm{OH})\right]$ (right) viewed from different perspectives $(\bullet=\mathrm{O}, \bullet=\mathrm{N}, \bullet=\mathrm{Ni}, \bullet=\mathrm{H}, \circ=$ C). (b) Side view of the molecular structures of $\left[\mathrm{L}^{\text {bisnac }} \mathrm{Ni}_{2}(\mu-\mathrm{OH})\right]$ (top) and $\left[\mathrm{L}^{\text {pyr }} \mathrm{Ni}_{2}(\mu-\mathrm{OH})\right]$ (bottom) in a ball and stick representation. Due to a twist the $\mu-\mathrm{OH}$ group in $\left[\mathrm{L}^{\text {bisnac }} \mathrm{Ni}_{2}(\mu-\mathrm{OH})\right]$ is more exposed than in $\left[\mathrm{L}^{\mathrm{pyr}} \mathrm{Ni}_{2}(\mu-\mathrm{OH})\right]$. 


\subsection{Summary}

Analogous to the previously discussed $\left[\mathbf{L}^{\mathbf{p y r}} \mathbf{N i}_{2}(\mu-\mathrm{OH})\right]$, the $\mu$-hydroxo complex $\left[\mathbf{L}^{\text {bisnac }} \mathbf{N i}_{\mathbf{2}}(\mu-\mathrm{OH})\right]$ was synthesized. Similarities between both ligand systems $\mathbf{H}_{\mathbf{3}} \mathbf{L}^{\mathbf{p y r}}$ and $\mathbf{H}_{3} \mathbf{L}^{\text {bisnac }}$ (hexadentate, trianionic) lead to comparable molecular structures and ${ }^{1} \mathrm{H}$ NMR chemical shifts. In particular, the arrangement of the DIPP moieties of $\left[\mathbf{L}^{\text {bisnac }} \mathbf{N i}_{\mathbf{2}}(\mu-\mathrm{OH})\right]$ leads to an upfield shift of the proton resonance of the hydroxo $\operatorname{group}\left(\delta\left\{{ }^{1} \mathrm{H}\right\}_{\mathrm{OH}}=-7.3 \mathrm{ppm}\right)$, which is consistent with $\left[\mathbf{L}^{\mathbf{p y r}} \mathbf{N i}_{\mathbf{2}}(\boldsymbol{\mu}-\mathbf{O H})\right]\left(\delta\left\{{ }^{1} \mathrm{H}\right\}_{\mathrm{OH}}=\right.$ $-6.7 \mathrm{ppm})$. The strong electronic shielding of the hydroxo-hydrogen is, besides the aromatic ring-currents of the DIPP moieties, mainly based on the electron-rich nature of both ligand systems. Despite the many similarities of both $\mu$-complexes, the exchange behaviour with water is different. While $\left[\mathbf{L}^{\mathrm{pyr}} \mathbf{N i}_{\mathbf{2}}(\mu-\mathbf{O H})\right]$ exhibits an extremely slow exchange with $\mathrm{D}_{2} \mathrm{O}$, the "nacnac"-based analogue $\left[\mathbf{L}^{\text {bisnac }} \mathbf{N i}_{\mathbf{2}}(\mu-\mathbf{O H})\right]$ undergoes rapid exchange. This effect can be explained by the larger $\mathrm{Ni} \cdots \mathrm{Ni}$ distance allowing for the facile formation of the $\mathrm{HO} \cdots \mathrm{HOH}$ intermediate and the steric shielding of the hydroxo group, which is less in $\left[\mathbf{L}^{\text {bisnac }} \mathbf{N i}_{\mathbf{2}}(\boldsymbol{\mu}-\mathbf{O H})\right]$ due to the large metal-metal distance and a slight twist of the complex. 



\section{A Dinuclear Nickel Dihydride Complex}

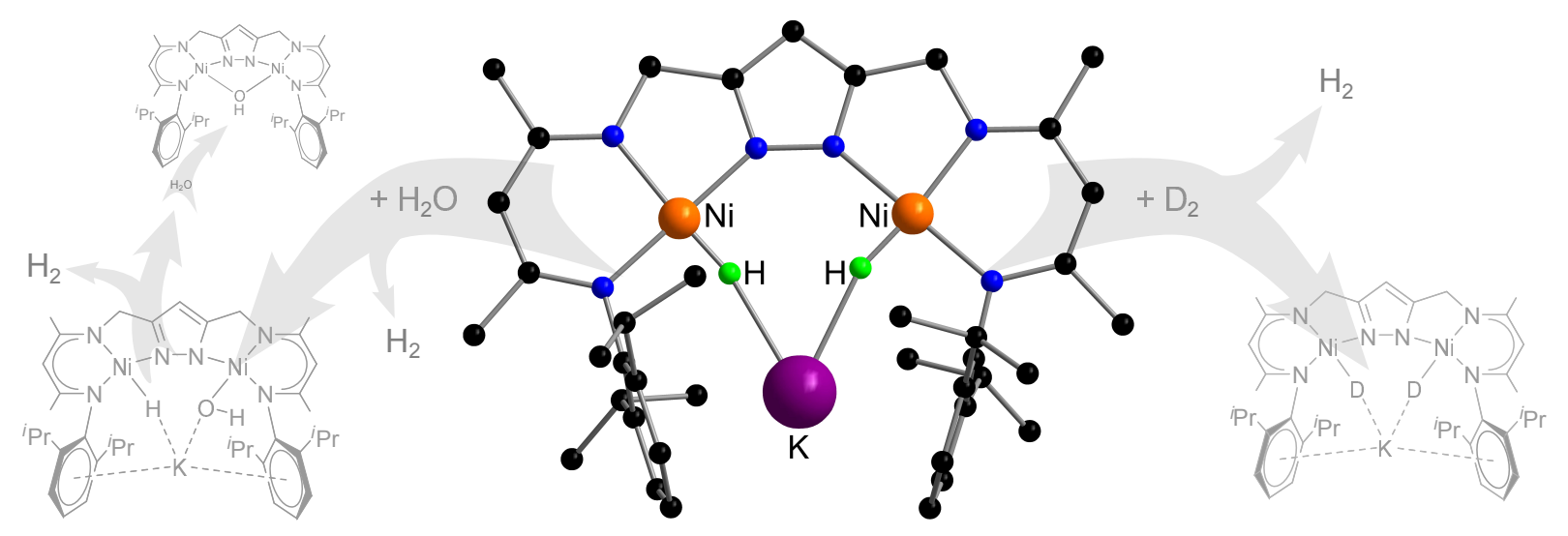

Abstract The bromide bridged complex $\left[\mathbf{L}^{\text {bisnac }} \mathbf{N i}_{2}(\mu-\mathbf{B r})\right]$ was converted with potassium triethylborohydride to give an anionic, dinuclear nickel dihydride complex $\mathbf{K}\left[\mathbf{L}^{\text {bisnac }} \mathbf{N i}_{\mathbf{2}}(\mathbf{H})_{\mathbf{2}}\right]$. Exposure to a $\mathrm{D}_{2}$ atmosphere led to an unusual H-D exchange behaviour eventually giving $\mathbf{K}\left[\mathbf{L}^{\text {bisnac }} \mathbf{N i}_{\mathbf{2}}(\mathbf{D})_{\mathbf{2}}\right]$. Furthermore, it was found that the reaction of $\mathbf{K}\left[\mathbf{L}^{\text {bisnac }} \mathbf{N i}_{\mathbf{2}}(\mathbf{H})_{\mathbf{2}}\right]$ with water resulted in the formation of a hydroxo-hydride species $\mathbf{K}\left[\mathbf{L}^{\text {bisnac }} \mathbf{N i}_{\mathbf{2}}(\mathbf{H})(\mathbf{O H})\right]$ and the release of $\mathrm{H}_{2}$. In addition to the characterization of the dihydride complex, this chapter deals with the investigation of the H-D exchange and the reaction with water as well. Isotopic labeling experiments were evaluated by means of different NMR techniques. DFT calculations provided insight into the mechanistic nature of these reactions. 


\subsection{State of Knowledge}

\section{Nickel Hydride Complexes}

The growing need to develop catalytic processes using earth-abundant metals including nickel, utilize dihydrogen as an energy carrier, and understand the active sites of nickelcontaining enzymes such as [NiFe] hydrogenase and methyl-coenzyme M reductase gave rise to a large variety of structures and reactivity of nickel hydride complexes. ${ }^{[03]}$

In particular, nickel hydride complexes of chelating ligands with bulky substituents which are remarkably stable have received great interest. The stability of these complexes is due to the sterical protection of the $\mathrm{Ni}-\mathrm{H}$ moiety, provided by the bulky substituens of the ligands. $\stackrel{[103.104]}{[}$ For example, nickel bromide complexes of the chelating alkylphosphine ligands dippe (dippe $\left.={ }^{i} \mathrm{Pr}_{2} \mathrm{PCH}_{2} \mathrm{CH}_{2} \mathrm{P}^{i} \mathrm{Pr}_{2}\right)$ and dcpe $\left(\right.$ dcpe $\left.=\mathrm{Cy}_{2} \mathrm{PCH}_{2} \mathrm{CH}_{2} \mathrm{PCy}_{2}\right)$ are converted cleanly to $\mathrm{Ni}^{\mathrm{II}}$ trihydride complexes with an excess of $\mathrm{NaBH}_{4}$ (Scheme 4.1) ${ }^{\underline{[105]}}$

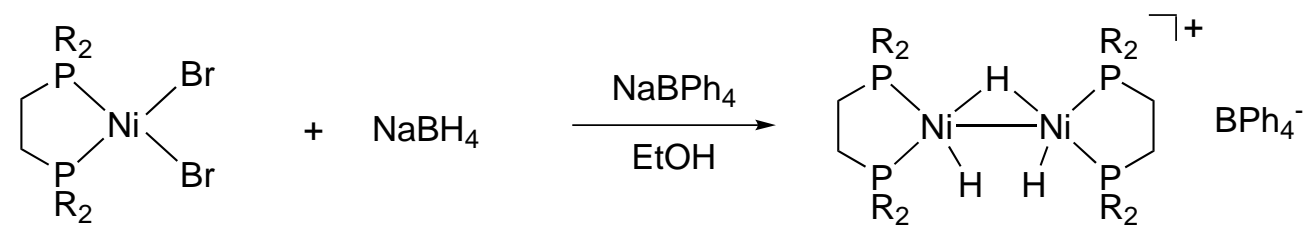
$\left(\mathrm{R}={ }^{\mathrm{i}} \mathrm{Pr}, \mathrm{Cy}\right)$

Scheme 4.1: Preparation of a Nill trihydride complex. $\stackrel{[103,105]}{ }$

Nitrogen-based ligands are also suitable for the formation of stable nickel hydride species, provided that the nitrogen substituents are sufficiently bulky. ${ }^{[103]}$ Bromide complexes of anionic $\beta$-diketiminate $\left(\mathrm{NacNac}\right.$ ) ligandsystems maintain the $\mathrm{Ni}^{\mathrm{II}}$ oxidation state after initial hydride transfer from $\mathrm{KHBEt}_{3}$ (Super-Hydride) resulting in a bridging nickel hydride (Scheme 4.2).

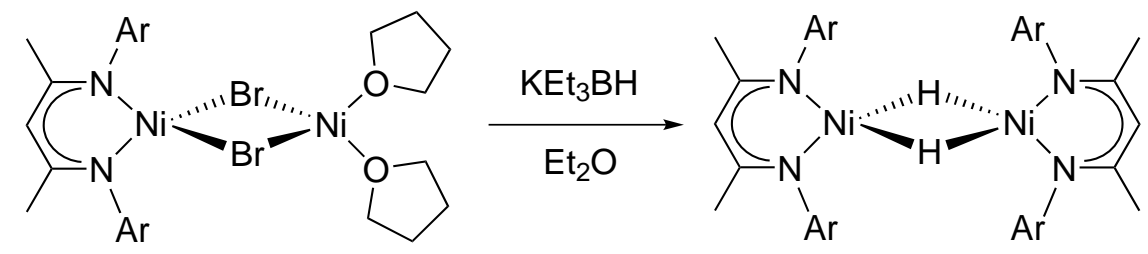

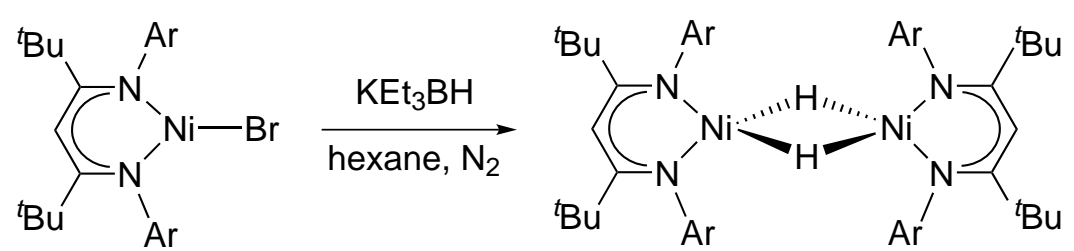<smiles>Brc1ccc(Br)c(CCC(Br)Br)c1Br</smiles>

Scheme 4.2: Dinuclear nickel hydride complexes of $\beta$-diketimininate ligands. 
As already mentioned in Chapter 2, a $\mu$-Br dinickel complex of a preorganized $\beta$-diketiminate ligandsystem with pyridine as a linker unit gave rise to a mixed valent hydride-bridged complex after treatment with $\mathrm{KHBEt}_{3}(\underline{\underline{\text { Scheme 2.4 }}}) .{ }^{[\underline{98}]}$

\section{Model Complexes for [NiFe] Hydrogenase}

Nature uses nickel to build [NiFe] hydrogenases for proton reduction and hydrogen oxidation. $\stackrel{[106.107]}{ }$ Since the nickel site is believed to be the primary dihydrogen binding site, $\underline{[108]}$ many chemists were inspired to design functional nickel complexes as models for [NiFe] hydrogenase. $\stackrel{[108-111]}{\underline{n}}$ Furthermore, due to the fact that the active site of hydrogenases rivals platinum as a catalyst for hydrogen production and uptake, $\stackrel{[112]}{\underline{[1}}$ there is much focus on the catalytic hydrogen production of these mimics. ${ }^{[113,114]}$ However, model complexes for catalytic proton reduction with water as a proton source are yet unknown. ${ }^{[106]}$

\section{Liberation of $\mathrm{H}_{2}$ from Water Mediated by Hydride Complexes}

Transition metal hydride complexes play an important catalytic role in a variety of synthetically important reactions and in biology. ${ }^{[115]}$ Since sustainable energy supply is the most important issue of the 21 st century, ${ }^{[116]}$ the search for clean and renewable sources of energy for the future has also insipired many chemists. $\stackrel{[117,118]}{ }$ In particular, the production of hydrogen from water under mild conditions has received great interest. ${ }^{[119,120]}$ In 2009 MiLstein $^{[121]}$ and coworkers established a monomeric pincer $\mathrm{Ru}^{\mathrm{II}}$ hydride catalyst capable of water splitting reactions. It was found that the Milstein catalyst facilitates the $\mathrm{H} \cdots \mathrm{H}$ interaction with water molecules leading to an outer sphere mechanism for $\mathrm{H}_{2}$ elimination.

\section{Exchange of Hydride Ligands with Coordinated $\mathrm{H}_{2}$}

An effective intramolecular site exchange of $\mathrm{H}$ atoms between $\eta^{2}-\mathrm{H}_{2}$ ligands and hydride ligands is commonly observed. $\stackrel{[122-127]}{=}$ Due to a low barrier of $\approx 5 \mathrm{kcal} \mathrm{mol}^{-1}$ or less the process is extremely facile. ${ }^{[128]}$ This can also be observed in ${ }^{1} \mathrm{H}$ NMR spectroscopy since the process leads to a single hydride resonance. Exchange reactions of hydride complexes in the protio form with $\mathrm{D}_{2}$ gas usually leads to the incorporation of deuterium and the formation of HD. $\stackrel{[128,129]}{ }$ According to Scheme 4.3 the $\mathrm{H}_{2} / \mathrm{H}^{-}$exchange can be considered as an example for heterolytic cleavage of the $\eta^{2}-\mathrm{H}_{2}$ ligand. It can be mediated by the protonation of the hydride ligand with $\eta^{2}-\mathrm{H}_{2}$ acting as an internal acid. The transition state structure for the rearrangement often features a linear or nearly linear orientation of three hydrogen atoms representing a $\mathrm{H}_{3}{ }^{-}$ligand. Eventually the exchange gives a new hydride and a new $\eta^{2}-\mathrm{H}_{2}$ ligand as well. 
Oxidative addition of the $\eta^{2}-\mathrm{H}_{2}$ ligand represents an alternative mechanism for the $\mathrm{H}_{2} / \mathrm{H}^{-}$ exchange. This mechanism leads to a tris-hydride intermediate and the pairwise reductive elimination of hydride ligands. ${ }^{[129]}$

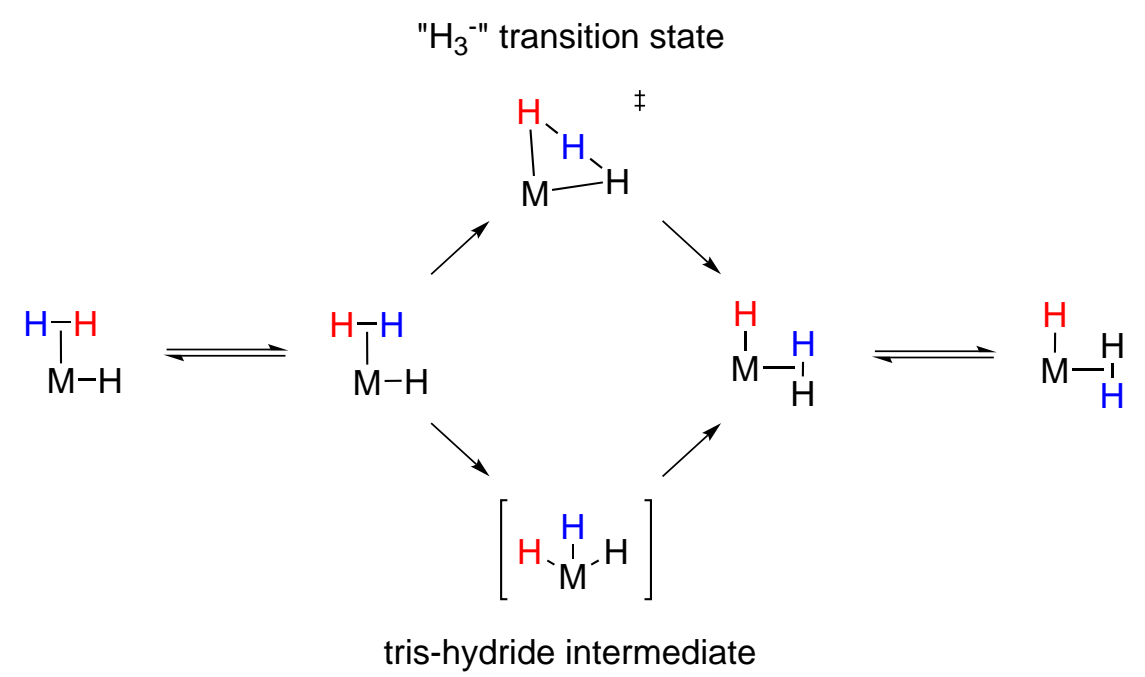

Scheme 4.3: Possible mechansims of $\mathrm{H}$-atom exchange for a complex featuring a hydride and $\mathrm{a} \eta^{2}-\mathrm{H}_{2}$ ligand. It can lead over $\mathrm{a}_{3}{ }^{-}$transition state (top) or a tris-hydride intermediate as a result of oxidative addition (bottom). In both mechanisms a rotation of the $\eta^{2}-H_{2}$ ligand is required for an exchange. ${ }^{[129]}$

\subsection{Motivation}

Since $\beta$-diketiminate ligands are capable of stabilizing high and low oxidation states and form complexes with low coordination numbers, the subsequent interest has given rise to new hydride complexes based on inexpensive nickel. ${ }^{[33,83,84,98]}$ As previously mentioned (Chapter 2) LIMBERG et al. reported a bimetallic nickel hydride complex comprised of preorganized $\beta$-diketiminato-moieties. ${ }^{[98]}$ The PYR ligand used, is the pyridine linked analogue to the pyrazole linked $\mathbf{H}_{3} \mathbf{L}^{\text {bisnac }}$, used in this work. Therefore, the preparation and isolation of a new nickel hydride complex starting from $\left[\mathbf{L}^{\text {bisnac }} \mathbf{N i}_{2}(\mu-\mathbf{B r})\right]$ was envisioned. However, the metal $\cdots$ metal distance in $\left[\mathbf{L}^{\mathbf{b i s n a c}} \mathbf{N i}_{\mathbf{2}}(\mu-\mathbf{B r})\right]\left(d_{\mathrm{Ni} \cdots \mathrm{Ni}}=\right.$ $3.81 \AA)$ is significantly longer than the one observed for the PYR based system $\left(d_{\mathrm{Ni} \cdots \mathrm{Ni}}=\right.$ $3.43 \AA) .{ }^{[98]}$ For this reason, in contrast to the PYR based hydride complex, the formation of a non-bridged system was expected. 


\subsection{Preparation of $\mathrm{K}\left[\mathrm{L}^{\text {bisnac }} \mathrm{Ni}_{2}(\mathrm{H})_{2}\right]$}

The reaction of $\left[\mathbf{L}^{\text {bisnac }} \mathbf{N i}_{\mathbf{2}}(\boldsymbol{\mu}-\mathbf{B r})\right]$ with an excess of $\mathrm{KHBEt}_{3}$ (potassium triethylborohydride) yielded an anionic dinuclear $\mathrm{Ni}^{\mathrm{II}}$ dihydride complex (Scheme 4.4). After addition of the hydride the yellowish THF suspension of the precursor complex turned into a deep orange clear solution within 10-60 minutes. The solution was filtered under inert conditions and either a slow diffusion of pentane vapours into the filtrate or layering with hexanes followed by cooling to $-30{ }^{\circ} \mathrm{C}$, led to the formation of orange crystals. The isolated rodor star-shaped single crystals were suitable for X-ray diffraction analysis. The ORTEP diagram of the molecular structure is shown in Figure 4.1 and an overview of selected bond lengths, distances and angles is given in Table 4.1.
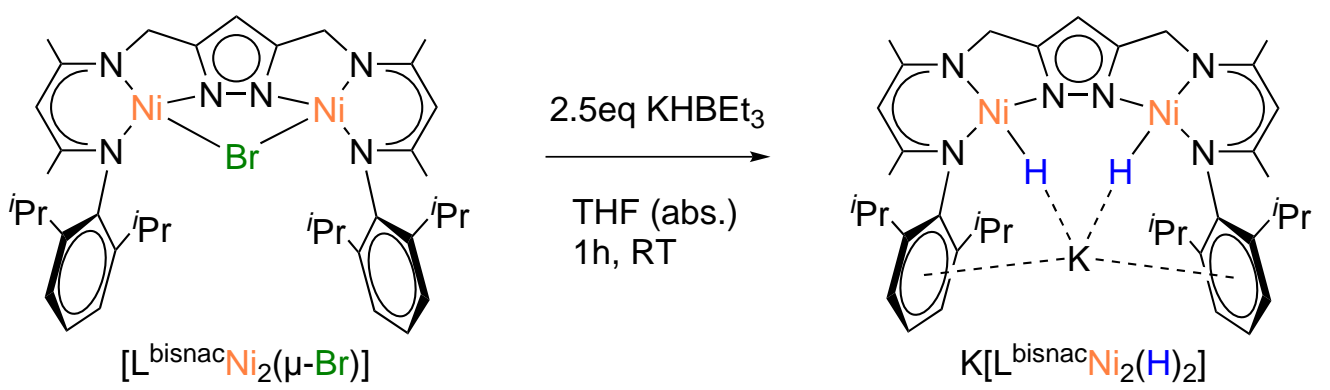

Scheme 4.4: Synthesis of the bimetallic nickel dihydride complex.
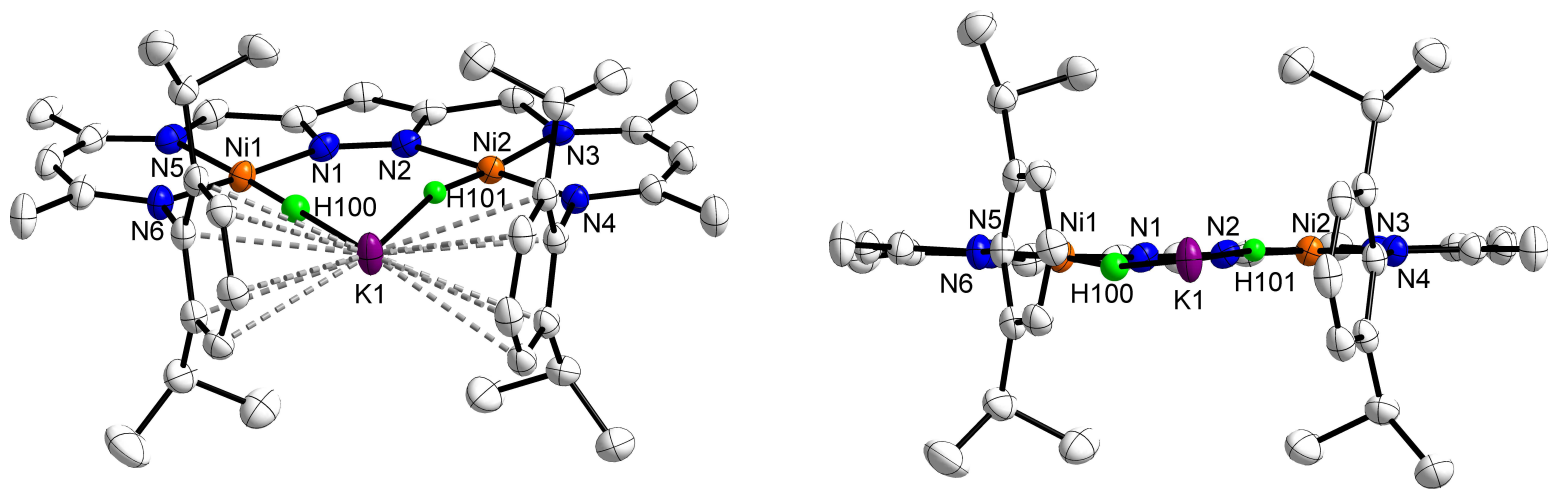

Figure 4.1.: Thermal displacement ellipsoids (shown at $50 \%$ probability) of the molecular structure of $\mathrm{K}\left[\mathrm{L}^{\text {bisnac }} \mathrm{Ni}_{2}(\mathrm{H})_{2}\right]$. All hydrogen atoms except the hydrides have been omitted for clarity (the hydrogen atoms are displayed as ball-and-stick representation with a fixed radius of $0.135 \AA$ ). Left: Top view of the molecular structure. Right: side view to illustrate the square-planar coordination environment.

$\mathbf{K}\left[\mathbf{L}^{\text {bisnac }} \mathbf{N i}_{\mathbf{2}}(\mathbf{H})_{\mathbf{2}}\right]$ contains two nickel centers in the +2 oxidation state. The angles between $\mathrm{Ni}$ and adjacent $\mathrm{N}$ or $\mathrm{H}$ atoms can be separated into two groups: those nearly $180^{\circ}$ (e.g. $\Varangle 172^{\circ}$ for $\mathrm{N} 5-\mathrm{Ni1}-\mathrm{H} 100$ and $\Varangle 178^{\circ}$ for N6-Ni1-N1) and those close to $90^{\circ}$ (e.g. N6-Ni1-N5, $\Varangle 94.93^{\circ}$, Table 4.1), indicative of square-planar geometry. This is in agreement with the expected environment for low-spin $\mathrm{d}^{8}$ metal centers.

The side view of $\mathbf{K}\left[\mathbf{L}^{\text {bisnac }} \mathbf{N i}_{\mathbf{2}}(\mathbf{H})_{\mathbf{2}}\right]$ (Figure 4.1, left) illustrates the nearly ideal square- 
Table 4.1.: Selected bond lengths, distances and angles of $\mathrm{K}\left[\mathrm{L}^{\text {bisnac }} \mathrm{Ni}_{2}(\mathrm{H})_{2}\right]$.

\begin{tabular}{|c|c|c|c|c|c|}
\hline \multicolumn{2}{|c|}{ Bond lengths / $\AA$} & \multicolumn{2}{|c|}{ Distances / Å } & \multicolumn{2}{|c|}{ Angles / ${ }^{\circ}$} \\
\hline Ni1-N6 & $1.881(2)$ & $\mathrm{H} 100 \cdots \mathrm{K} 1$ & $2.54(3)$ & N6-Ni1-N1 & $178.15(9)$ \\
\hline Ni1-N1 & $1.883(2)$ & $\mathrm{H} 101 \cdots \mathrm{K} 1$ & $2.49(3)$ & N6-Ni1-N5 & $94.93(9)$ \\
\hline Ni1-N5 & $1.9114(2)$ & $\mathrm{Ni} 1 \ldots \mathrm{K} 1$ & $3.8304(9)$ & N1-Ni1-N5 & $85.12(9)$ \\
\hline Ni2-N4 & $1.8811(2)$ & $\mathrm{Ni} 2 \ldots \mathrm{K} 1$ & $3.8164(1)$ & N6-Ni1-H100 & $89.638(1)$ \\
\hline Ni2-N2 & $1.8851(2)$ & $\mathrm{Ni} 1 \ldots \mathrm{Ni} 2$ & $4.1586(1)$ & $\mathrm{N} 1-\mathrm{Ni1}-\mathrm{H} 100$ & $90.505(1)$ \\
\hline Ni2-N3 & $1.909(2)$ & $\mathrm{H} 100 \cdots \mathrm{H} 101$ & $2.3485(4)$ & N5-Ni1-H100 & $172.447(1)$ \\
\hline $\mathrm{Ni1}-\mathrm{H} 100$ & $1.33(3)$ & $\mathrm{K} 1 \cdots \mathrm{Ar}^{\mathrm{N} 4 \dagger}$ & $2.8333(7)$ & N4-Ni2-N2 & 178.37(8) \\
\hline \multirow[t]{7}{*}{$\mathrm{Ni2}-\mathrm{H} 101$} & $1.37(3)$ & $\mathrm{K} 1 \cdots \mathrm{Ar}^{\mathrm{N} 6 \dagger}$ & $2.8502(1)$ & N4-Ni2-N3 & $94.81(8)$ \\
\hline & & & & N2-Ni2-N3 & $85.04(8)$ \\
\hline & & & & N4-Ni2-H101 & $91.664(1)$ \\
\hline & & & & N2-Ni2-H101 & $88.524(1)$ \\
\hline & & & & N3-Ni2-H101 & 173.381(1) \\
\hline & & & & $\mathrm{Ni1-H100-K1}$ & $163.368(2)$ \\
\hline & & & & $\mathrm{Ni2}-\mathrm{H} 101-\mathrm{K} 1$ & $162.332(2)$ \\
\hline
\end{tabular}

${ }^{\dagger}$ Measured distance of the aryl centroids (DIPP-N4 and -N6) to the potassium cation.

planar coordination of both nickel centers. As can be seen, the compound exhibits two hydrides that give the complex a negative charge. A potassium cation, which serves as a counterion, is positioned in the plane spanned by the two metal centers and their directly bound atoms. In addition to coordinating both hydride ligands, it is also coordinated to the two diisopropylphenyl sidearms via cation- $\pi$ interactions. Figure 4.2 illustrates the coordination of the DIPP moieties to the cation $(\bullet)$ with indicated distances $(\cdots)$ to their centroids $(\bullet)$.

The aryl-alkali metal distance is $\approx 2.84 \AA$, which is within the range for cation- $\pi$ bound potassium to aromatic systems. $\stackrel{[130,131]}{ }$ Moreover, the potassium to aryl-carbon distances ranging from 3.120 to $3.237 \AA\left(d(\varnothing)_{\mathrm{K} 1 \cdots \mathrm{C}}=3.17 \AA\right)$ compare to previously reported aryl sandwiched potassium cations. $\underline{\underline{[132} \underline{134.136]}]}$

A similar nacnac-based system has been reported by LIMBERG et al., which is a dimer of two mononuclear nacnac-based $\mathrm{Ni}^{\mathrm{I}}$ complexes bridged by a $\mathrm{N}_{2}$ unit with diazene like character. ${ }^{\left[{ }^{81]}\right]}$ In this case the dimer contains two potassium cations, which electrostatically interact with the $\pi$ clouds of two DIPP moieties. The distances between the cations to the centroids of the two adjacent DIPP units are 2.712 and $2.764 \AA$, slightly shorter than observed for $\mathbf{K}\left[\mathbf{L}^{\text {bisnac }} \mathbf{N i}_{2}(\mathbf{H})_{2}\right] .{ }^{[81]}$

$\left.\mathbf{K}\left[\mathbf{L}^{\text {bisnac }} \mathbf{N i}_{\mathbf{2}} \mathbf{( H}\right)_{2}\right]$ exhibits a $\mathrm{Ni} \cdots \mathrm{Ni}$ distance of $4.159 \AA$, which is within the range of other literature known dinuclear nickel complexes of pyrazolate based ligand systems $(3.501-4.587 \AA) . \stackrel{[10]}{=}$ Nonetheless, compared to its precursor $\left[\mathbf{L}^{\mathbf{b i s n a c}} \mathbf{N i}_{\mathbf{2}}(\mu-\mathbf{B r})\right]\left(d_{\mathrm{Ni} \cdots \mathrm{Ni}}\right.$ 


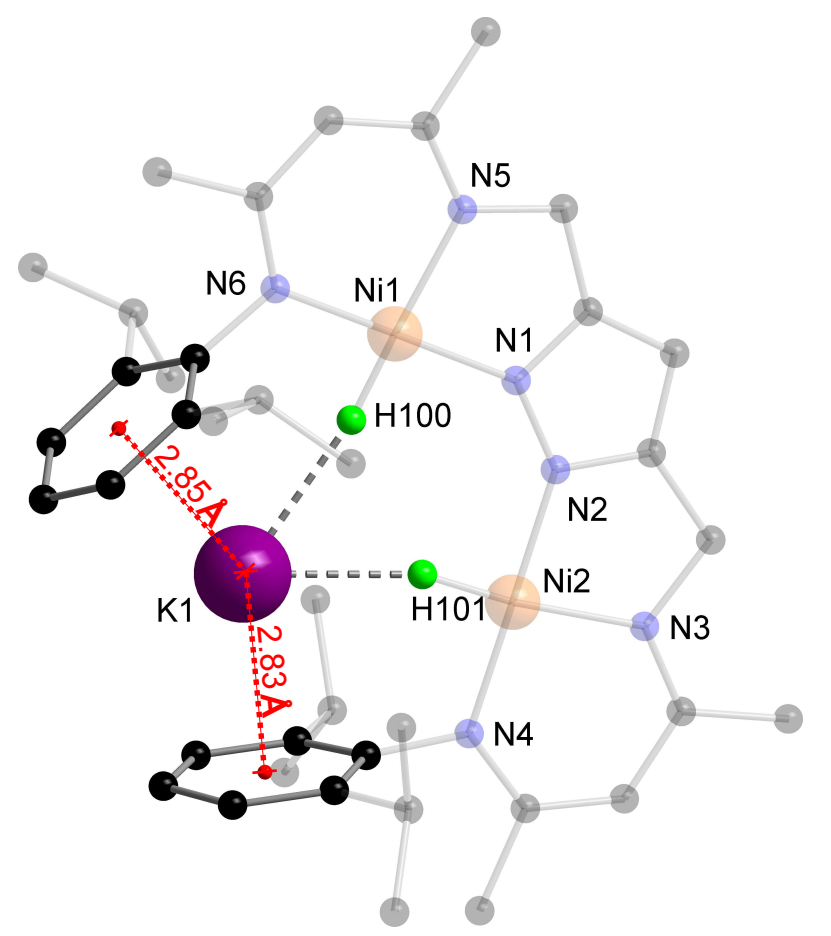

Figure 4.2.: The distances between the potassium cation $\mathrm{K} 1(\bullet)$ to the centroids $(\bullet)$ of both aryl sidearms illustrated on the molecular structure of $\mathrm{K}\left[\mathrm{L}^{\text {bisnac }} \mathrm{Ni}_{2}(\mathrm{H})_{2}\right]$. Remaining atoms are depicted in a transparent fashion.

$=3.807 \AA$ ) the distance is substantially longer. This observation can be explained by the potassium cation, which is bridging the $\mathrm{Ni}-\mathrm{H}$ hydrogen atoms. Due to the additional steric demand of the cation, a stretch of the cavity results, increasing the distance between the metal centers. An analoguous dihydride complex with sodium instead of potassium was prepared, $\stackrel{[135]}{\longrightarrow}$ showing a similar $\mathrm{Ni}$... Ni distance of $4.105 \AA$. Accordingly, the sodium cation is bridging both $\mathrm{Ni}-\mathrm{H}$ hydrogen atoms. However, in this case the alkali cation is not coordinated by the DIPP moieties but instead two THF molecules.

Like $\left[\mathbf{L}^{\text {bisnac }} \mathbf{N i}_{\mathbf{2}}(\mu-\mathbf{B r})\right]$ the dihydride complex contains two Ni centers in a $\mathrm{d}^{8}$ low spin configuration. NMR spectroscopy is a powerful tool for the characterization and investigation of reactions associated with the diamagnetic $\mathbf{K}\left[\mathbf{L}^{\text {bisnac }} \mathbf{N i}_{\mathbf{2}}(\mathbf{H})_{\mathbf{2}}\right]$. The ${ }^{1} \mathrm{H}$ NMR spectrum (measured in THF-D $_{8}$ ) revealed a sharp upfield shifted resonance at $-24.17 \mathrm{ppm}$ (green mark in Figure 4.3), corresponding to the hydride ligands with a normalized integral of two. Since the dihydride compound exhibits a symmetric geometry, the corresponding spectrum features a relatively simple set of signals with one upfield singlet representing both hydrides. The sodium analogue $\mathbf{N a}\left[\mathbf{L}^{\text {bisnac }} \mathbf{N i}_{\mathbf{2}}(\mathbf{H})_{2}\right]^{[135]}$ shows a comparable hydride associated resonance at $-23.54 \mathrm{ppm} . \stackrel{[135]}{.}$

The hydride chemical shift for $\mathbf{K}\left[\mathbf{L}^{\text {bisnac }} \mathbf{N i}_{\mathbf{2}}(\mathbf{H})_{\mathbf{2}}\right]$ and its sodium analogue lies within the typical range of -5 to $-30 \mathrm{ppm}$ of reported nickel-hydrides. $\stackrel{[136-143]}{\underline{3}}$ Such an upfield shifted resonance is indicative of strong electronic shielding, which is provided by the electron 


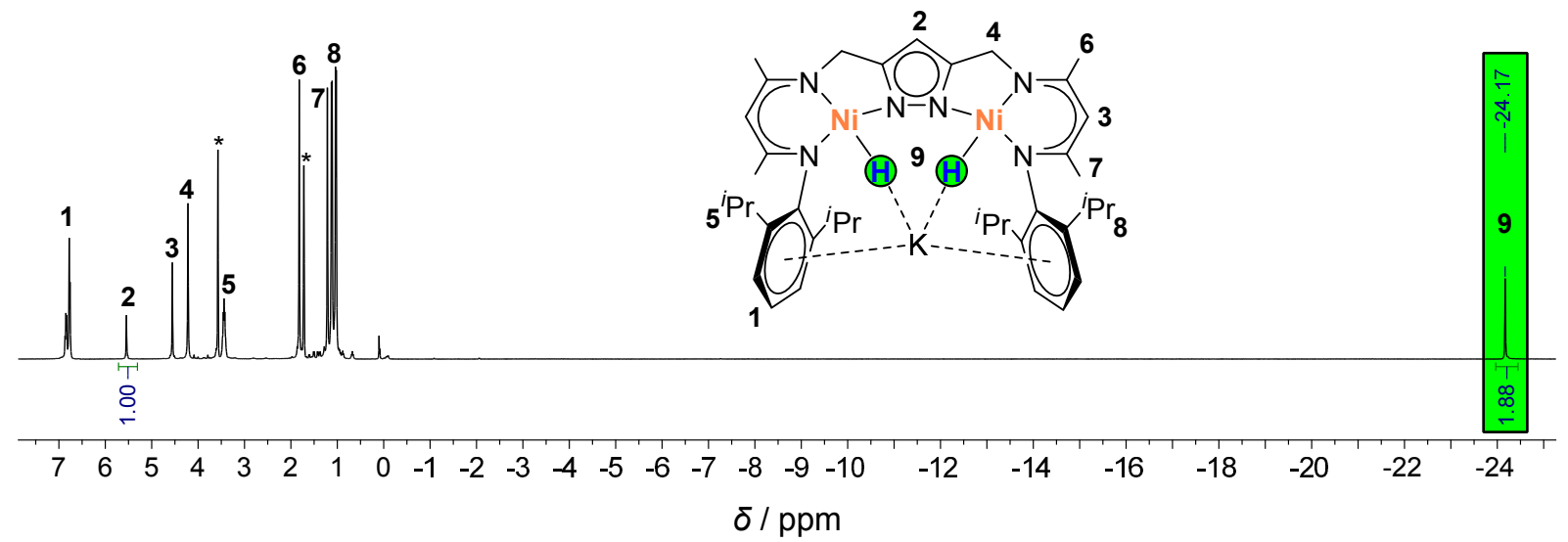

Figure 4.3.: ${ }^{1} \mathrm{H}$ NMR spectrum $(400 \mathrm{MHz})$ of $K\left[\mathrm{~L}^{\text {bisnac }} \mathrm{Ni}_{2}(\mathbf{H})_{2}\right]$. Residual solvent resonances $\left(T H F-D_{7}\right)$ are marked $\left({ }^{*}\right)$.

rich ligand system. This is also in accordance with the previously discussed results for the $\mu$-hydroxo complexes $\left[\mathrm{L}^{\mathrm{pyr}} \mathrm{Ni}_{\mathbf{2}}(\mu-\mathrm{OH})\right]$ and $\left[\mathrm{L}^{\text {bisnac }} \mathbf{N i}_{\mathbf{2}}(\mu-\mathrm{OH})\right]$. Furthermore, the alkali cation, which lies in a bridging position between the two hydrides also contributes to the pronounced upfield shift. The positive charge pulls electron density of the whole system into the proximity of the hydrides, which results in enhanced electronic shielding. This also results in a stronger metal-hydride bond which is reflected by the relatively short $\mathrm{Ni}-\mathrm{H}$ bond length (Table 4.1).

NMR spectroscopy also makes it possible to elucidate the surroundings of certain hydrogen atoms by NOESY experiments. A contour plot in Figure 4.4 illustrates NOE interactions between the metal bound hydrogen atoms and the ligand protons in close spatial proximity. The intenstiy of the corresponding cross correlations reflects the relative spatial distance between the hydrogen atoms. The strongest NOE interaction is observed for the protons of the isopropyl methyl groups (a) whereas the weakest cross peak signal was from the hydrogen atoms of the aromatic rings (c). This corresponds to the molecular structure of $\mathbf{K}\left[\mathbf{L}^{\text {bisnac }} \mathbf{N i}_{\mathbf{2}}(\mathbf{H})_{\mathbf{2}}\right]$ (Figure 4.1) and indicates structural integrity in solution.

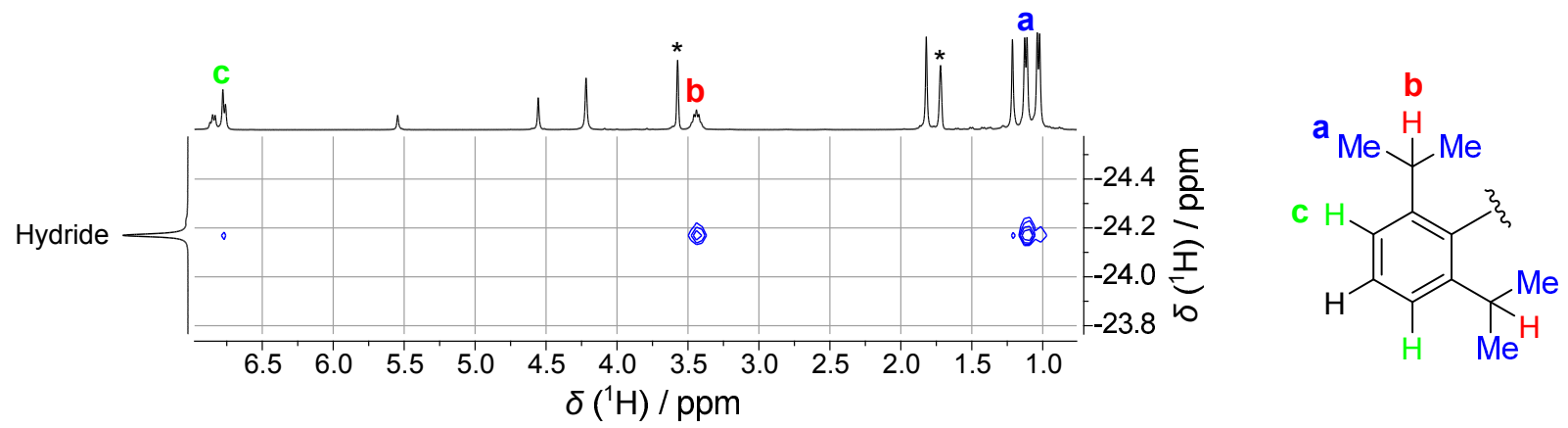

Figure 4.4.: Excerpt of the ${ }^{1} \mathrm{H}$ NOESY spectrum $\left(400 \mathrm{MHz}\right.$ ) recorded for $\mathrm{K}\left[\mathrm{L}^{\text {bisnac }} \mathrm{Ni}_{2}(\mathrm{H})_{2}\right]$ in THF- $\mathrm{D}_{8}$. Residual solvent resonances $\left(\mathrm{THF}-\mathrm{D}_{7}\right)$ are marked $\left({ }^{*}\right)$.

$\mathbf{K}\left[\mathbf{L}^{\text {bisnac }} \mathbf{N i}_{\mathbf{2}}(\mathbf{H})_{\mathbf{2}}\right]$ features a $\mathrm{Ni}-\mathrm{H}$ stretching vibration at $\tilde{\nu}=1961 \mathrm{~cm}^{-1}$, which is slightly larger than the $\mathrm{Ni}-\mathrm{H}$ bands for other reported hydride complexes 
$\left(\tilde{\nu}_{\mathrm{Ni}-\mathrm{H}} \approx 1768-1950 \mathrm{~cm}^{-1}\right) \cdot \stackrel{[144,145]}{1}^{2}$ Nonetheless, this is in accordance with the relatively short Ni-H bond length.

\subsection{Exchange with $D_{2}$}

Exposure of a degassed solution of $\mathbf{K}\left[\mathbf{L}^{\text {bisnac }} \mathbf{N i}_{\mathbf{2}}(\mathbf{H})_{\mathbf{2}}\right]$ in THF or THF-D 8 to a $\mathrm{D}_{2}$ atmosphere led to the $\mathrm{H}-\mathrm{D}$ exchange mediated formation of the corresponding dideuteride compound $\mathbf{K}\left[\mathbf{L}^{\text {bisnac }} \mathbf{N i}_{\mathbf{2}}(\mathbf{D})_{2}\right]$. Due to the susceptibility of the dihydride compound towards traces of water, the $\mathrm{D}_{2}$ gas had to be dried before use for the exchange experiments (a description of the used apparatus is found in D.1).

An exchange reaction with $\mathrm{D}_{2}$ can occur via two conceivable pathways which are illustrated in Scheme 4.6. One possible route describes a reaction which consists of two steps (pathway 1, Scheme 4.6). First, an intermediate of $\mathbf{K}\left[\mathbf{L}^{\mathbf{b i s n a c}} \mathbf{N i}_{\mathbf{2}}(\mathbf{H})(\mathbf{D})\right]$ is formed, which is thought to cause a slight shift of the hydride resonance in the ${ }^{1} \mathrm{H}$ NMR spectrum. After the release of HD, the intermediate can further react with another molecule of $\mathrm{D}_{2}$ (or to a lesser extent with HD) to the final compound. This is accompanied by the release of another equivalent of $\mathrm{HD}$.

Following the second probable route (pathway, Scheme 4.6), one molecule of $\mathrm{D}_{2}$ yields the dideuteride complex in a single step accompanied by the release of one molecule of $\mathrm{H}_{2}$.

NMR spectroscopic investigations showed that the exchange involves the pairwise substitution of both Ni-H atoms with the $\mathrm{D}_{2}$ (Scheme 4.5).
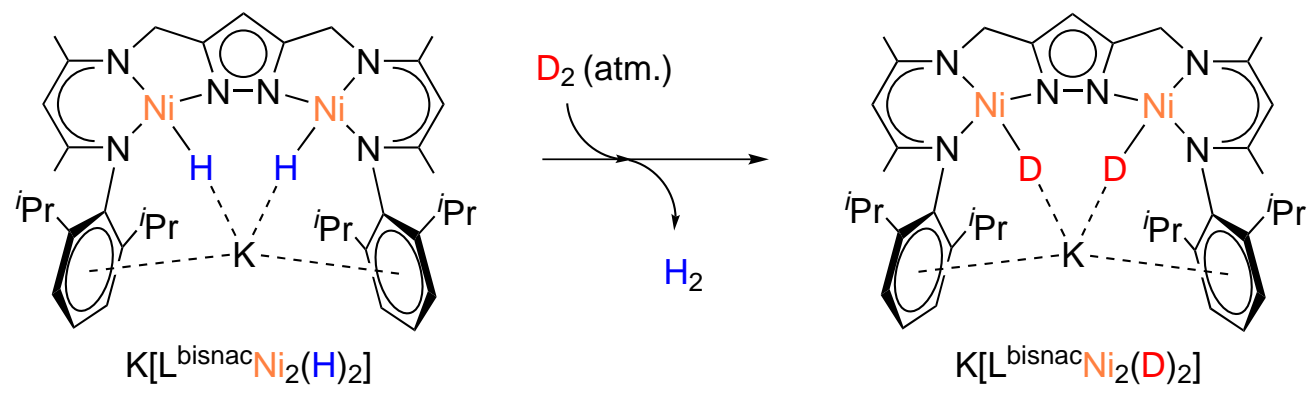

Scheme 4.5: Exchange reaction of the dihydride complex $K\left[L^{\text {bisnac }} \mathrm{Ni}_{2}(H)_{2}\right]$ with $D_{2}$ to the dideuteride complex $\mathrm{K}\left[\mathrm{L}^{\text {bisnac }} \mathrm{Ni}_{2}(\mathrm{D})_{2}\right]$. 
pathway 1
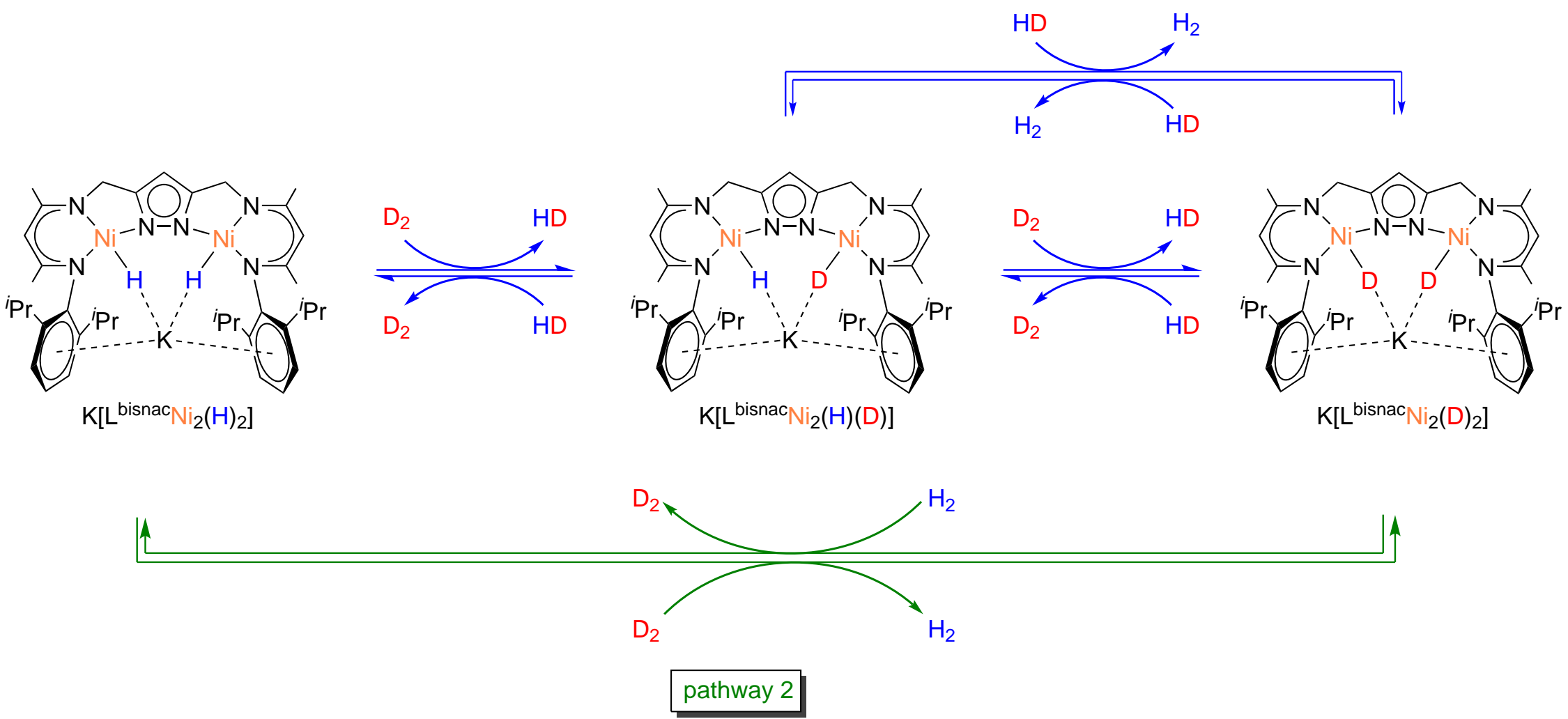

Scheme 4.6.: Proposed reaction pathways for the $\mathrm{H}-\mathrm{D}$ exchange of $\mathrm{K}\left[\mathrm{L}^{\text {bisnac }} \mathrm{Ni}_{2}(\mathrm{H})_{2}\right]$ to $\mathrm{K}\left[\mathrm{L}^{\text {bisnac }} \mathrm{Ni}_{2}(\mathrm{D})_{2}\right]$. The first pathway $\rightarrow$ describes the $\mathrm{H}-\mathrm{D}$ exchange by formation of a monodeuterated intermediate $K\left[L^{\text {bisnac }} \mathrm{Ni}_{2}(\mathrm{HD})\right]$ and concomitant release of $\mathrm{HD}$. This intermediate may then react further with one molecule of $\mathrm{D}_{2}$ or $\mathrm{HD}$ to give the dideuterated compound. The second assumed mechanism $\rightarrow$ describes the formation of $K\left[\mathrm{~L}^{\text {bisnac }} \mathrm{Ni}_{2}(\mathrm{D})_{2}\right]$ in just one step, that is the reaction with one molecule of $D_{2}$ and concomitant release of $H_{2}$. 


\section{Investigation of the Exchange by ${ }^{1} \mathrm{H}$ NMR spectroscopy}

The H-D exchange was followed by means of NMR spectroscopy. As shown in the ${ }^{1} \mathrm{H}$ NMR spectrum, the intensity of the hydride resonance at $-24.17 \mathrm{ppm}$ (spectrum (a) of Figure 4.5 decreases to a minimum immediately after starting the reaction by vigorous shaking of the sample (spectrum (b) Figure 4.5). Furthermore, chemical shifts corresponding to $\mathbf{K}\left[\mathbf{L}^{\text {bisnac }} \mathbf{N i}_{\mathbf{2}}(\mathbf{H})_{2}\right]$ remain unchanged. This indicates the clean transformation of the dihydride to the dideuteride complex.

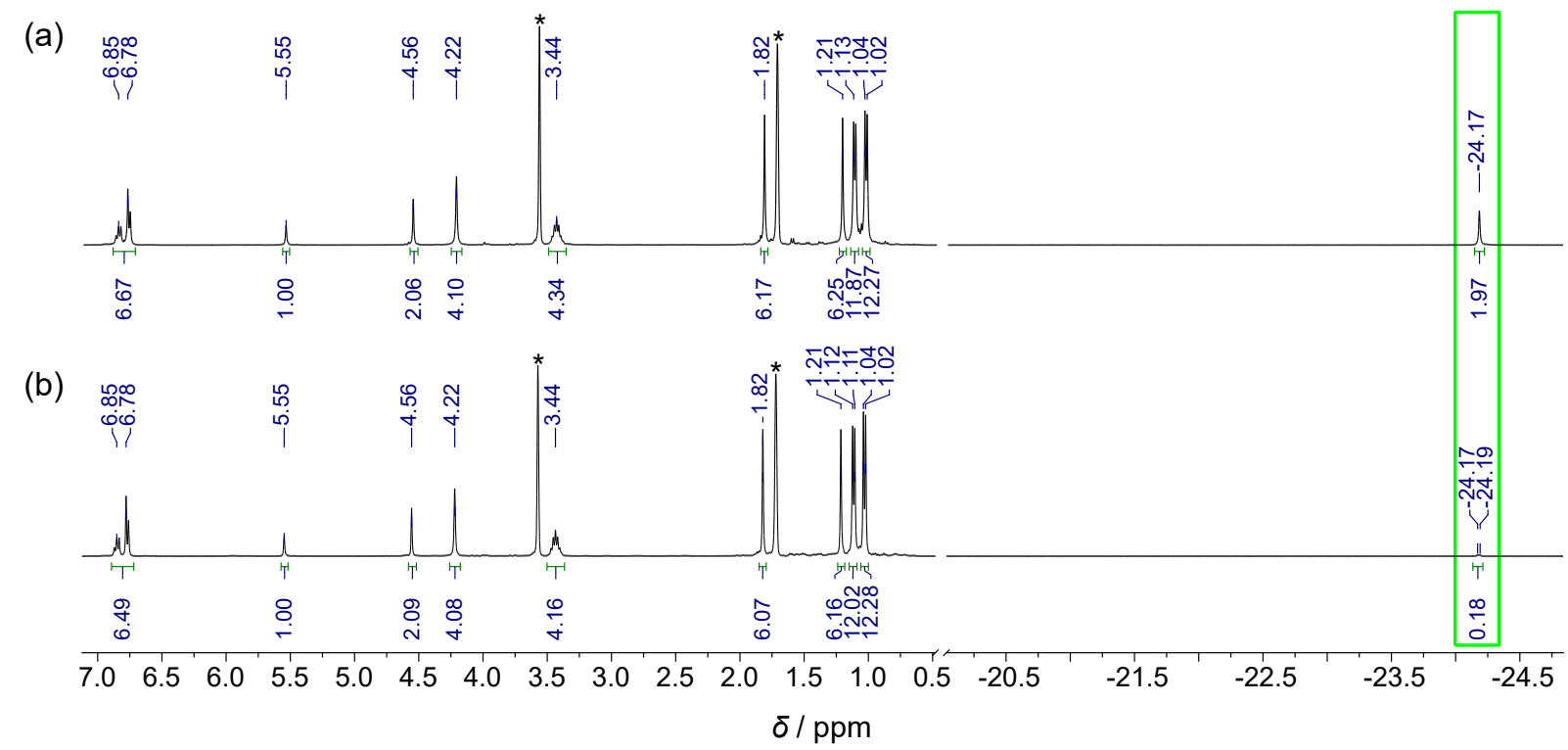

Figure 4.5.: $\mathrm{H}-\mathrm{D}$ exchange of $\mathrm{K}\left[\mathrm{L}^{\text {bisnac }} \mathrm{Ni}_{2}(\mathrm{H})_{2}\right]$ to $\mathrm{K}\left[\mathrm{L}^{\text {bisnac }} \mathrm{Ni}_{2}(\mathrm{D})_{2}\right]$ monitored by ${ }^{1} \mathrm{H} \mathrm{NMR}(400 \mathrm{MHz})$. (a): Spectrum of the THF- $\mathrm{D}_{8}$ solution of $\mathrm{K}\left[\mathrm{L}^{\text {bisnac }} \mathrm{Ni}_{2}(\mathbf{H})_{2}\right]$. (b): Spectrum of the degassed solution treated with atmospheric pressure of dried deuterium gas. The hydride resonance is highlighted $(\square)$ and residual solvents $\left(\right.$ THF- $\left._{7}\right)$ are marked $\left({ }^{*}\right)$.

Figure 4.6, which represents two regions of the ${ }^{1} \mathrm{H}$ NMR spectrum (Figure 4.5) reveals a new signal at $-24.19 \mathrm{ppm}(\square)$. After three days the intensity of this signal slightly increased while the resonance associated with $\mathbf{K}\left[\mathbf{L}^{\text {bisnac }} \mathbf{N i}_{\mathbf{2}}(\mathbf{H})_{\mathbf{2}}\right]$ further decreased. This indicates the formation of further conceivable $\mathrm{Ni}-\mathrm{H}$ species.

On the one hand $\mathbf{K}\left[\mathbf{L}^{\text {bisnac }} \mathbf{N i}_{\mathbf{2}}(\mathbf{H})(\mathbf{D})\right]$ can be formed, which is associated with a scrambling process (pathway 1, Scheme 4.6). On the other hand an intermediate can be formed due to the large excess of $\mathrm{D}_{2}$, which corresponds to $\mathbf{K}\left[\mathbf{L}^{\text {bisnac }} \mathbf{N i}_{\mathbf{2}}(\mathbf{H})_{\mathbf{2}}\left(\mathbf{D}_{\mathbf{2}}\right)\right]$ and involves the non-scrambling process (pathway 2, Scheme 4.6).

As a result of $\mathrm{D}_{2}$ scrambling to $\mathrm{HD}$, a characteristic triplet resonance in the ratio of 1:1:1 was expected..$^{[147-149]}$ However, the marked $(\square)$ region of Figure 4.6 only shows the $\beta$-diketiminato $\mathrm{CH}$-proton resonance at $4.56 \mathrm{ppm}$ with an unchanged normalized integral. Therefore, the resonance at $\delta\left\{{ }^{1} \mathrm{H}\right\}=-24.19 \mathrm{ppm}$ is attributed to $\mathbf{K}\left[\mathbf{L}^{\text {bisnac }} \mathbf{N i}_{\mathbf{2}}(\mathbf{H})_{\mathbf{2}}\left(\mathbf{D}_{\mathbf{2}}\right)\right]$ rather than $\mathbf{K}\left[\mathbf{L}^{\text {bisnac }} \mathbf{N i}_{\mathbf{2}}(\mathbf{H})(\mathbf{D})\right]$.

Suprisingly, no $\mathrm{H}_{2}$ release was observed in the ${ }^{1} \mathrm{H}$ NMR spectrum (b) of Figure 4.6. An as- 
sociated resonance was expected at $\delta\left\{{ }^{1} \mathrm{H}\right\}=4.55 \mathrm{ppm}^{[150]}$ (or 4.54 according to a reference experiment) in THF-D $\mathrm{D}_{8}$. The absence of a ${ }^{1} \mathrm{H}$ NMR signal associated to $\mathrm{H}_{2}$ is explainable by the low amount of released $\mathrm{H}_{2}$ compared to the high amount of $\mathrm{D}_{2}$. Indirect evidence for expulsion of $\mathrm{H}_{2}$ by $\mathrm{D}_{2}$ is provided by another experiment, which is mentioned below.

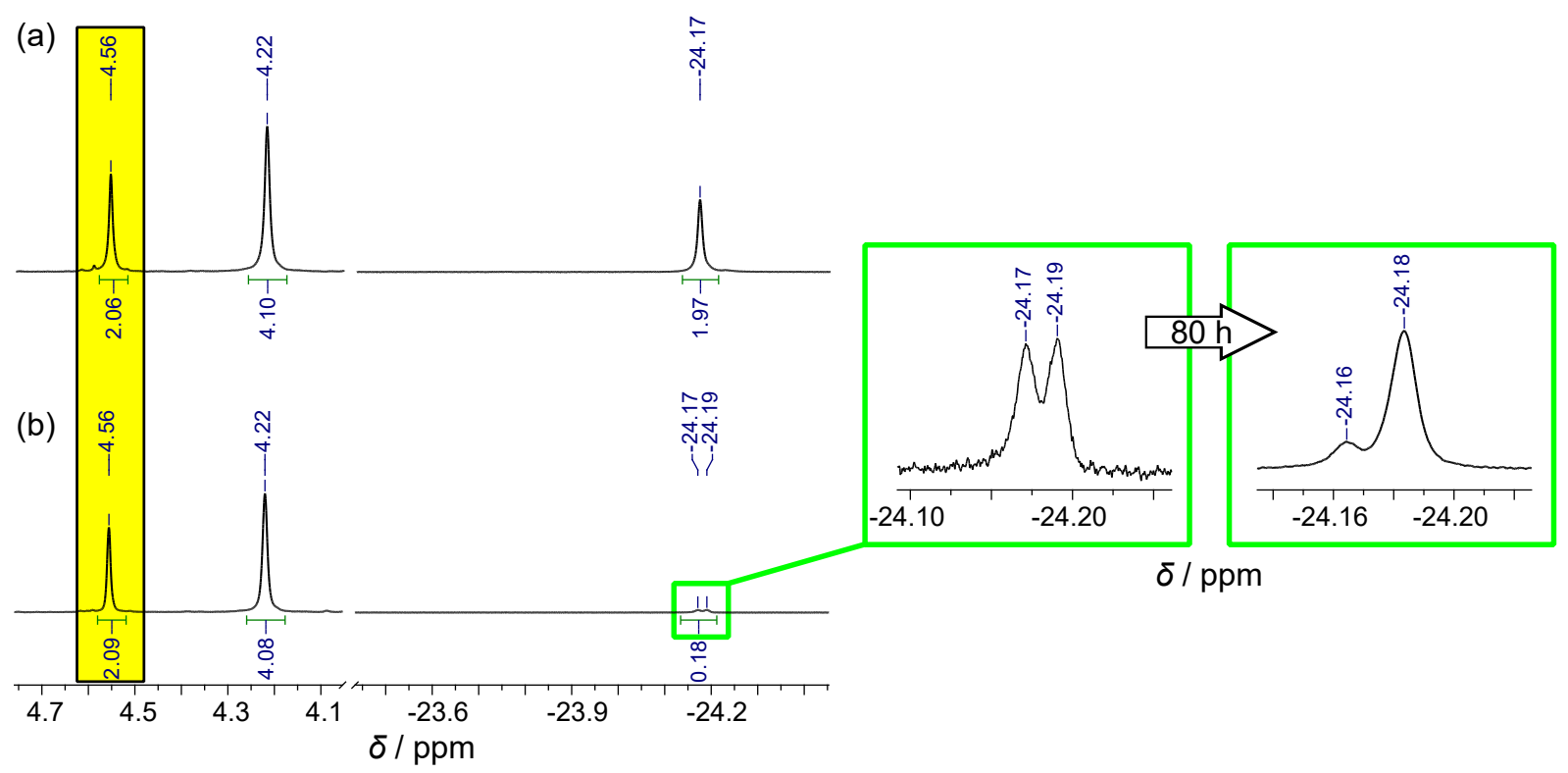

Figure 4.6.: Excerpt of Figure 4.5. Note that besides the resonance of residual hydride starting material at $-24.17 \mathrm{ppm}$, another signal at $-24.19 \mathrm{ppm}$ with same intensity developed (green marked). A control measurement of the sample after $80 \mathrm{~h}$ revealed that a change of intensity occured while the overall absolute integral of all signals remain the same. Furthermore, the $\mathrm{H}_{2}$ development would result in a resonance expected in the yellow marked range (besides the $\beta$-diketiminato $\mathrm{CH}$-proton resonance at $4.56 \mathrm{ppm}$ ) but could not be distinguished.

The composition of all hydridic and deuteridic compounds at the final state of the exchange represents an equilibrium. Therefore, an exchange experiment with a mixture of $\mathrm{D}_{2}$ and $\mathrm{H}_{2}$ was attempted to observe a shift of the equilibrium. After loading a threefold pump-freeze-thaw degassed sample of $\mathbf{K}\left[\mathbf{L}^{\text {bisnac }} \mathbf{N i}_{\mathbf{2}}(\mathbf{H})_{\mathbf{2}}\right]$ in $\mathrm{THF}-\mathrm{D}_{8}$ with an arbitrary mixture ${ }^{\dagger}$ of $\mathrm{D}_{2}$ and $\mathrm{H}_{2}$ the immediate exchange was monitored by ${ }^{1} \mathrm{H} \mathrm{NMR}$. The obtained spectrum is shown in Figure 4.7 and demonstrates the equilibrium shift of the hydridic compounds. After the incipiently established equilibrium no further changes were observed after two days.

At this state the equilibrium is shifted towards $\left.\mathbf{K}\left[\mathbf{L}^{\mathbf{b i s n a c}} \mathbf{N i}_{\mathbf{2}} \mathbf{(} \mathbf{H}\right)_{\mathbf{2}}\right]$ as indicated by the higher intensity of its characeristic hydride resonance $\left(\delta\left\{{ }^{1} \mathrm{H}\right\}=-24.17 \mathrm{ppm}\right.$, right box of Figure 4.7). Although the resonance of dissolved $\mathrm{H}_{2}$ can be observed $\left(\delta\left\{{ }^{1} \mathrm{H}\right\}=4.54 \mathrm{ppm}\right.$, middle box of Figure 4.7), no HD was observed. The expected chemical shift of the HD signal is indicated in the middle excerpt of Figure 4.7. This provides further evidence for

\footnotetext{
†The gas was obtained from the respective gas cylinders as is. The mixing was performed by consecutive transfer of $D_{2}$ and $H_{2}$ into a balloon which was then connected to the Young valve NMR tube.
} 

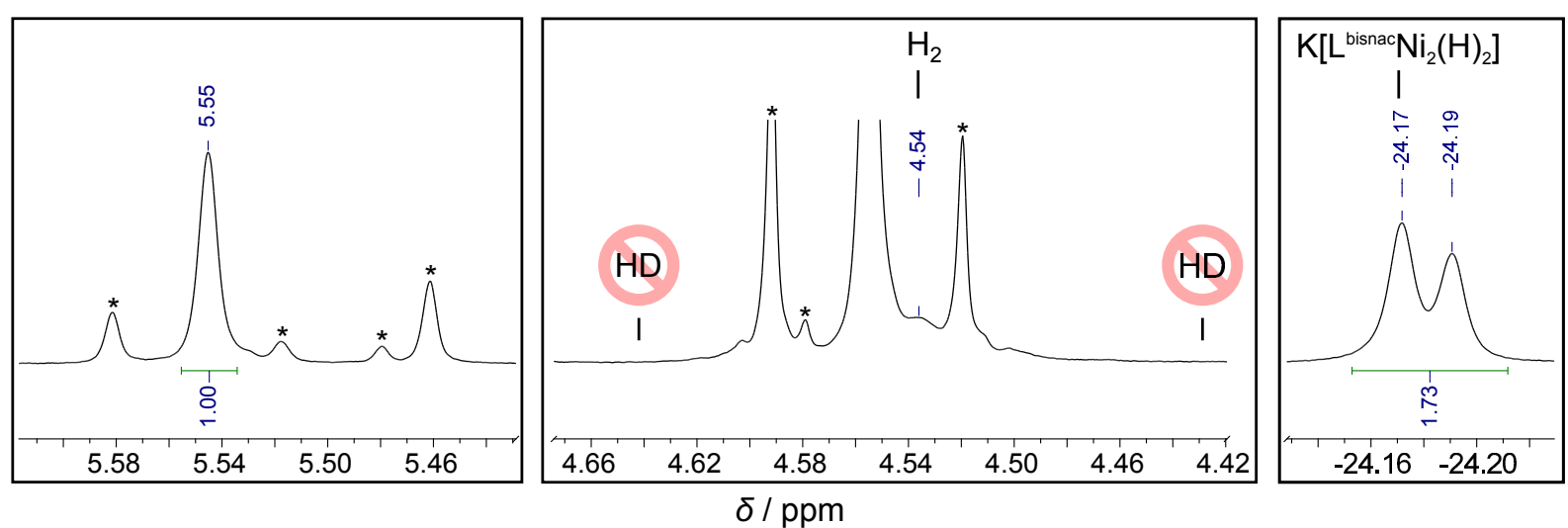

Figure 4.7.: Excerpt of the ${ }^{1} \mathrm{H}$ NMR spectrum $(400 \mathrm{MHz})$ of $\mathrm{K}\left[\mathrm{L}^{\text {bisnac }} \mathrm{Ni}_{\mathbf{2}}(\mathbf{H})_{2}\right]$ treated with a mixture of $\mathrm{D}_{2}$ and $\mathrm{H}_{2}$ after two days. The spectrum was measured in THF- $\mathrm{D}_{8}$ at room temperature. Left: the region including the pyrazolate backbone proton resonances is depicted. Middle: the region of $\mathrm{H}_{2}$ and $\mathrm{HD}$ is described. No resonance associated with $\mathrm{HD}\left({ }^{1} \mathrm{~J}_{\mathrm{HD}}=42 \mathrm{~Hz}\right)^{\left[\frac{[148,149]}{1}\right.}$ can be observed in the spectrum. Right: In addition to the dihydride resonance at $-24.17 \mathrm{ppm}$ another hydride signal at $-24.19 \mathrm{ppm}$ is observed. Side products are marked $\left({ }^{*}\right)$.

a non-scrambling exchange pathway (pathway 2, Scheme 4.6).

As can be seen in left box of Figure 4.7, the ${ }^{1} \mathrm{H}$ NMR signal at $5.55 \mathrm{ppm}$ accounts for the main species, which is the dihydride complex or its deuterated analogue. However, the right box of Figure 4.7 describes two signals of different intensity with a total normalized integral of 1.73. This implies that the amount of $\mathbf{K}\left[\mathbf{L}^{\text {bisnac }} \mathbf{N i}_{\mathbf{2}}(\mathbf{D})_{\mathbf{2}}\right]$ is relatively low compared to $\mathbf{K}\left[\mathbf{L}^{\text {bisnac }} \mathbf{N i}_{\mathbf{2}}(\mathbf{H})_{\mathbf{2}}\right]$ which can be due to a lower amount of $\mathrm{D}_{2}$ gas in comparison to $\mathrm{H}_{2}$. Since the signal at $-24.17 \mathrm{ppm}$ can be attributed to $\left.\mathbf{K}\left[\mathbf{L}^{\text {bisnac }} \mathbf{N i}_{\mathbf{2}} \mathbf{(} \mathbf{H}\right)_{\mathbf{2}}\right]$ the other signal at $-24.19 \mathrm{ppm}$ is possibly correlated to an intermediate species such as $\mathrm{K}\left[\mathrm{L}^{\text {bisnac }} \mathrm{Ni}_{2}(\mathrm{H})_{2}\left(\mathrm{D}_{2}\right)\right]$ or $\mathrm{K}\left[\mathrm{L}^{\text {bisnac }} \mathrm{Ni}_{2}(\mathrm{H})_{2}\left(\mathrm{H}_{2}\right)\right]$.

The contour plot from a ${ }^{1} \mathrm{H}-\mathrm{NOESY}$ experiment (Figure 4.8) shows the expected NOE correlations of the hydride resonances to the isopropyl groups (blue cross peaks, Figure 4.8). Additionally, the exchange between $\mathrm{H}_{2}$ and the hydrides becomes evident (red cross peak, Figure 4.8). 


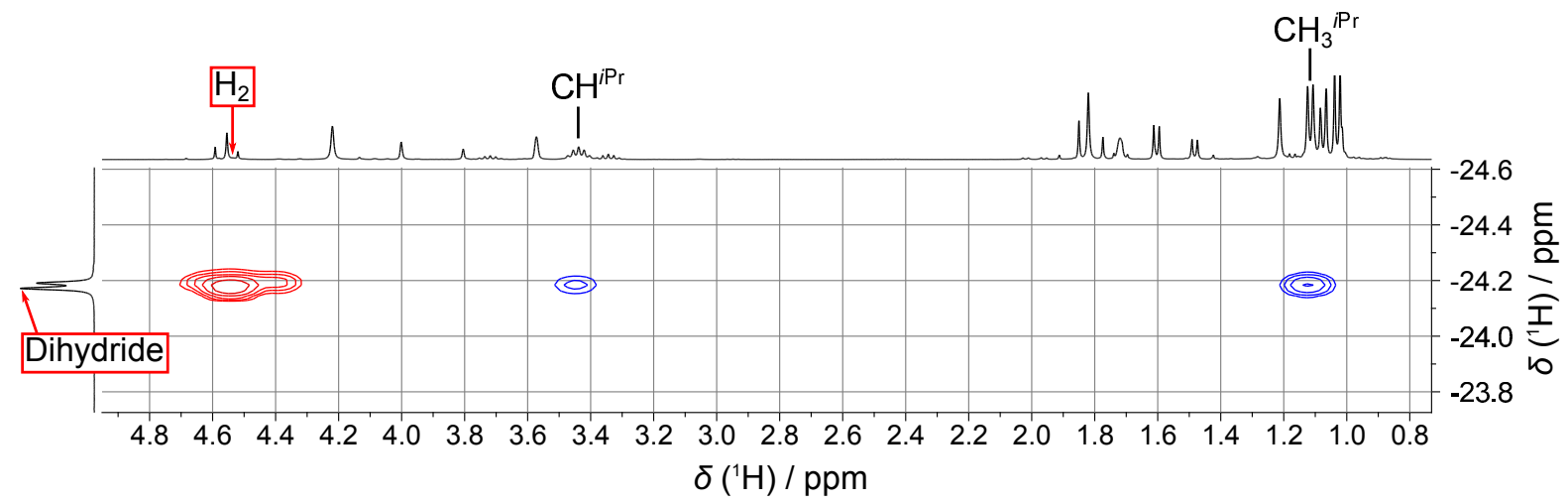

Figure 4.8.: ${ }^{1} \mathrm{H}$-NOESY spectrum $(400 \mathrm{MHz})$ of $\mathrm{K}\left[\mathrm{L}^{\text {bisnac }} \mathrm{Ni}_{2}(\mathbf{H})_{2}\right]$ treated with a mixture of $\mathrm{D}_{2} / \mathrm{H}_{2}$ measured in THF- $\mathrm{D}_{8}$ at room temperature. Besides the two known NOE correlations of the hydrides and the DIPP isopropylgroups a proton exchange correlation was observed. It was attributed to the exchange of $\mathrm{K}\left[\mathrm{L}^{\text {bisnac }} \mathrm{Ni}_{2}(\mathrm{H})_{2}\right]$ with $\mathrm{H}_{2}$.

\section{Investigation of the Exchange by ${ }^{2} \mathrm{H}$ NMR spectroscopy}

Conducting the same experiment in THF and monitoring by ${ }^{2} \mathrm{H}$ NMR revealed a new resonance arising at $-24.11 \mathrm{ppm}$ (Figure 4.9). The intensity and sharp shape of the singlet resonance suggests a correlation to the formed $\mathbf{K}\left[\mathbf{L}^{\mathbf{b i s n a c}} \mathbf{N i}_{\mathbf{2}}(\mathbf{D})_{2}\right]$. During the experiment an expected signal for $\mathrm{HD}\left(1: 1 \text { doublet, } J_{\mathrm{HD}}=42 \mathrm{~Hz}\right)^{\underline{[148,149]}}$ was not observed, which is in accordance with the results obtained by ${ }^{1} \mathrm{H}$ NMR and provides further evidence for a non-scrambling pathway.

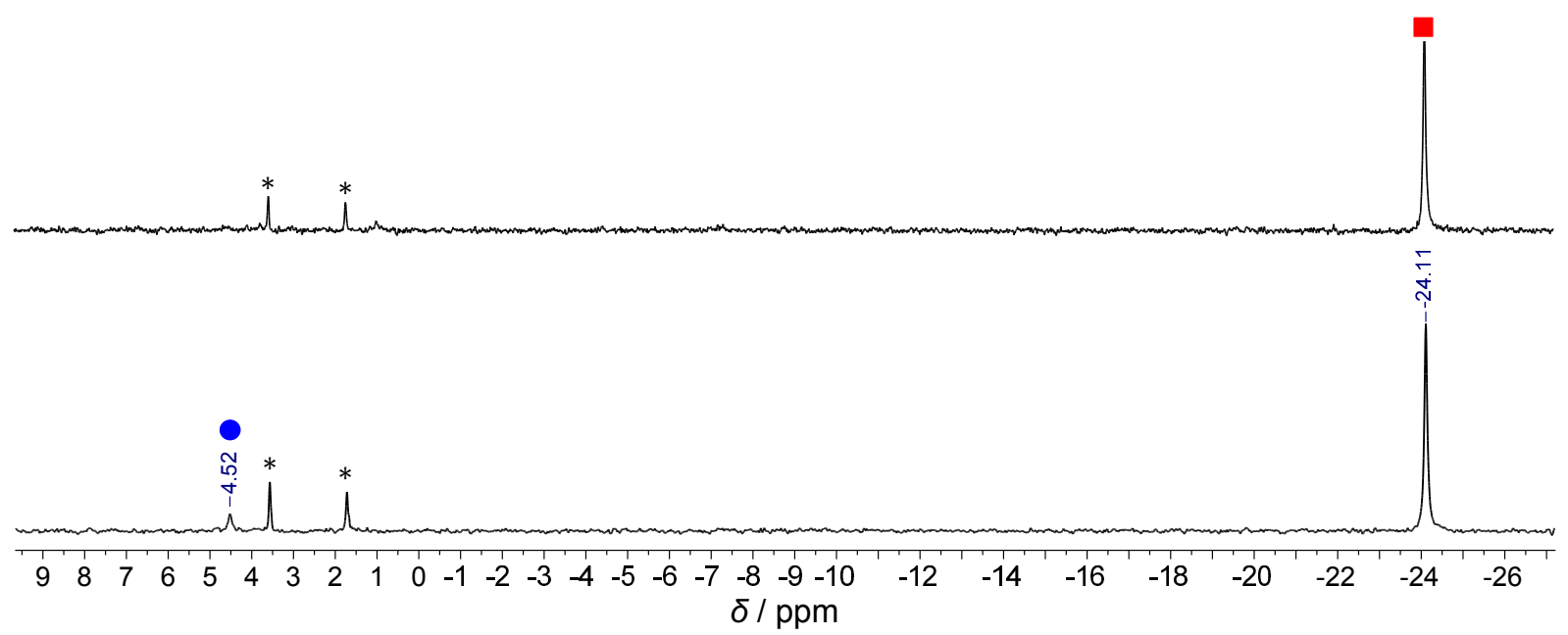

Figure 4.9.: ${ }^{2} \mathrm{H}$ NMR spectrum (77 MHz) of $\mathbf{K}\left[\mathrm{L}^{\text {bisnac }} \mathrm{Ni}_{2}(\mathrm{D})_{2}\right]$. Top: $\mathrm{K}\left[\mathrm{L}^{\text {bisnac }} \mathrm{Ni}_{2}(\mathrm{H})_{2}\right]$ was deuterated by first degasing a THF solution of the compound via pump-freeze-thaw procedure followed by loading of the schlenk tube with atmospheric pressure of dried $D_{2}$ gas. As can be seen, the deutero-hydride complex has formed $(\square)$. These cycles have been repeated 3 times. The NMR sample was filled into a Young NMR tube in a glovebox under $\mathrm{N}_{2}$ atmosphere. Bottom: $\mathrm{H}$-D exchange performed in a Young NMR tube by loading a pump-freeze-thaw degassed sample of the dihydride compound with atmospheric pressure of $D_{2}$ gas $(\bullet)$. Residual solvents (THF-D) are marked $\left(^{*}\right)$. 
Following a new approach, the opposite reaction was conducted. $\mathbf{K}\left[\mathbf{L}^{\text {bisnac }} \mathbf{N i}_{\mathbf{2}}(\mathbf{D})_{\mathbf{2}}\right]$ was produced by loading a pump-freeze-thaw degassed solution of $\mathbf{K}\left[\mathbf{L}^{\text {bisnac }} \mathbf{N i}_{\mathbf{2}}(\mathbf{H})_{\mathbf{2}}\right]$ in THF with dried $\mathrm{D}_{2}$. By layering the cooled THF solution with hexanes and further cooling $\left(-30{ }^{\circ} \mathrm{C}\right)$, crystals could be grown, which were then used for further experiments. About $10 \mathrm{mg}$ of the crystalline $\mathbf{K}\left[\mathbf{L}^{\text {bisnac }} \mathbf{N i}_{\mathbf{2}}(\mathbf{D})_{\mathbf{2}}\right]$ was dissolved in $0.5 \mathrm{~mL}$ of dry THF and the solution transferred into a Young valve NMR tube. The sample was degassed and treated with one atmosphere of dried $\mathrm{H}_{2}$ gas. The following exchange reaction was monitored by ${ }^{2} \mathrm{H}$ NMR (Figure 4.10).

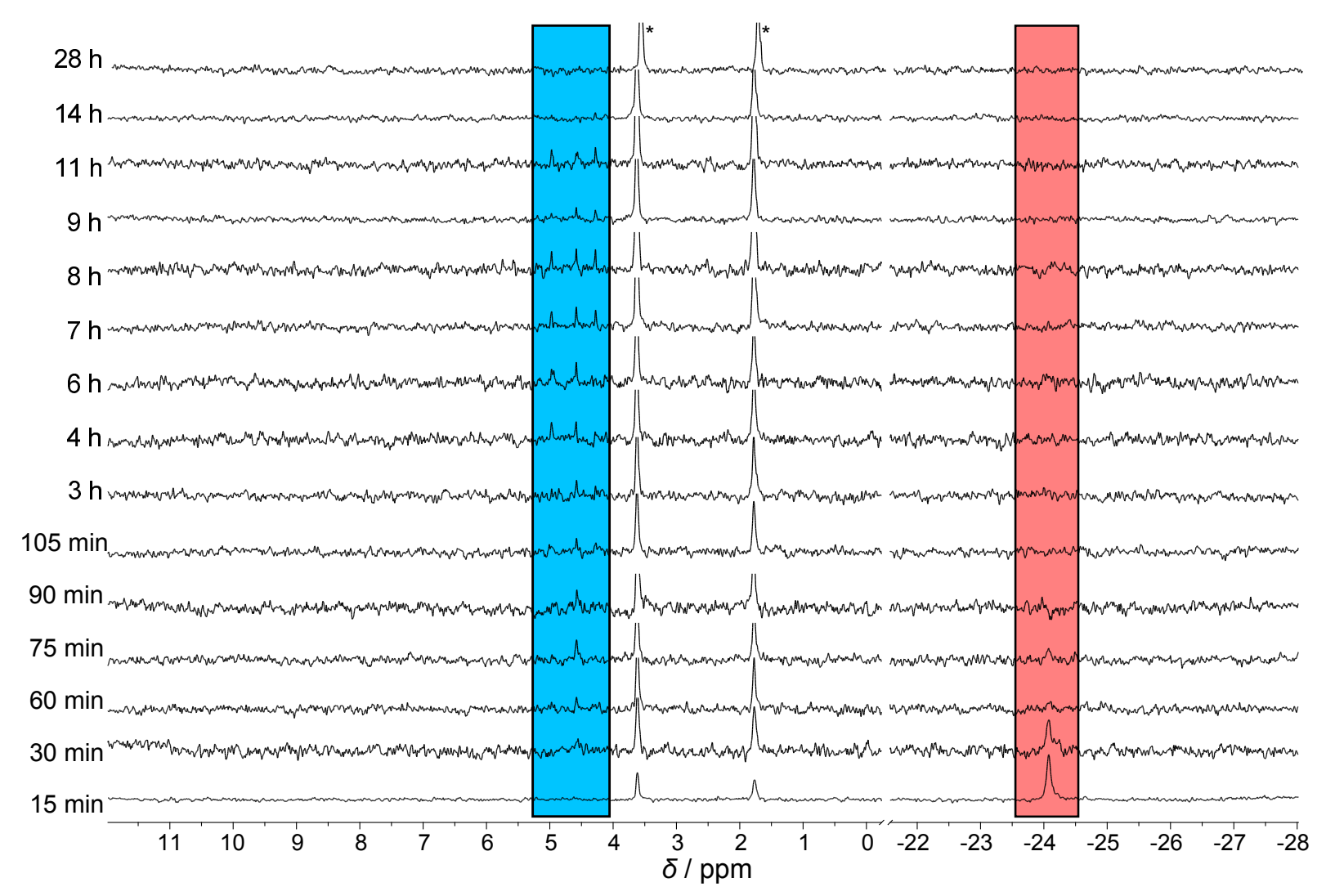

Figure 4.10.: $\mathrm{D}-\mathrm{H}$ exchange of $\mathrm{K}\left[\mathrm{L}^{\text {bisnac }} \mathrm{Ni}_{2}(\mathrm{D})_{2}\right]$ to $\mathrm{K}\left[\mathrm{L}^{\text {bisnac }} \mathrm{Ni}_{2}(\mathrm{H})_{2}\right]$ over a $28 \mathrm{~h}$ time frame, monitored by ${ }^{2} \mathrm{H}$ NMR spectroscopy $(61 \mathrm{MHz})$. During the course of time the dideutero-hydride signal $(\square)$ disappears while new signals for $D_{2}$ and HD develop ( $\square$ ). Residual solvents (THF-D) are marked $\left({ }^{*}\right)$.

Before starting the reaction, the resonance of the deutero-hydride compound was observed at $-24.11 \mathrm{ppm}$. After addition of $\mathrm{H}_{2}$ (atmospheric pressure), ${ }^{2} \mathrm{H}$ NMR spectra were recorded with a measurement time of 15 minutes at the beginning and after 6 hours with a measurement time of 1 hour. A rapid exchange occured, as indicated by the vanishing deutero-hydride signal. Although the amount of released $\mathrm{D}_{2}$ is small compared to $\mathrm{H}_{2}$ a new small singlet resonance appeared at $4.57 \mathrm{ppm}$ (Figure 4.11). The chemical shift differs $0.05 \mathrm{ppm}$ from the shift for free dideutrium $\left(\delta\left\{{ }^{2} \mathrm{H}\right\}\left(\mathrm{D}_{2}\right)=4.52 \mathrm{ppm}\right)$. This indicates weak coordination of $\mathrm{D}_{2}$ to $\mathbf{K}\left[\mathbf{L}^{\text {bisnac }} \mathbf{N i}_{\mathbf{2}}(\mathbf{H})_{\mathbf{2}}\right]$, giving the intermediate $\mathbf{K}\left[\mathbf{L}^{\text {bisnac }} \mathbf{N i}_{\mathbf{2}}(\mathbf{H})_{\mathbf{2}}\left(\mathbf{D}_{\mathbf{2}}\right)\right]$, which was proposed earlier. Due to a preferential coordination of $\mathrm{D}_{2}$ to $\mathbf{K}\left[\mathbf{L}^{\text {bisnac }} \mathbf{N i}_{\mathbf{2}}(\mathbf{H})_{\mathbf{2}}\right]$ forming $\mathbf{K}\left[\mathbf{L}^{\text {bisnac }} \mathbf{N i}_{\mathbf{2}}(\mathbf{H})_{\mathbf{2}}\left(\mathbf{D}_{\mathbf{2}}\right)\right]$ its slightly shifted reso- 
nance is observed in ${ }^{2} \mathrm{H}$ NMR. ${ }^{[151]}$ In accordance with previously conducted experiments, no signs of HD formation were detected during the incipient process.

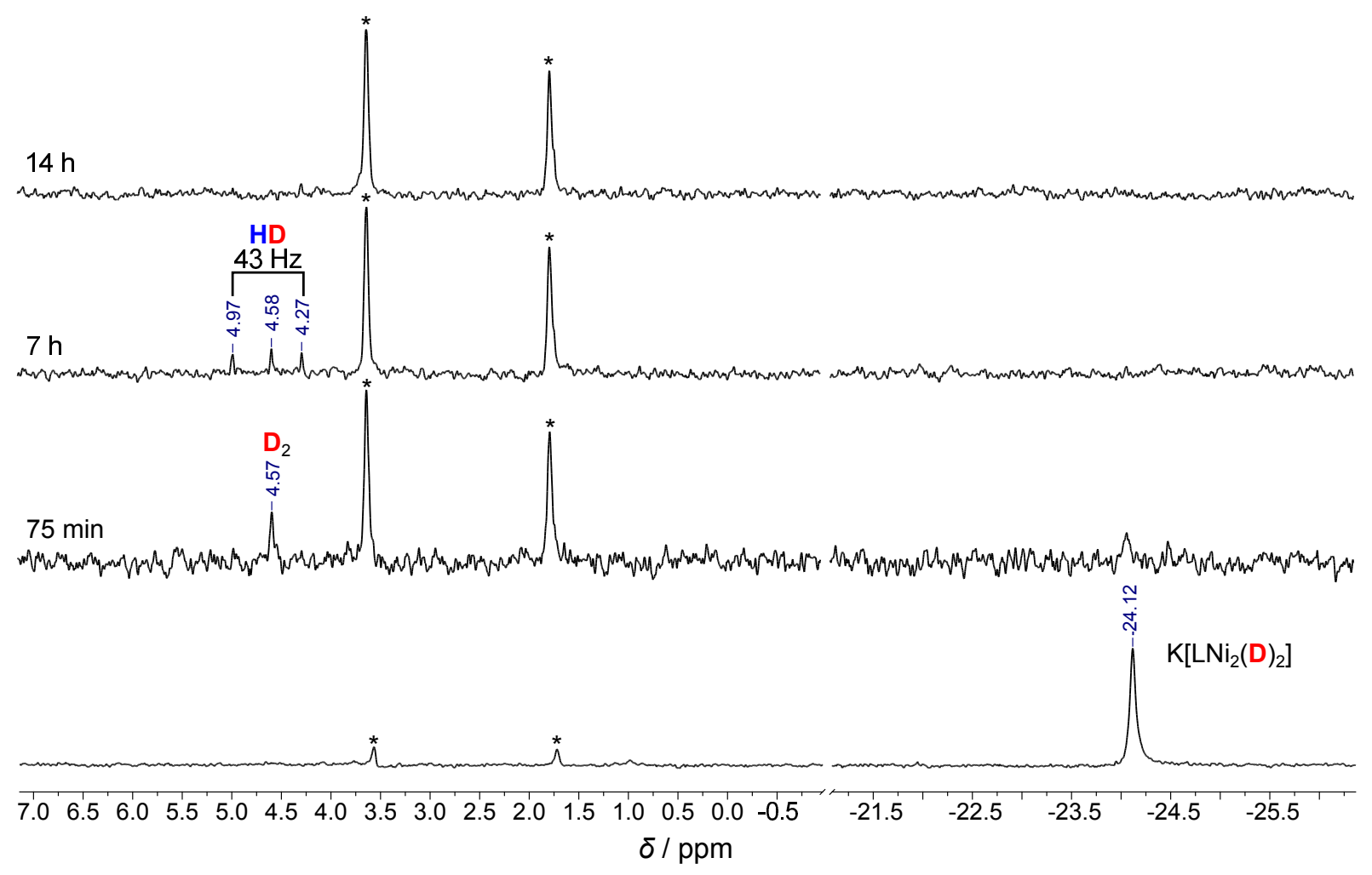

Figure 4.11.: Excerpt of Figure 4.10. The bottom spectrum shows the untreated sample of $K\left[L^{\text {bisnac }} \mathrm{Ni}_{2}(\mathbf{D})_{2}\right]$ in THF. After the degassed sample was loaded with $\mathrm{H}_{2}$ the deutero-hydride signal at $-24 \mathrm{ppm}$ vanished while a new signal occured at $4.57 \mathrm{ppm}\left(\mathrm{D}_{2}\right)$. After around 7 hours another doublet resonance was observed at $4.62 \mathrm{ppm}$ and with a coupling constant of $43 \mathrm{~Hz}$. This signal was attributed to HD. Residual solvents (THF-D) are marked (*).

Surprisingly, after recording further spectra up to $28 \mathrm{~h}$ after the start of the reaction, another set of signals slowly developed in addition to the dideuterium signal with peaks

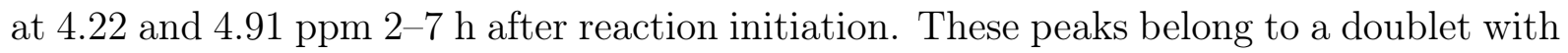
a coupling constant of $42.7 \mathrm{~Hz}$ which fits the ${ }^{1} J$ coupling constant reported for HD. $\stackrel{[148,149]}{ }$ The fact that the presence of HD was only observed long after the full exchange of $\mathbf{K}\left[\mathbf{L}^{\text {bisnac }} \mathbf{N i}_{\mathbf{2}}(\mathbf{D})_{\mathbf{2}}\right]$ with $\mathrm{H}_{2}$ indicates that its formation was not correlated to this H-D exchange process. Instead, the emergence of a $\mathrm{HD}$ associated resonance can be the result of a $\mathrm{H} / \mathrm{D}$ exchange mediated by present trace amounts of $\left[\mathbf{L}^{\text {bisnac }} \mathbf{N i}_{\mathbf{2}}(\mathbf{O H})\right]$ or even water with $\mathrm{D}_{2} \cdot{ }^{[152,153]}$ Accordingly, the formed $\left[\mathbf{L}^{\text {bisnac }} \mathbf{N i}_{\mathbf{2}}(\mathrm{OD})\right]^{\dagger}$ further exchanges with the present $\mathrm{H}_{2}$ in a subsequent step. This process repeats until $\mathrm{D}_{2}$ is eventually consumed. A direct $\mathrm{H}-\mathrm{D}$ exchange between $\mathrm{H}_{2}$ and the developed $\mathrm{D}_{2}$ is unlikely under these conditions. ${ }^{[154.155]}$ However, the second exchange process leading to the development of HD takes place at a very low rate. After $14 \mathrm{~h}$ the signals completely vanished, likely due to a statistic gas exchange in the solution with the dihydrogen atmosphere.

†Due to superposition with a pronounced artefact the region is not shown in Figure 4.11. 


\section{Anticipation: Reaction with Water}

The conversion of $\mathbf{K}\left[\mathbf{L}^{\text {bisnac }} \mathbf{N i}_{\mathbf{2}}(\mathbf{H})_{\mathbf{2}}\right]$ with water leads to the release of $\mathrm{H}_{2}$ and the formation of a hydroxo-hydrido complex, which is discussed in depth in the next section (4.5). Figure 4.12 is the ${ }^{1} \mathrm{H}$ NOESY contour diagram of a sample containing $\mathbf{K}\left[\mathbf{L}^{\text {bisnac }} \mathbf{N i}_{\mathbf{2}}(\mathbf{H})_{\mathbf{2}}\right]$, which was reacted with one equivalent of water. $\mathrm{H}_{2}$, which is formed during the process, shows a significant exchange correlation with the residual $\mathbf{K}\left[\mathbf{L}^{\text {bisnac }} \mathbf{N i}_{\mathbf{2}}(\mathbf{H})_{\mathbf{2}}\right]$. This demonstrates that even low amounts of $\mathrm{H}_{2}$ are observed in the spectrum. It further proves the coordination of the dihydrogen molecule towards $\mathbf{K}\left[\mathbf{L}^{\text {bisnac }} \mathbf{N i}_{\mathbf{2}}(\mathbf{H})_{\mathbf{2}}\right]$, which is sustained even after one week. The result is in accordance with observations made for the reaction of $\mathbf{K}\left[\mathbf{L}^{\text {bisnac }} \mathbf{N i}_{\mathbf{2}}(\mathbf{D})_{2}\right]$ with $\mathrm{H}_{2}$, in which the formed $\mathrm{D}_{2}$ also remained coordinated to the exchange product $\mathbf{K}\left[\mathbf{L}^{\text {bisnac }} \mathbf{N i}_{\mathbf{2}}(\mathbf{H})_{\mathbf{2}}\right]$.

Furthermore, the abscence of released $\mathrm{H}_{2}$ (monitored by ${ }^{1} \mathrm{H}$ NMR) in the analogous reaction of $\mathbf{K}\left[\mathbf{L}^{\text {bisnac }} \mathbf{N i}_{\mathbf{2}}(\mathbf{H})_{2}\right]$ with $\mathrm{D}_{2}$ can now be explained. In this case the produced $\mathrm{H}_{2}$ is entirely released from the coordination sphere of $\mathbf{K}\left[\mathbf{L}^{\mathbf{b i s n a c}} \mathbf{N i}_{\mathbf{2}}(\mathbf{D})_{\mathbf{2}}\right]$, due to a much more favourable coordination of $\mathrm{D}_{2}$. Subsequently, the low amount of free $\mathrm{H}_{2}$ is displaced from the solution by the $\mathrm{D}_{2}$ atmosphere. Only if coordinated to the hydride complex or its deuterium congener, low amounts of $\mathrm{H}_{2}$ or $\mathrm{D}_{2}$ will be visible in the NMR spectrum. The chemical shift difference between coordinated $\mathrm{H}_{2}\left(\delta\left\{{ }^{1} \mathrm{H}\right\}=4.54 \mathrm{ppm}\right)$ and $\mathrm{D}_{2}\left(\delta\left\{{ }^{2} \mathrm{H}\right\}\right.$ $=4.57 \mathrm{ppm}$ ) can be explained by the stronger interactions which arise from $\mathrm{D}_{2}$ coordination. Therefore, the resonance of the weakly coordinated $\mathrm{H}_{2}$ molecule is nearly the same as free $\mathrm{H}_{2}$.

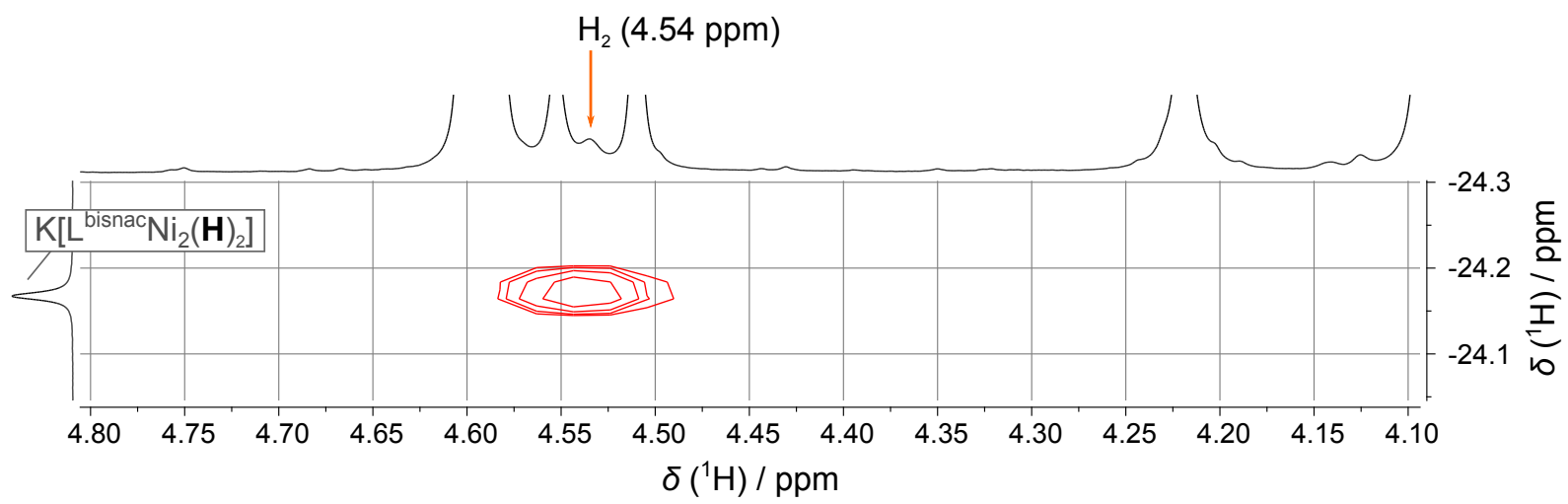

Figure 4.12.: ${ }^{1} \mathrm{H}$ NOESY spectrum of $\mathrm{K}\left[\mathrm{L}^{\text {bisnac }} \mathrm{Ni}_{2}(\mathrm{H})_{2}\right]$ converted with one equivalent of $\mathrm{H}_{2} \mathrm{O}$. During the process $\mathrm{H}_{2}$ is released. The spectrum shows the exchange correlation of the dihydride complex with $\mathrm{H}_{2}$ (4.54 ppm). 


\section{Experiments with Parahydrogen}

It was envisioned that further evidence for the one step substitution (non-scrambling) of the hydrides in $\mathbf{K}\left[\mathbf{L}^{\text {bisnac }} \mathbf{N i}_{\mathbf{2}}(\mathbf{H})_{\mathbf{2}}\right]$ with parahydrogen can be obtained. Unfortunately, from the experiments a clear statement in either direction could not be made. The detailed description and discussion of these experiments is found in the appendix (D.2).

\section{Mechanistic Proposal}

The experimental results suggest that after the incipient exchange of $\mathbf{K}\left[\mathbf{L}^{\text {bisnac }} \mathbf{N i}_{\mathbf{2}}(\mathbf{D})_{\mathbf{2}}\right]$ with $\mathrm{H}_{2}, \mathrm{D}_{2}$ is released. However, the observed ${ }^{2} \mathrm{H}$ resonance, which was shifted in comparison to free $\mathrm{D}_{2}$ indicates a coordination of $\mathrm{D}_{2}$ to the formed exchange product $\mathbf{K}\left[\mathbf{L}^{\text {bisnac }} \mathbf{N i}_{\mathbf{2}}(\mathbf{H})_{2}\right]$ giving $\mathbf{K}\left[\mathbf{L}^{\text {bisnac }} \mathbf{N i}_{\mathbf{2}}(\mathbf{H})_{\mathbf{2}}\left(\mathbf{D}_{\mathbf{2}}\right)\right]$. The experiment conducted with a mixture of $\mathrm{D}_{2}$ and $\mathrm{H}_{2}$ revealed a new hydride resonance at $-24.19 \mathrm{ppm}$ in the ${ }^{1} \mathrm{H}$ NMR that is attributable to such an intermediate. Considering the opposite reaction of $\mathbf{K}\left[\mathbf{L}^{\text {bisnac }} \mathbf{N i}_{\mathbf{2}}(\mathbf{H})_{\mathbf{2}}\right]$ with $\mathrm{D}_{2}$, the respective $\mathrm{H}_{2}$ coordinated analogue $\mathbf{K}\left[\mathbf{L}^{\text {bisnac }} \mathbf{N i}_{\mathbf{2}^{-}}\right.$ $(\mathrm{D})_{\mathbf{2}}\left(\mathbf{H}_{\mathbf{2}}\right)$ ] was not observed in the ${ }^{1} \mathrm{H}$ NMR spectrum. This can be explained by the preferred coordination of $\mathrm{D}_{2}$ over $\mathrm{H}_{2}$. The low amount of released $\mathrm{H}_{2}$ is then displaced by the excess amount of $\mathrm{D}_{2}$, which further explains the absence of a $\mathrm{H}_{2}$ resonance in the NMR spectrum.

First results from DFT calculations conducted by MATA et al. support the formation of an intermediate $\mathbf{K}\left[\mathbf{L}^{\text {bisnac }} \mathbf{N i}_{\mathbf{2}}(\mathbf{H})_{\mathbf{2}}\left(\mathbf{H}_{\mathbf{2}}\right)\right]$ during the exchange process. The calculated structure of the intermediate is illustrated in a) of Figure 4.13 (details on the calculation procedure are found in the experimental part 7). $\mathrm{H}_{2}$ coordinates to one $\mathrm{Ni}$ ion in an endon fashion. In the transition state $\mathrm{b}$ ) the distance of coordinated $\mathrm{H}_{2}$ further decreases while the Ni-hydride bond at the $\mathrm{H}_{2}$-coordinated $\mathrm{Ni}$ weakens $(\rightarrow)$. It is assumed that this eventually leads to the formation of a bond between the former hydrides and a symmetric structure with each metal ion coordinated by molecular $\mathrm{H}_{2}$. Based on these considerations a mechanistic proposal was established (Scheme 4.7). 
a)

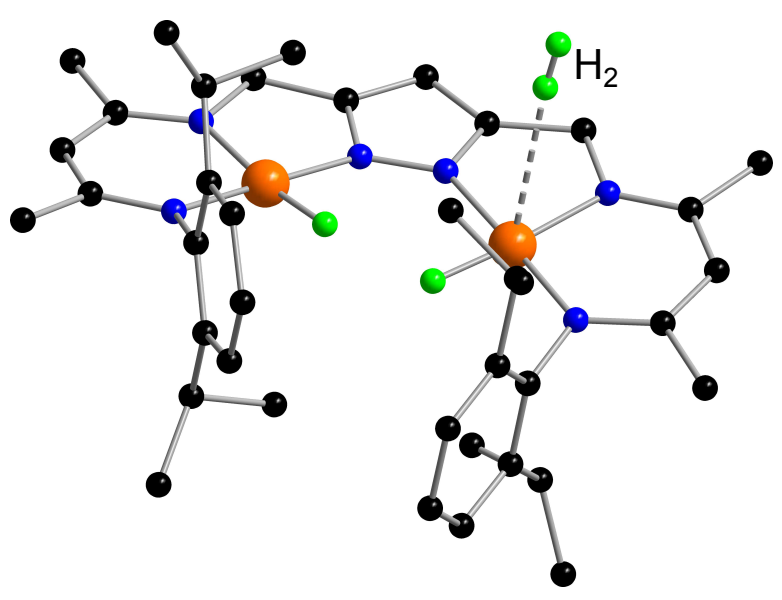

$\mathrm{K}\left[\mathrm{L}^{\text {bisnac }} \mathrm{Ni}_{2}(\mathrm{H})_{2}\left(\mathrm{H}_{2}\right)\right]$

Intermediate b)

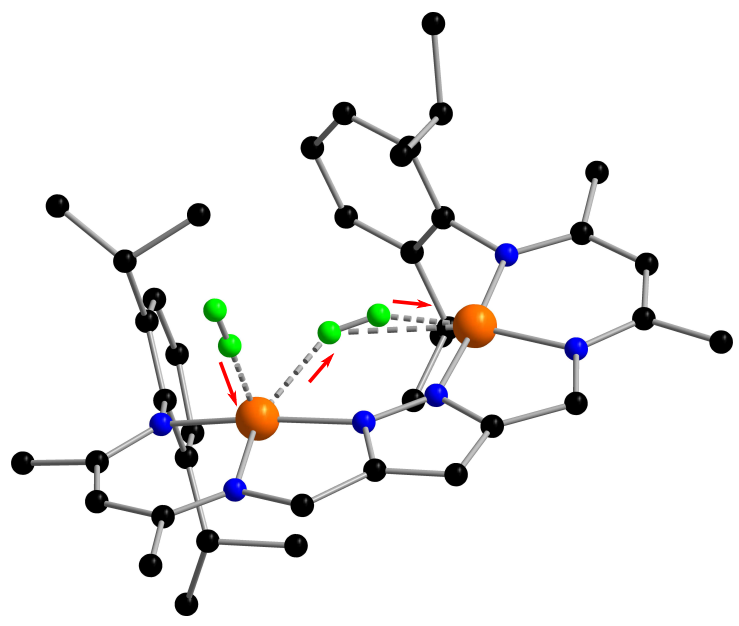

$\mathrm{K}\left[\mathrm{L}^{\text {bisnac }} \mathrm{Ni}_{2}\left(\mathrm{H}_{2}\right)\left(\mathrm{H}_{2}\right)\right]$

Transition State

Figure 4.13.: Calculated structures for the intermediate $\left[\mathrm{L}^{\text {bisnac }} \mathrm{Ni}_{2}(\mathrm{H})_{2}\left(\mathrm{H}_{2}\right)\right]$ a) and the transition state [ $\left.{ }^{\text {bisnac }} \mathrm{Ni}_{2}\left(\mathrm{H}_{2}\right)\left(\mathrm{H}_{2}\right)\right]$ b).

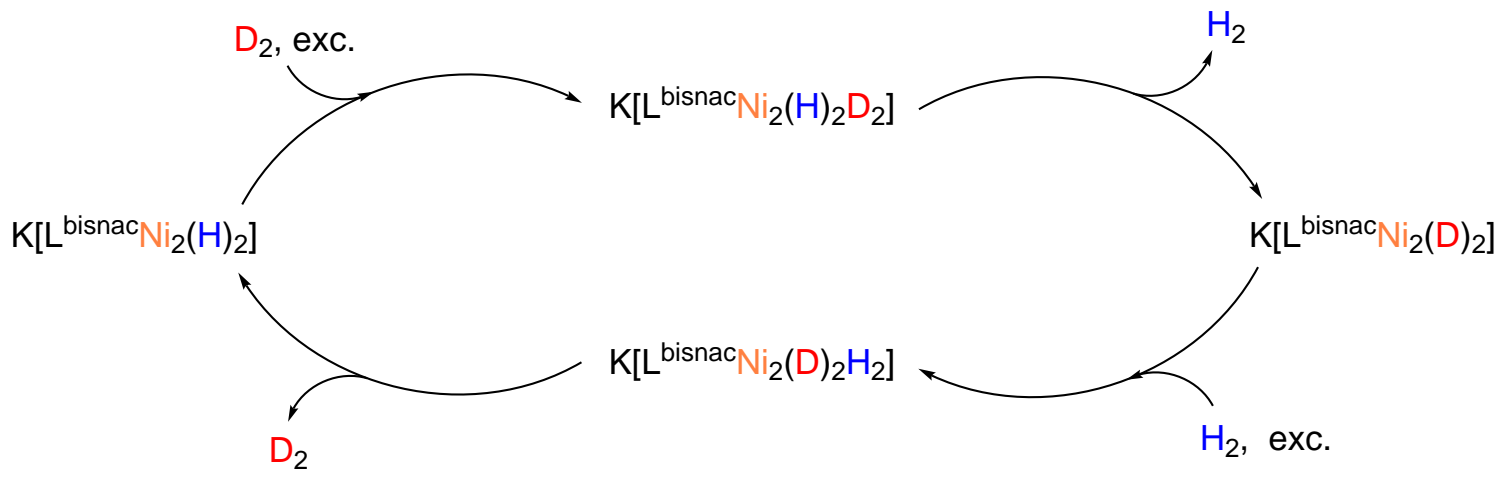

Scheme 4.7: Proposed pathway for the exchange of $K\left[L^{\text {bisnac }} \mathrm{Ni}_{2}(\mathbf{H})_{2}\right]$ with $D_{2}$. In the first step a $D_{2}$ coordinated intermediate $K\left[L^{\text {bisnac }} \mathbf{N i}_{2}\left(H_{2}\right)_{2}\left(D_{2}\right)\right]$ is formed. The subsequent exchange leads to $\mathrm{K}\left[\mathrm{L}^{\text {bisnac }} \mathrm{Ni}_{2}(\mathrm{D})_{2}\right]$ and the release of $\mathrm{H}_{2}$. 


\section{Summary}

The dihydride $\left.\mathbf{K}\left[\mathbf{L}^{\text {bisnac }} \mathbf{N i}_{\mathbf{2}} \mathbf{( H}\right)_{\mathbf{2}}\right]$ exchanges with $\mathrm{D}_{2}$ resulting in $\mathbf{K}\left[\mathbf{L}^{\text {bisnac }} \mathbf{N i}_{\mathbf{2}}(\mathbf{D})_{\mathbf{2}}\right]$. This exchange is reversible under an $\mathrm{H}_{2}$ atmosphere. The pathway for the exchange leads to an intermediate, which is described by the $\mathrm{D}_{2}$ coordinated $\mathbf{K}\left[\mathbf{L}^{\text {bisnac }} \mathbf{N i}_{\mathbf{2}}(\mathbf{H})_{\mathbf{2}}\right]$ giving $\mathbf{K}\left[\mathbf{L}^{\text {bisnac }} \mathbf{N i}_{\mathbf{2}}(\mathbf{H})_{\mathbf{2}}\left(\mathbf{D}_{\mathbf{2}}\right)\right]$ or $\mathbf{K}\left[\mathbf{L}^{\text {bisnac }} \mathbf{N i}_{\mathbf{2}}(\mathbf{D})_{\mathbf{2}}\left(\mathbf{H}_{\mathbf{2}}\right)\right]$ with $\mathrm{H}_{2}$.

This intermediate was observed in the ${ }^{1} \mathrm{H}$ NMR spectrum exhibiting a slightly shifted hydride resonance. After the pairwise substitution of the $\mathrm{Ni}-\mathrm{H}$ atoms with one molecule of $\mathrm{D}_{2}$ or $\mathrm{H}_{2}, \mathrm{H}_{2}$ and $\mathrm{D}_{2}$, respectively, is produced. Due to a preferred coordination of $\mathrm{D}_{2}$, produced $\mathrm{H}_{2}$ (free or coordinated) was not observed under a $\mathrm{D}_{2}$ atmosphere. In the absence of $\mathrm{D}_{2}$ the presence of $\mathrm{H}_{2}$ was proven by ${ }^{1} \mathrm{H}$ NMR and a ${ }^{1} \mathrm{H}-{ }^{1} \mathrm{H}$ NOESY experiment. The exchange process was followed by ${ }^{1} \mathrm{H}$ and ${ }^{2} \mathrm{H}$ NMR leading to consistent results. Conversion of $\mathbf{K}\left[\mathbf{L}^{\text {bisnac }} \mathbf{N i}_{\mathbf{2}}(\mathbf{D})_{\mathbf{2}}\right]$ in a $\mathrm{H}_{2}$ atmosphere showed the coordination of the produced $\mathrm{D}_{2}$ to the substitution product $\mathbf{K}\left[\mathbf{L}^{\text {bisnac }} \mathbf{N i}_{\mathbf{2}}(\mathbf{H})_{\mathbf{2}}\right]$ in ${ }^{2} \mathrm{H} \mathrm{NMR}$. In this case the $\mathrm{D}_{2}$ associated resonance was slightly shifted (0.05 ppm downfield) compared to free $\mathrm{D}_{2}$. During all conducted experiments no signs of an incipient HD formation as a conceivable result of a $\mathrm{D}_{2}$ or $\mathrm{H}_{2}$ scrambling process were found, confirming the direct exchange. 


\subsection{Reaction with Water}

The dihydride complex $\mathbf{K}\left[\mathbf{L}^{\text {bisnac }} \mathbf{N i}_{\mathbf{2}}(\mathbf{H})_{\mathbf{2}}\right]$ is particularly susceptible towards traces of moisture. As a result of the reaction with water eventually the $\mu$-hydroxo compound $\left[\mathbf{L}^{\text {bisnac }} \mathbf{N i}_{\mathbf{2}}(\mu-\mathrm{OH})\right]$ emerges. It can be identified by its characteristic upfield resonance in the ${ }^{1} \mathrm{H}$ NMR spectrum $\left(\delta\left\{{ }^{1} \mathrm{H}\right\}_{\mathrm{OH}}=-7.26 \mathrm{ppm}\right)$. Nonetheless, the reaction path to the $\mu$-hydroxo complex appears to go through an intermediate species, as was discovered during reactions like the $\mathrm{H}-\mathrm{D}$ exchange with $\mathrm{H}_{2}$ and $\mathrm{D}_{2}$ by means of immediate NMR spectroscopic screening. This intermediate was described as the hydrido-hydroxo species $\mathbf{K}\left[\mathrm{L}^{\text {bisnac }} \mathrm{Ni}_{2}(\mathrm{OH})(\mathbf{H})\right]$ (Scheme 4.8) .
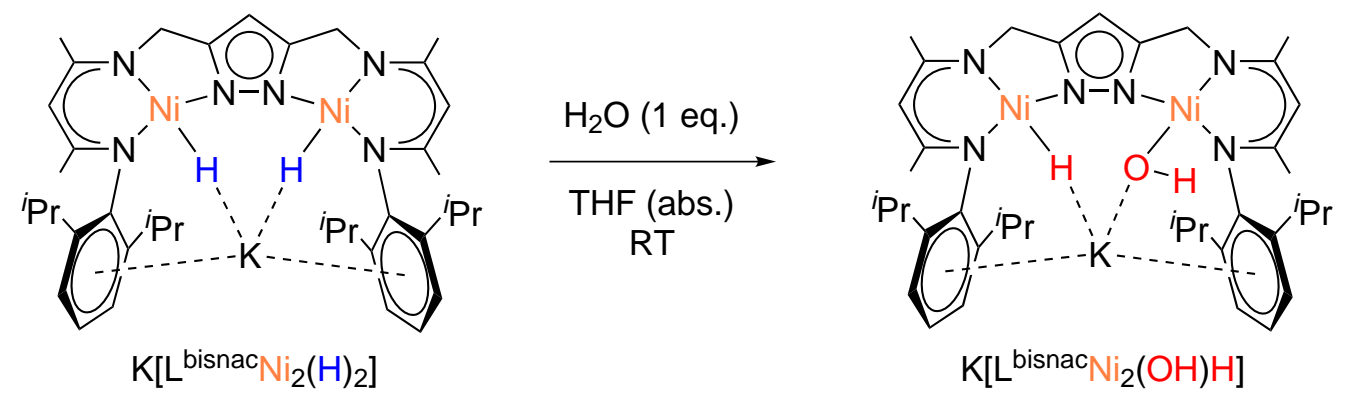

Scheme 4.8: Reaction of the dihydride complex $K\left[\mathrm{~L}^{\text {bisnac }} \mathrm{Ni}_{2}(\mathrm{H})_{2}\right]$ with $\mathrm{H}_{2} \mathrm{O}$ to the hydroxo-hydrido complex $\mathrm{K}\left[\mathrm{L}^{\text {bisnac }} \mathrm{Ni}_{2}(\mathrm{OH})(\mathrm{H})\right]$. A coordination of the potassium cation to the hydride and one of the free electron pairs of the hydroxo-oxygen atom is indicated.

A ${ }^{1} \mathrm{H}$ NMR experiment with $\mathbf{K}\left[\mathbf{L}^{\text {bisnac }} \mathbf{N i}_{\mathbf{2}}(\mathbf{H})_{\mathbf{2}}\right]$ and a stoichiometric amount of water in THF-D 8 was performed to reveal the formation of the intermediate. After the addition of water, $\mathbf{K}\left[\mathbf{L}^{\text {bisnac }} \mathbf{N i}_{\mathbf{2}}(\mathbf{H})_{\mathbf{2}}\right]$ is consumed, as can be observed by the decrease of the characeristic hydride resonance ( $\bullet$, spectrum a of Figure 4.14).

At the same time, the characteristic $\mu$-hydroxo resonance at $-7.26 \mathrm{ppm}(\boldsymbol{\square})$ emerged alongside other resonances in the upfield at $\delta\left\{{ }^{1} \mathrm{H}\right\}=-2.06 \mathrm{ppm}$ and $\delta\left\{{ }^{1} \mathrm{H}\right\}=-26.01 \mathrm{ppm}$ $(\Delta$, spectrum a, Figure 4.14). The equal integrals of the observed resonances and a correlation in the ${ }^{1} \mathrm{H}-{ }^{1} \mathrm{H}$ COSY experiment imply that the signals belong to the same compound. The formation of several complexes is reflected by the additional resonances in the region of the pyrazolate backbone proton shift (5-6 ppm, Figure 4.14).

The signal at $\delta\left\{{ }^{1} \mathrm{H}\right\}=-26.01 \mathrm{ppm}$ was attributed to the hydride and indicates a stronger hydrogen shielding than in $\mathbf{K}\left[\mathbf{L}^{\text {bisnac }} \mathbf{N i}_{\mathbf{2}}(\mathbf{H})_{\mathbf{2}}\right]\left(\delta\left\{{ }^{1} \mathrm{H}\right\}=-24.2 \mathrm{ppm}\right)$. Association of the signal at $\delta\left\{{ }^{1} \mathrm{H}\right\}=-2.06 \mathrm{ppm}$ to a hydroxide was proven by ${ }^{17} \mathrm{O}$ labeling experiments using $\mathrm{H}_{2}{ }^{17} \mathrm{O}$ (Figure 4.15 and Figure 4.16). The excerpt of a ${ }^{1} \mathrm{H}$ NMR spectrum recorded after one week (Figure 4.16) shows the significant line broadening caused by the coupling between the proton and the ${ }^{17} \mathrm{O}$ nucleus in $\mathbf{K}\left[\mathbf{L}^{\text {bisnac }} \mathbf{N i}_{\mathbf{2}}\left({ }^{17} \mathbf{O H}\right)(\mathbf{H})\right]$ and $\left[\mathbf{L}^{\text {bisnac }} \mathbf{N i}_{\mathbf{2}}(\mu-\right.$ $\mathrm{OH})]$.

In comparison to $\left[\mathbf{L}^{\text {bisnac }} \mathbf{N i}_{\mathbf{2}}(\mu-\mathrm{OH})\right]\left(\delta\left\{{ }^{1} \mathrm{H}\right\}_{\mathrm{OH}}=-7.26 \mathrm{ppm}\right)$, the resonance of the 
hydroxo bridge in $\mathbf{K}\left[\mathbf{L}^{\text {bisnac }} \mathbf{N i}_{\mathbf{2}}(\mathbf{O H})(\mathbf{H})\right]$ is shifted downfield $\left(\delta\left\{{ }^{1} \mathrm{H}\right\}=-2.06 \mathrm{ppm}\right)$ due to less electronic shielding.

(e)

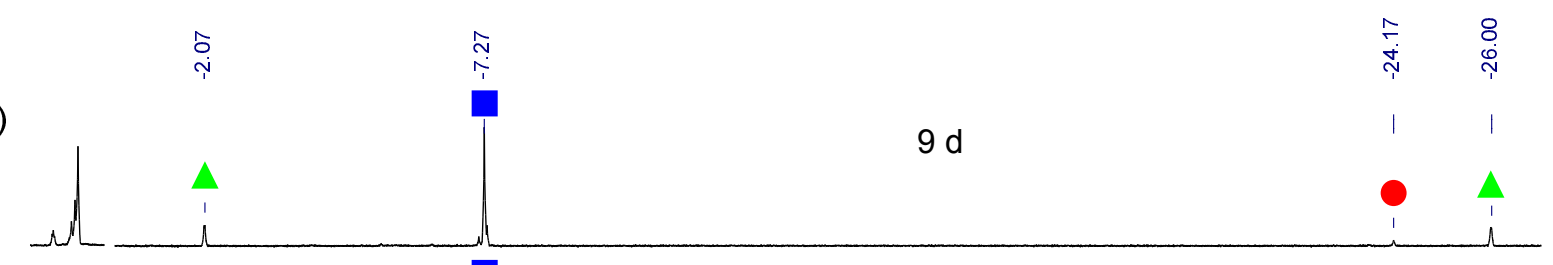

(d)

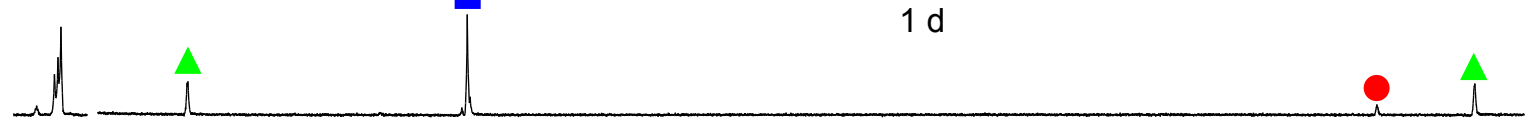

(c)

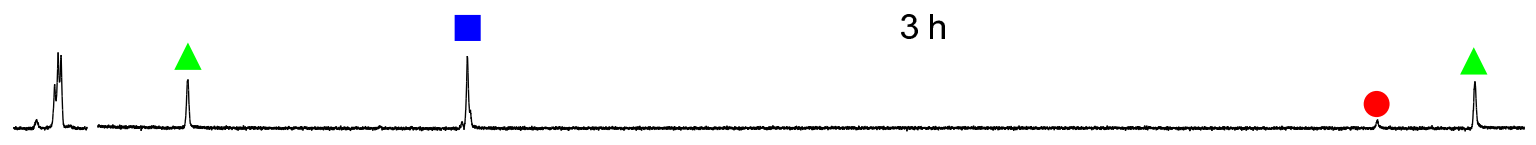

(b)

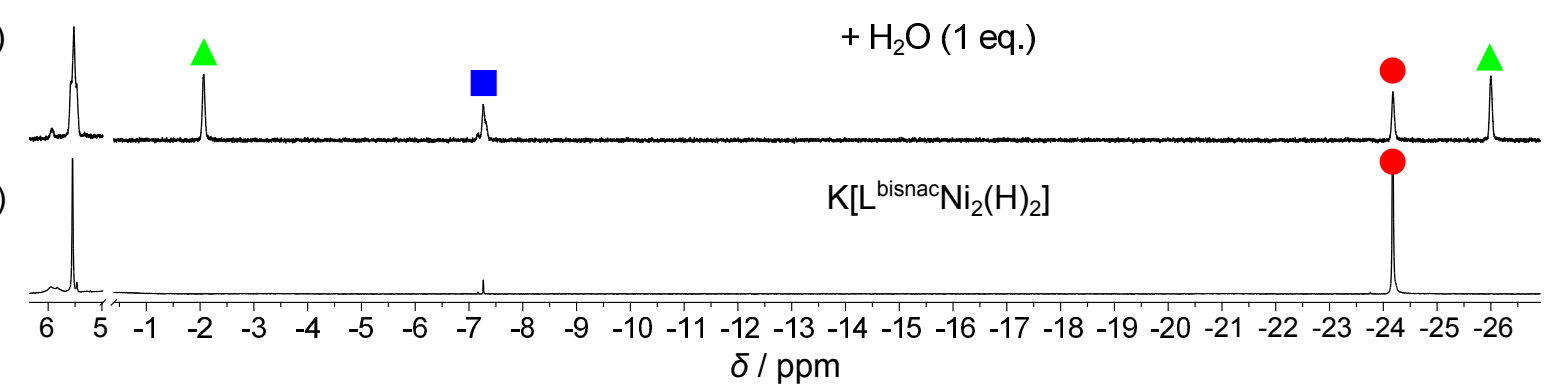

Figure 4.14.: The reaction of $\mathrm{K}\left[\mathrm{L}^{\text {bisnac }} \mathrm{Ni}_{2}(\mathrm{H})_{2}\right]$ with one equivalent of $\mathrm{H}_{2} \mathrm{O}$ monitored by ${ }^{1} \mathrm{H}$ NMR. Spectrum (a) represents the untreated sample of $K\left[L^{\text {bisnac }} \mathrm{Ni}_{2}(\mathrm{H})_{2}\right](\bullet)$. Directly after treatment with one equivalent of water a spectrum was recorded (b). Afterwards control measurements were conducted at (c) $3 \mathrm{~h}$, (d) $1 \mathrm{~d}$ and (e) $9 \mathrm{~d}$. The characteristic dihydride resonance $\bullet$ of $\mathrm{K}\left[\mathrm{L}^{\text {bisnac }} \mathrm{Ni}_{2}(\mathrm{H})_{2}\right]$ decreases upon reaction with water while the characteristic hydroxide resonance $\square$ of $\left[\mathrm{L}^{\text {bisnac }} \mathrm{Ni}_{2}(\mu-\mathrm{OH})\right]$ arises. At the same time two new upfield signals $\Delta$ emerge which are of the same intensity. These signals decrease over time indicating the final transformation to $\left[\mathrm{L}^{\text {bisnac }} \mathrm{Ni}_{2}(\mu-\mathrm{OH})\right]$.

Strong signal broadening for the respective hydroxo groups caused by ${ }^{17} \mathrm{O}$ was also observed in a ${ }^{17} \mathrm{O}$ NMR experiment (Figure 4.17). Compared to the ${ }^{17} \mathrm{O}$ chemical shift of $\left[\mathbf{L}^{\text {bisnac }} \mathbf{N i}_{\mathbf{2}}\left(\mu-{ }^{17} \mathrm{OH}\right)\right] \quad\left(\delta\left\{{ }^{17} \mathrm{O}\right\}_{\mathrm{OH}}=-168.71 \mathrm{ppm}\right)$, the resonance for $\mathbf{K}\left[\mathbf{L}^{\text {bisnac }} \mathbf{N i}_{\mathbf{2}}\left({ }^{17} \mathrm{OH}\right)(\mathbf{H})\right]\left(\delta\left\{{ }^{17} \mathrm{O}\right\}_{\mathrm{OH}}=-88.58 \mathrm{ppm}\right)$ is significantly shifted towards lower field. This is in accordance with the ${ }^{1} \mathrm{H}$ NMR results and provides further evidence for a coordination of the potassium cation to one free electron pair of the hydroxo oxygen.

A similar result was reported by MILSTEIN and coworkers for a reaction of a mononuclear pincer $\mathrm{Ru}^{\mathrm{II}}$ hydride complex with water. $\stackrel{[121]}{\underline{[1}}$ During the reaction a water molecule was activated, probably caused by a proton migration to the ligand system of the compound, forming a hydrido-hydroxo complex in the process. The chemical shifts for the hydride ligand $\left(\delta\left\{{ }^{1} \mathrm{H}\right\}=-14.9 \mathrm{ppm}\right)$ and the hydroxo ligand $\left(\delta\left\{{ }^{1} \mathrm{H}\right\}=-1.4 \mathrm{ppm}\right)$ of the mononuclear octahedral complex are comparable to the shifts observed for $\mathbf{K}\left[\mathbf{L}^{\text {bisnac }} \mathbf{N i}_{\mathbf{2}}(\mathbf{O H})(\mathbf{H})\right]$, despite the fact that the latter compound is dinuclear and expected to have a square 
planar metal coordination environment.

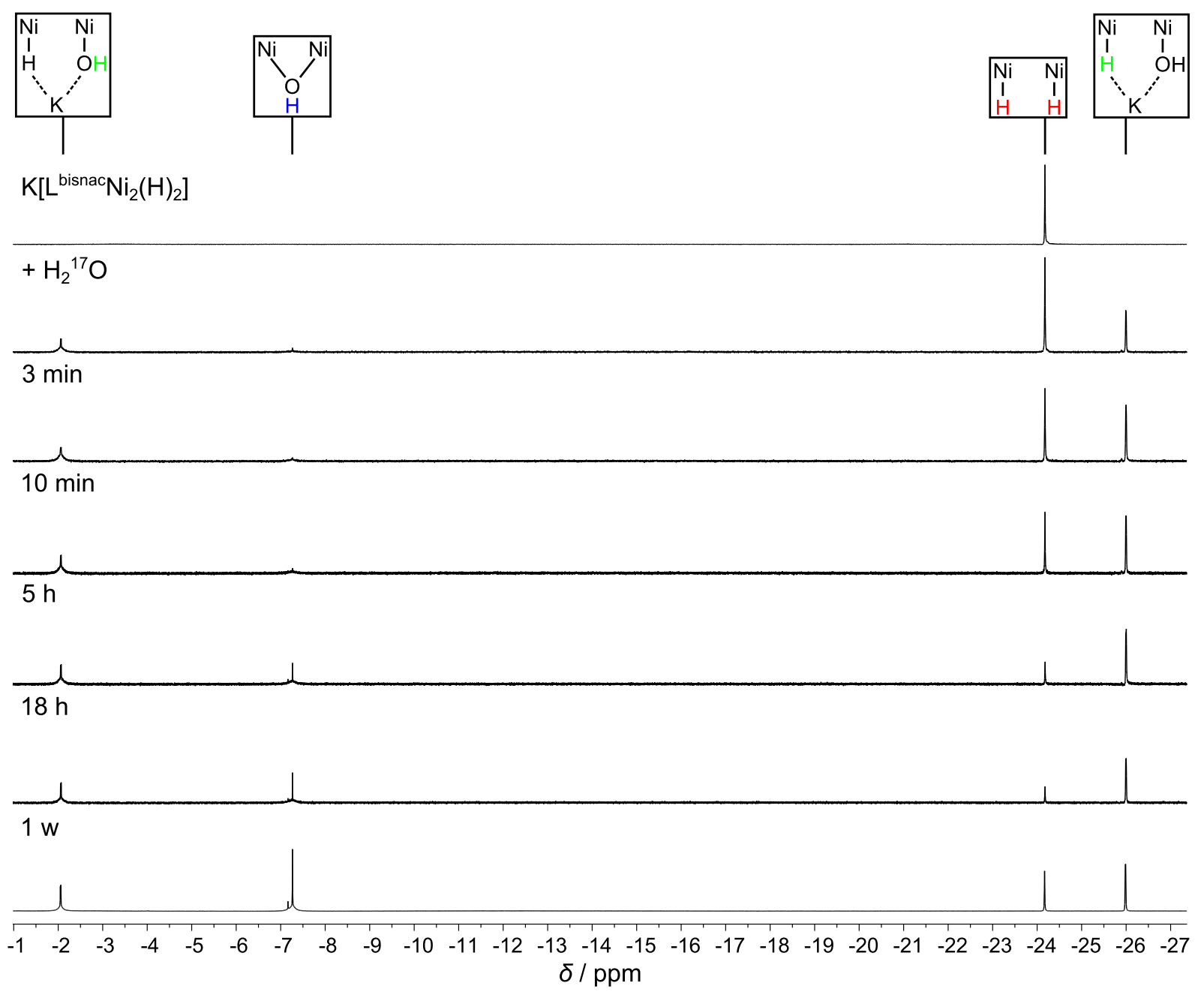

Figure 4.15.: ${ }^{1} \mathrm{H}$ NMR spectra $(400 \mathrm{MHz})$ of $\mathrm{K}\left[\mathrm{L}^{\text {bisnac }} \mathrm{Ni}_{2}(\mathrm{H})_{2}\right]$ converted with one equivalent of $\mathrm{H}_{2}{ }^{17} \mathrm{O}$ measured in THF- $\mathrm{D}_{8}$ at room temperature. Shown are the excerpts of the spectra associated with the characteristic resonances of $K\left[L^{\text {bisnac }} \mathrm{Ni}_{2}(\mathrm{H})_{2}\right]$, $\left[\mathrm{L}^{\text {bisnac }} \mathrm{Ni}_{2}(\mu-\mathrm{OH})\right]$ and $\mathrm{K}\left[\mathrm{L}^{\text {bisnac }} \mathrm{Ni}_{2}(\mathrm{OH})(\mathrm{H})\right]$ and their ${ }^{17} \mathrm{O}$ labeled analogues. The experiment was monitored over a one week timescale.

Furthermore, Milstein et al. observed a downfield shift of the hydride ligand from $-26.5 \mathrm{ppm}$ to $-14.9 \mathrm{ppm}$ after the reaction of the hydride complex with water, $\stackrel{[121]}{=}$ whereas the reaction of $\mathbf{K}\left[\mathbf{L}^{\text {bisnac }} \mathbf{N i}_{\mathbf{2}}(\mathbf{H})_{\mathbf{2}}\right]$ with water lead to a upfield shift of the remaining hydride $\left(\delta\left\{{ }^{1} \mathrm{H}\right\}=-24.2 \mathrm{ppm} \longrightarrow-26.0 \mathrm{ppm}\right)$.

Although introduction of an electron withdrawing group like $\mathrm{OH}$ should lead to a decrease in electronic shielding as seen in the reported ruthenium complex, ${ }^{[121]}$ substitution of a hydride with a hydroxo group in $\mathbf{K}\left[\mathbf{L}^{\text {bisnac }} \mathbf{N i}_{\mathbf{2}}(\mathbf{H})_{\mathbf{2}}\right]$ still retains the coordination of the potassium counterion due to the unchanged complex charge. In this scenario the cation is most likely coordinated by the two aryl sidearms of the system via $\pi$-cationic interactions similar to the precursor $\mathbf{K}\left[\mathbf{L}^{\text {bisnac }} \mathbf{N i}_{\mathbf{2}}(\mathbf{H})_{\mathbf{2}}\right]$. This cationic bridge between the hydroxide and the hydride allows for the transfer of electron density, particularly that originating from the free electron pairs belonging to the hydroxide oxygen. The transfer consequently 


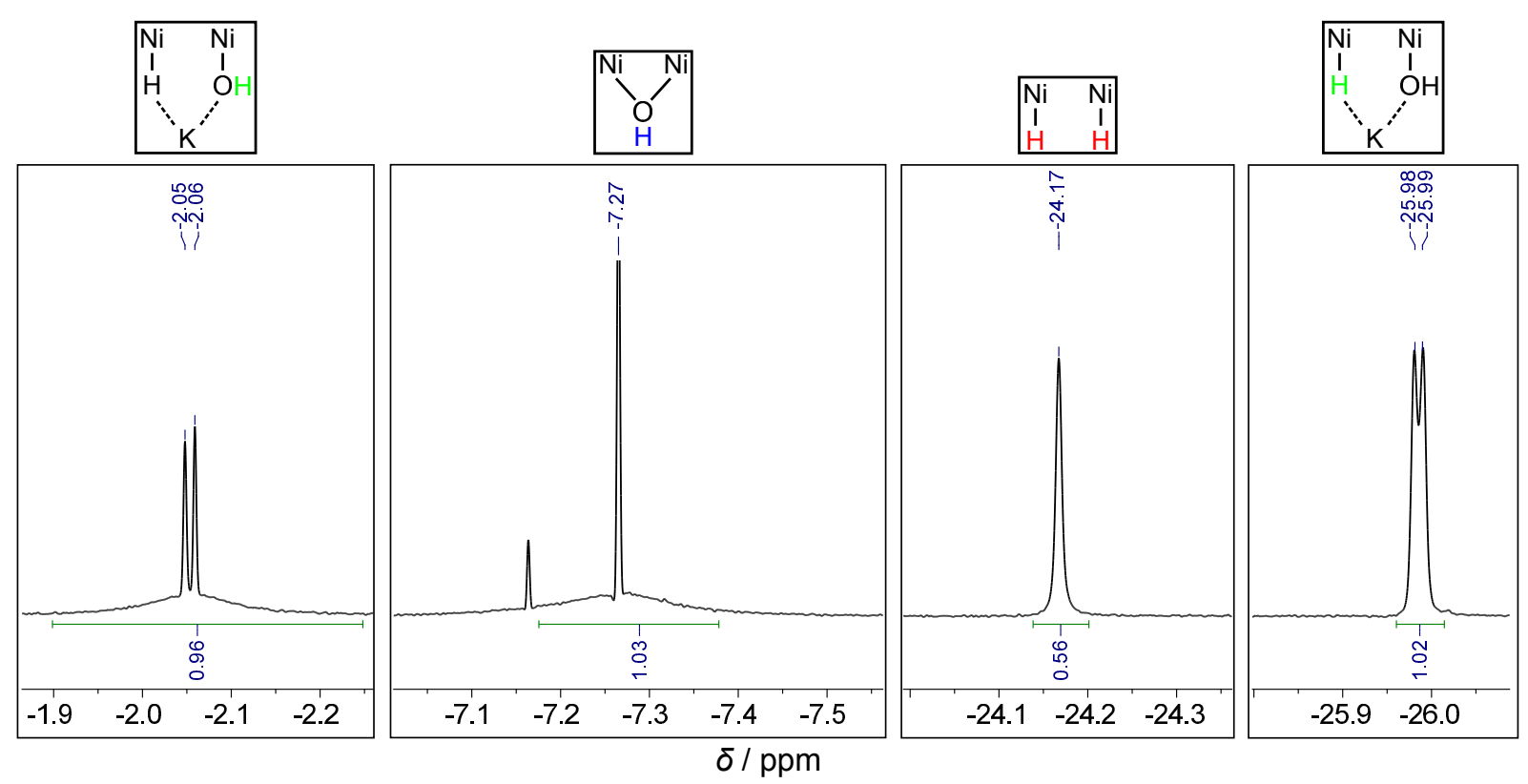

Figure 4.16.: Excerpt of the spectrum recorded after one week. Considerable line broadening of the ${ }^{17}$ O-labeled hydroxo groups is observed.

leads to a shielding effect on the hydride ligand. At the same time the hydroxide proton experiences less electronic shielding but due to the anionic nature of the complex its resonance is still observed in the upfield region of the ${ }^{1} \mathrm{H}$ NMR spectrum.

Coupling $\left(J_{\mathrm{HH}}=5.6 \mathrm{~Hz}\right)$ between both upfield resonances further substantiates the presence of a $\mathrm{H} \cdots \mathrm{K} \cdots \mathrm{OH}$ constellation (Figure 4.18). The observed $J_{\mathrm{HH}}$ coupling constant differs from the proton coupling constant of a water molecule $(7.34 \mathrm{~Hz}) .{ }^{[156]}$ Moreover, $\mathrm{H} \cdots \mathrm{H}$ interactions in hydrido complexes were reported in a range of $2-4 \mathrm{~Hz}$ and are therefore also unlikely for $\mathbf{K}\left[\mathbf{L}^{\text {bisnac }} \mathbf{N i}_{\mathbf{2}}(\mathbf{O H})(\mathbf{H})\right] .{ }^{[157,158]}$

Another measurement after three hours showed that $\mathbf{K}\left[\mathbf{L}^{\text {bisnac }} \mathbf{N i}_{\mathbf{2}}(\mathbf{H})_{\mathbf{2}}\right]$ was almost completely consumed (๑, spectrum b, Figure 4.14). Furthermore, the resonances of the intermediate $(\Delta)$ decreased while the signal of $\left[\mathbf{L}^{\mathbf{b i s n a c}} \mathbf{N i}_{2}(\mu-\mathbf{O H})\right](\mathbf{\square})$ increased. After 9 days almost complete conversion to $\left[\mathbf{L}^{\text {bisnac }} \mathbf{N i}_{\mathbf{2}}(\mu-\mathbf{O H})\right]$ is observed $(\boldsymbol{\square}$, spectrum e, Figure 4.14).

The contour plot of a ${ }^{1} \mathrm{H}^{1}{ }^{1} \mathrm{H}$ NOESY experiment (Figure 4.19), which was recorded one week after the start of the reaction shows the NOE interaction between the proton resonance of the $\mathrm{OH}$ group and the hydride $(\square)$. During conversion of $\mathbf{K}\left[\mathbf{L}^{\text {bisnac }} \mathbf{N i}_{\mathbf{2}}(\mathbf{H})_{\mathbf{2}}\right]$ with one equivalent of water, $\mathrm{H}_{2}$ is produced in an acid-base reaction, in which the protons are provided by water. The small resonance at $4.54 \mathrm{ppm}$ exhibits a strong correlation $(\square)$ to residual $\mathbf{K}\left[\mathbf{L}^{\text {bisnac }} \mathbf{N i}_{\mathbf{2}}(\mathbf{H})_{\mathbf{2}}\right]$, which is expected for the rapid exchange between molecular hydrogen and the hydrides of the complex. Remarkably, the small amount of $\mathrm{H}_{2}$ formed is still observed in the spectrum after one week, implying its coordination to the complex rather than equilibration with the $\mathrm{N}_{2}$ atmosphere of the sample. 


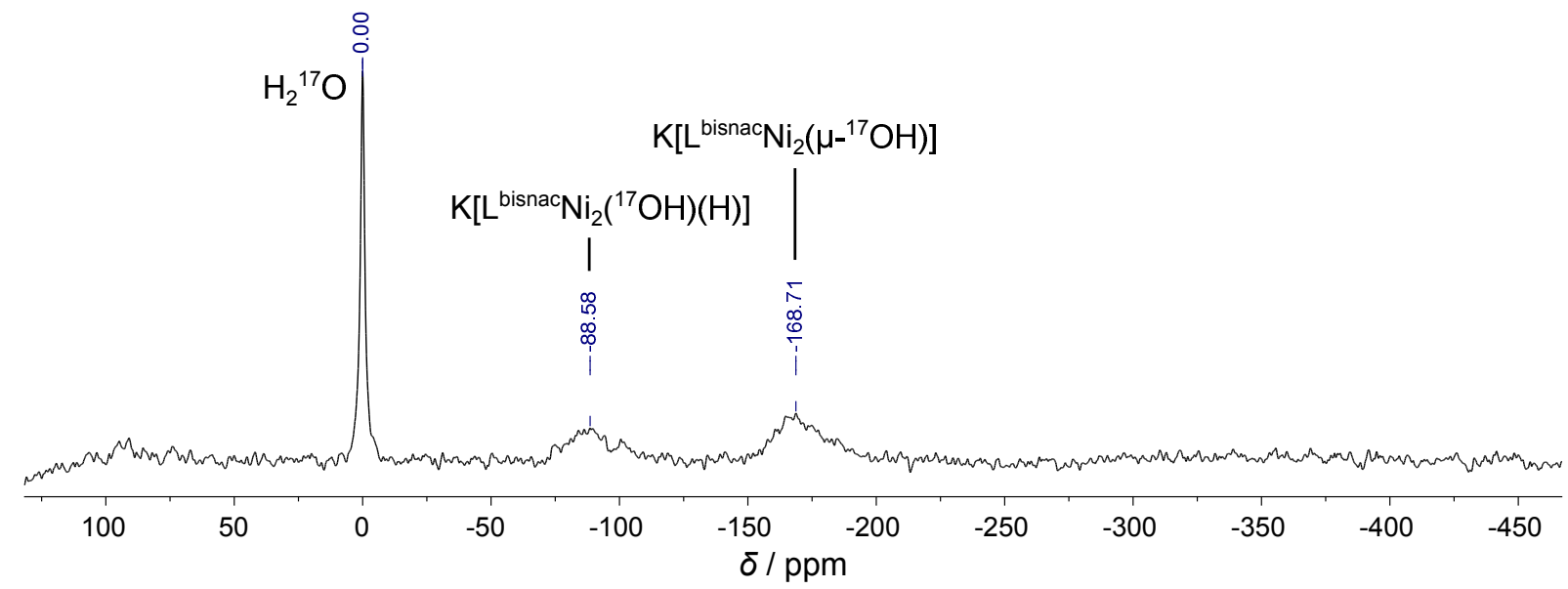

Figure 4.17.: ${ }^{17} \mathrm{O}$ NMR spectrum (54 MHz) recorded after two weeks. While the $\mathrm{H}_{2}{ }^{17} \mathrm{O}$ associated signal is sharp, the signals correlated to $\mathrm{K}\left[\mathrm{L}^{\text {bisnac }} \mathrm{Ni}_{2}\left({ }^{17} \mathrm{OH}\right)(\mathrm{H})\right]$ and $\mathrm{K}\left[\mathrm{L}^{\text {bisnac }} \mathrm{Ni}_{2}\left(\mu{ }^{-17} \mathrm{OH}\right)\right]$ exhibit significant broadening.

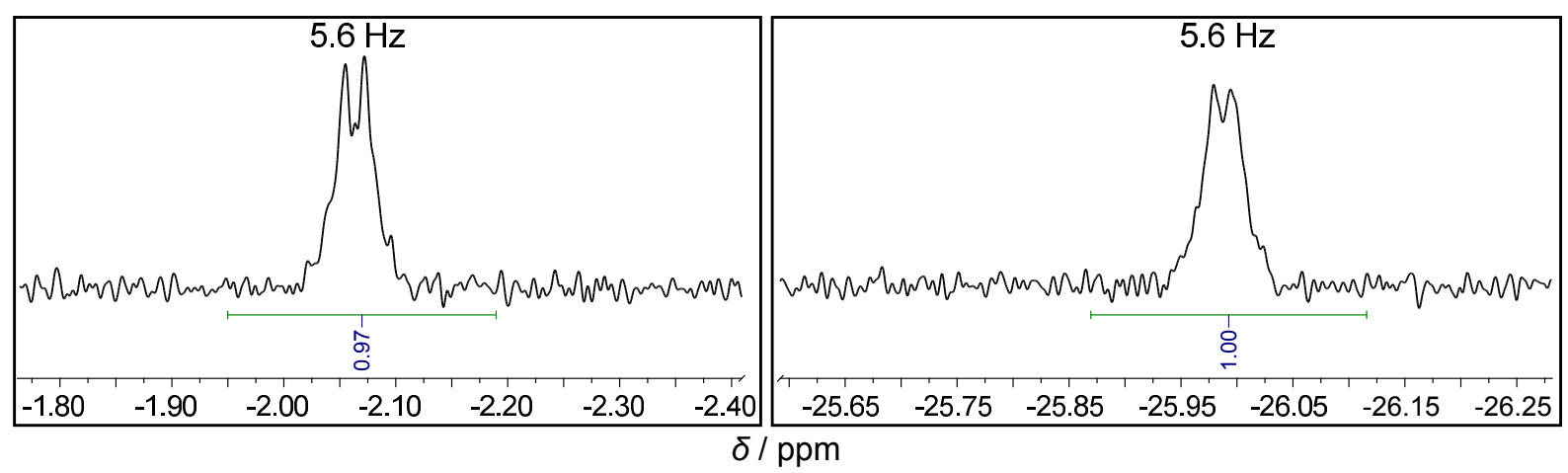

Figure 4.18.: Excerpt of a ${ }^{1} \mathrm{H}$ NMR spectrum recorded after one week emphasizing the two signals occuring at $-2.1 \mathrm{ppm}$ and $-26.0 \mathrm{ppm}$. Both signals exhibit a coupling constant of $5.56 \mathrm{~Hz}$.

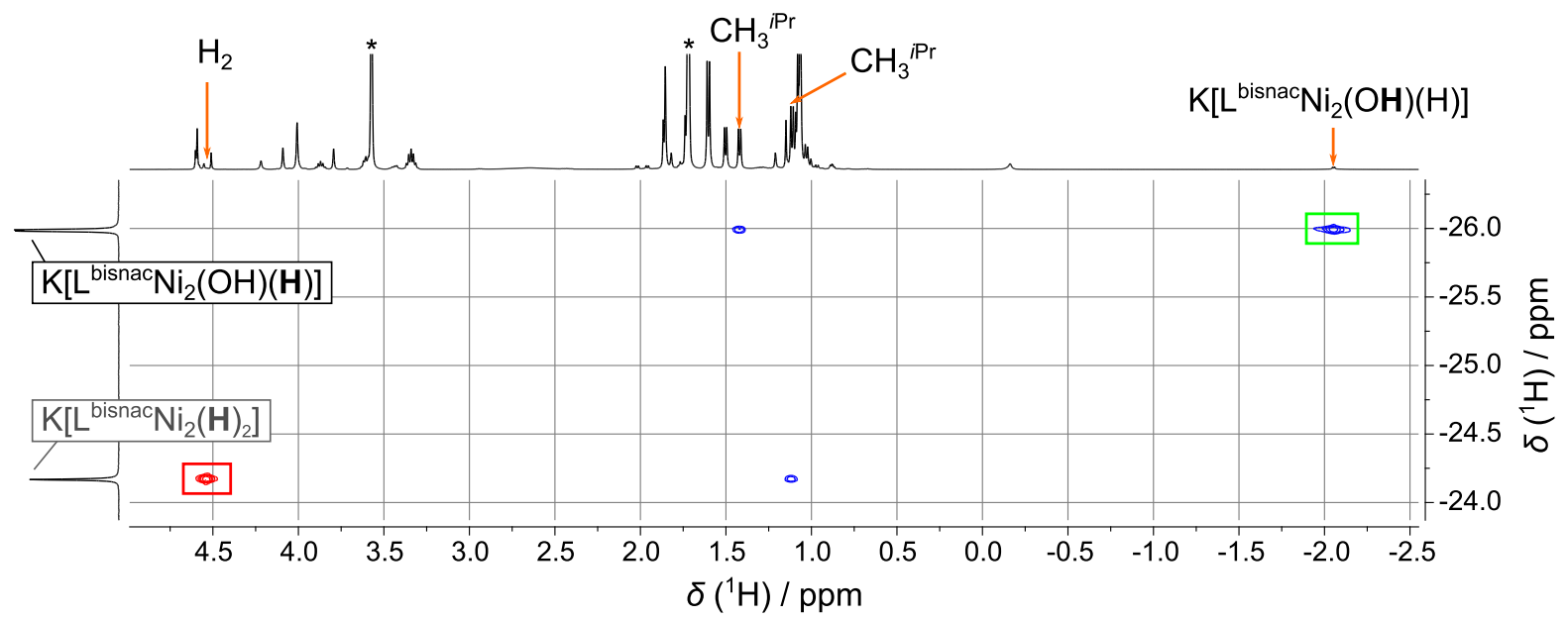

Figure 4.19.: ${ }^{1} \mathrm{H}$ NOESY spectrum $(500 \mathrm{MHz})$ recorded after one week. The NOE interaction between the hydroxo group and the hydride of $\left.\mathrm{K}_{[}{ }^{\text {bisnac }} \mathbf{N i}_{2}(\mathbf{O H})(\mathbf{H})\right]$ is indicated $(\square)$. Furthermore, an exchange correlation can be observed $(\square)$. It is attributed to the exchange between unconverted $K\left[L^{\text {bisnac }} \mathrm{Ni}_{2}(\mathbf{H})_{2}\right]$ and $\mathrm{H}_{2}$, which was produced during the reaction with water. Residual solvents are marked $\left({ }^{*}\right)$. 
The excerpt of a recorded IR spectrum frigure 4.20) from the reaction mixture shows the $\mathrm{O}-\mathrm{H}$ stretching vibration of $\left[\mathbf{L}^{\text {bisnac }} \mathbf{N i}_{\mathbf{2}}(\mu-\mathbf{O H})\right]\left(\tilde{\nu}=3608 \mathrm{~cm}^{-1}\right)$. An additional band can be observed at $\tilde{\nu}=3622 \mathrm{~cm}^{-1}$, attributed to the hydroxo group of $\mathbf{K}\left[\mathbf{L}^{\text {bisnac }} \mathbf{N i}_{\mathbf{2}}(\mathbf{O H})(\mathbf{H})\right]$. It corresponds to the $\mathrm{OH}$ stretching vibration reported for hydroxo-hydrido complexes $\left(\tilde{\nu}_{\mathrm{O}-\mathrm{H}}=3620 \mathrm{~cm}^{-1}\right) \cdot{ }^{[159,160]} \mathrm{A}$ band associated to the Ni-H stretching vibration could not be determined in the expected region $\left(\tilde{\nu}_{\mathrm{Ni}-\mathrm{H}} \approx 1768-\right.$ $\left.1950 \mathrm{~cm}^{-1}\right) . \underline{[144,145]}$

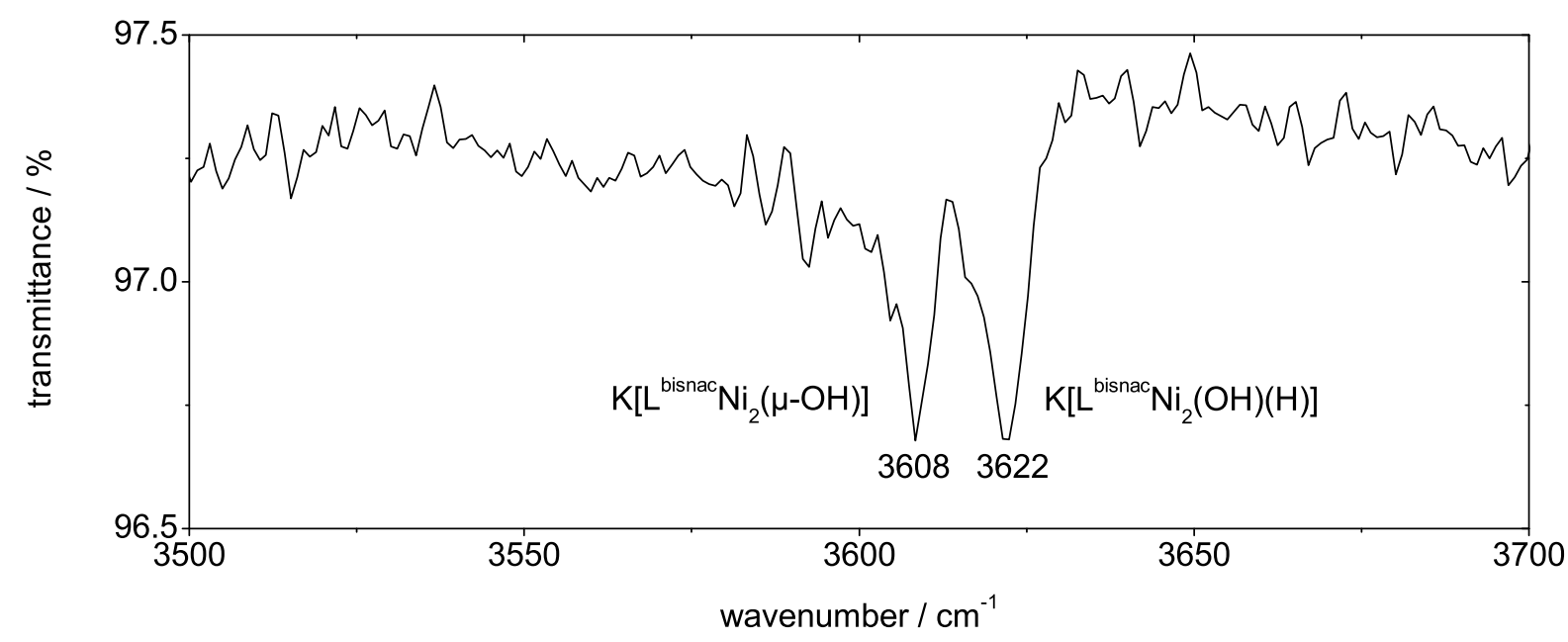

Figure 4.20.: Excerpt of the IR spectrum recorded from the reaction solution $\left(K\left[L^{\text {bisnac }} \mathbf{N i}_{2}\left(\mathbf{H}_{2}\right]+\mathrm{H}_{2} \mathrm{O}\right)\right.$. In addition to the $\mathrm{O}-\mathrm{H}$ stretching vibration of the final product $\left[\mathrm{L}^{\text {bisnac }} \mathrm{Ni}_{2}(\mu-\mathrm{OH})\right]\left(\tilde{\nu}=3608 \mathrm{~cm}^{-1}\right)$, a band at $\tilde{\nu}=3622 \mathrm{~cm}^{-1}$ attributed to $\mathrm{K}\left[\mathrm{L}^{\text {bisnac }} \mathrm{Ni}_{2}(\mathrm{OH})(\mathrm{H})\right]$ also appeared.

\section{Experiments with $\mathrm{D}_{2} \mathrm{O}$}

To gain more insight into the reaction process, the experiment was repeated with $\mathrm{D}_{2} \mathrm{O}$ and immediately monitored by ${ }^{2} \mathrm{H}$ NMR spectroscopy. The result of the experiment is illustrated in Figure 4.21. The first measurement revealed the new deuterium resonance at $-26 \mathrm{ppm}$ attributed to the hydride, but a deuterium hydroxide resonance was observed neither at $-2 \mathrm{ppm}$ nor at $-7.3 \mathrm{ppm}\left(\boldsymbol{\square}, \mathbf{\square}\right.$, Figure 4.21). Furthermore, $\mathrm{D}_{2} \mathrm{O}$ is still not fully converted as indicated by the broadened signal at 2-4 ppm (0).

Surprisingly, another resonance arose at $-24 \mathrm{ppm}$, which was assigned to the deuterated form of $\mathbf{K}\left[\mathbf{L}^{\text {bisnac }} \mathbf{N i}_{\mathbf{2}}(\mathbf{H})_{\mathbf{2}}\right]$ ( $\mathbf{\square}$, Figure 4.21).

Over time the broad signal at 2-4 ppm (O) vanished while at the same time a singlet at $4.53 \mathrm{ppm}$ and a doublet at $4.57 \mathrm{ppm}$ arose $(\mathbf{O})$. This indicates the formation of $\mathrm{D}_{2}$ and HD ( $\square$, Figure 4.21). 


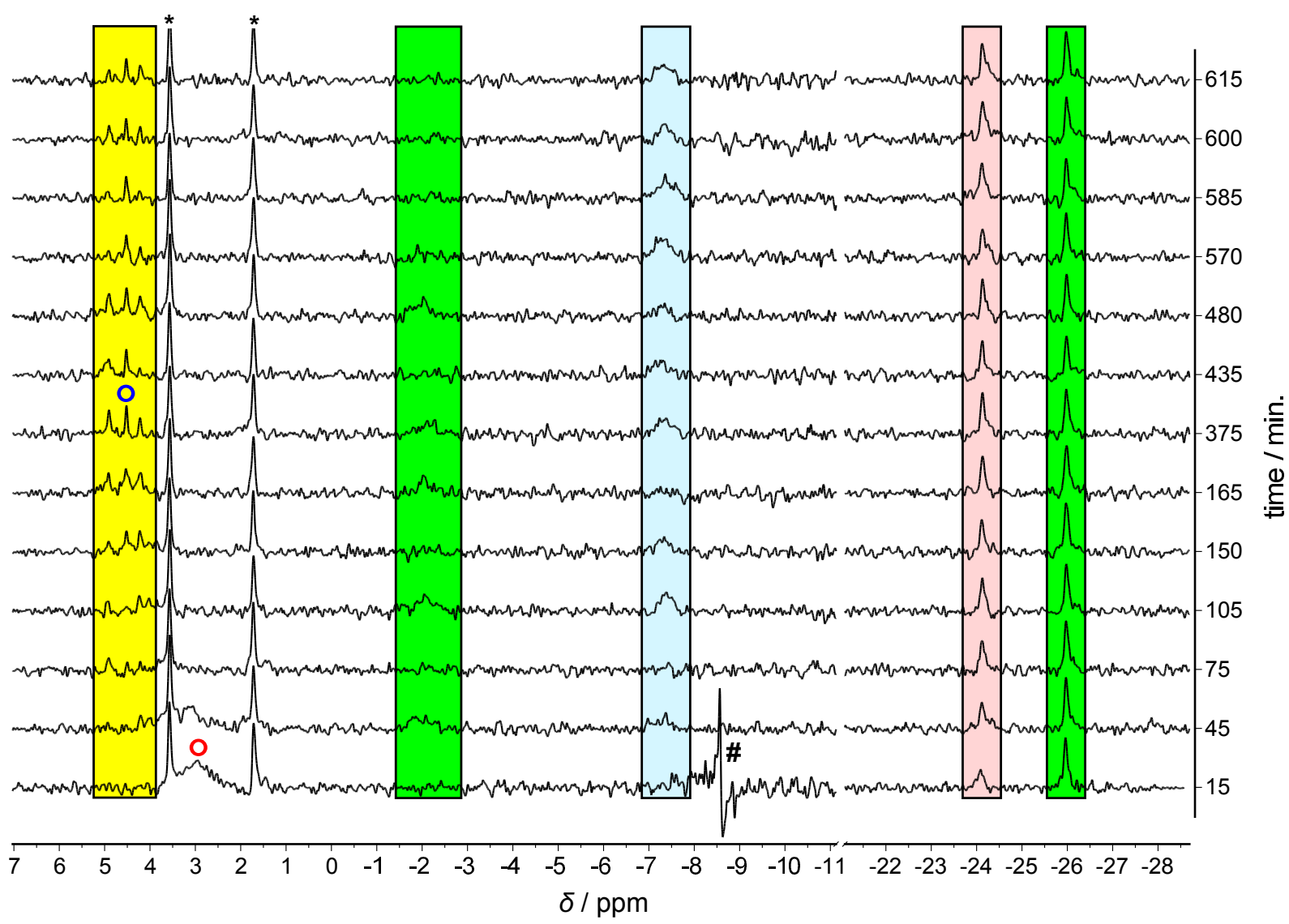

Figure 4.21.: The reaction of $\mathrm{K}\left[\mathrm{L}^{\text {bisnac }} \mathrm{Ni}_{2}(\mathbf{H})_{2}\right]$ with $\mathrm{D}_{2} \mathrm{O}$ monitored by ${ }^{2} \mathrm{H}$ NMR over a $10 \mathrm{~h}$ time frame. The chemical shift regions for the different species are marked $\left(\boldsymbol{\square}: \mathbf{K}\left[\mathrm{L}^{\text {bisnac }} \mathbf{N i}_{2}(\mu-\mathrm{OD})\right]\right.$; $\mathbf{\square}$ :

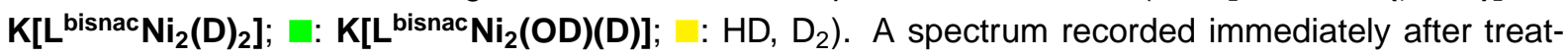
ment with $\mathrm{D}_{2} \mathrm{O}$ revealed the hydridic resonanes at $-24 \mathrm{ppm}$ and $-26 \mathrm{ppm}$ and another broadened signal from 2-4 ppm $0\left(D_{2} O\right)$. The OD resonances at -2.1 and $-7.3 \mathrm{ppm}$ were observed after $45 \mathrm{~min}$. After $75 \mathrm{~min}$, a small singlet $\left(4.53 \mathrm{ppm}, \mathrm{D}_{2}\right)$ and a doublet (HD) emerged at $4.57 \mathrm{ppm}$. These signals increased in a $6 \mathrm{~h}$ timeframe 0 . The trace associated with the first measurement shows an $\operatorname{artifact}^{\dagger}(\#)$. Residual solvent (THF-D) is marked $\left(^{*}\right)$.

Furthermore, the weak hydroxo resonance of $\mathbf{K}\left[\mathbf{L}^{\text {bisnac }} \mathbf{N i}_{\mathbf{2}}(\mathrm{OD})\right]$ can be observed at $\delta\left\{{ }^{2} \mathrm{H}\right\}=-7.26 \mathrm{ppm}(\boldsymbol{\square})$. The hydroxo resonance of $\mathbf{K}\left[\mathbf{L}^{\text {bisnac }} \mathbf{N i}_{\mathbf{2}}(\mathbf{O D})(\mathbf{D})\right]$, which was expected at $\delta\left\{{ }^{2} \mathrm{H}\right\}=-2.1 \mathrm{ppm}(\square)$, was not clearly visible due to very low intensity. This phenomenon can be caused by a continual H-D exchange process between the OD substituent and the formed byproducts $\left(\mathrm{HD}, \mathrm{D}_{2}, \mathrm{H}_{2}\right)$. The deuterium atom of the hydroxo group in $\mathbf{K}\left[\mathbf{L}^{\text {bisnac }} \mathbf{N i}_{\mathbf{2}}(\mathbf{O D})(\mathbf{D})\right]$ is supposed to be more acidic due to the lower electronic shielding, while the $\mu$-OD of $\mathbf{K}\left[\mathbf{L}^{\text {bisnac }} \mathbf{N i}_{2}(\mathrm{OD})\right]$ resonating at a higher field is less acidic. Therefore, a preferable exchange of $\mathbf{K}\left[\mathbf{L}^{\text {bisnac }} \mathbf{N i}_{2}(\mathrm{OD})(\mathrm{D})\right]$ with the byproducts to form $\mathbf{K}\left[\mathbf{L}^{\text {bisnac }} \mathbf{N i}_{\mathbf{2}}(\mathbf{O H})(\mathrm{D})\right]$ can occur, providing an explanation for the low deuterium signal intensity observed.

† During the first measurement an artifact occured at the point of irradiation ( $-8.5 \mathrm{ppm})$. For the following measurements the point of irradiation was set to $-12 \mathrm{ppm}$. 


\section{Mechanistic Considerations}

Based on the results, a mechanistic proposal for the formation of $\left[\mathbf{L}^{\text {bisnac }} \mathbf{N i}_{\mathbf{2}}(\mu-\mathbf{O H})\right]$ from $\mathbf{K}\left[\mathbf{L}^{\text {bisnac }} \mathbf{N i}_{\mathbf{2}}(\mathbf{H})_{\mathbf{2}}\right]$ was established (Scheme 4.22). In the first step $\mathbf{K}\left[\mathbf{L}^{\text {bisnac }} \mathbf{N i}_{\mathbf{2}}(\mathbf{O H})(\mathbf{H})\right]$ is formed as an intermediate. The experiments with deuterium labeled water revealed the formation of $\mathrm{D}_{2}$ and $\mathrm{HD}$. This indicated two probable pathways (a) and (b), which are described in Scheme 4.22. An acid-base reaction of $\mathrm{D}_{2} \mathrm{O}$ (Figure 4.21) with $\left.\mathbf{K}\left[\mathbf{L}^{\text {bisnac }} \mathbf{N i}_{\mathbf{2}} \mathbf{( H}\right)_{\mathbf{2}}\right]$ leads to the release of HD (a). At the same time, $\mathrm{H}_{2}$ is produced by a non-scrambling reaction decribed by pathway (b). In this case the whole water molecule is incorporated into the complex. The mechanism for the substitution in pathway (b) is comparable to the direct exchange of $\mathbf{K}\left[\mathbf{L}^{\text {bisnac }} \mathbf{N i}_{\mathbf{2}}(\mathbf{H})_{\mathbf{2}}\right]$ with $\mathrm{D}_{2}$. The following step describes the release of another molecule of HD or $\mathrm{D}_{2}$ (Figure 4.21) from the formed intermediates resulting in the $\mu$-oxo complex $\mathbf{K}\left[\mathbf{L}^{\mathbf{b i s n a c}} \mathbf{N i}_{\mathbf{2}}(\mu-\mathrm{O})\right]$. Subsequently, $\mathbf{K}\left[\mathbf{L}^{\text {bisnac }} \mathbf{N i}_{\mathbf{2}}(\mu-\mathrm{O})\right]$ reacts with another molecule of water eventually leading to $\left[\mathbf{L}^{\text {bisnac }} \mathbf{N i}_{\mathbf{2}}(\mu-\mathrm{OD})\right]$ (Scheme 4.22). This last conversion is fast, since the $\mu$-oxo species was not observed in ${ }^{17} \mathrm{O}$ NMR during experiments with $\mathrm{H}_{2}{ }^{17} \mathrm{O}$.

While the rapidly formed complexes were observed immediately in the ${ }^{2} \mathrm{H}$ NMR experiment, both $\mathrm{D}_{2}$ and HD could not be determined at the same time (Figure 4.21). This is due to a rapid exchange with residual $\mathbf{K}\left[\mathbf{L}^{\text {bisnac }} \mathbf{N i}_{\mathbf{2}}(\mathbf{H})_{2}\right]$ giving $\mathbf{K}\left[\mathbf{L}^{\text {bisnac }} \mathbf{N i}_{\mathbf{2}}(\mathbf{D})_{2}\right]$ or $\mathbf{K}\left[\mathbf{L}^{\text {bisnac }} \mathbf{N i}_{\mathbf{2}}(\mathbf{H D})\right]$, respectively. The latter was not distinguishable by a chemical shift difference of the $(\mathrm{D})_{2}$ species due to the low resolution in ${ }^{2} \mathrm{H}$ NMR. During the process $\mathrm{H}_{2}$ is produced, which is not observed in ${ }^{2} \mathrm{H}$ NMR. After conversion of residual $\mathbf{K}\left[\mathbf{L}^{\text {bisnac }} \mathbf{N i}_{\mathbf{2}}(\mathbf{H})_{\mathbf{2}}\right]$, further $\mathrm{HD}$ and $\mathrm{D}_{2}$ production was visible in the ${ }^{2} \mathrm{H}$ NMR spectrum (Figure 4.21). 


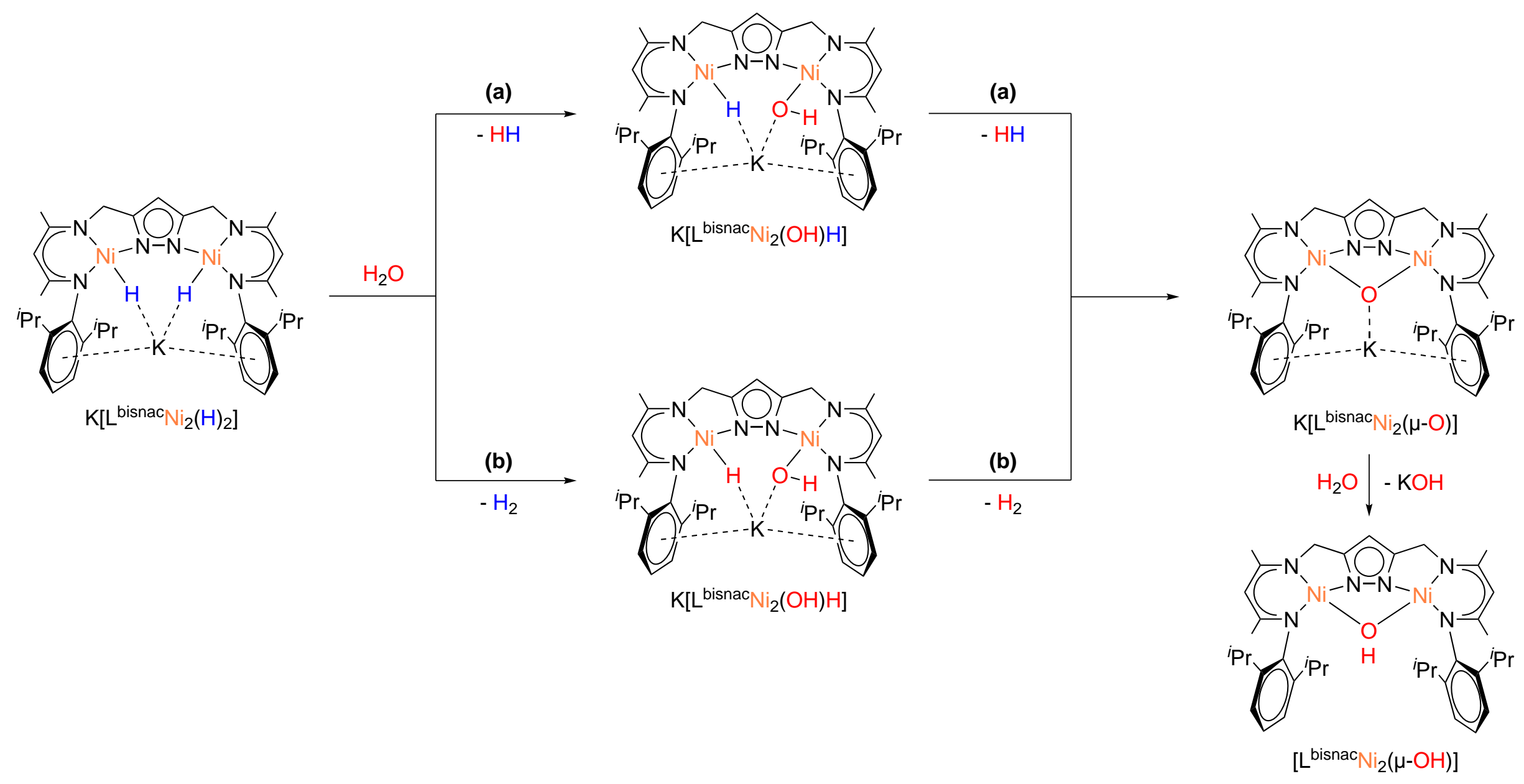

Scheme 4.22.: Proposed reaction pathways for the conversion of $K\left[\mathrm{~L}^{\text {bisnac }} \mathbf{N i}_{2}(\mathrm{H})_{2}\right]$ with $\mathrm{H}_{2} \mathrm{O}$ to $\left[\mathrm{L}^{\text {bisnac }} \mathrm{Ni}_{2}(\mu-\mathrm{OH})\right]$. The pathway (a) represents the formation of the intermediate $\mathrm{K}\left[\mathrm{L}^{\text {bisnac }} \mathrm{Ni}_{2}(\mathrm{OH})(\mathrm{H})\right]$ as a result of an acid-base reaction with water. At the same time $\mathrm{HH}$ is produced. Pathway (b) describes a non-scrambling mediated formation of the intermediate $\left[\mathrm{L}^{\text {bisnac }} \mathrm{Ni}_{2}(\mathrm{OH})(\mathrm{H})\right.$ ], which is accompanied by the release of $\mathrm{H}_{2}$. In the next step the intermediates release another molecule of $\mathrm{HH}$ (a) or $\mathrm{H}_{2}$ (b) giving the oxo-bridged $\mathbf{K}\left[\mathrm{L}^{\text {bisnac }} \mathrm{Ni}_{2}(\mu-\mathrm{O})\right]$. The subsequent reaction with $\mathrm{H}_{2} \mathrm{O}$ leads to the final formation of [ $\left.\mathrm{L}^{\text {bisnac }} \mathrm{Ni}_{2}(\mu-\mathrm{OH})\right]$. 


\subsection{Summary and Outlook}

The anionic dihydride complex $\mathbf{K}\left[\mathbf{L}^{\text {bisnac }} \mathbf{N i}_{\mathbf{2}}(\mathbf{H})_{\mathbf{2}}\right]$ was established and fully characterized. $\mathbf{K}\left[\mathbf{L}^{\text {bisnac }} \mathbf{N i}_{\mathbf{2}}(\mathbf{H})_{\mathbf{2}}\right]$ exchanges with $\mathrm{D}_{2}$ to yield $\mathrm{H}_{2}$ in the process. It was shown that the exchange involves the direct pairwise substitution of the $\mathrm{Ni}-\mathrm{H}$ atoms with $\mathrm{D}_{2}$ instead of a two step exchange process including scrambling to HD. This exchange behaviour was not expected and may therefore provide new insights into the exchange processes of bimetallic enzymes.

Furthermore, it was shown that $\mathbf{K}\left[\mathbf{L}^{\text {bisnac }} \mathbf{N i}_{\mathbf{2}}(\mathbf{H})_{\mathbf{2}}\right]$ is susceptible towards water giving rise to a hydroxo-hydrido intermediate $\mathbf{K}\left[\mathbf{L}^{\text {bisnac }} \mathbf{N i}_{2}(\mathbf{O H})(\mathbf{H})\right]$. The hydroxide substituent was determined via ${ }^{17} \mathrm{O}$ labeling, while an experiment with $\mathrm{D}_{2} \mathrm{O}$ provided further insight into the reaction mechanism. $\mathrm{H}_{2}, \mathrm{D}_{2}$ and $\mathrm{HD}$ were formed during the reaction with $\mathrm{D}_{2} \mathrm{O}$ due to two proposed reaction pathways. In one pathway water provides protons to furnish the liberation of $\mathrm{H}_{2}$. The second pathway involves a direct substitution of the $\mathrm{Ni}-\mathrm{H}$ hydrogen atoms with the water molecule and leads to the pairwise release of the $\mathrm{Ni}-\mathrm{H}$ hydrogen atoms as $\mathrm{H}_{2}$, which is consistent with the exchange behavior of $\left.\mathbf{K}\left[\mathbf{L}^{\text {bisnac }} \mathbf{N i}_{\mathbf{2}} \mathbf{(} \mathbf{H}\right)_{\mathbf{2}}\right]$ with $\mathrm{D}_{2}$. Subsequently, the proposed $\left[\mathbf{L}^{\mathbf{b i s n a c}} \mathbf{N i}_{\mathbf{2}}(\mu-\mathrm{O})\right]$ is formed upon liberation of another molecule of $\mathrm{H}_{2}$. Remarkably, the liberated $\mathrm{H}_{2}$ entirely originates from the previously incorporated water molecule. $\left[\mathbf{L}^{\text {bisnac }} \mathbf{N i}_{\mathbf{2}}(\mu-\mathrm{O})\right]$ was not observed by NMR techniques and is eventually converted to $\left[\mathbf{L}^{\text {bisnac }} \mathbf{N i}_{\mathbf{2}}(\mu-\mathbf{O H})\right]$ with another molecule of $\mathrm{H}_{2} \mathrm{O}$ in a rapid fashion.

The remarkable outcome of the reaction of $\mathbf{K}\left[\mathbf{L}^{\text {bisnac }} \mathbf{N i}_{\mathbf{2}}(\mathbf{H})_{2}\right]$ with water provides valuable information for water splitting processes and the liberation of $\mathrm{H}_{2}$. Furthermore, $\mathbf{K}\left[\mathbf{L}^{\text {bisnac }} \mathbf{N i}_{\mathbf{2}}(\mathrm{OH})(\mathbf{H})\right]$ can serve as a bimetallic model complex for [NiFe] hydrogenase and may represent the first example of hydrogen production with protons derived from water.

$\left.\mathbf{K}\left[\mathbf{L}^{\text {bisnac }} \mathbf{N i}_{\mathbf{2}} \mathbf{( H}\right)_{\mathbf{2}}\right]$ has already shown remarkable reactivity towards $\mathrm{H}_{2}$ and water, yet the scope of further possible reactions with this promising nickel dihydride complex still remains to be explored fully. A small preview of further conversions is granted in the following chapters. It is anticipated that the promise of these initial studies will provide motivation for future works to discover the full spectrum of capabilities associated with the $\mathbf{K}\left[\mathbf{L}^{\text {bisnac }} \mathbf{N i}_{\mathbf{2}}(\mathbf{H})_{\mathbf{2}}\right]$ system. 


\section{A Phenylvinyl-Bridged Dinuclear Nickel(II) Complex}

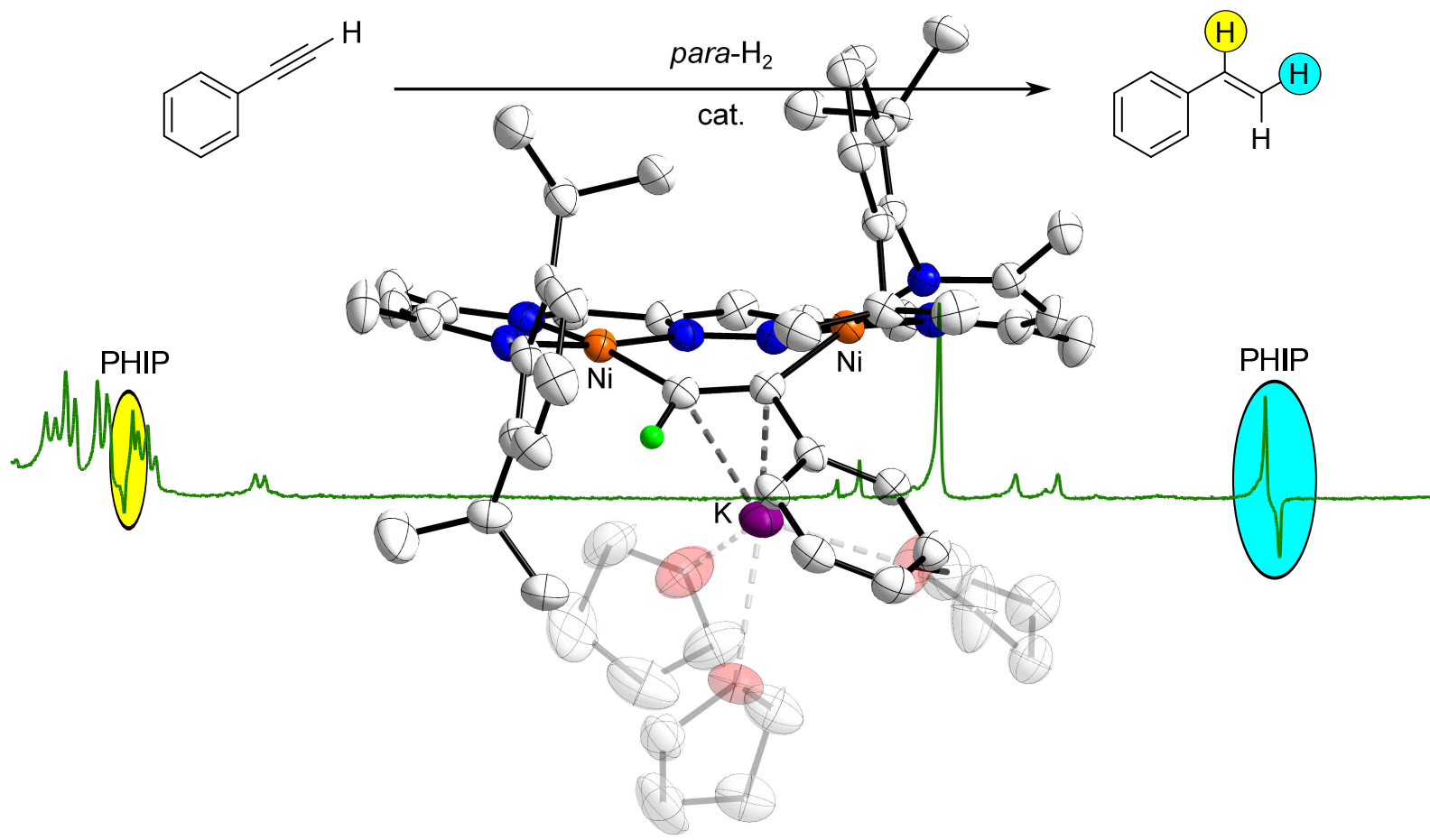

Abstract $\mathbf{K}\left[\mathbf{L}^{\text {bisnac }} \mathrm{Ni}_{2}(\mathbf{H})_{2}\right]$ was converted with phenylacetylene to give a phenylvinyl-bridged complex. This chapter deals with the detailed characterization of the complex using different NMR techniques and provides insight into the reaction pathway by means of deuterium labeling experiments. Furthermore, a catalytic semihydrogenation of phenylacetylene to styrene in presence of the complex was investigated. Isotopic labeling and para-hydrogen induced polarization (PHIP), monitored by NMR spectroscopy, came into use to elucidate mechanistic pathways and selectivities. 


\subsection{State of Knowledge}

Among the various possibilities to utilize hydride complexes of transition metals, the reaction with unsaturated hydrocarbon compounds is of significant interest due to its involvement in the catalytic cycles of reduction processes such as hydrogenation or hydroboration as well as hydrosilylation or oligomerization processes. ${ }^{[161]}$ For instance, several systems suitable for catatalytic alkyne dimerization-,$\stackrel{[162-164]}{\underline{160]}}$ oligomerization-,$\stackrel{[165]}{,}$ hydro-

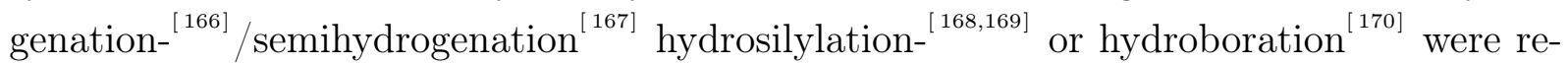
ported. BIANCHIN et al. investigated the catalytic alkyne hydrogenation activity of hydride complexes with various different metals. $\stackrel{[171-173]}{ }$

A few reactions of nickel hydride complexes with substituted alkynes are reported. $\stackrel{[181-183]}{[}$ The reaction of a dinuclear nickel hydride complex with internal alkynes led to the formation of mononuclear $\eta^{2}$ - alkyne adducts (Scheme 5.1) ${ }^{[181]}$ Years later in 2011 REYES reported a similar complex with a modified alkyl substituent (isopropyl instead of tertbutyl). This complex was able to hydrogenate diphenylacetylene in a catalytical fashion by addition of dihydrogen. ${ }^{[182]}$ Recently, WILSON et al. described nickel POCOP$\left(\left[2,6-\left(\mathrm{R}_{2} \mathrm{PO}\right)_{2} \mathrm{C}_{6} \mathrm{H}_{3}\right] \mathrm{R}={ }^{i} \mathrm{Pr},{ }^{c} \mathrm{Pe}\right)^{[161]}$ pincer hydride complexes and their reactivity towards phenylacetylene (S. Scheme 5.1). ${ }^{[183]}$

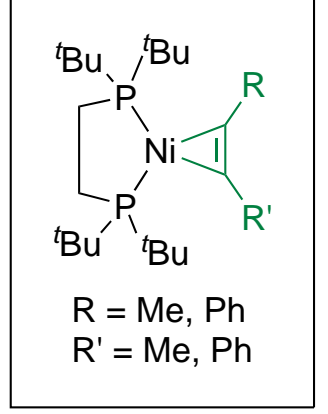

WATERMAN, 2003

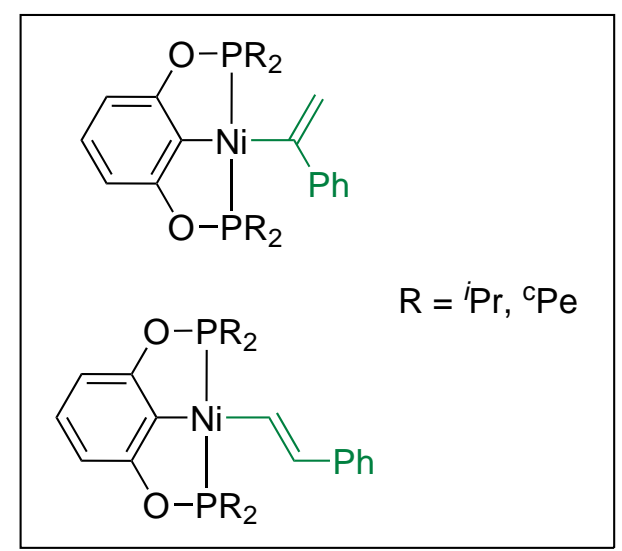

WILSON, 2015

Scheme 5.1: Examples of nickel alkenyl-complexes prepared from hydride complexes. ${ }^{[181,183]}$

\section{Styrene}

Styrene represents a simple aromatic hydrocarbon but nonetheless is included in many chemical industrial processes. Besides the use as a component in hydroformylation, hydroamination, organic synthesis and metathesis reactions, the compound serves as an important substrate in the polymer industry. ${ }^{[174]}$ The very versatile styrene monomer, also commercially known as SM, is produced in large quantities for the manufacture of 
plastics such as acrylonitrile-butadiene-styrene copolymer (ABS), styrene-arylonitrile resins (SAN), styrene-butadiene (SB) latex, styrene-butadiene-rubber (SBR), unsaturated polyester resins (UPR) and, most importantly, polystyrene, which accounts for $64 \%$ of the worldwide demand of styrene. ${ }^{[175]}$ So far, the production of styrene in an industrial scale is performed by coproduction of propylene oxide (PO) and SM or by catalytic dehydrogenation of ethylbenzene $(\mathrm{EBDH})$ using a potassium-promoted iron oxide catalyst. ${ }^{[175,176]}$ The styrene produced in the latter way accounts for $\geq 90 \%$ of the world's capacity, resulting in an anual amount of 25 million tons of the monomer. ${ }^{[177]}$ Due to the harsh conditions particularly in the arene alkylation process, which is usually performed using a Friedel-Crafts or zeolite catalyst, problems regarding alkylation selectivity or stoichiometric waste are often an issue. Thus, other more efficient methods are sought-after like the direct formation of styrene from benzene and ethylene via aromatic $\mathrm{C}-\mathrm{H}$ bond activation and C-C bond formation. $\stackrel{[178,179]}{ }$

\section{Semihydrogenation of Phenylacetylene to Styrene}

An alternative approach to synthesize terminal alkenes is the selective hydrogenation of alkynes (semihydrogenation) catalyzed by transition metal complexes (Scheme 5.2).

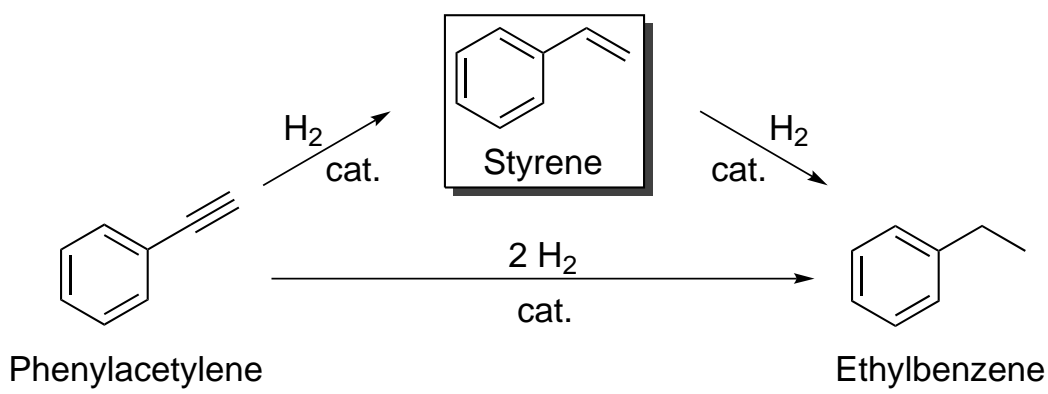

Scheme 5.2: The hydrogenation of phenylacetylene to styrene (semihydrogenation) and ethylbenzene.

A variety of Pd-, Rh-, Ir-, Ru- and V-catalyzed systems for the hydrogenation of alkynes to give alkenes have been reported in the past decades. ${ }^{[180]}$ However there are still key issues for this type of hydrogenation reaction such as the replacement of precious metals and over-hydrogenation. ${ }^{[180]}$ The group of BIANCHINI reported the catalytic generation of styrene from phenylacetylene with a cis-hydrido( $\eta^{2}$-dihydrogen)ruthenium(II)- and a similar iron(II)- based complex. $\stackrel{[172,173]}{[}$ 


\subsection{Motivation}

Organonickel complexes are frequently used in industrial homogenous catalysis. The large variety of carbon-carbon-bond formation reactions, which can be carried out with high selectivity, includes linear and cyclic oligomerization and polymerization reactions of monoenes and dienes as well as hydrocyanation reactions. In particular, complexes, serving as active catalysts for oligomerization and isomerization reactions are supposed to also be active as hydrogenation catalysts. Despite the fact that hydrogenase enzymes, containing the abundantly available transition metals iron and nickel, are used for the activation of dihydrogen in nature, and that Raney-Ni is used as one of the oldest and most efficient heterogenous hydrogenation catalysts, very few nickel complexes which are able to catalyze homogenous hydrogenation reactions, are known. ${ }^{[184,185]}$

Most of these homogeneous hydrogenation catalysts are based on mononuclear complexes and associated dinclear systems are rarely studied. A potential cooperative effect between two metal centers could prove advantageous for selectivity towards formed products. The semihydrogenation of phenylacetylene to styrene is a selective hydrogenation process. Therefore, and due to the importance of industrial styrene production the interest in a preorganized bimetallic system arose. Due to the rarity of such compounds, an establishment may provide the opportunity to study new mechanistical aspects. 


\subsection{Preparation and Characterization of the Complex}

\subsubsection{Synthesis and Molecular Structure}

Treatment of an orange solution of $\mathbf{K}\left[\mathbf{L}^{\text {bisnac }} \mathbf{N i}_{\mathbf{2}}(\mathbf{H})_{\mathbf{2}}\right]$ in THF with a stoichiometric amount of phenylacetylene $(\mathrm{PA})$ at $-30{ }^{\circ} \mathrm{C}$ resulted in an immediate color change to dark green and the formation of $\mathbf{K}\left[\mathbf{L}^{\text {bisnac }} \mathbf{N i}_{2}\left(\mu-\eta^{1}: \eta^{1}-\mathbf{C H C P h}\right)\right]$ (Scheme 5.3) .
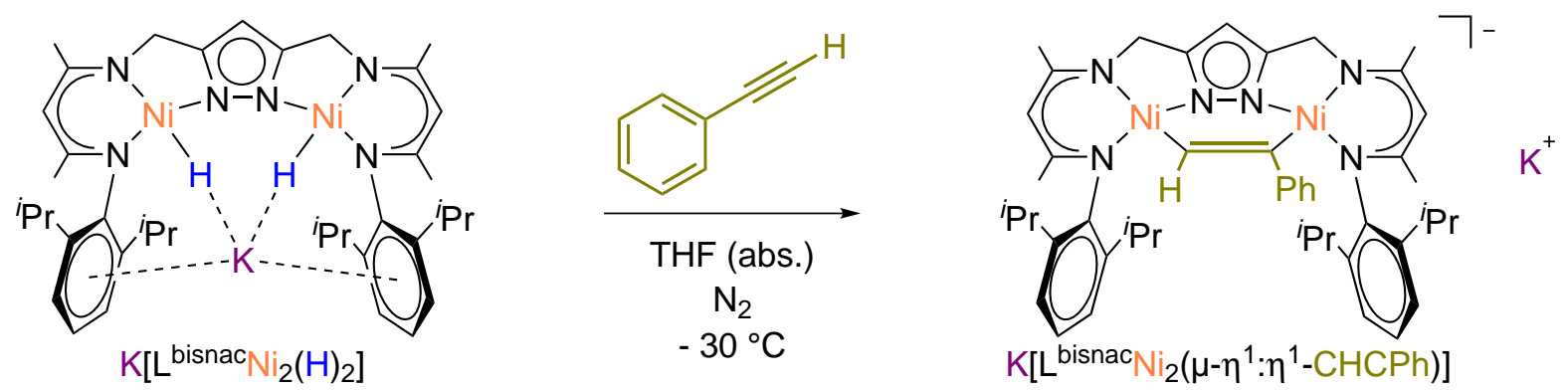

Scheme 5.3: Reaction of $\mathrm{K}\left[\mathrm{L}^{\text {bisnac }} \mathrm{Ni}_{2}(\mathrm{H})_{2}\right]$ with one equivalent of phenylacetylene.

Layering of the cooled $\left(-30^{\circ} \mathrm{C}\right)$ solution with hexane led to formation of dark green, rod shaped crystals, which were suitable for X-ray analysis. The molecular structure is depicted in Figure 5.1. Selected bond lengths, distances and angles are shown in Table 5.1. Conversion of the hydride compound with phenylacetylene led to the formation of a $\mu-\eta^{1}: \eta^{1}$ phenylvinyl-bridged complex. Although so far $\left(\mu-\eta^{1}: \eta^{1}\right)$ metal-metal-bridged vinyl complexes based on $\mathrm{Al}^{\left[\frac{[186]}{\underline{10}}\right.}$ or $\mathrm{Rh}^{\left[\frac{[187]}{\underline{1}}\right.}$ or in the form of metalla-cyclobutene complexes ${ }^{[188-194]}$ are reported in the literature, $\mu-\eta^{1}: \eta^{1}$-vinyl-bridged dinickel complexes are novel.

$\mathbf{K}\left[\mathbf{L}^{\text {bisnac }} \mathbf{N i}_{\mathbf{2}}\left(\mu-\eta^{1}: \eta^{1}-\mathbf{C H C C}_{6} \mathbf{H}_{5}\right)\right] \cdot(\mathbf{T H F})_{3}$ crystallizes in the triclinic spacegroup $P \overline{1}$ with four molecules in the unit cell. The metal centers are coordinated in a slightly distorted square-planar fashion (Figure 5.1). The angles between one nickel atom and two adjacent atoms confirm this: For Ni1, the two largest angles correspond to $\Varangle \mathrm{N} 3-\mathrm{Ni1}-\mathrm{C} 41$ $=167^{\circ}$ and $\Varangle \mathrm{N} 1-\mathrm{Ni1}-\mathrm{N} 4=173^{\circ}$. The other four angles range from 83 to $95^{\circ}$. For instance $\Varangle \mathrm{N} 1-\mathrm{Ni1}-\mathrm{C} 41$ is $89.74^{\circ}$.

A similar metal coordination environment was found in $\left[\mathbf{L}^{\text {bisnac }} \mathbf{N i}_{\mathbf{2}}(\mu-\mathbf{B r})\right]$. On the other hand the two largest angles for Ni2 correspond to $\Varangle \mathrm{N} 5$-Ni1-C42 $=154^{\circ}$ and $\Varangle \mathrm{N} 2-\mathrm{Ni1}-\mathrm{N} 6$ $=162^{\circ}$ while the four smaller angles range from 82 to $101^{\circ}$. This shows that the environment of the Ni2 center is more distorted from square-planar. A look at the non-symmetric bridging phenylvinyl unit of the compound gives the reason for the distortion of the Ni2 center: due to the steric demand of the phenyl group, the adjacent diisopropylphenyl side arm is pushed away hence moving the aryl-connected nitrogen atom N6 out of the ideal square-planar coordination of the metal center. 


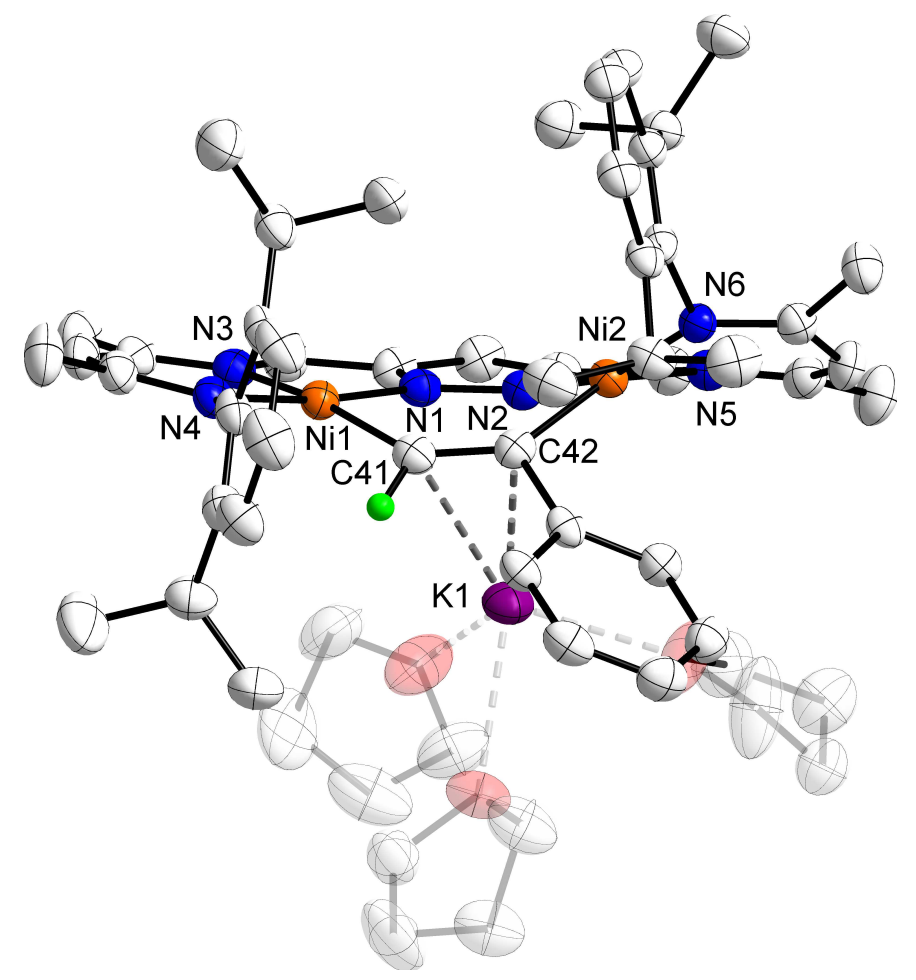

Figure 5.1.: Thermal displacement ellipsoids (shown at $50 \%$ probability) of the molecular structure of $\mathrm{K}\left[\mathrm{L}^{\text {bisnac }} \mathrm{Ni}_{2}\left(\mu-\eta^{1}: \eta^{1}-\mathrm{CHCC}_{6} \mathrm{H}_{5}\right)\right] \cdot(\mathrm{THF})_{3}$. All hydrogen atoms except the vinyl hydrogen atom have been omitted for the sake of clarity (the hydrogen atom is displayed with a fixed radius of $0.135 \AA$ ). For more clarity, the coordinated THF molecules are displayed in a transparent fashion.

The bridging unit comprises a dianionic phenylvinyl group, resulting in an overall monoanionic compound. However, the negative charge is compensated by a potassium cation similar to $\mathbf{K}\left[\mathbf{L}^{\text {bisnac }} \mathbf{N i}_{\mathbf{2}}(\mathbf{H})_{\mathbf{2}}\right]$. In $\mathbf{K}\left[\mathbf{L}^{\text {bisnac }} \mathbf{N i}_{\mathbf{2}}\left(\mu-\eta^{\mathbf{1}}: \eta^{\mathbf{1}}-\mathbf{C H C P h}\right)\right]$ the cation is not coordinated between the DIPP moieties as in $\mathbf{K}\left[\mathbf{L}^{\text {bisnac }} \mathbf{N i}_{\mathbf{2}}(\mathbf{H})_{\mathbf{2}}\right]$ due to the largely increased distance between the sidearms. Instead, it is coordinated to the vinyl unit of the bridge and further stabilized by three solvent (THF) molecules.

The $\mathrm{C}=\mathrm{C}$ bond length of the bridging vinyl unit is $1.358 \AA$, which fits the length of a $\mathrm{C}=\mathrm{C}$ double bond and is consitent with the $\mathrm{C}=\mathrm{C}$ bond length of other $\mu-\eta^{1}: \eta^{1}$ vinyl complexes $(1.30-1.36 \AA) . \stackrel{[186,190,192,194-196]}{\underline{1}}$ Despite the bulkyness of the entire bridge, the short vinyl unit brings the two nickel centers into close vicinity with a distance of $d_{\mathrm{Ni}-\mathrm{Ni}}$ $=3.88 \AA$, which is within the range of other literature known dinuclear nickel complexes of pyrazolate-based ligand systems $(3.501-4.587 \AA) .{ }^{[10]}$ 
Table 5.1.: Selected bond lengths, distances and angles of $K\left[\mathrm{~L}^{\text {bisnac }} \mathrm{Ni}_{2}\left(\mu-\eta^{1}: \eta^{1}-\mathrm{CHCC}_{6} \mathrm{H}_{5}\right)\right] \cdot(\mathrm{THF})_{3}$.

\begin{tabular}{|c|c|c|c|}
\hline \multicolumn{2}{|c|}{ Bond lengths / $\AA$} & \multicolumn{2}{|c|}{ Angle / ${ }^{\circ}$} \\
\hline $\mathrm{Ni1-N1}$ & $1.855(2)$ & N1-Ni1-N4 & $173.44(9)$ \\
\hline $\mathrm{Ni1-N4}$ & $1.903(2)$ & N1-Ni1-C41 & $89.74(10)$ \\
\hline Ni1-C41 & $1.904(3)$ & N4-Ni1-C41 & $94.56(10)$ \\
\hline Ni1-N3 & $1.941(2)$ & N1-Ni1-N3 & $82.88(9)$ \\
\hline $\mathrm{Ni} 1 \ldots \mathrm{K} 1$ & $3.7857(7)$ & N4-Ni1-N3 & $93.77(9)$ \\
\hline $\mathrm{Ni2}-\mathrm{N} 2$ & $1.853(2)$ & C41-Ni1-N3 & $167.12(9)$ \\
\hline $\mathrm{Ni2}-\mathrm{C} 42$ & $1.921(2)$ & N2-Ni2-C42 & $90.61(9)$ \\
\hline $\mathrm{Ni2}-\mathrm{N} 6$ & $1.9284(19)$ & N2-Ni2-N6 & $162.04(8)$ \\
\hline $\mathrm{Ni2}-\mathrm{N} 5$ & $1.945(2)$ & C42-Ni2-N6 & $100.78(9)$ \\
\hline $\mathrm{Ni} 2 \ldots \mathrm{K} 1$ & $3.4545(6)$ & N2-Ni2-N5 & $82.24(8)$ \\
\hline K1-O1 & $2.634(2)$ & N5-Ni2-N6 & $93.04(8)$ \\
\hline $\mathrm{K} 1-\mathrm{O} 2$ & $2.707(2)$ & C42-Ni2-N5 & $153.62(9)$ \\
\hline K1-O3 & $2.743(3)$ & C41-C42-Ni2 & $127.68(18)$ \\
\hline K1-N2 & $2.875(2)$ & Ni1-C41-C42 & $134.73(19)$ \\
\hline K1-C42 & $3.005(2)$ & C41-C42-C43 & $117.8(2)$ \\
\hline K1-C41 & $3.112(2)$ & & \\
\hline K1-N1 & $3.138(2)$ & & \\
\hline $\mathrm{K} 1-\mathrm{C} 43$ & $3.274(2)$ & & \\
\hline C41-C42 & $1.358(3)$ & & \\
\hline $\mathrm{C} 41-\mathrm{H} 41$ & $0.9910(286)$ & & \\
\hline $\mathrm{Ni} 1 \ldots \mathrm{Ni} 2$ & $3.8759(11)$ & & \\
\hline
\end{tabular}




\subsubsection{Characterization by NMR Spectroscopy}

The ${ }^{1} \mathrm{H}$ NMR spectrum of $\mathbf{K}\left[\mathbf{L}^{\text {bisnac }} \mathbf{N i}_{\mathbf{2}}\left(\mu-\eta^{\mathbf{1}}: \eta^{\mathbf{1}} \mathbf{- C H C P h}\right)\right]$ exhibits a larger number of resonances (Figure 5.2 in comparison to the starting material $\mathbf{K}\left[\mathbf{L}^{\text {bisnac }} \mathbf{N i}_{\mathbf{2}}(\mathbf{H})_{\mathbf{2}}\right]$, indicating the formation of a compound with lower symmetry, which is consistent with X-ray analysis.

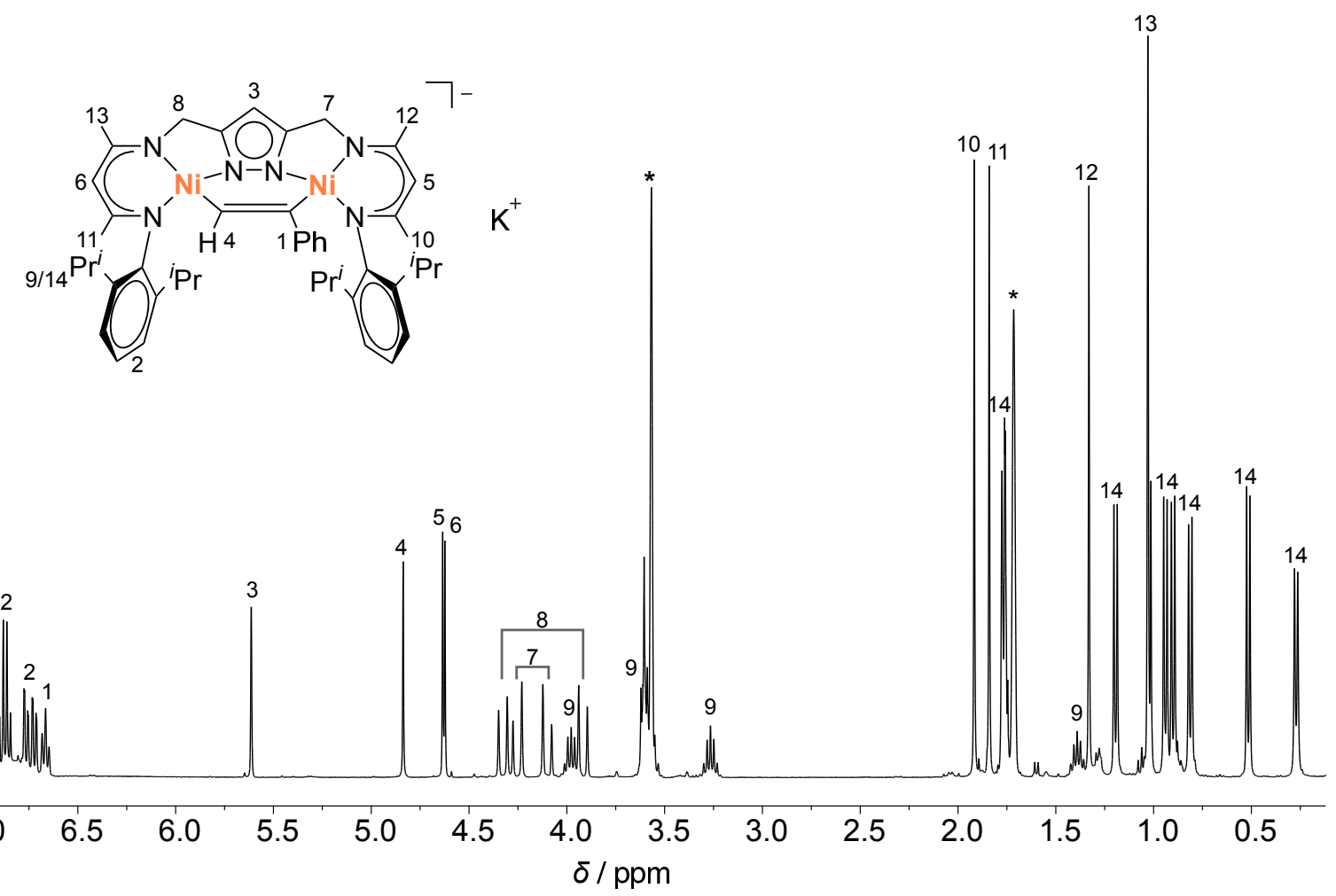

Figure 5.2.: ${ }^{1} \mathrm{H}$ NMR spectrum $(400 \mathrm{MHz})$ of $\mathrm{K}\left[\mathrm{L}^{\text {bisnac }} \mathrm{Ni}_{2}\left(\mu-\eta^{1}: \eta^{1}-\mathrm{CHCPh}\right)\right]$ recorded at room temperature. Residual solvent signals (THF) are marked $\left(^{*}\right)$.

The protons belonging to the methylene groups, which only give a single resonance for the symmetric $\mathbf{K}\left[\mathbf{L}^{\text {bisnac }} \mathbf{N i}_{\mathbf{2}}(\mathbf{H})_{\mathbf{2}}\right]$ now show a signal pattern typical of an AB-spin-system (Figure 5.3). In this case, the protons are diastereotopic, which is reflected by the pronounced roof pattern.

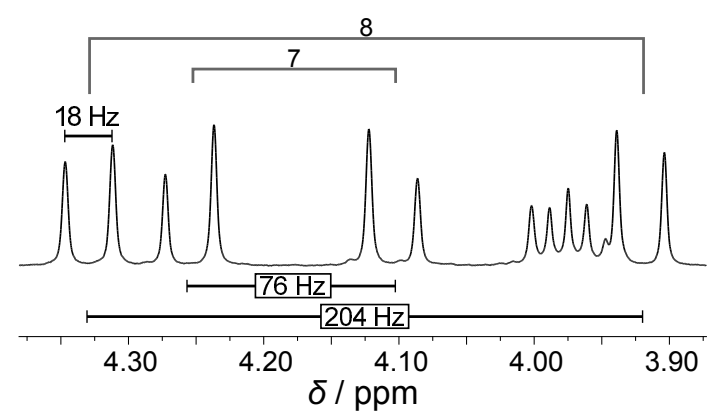

Figure 5.3.: Roof pattern in the ${ }^{1} \mathrm{H}$ NMR spectrum of $\mathrm{K}\left[\mathrm{L}^{\text {bisnac }} \mathrm{Ni}_{2}\left(\mu-\eta^{1}: \eta^{1}-\mathrm{CHCPh}\right)\right]$ attributable to the methylene groups 7 and 8 . 
At first sight the integration of the signals corresponding to the aryl-proton resonances was short by two protons. At significantly higher intensity, two further signals featuring a significant linebroadening were determined in the ${ }^{1} \mathrm{H}$ NMR spectrum, which each correspond to one proton (Figure 5.4).

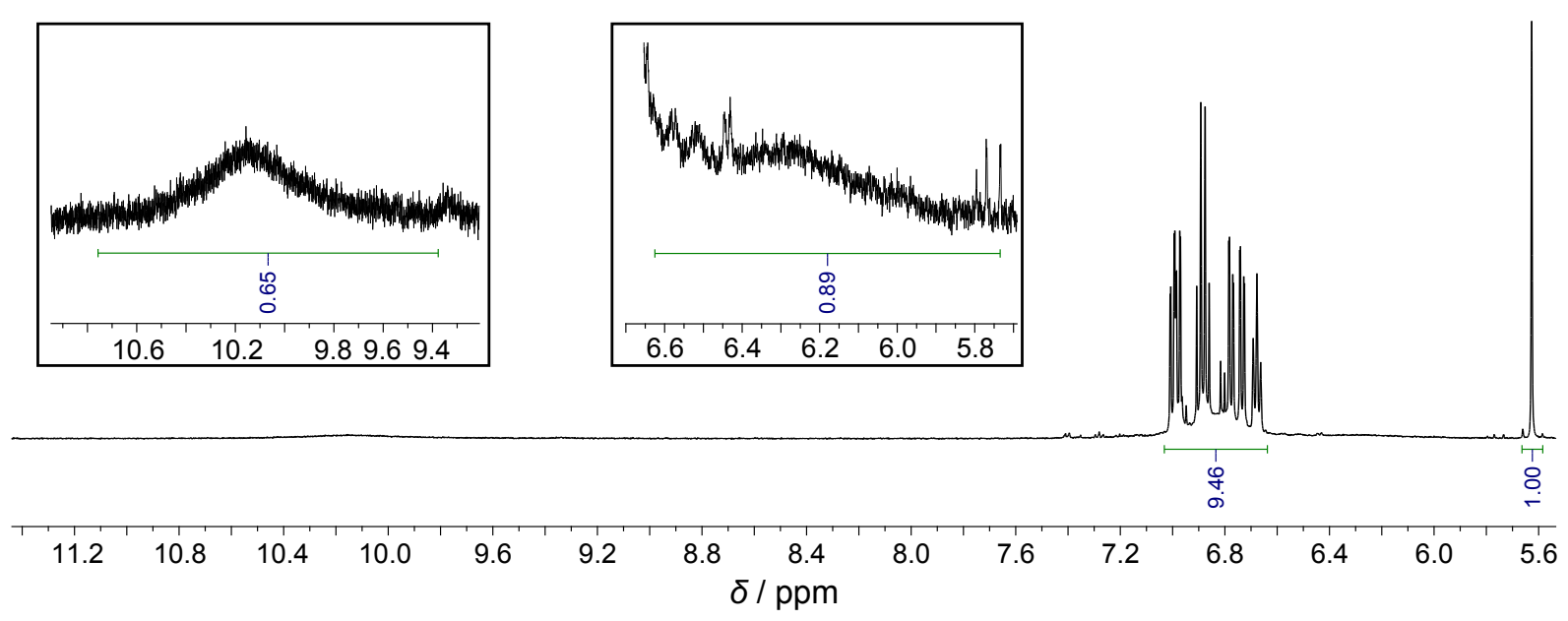

Figure 5.4.: Aromatic region of the ${ }^{1} \mathrm{H}$ NMR spectrum (500 MHz) of $K\left[L^{\text {bisnac }} \mathrm{Ni}_{2}\left(\mu-\eta^{1}: \eta^{1}-\mathrm{CHCPh}\right)\right]$ recorded at room temperature. At increased intensities two single proton resonances with a significant line broadening were revealed as seen in the two excerpts.

The broadening of the signals originates from a dynamic effect associated with a rotation of the phenyl substituent of the bridging unit. Spectra recorded at lower temperatures show the broadened resonances resolved as doublets. The resonances were assigned to the hydrogen atoms in ortho position of the phenyl ring. Upon lowering the temperature, the dynamic effect caused by the rotation of the phenyl ring is minimized (Figure 5.5).

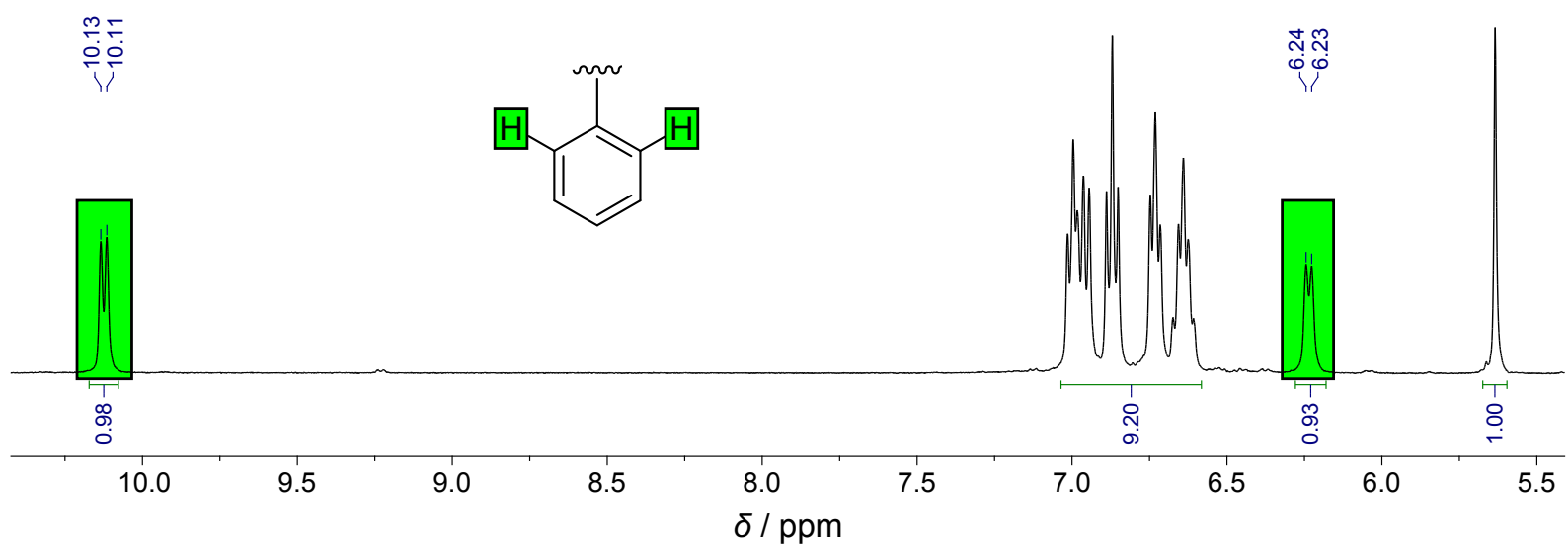

Figure 5.5.: ${ }^{1} \mathrm{H}$ NMR spectrum $(400 \mathrm{MHz})$ of $\mathrm{K}\left[\mathrm{L}^{\text {bisnac }} \mathrm{Ni}_{2}\left(\mu-\eta^{1}: \eta^{1}-\mathrm{CHCPh}\right)\right]$ recorded at $-50{ }^{\circ} \mathrm{C}$. In particular, the two resonances exhibiting a line broadening at room temperature are now resolved as sharp doublets. As can be seen the determined normalized integrals of all aromatic resonances sum up to 11 as expected for the phenyl- and the two DIPP substituents of the ligand.

A ${ }^{1} \mathrm{H}-{ }^{13} \mathrm{C}$ HSQC experiment performed at $-35{ }^{\circ} \mathrm{C}$ shows the carbon-proton correlations (Figure 5.6). The two sharp doublet proton signals at $10.14 \mathrm{ppm}$ and $6.24 \mathrm{ppm}$ show 
cross correlations with aromatic carbons. A comparison of the aromatic carbon signals in the ${ }^{13} \mathrm{C}\{\mathrm{H}\}$ NMR spectra, which were recorded at room temperature and at $-35{ }^{\circ} \mathrm{C}$, additionally provides evidence for dynamic effects. The proton-bound aromatic carbon atoms of the DIPP and phenyl substituents are expected to give a total of 11 signals in the ${ }^{13} \mathrm{C}$ NMR. Although this number of signals could not clearly be distinguished at room temperature, a spectrum recorded at $-35{ }^{\circ} \mathrm{C}$ led to the expected 11 signals as can be seen in spectrum (b) of Figure 5.7. As a result of sample cooling the ortho- ( $\square$ ) and meta-carbon $(\mathbf{\square})$ resonances are resolved.

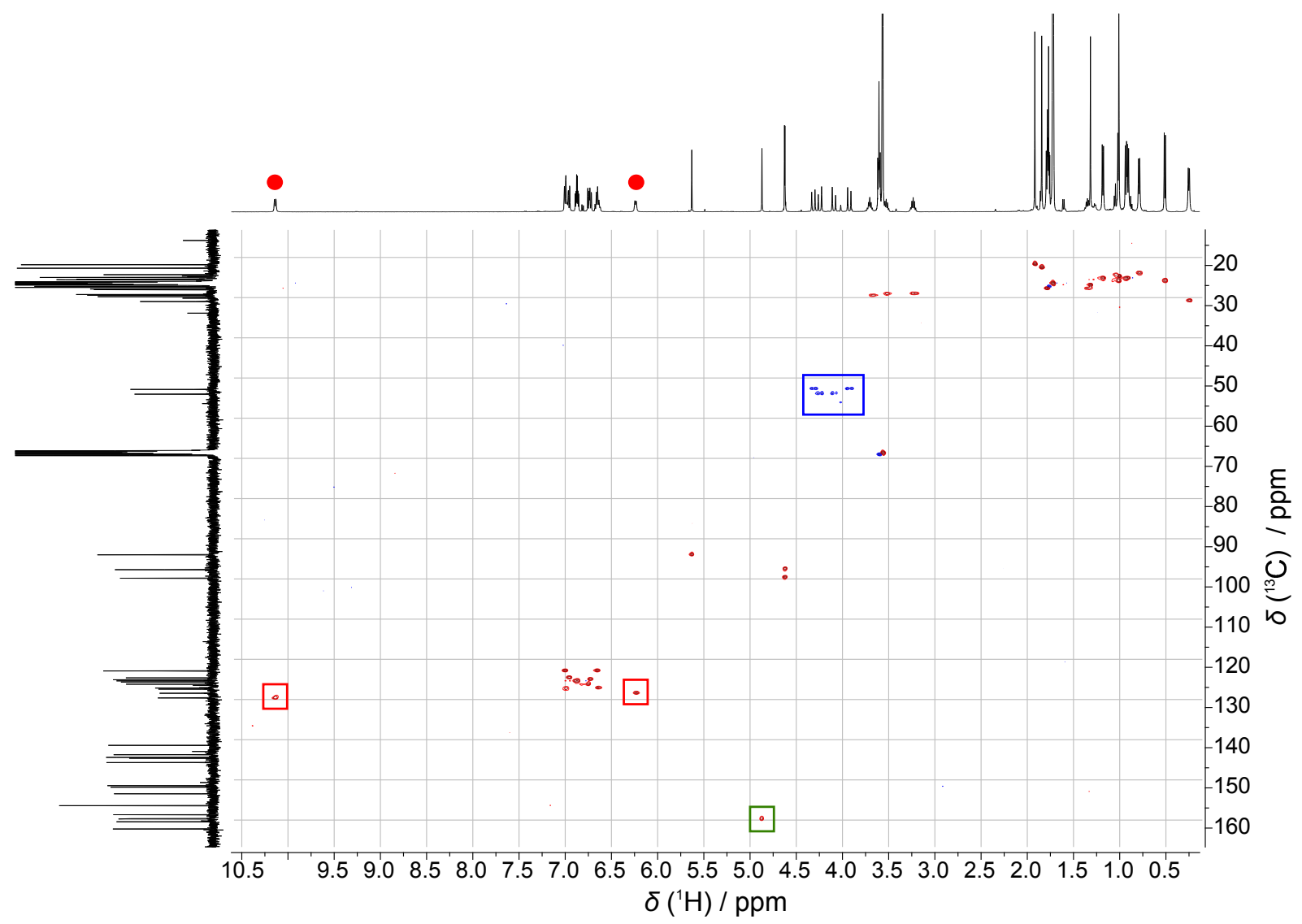

Figure 5.6.: ${ }^{1} \mathrm{H}^{13} \mathrm{C}$ HSQC spectrum of $\mathrm{K}\left[\mathrm{L}^{\text {bisnac }} \mathrm{Ni}_{2}\left(\mu-\eta^{1}: \eta^{1}-\mathrm{CHCPh}\right)\right]$ at $-35^{\circ} \mathrm{C}$. At this temperature, the cross peaks representing the $\mathrm{CH}$ correlations of the aryl protons $(\bullet)$ are well resolved $(\square)$. Furthermore, the proton-carbon correlations for the methylene groups are observed as blue cross peaks with a negative phase (ם). Note the cross peak for the vinyl-CH group in the carbon downfield region ( $\square$ ).

Furthermore, a ${ }^{1} \mathrm{H}-{ }^{1} \mathrm{H}$ COSY spectrum (see Figure 5.8) measured at $-35{ }^{\circ} \mathrm{C}$ revealed the scalar couplings between the two ortho aryl proton signals to adjacent protons. For instance, the ${ }^{3} J_{\mathrm{HH}}$ coupling of both protons in ortho position to their neighbouring protons in meta position gives a strong correlation cross peak whereas the ${ }^{4} J_{\mathrm{HH}}$ couplings to each other as well as to the proton in para position is significantly weaker. 


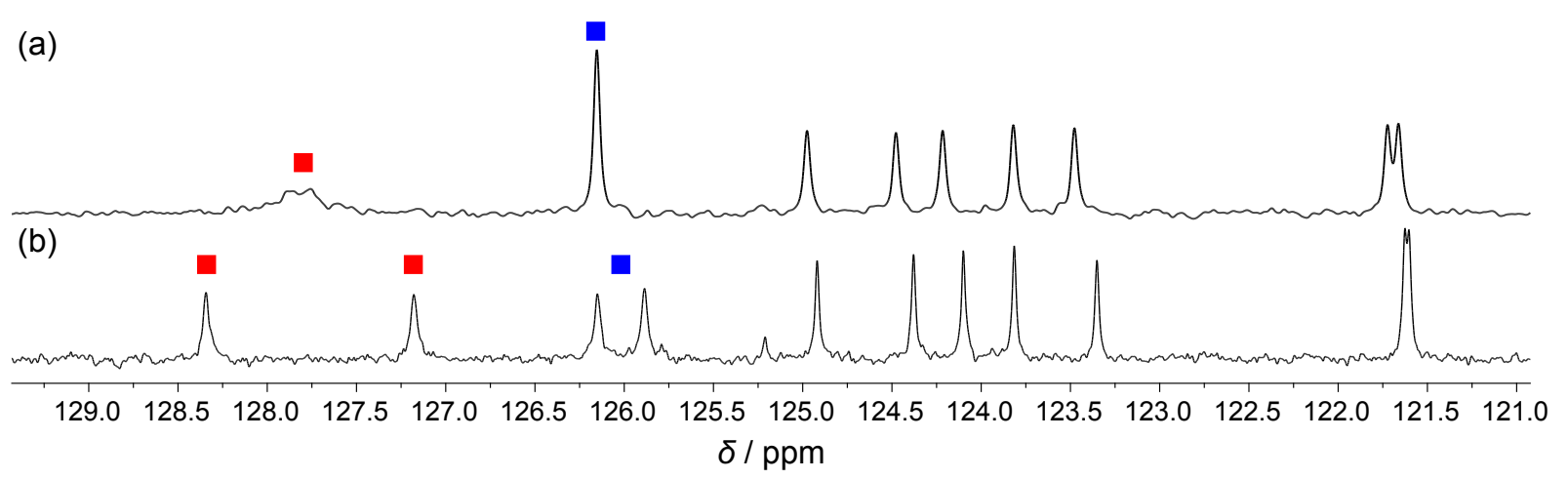

Figure 5.7.: Comparison of the ${ }^{13} \mathrm{C}$ NMR spectra of $\mathrm{K}\left[\mathrm{L}^{\text {bisnac }} \mathrm{Ni}_{2}\left(\mu-\eta^{1}: \eta^{1}-\mathrm{CHCPh}\right)\right]$ at $25^{\circ} \mathrm{C}$ (a) and at $-35{ }^{\circ} \mathrm{C}$ (b). At room temperature, a signal with pronounced linebroadening occurs at $128.0 \mathrm{ppm}$ representing both carbon atoms in ortho-position to the substituted phenyl carbon of the bridging unit. Cooling led to distinct signals at 127.62 and 128.45 ppm ( $\square$ ). The carbon atom in meta-position also exhibits this effect (ם).
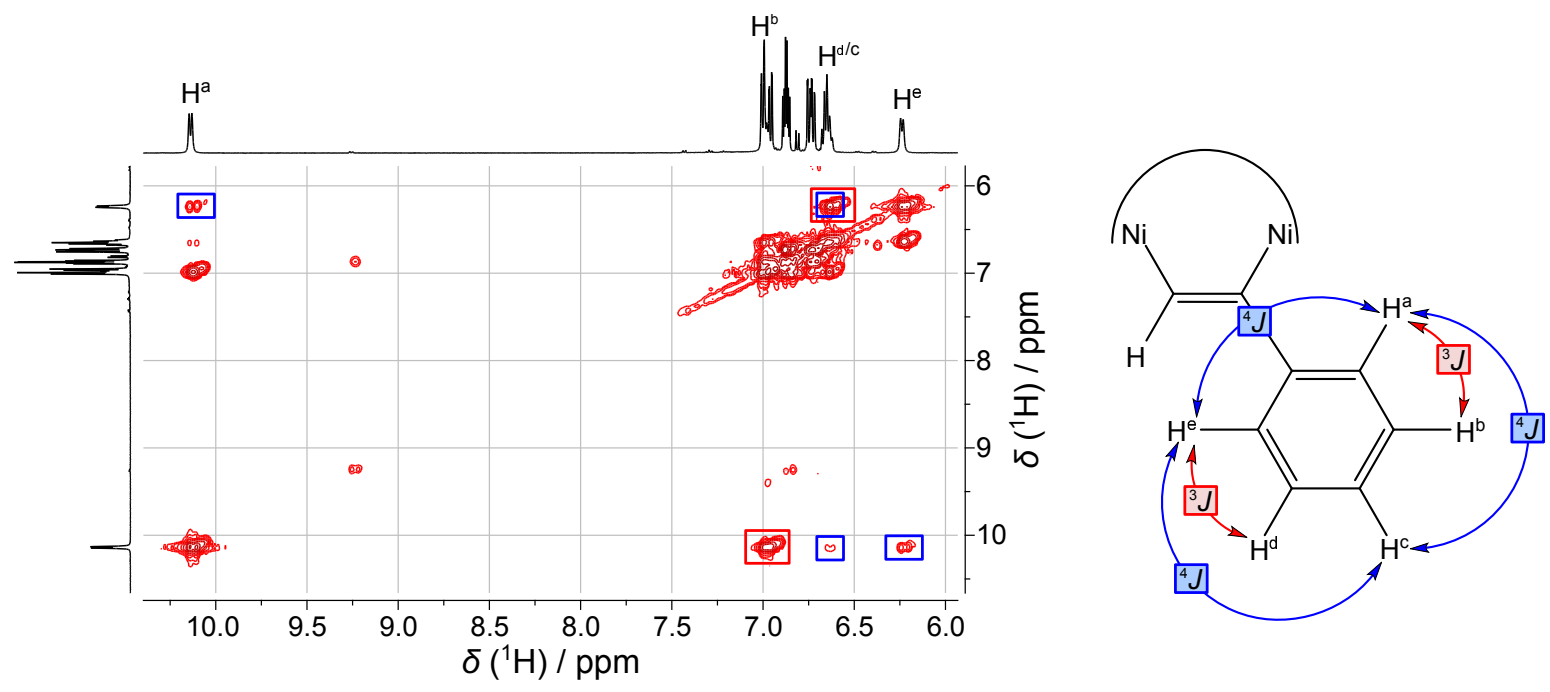

Figure 5.8.: ${ }^{1} \mathrm{H}$ COSY spectrum of $\mathrm{K}\left[\mathrm{L}^{\text {bisnac }} \mathrm{Ni}_{2}\left(\mu-\eta^{1}: \eta^{1}-\mathrm{CHCPh}\right)\right]$ at $-35^{\circ} \mathrm{C}$. The ${ }^{3} J_{\mathrm{HH}}$ proton correlations are indicated by red marks ( $\square$ ) and the ${ }^{4} J_{H H}$ proton couplings by blue marks $(\square)$.

Even at low temperature (i. e. $-35^{\circ} \mathrm{C}$ ), although exhibiting relatively sharp signals for the hydrogen atoms in ortho position of the phenyl ring in the ${ }^{1} \mathrm{H}$ NMR spectrum, an exchange of the proton positions is caused by the rotation of the phenyl substituent around the phenyl-vinyl C-C bond. This effect is observed in the ${ }^{1} \mathrm{H}-{ }^{1} \mathrm{H}$ NOESY experiment (Figure 5.9).

NOE correlations result in a cross peak signal with negative phase. On the other hand rotational or chemical exchanges show correlation signals with positive phase. The intense cross peaks with positive phase indicate a rotational exchange of the involved protons and were attributed to the aryl protons in ortho-position $\left(\mathrm{H}^{\mathrm{a}}\right.$ and $\left.\mathrm{H}^{\mathrm{e}}\right)$.

Furthermore, rotation of the phenyl around the phenyl-vinyl bond also causes protons in meta position to be exchanged although it is observed to a lesser extent in ${ }^{1} \mathrm{H} \mathrm{NMR}$. Nonetheless, in the NOESY experiment fast rotational exchange of these protons $\left(\mathrm{H}^{\mathrm{b}}\right.$ and 


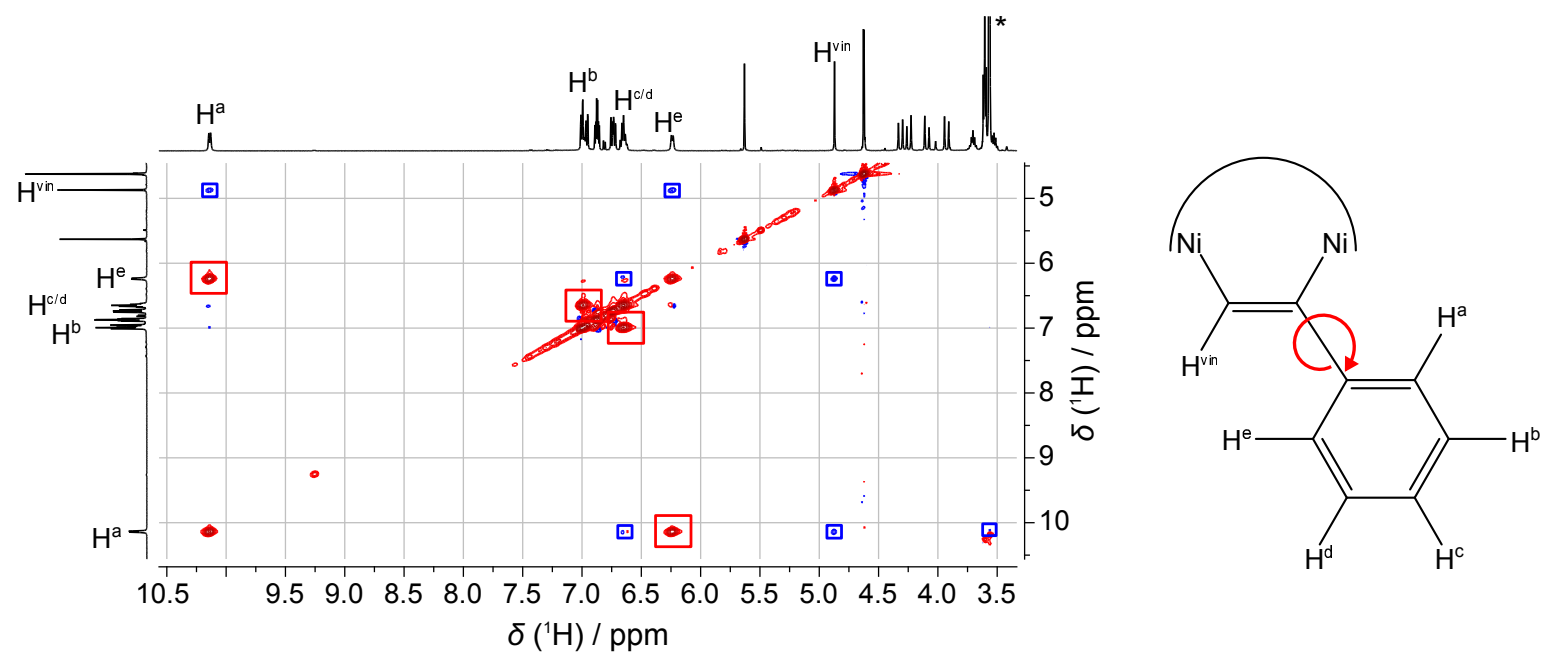

Figure 5.9.: ${ }^{1} \mathrm{H}$ NOESY spectrum of $\mathrm{K}\left[\mathrm{L}^{\text {bisnac }} \mathrm{Ni}_{2}\left(\mu-\eta \eta^{1}: \eta^{1}-\mathrm{CHCPh}\right)\right]$ at $-35{ }^{\circ} \mathrm{C}$. Rotational exchange correlations of protons (resulting in red cross peak signals with a positive phase) are indicated by red marks ( $\square$ ) and NOE correlations (blue cross peaks with negative phase) by blue marks ( $\square$ ).

$\mathrm{H}^{\mathrm{d}}$ ) is indicated by red marks in the standard aromatic region around $7 \mathrm{ppm}$.

NOE correlations can also be observed: both protons in ortho position of the phenyl ring $\left(\mathrm{H}^{\mathrm{a}}\right.$ and $\left.\mathrm{H}^{\mathrm{e}}\right)$ show a spatial correlation with the phenyl hydrogen in para position (though very weak) and with the vinyl hydrogen $\mathrm{H}^{\mathrm{vin}}$, which can be caused by rotation of the aryl substituent. Furthermore, a very weak NOE correlation to a THF solvent molecule, coordinating to the potassium cation, is observed (small cross signal at the bottom right of the spectrum). Figure 5.10 illustrates the distances between the protons exhibiting NOE.

The hydrogen atoms of the rotating phenyl group of the bridging unit in $\mathbf{K}\left[\mathbf{L}^{\mathbf{b i s n a c}} \mathbf{N i}_{\mathbf{2}}(\mu-\right.$ $\left.\left.\eta^{1}: \eta^{1}-\mathbf{C H C P h}\right)\right]$ could unambiguously be assigned.

A large difference in chemical shift of $\mathrm{H}^{\mathrm{a}}$ and $\mathrm{H}^{\mathrm{e}}$ in ortho position can be better understood by taking a closer look at the molecular structure of $\mathbf{K}\left[\mathbf{L}^{\text {bisnac }} \mathbf{N i}_{\mathbf{2}}\left(\mu-\eta^{\mathbf{1}}: \eta^{\mathbf{1}}-\mathbf{C H C P h}\right)\right]$ (Figure 5.10) and at the bridging phenylvinyl group in particular. The negative charge of the compound is stabilized by a potassium cation, which is further coordinated by three solvent (THF) molecules. One of these solvent molecules is positioned in such close proximity to the briding unit that correlations between corresponding hydrogen atoms are visible in the NOESY spectrum. Coordination of the large potassium cation to the double bond of the vinyl unit and especially to one $\mathrm{C}-\mathrm{C}$ bond of the phenyl group results in a significant decrease of electron density in this region. Consequently, $\left(\mathrm{H}^{\mathrm{a}}\right)$ is much more deshielded than all the other hydrogen atoms of the aromatic ring leading to a strongly downfield shifted resonance.

The opposite is observed for $\mathrm{H}^{\mathrm{b}}$ (Figure 5.10). One of the DIPP sidearms of the ligand system is positioned in close proximity to $\mathrm{H}^{\mathrm{b}}$, in such a way that the ring current caused 


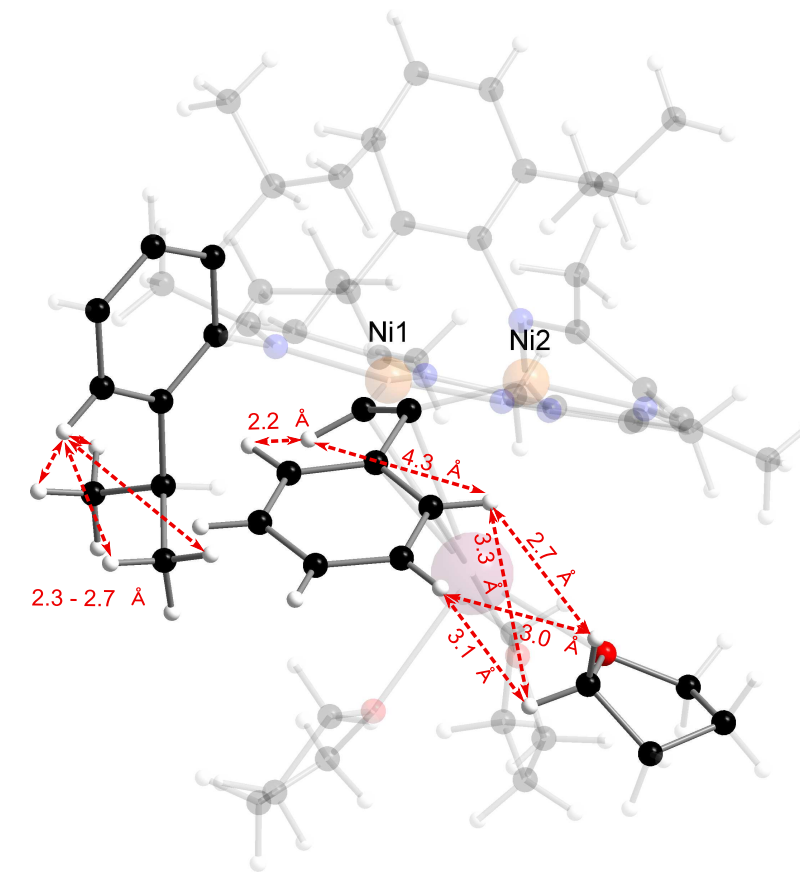

Figure 5.10.: Distances between hydrogen atoms belonging to the isopropyl groups and the bridging phenylvinyl unit of $\mathrm{K}\left[\mathrm{L}^{\text {bisnac }} \mathrm{Ni}_{2}\left(\mu-\eta^{1}: \eta^{1}-\mathrm{CHCPh}\right)\right]$ indicated by red arrows. For more clarity all other atoms and bonds are depicted in a transparent fashion.

by its aromatic $\pi$-system effectively shields the hydrogen atom. This eventually leads to a resonance shifted to higher fields.

A hindered rotation of an aryl group around a $\mathrm{C}\left(\mathrm{sp}^{2}\right)-\mathrm{C}\left(\mathrm{sp}^{2}\right)$ bond has been reported before. ${ }^{[197]}$ Although, in that case a mononuclear, cationic ruthenium complex with a styrene coordinated in a $\eta^{2}$ fashion was involved, the observations made were very similar to those of this study. For both hydrogen atoms in ortho-position of the aryl group different ${ }^{1} \mathrm{H}$ NMR shifts were observed which were only resolved at lower temperatures. The significant difference in chemical shift between both resonances (though not as large as those observed for this work) were explained by the close spatial proximity of one ortho hydrogen to a ligand aryl group resulting in an aromatic edge-face interaction. ${ }^{[197]}$ 


\subsubsection{Rotational Barrier of the Phenyl Substituent}

In order to establish the rotational barrier for the phenyl substituent of the bridging unit a variable temperature ${ }^{1} \mathrm{H}$ NMR screening experiment between -50 and $70{ }^{\circ} \mathrm{C}$ was conducted. The result of the screening experiment below room temperature is depicted in Figure 5.11.

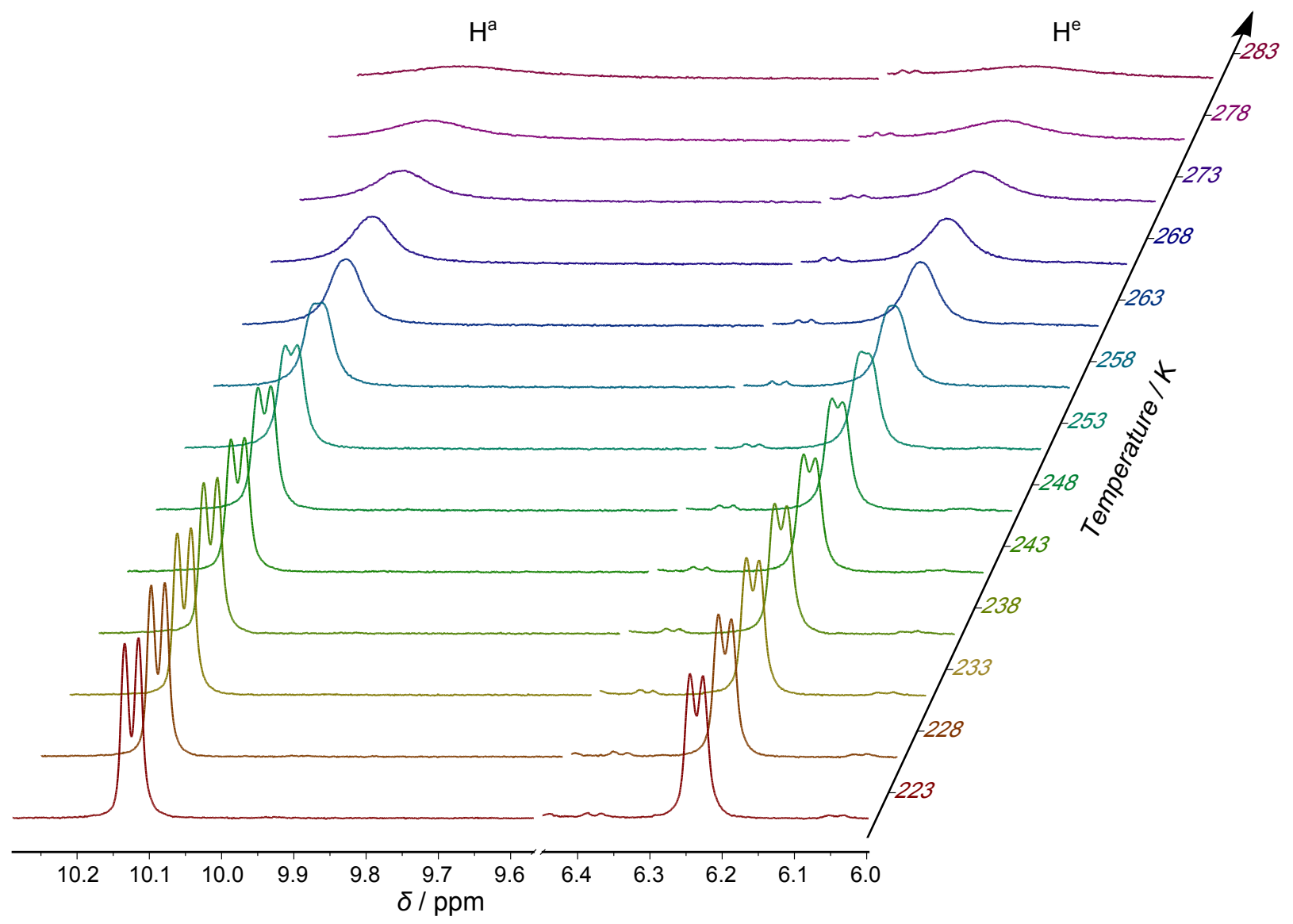

Figure 5.11.: Low temperature ${ }^{1} \mathrm{H}$ NMR screening (400 MHz) of $\mathrm{K}\left[\mathrm{L}^{\text {bisnac }} \mathrm{Ni}_{2}\left(\mu-\eta^{1}: \eta^{1}-\mathrm{CHCPh}\right)\right]$ measured in a range of 223-283 K. Depicted are the two hydrogen atoms in ortho-position of the phenyl ring $\left(\mathrm{H}^{\mathrm{a}}\right.$ and $\left.\mathrm{H}^{\mathrm{e}}\right)$.

At room temperature and above line broadening of the resonances for $\mathrm{H}^{\mathrm{a}}$ and $\mathrm{H}^{\mathrm{b}}$ becomes very pronounced, which is attributable to the large difference in chemical shift $(\Delta \delta \approx$ $1600 \mathrm{~Hz}$ ). Therefore, a reasonable line shape analysis was difficult.

Furthermore, a coalescence point could not be clearly identified from the measured spectra, due to several difficulties: upon raising the temperature the aromatic resonance lying in the upper field of the spectrum shifts to lower field while broadening. At temperatures above $30{ }^{\circ} \mathrm{C}$ it was difficult to determine the line shape of the signal due to superposition by other aromatic signals. ${ }^{1} \mathrm{H}$ NMR spectra collected at elevated temperatures are shown in (Figure 5.12). Nonetheless, spectra recorded up to a temperature of $50{ }^{\circ} \mathrm{C}$ were used to estimate the rate constant for the exchange at the corresponding temperature by 
performing a lineshape analysis. From obtained spectra, the signal width at half-height $\Delta \nu_{1 / 2}$, the coupling constant $J_{\mathrm{AB}}$ and the frequencies $\nu_{\mathrm{A}}$ and $\nu_{\mathrm{B}}$ were determined to calculate rate constants $k$ for the exchange at the particular temperatures. Since only spectra recorded at temperatures below coalescence temperature were used, equation (5.1) was applied. ${ }^{[198]}$ The exact coalescence point was not observed in ${ }^{1} \mathrm{NMR}$ experiments for the above mentioned reasons. However, at a temperature of $70{ }^{\circ} \mathrm{C}$ (top trace of Figure 5.12 a signal at $\approx 8.2 \mathrm{ppm}$ exhibiting less broadening is observed, indicating a coalescence point at lower tempearture.

$$
k=\pi\left[\left(\Delta \nu_{\mathrm{e}}\right)_{1 / 2}-\left(\Delta \nu_{0}\right)_{1 / 2}\right]
$$

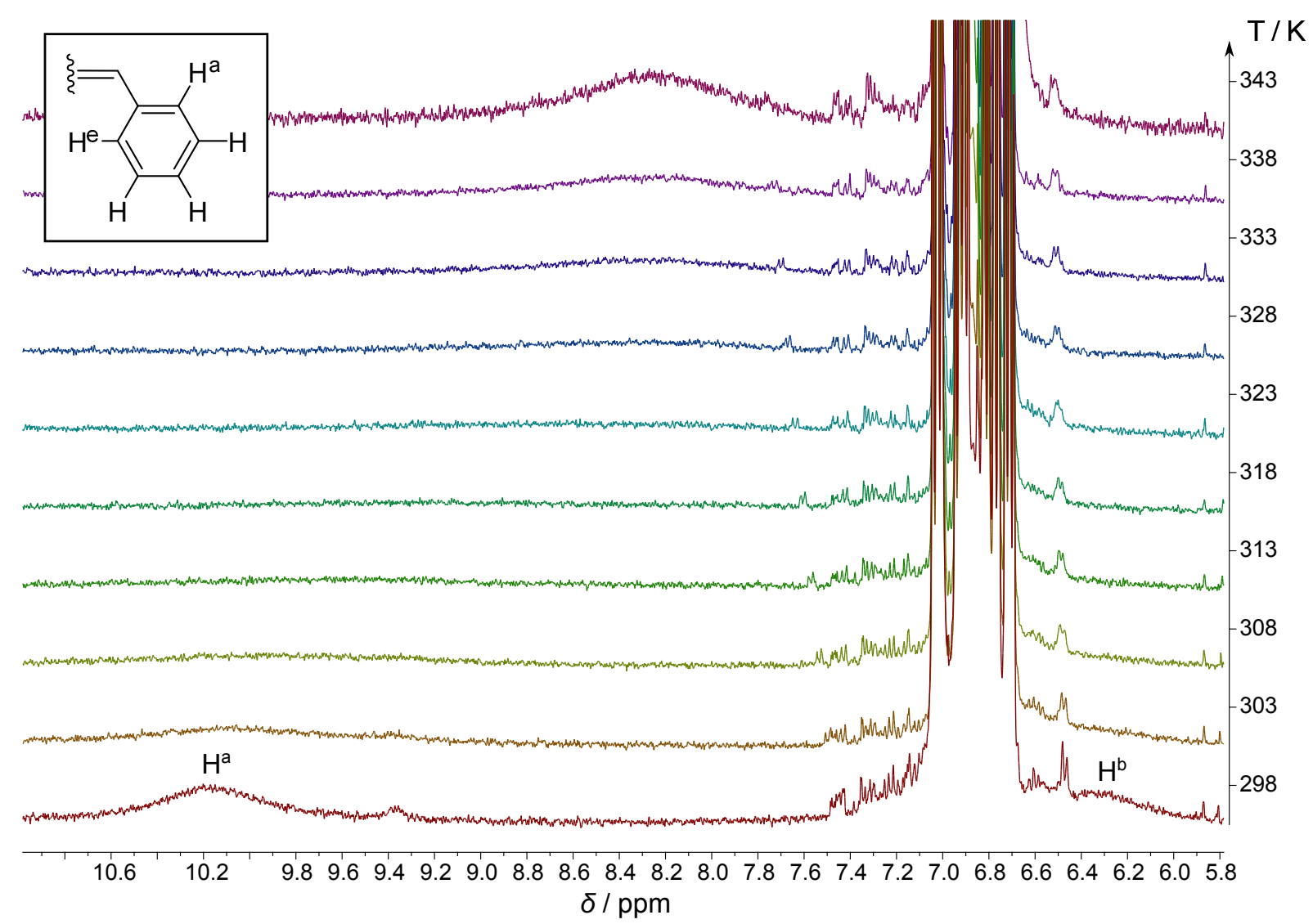

Figure 5.12.: High temperature ${ }^{1} \mathrm{H}$ NMR screening (400 MHz) of $K\left[L^{\text {bisnac }} \mathrm{Ni}_{2}\left(\mu-\eta^{1}: \eta^{1}-\mathrm{CHCPh}\right)\right]$. Depicted is the detail of the recorded spectra showing a dynamic effect for the two hydrogen atoms in ortho-position of the phenyl substituent $\left(\mathrm{H}^{\mathrm{a}}\right.$ and $\left.\mathrm{H}^{\mathrm{b}}\right)$. Above coalescence temperature line broadening of the collapsed signal decreases (343 K).

$\left(\Delta \nu_{\mathrm{e}}\right)_{1 / 2}$ represents the half-height width of observed signals. The determined half-height width obtained from the spectrum measured at the lowest temperature $\left(-50{ }^{\circ} \mathrm{C}\right)$ is considered as half-height width at zero exhange $\left(\Delta \nu_{0}\right)_{1 / 2}$. Furthermore, this spectrum provided the coupling constant $J_{\mathrm{AB}}$, which was used in equation 5.2 to determine the exchange rate $k_{\mathrm{C}}$ at coalescence temperature $T_{\mathrm{C}}$. As mentioned above $T_{\mathrm{C}}$ could not be determined 
from ${ }^{1} \mathrm{H}$ NMR spectra within the particular temperature range. ${ }^{[146,199]}$ Calculated rate constants for different temperatures are shown in Table 5.2.

$$
k_{\mathrm{C}}=\frac{\pi \sqrt{\left(\nu_{\mathrm{A}}-\nu_{\mathrm{B}}\right)^{2}+6 J_{\mathrm{AB}}^{2}}}{\sqrt{2}}
$$

Table 5.2.: Determined rate constants $k^{[\mathrm{a}]}$ and the calculated coalescence rate constant $k_{\mathrm{C}}{ }^{[\mathrm{b}]}$.

\begin{tabular}{cccc}
\hline$T / \mathrm{K}$ & $k / \mathrm{s}^{-1}$ & $T / \mathrm{K}$ & $k / \mathrm{s}^{-1}$ \\
\hline 223 & 0 & 273 & 101 \\
228 & 0.63 & 278 & 156 \\
233 & 1.16 & 283 & 252 \\
238 & 2.27 & 298 & $545^{[\mathrm{c}]}$ \\
243 & 4.07 & 303 & $1034^{[\mathrm{c}]}$ \\
248 & 8.55 & 308 & $1211^{[\mathrm{c}]}$ \\
253 & 15.1 & 313 & $1491^{[\mathrm{c}]}$ \\
258 & 26.2 & 318 & $2165^{[\mathrm{c}]}$ \\
263 & 47.6 & 323 & $2400^{[\mathrm{c}]}$ \\
268 & 67.6 & $T_{\mathrm{C}}$ & 3458 \\
\hline
\end{tabular}

${ }^{[a]}$ Experimental errors are $\approx 5 \%$.

${ }^{[b]}$ The value represents the average calculated with equation (5.2) for the temperatures from 223-248 K. ${ }^{[c]}$ Pronounced line broadening effects

With the determined rate constants $k$, a DNMR simulation ${ }^{\dagger}$ was conducted. A comparison between the experimentally obtained and simulated spectra is shown in Figure 5.13. Considering the Arrhenius equation (5.3), $\ln (k)$ vs. $1 / T$ was plotted, as illustrated in Figure 5.14 (a). A linear fit was applied, exhibiting a slope equal to $E_{\mathrm{a}} / R$. With the previously determined rate constant $T_{\mathrm{C}}(323 \mathrm{~K})$ could be estimated by extrapolation of the linear fit. The value is consistent with the already decreasing line broadening at $343 \mathrm{~K}$ (Figure 5.12).

Moreover, based on the Eyring equation (5.4) and its transformations (5.5) and (5.6), $\ln \left(\frac{k}{T}\right)$ was plotted against $1 / T$, as illustrated in Figure 5.14 (b). The slope $\left(-\frac{\Delta H^{\ddagger}}{R}\right)$ and the intercept $\left(23.76+\frac{\Delta S^{\ddagger}}{R}\right)$ of the linear fit yielded the parameters for the activation enthalpy $\Delta H^{\ddagger}$ and the activation entropy $\Delta S^{\ddagger} . \underline{\underline{[146]}}$

$$
\begin{aligned}
& \ln k=-\frac{E_{a}}{R T}+\ln A \\
& k=\kappa \frac{k_{b} T}{h} \exp \left(-\frac{\Delta G^{\ddagger}}{R T}\right)
\end{aligned}
$$

\footnotetext{
${ }^{\dagger}$ H. J. Reich 2011, WINDNMR-Pro (Version 7.1) [Software].

Available from http://www.chem.wisc.edu/areas/reich/plt/windNMR.htm.
} 


\begin{tabular}{|c|c|}
\hline Experimental & Induh \\
\hline & Whe \\
\hline & MUn \\
\hline & $M$ \\
\hline & MU \\
\hline & MM \\
\hline$\Omega$ & man \\
\hline$r$ & moln \\
\hline r & Jull \\
\hline$儿$ & MH \\
\hline 儿 & Jillar \\
\hline 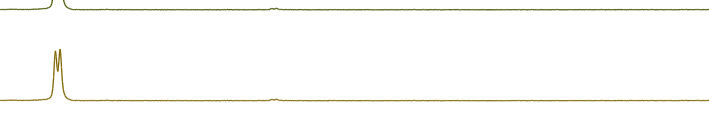 & mollar \\
\hline h & man \\
\hline h & mons \\
\hline \begin{tabular}{c|c:c|c|}
10.0 & 9.5 & 9.0 & $8.5 / \mathrm{s} / \mathrm{pm}^{\prime}$ \\
\end{tabular} & \begin{tabular}{l|l|l}
7.0 & 6.5 & 6.4 \\
\end{tabular} \\
\hline
\end{tabular}

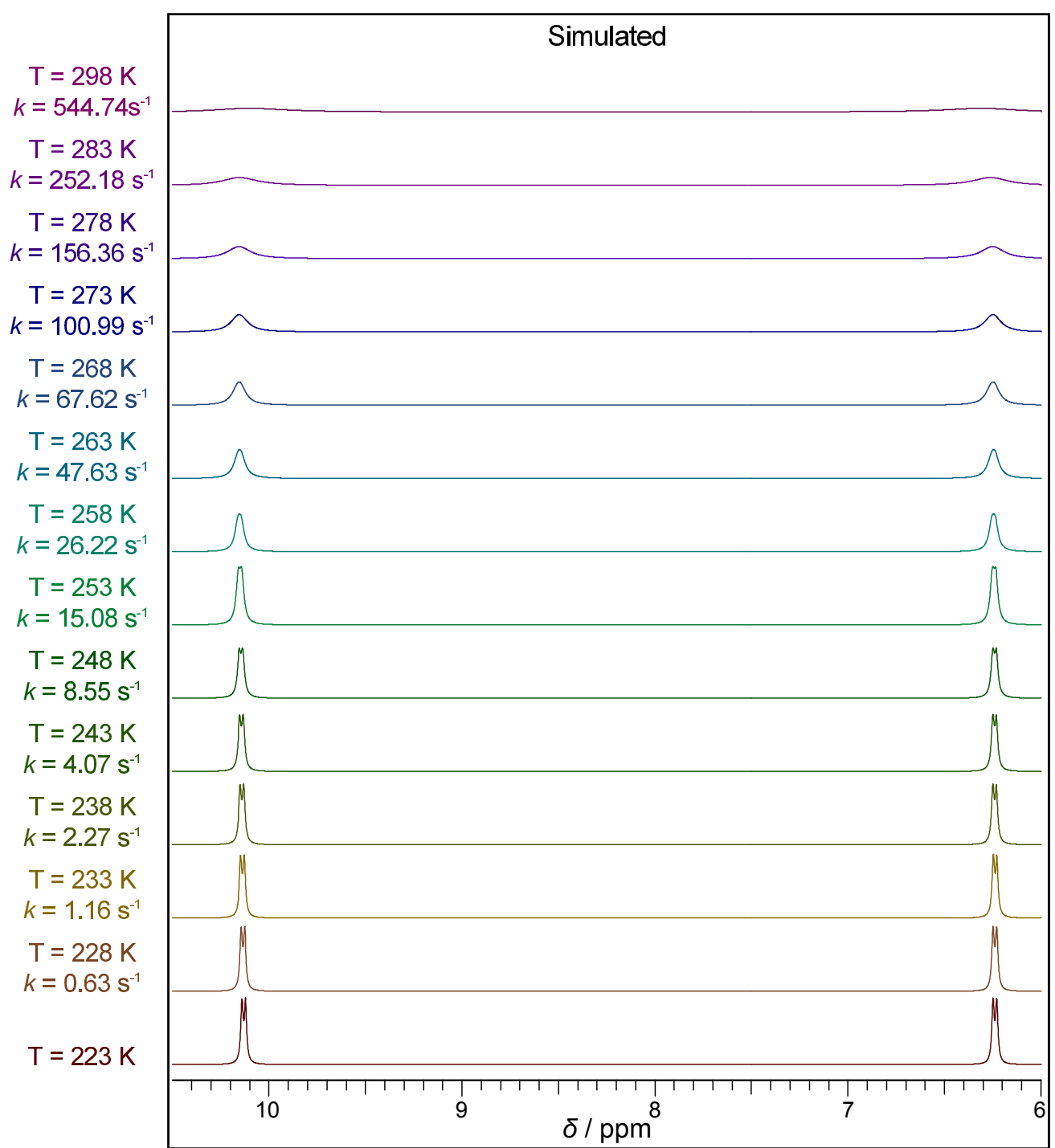

Figure 5.13.: Temperature dependence of lineshapes corresponding to exchanging hydrogens of the phenyl ring and exchange rates $k$. Experimental results are depicted on the left side while on the right side a simulation is shown. 
$\Delta G^{\ddagger}=R T\left(23.76-\ln \frac{k}{T}\right)$

$\ln \left(\frac{k}{T}\right)=23.76-\left(\frac{\Delta H^{\ddagger}}{R}\right) \frac{1}{T}+\left(\frac{\Delta S^{\ddagger}}{R}\right)$

with

$T$ absolute temperature

$R \quad$ general gas constant $\left(8.3144 \mathrm{~J} \mathrm{~K}^{-1}\right)$

A frequency factor

$k_{\mathrm{b}} \quad$ Boltzmann constant $\left(1.3805 \cdot 10^{-23} \mathrm{~J} \mathrm{~K}^{-1}\right)$

$h \quad$ Planck quantum of action $\left(6.6256 \cdot 10^{-34} \mathrm{Js}\right)$

$\kappa \quad$ transmission coefficient (usually set to 1 )

(a)

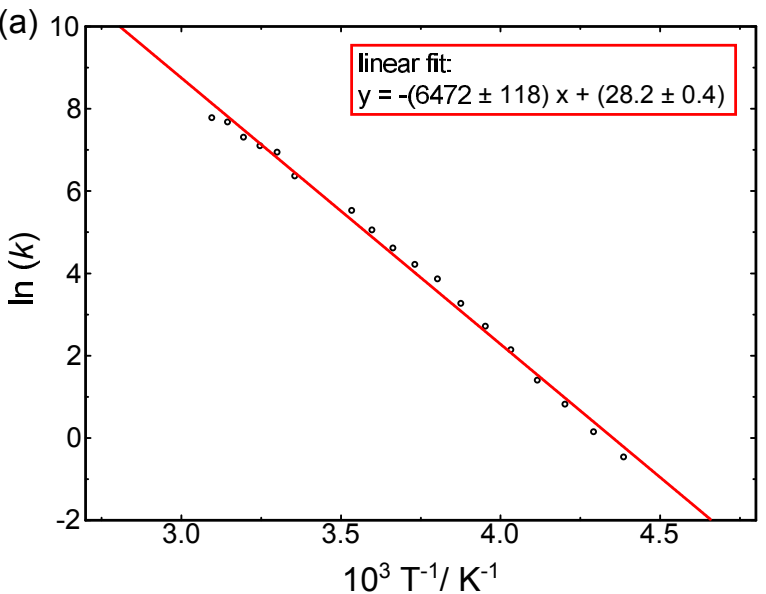

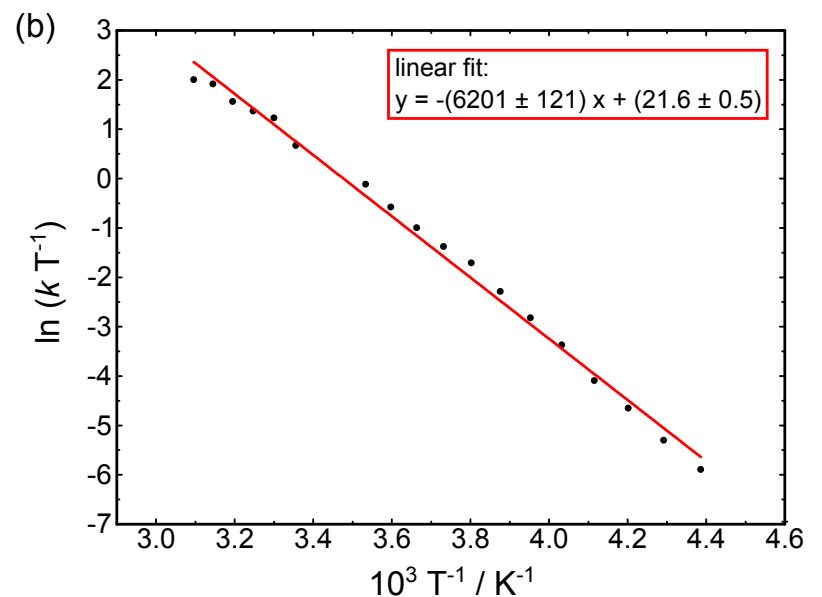

Figure 5.14.: (a) Arrhenius plot of aquired data with linear fit ${ }^{\S}$ applied. (b) Eyring plot with an applied linear fit.

With the parameters of activation enthalpy and entropy, the free activation enthalpy $\Delta G^{\ddagger} 298 \mathrm{~K}$ was calculated by applying equation 5.7. Determined activation parameters are summarized in Table 5.3.

$$
\Delta G^{\ddagger}=\Delta H^{\ddagger}-T \Delta S^{\ddagger}
$$

Comparable information on the barrier of rotation of the phenyl group in a $\mu-\eta^{1}: \eta^{1}$ phenylvinyl ligand was not found. Nonetheless, the obtained rotational barrier was compared to $\alpha$ - and $\beta$-substituted styrenes. ${ }^{[200]}$ With a hydrogen in the cis- $\beta$ position, a barrier of less than $6.0 \mathrm{kcal} \mathrm{mol}^{-1}$ at coalescence temperature was reported, which is significantly lower

\footnotetext{
$\S$ The linear fit of the data in the Arrhenius plot was extrapolated in order to estimate $T_{\mathrm{C}}$.
} 
Table 5.3.: Determined activation parameters.

\begin{tabular}{ccccccc}
\hline \multicolumn{2}{c}{$\mathrm{E}_{\mathrm{A}}$} & \multicolumn{2}{c}{$\Delta H^{\ddagger}$} & \multicolumn{2}{c}{$\Delta S^{\ddagger}$} & \multicolumn{2}{c}{$\Delta G^{\ddagger} 298 \mathrm{k}$} \\
$\mathrm{kJ} \mathrm{mol}^{-1}$ & $\mathrm{kcal} \mathrm{mol}$ & $\mathrm{kJ} \mathrm{mol}^{-1}$ & $\mathrm{kcal} \mathrm{mol}$ & $\mathrm{J} \mathrm{mol}^{-1} \mathrm{~K}^{-1}$ & $\mathrm{~kJ} \mathrm{~mol}^{-1}$ & $\mathrm{kcal} \mathrm{mol}^{-1}$ \\
\hline $54 \pm 1$ & $12.9 \pm 0.3$ & $52 \pm 1$ & $12.3 \pm 0.2$ & $-18 \pm 4$ & $57 \pm 1$ & $13.6 \pm 0.3$ \\
\hline
\end{tabular}

than the herein determined value of $\Delta G^{\ddagger} 323 \mathrm{~K}=13.7 \mathrm{kcal} \mathrm{mol}^{-1}$. However, considering the steric influence of the bulky DIPP substituents and solvent coordinated to the potassium, the higher barrier can be reasonably explained. Furthermore, the value calculated for the anionic phenylvinyl bridge at room temperature $\left(\Delta G^{\ddagger} 298 \mathrm{~K}=13.6 \mathrm{kcal} \mathrm{mol}^{-1}\right)$ compares with rotational barriers of phenylallyl alkali metal salts, which have been reported within a range of $10.3-12.9 \mathrm{kcal} \mathrm{mol}^{-1} .^{[201]}$ 


\subsection{Studies on the Reaction}

Since the reaction of the dihydride complex $\mathbf{K}\left[\mathbf{L}^{\text {bisnac }} \mathbf{N i}_{\mathbf{2}}(\mathbf{H})_{\mathbf{2}}\right]$ with phenylacetylene led to a phenylvinyl-bridged complex the question of the whereabouts of the formerly metal bound hydrogen atoms arises.

WAILES et al. reported a similar reaction with a bis(cyclopentadienyl)-dihydrido zirconium complex $\left[\left(\mu^{5}-\mathrm{C}_{5} \mathrm{H}_{5}\right)_{2} \mathrm{Zr}(\mathrm{H})_{2}\right] .{ }^{[202]}$ Among other alkyne substrates the hydrido complex was also reacted with phenylacetylene resulting in formation of olefin complexes accompanied by release of dihydrogen.

One year later, it was found that the complex was capable of catalytically hydrogenating alkynes and olefins. In these reactions, $\mathrm{H}_{2}$ pressures of 50-100 atm and temperatures of $80-120{ }^{\circ} \mathrm{C}$ were applied to fully hydrogenate various subtrates in quantitative yields. However, under these conditions hydrogenated products appeared to suffer from pyrolysis, which led to deactivation of the catalyst. ${ }^{[203]}$

The reaction of a related zirconium dimer complex $\left[\left(\mu^{5}-\mathrm{C}_{5} \mathrm{H}_{5} \mathrm{CH}_{3}\right)_{2} \mathrm{ZrH}(\mu-\mathrm{H})\right]_{2}$ with diphenylacetylene and phenylacetylene was investigated more than a decade later by JONES et al. In this case it was found that a zirconacyclopentadiene compound and nearly stoichiometric amounts of $\mathrm{H}_{2}$ were produced. If formed $\mathrm{H}_{2}$ was not removed, it led to hydrogenation of unsaturated hydrocarbons. Use of phenylacetylene led to the principal formation of ethylbenzene. ${ }^{[204]}$ Some years later, BiANCHINI et al. investigated a $\mathrm{Rh}^{\mathrm{I}}$ monohydride complex which reacted with phenylacetylene to form $\sigma$-acetylide and $\sigma$-alkenyl complexes accompanied by styrene formation. ${ }^{[205]}$

\subsubsection{Formation of Styrene}

The ${ }^{1} \mathrm{H}$ NMR spectrum of a sample containing $\mathbf{K}\left[\mathbf{L}^{\mathbf{b i s n a c}} \mathbf{N i}_{\mathbf{2}}(\mathbf{H})_{\mathbf{2}}\right]$, which was reacted with phenylacetylene (2 eq) directly in a J. Young NMR tube revealed additional signals (Figure 5.15). These signals do not belong to the main compound $\mathbf{K}\left[\mathbf{L}^{\text {bisnac }} \mathbf{N i}_{\mathbf{2}}\left(\mu-\eta^{\mathbf{1}}: \eta^{\mathbf{1}}\right.\right.$ $\mathrm{CHCPh})]$.

It was found that these resonances stem from styrene, which was formed during the course of the reaction. Conduction of the reaction with stoichiometric amounts of phenylacetylene led to complete conversion to $\mathbf{K}\left[\mathbf{L}^{\text {bisnac }} \mathbf{N i}_{2}\left(\mu-\eta^{1}: \eta^{1}-\mathbf{C H C P h}\right)\right]$ but no resonances associated with styrene were observed. This indicates the preferential reaction to $\mathrm{K}\left[\mathrm{L}^{\text {bisnac }} \mathrm{Ni}_{2}\left(\mu-\eta^{1}: \eta^{1}-\mathrm{CHCPh}\right)\right]$.

If two equivalents of phenylacetylene are used, the second equivalent is hydrogenated to styrene. During the course of a week the entire amount of PA was converted (after 48 hours $\approx 0.44$ eq of styrene were produced $)$. Since $\mathbf{K}\left[\mathbf{L}^{\text {bisnac }} \mathbf{N i}_{\mathbf{2}}\left(\mu-\eta^{\mathbf{1}}: \eta^{\mathbf{1}}-\mathbf{C H C P h}\right)\right]$ is quantitatively formed with one equivalent of phenylacetylene, it was thought that the 


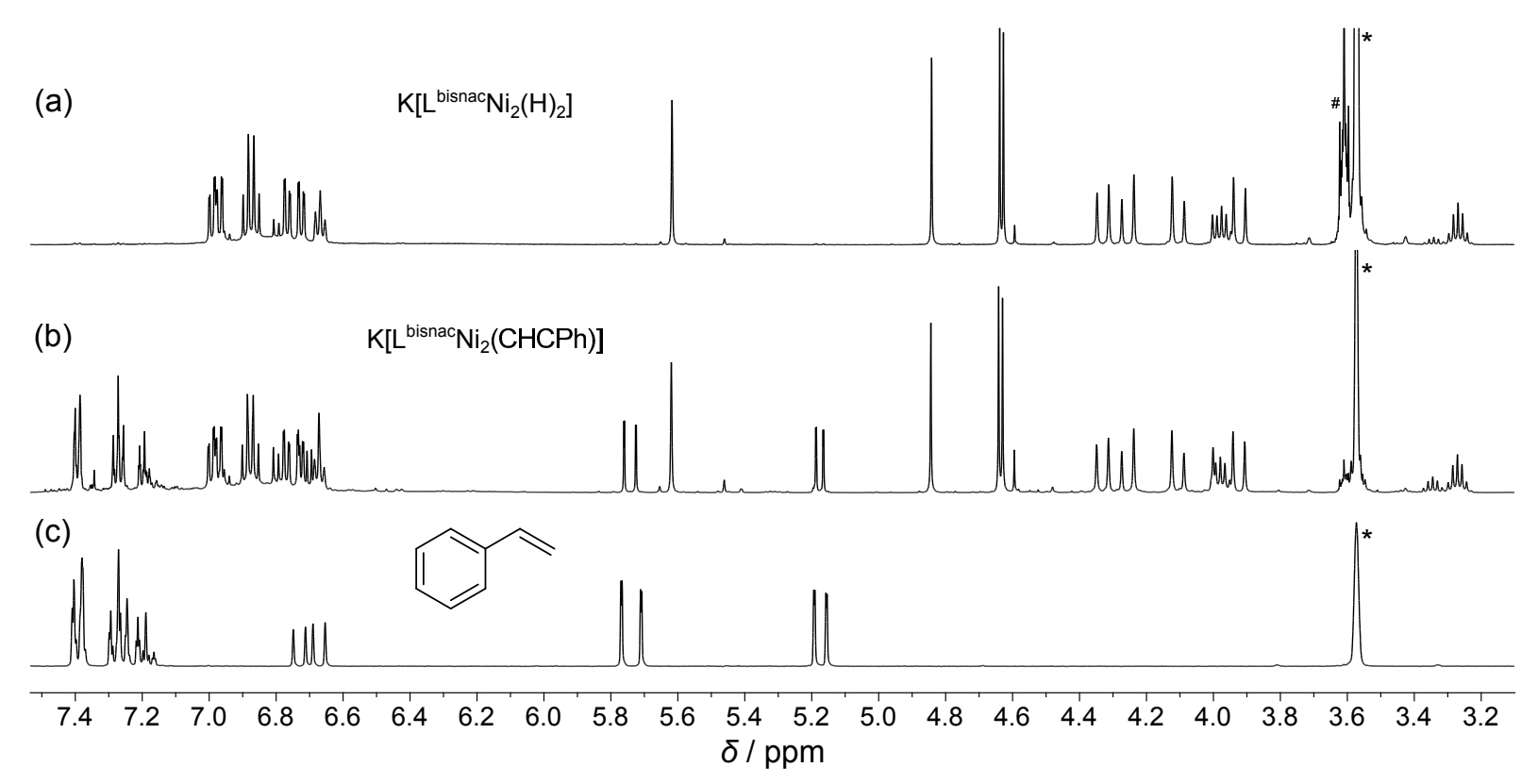

Figure 5.15.: Comparison of the ${ }^{1} \mathrm{H}$ NMR spectrum $(500 \mathrm{MHz})$ of crystalline $K\left[L^{\text {bisnac }} \mathbf{N i}_{2}\left(\mu-\eta \eta^{1}: \eta^{1}-\right.\right.$ CHCPh)] (a) with the spectrum $(500 \mathrm{MHz})$ of $\mathrm{K}\left[\mathrm{L}^{\text {bisnac }} \mathrm{Ni}_{2}(\mathbf{H})_{2}\right]$ with 2 equivalents of phenylacetylene after complete reaction (b). To show that during the reaction process styrene was formed, a benchmark spectrum (c) is also depicted ( $300 \mathrm{MHz}$ ). All samples were measured in THF- $\mathrm{D}_{8}$ at room temperature. Residual solvent resonances of THF and THF- $D_{7}$ were marked $\left(\#,{ }^{*}\right)$.

two hydrides yield molecular dihydrogen during the process. A dihydrogen resonance is expected at $4.55 \mathrm{ppm}$ in THF- $\mathrm{D}_{8} \cdot{ }^{[150]}$ Unfortunately, reaction in a closed J. Young valve NMR tube followed by ${ }^{1} \mathrm{H}$ NMR monitoring did not reveal a dihydrogen resonance.

However, it can be explained due to several effects: $\mathrm{H}_{2}$ is a very light gas compared to the $\mathrm{N}_{2}$ gas present in the sample and is formed in a maximum amount of one equivalent, which is very low compared to the excess $\mathrm{N}_{2}$. Therefore, the amount of dissolved $\mathrm{H}_{2}$ is expected to be low. Furthermore, a superposition by adjacent resonances can occur. For example, the signal at $4.63 \mathrm{ppm}$, corresponding to the $\beta$-diketiminato $\mathrm{CH}$ or signals stemming from impurities or side products can conceal the small $\mathrm{H}_{2}$ signal.

Nonetheless, reaction in an open vessel containing a concentrated solution of the starting material (around $60 \mathrm{mg}$ dissolved in $1 \mathrm{~mL}$ of THF) led to the visible formation of gas. 


\subsubsection{Deuterium Labeling}

\section{Investigation by ${ }^{2} \mathrm{H}$ NMR spectroscopy}

In order to find clear analytic evidence for the release of dihydrogen during reaction of $\mathbf{K}\left[\mathbf{L}^{\text {bisnac }} \mathbf{N i}_{\mathbf{2}}(\mathbf{H})_{2}\right]$ with $\mathrm{PA}$, an isotopic labeling experiment was conducted. The previously described $\mathbf{K}\left[\mathbf{L}^{\text {bisnac }} \mathbf{N i}_{2}(\mathbf{D})_{2}\right]$ (Chapter 4.4) is well suited, not only to possibly "indirectly" prove the generation of $\mathrm{H}_{2}$ by formation of $\mathrm{D}_{2}$, but also to use as a probe to gain insight into the reaction mechanism. With $\mathrm{D}_{2}$ having twice the mass as dihydrogen there would be a chance of observing formed $\mathrm{D}_{2}$ gas in a solution NMR experiment.

A sample of a solution containing $\mathbf{K}\left[\mathbf{L}^{\text {bisnac }} \mathbf{N i}_{\mathbf{2}}(\mathbf{D})_{\mathbf{2}}\right]$ in dry THF with two equivalents of phenylacetylene was prepared and monitored by ${ }^{2} \mathrm{H}$ NMR spectroscopy. Selected spectra collected after different times are shown as a stack plot in Figure 5.16.

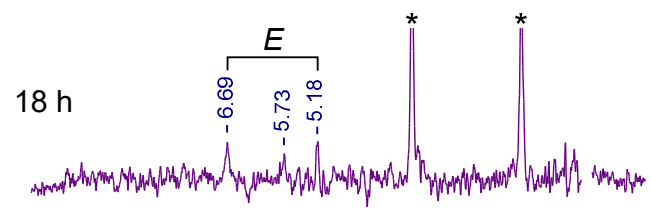<smiles>[2H]/C(=C/c1ccccc1)c1ccccc1</smiles>

38 min 19 min start

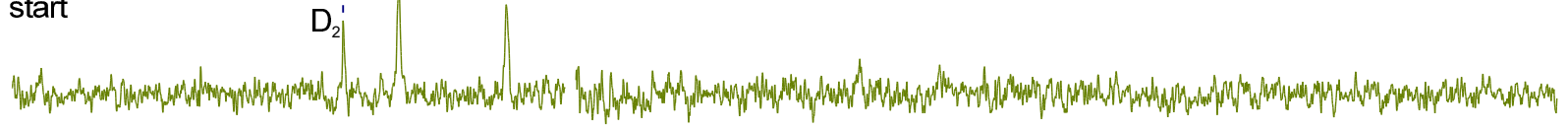

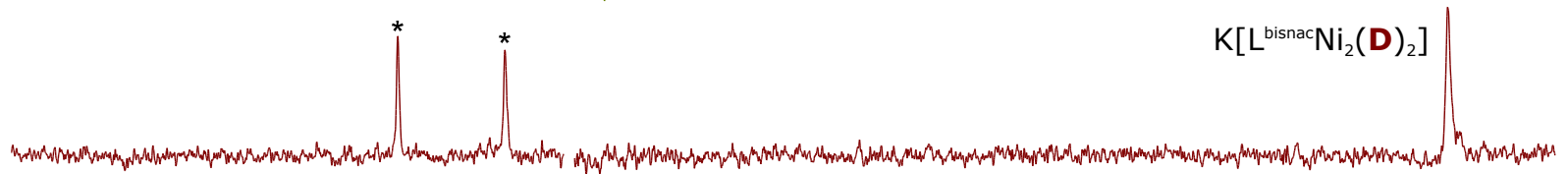

$\begin{array}{llllllllllllllllll}9 & 8 & 7 & 6 & 5 & 4 & 3 & 2 & 1 & -10 & -12 & -14 & -16 & -18 & -20 & -22 & -24 \\ \delta / \mathrm{ppm} & & & & \end{array}$

Figure 5.16.: Formation of $\mathrm{K}\left[\mathrm{L}^{\text {bisnac }} \mathrm{Ni}_{2}\left(\mu-\eta^{1}: \eta^{1}-\mathrm{CHCPh}\right)\right]$ from $\mathrm{K}\left[\mathrm{L}^{\text {bisnac }} \mathrm{Ni}_{2}(\mathrm{D})_{2}\right]$ in presence of an excess amount of phenylacetylene (2 eq) monitored by ${ }^{2} \mathrm{H}$ NMR $(61 \mathrm{MHz})$ at room temperature. After formation of the phenylvinyl-bridged complex, indicated by the vanished characteristic dideuteride resonance of $K\left[L^{\text {bisnac }} \mathrm{Ni}_{2}(D)_{2}\right], \mathrm{D}_{2}$ was released and subsequently consumed during the deuterohydrogenation of the second equivalent of phenylacetylene to yield styrene.

The absence of the ${ }^{2} \mathrm{H}$ NMR signal at $-24 \mathrm{ppm}$ in the first spectrum after initiation of the reaction (light green trace in Figure 5.16) indicates the full conversion of $\mathbf{K}\left[\mathbf{L}^{\mathbf{b i s n a c}} \mathbf{N i}_{\mathbf{2}}(\mathbf{D})_{\mathbf{2}}\right]$ 
to $\mathbf{K}\left[\mathbf{L}^{\text {bisnac }} \mathbf{N i}_{\mathbf{2}}\left(\mu-\eta^{1}: \eta^{1}-\mathbf{C H C P h}\right)\right]$. At the same time the incipient release of $\mathrm{D}_{2}$ becomes evident $\left(\delta\left\{{ }^{2} \mathrm{H}\right\}=4.54 \mathrm{ppm}\right)$. This is consistent with visual experience made during the experiment (immediate color change of the reaction solution to dark green) and further shows that all components $\left(\mathbf{K}\left[\mathbf{L}^{\text {bisnac }} \mathbf{N i}_{\mathbf{2}}\left(\mu-\eta^{1}: \eta^{1}-\mathbf{C H C P h}\right)\right]\right.$, phenylacetylene and $\mathrm{D}_{2}$ ) are separate in the reaction mixture shortly after incorporation of the first equivalent of phenylacetylene.

The dissolved $\mathrm{D}_{2}$ slowly diffuses into the gaseous phase of the sample until an equilibrium is reached. At the same time deuterium hydrogenation of the excess amount of phenylacetylene takes place. However, the rate of this reaction is very low compared to the hydrogenation with $\mathrm{H}_{2}$, since characteristic resonances of styrene appeared many hours after reaction start (purple trace in Figure 5.16). This may be attributed to a kinetic isotope effect.

One of the substrates (phenylacetylene or more likely $\mathrm{D}_{2}$ ) may coordinate to the metal ions of the formed complex $\mathbf{K}\left[\mathbf{L}^{\text {bisnac }} \mathbf{N i}_{2}\left(\mu-\eta^{1}: \eta^{1}-\mathbf{C H C P h}\right)\right]$ followed by the other substrate. This then results in hydrogenation of excess amounts of phenylacetylene to styrene with $\mathbf{K}\left[\mathbf{L}^{\text {bisnac }} \mathbf{N i}_{\mathbf{2}}\left(\mu-\eta^{1}: \eta^{1}-\mathbf{C H C P h}\right)\right]$ acting as a potential catalyst.

In a further experiment integration of relevant resonances at $6.68,5.72$ and 5.16 ppm corresponding to $\mathrm{D}^{\mathrm{a}}, \mathrm{D}^{\mathrm{c}}$ and $\mathrm{D}^{\mathrm{b}}$ (Figure 5.17) gave a ratio of 2:1:2. This indicates the formation of several deuterated styrene species. Since the most prominent signals are associated to $\mathrm{D}^{\mathrm{a}}$ and $\mathrm{D}^{\mathrm{b}}$, the cis deutero-hydrogenated product, that is $E$-(1,2-dideuterovinyl)benzene accounts for the main species. The presence of the signal at $5.72 \mathrm{ppm}$, which is correlated to a deuterium resonance in the $\mathrm{D}^{\mathrm{c}}$ position implies additional formation of styrene with a fully deuterated vinyl substituent (Scheme 5.4).

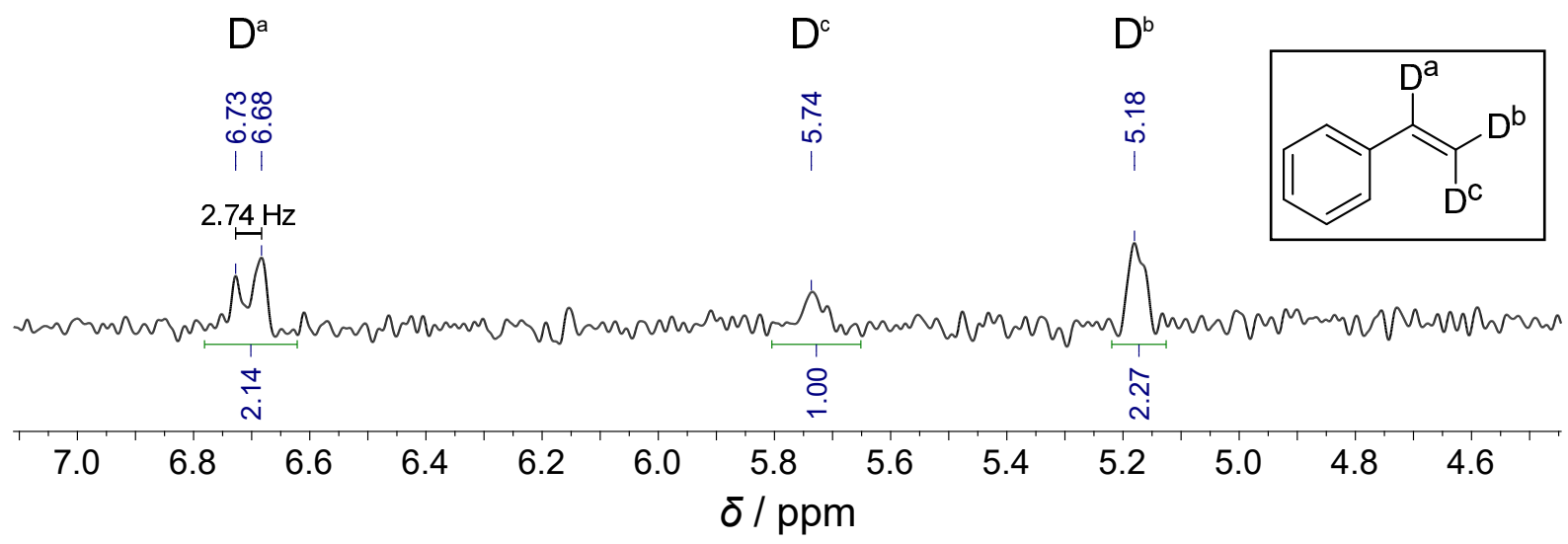

Figure 5.17.: ${ }^{2} \mathrm{H}$ NMR spectrum $(62 \mathrm{MHz})$ measured several hours after the conversion of $K\left[L^{\text {bisnac }} \mathrm{Ni}_{2}(\mathrm{D})_{2}\right]$ with an excess amount of phenylacetylene (2 eq). The spectrum shows characteristic resonances of deutero-styrene. Positions of deuterium atoms in the styrene molecule are indicated.

An $\mathrm{H} / \mathrm{D}$-exchange reaction likely caused by another compound, can result in the fully deuterated vinyl substituent. Such an H/D exchange in the $\beta$-position of $\alpha$-aryl olefins 
like styrene was reported before. ${ }^{[206]}$ In this case, the exchange was catalyzed by a rhodium deuteride complex yielding the geminally deuterated product (i.e. deuteration in $\mathrm{D}^{\mathrm{b}}$ and $\mathrm{D}^{\mathrm{c}}$ position).

This step, however, requires that deutero-hydrogenation of phenylacetylene competes with $\mathrm{H} / \mathrm{D}$ exchange, occuring at a similar rate, since during reaction of $\mathbf{K}\left[\mathbf{L}^{\text {bisnac }} \mathbf{N i}_{\mathbf{2}}(\mathbf{D})_{\mathbf{2}}\right]$ with two equivalents of PA only one equivalent of $\mathrm{D}_{2}$ can be formed. The initially formed $E$-(1,2-dideuterovinyl)benzene may not be immediately substituted by another phenylacetylene molecule allowing for a complex mediated $\mathrm{H} / \mathrm{D}$ exchange in the styrene molecule.

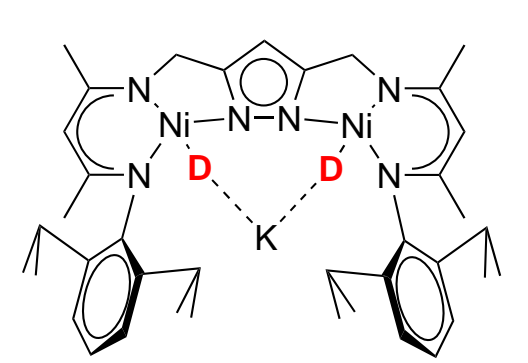

$\mathrm{K}\left[\mathrm{L}^{\text {bisnac }} \mathrm{Ni}_{2}(\mathrm{D})_{2}\right]$

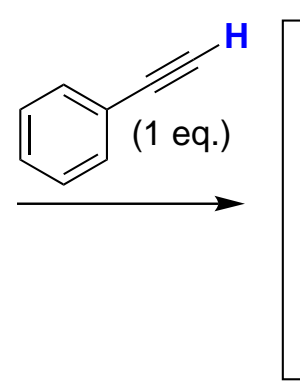

$\leftarrow$

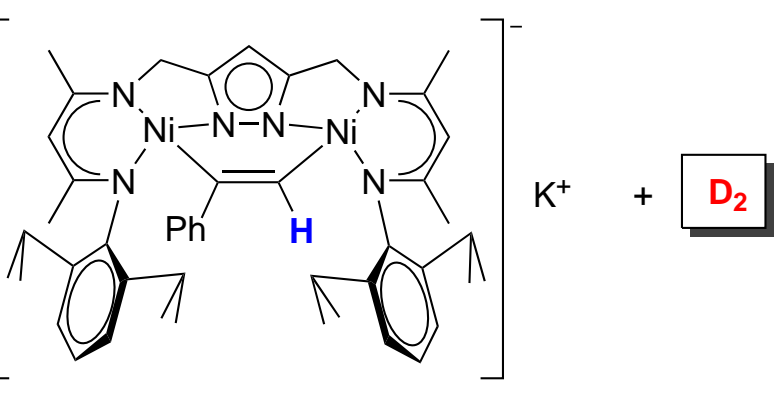
$\mathrm{K}\left[\mathrm{L}^{\text {bisnac }} \mathrm{Ni}_{2}(\mathrm{CHCPh})\right]$<smiles>C#Cc1ccccc1</smiles>

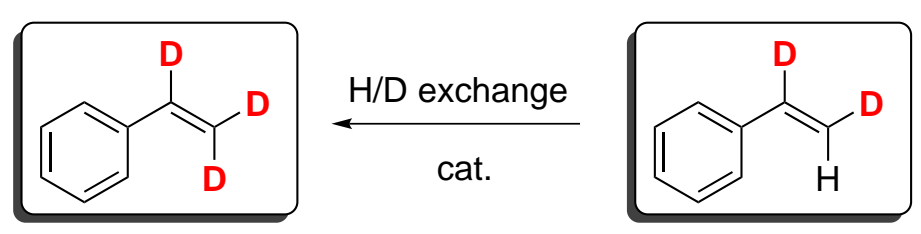

Scheme 5.4: Proposed pathway of the reaction of $K\left[L^{\text {bisnac }} \mathrm{Ni}_{2}(\mathrm{D})_{2}\right]$ with phenylacetylene to $\mathrm{K}\left[\mathrm{L}^{\text {bisnac }} \mathrm{Ni}_{2}\left(\mu-\eta^{1}: \eta^{1}-\mathrm{CHCPh}\right)\right]$ accompanied by release of dideuterium. Afterwards, another equivalent of phenylacetylene is hydrogenated to styrene. In a competing $H / D$ exchange step styrene with its fully deuterated vinyl substituent presumably forms.

Formation of the trans hydrogenation product (i.e. Z-(1,2-dideuterovinyl)benzene) was considered but did not fit into the occuring ratio (in this case a ratio of 2:2:1 was expected). Another possible product is the geminally deuterated styrene (2,2-deuterovinyl)benzene, but its formation was also very unlikely since an expected ratio of 1:2:2 was not observed. Nonetheless, other monodeuterated or even non-deuterated styrene isomers have to be taken into account. For instance, traces of water can lead to formation of small amounts of HD upon conversion with $\mathbf{K}\left[\mathbf{L}^{\text {bisnac }} \mathbf{N i}_{\mathbf{2}}(\mathbf{D})_{2}\right]$ (chapter 4 ). Therefore, the sketch shown in Scheme 5.4 only describes a tentative suggestion of formed products and does not reflect the overall composition of styrene isomers.

By carefully examining the resonance at $6.68 \mathrm{ppm}$, corresponding to deuterium in $\alpha$-pos- 
ition of formed styrene, a coupling constant of $2.7 \mathrm{~Hz}$ was determined (Figure 5.17). This coupling constant is associated with ${ }^{3} J_{\mathrm{HD}}$ trans-coupling of the $\alpha$-deuterium to the hydrogen atom in $\beta$-position $\left(\mathrm{H}^{\mathrm{c}}\right)$, which further substantiates the principal formation of the cis deutero-hydrogenated product. $\stackrel{[207,208]}{ }$

Regarding the formation of $\mathbf{K}\left[\mathbf{L}^{\text {bisnac }} \mathbf{N i}_{\mathbf{2}}\left(\mu-\eta^{\mathbf{1}}: \eta^{\mathbf{1}} \mathbf{C H C P h}\right)\right]$ both experiments did not show a vinylic deuterium resonance in the ${ }^{2} \mathrm{H}$ NMR experiment. This implies that the hydrogen atom from the substrate (PA) is maintained in the complex (Scheme 5.4).

\section{Investigation by ${ }^{1} \mathrm{H}$ NMR spectroscopy}

In another experimental setup, the in situ generated $\mathrm{Ni}-\mathrm{D}$ complex was converted with

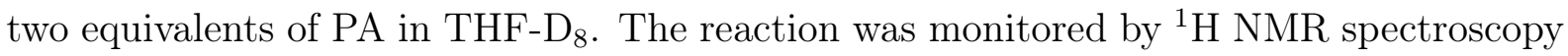
to further elucidate the fate of the deuterium atoms.

If generated $\mathrm{D}_{2}$ reacts with the second equivalent of phenylacetylene, the corresponding deuterium atoms in the formed styrene remain silent in the proton NMR while other proton resonances are observed. Figure 5.18 illustrates the result of the ${ }^{1} \mathrm{H}$ NMR experiment.

After addition of two equivalents of phenylacetylene to $\mathbf{K}\left[\mathbf{L}^{\text {bisnac }} \mathbf{N i}_{\mathbf{2}}(\mathbf{D})_{2}\right]$ at room temperature the expected rapid formation of the phenylvinyl-bridged compound $\mathbf{K}\left[\mathbf{L}^{\text {bisnac }} \mathbf{N i}_{\mathbf{2}}\left(\mu-\eta^{\mathbf{1}}: \eta^{\mathbf{1}}-\mathbf{C H C P h}\right)\right]$ occured. Similar to the analogous reaction with the dihydride complex rapid formation of the product was indicated by an immediate color change from the clear orange solution of the starting material to a clear dark green solution. As can be further seen, one equivalent of phenylacetylene was consumed during this first reaction step, leaving the remaining equivalent in the sample solution as indicated by the present characteristic resonances ( $\Delta$ ) in spectrum (b) of Figure 5.18.

However, reaction of the excess amount of phenylacetylene with $\mathrm{D}_{2}$ proceeded very slowly compared to the conversion with $\mathrm{H}_{2}$, which may be attributable to a kinetic isotope effect as described in the previous section.

Another remarkable observation in spectrum (b) and (c) Figure 5.18 was the signal of the vinyl proton bound to the bridging unit which resonates at $\delta=4.84 \mathrm{ppm}(\bullet)$. This finding is consistent with that from the ${ }^{2} \mathrm{H}$ NMR investigations and confirms the hydrogen originating from the alkyne. It also substantiates the formation of $\mathrm{D}_{2}$ (with no formed HD). In contrast to the deuterium NMR experiment, ${ }^{1} \mathrm{H}$ NMR monitoring allowed for better estimation of substrate consumption via comparison of corresponding proton signal integrals with benchmark signal integrals.

After three weeks, a ${ }^{1} \mathrm{H}$ NMR measurement of the sample yielded spectrum (c) shown in Figure 5.18, Characteristic resonances of PA ( $\mathbf{\Delta})$ disappeared, indicating consumption of the substrate.

At the same time new resonances arose, which may be correlated to formed styrene as 


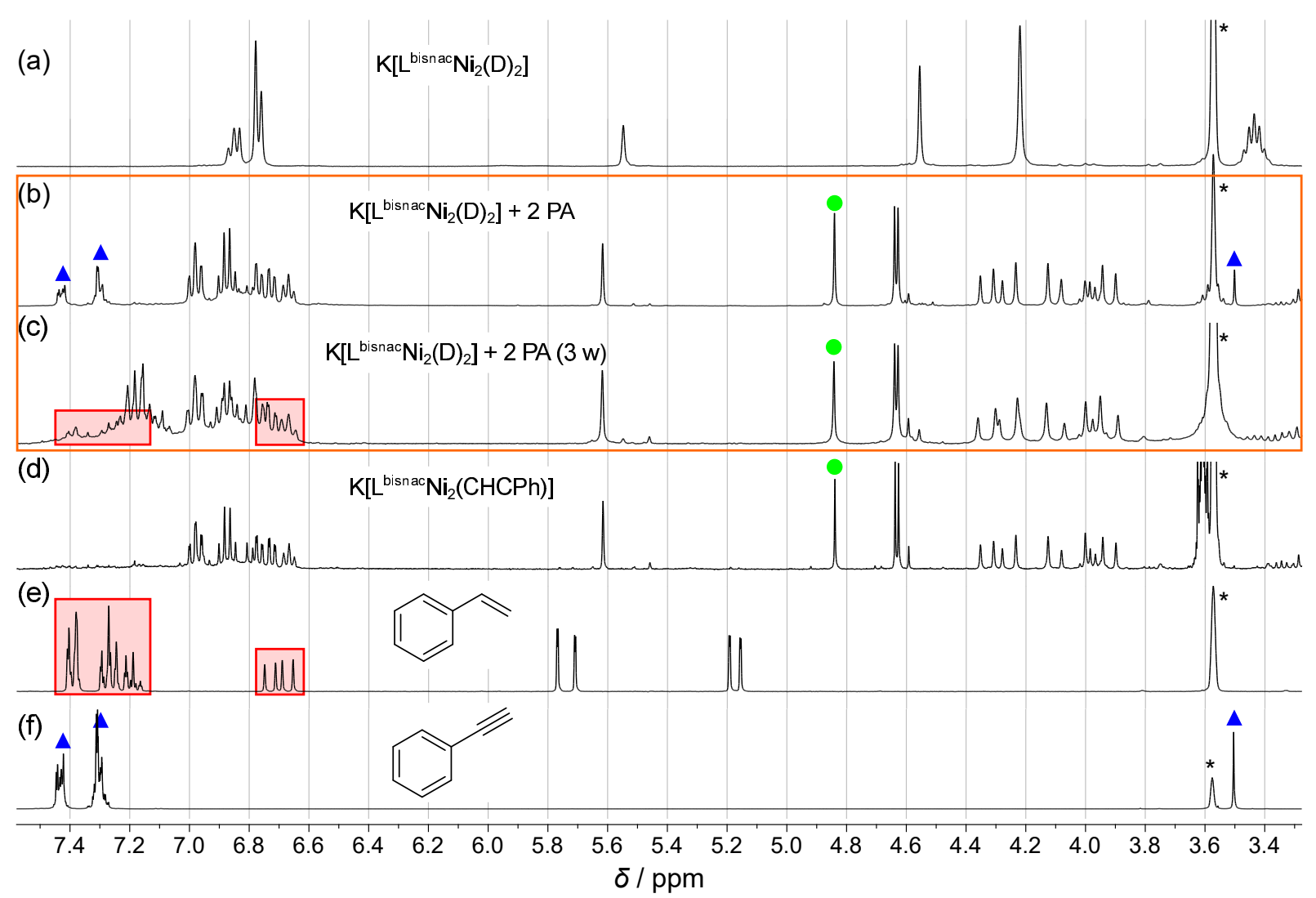

Figure 5.18.: ${ }^{1} \mathrm{H}$ NMR spectra measured in THF- $\mathrm{D}_{8}\left({ }^{*}\right)$. (a) untreated sample of the dideuteride complex $\left.\mathrm{K}_{[} \mathrm{L}^{\text {bisnac }} \mathrm{Ni}_{2}(\mathrm{D})_{2}\right](400 \mathrm{MHz})$. (b) $\mathbf{K}\left[\mathrm{L}^{\text {bisnac }} \mathrm{Ni}_{2}(\mathrm{D})_{2}\right]$ treated with 2 equivalents of phenylacetylene $(400 \mathrm{MHz})$. Initially after addition of the substrate to the dideuteride complex $\mathrm{K}\left[\mathrm{L}^{\text {bisnac }} \mathrm{Ni}_{2}\left(\mu-\eta^{1}: \eta^{1}\right.\right.$ CHCPh)] was formed. No signs of styrene formation were observed. The second equivalent of phenylacetylene is still present $\Delta$. The vinyl hydrogen resonance of the resulting $\left.\mathrm{K}_{\left[L^{\mathrm{bisnac}}\right.} \mathrm{Ni}_{2}\left(\mu-\eta^{1}: \eta^{1}-\mathrm{CHCPh}\right)\right]$ is marked with $\bullet$. (c) represents the sample after 3 weeks $(300 \mathrm{MHz})$. PA was consumed and new resonances in the aromatic regions are observed. (d), (e) and (f) represent benchmark spectra of $\mathrm{K}\left[\mathrm{L}^{\text {bisnac }} \mathrm{Ni}_{2}\left(\mu-\eta^{1}: \eta^{1}-\mathrm{CHCPh}\right)\right]$ (400 MHz), styrene (300 MHz) and phenylacetylene (400 MHz). 
indicated by red markings in the ${ }^{1} \mathrm{H}$ NMR spectrum (c). Missing characteristic styrene resonances at $5.18 \mathrm{ppm}$ and $5.73 \mathrm{ppm}$ can be correlated to formation of corresponding deuterated styrene isomers. However, the absolute absence of corresponding vinyl-resonances not only indicates the formation of styrene as result of cis hydrogenation, but also correlates to complete deuteration of the vinyl substituent.

This supports the results of ${ }^{2} \mathrm{H}$ NMR experiments, in which a H/D - exchange was assumed shortly after formation of the cis deuterated product, leading to the fully deuterated vinyl substituent. However, considering the very low intensity of signals corresponding to formed deutero-styrene, the amount of semihydrogenated substrate was considerably low which is also in accordance with the ${ }^{2} \mathrm{H}$ NMR experimental results.

The aromatic region in the ${ }^{1} \mathrm{H}$ NMR spectrum (c) of Figure 5.18 shows another resonance pattern exhibiting a significant intensity. Due to their appearance in the aromatic region (7.08-7.23 ppm), these signals were attributed to a new aromatic species. The formation of deuterated ethylbenzene was considered (Figure 5.19) but was not confirmed by ${ }^{13} \mathrm{C}$ NMR (Figure D.6).

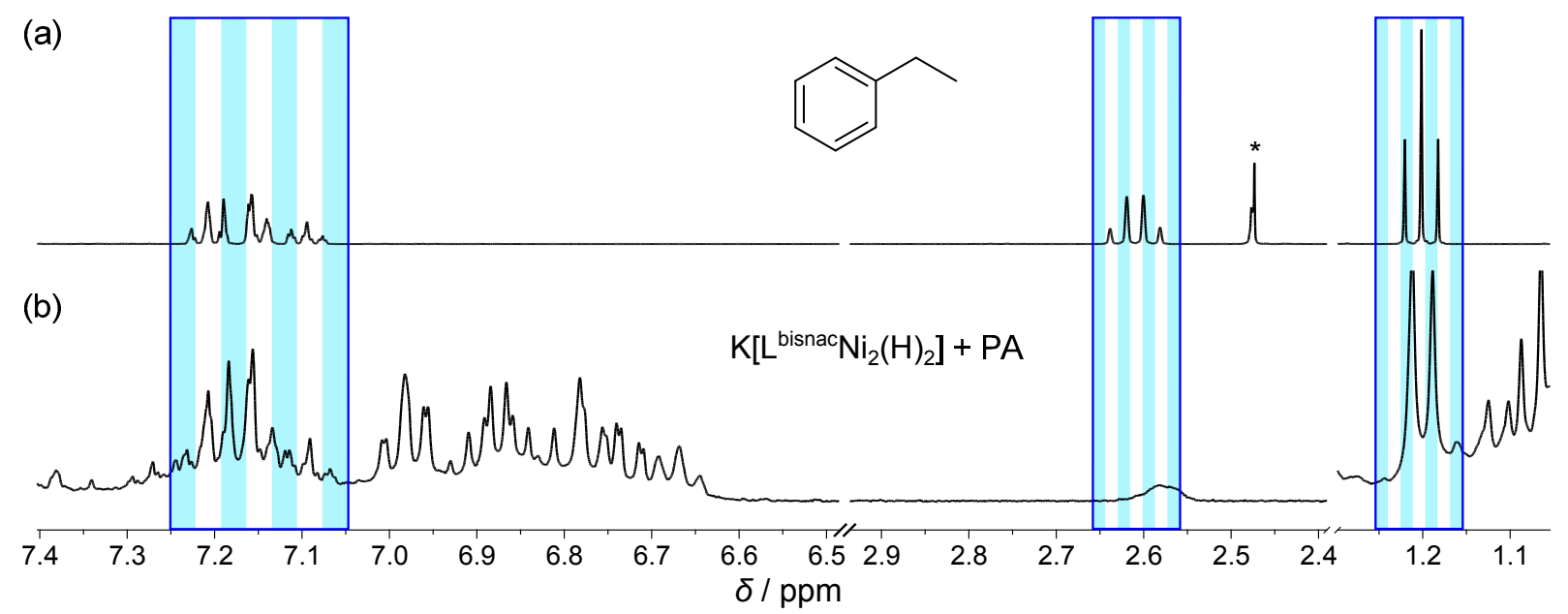

Figure 5.19.: ${ }^{1} \mathrm{H}$ NMR spectra measured in THF- $\mathrm{D}_{8}$. Residual amounts of solvents are marked $\left({ }^{*}\right)$. (a) describes a sample of ethylbenzene. In (b) the spectrum of the reaction mixture containing $K\left[L^{\text {bisnac }} \mathrm{Ni}_{2}\left(\mu-\eta^{1}: \eta^{1}-\mathrm{CHCPh}\right)\right]$ synthesized from $\mathrm{K}\left[\mathrm{L}^{\text {bisnac }} \mathrm{Ni}_{2}(\mathrm{D})_{2}\right]$ and two equivalents of phenylacetylene (after 3 weeks) is shown. Specific regions were omitted from both spectra for the sake of clarity. Resonance regions of ethylbenzene are encased. The absence of aliphatic ethylbenzene resonances in the ${ }^{1} \mathrm{H}$ NMR spectrum (b) indicate full deuteration.

Unfortunately, the identity of this species could not be elucidated so far and is probably correlated to side or decomposition reactions. Since the deuterium hydrogenation process occurs very slowly, faster side reactions are likely to occur. This can lead to the consumption of the second equivalent of phenylacetylene over the course of time without a hydrogenation with $\mathrm{D}_{2}$ to come into effect. 


\subsubsection{Para Hydrogen Induced Polarization Experiments}

\section{Introduction}

In 1986 BOWERS and WEITEKAMP independently published a theoretical prediction to dramatically enhance NMR signal intensity specifically by using para-hydrogen enriched $\mathrm{H}_{2}$ in hydrogenation reactions. ${ }^{[209]}$ A year later these predictions could be experimentally verified. $\stackrel{[210,211]}{=}$ The paper published by BOWERS and WEITEKAMP was entitled "Parahydrogen and Synthesis Allow Dramatically Enhanced Nuclear Alignment". To describe the formation of non-equilibrium magnetizations by using parahydrogen at upfield the abbreviation PASADENA would be later used. ${ }^{[212]}$

In 1988 PRAVICA and WEITEKAMP demonstrated that the conduction of substrate hydrogenation at low magnetic field and subsequent adiabatic transport of obtained products to upfield led to net NMR alignment. This phenomenon would later be called adiabatic longitudinal transport after dissociation endegers nuclear alignment (ALTADENA). $\stackrel{[212]}{ }$

Both PASDENA and ALTADENA require incorporation of parahydrogen into the molecule of interest. An alternative method relies on the scalar coupling network which modulates the transfer of polarization from parahydrogen derived hydrides to the observed analyte. This method was termed signal amplification by reversible exchange (SABRE) and is, similar to ALTADENA conducted at low magnetic field followed by rapid transport into the NMR spectrometer. ${ }^{[212,213]}$

\section{Parahydrogen}

Molecular hydrogen consists of four nuclear spin isomers. Since protons are considered as fermions, these spin isomers must satisfy Pauli's principle, which states that the overall wave function has to be antisymmetric with respect to exchange of nuclei. As a consequence, symmetric rotational states must be combined with antisymmetric nuclear spin states. Accordingly, antisymmetric rotational states must be combined with symmetric nuclear spin states. The symmetric nuclear spin states are 3-fold degenerate and contain the terms $\alpha \alpha, \alpha \beta+\beta \alpha$ and $\beta \beta$, representing orthohydrogen. On the other hand parahydrogen molecules solely exist as antisymmetric $\alpha \beta-\beta \alpha$ spin isomers. ${ }^{[214]}$ At ambient temperature $\left(25^{\circ} \mathrm{C}\right)$, dihydrogen gas is composed of around $75 \%$ orthohydrogen and $25 \%$ parahydrogen. Due to the energetic difference between triplet and singlet states, with the latter representing the lowest energy state, the statistical ratio can be shifted towards parahydrogen by lowering the temperature. ${ }^{[215]}$ As a result the ratio between ortho- and parahydrogen is shifted from 3:1 (298 K) to around 1:1 at $80 \mathrm{~K}$ and at $20 \mathrm{~K}$ the dihydrogen gas mainly consists of parahydrogen $(99.82 \%) . \underline{[.216]}$

Because transitions between symmetric and antisymmetric spin states are forbidden, a change of the ortho- and parahydrogen distribution can only be facilitated in presence 
of a catalyst. Among other materials, paramagnetic solids (e.g. charcoal) are the most effective to mediate the transition. $\stackrel{[212,216]}{ }$

This also implies that once formed, $\mathrm{H}_{2}$ out of thermal equilibrium is stable for a long time, provided that catalytic substances are absent. ${ }^{[212]}$

\section{Parahydrogen Induced Polarization}

The nuclear wavefunction of parahydrogen is, as demanded, antisymmetric in exchange of the nuclei. Therefore, the total nuclear spin equals zero (singlet state) which means that parahydrogen does not show a Zeeman effect. As a consequence it can not be observed in the NMR spectrum. ${ }^{[209]}$

In order to observe parahydrogen induced polarization (PHIP) in high magnetic field, firstly the nuclear spin alignment of the parahydrogen molecule has to be transferred to the molecule of interest. This can be accomplished either by complete pairwise transfer of the hydrogen molecule or by hyperpolarization transfer mediated by reversible exchange. $\stackrel{[217]}{[}$ In the former case, which refers to the previously mentioned PASADENA protocol, the singlet symmetry of the transferred parahydrogen molecule has to be broken. ${ }^{[214]}$ However, in all cases spin-spin coupling has to be retained and the hydrogen transfer has to occur in a faster rate than the proton relaxation. ${ }^{[210]}$ Especially the latter point limits the PHIP effect due to loss of hyperpolarization.

PHIP (Para Hydrogen Induced Polarization) provides the opportunity for non-thermal spin polarization leading to characteristic anomalous signal patterns in proton NMR experiments. These signals are usually enhanced by several orders of magnitude due to large population differences of associated transitions compared to non-thermal transitions. If hydrogen atoms stemming from para- $\mathrm{H}_{2}$ are transferred pairwise to magnetically inequivalent positions of the substrate molecule, in addition to strong enhancement a signal with different phases is observed. The phase difference in turn is caused by overpopulation (relative to a normal Boltzmann distribution) of energy states of the product molecule,

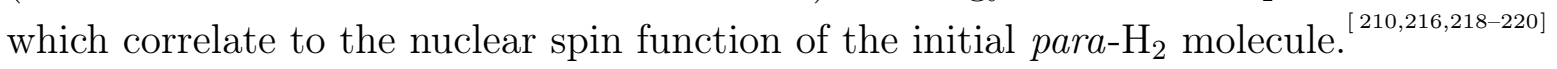

\section{Motivation}

Since PHIP represents a valuable tool for obtaining structural and dynamic information particularly during the process of olefin and alkyne hydrogenation, a respective experiment was considered to illuminate the generation of styrene from phenylacetylene. The two protocols PASADENA and ALTADENA came into use. In the PASADENA case, after hydrogenation the aligned parahydrogen atoms in the product molecule styrene were hyperpolarized at upfield resulting in antiphase doublets. ALTADENA further 
allows for the transfer of polarization to other atoms in the product molecule than the initial parahydrogen atoms due to the strong $J$-coupling $(J>\delta \nu)$ exhibited under these conditions. $\stackrel{[218,221]}{ }$

In the particular case of phenylacetylene semihydrogenation with parahydrogen several styrene isomers are expected. Scheme 5.5 shows the ${ }^{1} \mathrm{H}$ NMR spectrum of styrene measured in THF- $\mathrm{D}_{8}$. The expected chemical shift positions for the possible styrene isomers exhibiting hyperpolarized hydrogens is indicated by markings (cis $=*$, trans $=*$, geminal $=*)$.<smiles>[PH3+]C=Cc1ccccc1</smiles>

cis<smiles>[1H]C=Cc1ccccc1</smiles>

trans<smiles>[PH3+]C=Cc1ccccc1</smiles>

geminal

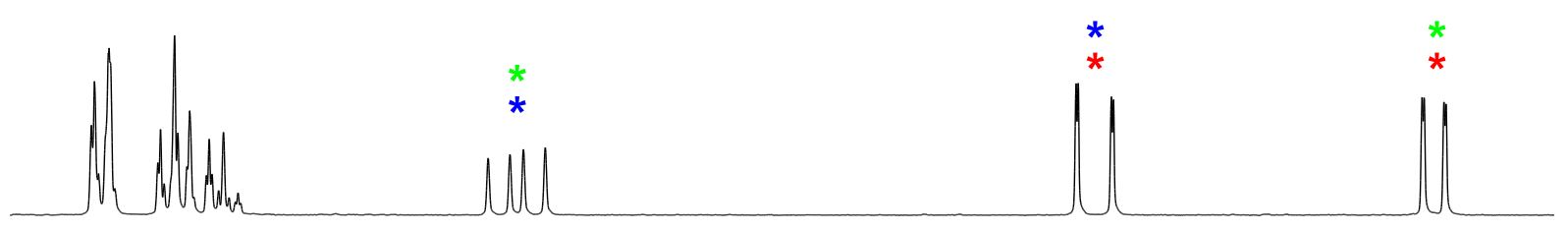

$\begin{array}{llllllllllllllllllllllllllll}7.5 & 7.4 & 7.3 & 7.2 & 7.1 & 7.0 & 6.9 & 6.8 & 6.7 & 6.6 & 6.5 & 6.4 & 6.3 & 6.2 & 6.1 & 6.0 & 5.9 & 5.8 & 5.7 & 5.6 & 5.5 & 5.4 & 5.3 & 5.2 & 5.1 & 5.0\end{array}$ $\delta / \mathrm{ppm}$

Scheme 5.5: ${ }^{1} \mathrm{H}$ NMR spectrum $(400 \mathrm{MHz})$ of styrene measured in THF-D $\mathrm{D}_{8}$. The expected signal positions enhanced by a hyperpolarization in the cis- $(*)$, trans $(*)$ and geminal- $(*)$ hydrogenated styrene isomer are marked.

\section{Experimental Setup}

In order to deterimine the rate of conversion of the used parahydrogen back to orthohydrogen in solution, a blank degassed NMR sample of THF-D 8 was loaded with two atmospheres of freshly synthesized para- $\mathrm{H}_{2}$ gas $\left(\right.$ para $-\mathrm{H}_{2} \geq 92 \%$ at $\left.36 \mathrm{~K}\right)$. The sample tube was shaken, transferred into the NMR spectrometer and monitored by ${ }^{1} \mathrm{H}$ NMR over a 18 hour time frame. Figure D.1 illustrates the progression of the ortho-dihydrogen signal intensity at $4.53 \mathrm{ppm}$ over the course of time.

As a benchmark for total ortho- $\mathrm{H}_{2}$ gas saturation of the solvent another degassed sample of THF-D 8 loaded with two atmospheres of dihydrogen (under standard conditions i.e. $o-\mathrm{H}_{2}$ ) was prepared and a ${ }^{1} \mathrm{H}$ NMR spectrum recorded. In order to determine the conversion percentage, the absolute integral for the ortho-dihydrogen singlet resonance was measured and all integrals set into ratio to the benchmark integral (100\%). The integral slowly increases over time according to the expected slow conversion of para- $\mathrm{H}_{2}$ back to ortho- $\mathrm{H}_{2}$. Furthermore, the plot shows that the starting amount of $o-\mathrm{H}_{2}$ in the sample corresponds to around $7 \%$. This finding well confirms the estimated amount of freshly produced 


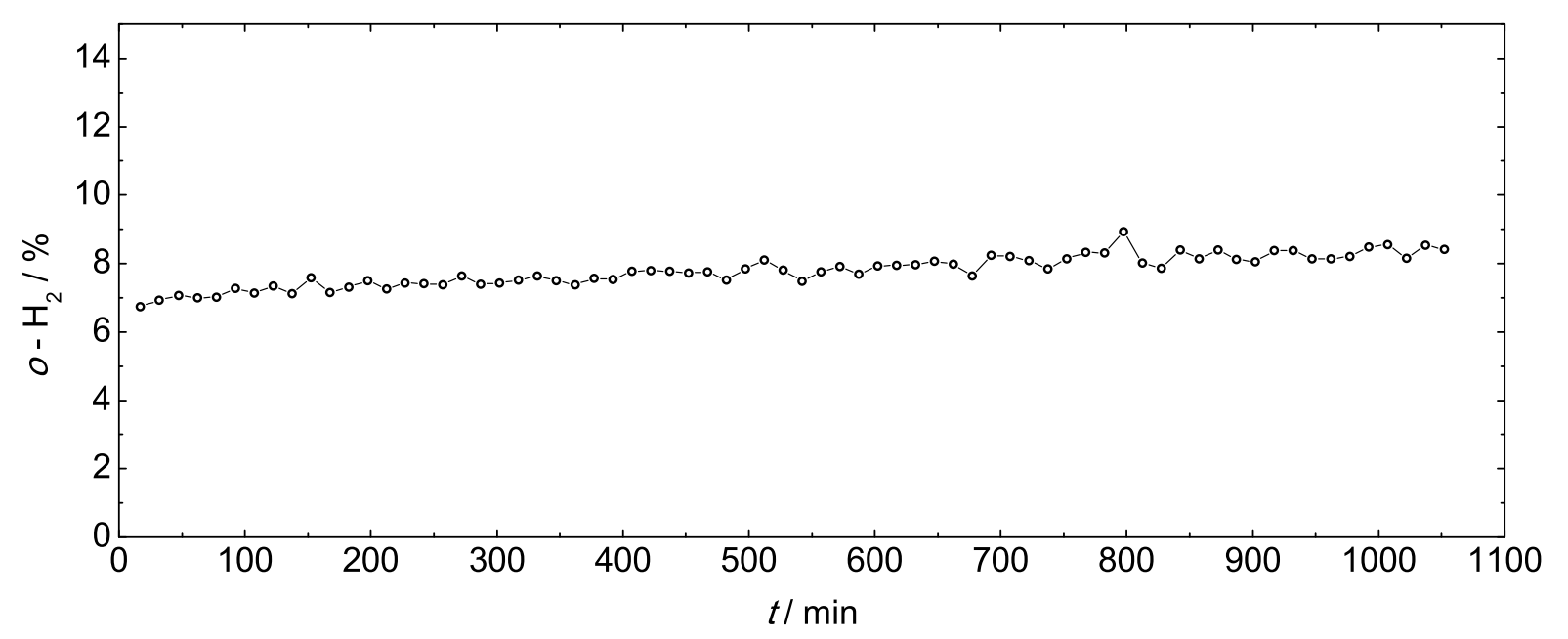

Figure 5.20.: Increase of ortho- $\mathrm{H}_{2}$ in a sample of THF-D $\mathrm{D}_{8}$ over a $18 \mathrm{~h}$ time frame. Data was aquired by ${ }^{1} \mathrm{H}$ NMR monitoring. The depicted ratio was determined by measurement of absolute integrals of the dihydrogen signal at $4.53 \mathrm{ppm}$ adjusted to the absolute integral of the benchmark (determined by a seperate experiment with a THF- $\mathrm{D}_{8}$ solvent sample loaded with 2 atmospheres of dihydrogen gas, set to $100 \%)$.

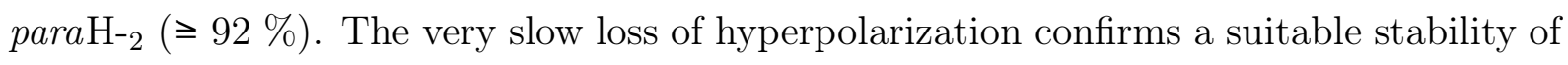
para- $\mathrm{H}_{2}$ in $\mathrm{THF}-\mathrm{D}_{8}$, thus allowing for further experiments.

A Wilmad overpressure NMR tube containing phenylacetylene and $\mathbf{K}\left[\mathbf{L}^{\text {bisnac }} \mathbf{N i}_{\mathbf{2}}\left(\mu-\eta^{\mathbf{1}}: \eta^{\mathbf{1}} \mathbf{-}\right.\right.$ CHCPh)] (10\%) in THF- $\mathrm{D}_{8}$ was degassed by applying vacuum while cooling the solution in an acetone / dry ice bath. Subsequent treatment with para-hydrogen (around $92 \%$ para-hydrogen, 6 atm.) followed by vigorous shaking of the tube initiated the hydrogenation reaction. The sample was rapidly transferred into the spectrometer and single fid's were recorded immediately. Although the reaction was initiated outside the spectrometer and thus at low field environment (ALTADENA), the reaction to styrene progressed at a relatively low rate. Consequently, the hydrogenation process still went on after transferring the sample into the spectrometer, allowing the experiment to take place at upfield (PASADENA) conditions. In order to obtain a maximum signal amplitude, data aquisition was performed by applying a flip angle of $45^{\circ}$. 


\section{Results and Discussion}

As indicated in Scheme 5.6, a hydrogenation of phenylacetylene catalyzed by $\mathbf{K}\left[\mathbf{L}^{\text {bisnac }} \mathbf{N i}_{\mathbf{2}}\left(\mu-\eta^{1}: \eta^{\mathbf{1}}-\mathbf{C H C P h}\right)\right]$ is expected to yield cis hydrogenated styrene if the reaction occurs stereoselectively as concluded from deuterium labeling experiments. Thus, hydrogenation with para- $\mathrm{H}_{2}$ is expected to yield a product with $\mathrm{H}^{\mathrm{a}}$ and $\mathrm{H}^{\mathrm{b}}$ being aligned

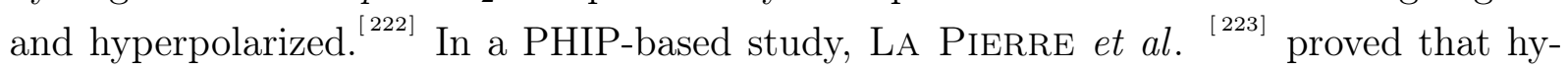
drogenation of internal alkynes involved participation of one molecule of para- $\mathrm{H}_{2}$ as was seen by the polarization transfer to the product alkene. Thus, a hydrogen splitting into two distinct molecules, as in hydrogenation by frustrated Lewis pairs, could be excluded.

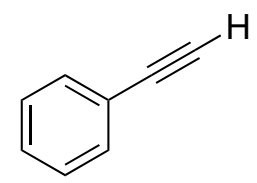

phenylacetylene

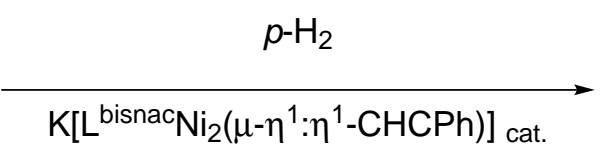

$\mathrm{K}\left[\mathrm{L}^{\text {bisnac }} \mathrm{Ni}_{2}\left(\mu-\eta^{1}: \eta^{1}-\mathrm{CHCPh}\right)\right]_{\text {cat. }}$<smiles>[PH3+]C=Cc1ccccc1</smiles>

styrene

Scheme 5.6: The hydrogenation of phenylacetylene to styrene. Numbering of respective styrene hydrogen atoms $\left(\mathrm{H}_{\mathrm{a}}, \mathrm{H}_{\mathrm{b}}\right.$ and $\left.\mathrm{H}_{\mathrm{c}}\right)$ is indicated.

In the spectra recorded under PASADENA conditions (s. Figure 5.21), two antiphase doublet signals were determined at $6.70 \mathrm{ppm}$ and $5.17 \mathrm{ppm}$ after 30 seconds. These are associated with the hydrogen atoms $\mathrm{H}^{\mathrm{a}}$ and $\mathrm{H}^{\mathrm{b}}$ of the formed styrene molecule. Signal intesities were amplified with hydrogenation progressing, as was observed in further recorded spectra.

This experimental result demonstrated that a complete transfer of a $\mathrm{H}_{2}$ molecule to the alkyne was involved, which led to a polarization transfer to the product. Furthermore, the outcome was in agreement with the theory as mentioned above and showed that mainly a stereoselective addition of the para- $\mathrm{H}_{2}$ to the triple bond of phenylacetylene in cis fashion occured. In the case of a non-stereoselective hydrogenation process, a respective hyperpolarized signal of similar intensity for $\mathrm{H}^{\mathrm{c}}$ would be expected at $5.73 \mathrm{ppm}$. For instance, this phenomenon was reported for hydrogenations using Noyori's asymmetric hydrogenation

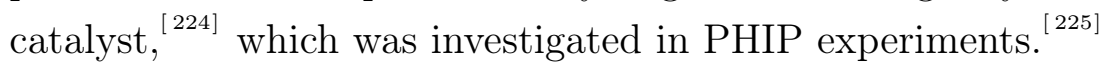

Moreover, the obtained spectra yielded clear evidence that in a selective hydrogenation phenylacetylene is exclusively converted to styrene. A considered hydrogenation of styrene is expected to give rise to hyperpolarization in the product molecule, which is seen due to a significant enhancement of associated signals. 


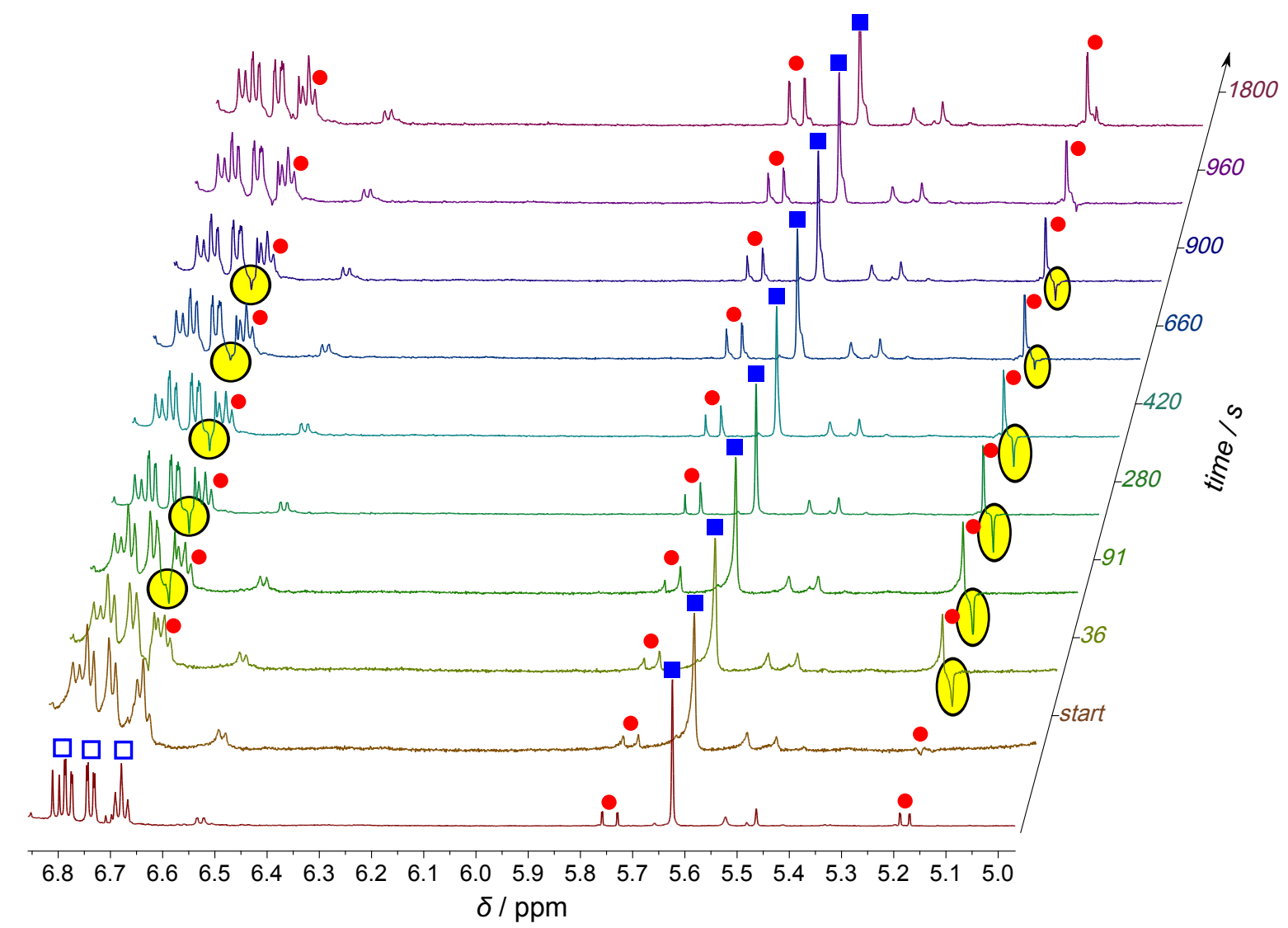

Figure 5.21.: Selected regions of ${ }^{1} \mathrm{H}$ NMR spectra $(600 \mathrm{MHz})$ of a sample containing phenylacetylene, $\mathrm{K}\left[\mathrm{L}^{\text {bisnac }} \mathrm{Ni}_{2}\left(\mu-\eta^{1}: \eta^{1}-\mathrm{CHCPh}\right)\right](0.1 \mathrm{eq})$ and $6 \mathrm{~atm}$. of para- $\mathrm{H}_{2}$ recorded over a 30 minute timescale. The spectra were recorded with an applied flip angle of $45^{\circ}$. The bottom spectrum represents conditions without dihydrogen atmosphere. Due to the relatively high amount of phenylacetylene in the sample ( $>10$ fold in comparison to the catalyst), impurities like traces of styrene, which were contained in the used substrate are detectable in the spectrum of unreacted starting materials. The typical PASADENA spectral pattern exhibiting antiphase doublet signals for the hyperpolarized hydrogen atoms $\mathrm{H}^{\mathrm{a}}$ and $\mathrm{H}^{\mathrm{b}}$ was observed after 30 seconds (yellow circles). Characteristic resonances of styrene $\bullet$ and the pyrazolate backbone proton resonance $\boldsymbol{\square}$ of $\mathrm{K}\left[\mathrm{L}^{\text {bisnac }} \mathrm{Ni}_{2}\left(\mu-\eta^{1}: \eta^{1}-\mathrm{CHCPh}\right)\right] \mathrm{Q}$ are indicated.

Almost 20 years ago, the mechanism for the catalytic homogeneous hydrogenation of phenylacetylene to styrene was studied in detail by HARTHUN et al. ${ }^{[207]}$ In this work, $\mathrm{Rh}^{\mathrm{I}}$ and $\mathrm{Pd}^{0}$ catalysts were used in a hydrogenation process involving para- $\mathrm{H}_{2}$. Similar to the results obtained in this work, a PHIP was observed in the hydrogenation product styrene. The phenomenon was associated with mechanistic reasons, that is the continuance of the monohydrogenated substrate in the coordination sphere of the catalyst, probably caused by coordination of the phenyl group of the substrate. Since in $\mathbf{K}\left[\mathbf{L}^{\text {bisnac }} \mathbf{N i}_{\mathbf{2}}(\mu-\right.$ $\left.\left.\eta^{1}: \eta^{1}-\mathbf{C H C P h}\right)\right]$ the substrate is already coordinated to both metal centers in a bridging fashion, a similar mechanistic reason can lead to pairwise transfer of the $\mathrm{H}_{2}$ molecule, consequently leading to the observed PHIP.

Another feature in the work of HARTHUN was, that besides the cis hydrogenation also a geminal hydrogenation of the substrate took place, which caused an associated hyperpo- 
larization in the obtained proton NMR spectra. Hydrogen exchange mechanisms occuring at the same time as the cis hydrogenation were quoted as a main reason for the geminally hydrogenated product formation. Although the corresponding resonance of the hydrogen $\left(\mathrm{H}^{\mathrm{c}}\right)$ related to the geminal hydrogenation product was quite small in comparison to the cis hydrogenated styrene, the PHIP was still well observed in the spectra. In particular, HARTHUN could prove a simultaneous generation of geminally hydrogenated phenylacetylene via deuterium labeling experiments. ${ }^{[207.208]}$

In this work, however, no evidence was found that can be associated with a geminal hydrogenation process. As seen in Figure 5.21, the resonance at $5.73 \mathrm{ppm}$ remained non-hyperpolarized during the whole screening experiment. This is consistent with the results obtained from deuterium labeling experiments, which were discussed in the previous sections. In particular, during a labeling experiment with deuterium, the geminally deuterated product would not result in a signal at $6.68 \mathrm{ppm}$. In fact, this signal was observed in the measured ${ }^{2} \mathrm{H}$ NMR spectrum. Furthermore, the unpolarized hydrogen resonating at $5.73 \mathrm{ppm}$ allows for the exclusion of a trans hydrogenation mechanism. This observation is also in accordance with the deuterium labeling experiments, in which the formation of the trans hydrogenated product was excluded due to a mismatch in the found integral ratio of signals.

Hyperpolarization of the aligned hydrogen atoms in formed styrene did not linger for long, which is reflected by a decrease of intensity of the antiphase signal. PHIP is usually short-lived resulting in a decay during a 10 minute time scale. The strongest factor contributing to this decay is represented by dipolar relaxation, which in turn is correlated to the $\mathrm{T} 1$ time constant of the transferred para- $\mathrm{H}_{2}$ molecule. ${ }^{[216]}$ The fact that PHIP is still observed in the spectra even after around 16 minutes shows that the hydrogenation process still went on.

Eventually, at around 30 minutes, the resonances of hyperpolarized compound matched the resonances of styrene with $\mathrm{H}^{\mathrm{a}}$ and $\mathrm{H}^{\mathrm{b}}$ being in the decayed non-thermal state again. This results in a superimposed doublet signal whose right peak is barely visible due to the average of positive and negative phase originating from the newly induced PHIP.

The comparison of relative signal intensities of styrene formed with $p a r a-\mathrm{H}_{2}$ and styrene, generated with ortho- $\mathrm{H}_{2}$, respectively, is depicted in Figure 5.22. While for styrene in non-thermal state an average of both $\mathrm{H}^{\mathrm{a}}$ - and $\mathrm{H}^{\mathrm{b}}$-resonances was taken to obtain respective data points, for the styrene in hyperpolarized state the normalized intensities of the associated proton resonances were calculated and plotted separately. 


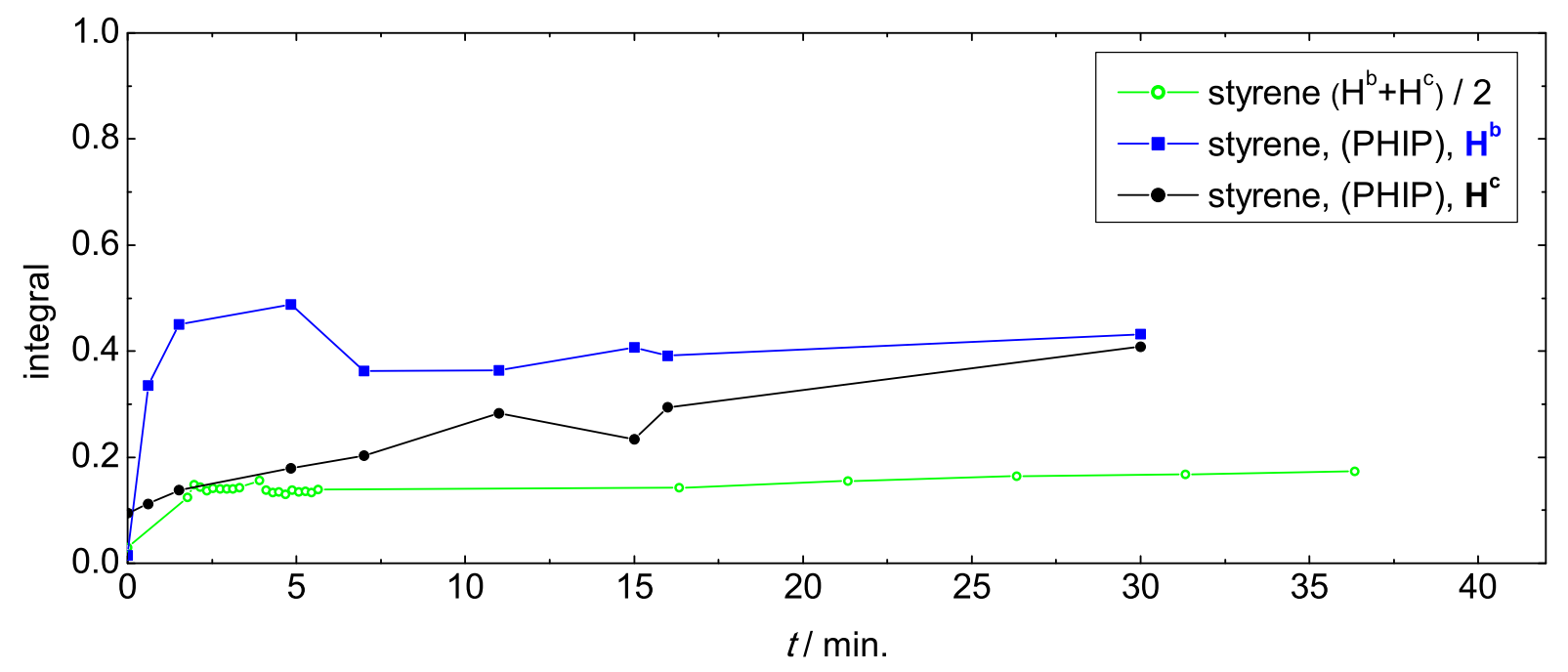

Figure 5.22.: Comparison of relative integrals $\mathrm{s}^{\ddagger}$ of the proton resonances $\mathrm{H}^{\mathrm{b}}$ and $\mathrm{H}^{\mathrm{c}}$ in hyperpolarized styrene with an average integral ratio of respective styrene resonances obtained in an experiment with $\mathrm{o}-\mathrm{H}_{2}$.

To determine the intensity enhancement factor caused by PHIP, the spectrum that shows maximum hyperpolarization for the resonance at $5.17 \mathrm{ppm}$ was compared to a spectrum of a sample which was treated with normal state $\mathrm{H}_{2}$ recorded after the same time $(90 \mathrm{~s})$. The overlay of both spectra in the specific region is shown in Figure 5.23. By means of normalized integration of associated signals an enhancement factor of three was calculated. The small enhancement factor is attributable to the fast decay of hyperpolarization in the styrene molecule under the given conditions.

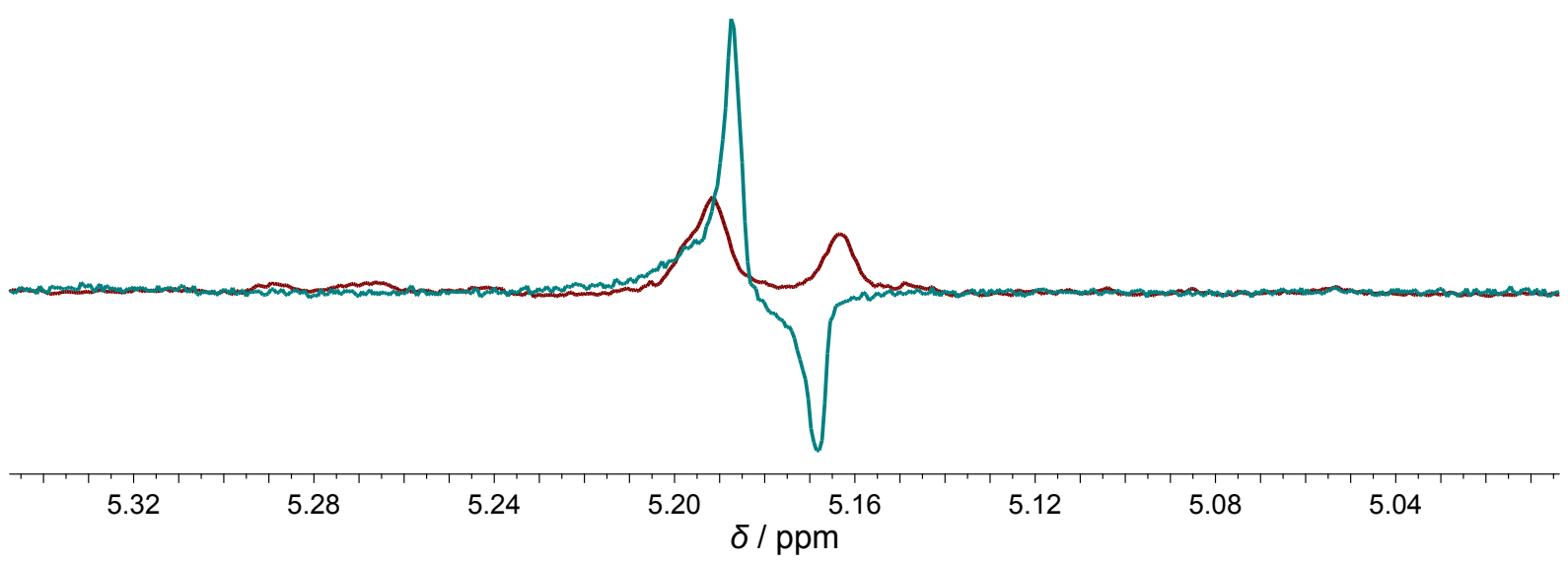

Figure 5.23.: Overlay of the hyperpolarized signal (-) with a reference signal (-). Both spectra were recorded $90 \mathrm{~s}$ after the reaction initiation collecting one scan.

\footnotetext{
The integrals of the characteristic resonances for the styrene protons in cis- and trans-position have been normalized to the sum of integrals associated with the pyrazolate backbone hydrogen atoms of all occuring complex species. This sum of integrals equals the integral of the singlet resonance correlated to the backbone proton resonance of the starting material before initiation of the reaction.
} 


\section{Conclusion}

Conduction of experiments with parahydrogen exploiting PHIP and ${ }^{1} \mathrm{H}$ NMR provided valuable information regarding the hydrogenation process of phenylacetylene. The assumption that the semihydrogenation process of phenylacetylene takes place exclusively with no signs of complete hydrogenation to ethylbenzene, could be confirmed. Furthermore, it could be proven that in the initial phase of semihydrogenation a cis hydrogenation takes place with no indications of trans- or geminal- hydrogenation. Therefore, the herein obtained results are consistent with the interpretation of the isotopic labeling experiments.

\subsubsection{Mechanistic Considerations}

The results obtained from isotopic labeling experiments allowed for a mechanistic proposal of the formation of $\mathbf{K}\left[\mathbf{L}^{\text {bisnac }} \mathbf{N i}_{2}\left(\mu-\eta^{1}: \eta^{1}-\mathbf{C H C P h}\right)\right]$ (Scheme 5.7).

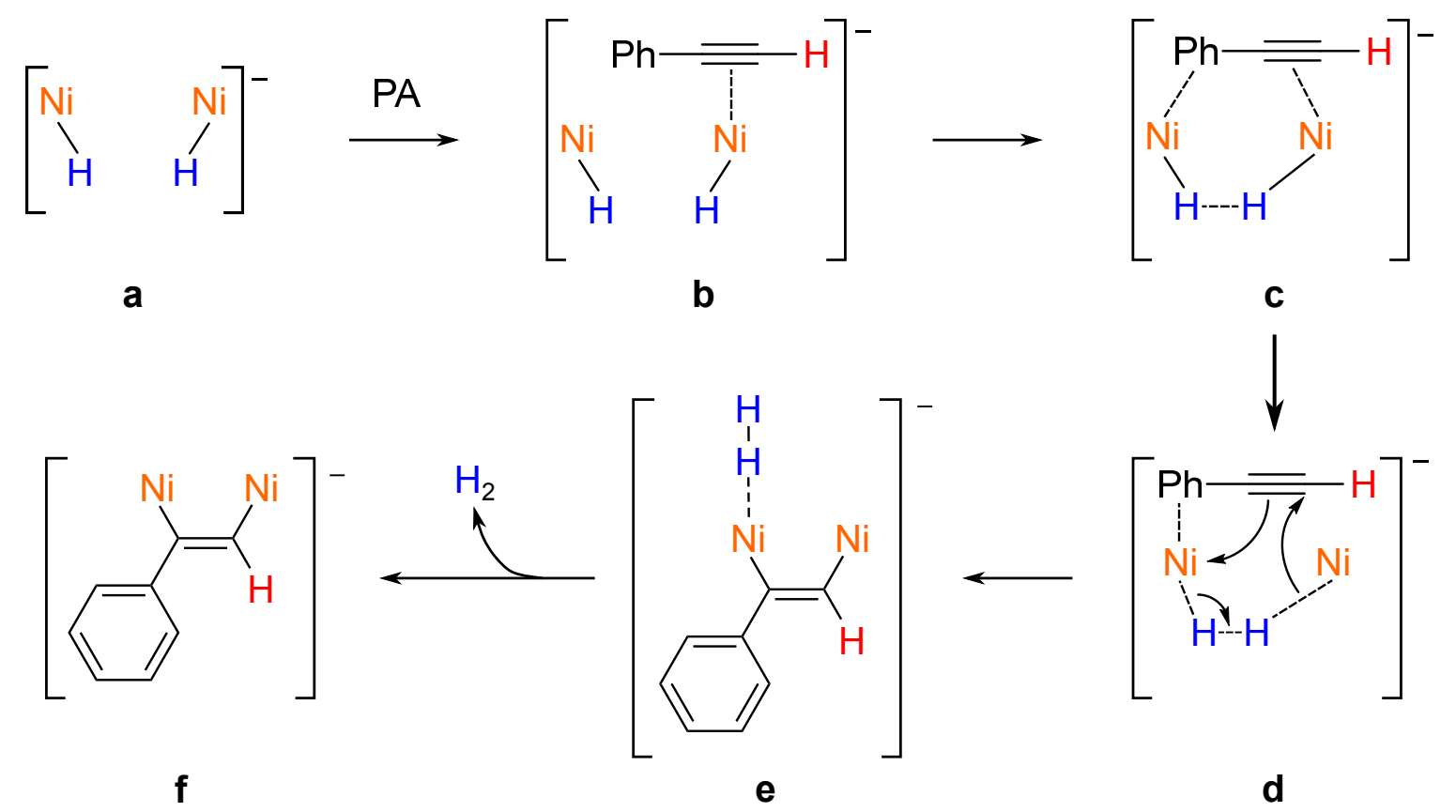

Scheme 5.7: Mechanistic hypothesis for the formation of $\mathrm{K}\left[\mathrm{L}^{\text {bisnac }} \mathrm{Ni}_{2}\left(\mu-\eta^{1}: \eta^{1}-\mathrm{CHCPh}\right)\right]$. For more clarity the ligand skeleton is omitted and participating hydrogen atoms are marked by different colors. In the first step phenylacetylene (PA) coordinates to $K\left[L^{\text {bisnac }} \mathbf{N i}_{2}(\mathbf{H})_{2}\right]$ (a) to form species (b). The coordinated substrate further moves into the cavity, while the distance between both hydrogen atoms $(H)$ decreases (c). This leads to a 6-center-6-electron transition state (d), in which the hydrogen atoms are substituted by $P A$, forming $\mathrm{H}_{2}(e) . \mathrm{H}_{2}$ is released and $\mathbf{K}\left[\mathrm{L}^{\text {bisnac }} \mathrm{Ni}_{2}\left(\mu-\eta^{1}: \eta^{1}-\mathrm{CHCPh}\right)\right]$ formed (f).

In the first step phenylacetylene coordinates to one of the $\mathrm{Ni}$ ions of $\left.\mathbf{K}\left[\mathbf{L}^{\mathbf{b i s n a c}} \mathbf{N i}_{\mathbf{2}} \mathbf{(} \mathbf{H}\right)_{\mathbf{2}}\right]$ (a) via the $\pi$-system of either the triple bond or the aryl-group forming (b). The substrate further moves into the cavity, while the distance between the hydrogen atoms $(\mathrm{H})$ decreases (c). This leads to a 6-center-6-electron transition state (d), in which the hydrogen atoms are substituted by PA, forming $\mathrm{H}_{2}$ (e). Release of $\mathrm{H}_{2}$ from the complex 
eventually leads to $\mathbf{K}\left[\mathbf{L}^{\text {bisnac }} \mathbf{N i}_{\mathbf{2}}\left(\mu-\eta^{\mathbf{1}}: \eta^{\mathbf{1}} \mathbf{- C H C P h}\right)\right]$ (f). The hydrogen atom (H) belonging to the phenylacetylene is retained in $\mathbf{K}\left[\mathbf{L}^{\text {bisnac }} \mathbf{N i}_{\mathbf{2}}\left(\mu-\eta^{\mathbf{1}}: \eta^{\mathbf{1}}-\mathbf{C H C P h}\right)\right]$. This was proven by reaction of $\mathbf{K}\left[\mathbf{L}^{\text {bisnac }} \mathbf{N i}_{2}(\mathbf{D})_{2}\right]$ with two equivalents of PA. ${ }^{1} \mathrm{H}$ NMR showed the resonance of the vinyl-hydrogen in a normalized integral of one. The same experiment monitored by ${ }^{2} \mathrm{H}$ NMR further showed the release of $\mathrm{D}_{2}$, proving the pairwise substitution of the hydrogen atoms of $\mathbf{K}\left[\mathbf{L}^{\text {bisnac }} \mathbf{N i}_{\mathbf{2}}(\mathbf{H})_{\mathbf{2}}\right]$. The proposed mechanism is consistent with the hydrogen exchange observed in $\mathbf{K}\left[\mathbf{L}^{\text {bisnac }} \mathbf{N i}_{\mathbf{2}}(\mathbf{H})_{\mathbf{2}}\right]$ with $\mathrm{D}_{2}$ and vice versa (44).

\subsubsection{Summary}

Based on the ${ }^{1} \mathrm{H}$ and ${ }^{2} \mathrm{H}$ NMR results obtained from experiments performed with $\mathbf{K}\left[\mathbf{L}^{\text {bisnac }} \mathbf{N i}_{\mathbf{2}}(\mathbf{H})_{2}\right]$ and $\mathbf{K}\left[\mathbf{L}^{\text {bisnac }} \mathbf{N i}_{\mathbf{2}}(\mathbf{D})_{\mathbf{2}}\right]$, which were reacted with an excess of phenylacetylene, a proposal for the pathway of reaction was established. In the initial step, formation of the phenylvinylcomplex $\mathbf{K}\left[\mathbf{L}^{\text {bisnac }} \mathbf{N i}_{2}\left(\mu-\eta^{1}: \eta^{1}-\mathbf{C H C P h}\right)\right]$ takes place, which is shown by ${ }^{1} \mathrm{H}$ NMR experiments. This reaction is fast (several seconds) and accompanied by the pairwise release of the $\mathrm{Ni}-\mathrm{H}$ hydrogen atoms in the form of $\mathrm{H}_{2}$ or $\mathrm{D}_{2}$ from the dihydrido complex or its deuterium labeled analogue, respectively. While the generation of dihydrogen could not explicitly be proven by NMR, the formation of dideuterium, which was obtained after the analogous reaction with $\mathbf{K}\left[\mathbf{L}^{\text {bisnac }} \mathbf{N i}_{\mathbf{2}}(\mathbf{D})_{\mathbf{2}}\right]$ was demonstrated. The concentration of $\mathrm{D}_{2}$ in the solution relatively slowly decreased until an equilibrium of the gas amount in solution and the gas phase was reached (after ca. 30 minutes). During this time, no further reaction was observed, due to the fact that the formation of new deuterium based species was not detected in the ${ }^{2} \mathrm{H}$ NMR experiment for up to 18 hours.

In a reaction of the hydrido or deuterido complex with two equivalents of PA after the release of dihydrogen or dideuterium, respectively, the second equivalent of substrate is hydrogenated or deutero-hydrogenated. It could be shown by ${ }^{1} \mathrm{H}-$ and ${ }^{2} \mathrm{H}-\mathrm{NMR}$ that the hydrogenation selectively yields styrene. The semihydrogenation of PA was further confirmed by studies including the use of parahydrogen.

The hydrogenation most likely occured with support of the incipiently formed phenylvinyl complex in a subsequent slower reaction. Since no intermediate complex species was observed during this reaction process, the necessary interaction of the involved compounds comprising the substrates phenylacetylene and $\mathrm{H}_{2}$ and $\mathbf{K}\left[\mathbf{L}^{\text {bisnac }} \mathbf{N i}_{\mathbf{2}}\left(\mu-\eta^{\mathbf{1}}: \eta^{\mathbf{1}}-\mathbf{C H C P h}\right)\right]$ is considered as the rate determining step. Therefore, after the coordination of the substrate to the active site of the complex the following hydrogenation and subsequent release of styrene is expected to be fast.

The formation of styrene was the result of a cis hydrogenation with no further indications of trans- or geminal hyrogenations, which was confirmed by deuterium labeling and parahydrogen induced polarization experiments. However, an H/D exchange appeared to 
take place after formation of the semihydrogenation product, yielding the fully deuterated vinyl moiety. The whole process of hydrogenation with $\mathrm{D}_{2}$ was slow, since the full consumption of substrate covered a period of over three weeks. This indicates a kinetic isotope effect. Furthermore, during this time not only the deuterium hydrogenated species were generated but also a significant amount of other unidentified aromatic species as a result of side reactions.

Based on obtained results a hypothetical reaction pathway for the formation of $\mathbf{K}\left[\mathbf{L}^{\text {bisnac }} \mathbf{N i}_{2}\left(\mu-\eta^{1}: \eta^{1}-\mathbf{C H C P h}\right)\right]$ was established. It is proposed to be similar to the DFT supported mechanistic pathway, established for the H-D-exchange of $\mathbf{K}\left[\mathbf{L}^{\text {bisnac }} \mathrm{Ni}_{2}(\mathbf{H})_{2}\right]$ with $\mathrm{D}_{2}$ and includes a substitution of both metal-bound hydrogen atoms leading to the release of one molecule of $\mathrm{H}_{2}$. 


\subsection{Investigation of Phenylacetylene Semihydrogenation}

Because the previously conducted experiments indicated the semihydrogenation of PA to styrene to be catalyzed by $\mathbf{K}\left[\mathbf{L}^{\text {bisnac }} \mathbf{N i}_{\mathbf{2}}\left(\mu-\eta^{\mathbf{1}}: \eta^{\mathbf{1}}-\mathbf{C H C P h}\right)\right]$ or a related active species, explicit experiments under catalytic conditions were attempted. General conditions for the catalytic semihydrogenation of phenylacetylene to styrene are illustrated in Scheme 5.8
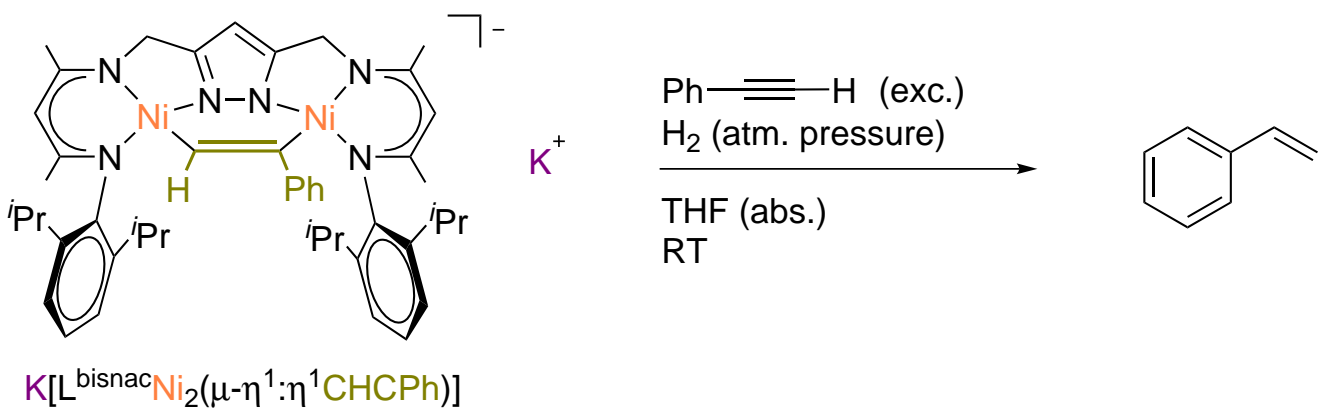

Scheme 5.8: General conditions for a catalytic semihydrogenation experiment.

Before the investigation of a potential catalytic activity of $\mathbf{K}\left[\mathbf{L}^{\text {bisnac }} \mathbf{N i}_{\mathbf{2}}\left(\mu-\eta^{\mathbf{1}}: \eta^{\mathbf{1}}-\mathbf{C H C}-\right.\right.$ $\mathbf{P h})$ ] several blind experiments were conducted in advance to exclude a catalytic reaction caused by impurities in used substrates or other factors.
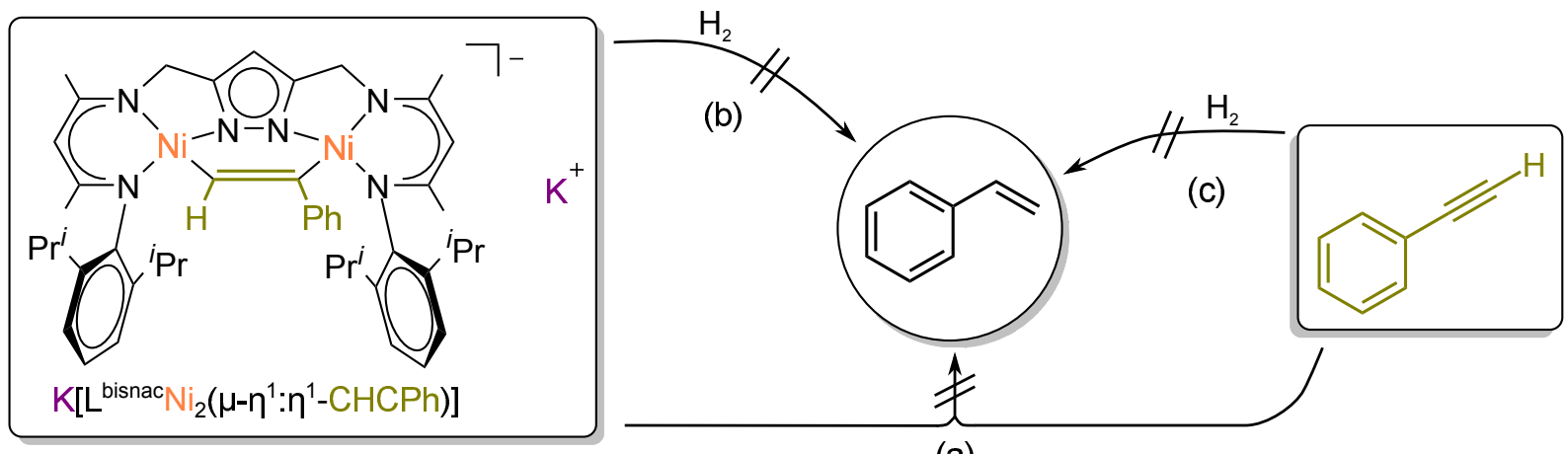

(a)

Scheme 5.9: Exclusion of catalytic activity under the abscence of a single component.

Solutions of a) $\mathbf{K}\left[\mathbf{L}^{\text {bisnac }} \mathbf{N i}_{\mathbf{2}}\left(\mu-\eta^{\mathbf{1}}: \eta^{\mathbf{1}}-\mathbf{C H C P h}\right)\right]$ and phenylacetylene (15 eq) b) $\mathbf{K}\left[\mathbf{L}^{\text {bisnac }} \mathbf{N i}_{\mathbf{2}}\left(\mu-\eta^{\mathbf{1}}: \eta^{\mathbf{1}}-\mathbf{C H C P h}\right)\right]$ and $\mathrm{H}_{2}$ (atmospheric pressure) and c) phenylacetylene and dihydrogen (atmospheric pressure) were prepared and monitored by ${ }^{1} \mathrm{H}$ NMR for several days. During this time no styrene development could be detected and no further changes occured in the measured samples (Scheme 5.9). Therefore, the catalytic semihydrogenation of phenylacetylene to styrene under these conditions was excluded. The ${ }^{1} \mathrm{H}$ NMR spectra associated with the blind experiments are described in the appendix (Figure D.7, Figure D.8 and Figure D.9. 
Since any catalytic reactivity of the separated starting materials could be excluded, an experiment was conducted under catalytic conditions: a pump-freeze-thaw degassed sample of $\mathbf{K}\left[\mathbf{L}^{\text {bisnac }} \mathbf{N i}_{\mathbf{2}}\left(\mu-\eta^{\mathbf{1}}: \eta^{\mathbf{1}} \mathbf{- C H C P h}\right)\right]$ and an excess of phenylacetylene (15 eq) in THF-D was loaded with dried dihydrogen gas (atmospheric pressure). The reaction was then followed via ${ }^{1} \mathrm{H}$ NMR spectroscopy at room temperature, revealing the formation of styrene (Figure 5.24, •).

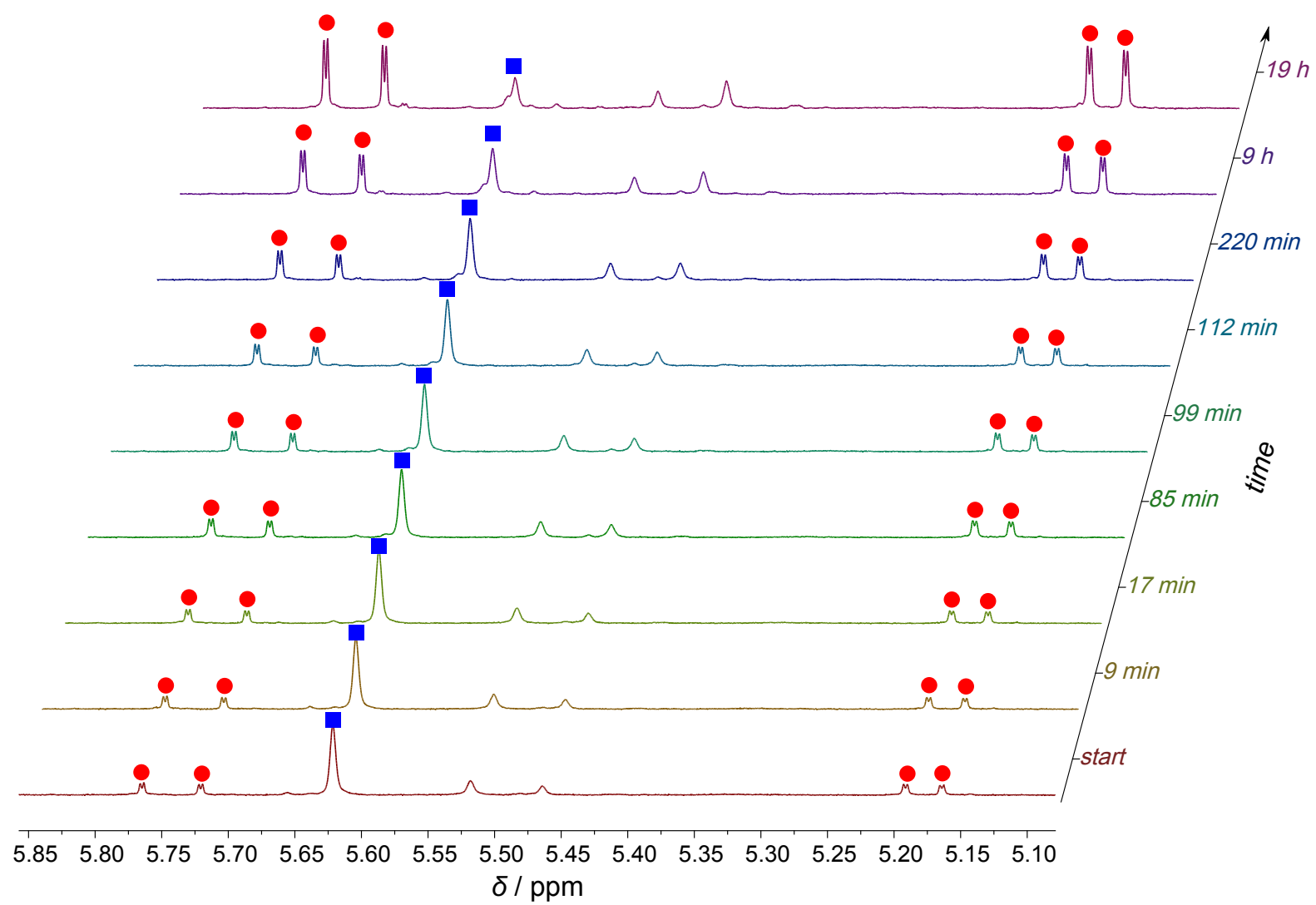

Figure 5.24.: Catalytically generated styrene $\bullet$ from phenylacetylene $(15 \mathrm{eq})$ in the presence of $\mathrm{H}_{2}$

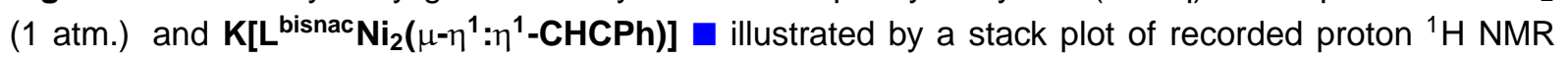
spectra $(400 \mathrm{MHz})$.

However, the generation is very slow probably due to the low concentration of dissolved dihydrogen gas in the solution. A plot illustrating the formation of styrene over the course of time is shown in Figure 5.25.

A ${ }^{1} \mathrm{H}$ NMR control measurement after 60 days showed no more phenylacetylene resonances (Figure 5.26). Furthermore, in addition to significant resonances belonging to the generated styrene, other major resonances at $2.61 \mathrm{ppm}$ and $1.20 \mathrm{ppm}$ were observed in the spectrum. As mentioned before, these quartet and triplet signals are characteristic for ethylbenzene. 


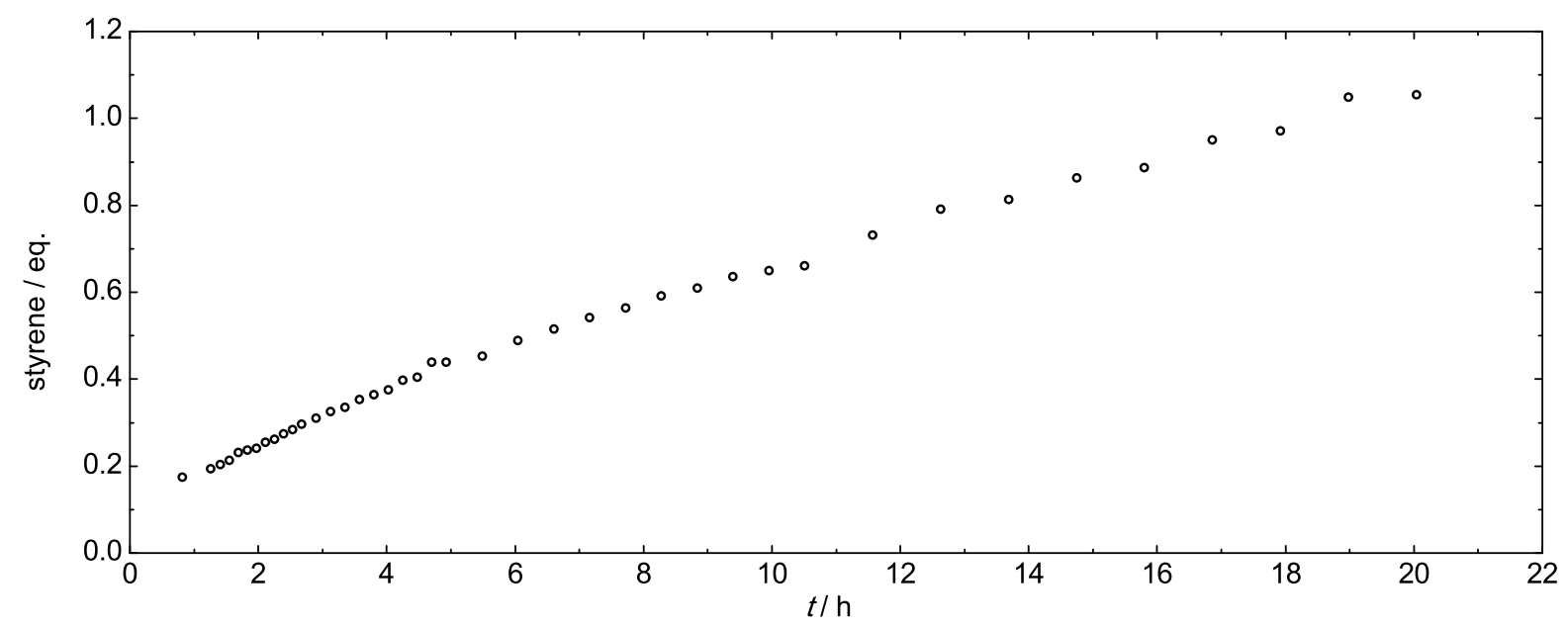

Figure 5.25.: Plot representing the styrene production (in equivalents with respect to the used complex) over time by catalytic hydrogenation of phenylacetylene with $\mathrm{K}\left[\mathrm{L}^{\text {bisnac }} \mathrm{Ni}_{2}\left(\mu-\eta^{1}: \eta^{1}-\mathrm{CHCPh}\right)\right]$.

The excess amount of the substrate phenylacetylene was fully consumed (according to an absence of corresponding signals in ${ }^{1} \mathrm{H}$ NMR). This proves the sustained activity of the catalyst after reaching an observed turnover number $(\mathrm{TON})^{\ddagger}$ of about 11 with respect to formed styrene, which is near the calculated limit of $\mathrm{TON}_{\max }=15$ under these specific conditions. $^{\S}$ Figure 5.27 illustrates a plot of the styrene production (in equivalents) as a function of time.

Considering the fact that the corresponding control spectrum, recorded after 28 days (Fig-

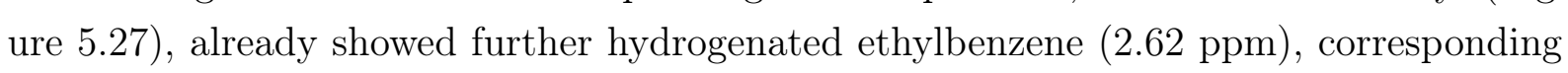
to 2.1 equivalents, the total TON is $\approx 13$. Furthermore, downfield shifted aromatic resonances $(7.85-7.61 \mathrm{ppm})$ were observed in the spectrum, which most likely correspond to benzaldehyde, a side product which may have formed by oxidation of styrene (Figure 5.28 The presence of oxygen was probably due to impurites in the used $\mathrm{H}_{2}$. However the production of this particular side product ceased after 7 days, indicating the consumption of oxygen. After 7 days the integrals of the associated resonances remain more or less stable, indicating that the oxidant was fully consumed. At this point the multipletts of this side product correspond to an amount of roughly two equivalents, consequently leading to an associated turnover number of $\mathrm{TON}_{\max }=15$.

\footnotetext{
The turnover number (TON) represents the catalytic productivity of a catalyst before its complete inactivation. For instance a TON of 1 equals the conversion of one equivalent of substance in regard to the amount of catalyst.

$\S$ The maximum possible turnover number is correlated to the total amount of substrate (PA) used in the catalysis experiment. The amount of substrate was determined by integration of its signals in $\mathrm{a}^{1} \mathrm{H}$ NMR spectrum measured before the start of the reaction i.e. the loading with $\mathrm{H}_{2}$.
} 
(a) mixture after 60 days

(b)

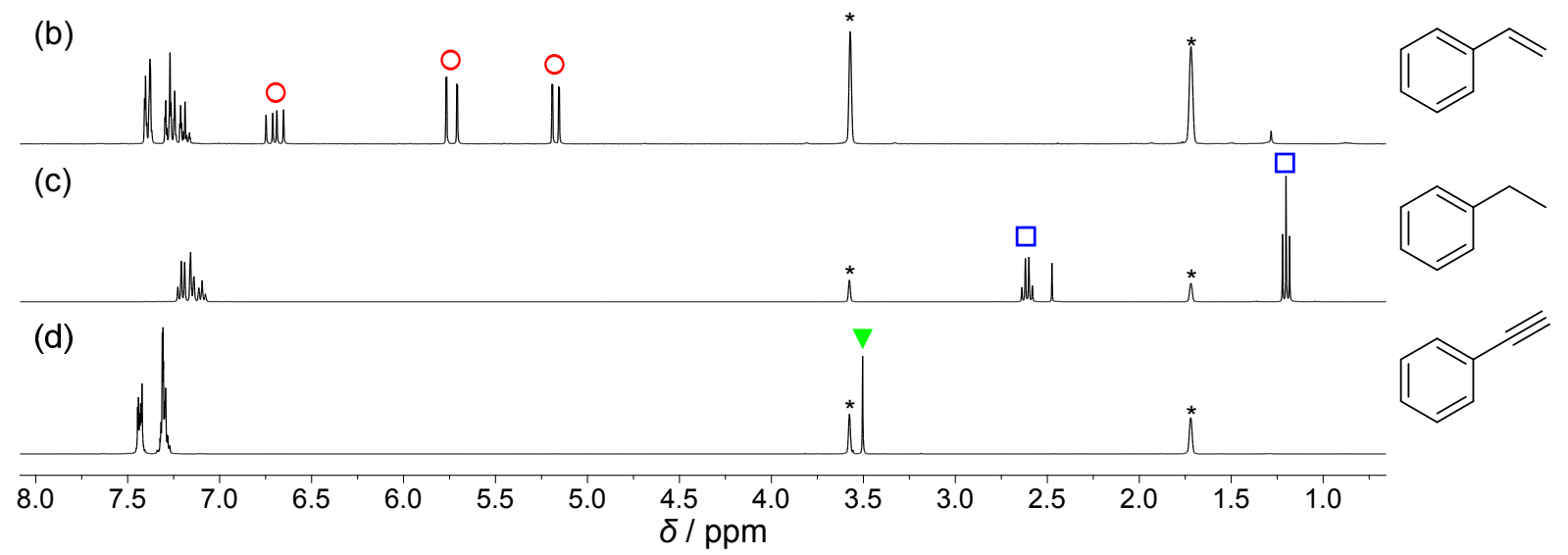

Figure 5.26.: ${ }^{1} \mathrm{H}$ NMR spectrum $(400 \mathrm{MHz})$ of a control measurement of the catalytic hydrogenation experiment after 60 days (a). Reference spectra of (b) styrene (300 MHz), (c) ethylbenzene $(400 \mathrm{MHz})$ and (d) phenylacetylene $(400 \mathrm{MHz})$ are shown. No resonances correlated to the substrate phenylacetylene $\nabla$ could be observed after this time. Furthermore, characteristic resonances of styrene 0 and ethylbenzene $\square$ are observed in (a).

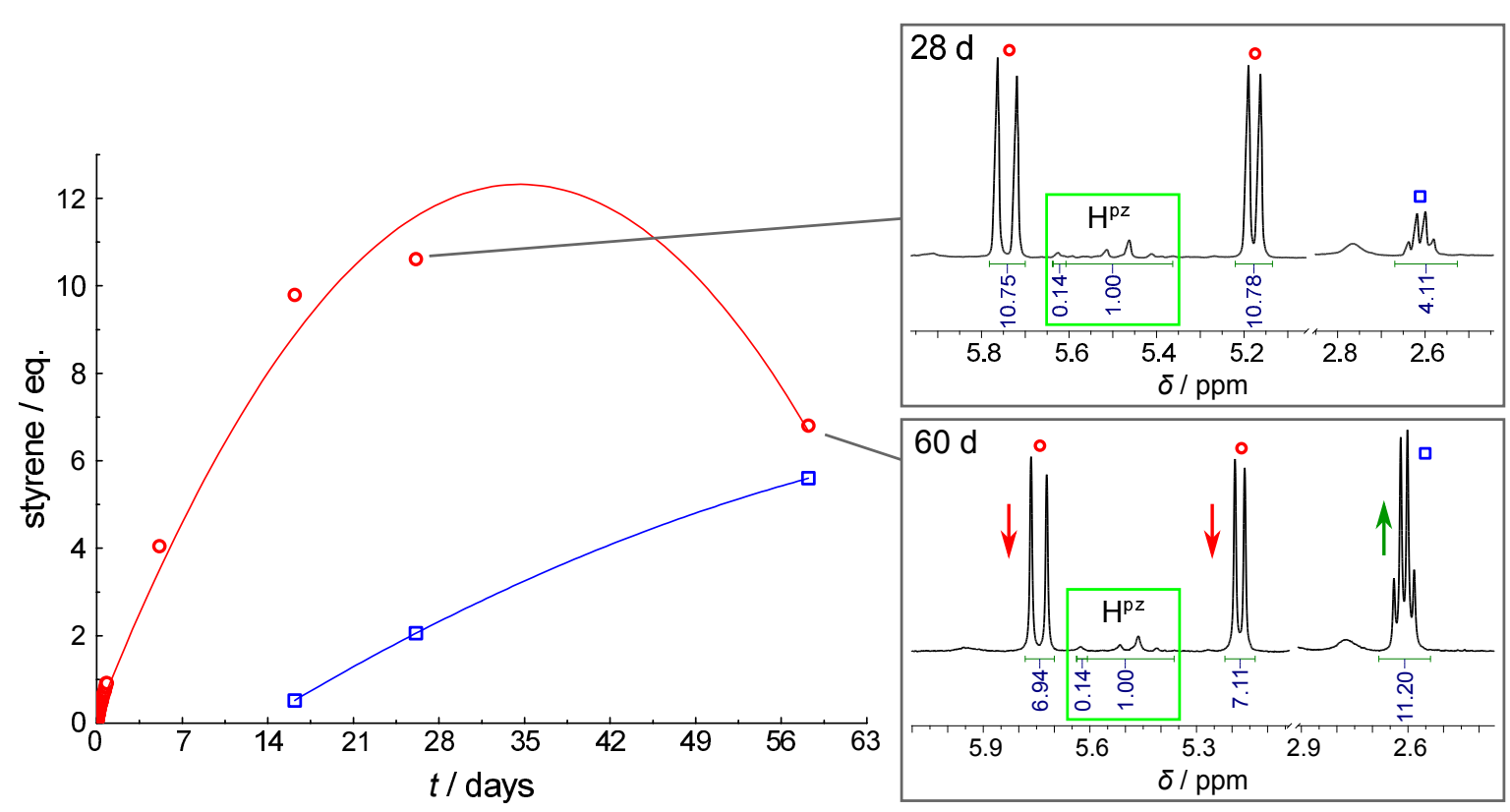

Figure 5.27.: Plot of styrene equivalents present in the sample against the time. After 28 days the amount of the fully hydrogenated substrate $(\square)$ increases while a decrease of the semihydrogenated substrate (0) could be observed in proton NMR as indicated by the depicted boxes. The merged integrals of pyrazolate backbone proton resonances were set as a benchmark $\square$. 


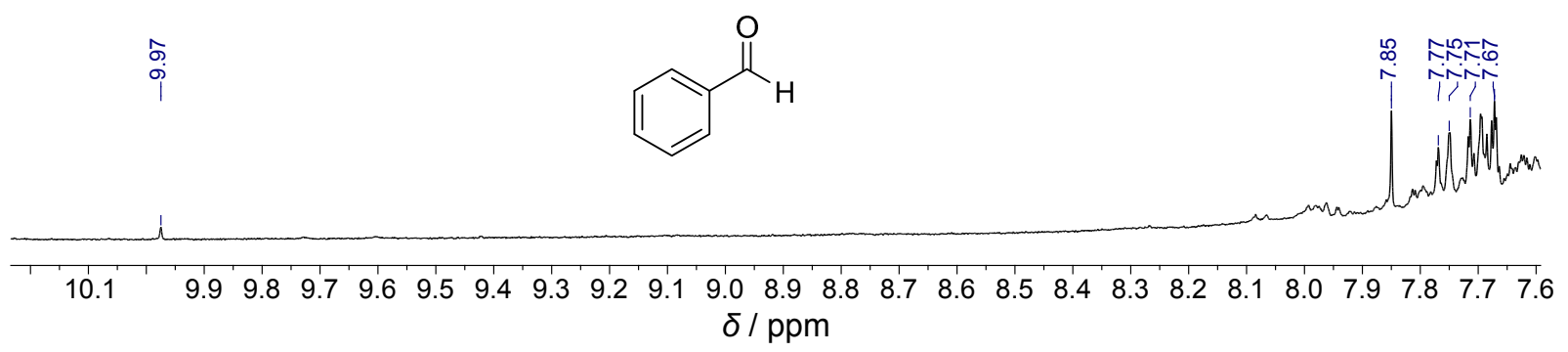

Figure 5.28.: ${ }^{1} \mathrm{H}$ NMR spectrum of the reaction mixture recorded one week after initiation of the reaction. Resonances at the lowfield of the spectrum indicate the formation of benzaldehyde.

Remarkably, even with a minimal amount of $\mathrm{H}_{2}$, as indicated by the very small signal at $4.54 \mathrm{ppm}$, the hydrogenation of styrene progresses further leading to a significant amount of the completely hydrogenated substrate. It is assumed that the phenylvinyl-bridged compound $\mathbf{K}\left[\mathbf{L}^{\text {bisnac }} \mathbf{N i}_{\mathbf{2}}\left(\mu-\eta^{\mathbf{1}}: \eta^{\mathbf{1}}-\mathbf{C H C P h}\right)\right]$ is able to selectively generate styrene from an excess amount of phenylacetylene through a semihydrogenation process in a catalytic fashion. At lower concentrations of PA formed styrene is further hydrogenated to ethylbenzene. However, after the entire consumption of PA, full hydrogenation of styrene to ethylbenzene was accelerated. This further shows, that the semihydrogenation is dominant.

\subsubsection{Mechanism of Phenylacetylene Semihydrogenation}

The establishment of a mechanistic proposal (Scheme 5.10) for the catalytic selective hydrogenation of phenylacetylene to styrene is based on the following considerations:

a) In the context of the previous chapter it could be shown by ${ }^{1} \mathrm{H}$ NMR spectroscopic studies including the use of parahydrogen that the exchange with $\mathbf{K}\left[\mathbf{L}^{\text {bisnac }} \mathbf{N i}_{\mathbf{2}}(\mathbf{H})_{\mathbf{2}}\right]$ leads to a loss of hyperpolarization (appendix D.2). Deuterium labeling experiments further proved that this exchange occured in a non-scrambling process. Thus, it was concluded that a regeneration of $\mathbf{K}\left[\mathbf{L}^{\text {bisnac }} \mathbf{N i}_{\mathbf{2}}(\mathbf{H})_{2}\right]$ from $\mathbf{K}\left[\mathbf{L}^{\text {bisnac }} \mathbf{N i}_{\mathbf{2}}\left(\mu-\eta^{1}: \eta^{1}-\mathbf{C H C P h}\right)\right]$ with parahydrogen also results in a loss of hyperpolarization. The fact that PHIP is observed in the product molecule after the catalytic reaction with parahydrogen proved the retention of hyperpolarization in the $\mathrm{H}_{2}$ molecule over the entire hydrogenation process.

Furthermore, experiments only including $\mathbf{K}\left[\mathbf{L}^{\text {bisnac }} \mathbf{N i}_{2}\left(\mu-\eta^{1}: \eta^{1}-\mathbf{C H C P h}\right)\right]$ and $\mathrm{H}_{2}$ showed that neither formation of styrene nor regeneration to $\mathbf{K}\left[\mathbf{L}^{\text {bisnac }} \mathbf{N i}_{\mathbf{2}}(\mathbf{H})_{2}\right]$ occured. These two findings strongly suggest that $\mathbf{K}\left[\mathbf{L}^{\text {bisnac }} \mathbf{N i}_{\mathbf{2}}(\mathbf{H})_{\mathbf{2}}\right]$ is not participating in the catalytic cycle.

b) Experiments with parahydrogen showed that the $\mathrm{H}_{2}$ molecule is transferred to the alkyne in a pairwise fashion (PHIP). 
c) Deuterium labeling- and parahydrogen experiments monitored by ${ }^{1} \mathrm{H}$ and ${ }^{2} \mathrm{H}$ NMR revealed that semihydrogenation yielded cis- styrene. The incipient formation of transor geminally hydrogenated isomers could be excluded.

d) The approximation of a bridging ligand retention in $\mathbf{K}\left[\mathbf{L}^{\text {bisnac }} \mathbf{N i}_{\mathbf{2}}\left(\mu-\eta^{1}: \eta^{\mathbf{1}}-\mathbf{C H C P h}\right)\right]$ during the whole catalytic cycle was made.

e) A possible role of $\mathrm{K}^{+}$in the whole process was neglected.

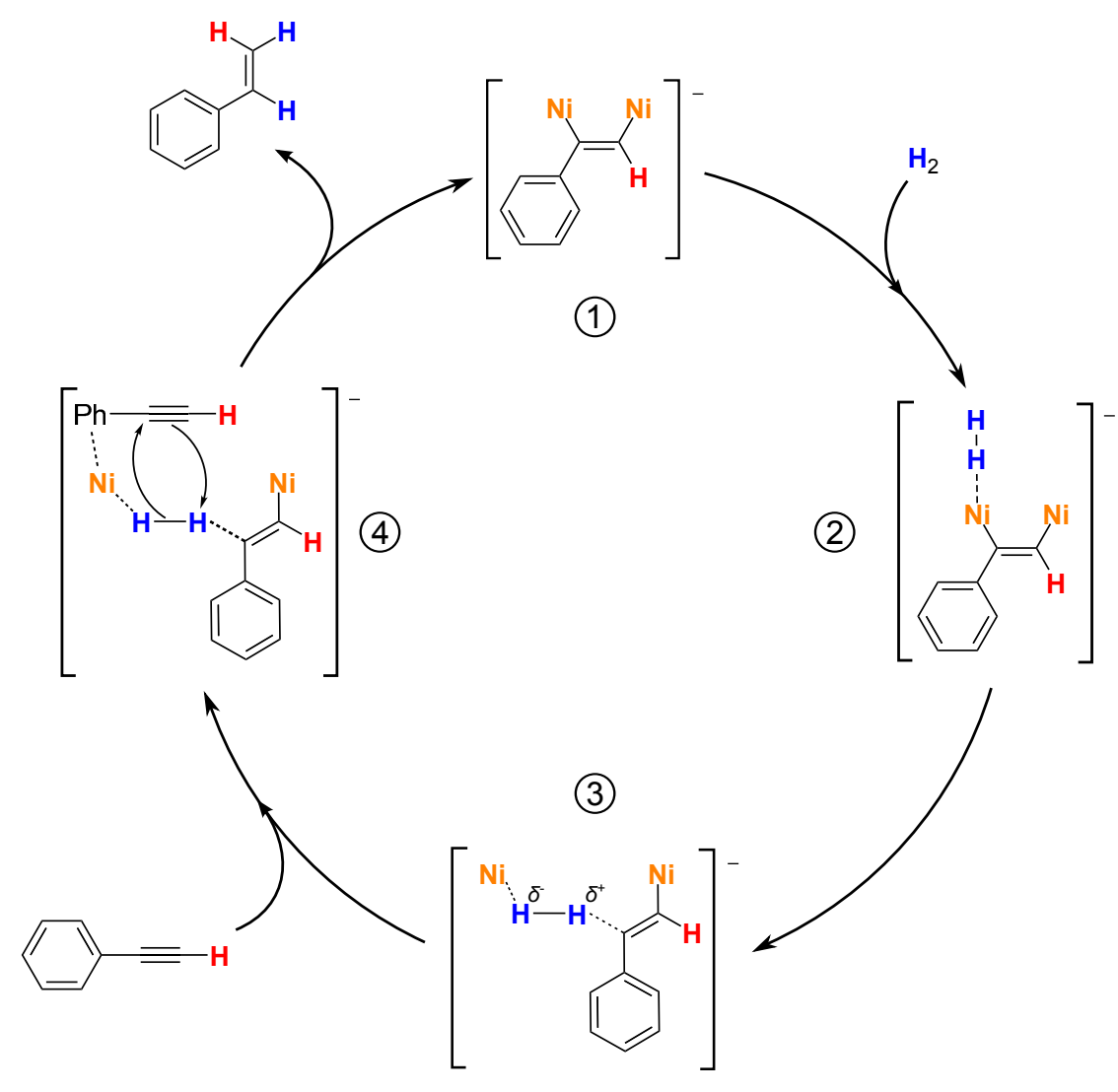

Scheme 5.10: Mechanistic proposal for the catalytic generation of styrene from phenylacetylene. $\mathrm{H}_{2}$ coordinates to $\mathrm{K}\left[\mathrm{L}^{\text {bisnac }} \mathrm{Ni}_{2}\left(\mu-\eta^{1}: \eta^{1}-\mathrm{CHCPh}\right)\right]$ (1) in an end-on fashion yielding the intermediate (2). Subsequently, the $\mathrm{H}_{2}$ molecule is inserted into the $\mathrm{Ni}-\mathrm{C}$ bond leading to the polarization of $\mathrm{H}_{2}$ (3) The coordination of the substrate phenylacetylene mediated by cation- $\pi$ interactions would enable a 4-center4-electron transition state between $\mathrm{H}_{2}$ and the substrate (4)). Elimination of formed styrene from the system and reformation of $\mathrm{K}\left[\mathrm{L}^{\text {bisnac }} \mathrm{Ni}_{2}\left(\mu-\eta^{1}: \eta^{1}-\mathrm{CHCPh}\right)\right]$ (1) closes the cycle.

The first step of the proposed catalytic cycle is described by the coordination of $\mathrm{H}_{2}$ yielding the intermediate (2). In the previous chapter DFT calculations regarding the exchange of $\mathbf{K}\left[\mathbf{L}^{\text {bisnac }} \mathbf{N i}_{\mathbf{2}}(\mathbf{H})_{\mathbf{2}}\right]$ with $\mathrm{D}_{2}$ or $\mathrm{H}_{2}$ yielded a preferred end-on coordination of the dihydrogen molecule to one of the $\mathrm{Ni}^{\mathrm{II}}$ centers. It was therefore assumed, that in the semihydrogenation process the $\mathrm{H}_{2}$ molecule coordinates in a similar fashion. Coordination of $\mathrm{H}_{2}$ to $\mathbf{K}\left[\mathbf{L}^{\text {bisnac }} \mathbf{N i}_{\mathbf{2}}\left(\mu-\eta^{\mathbf{1}}: \eta^{\mathbf{1}} \mathbf{- C H C P h}\right)\right]$ is considered as rate determining since no intermediate was observed during the catalytic process.

Subsequently, insertion of $\mathrm{H}_{2}$ into the $\mathrm{Ni}-\mathrm{C}$ bond leads to polarization of dihydrogen (3). 
At the same time the cavity between the two nickel centers is opened up possibly providing enough space for the approaching substrate PA. The resulting species (4) is stabilized via cation- $\pi$ interactions of the substrate phenyl group to the $\mathrm{Ni}^{\mathrm{II}}$ center. In the following hydrogenation step mediated by a 4-center-4-electron transition state styrene is formed. After release of the semihydrogenated product $\mathbf{K}\left[\mathbf{L}^{\text {bisnac }} \mathbf{N i}_{\mathbf{2}}\left(\mu-\eta^{\mathbf{1}}: \eta^{\mathbf{1}}-\mathbf{C H C P h}\right)\right]$ (1) is reformed.

\section{Outlook}

Although the full hydrogenation of significant amounts of styrene to ethylbenzene occured under the presence of $\mathbf{K}\left[\mathbf{L}^{\text {bisnac }} \mathbf{N i}_{\mathbf{2}}\left(\mu-\eta^{\mathbf{1}}: \eta^{\mathbf{1}} \mathbf{- C H C P h}\right)\right]$, it has to be shown in a separate experiment that $\mathbf{K}\left[\mathbf{L}^{\text {bisnac }} \mathbf{N i}_{\mathbf{2}}\left(\mu-\eta^{\mathbf{1}}: \eta^{\mathbf{1}}-\mathbf{C H C P h}\right)\right]$ represents the active catalyst in the cycle. Therefore, future works include the reaction of $\mathbf{K}\left[\mathbf{L}^{\text {bisnac }} \mathbf{N i}_{2}\left(\mu-\eta^{1}: \eta^{1}-\mathbf{C H C P h}\right)\right]$ with isotopically labeled phenylacetylene or other alkyne substrates (e.g. 1-hexyne). These experiments are expected to provide evidence for the retention of the phenylvinyl bridging ligand during the catalytic process. Moreover, the influence of the potassium cation on the mechanism has to be studied. For instance, investigation of the reaction with a sodium analogue $\left(\mathbf{N a}\left[\mathbf{L}^{\text {bisnac }} \mathbf{N i}_{\mathbf{2}}\left(\mu-\eta^{\mathbf{1}}: \eta^{\mathbf{1}}-\mathbf{C H C P h}\right)\right]\right)$ can show a possible dependence on the alkali cation. Furthermore, in order to understand the role of $\mathbf{K}\left[\mathbf{L}^{\text {bisnac }} \mathbf{N i}_{\mathbf{2}}(\mu-\right.$ $\left.\left.\eta^{1}: \eta^{1}-\mathbf{C H C P h}\right)\right]$ in the hydrogenation of styrene to ethylbenzene, separate experiments have to be performed. 


\subsubsection{Influences on the Catalytic Semihydrogenation Process}

\subsubsection{High Pressure}

In order to investigate a possible dependency of the rate of styrene generation on the applied pressure of $\mathrm{H}_{2}$, high pressure experiments were conducted. For this purpose a sample containing a pump-freeze-thaw degassed $\mathrm{THF}^{-\mathrm{D}_{8}}$ solution of $\mathbf{K}\left[\mathbf{L}^{\mathbf{b i s n a c}} \mathbf{N i}_{\mathbf{2}}(\boldsymbol{\mu}-\right.$ $\left.\left.\eta^{1}: \eta^{1}-\mathbf{C H C P h}\right)\right]$ and an excess of phenylacetylene (15 eq) was loaded with dihydrogen gas (6 atm.). After applying pressure, the sample was frozen in liquid nitrogen to slow down any reaction to minimum before the measurement. To initiate the hydrogenation of styrene the sample was warmed up, vigorously shaken and transferred into the spectrometer. The beginning of the reaction was monitored by recording one scan proton NMR spectra at room temperature over a timescale of 6 minutes. After that, regular 16 scan spectra were collected applying an increasing interval between measurements.

Surprisingly, the reaction progressed only slightly faster in comparison to the reaction performed at one atmosphere of $\mathrm{H}_{2}$. This is described by a plot of styrene production vs time (Figure 5.29). Furthermore, Figure 5.30 illustrates the graphically determined catalytic turnover frequency $(\mathrm{TOF})^{\S}$ for both conditions.

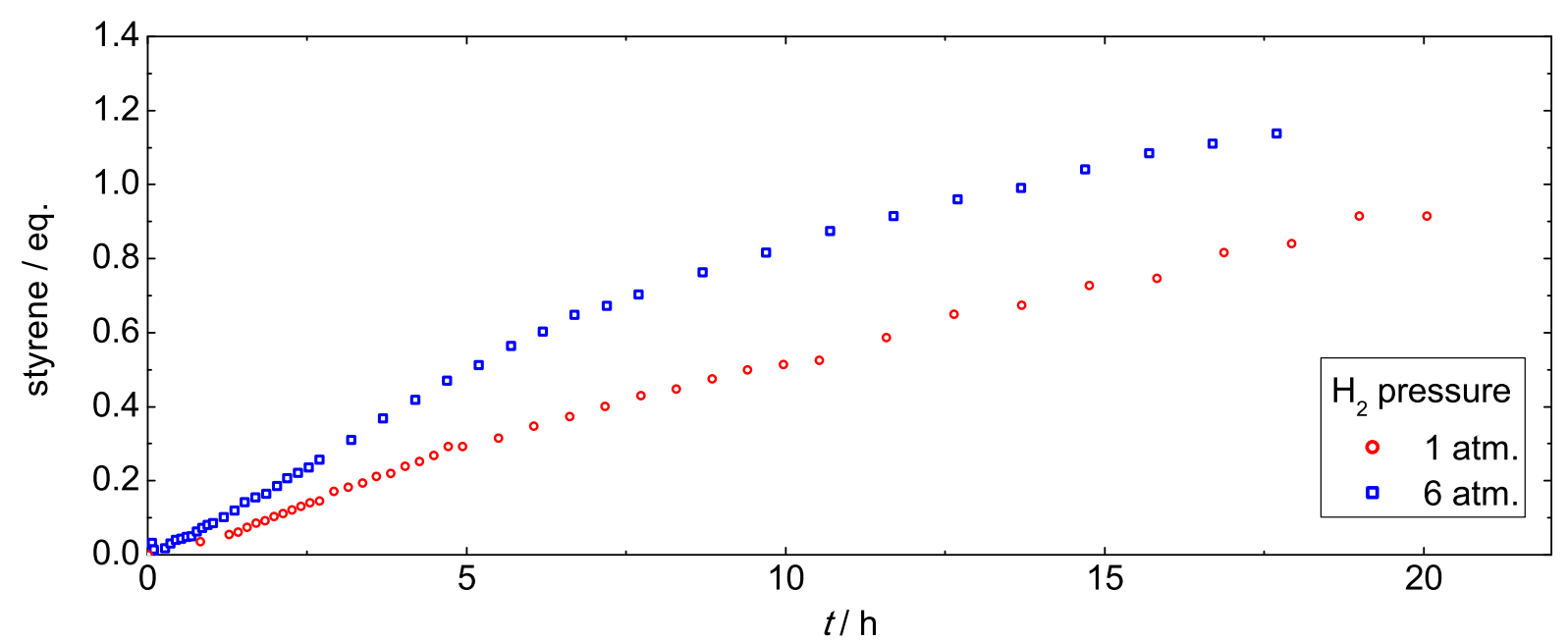

Figure 5.29.: Comparison of the initial progress for the catalytic semihydrogenation with 1 atm. and 6 atm. of $\mathrm{H}_{2}$.

In contrast to the reaction performed at atmospheric pressure of $\mathrm{H}_{2}$, during the high pressure experiment production of ethylbenzene was observed (Figure 5.31). Although the full hydrogenation occured to a much lesser extent than the semihydrogenation it was progressing from the start of the reaction.

$\S$ The turnover frequency TOF reflects the efficiency of a catalyst. It is described as the quotient of the turnover number and the time. 


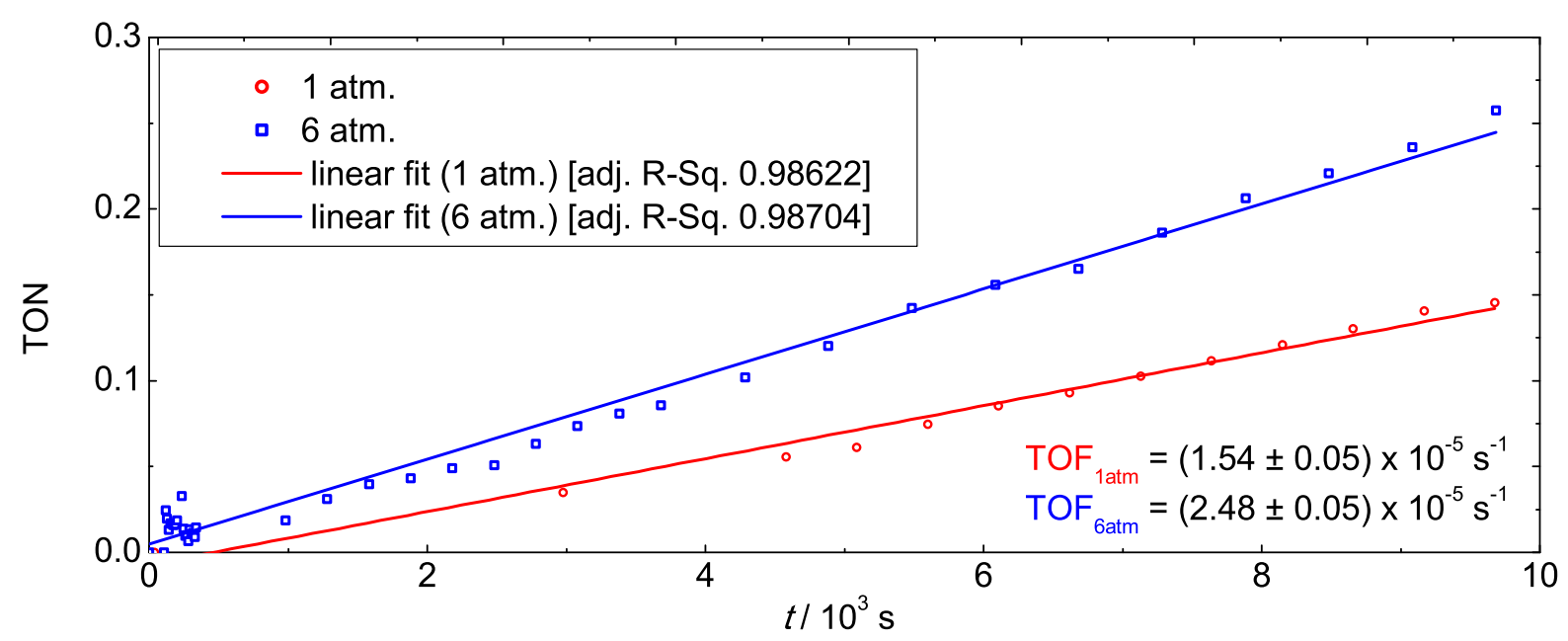

Figure 5.30.: Plot of catalytic turnovers (TON) against the time. The turnover frequency (TOF) for the initial progress of semihydrogenation with $\mathrm{H}_{2}$ (atmospheric pressure and $6 \mathrm{~atm}$.) was determined graphically.

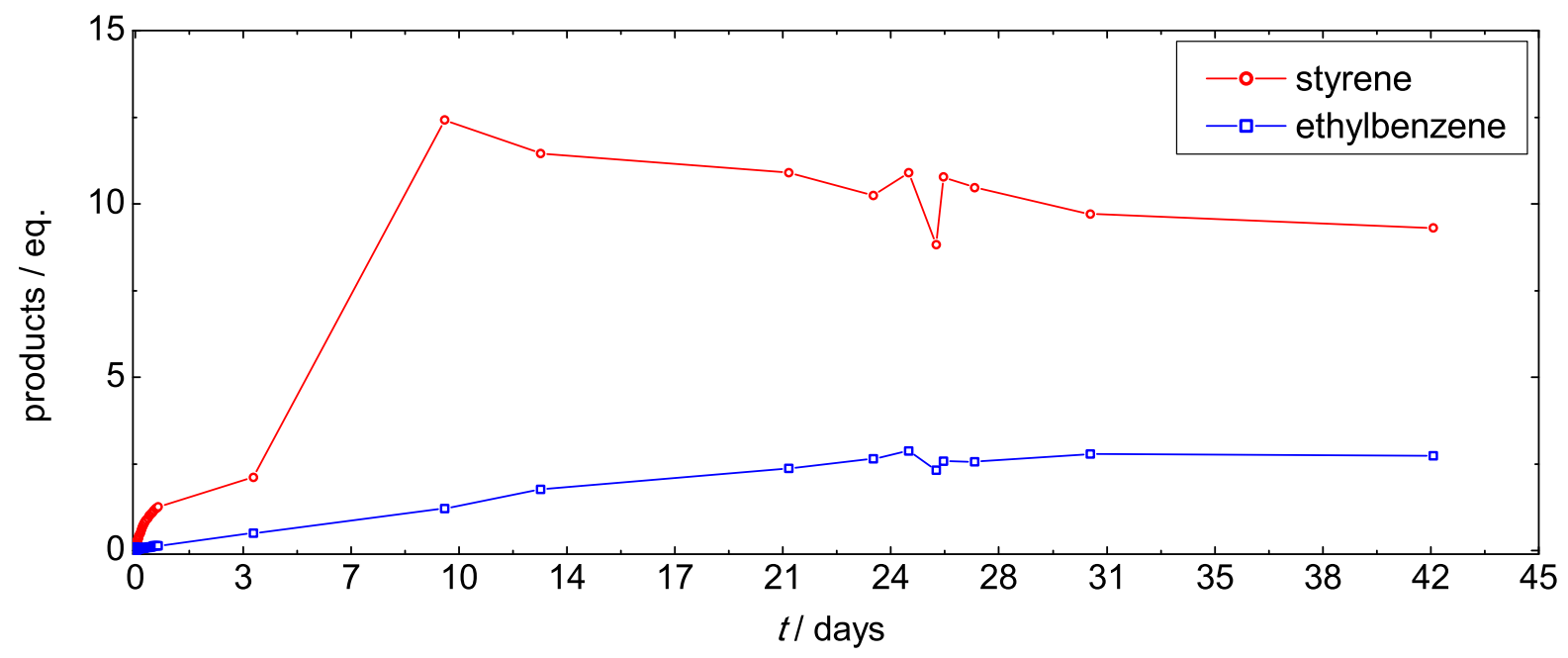

Figure 5.31.: The changing amount (in equivalents) of the hydrogenation products styrene $(0)$ and ethylbenzene ( $\square$ ) over a six week timescale under high pressure ( 6 atmospheres) of $\mathrm{H}_{2}$. After one day 0.12 eq of ethylbenzene were generated. Full consumption of phenylacetylene led to a decrease of the amount of formed styrene due to further hydrogenation to ethylbenzene. 
${ }^{1} \mathrm{H}$ NMR spectra Figure 5.32) collected after initiation of the reaction revealed a difference in complex distribution compared to the reaction under 1 atm of $\mathrm{H}_{2}$ : the resonance of the pyrazolate backbone at $5.62 \mathrm{ppm}(\bullet)$ associated with $\mathbf{K}\left[\mathbf{L}^{\text {bisnac }} \mathbf{N i}_{\mathbf{2}}\left(\mu-\eta^{\mathbf{1}}: \eta^{\mathbf{1}}-\mathbf{C H C P h}\right)\right]$ substantially decreased shortly after start of the reaction.

At the same time, in addtition to the formation of $\left[\mathbf{L}^{\text {bisnac }} \mathbf{N i}_{\mathbf{2}}(\mu-\mathbf{O H})\right](\mathbf{\square})$ two other species $\mathbf{X}$ and $\mathbf{Y}$ developed. In particular, the resonance at $5.52 \mathrm{ppm}(\mathbf{X})$ significantly increased representing the major compound.

The first assumption after this observation was that the low rate of the hydrogenation in this particular case was probably correlated to a poisoning of the catalyst. However, a control measurement after ten days revealed an increase in the amount of styrene and full consumption of the substrate (no PA associated resonances were observed in the ${ }^{1} \mathrm{H}$ NMR spectrum). This showed that semihydrogenation of phenylacetylene was either promoted by residual amounts of $\mathbf{K}\left[\mathbf{L}^{\text {bisnac }} \mathbf{N i}_{\mathbf{2}}\left(\mu-\eta^{\mathbf{1}}: \eta^{\mathbf{1}}-\mathbf{C H C P h}\right)\right]$ or another catalytically active species.

The transformation of $\mathbf{K}\left[\mathbf{L}^{\text {bisnac }} \mathbf{N i}_{\mathbf{2}}\left(\mu-\eta^{\mathbf{1}}: \eta^{\mathbf{1}} \mathbf{- C H C P h}\right)\right]$ to $\mathbf{X}$ and $\mathbf{Y}$ was also observed in the experiment conducted at atmospheric $\mathrm{H}_{2}$ pressure but to a much lesser extent.

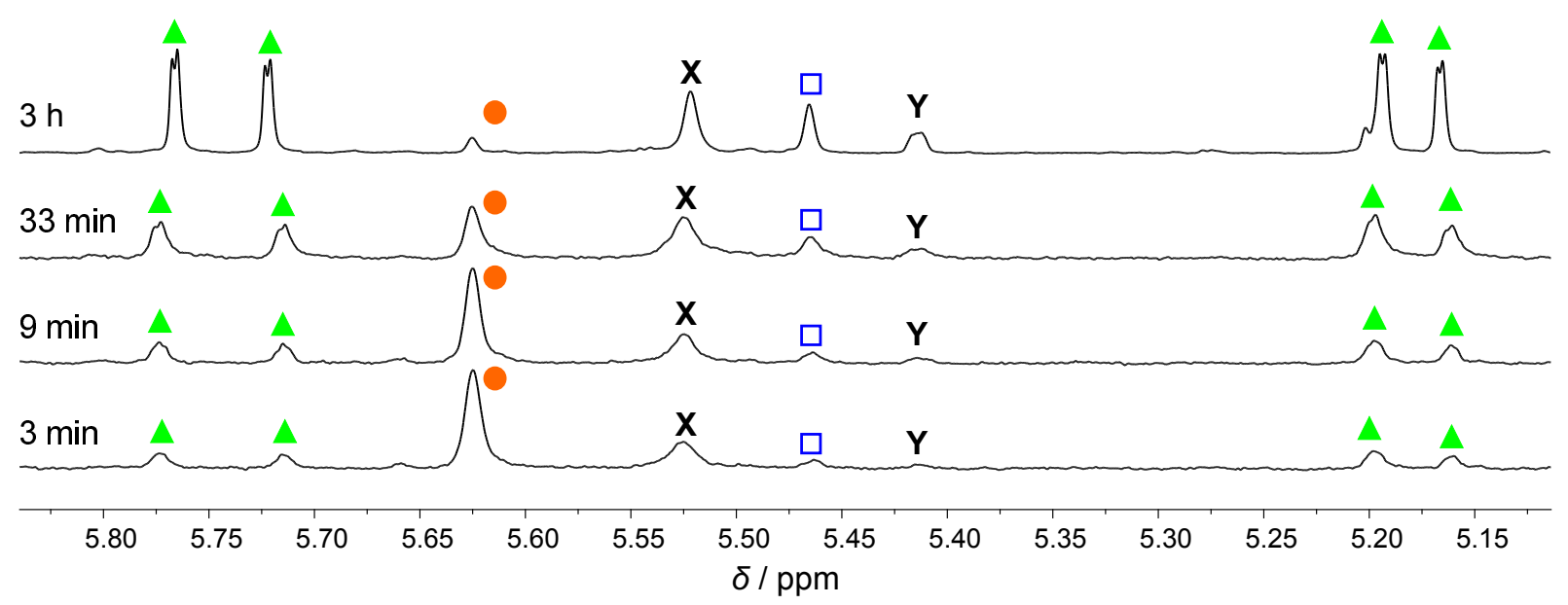

Figure 5.32.: ${ }^{1} \mathrm{H}$ NMR spectra recorded during the catalytic semihydrogenation experiment at high pressure (6 atm.) after 3, 9, 33 minutes $(300 \mathrm{MHz})$ and 3 hours $(400 \mathrm{MHz})$. The resonances associated with styrene are marked $(\Delta)$. During the course of this time the characteristic resonance of $K\left[L^{\text {bisnac }} \mathbf{N i}_{2}(\mu-\right.$ $\left.\left.\eta^{1}: \eta^{1}-\mathrm{CHCPh}\right)\right]$ (•) significantly decreases while the amount of [ [ $\left.{ }^{\text {bisnac }} \mathrm{Ni}_{2}(\mu-\mathrm{OH})\right](\square)$ and two further unidentified species $\mathbf{X}$ and $\mathbf{Y}$ increased.

The ratio between different complexes in solution differs upon change of applied $\mathrm{H}_{2}$ pressure. Therefore, two possible scenarios are proposed for the increased rate of the semihydrogenation process:

a) $\mathbf{K}\left[\mathbf{L}^{\text {bisnac }} \mathbf{N i}_{\mathbf{2}}\left(\mu-\eta^{\mathbf{1}}: \eta^{\mathbf{1}}-\mathbf{C H C P h}\right)\right]$ is the active catalyst, which was mainly present in the experiment with one atmosphere of dihydrogen. Considering the in situ generation of $\mathbf{K}\left[\mathbf{L}^{\text {bisnac }} \mathbf{N i}_{2}\left(\mu-\eta^{1}: \eta^{1}-\mathbf{C H C P h}\right)\right]$ from $\mathbf{K}\left[\mathbf{L}^{\text {bisnac }} \mathbf{N i}_{2}(\mathbf{H})_{2}\right]$ with two equivalents of PA and under abscence of $\mathrm{H}_{2}$, semihydrogenation of the second equivalent of substrate took 
place in a clean fashion. No formation of $\left[\mathbf{L}^{\text {bisnac }} \mathbf{N i}_{\mathbf{2}}(\mu-\mathrm{OH})\right], \mathbf{X}$ and $\mathbf{Y}$ was observed in ${ }^{1} \mathrm{H}$ NMR experiments during the process. This excludes catalytic activity of the latter species.

Addition of $\mathrm{H}_{2}$ (atmospheric to high pressure conditions) can lead to poisoning of the catalyst thus lowering the rate of reaction. Following this assumptions, the increased rate of hydrogenation under high pressure conditions and lower concentrations of $\mathbf{K}\left[\mathbf{L}^{\mathbf{b i s n a c}} \mathbf{N i}_{\mathbf{2}}(\mu-\right.$ $\left.\left.\eta^{1}: \eta^{1}-\mathbf{C H C P h}\right)\right]$ implies a dependency on the $\mathrm{H}_{2}$ pressure.

b) at least one of the complexes resulting from transformation of $\mathbf{K}\left[\mathbf{L}^{\mathbf{b i s n a c}} \mathbf{N i}_{\mathbf{2}}\left(\mu-\eta^{\mathbf{1}}: \eta^{\mathbf{1}}\right.\right.$ CHCPh)] possesses a catalytic activity. In this case the semihydrogenation of phenylacetylene will also be sustained. To investigate a catalytic activity of the side products, the isolation of the respective species is necessary. So far isolation attempts were not succesful.

In order to investigate the dependency of the hydrogenation process on the concentration of substrate, another NMR experiment was conducted under high pressure conditions (6 atm. of $\mathrm{H}_{2}$ ). In this case a lower excess of five equivalents of phenylacetylene was added to the THF-D $D_{8}$ solution of $\mathbf{K}\left[\mathbf{L}^{\text {bisnac }} \mathbf{N i}_{2}\left(\mu-\eta^{1}: \eta^{1}-\mathbf{C H C P h}\right)\right]$. The hydrogenation rate was lower with a smaller amount of substrate (Figure 5.33) under the same pressure conditions.

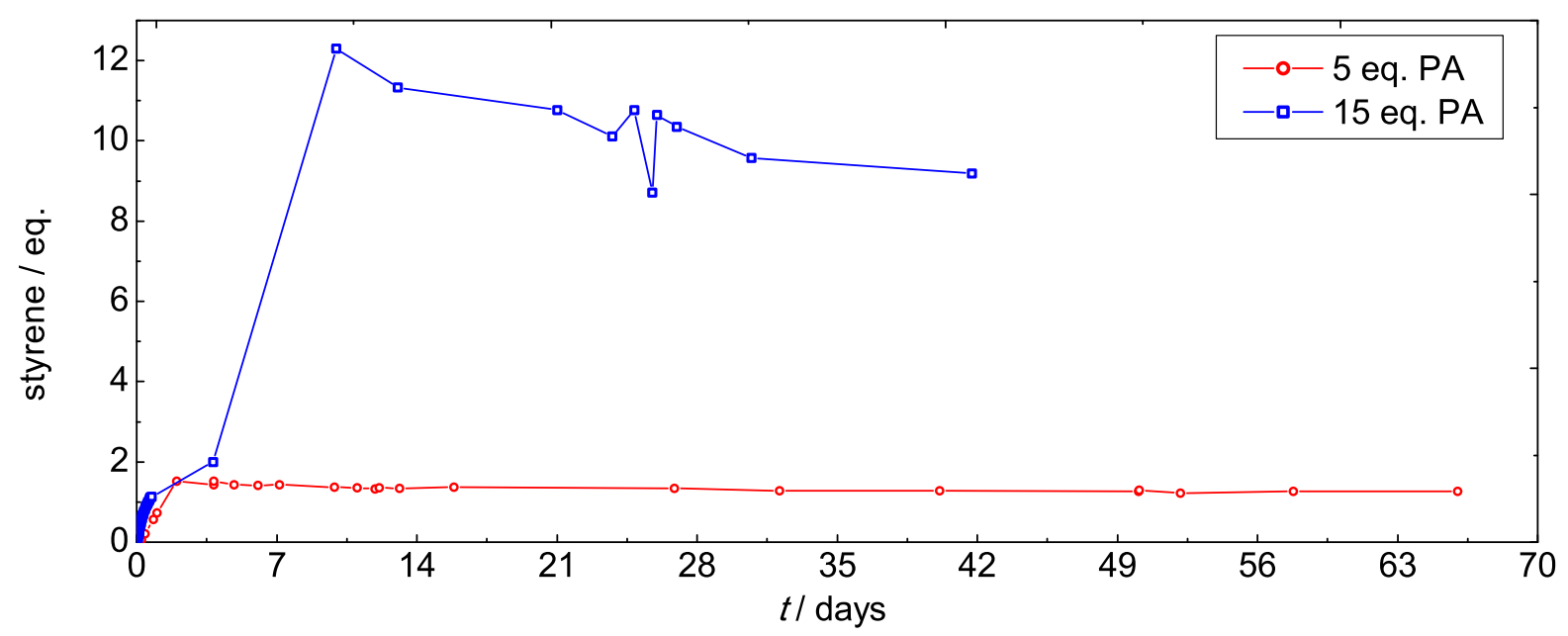

Figure 5.33.: Comparison of the hydogenation rates in samples containing different excess amounts ( 5 eq and $15 \mathrm{eq}$ ) of substrate. Both reactions were performed under the same pressure conditions (6 atmospheres of $\mathrm{H}_{2}$ ). 
Surprisingly, during this experiment the catalytic production of styrene ceased with high amounts of $\mathrm{H}_{2}$ and phenylacetylene still present in the sample mixture as illustrated in Figure 5.34. The plot Figure 5.34 further shows that $\mathbf{K}\left[\mathbf{L}^{\text {bisnac }} \mathbf{N i}_{\mathbf{2}}\left(\mu-\eta^{\mathbf{1}}: \eta^{\mathbf{1}}-\mathbf{C H C P h}\right)\right]$ drastically decreased after initiation of the reaction while the amount of $\mathbf{X}$ increased. However, after reaching a maximum of $\approx 0.6$ eq after 10 hours $\mathbf{X}$ also decreased. In the ${ }^{1} \mathrm{H}$ NMR spectra (Figure 5.35 the decrease of resonances related to complexes is observed. This is explained by the formation of a precipitate in the sample.

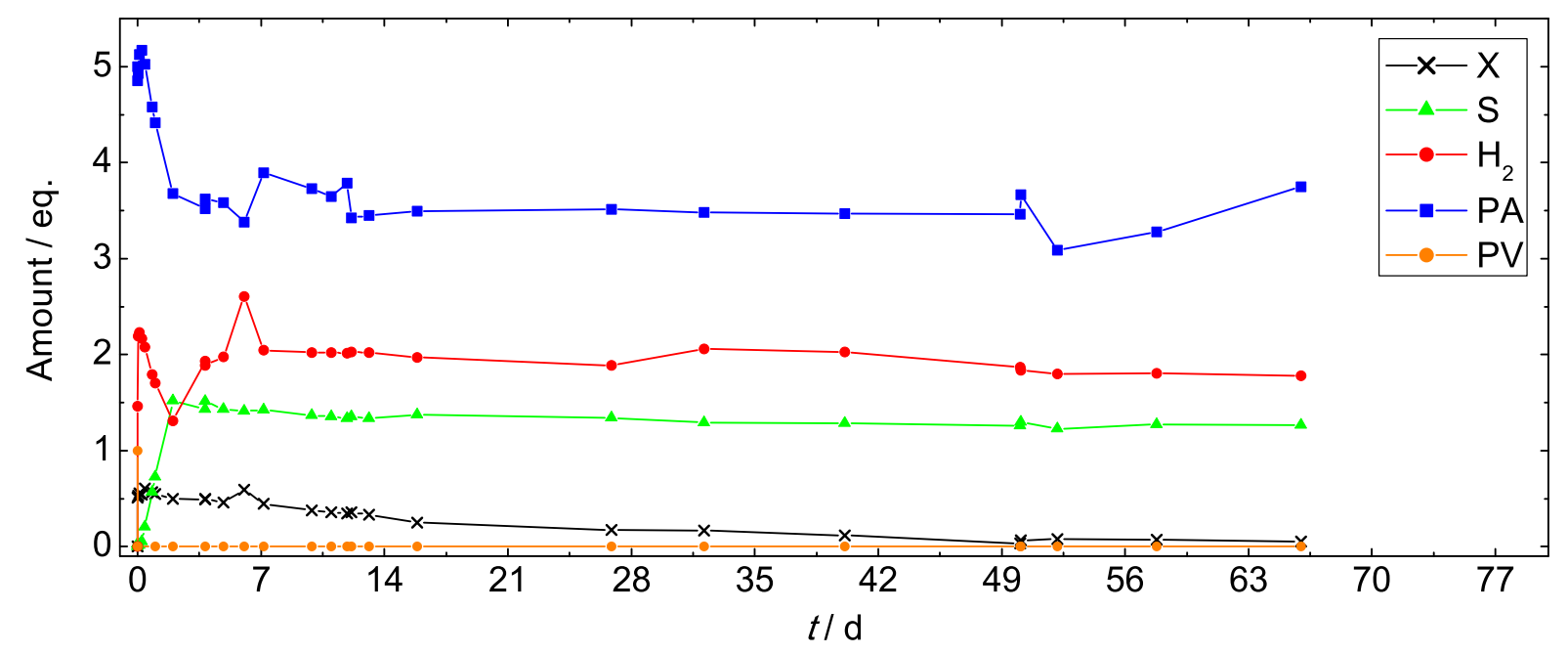

Figure 5.34.: Time course for the catalytic hydrogenation experiment performed with 5 equivalents of $P A$ and 6 atmospheres of $H_{2}$. After the start of the reaction the amount of $K\left[L^{\text {bisnac }} \mathbf{N i}_{2}\left(\mu-\eta^{1}: \eta^{1}-C H C P h\right)\right]$ $(P V)$ drastically decreases while $\mathbf{X}$ builds up to $\approx 0.5$ eq before also decreasing. During the first days $\approx 1.5$ eq of $P A$ were converted to styrene $(S)$ before the hydrogenation ceased.

Since the catalytic semihydrogenation of phenylacetylene ceased under these conditions the following conclusions were made. Firstly, the exclusive presence of $\mathbf{X}$ after one week and no further occuring hydrogenation processes prove the catalytic inactivity of $\mathbf{X}$. However, shortly after start of the reaction $\mathbf{K}\left[\mathbf{L}^{\text {bisnac }} \mathbf{N i}_{\mathbf{2}}\left(\mu-\eta^{\mathbf{1}}: \eta^{\mathbf{1}}-\mathbf{C H C P h}\right)\right]$ was consumed (related resonances were undetectable in ${ }^{1} \mathrm{H}$ NMR after 1 hour). This implies the presence of another catalytically active species, which was inactive, too, after four days (Figure 5.34). Secondly, the lower concentration of PA led to a faster increase of $\mathbf{X}$ indicating that its formation is possibly dependent on accessibility to the active center of the precursor $^{\S}$. The experimental conditions further show that generation of $\mathbf{X}$ is probably dependent on the pressure of $\mathrm{H}_{2}$.

\footnotetext{
${ }^{\S}$ At this point the origin of $\mathrm{X}$ is not understood. One of the at least three observed complexes $\mathrm{K}\left[\mathrm{L}^{\text {bisnac }} \mathrm{Ni}_{2}\left(\mu-\eta^{1}: \eta^{1}-\mathrm{CHCPh}\right)\right]$, [ $\left.\mathrm{L}^{\text {bisnac }} \mathrm{Ni}_{2}(\mu-\mathrm{OH})\right]$ or $\mathrm{Y}$ are potential precursors.
} 

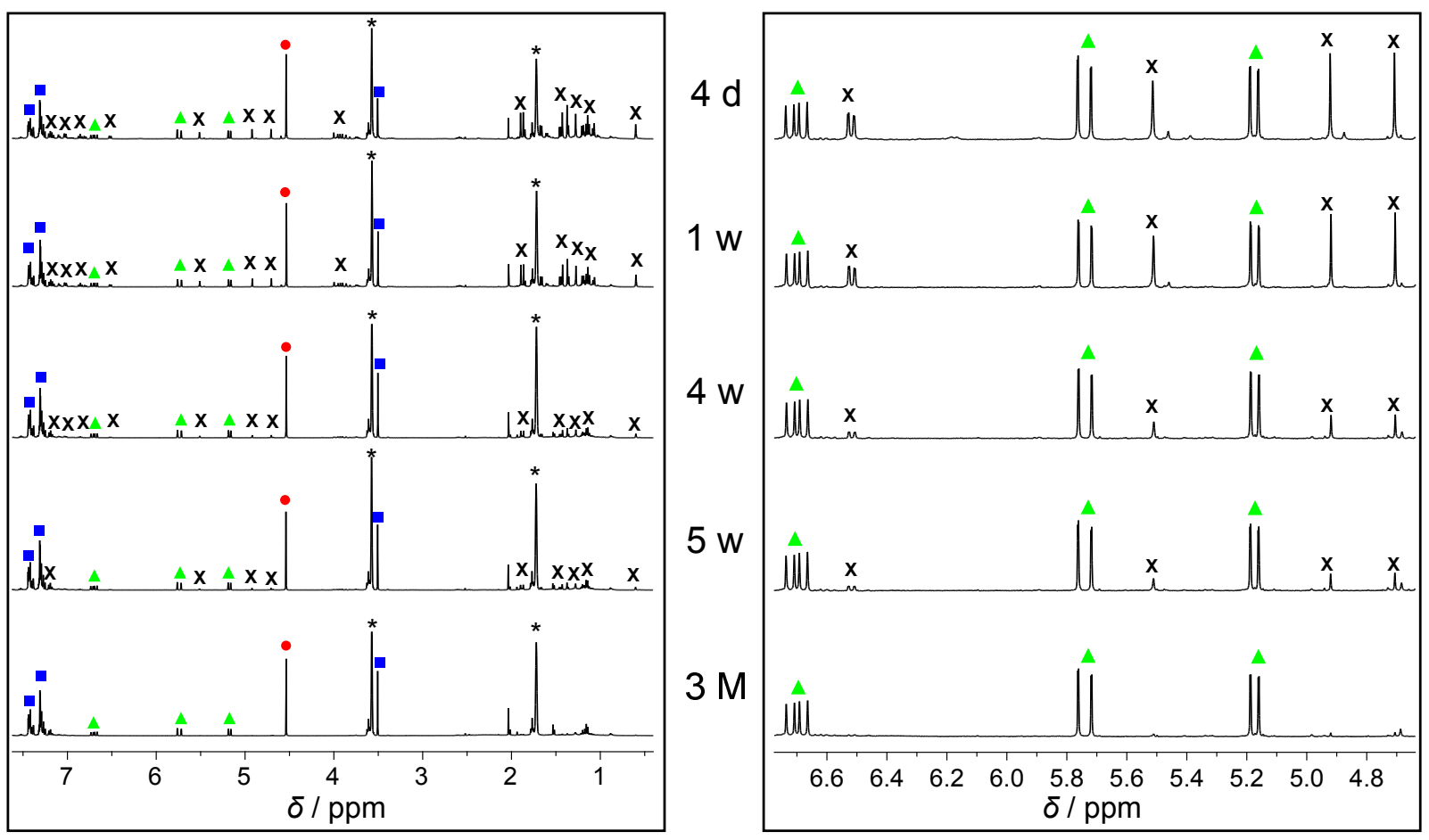

Figure 5.35.: A catalytic experiment performed under 6 atm. of $\mathrm{H}_{2}$ with 5 eq of phenylacetylene, monitored by ${ }^{1} \mathrm{H}$ NMR. Shown are the spectra associated with control measurements after a certain time. Left: the whole range of the measured spectrum to illustrate the cease of the catalytic reaction. While the intensities of the resonances correlated to the substrate phenylacetylene $(\square)$ and the hydrogenation product styrene $(\Delta)$ do not change any further, the intensity of complex associated signals $(\mathbf{X})$ decreases. Residual solvents $\left(^{*}\right)$ and $\mathrm{H}_{2}(\bullet)$ are marked. Right: detail of the main spectra illustrated with adjusted intensity. The decrease of the complex species can be observed. 


\subsubsection{Mixing Issues}

Effective mixing of the reactands by means of vigorous shaking of the NMR sample tube led to rapid formation of $\mathbf{X}$. This is illustrated by comparison of ${ }^{1} \mathrm{H}$ NMR spectra immediately recorded after initiation of the catalytic semihydrogenation reaction (Figure 5.36). Both spectra are collected from samples containing the same ratio of reactands (i.e. 15 eq of PA and 6 atm. of $\mathrm{H}_{2}$ ). Spectrum (a) of Figure 5.36 represents a sample, which was not shaken before the measurement. Although $\mathbf{K}\left[\mathbf{L}^{\text {bisnac }} \mathbf{N i}_{\mathbf{2}}\left(\mu-\eta^{\mathbf{1}}: \eta^{\mathbf{1}}-\mathbf{C H C P h}\right)\right]$ (assigned to the pyrazole-backbone resonance at $5.62 \mathrm{ppm}$, ๑) still accounts for the major complex in the mixture a significant increase in species $\mathbf{X}$ at $5.52 \mathrm{ppm}$ can already be observed. Vigorous shaking of a sample before the measurement led to the instant consumption of $\mathbf{K}\left[\mathbf{L}^{\text {bisnac }} \mathbf{N i}_{2}\left(\mu-\eta^{\mathbf{1}}: \eta^{\mathbf{1}}-\mathbf{C H C P h}\right)\right]$, giving rise to major formation of $\mathbf{X}$ as can be seen in spectrum (b) of Figure 5.36. Furthermore, several other resonances besides the $\left[\mathbf{L}^{\text {bisnac }} \mathbf{N i}_{2}(\boldsymbol{\mu}-\mathbf{O H})\right](\mathbf{\square})$ or $\mathbf{Y}$ associated, were observed in the spectrum $(\mathbf{y})$.

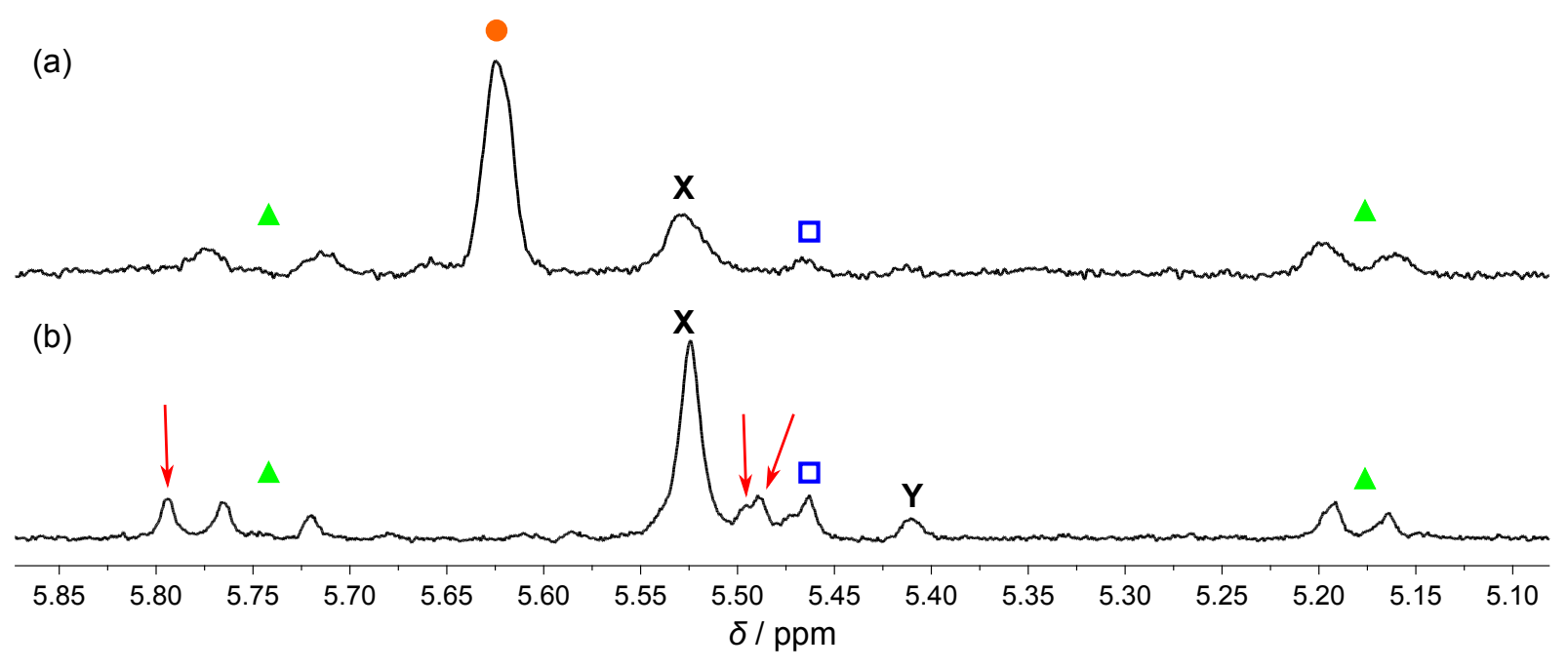

Figure 5.36.: Excerpts of initial ${ }^{1} \mathrm{H}$ NMR spectra ( $300 \mathrm{MHz}$, a; $400 \mathrm{MHz}$, b) collected from samples containing $K\left[L^{\text {bisnac }} \mathrm{Ni}_{2}\left(\mu-\eta^{1}: \eta^{1}-\mathrm{CHCPh}\right)\right], 15$ eq of phenylacetylene and $\mathrm{H}_{2}\left(6\right.$ atm.) in THF-D $\mathrm{D}_{8}$. Spectrum (a) represents a sample, which was not vigorously shaken before. Besides the major pyrazole-backbone proton resonance of $K\left[L^{\text {bisnac }} \mathrm{Ni}_{2}\left(\mu-\eta^{1}: \eta^{1}-\mathrm{CHCPh}\right)\right](\bullet)$, the formation of $\mathrm{X}$ and $\left[\mathrm{L}^{\text {bisnac }} \mathrm{Ni}_{2}(\mu-\mathrm{OH})\right](\square)$ and $\mathbf{Y}$ is observed. Spectrum (b) indicates the entire conversion of $\left.\mathrm{K} \mathrm{L}^{\mathrm{bisnac}} \mathrm{Ni}_{2}\left(\mu-\eta^{1}: \eta^{1}-\mathrm{CHCPh}\right)\right]$ and major formation of $\mathbf{X}$ along with various other species $(\searrow)$. Characteristic $\beta$-hydrogen resonances of styrene are marked $(\Delta)$.

The result shows that effective mixing via vigorous shaking of the sample tube immediately gave rise to a variety of side products at the cost of $\mathbf{K}\left[\mathbf{L}^{\text {bisnac }} \mathbf{N i}_{\mathbf{2}}\left(\mu-\eta^{\mathbf{1}}: \eta^{\mathbf{1}}\right.\right.$ $\mathbf{C H C P h})]$. In order to investigate a sustained catalytic activity after the consumption of $\mathbf{K}\left[\mathbf{L}^{\text {bisnac }} \mathbf{N i}_{\mathbf{2}}\left(\mu-\eta^{\mathbf{1}}: \eta^{\mathbf{1}}-\mathbf{C H C P h}\right)\right]$ the development of styrene resonances was monitored over time. Figure 5.37 shows ${ }^{1} \mathrm{H}$ NMR spectra recorded after 5 hours.

In the unshaken sample represented by ${ }^{1} \mathrm{H}$ NMR spectrum (a) of Figure 5.37 a reasonable amount amount of styrene was formed, which is due to the slower deacativa- 

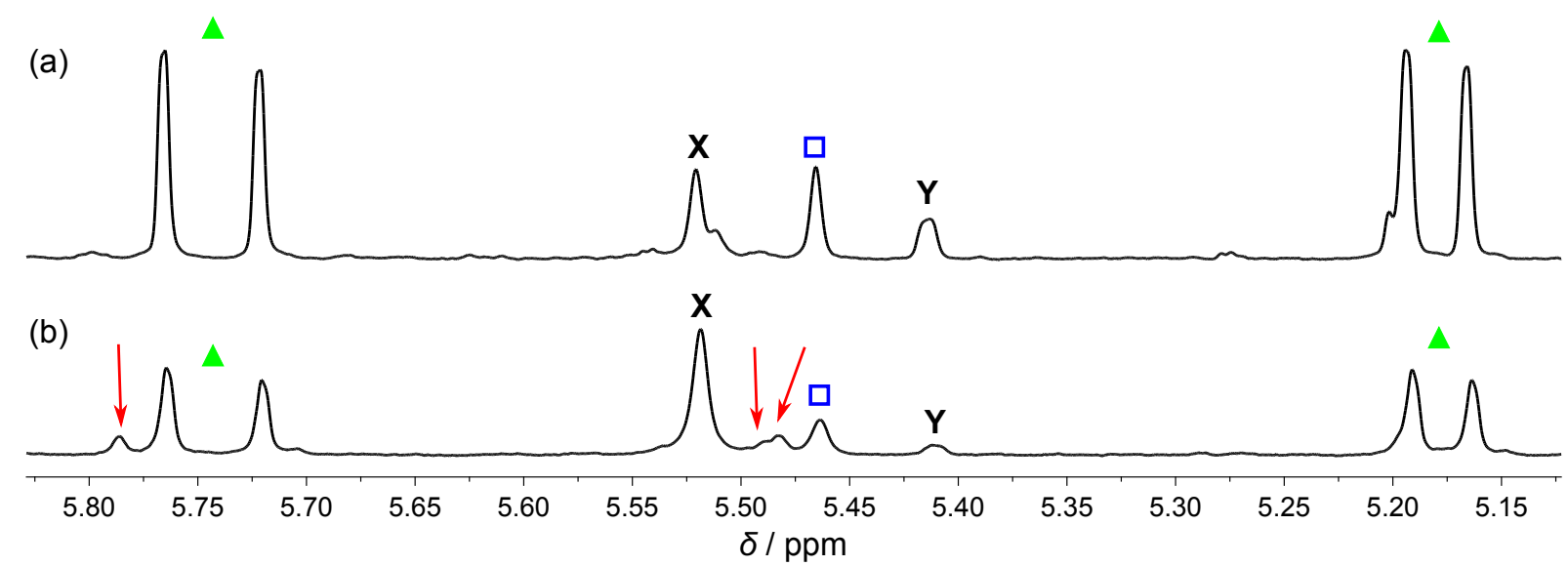

Figure 5.37.: Excerpt of initial ${ }^{1} \mathrm{H}$ NMR spectra $(400 \mathrm{MHz})$ collected after a reaction time of $5 \mathrm{~h}$ from a sample of $\left.\mathrm{K}^{\mathrm{b}} \mathrm{L}^{\text {bisnac }} \mathrm{Ni}_{2}\left(\mu-\eta^{1}: \eta^{1}-\mathrm{CHCPh}\right)\right], 15$ eq of phenylacetylene and 6 atmospheres of $\mathrm{H}_{2}$ in THF$\mathrm{D}_{8}$, which was not shaken before the monitoring (a) and from a sample which was shaken vigorously before (b). Associations to respective resonances are indicated by the same colored symbols used in Figure 5.36.

tion of $\mathbf{K}\left[\mathbf{L}^{\text {bisnac }} \mathbf{N i}_{\mathbf{2}}\left(\mu-\eta^{\mathbf{1}}: \eta^{\mathbf{1}}-\mathbf{C H C P h}\right)\right]$. Interestingly, spectrum (b) of Figure 5.37 also showed a recognizable increase in semihydrogenated product despite the fact that $\mathbf{K}\left[\mathbf{L}^{\text {bisnac }} \mathbf{N i}_{\mathbf{2}}\left(\mu-\eta^{\mathbf{1}}: \eta^{\mathbf{1}}-\mathbf{C H C P h}\right)\right]$ was already consumed (s. spectrum b of Figure 5.36) at the early stage. This observation implies that besides $\mathbf{K}\left[\mathbf{L}^{\text {bisnac }} \mathbf{N i}_{\mathbf{2}}\left(\mu-\eta^{\mathbf{1}}: \eta^{\mathbf{1}}-\mathbf{C H C P h}\right)\right]$ at least one of the formed side products posseses a catalytic activity.

Although a larger fraction of $\left[\mathbf{L}^{\text {bisnac }} \mathbf{N i}_{\mathbf{2}}(\boldsymbol{\mu}-\mathbf{O H})\right](\mathbf{\square})$ was formed in the unshaken sample as described in (a) of Figure 5.37, a larger amount of styrene was observed in comparison to the shaken reaction mixture (b). Generation of a larger fraction of hydroxo complex can be caused by several factors: firstly, higher amounts of residual water in this particular sample (NMR tube, preparation apparatus, etc.) lead to a faster transformation to $\left[\mathbf{L}^{\text {bisnac }} \mathbf{N i}_{\mathbf{2}}(\mu-\right.$ $\mathbf{O H})$ ]. Secondly, species $\mathbf{X}$ is less susceptible or even inert towards further conversion to $\left[\mathbf{L}^{\text {bisnac }} \mathbf{N i}_{2}(\mu-\mathrm{OH})\right]$. Thirdly, $\mathbf{X}$ originates from $\left[\mathbf{L}^{\text {bisnac }} \mathbf{N i}_{2}(\mu-\mathrm{OH})\right]$.

Although not many spectra were collected during this time, plotted normalized integrals of characteristic styrene doublet resonances as a function of time indicate a much higher initial conversion rate of the substrate PA in the unshaken sample ( $\boldsymbol{\Delta}$ in Figure 5.38).

Comparison of high pressure experiments $\boldsymbol{\Delta}$ and $\boldsymbol{\square}$ with an experiment conducted at atmospheric pressure $(\bullet)$ show that elevated pressure of $\mathrm{H}_{2}$ results in a faster production of styrene. This effect was observed despite the fact that the amount of $\mathbf{K}\left[\mathbf{L}^{\text {bisnac }} \mathbf{N i}_{\mathbf{2}}(\mu-\right.$ $\left.\left.\eta^{1}: \eta^{1}-\mathbf{C H C P h}\right)\right]$ was much less in the high pressure experiments due to the transformation to side products. The sustained catalytic activity possibly mediated by one of the side products is indicated by continuous styrene production beyond entire consumption of $\mathbf{K}\left[\mathbf{L}^{\text {bisnac }} \mathbf{N i}_{\mathbf{2}}\left(\mu-\eta^{1}: \eta^{1}-\mathbf{C H C P h}\right)\right]$ after around five hours (according to Figure 5.37). 


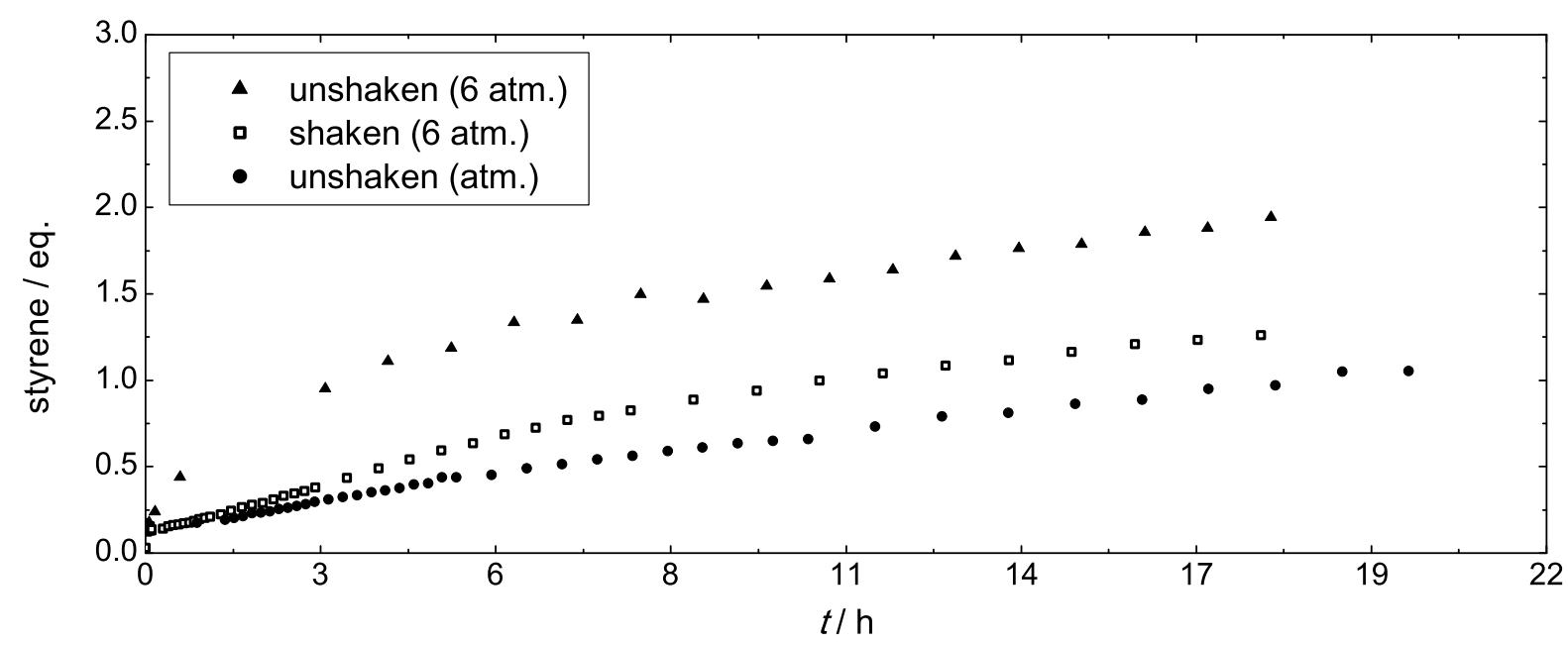

Figure 5.38.: Comparison of styrene production for the unshaken $(\boldsymbol{\Delta})$ and shaken sample $(\boldsymbol{\square})$ containing $\mathrm{K}\left[\mathrm{L}^{\text {bisnac }} \mathrm{Ni}_{2}\left(\mu-\eta^{1}: \eta^{1}-\mathrm{CHCPh}\right)\right], \mathrm{PA}(15 \mathrm{eq})$ and $\mathrm{H}_{2}$ (6 atm.). Furthermore, the production in an unshaken sample under atmospheric $\mathrm{H}_{2}$ pressure is depicted $(\bullet)$.

\subsubsection{Conclusion}

Considering the compiled information obtained by these experiments it can be concluded that higher pressure of $\mathrm{H}_{2}$ leads to a faster semihydrogenation of phenylacetylene. It was further observed that $\mathbf{K}\left[\mathbf{L}^{\text {bisnac }} \mathbf{N i}_{\mathbf{2}}\left(\mu-\eta^{\mathbf{1}}: \eta^{\mathbf{1}}-\mathbf{C H C P h}\right)\right]$ undergoes a transformation to another dominant species during catalytic experiments. Elevated pressure of $\mathrm{H}_{2}$ showed rapid transformation which was promoted by effective mixing of the sample, eventually leading to the entire conversion of the complex. The formed species $\mathbf{X}$ did not show catalytic activity in regards of PA hydrogenation. Nonetheless, the formation of smaller amounts of side products led to a sustained production of styrene. 


\subsubsection{Investigation of Species $X$}

During the catalytic semihydrogenation of phenylacetylene to styrene a conversion of $\mathbf{K}\left[\mathbf{L}^{\text {bisnac }} \mathbf{N i}_{\mathbf{2}}\left(\mu-\eta^{\mathbf{1}}: \eta^{\mathbf{1}}-\mathbf{C H C P h}\right)\right]$ to an unknown species occured, which was referred to as species $\mathbf{X}$. Since the catalytically inactive compound represents a major side product, its identification was focussed.

It turned out that $\mathbf{X}$ was the result of internal $\mathrm{CH}$-acitvation, which was promoted at high pressures. The proposed structure of the $\mathrm{CH}$-activation product is illustrated in Scheme 5.11, Its identification was based on in-depth NMR spectroscopic investigations, which are discussed in this section.

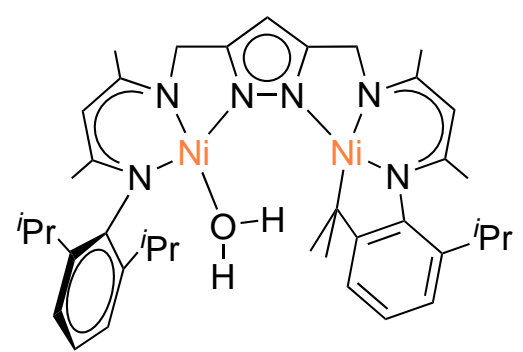

Scheme 5.11: The proposed structure of $X$.

\subsubsection{The Formation}

As mentioned in the catalysis section (5.5) of this work, treatment of a THF- $\mathrm{D}_{8}$ solution of $\mathbf{K}\left[\mathbf{L}^{\text {bisnac }} \mathbf{N i}_{\mathbf{2}}\left(\mu-\eta^{\mathbf{1}}: \eta^{\mathbf{1}}-\mathbf{C H C P h}\right)\right]$ with one atmosphere of $\mathrm{H}_{2}$ did not lead to any change. However, after applying higher pressures (6 atm) of $\mathrm{H}_{2}$, an immediate change was observed: The color of the formerly low green solution changed to brownish. A reestablishment of $\mathbf{K}\left[\mathbf{L}^{\text {bisnac }} \mathbf{N i}_{\mathbf{2}}(\mathbf{H})_{\mathbf{2}}\right]$ from $\mathbf{K}\left[\mathbf{L}^{\text {bisnac }} \mathbf{N i}_{\mathbf{2}}\left(\mu-\eta^{\mathbf{1}}: \eta^{\mathbf{1}}-\mathbf{C H C P h}\right)\right]$ was not occuring since no hydride associated resonance was found in the ${ }^{1} \mathrm{H}$ NMR.

Instead, the NMR experiment revealed rapid transformation of the starting material to two main pyrazolate-based complex species. The compounds were determined by singlet resonances in the typical pyrazolate-backbone proton region of the spectrum (s. Figure 5.39). Besides the known pyrazolate backbone resonance of $\left[\mathbf{L}^{\text {bisnac }} \mathbf{N i}_{\mathbf{2}}(\mu-\mathbf{O H})\right]$ at $5.46 \mathrm{ppm}(\boldsymbol{\square})$ another new resonance emerged at $5.52 \mathrm{ppm}$ (Figure 5.39), which was assigned to the new species $\mathbf{X}$. By integration of these resonances a fraction of $11 \%$ for the hydroxo-bridged and a fraction of $59 \%$ for the new species $\mathbf{X}$ could be calculated (corresponding to the sum of all pyrazolate based signals, which equals the integral of the clean starting material). However, another smaller signal was observed, which was superimposed by the resonance associated with $\mathbf{X}(\mathbf{y})$. It has to be mentioned that this result represents the incipient state of the sample directly after initiation of the transformation reaction. With time progressing the equilibrium is almost entirely shifted to species $\mathbf{X}$. 


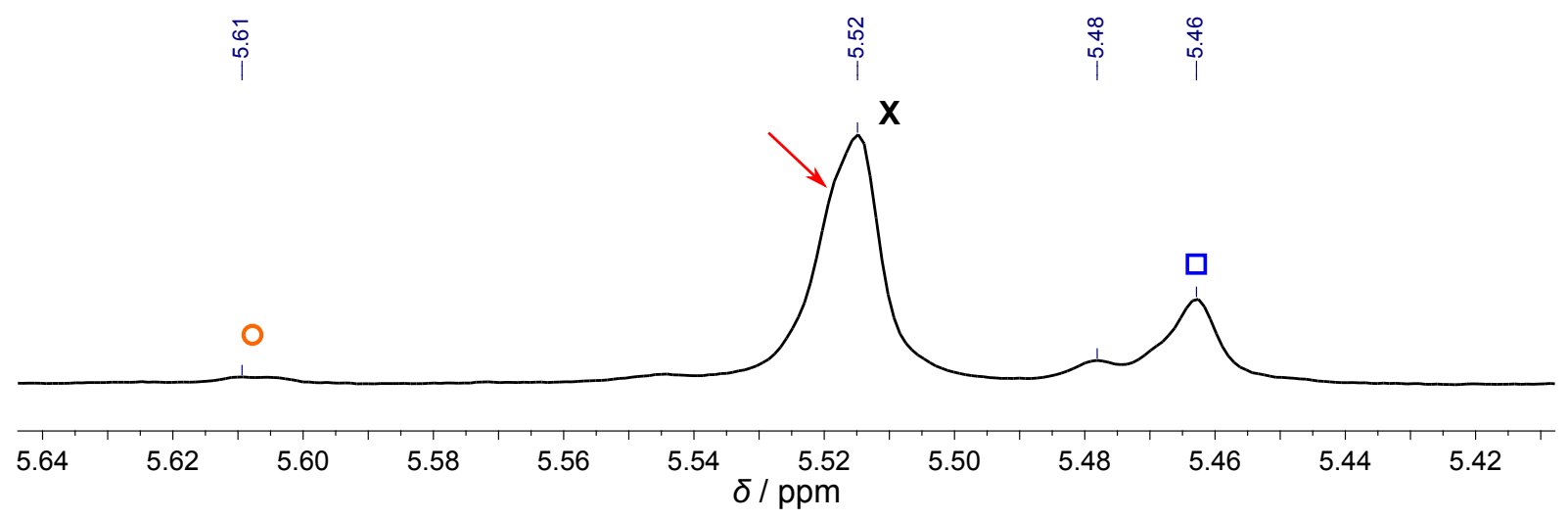

Figure 5.39.: Detail of the ${ }^{1} \mathrm{H}$ NMR spectrum $(400 \mathrm{MHz})$ immediately recorded after treatment of a sample of $\mathrm{K}\left[\mathrm{L}^{\text {bisnac }} \mathrm{Ni}_{2}\left(\mu-\eta^{1}: \eta^{1}-\mathrm{CHCPh}\right)\right](\mathrm{O})$ dissolved in THF-D $\mathrm{D}_{8}$ with $6 \mathrm{~atm}$. of $\mathrm{H}_{2}$. Besides the occurence of the $\mu$-hydroxo-bridged complex $\left(\mathrm{H}^{\mathrm{pz}}\right.$ at $\left.5.48 \mathrm{ppm}, \mathbf{\square}\right)$ the formation of a new species $\mathbf{X}$ is indicated by the major resonance at $5.52 \mathrm{ppm}$. A very small amount of another side product can also be observed at $4.48 \mathrm{ppm}$.

In order to obtain a complete picture of the newly formed species, the full set of resonances was assigned. Integration of the signals was performed in a ${ }^{1} \mathrm{H}$ NMR spectrum recorded several weeks after initiation of the experiment. At this point no further significant change occured. According to Figure 5.40 the $\beta$-diketiminate $\mathrm{CH}$ functions $\left(\mathrm{CH}^{\text {nac }}\right)$ could be assigned as well as the two methylene $\left(\mathrm{CH}_{2}\right)$ and isopropyl $\mathrm{CH}$ groups $\left(\mathrm{CH}^{i \operatorname{Pr}}\right)$. With this information the amount of $\mathbf{X}$, which was immediately formed (Figure 5.39), was calculated to $38 \%$.

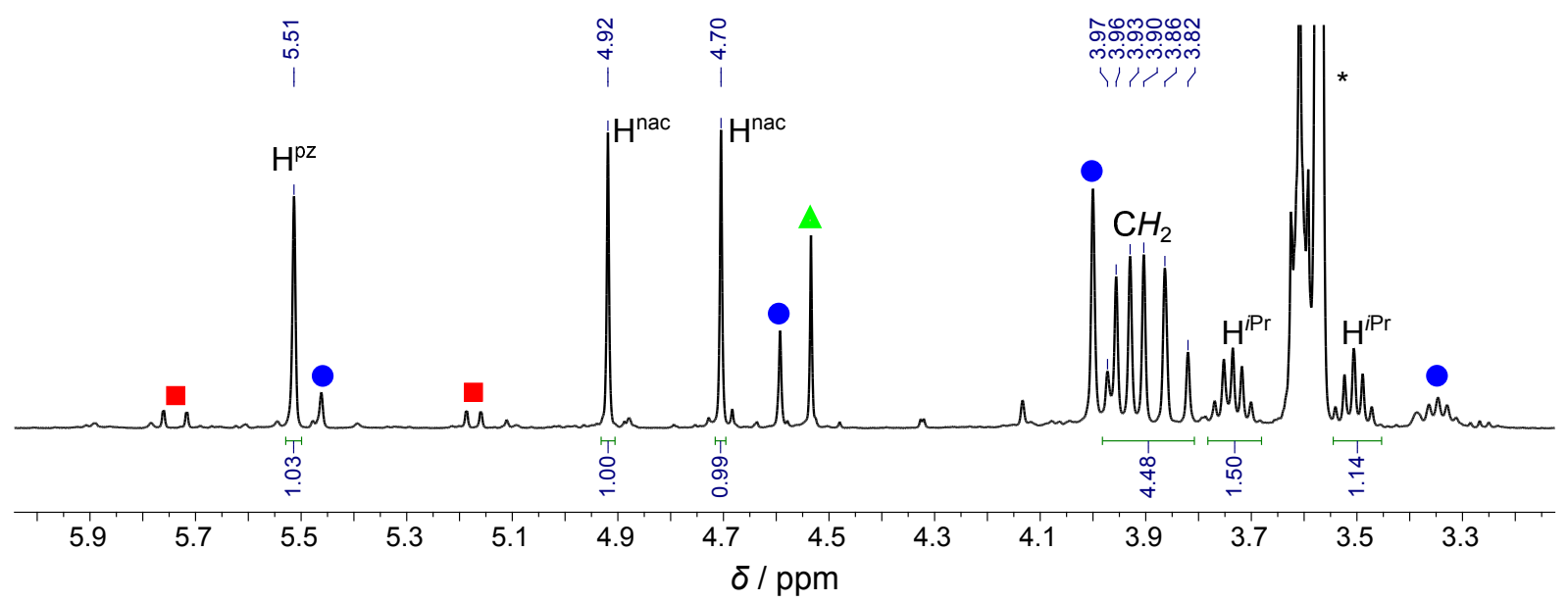

Figure 5.40.: Excerpt of the ${ }^{1} \mathrm{H}$ NMR spectrum $(400 \mathrm{MHz})$ recorded several weeks after treatment of a sample of $K\left[\mathrm{~L}^{\text {bisnac }} \mathrm{Ni}_{2}\left(\mu-\eta^{1}: \eta^{1}-\mathrm{CHCPh}\right)\right]$ dissolved in THF- $\mathrm{D}_{8}$ with 6 atm. of $\mathrm{H}_{2}(\Delta)$. Besides the major species $X$, the hydroxo-bridged compound [ $\left[\mathrm{L}^{\text {bisnac }} \mathrm{Ni}_{2}(\mu-\mathrm{OH})\right](\bullet)$, traces of styrene ( $\square$ ) which stem from the crystalline material of $\mathbf{K}\left[\mathrm{L}^{\text {bisnac }} \mathbf{N i}_{2}\left(\mu-\eta \eta^{1}: \eta^{1}-\mathbf{C H C P h}\right)\right]$ were present in the reaction mixture. Residual solvent is marked $\left({ }^{*}\right)$. 
Considering the fact that $\mathrm{H}_{2}$, which was used for this experiment, was added at high pressure (6 atm.), it was hardly possible to remove the residual moisture from the gas with the standard procedure (appendix, D.1). Under these conditions the formation of hydroxo based species was promoted. At the start of the experiment the second largest amount of unassigned complex $(21 \%)$ represented by the shoulder of the signal at $5.52 \mathrm{ppm}$ (Figure 5.39) was associated with a new upfield shifted resonance at $-7.31 \mathrm{ppm}$ by integral relation. This signal lies in close proximity to the resonance of $\left[\mathbf{L}^{\mathbf{b i s n a c}} \mathbf{N i}_{\mathbf{2}}(\mu-\mathbf{O H})\right]$ $(-7.26 \mathrm{ppm}$, Figure 5.41). Therefore, it was assumed that the second set of resonances belongs to a complex with similar properties like $\left[\mathrm{L}^{\text {bisnac }} \mathbf{N i}_{\mathbf{2}}(\mu-\mathrm{OH})\right]$.

Figure 5.41 shows ${ }^{1} \mathrm{H}$ NMR spectra of three performed experiments including $\mathbf{K}\left[\mathbf{L}^{\text {bisnac }} \mathbf{N i}_{\mathbf{2}}\left(\mu-\eta^{\mathbf{1}}: \eta^{\mathbf{1}} \mathbf{- C H C P h}\right)\right]$ and $\mathrm{H}_{2}(6$ atm). Spectrum (a) was recorded 10 hours after initiation. It was observed that a significant amount of $\mathbf{X}$ was formed $\left(\mathbf{K}\left[\mathbf{L}^{\text {bisnac }} \mathbf{N i}_{\mathbf{2}}(\mu-\right.\right.$ $\left.\left.\eta^{1}: \eta^{1}-\mathbf{C H C P h}\right)\right]$ was consumed). Nontheless, the amount of the proposed hydroxide-species $O$ was higher than that of $\left[\mathbf{L}^{\text {bisnac }} \mathbf{N i}_{2}(\mu-\mathbf{O H})\right]$. In another experiment (b), which was measured after one month the signal intensities of $\bigcirc$ and $\bullet\left(\left[\mathbf{L}^{\text {bisnac }} \mathbf{N i}_{\mathbf{2}}(\mu-\mathbf{O H})\right]\right)$ are similar. The third experiment (c) was measured after one month, similar to (b). However, in contrast to (a) and (b) the pressure-sample-tube exhibited a smaller diameter, which made an effective mixing by vigorous shaking impossible. A significant amount of $\left[\mathbf{L}^{\text {bisnac }} \mathbf{N i}_{\mathbf{2}}(\mu-\mathrm{OH})\right](\bigcirc)$ was still present while the amount of $\bigcirc$ was small.
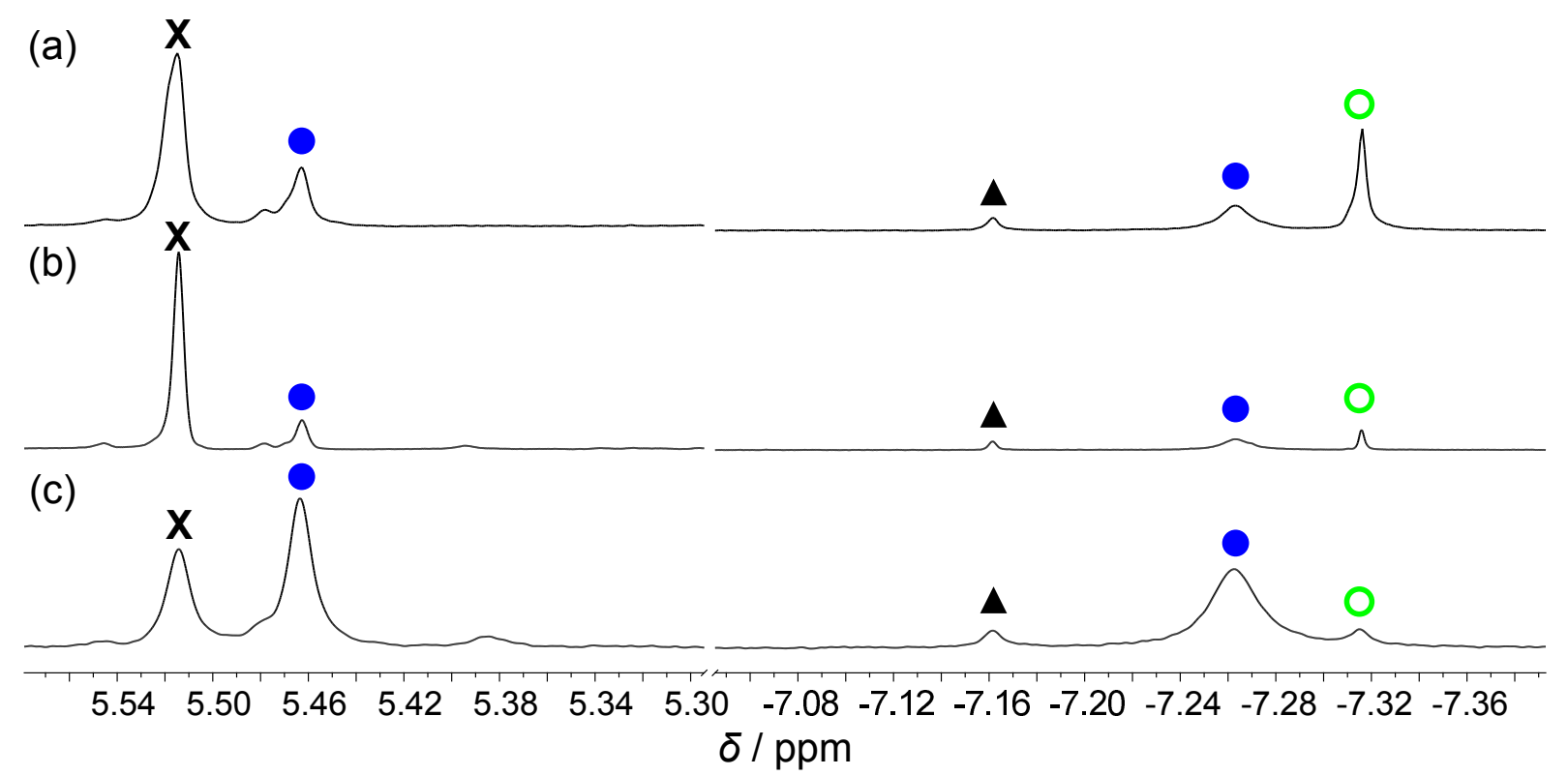

Figure 5.41.: ${ }^{1} \mathrm{H}$ NMR spectra $(400 \mathrm{MHz})$ of the conversion of $\mathrm{K}\left[\mathrm{L}^{\text {bisnac }} \mathrm{Ni}_{2}\left(\mu-\eta^{1}: \eta^{1}-\mathrm{CHCPh}\right)\right]$ under high pressure of $\mathrm{H}_{2}(6 \mathrm{~atm})$. The spectra are associated with three different experiments and were recorded after 10 hours (a) and one month (b) and (c). Samples (a) and (b) were shaken while (c) was not. Besides $\mathbf{X}$ and [ $\left.{ }^{\text {bisnac }} \mathrm{Ni}_{2}(\mu-\mathrm{OH})\right](\bullet)$, two other (probably hydroxo associated) species are observed $(\mathbf{\Lambda}$, O).

This indicates that $\mathbf{X}$ is formed from $O$, which in turn orignates from $\left[\mathbf{L}^{\text {bisnac }} \mathbf{N i}_{\mathbf{2}}(\mu-\mathrm{OH})\right]$ (๑). Furthermore, the build-up of $\bigcirc$ seems to be dependent on effective mixing of the 
solution. The third resonance $\boldsymbol{\Delta}$ may be attributable to another hydroxo-based species. It can be associated with an intermediate step either in the formation of $\left[\mathbf{L}^{\mathbf{b i s n a c}} \mathbf{N i}_{\mathbf{2}}(\mu-\right.$ $\mathrm{OH})]$ from $\mathrm{K}\left[\mathrm{L}^{\text {bisnac }} \mathbf{N i}_{2}\left(\mu-\eta^{\mathbf{1}}: \eta^{\mathbf{1}}-\mathbf{C H C P h}\right)\right]$ or $\mathrm{X}$ from $\left[\mathrm{L}^{\mathrm{bisnac}} \mathbf{N i}_{\mathbf{2}}(\mu-\mathrm{OH})\right]$.

Heating of sample $(\mathrm{c})\left(50^{\circ} \mathrm{C}\right)$ led to further generation of $\mathbf{X}$. The time course (Figure 5.42) shows that $\left[\mathbf{L}^{\text {bisnac }} \mathbf{N i}_{\mathbf{2}}(\boldsymbol{\mu}-\mathbf{O H})\right](\mathbf{O})$ is consumed while $\mathbf{X}$ increases. This provides further evidence that $\mathbf{X}$ originates from $\left[\mathbf{L}^{\text {bisnac }} \mathbf{N i}_{2}(\mu-\mathbf{O H})\right]$. The amount of the proposed intermediate $\mathbf{O}$ only slightly increases due to conversion to $\mathbf{X}$. After a short time, an equilibrium was formed at this temperature and production of $\mathbf{X}$ ceased again (control measurements after several weeks of heating did not indicate further progress).

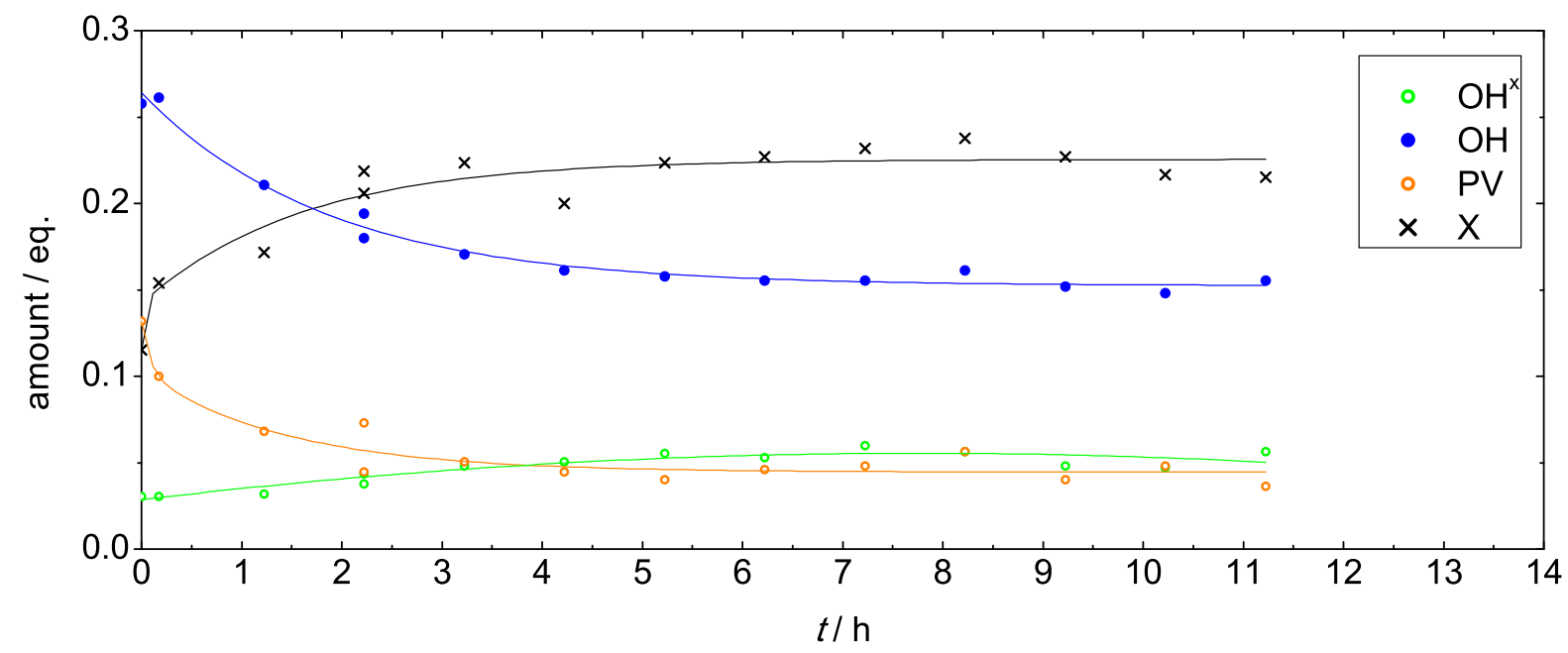

Figure 5.42.: Time course for the conversion of $\mathrm{K}\left[\mathrm{L}^{\text {bisnac }} \mathrm{Ni}_{2}\left(\mu-\eta^{1}: \eta^{1}-\mathrm{CHCPh}\right)\right]$ under high pressure of $\mathrm{H}_{2}(6 \mathrm{~atm})$ and at $50^{\circ} \mathrm{C}$ displayed as fitted plots. The unshaken sample was heated to promote the reaction, which ceased after one month at room temperature. At elevated temperature $\mathbf{K}\left[\mathrm{L}^{\text {bisnac }} \mathbf{N i}_{2}(\mu-\right.$ $\left.\left.\eta^{1}: \eta^{1}-\mathrm{CHCPh}\right)\right](0)$ and [ $\left.\mathrm{L}^{\text {bisnac }} \mathrm{Ni}_{2}(\mu-\mathrm{OH})\right](0)$ were further consumed while more $\mathbf{X}$ was formed. The proposed hydroxo-based species $\mathrm{OH}^{\mathrm{x}}(0)$ only showed a very small increase possibly due to a conversion to $\mathbf{X}$.

\subsubsection{Characterization}

${ }^{1} \mathrm{H}$ NMR spectra indicate the formation of a non-symmetric compound similar to the precursor $\mathbf{K}\left[\mathbf{L}^{\text {bisnac }} \mathbf{N i}_{\mathbf{2}}\left(\mu-\eta^{\mathbf{1}}: \eta^{\mathbf{1}}-\mathbf{C H C P h}\right)\right]$ (vide supra, Figure 5.40). In particular, the occurence of a doublet of doublet resonance between $3.82-3.97 \mathrm{ppm}$ with a prominent roof pattern was attributed to diastereotopic protons. A DEPT-135 measurement in combination with a ${ }^{1} \mathrm{H}_{-}{ }^{13} \mathrm{C}$ HSQC experiment confirmed the association to methylene groups containing diasterotopic protons (Figure 5.43). The asymmetry of the complex is also reflected by its complex resonance pattern in the aliphatic region of the ${ }^{1} \mathrm{H}$ NMR spectrum (Figure 5.44). To shed further light on the cause for asymmetry of $\mathbf{X}$ the aliphatic signals were assigned with additional support of $2 \mathrm{D}-\mathrm{NMR}$ data. It turned out that $\mathbf{X}$ contains four $\mathrm{CH}_{3}$ groups attributable to the methyl substituents of the $\beta$-diketiminate 
units of the system (grey markings in Figure 5.44). Six doublet resonances were assigned to isopropyl substituents of the DIPP sidearms (red markings in Figure 5.44). Two further singlet resonances, which were further shifted to the upfield are observed at $1.27 \mathrm{ppm}$ and $0.6 \mathrm{ppm}$.

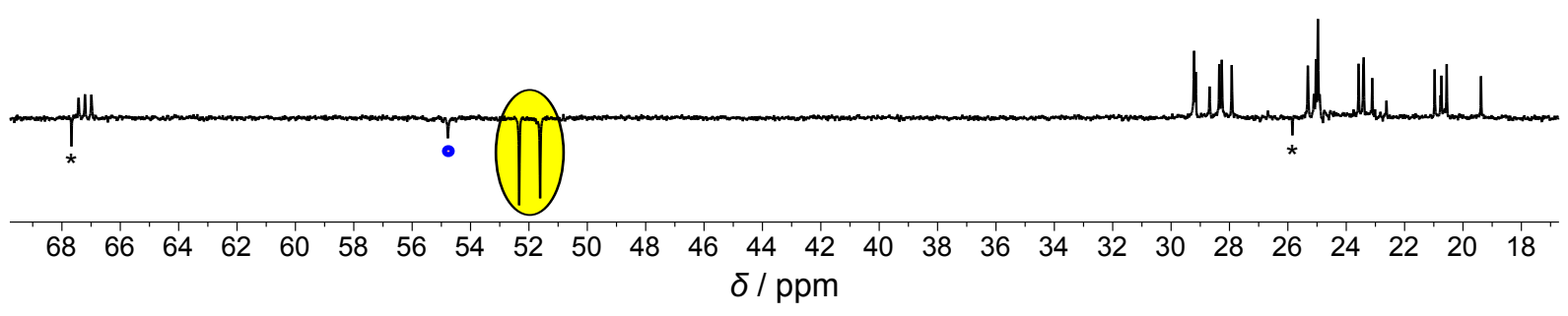

Figure 5.43.: Excerpt of the DEPT-135 spectrum (101 MHz) recorded several weeks after treatment of a sample of $\mathrm{K}\left[\mathrm{L}^{\text {bisnac }} \mathrm{Ni}_{2}\left(\mu-\eta^{1}: \eta^{1}-\mathrm{CHCPh}\right)\right]$ dissolved in THF-D $\mathrm{D}_{8}$ with $\mathrm{H}_{2}$ (6 atm.). Besides the $\mathrm{CH}_{2}$ carbon resonances of [ $\mathrm{L}^{\text {bisnac }} \mathrm{Ni}_{2}(\mu-\mathrm{OH})$ ] $(\bullet)$, two major resoances of same intensity are observed which were attributed to $\mathbf{X}$ (yellow marking). $\mathrm{CH}_{2}$ resonances of residual solvent are marked $\left({ }^{*}\right)$. .

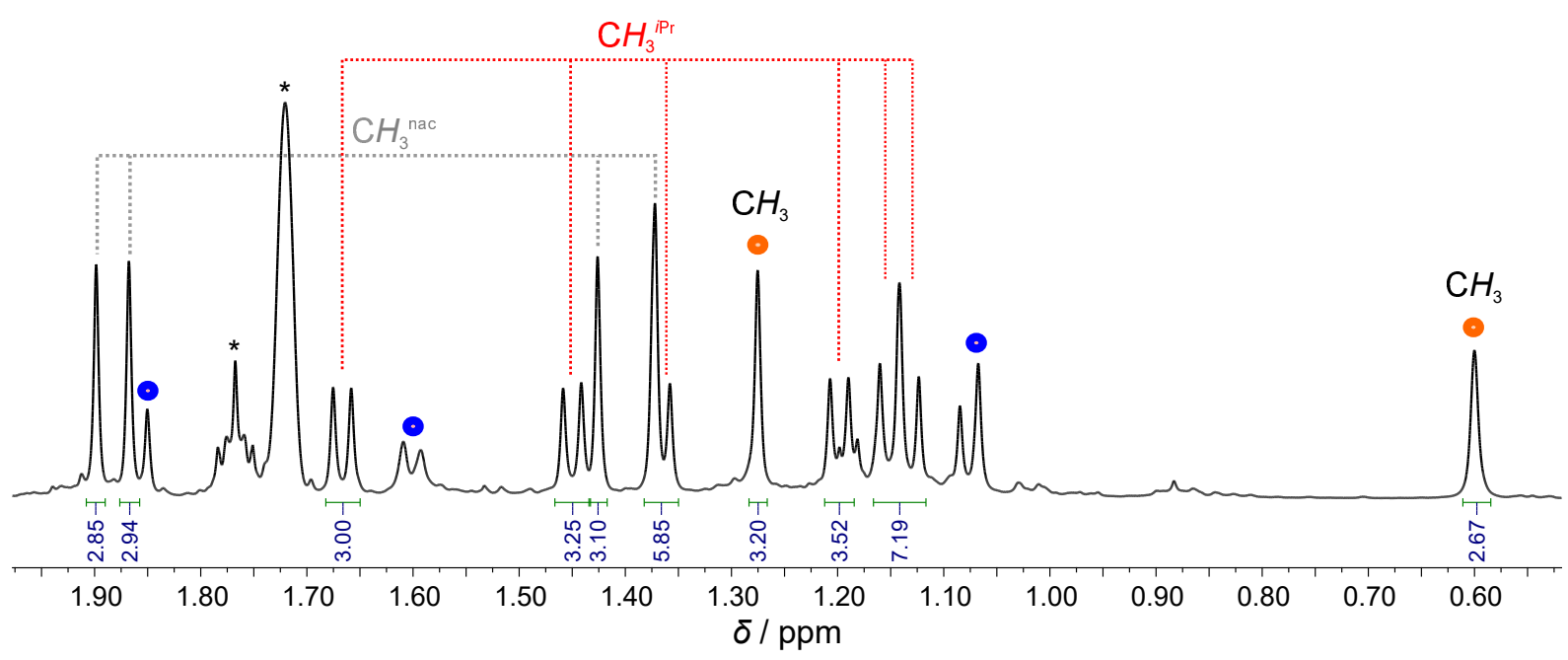

Figure 5.44.: Excerpt of the ${ }^{1} \mathrm{H}$ NMR spectrum (400 MHz) recorded several weeks after treatment of a sample of $K\left[L^{\text {bisnac }} \mathrm{Ni}_{2}\left(\mu-\eta^{1}: \eta^{1}-\mathrm{CHCPh}\right)\right]$ dissolved in THF-D $\mathrm{D}_{8}$ with $\mathrm{H}_{2}$ (6 atm.). Assignments of the resonances correlated to the new complex are indicated. In addition to the $\beta$-diketiminate $\left(\mathrm{CH}_{3}{ }^{\text {nac }}\right)$ and isopropyl methyl substituents $\mathrm{CH}_{3}{ }^{i P r}$ two unusual $\mathrm{CH}_{3}$ resonances are observed $(\Theta)$. Resonances associated with the side product $\left[\mathrm{L}^{\text {bisnac }} \mathrm{Ni}_{2}(\mu-\mathrm{OH})\right]$ are marked $(\bullet)$. Residual solvents are marked $\left(^{*}\right)$.

A ${ }^{1} \mathrm{H}-{ }^{1} \mathrm{H}$ COSY experiment revealed the correlation of these two signals by means of a ${ }^{4} J_{\mathrm{HH}}$ coupling. Additionally, NOE correlations between both signals were observed in a ${ }^{1} \mathrm{H}$ NOESY experiment ( $\square$ on the right in Figure 5.45), indicating a close spatial proximity of the groups. Moreover, the signal at $0.6 \mathrm{ppm}$ shows a NOE correlation ( $\square$ on the left in Figure 5.45 towards an aromatic doublet resonance at $6.5 \mathrm{ppm}$ while the resonance at $1.27 \mathrm{ppm}$ does not exhibit a correlation.

More information was obtained by a ${ }^{1} \mathrm{H}-{ }^{13} \mathrm{C}$ HMBC experiment. The detail of the contour plot illustrated in Figure 5.46 shows, besides the long range correlations between both of the methyl groups $(\bigcirc)$, even stronger correlations of the latter to a quarternary carbon 


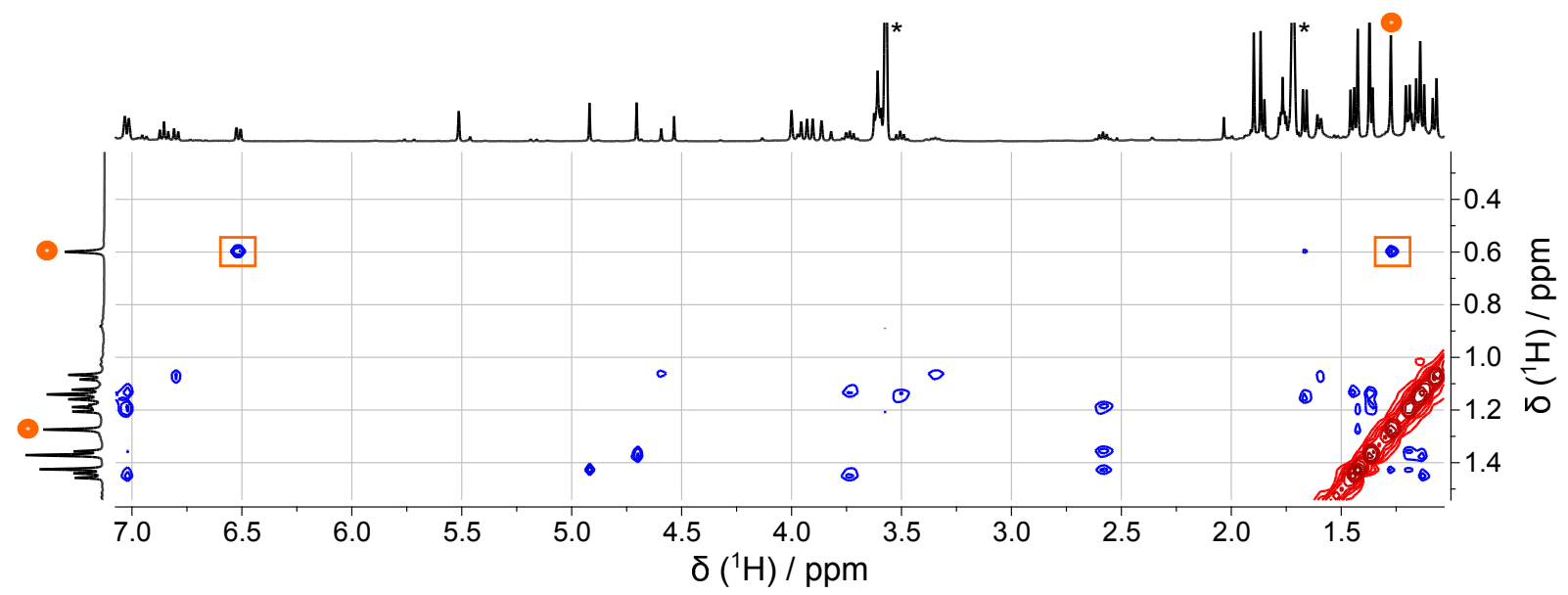

Figure 5.45.: Detail of the ${ }^{1} \mathrm{H}$ NOESY spectrum $(400 \mathrm{MHz})$ recorded several weeks after treatment of a sample of $K\left[L^{\text {bisnac }} \mathbf{N i}_{2}\left(\mu-\eta^{1}: \eta^{1}-\mathrm{CHCPh}\right)\right]$ dissolved in THF-D $\mathrm{D}_{8}$ with $\mathrm{H}_{2}$ (6 atm.). Correlations of one methyl group $(0.6 \mathrm{ppm})$ with an aromatic $\mathrm{CH}$ and the second methyl group $(\odot)$ are indicated.

atom, with a chemical shift of $67.78 \mathrm{ppm}(\boldsymbol{\square})$. Furthermore, a very weak correlation to a carbon atom lying in the aromatic range, was determined $(\square)$.

The information obtained by these NMR experiments, in particular the connection of both methyl substituents to a quarternary carbon atom, led to the conclusion that a metal-carbon bond was formed as a result of an internal $\mathrm{CH}$-activation.

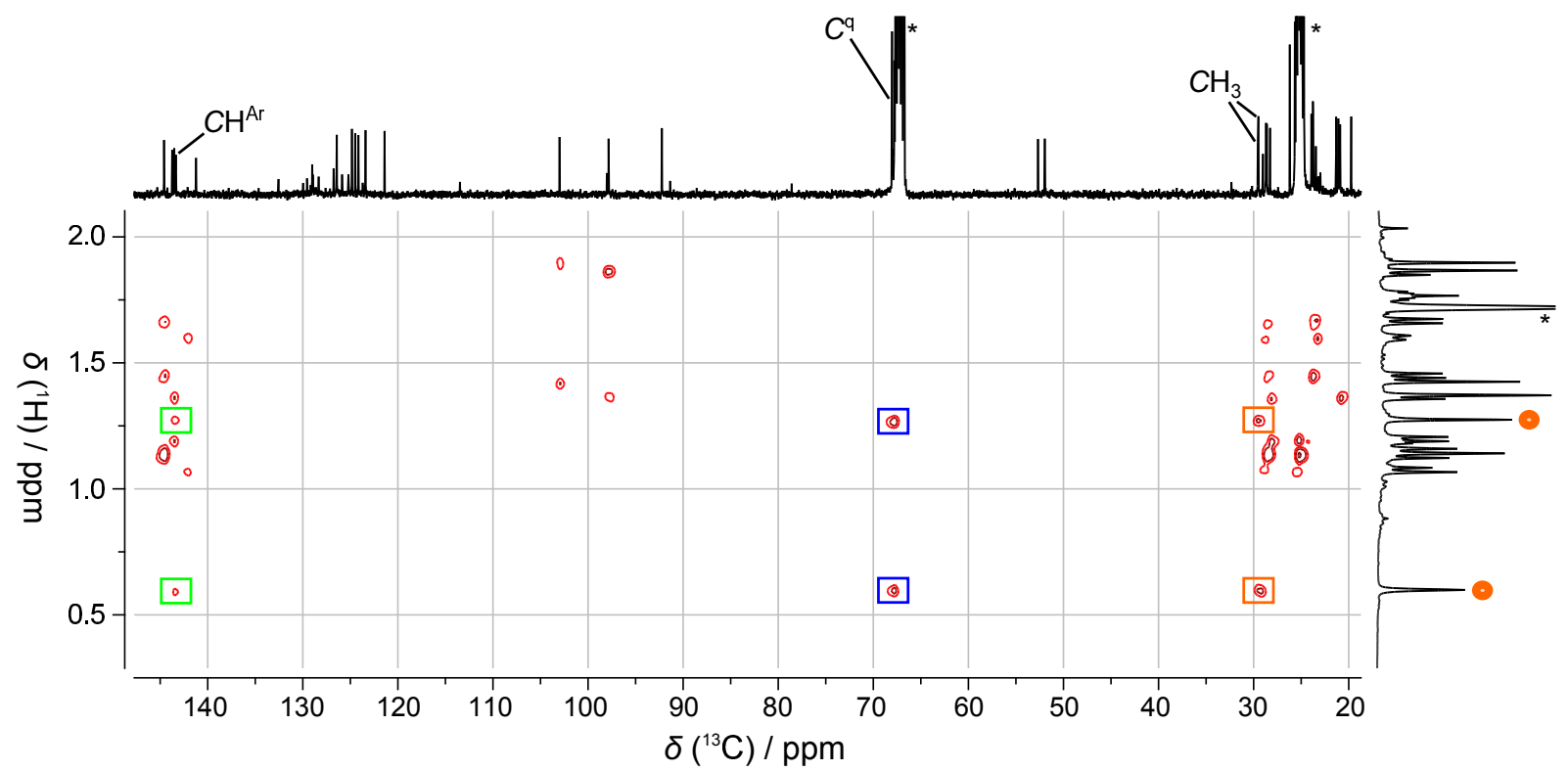

Figure 5.46.: Excerpt of the ${ }^{1} \mathrm{H}-{ }^{13} \mathrm{C}-\mathrm{HMBC}$ spectrum $(400 \mathrm{MHz}, 101 \mathrm{MHz})$ recorded several weeks after treatment of a sample of $K\left[L^{\text {bisnac }} \mathbf{N i}_{2}\left(\mu-\eta^{1}: \eta^{1}-\mathrm{CHCPh}\right)\right]$ dissolved in $T H F-D_{8}$ with $\mathrm{H}_{2}$ (6 atm.). Long range correlations of the two methyl groups $(\odot)$ to each other $(\square)$, to a quarternary carbon atom $(\square)$ and to an aromatic carbon atom $(\square)$ are indicated. Residual solvent resonances are marked $\left({ }^{*}\right)$. 


\subsubsection{Unusual Dynamic Effects}

At $-50{ }^{\circ} \mathrm{C}$ two new resonances at $13.21 \mathrm{ppm}$ and $-3.09 \mathrm{ppm}$ were observed, which are highlighted in spectrum (b) of Figure 5.47. This indicates that $\mathbf{X}$ features a dynamic effect. Both resonances correspond to a normalized integral of one. Further lowering the temperature led to a shift of the signals towards each other (at $-80{ }^{\circ} \mathrm{C}$ the chemical shifts were at 13.05 and $-1.94 \mathrm{ppm})$.

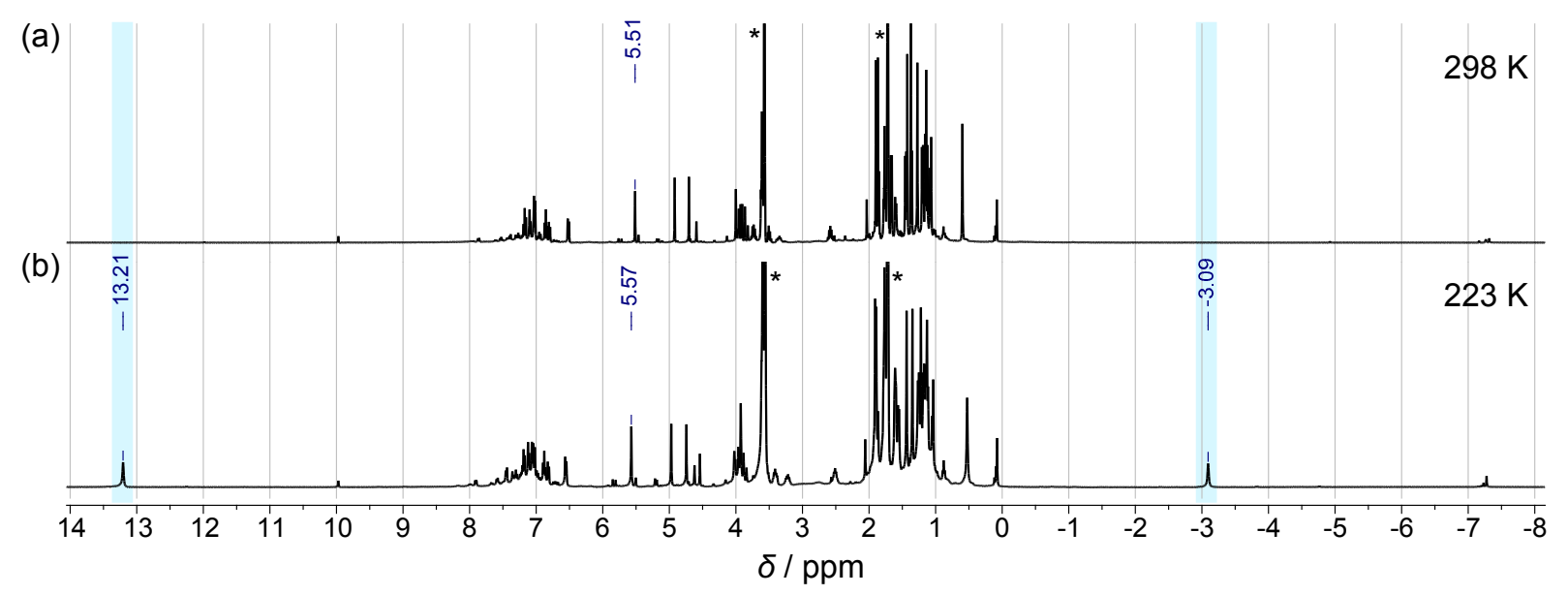

Figure 5.47.: Comparison of ${ }^{1} \mathrm{H}$ NMR spectra $(400 \mathrm{MHz})$, recorded several weeks after treatment of a sample of $\mathrm{K}\left[\mathrm{L}^{\text {bisnac }} \mathrm{Ni}_{2}\left(\mu-\eta^{1}: \eta^{1}-\mathrm{CHCPh}\right)\right]$ dissolved in THF- $\mathrm{D}_{8}$ with $\mathrm{H}_{2}$ (6 atm.). (a) represents a spectrum recorded at room temperature. At $-50{ }^{\circ} \mathrm{C}$ (b) two resonances at $-3.09 \mathrm{ppm}$ and $13.21 \mathrm{ppm}$ resolved. Residual solvent resonances are marked $\left({ }^{*}\right)$. .

In order to investigate the relation between the two signals ${ }^{1} \mathrm{H}-{ }^{1} \mathrm{H}$ COSY experiments at $-50{ }^{\circ} \mathrm{C}$ and $-100{ }^{\circ} \mathrm{C}$ were performed, revealing a coupling of the upfield and downfield shifted singlet resonances. The scalar coupling between both protons was confirmed by a double-quantum filtered ${ }^{1} \mathrm{H}-{ }^{1} \mathrm{H}$ COSY performed at $-100{ }^{\circ} \mathrm{C}$.

${ }^{1} \mathrm{H}$ NOESY spectra recorded at low temperature $\left(-50{ }^{\circ} \mathrm{C}\right.$ to $\left.-100{ }^{\circ} \mathrm{C}\right)$ revealed an extremely fast exchange between both protons. Lowering the temperature to $-100{ }^{\circ} \mathrm{C}$ yielded the spectrum shown in Figure 5.48.

The resonances exhibit NOE correlations to two methyl groups belonging to a metal coordinated isopropylmethyl-substituent of the DIPP sidearm and two hydrogen atoms belonging to the aryl ring of the latter. Three other stronger correlations were assigned to an isopropyl substituent, belonging to the other DIPP sidearm unit. The phase of correlations completely changed to positive sign (red cross peaks), making a discrimination between NOE and exchange directly from the 2D diagram difficult. To investigate a possible exchange between the yet unassigned protons, the setup of the NOESY measurement was changed to EXSY conditions with an applied mixing time of $t_{\mathrm{m}}=0.1 \mathrm{~s}$. Under these conditions the fast exchange was still occuring. Further lowering of $t_{\mathrm{m}}$ to even $10 \mathrm{~ms}$ still yielded a cross correlation in the recorded spectrum confirming a rapid exchange of both 
protons at $-100{ }^{\circ} \mathrm{C}$. These observations imply a fast exchange of both protons with each other. This can be caused by a facile rotation of the respective substituent.

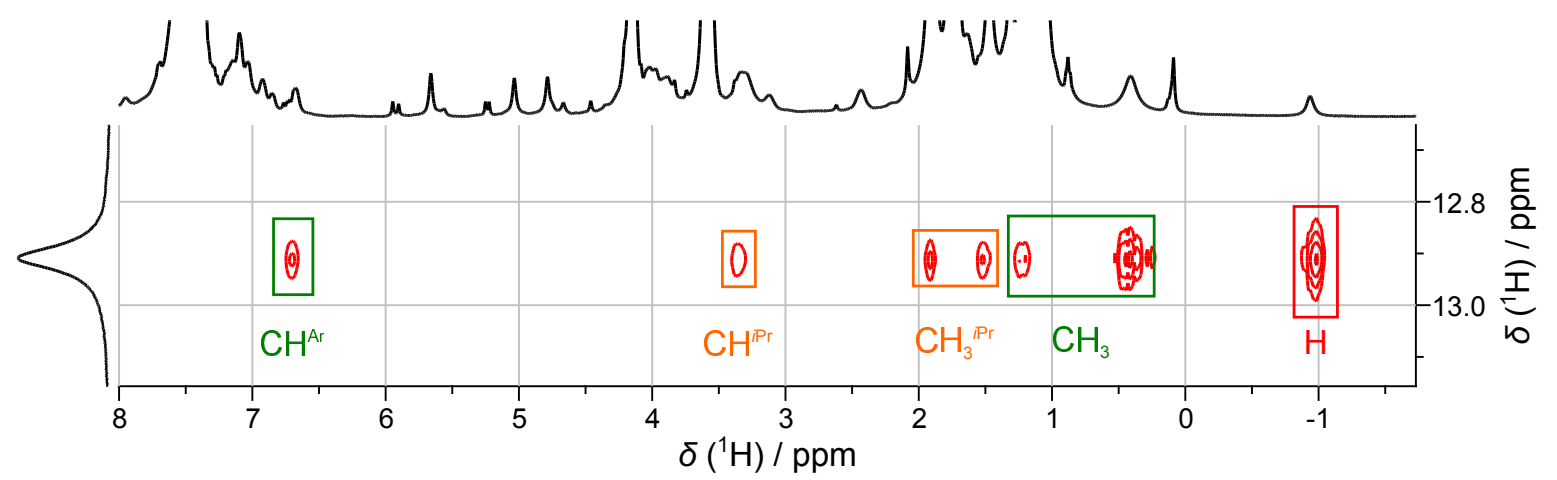

Figure 5.48.: Excerpt of the ${ }^{1} \mathrm{H}$ NOESY spectrum ${ }^{\ddagger}(400 \mathrm{MHz})$ measured at $-100{ }^{\circ} \mathrm{C}$ from a sample of $\mathrm{K}\left[\mathrm{L}^{\text {bisnac }} \mathrm{Ni}_{2}(\mathrm{CHCPh})\right]$ dissolved in THF-D 8 , treated with 6 atm. of $\mathrm{H}_{2} .^{\S}$

A similar phenomenon was discovered by CURTIS et al. with a $\left[\mathrm{Ni}(\text { diphosphine })_{2}\right]^{2+}$ complex, which reacts with dihydrogen to give a nickel hydride species with a proton bound to a bridging nitrogen atom of the diphosphine ligand. Upon cooling a solution of the compound in $\mathrm{CD}_{2} \mathrm{Cl}_{2}$ to $-90{ }^{\circ} \mathrm{C}$ two proton resonances at -15.2 and $7.4 \mathrm{ppm}$ were observed, which were assigned to the hydride ligand and the NH-proton. It was also found that a rapid intramolecular exchange of these protons occured. $\underline{[230]}$

For species $\mathbf{X}$ a ${ }^{1} J_{\mathrm{NH}}$ or ${ }^{1} J_{\mathrm{CH}}$ correlation was excluded by means of ${ }^{1} \mathrm{H}_{-}{ }^{13} \mathrm{C}$ HSQC and ${ }^{1} \mathrm{H}$ ${ }^{15} \mathrm{~N}-\mathrm{HMBC} / \mathrm{HSQC}$. Both experiments were performed at low temperature $\left(-50{ }^{\circ} \mathrm{C}\right.$ to $\left.-100{ }^{\circ} \mathrm{C}\right)$. Therefore, it was assumed that the hydrogen atoms belong to a $\mathrm{H}_{2} \mathrm{O}$ ligand. This assumption is corroborated by the fact that $\mathrm{X}$ originates from $\left[\mathrm{L}^{\mathbf{b i s n a c}} \mathbf{N i}_{\mathbf{2}}(\mu-\mathbf{O H})\right]$. A fast rotation of the small nickel coordinated $\mathrm{H}_{2} \mathrm{O}$ ligand around the metal-oxygen bond serves as an explanation for the fast exchange observed in the low temperature EXSY experiments.

\subsubsection{Intramolecular $\mathrm{C}-\mathrm{H}$ Activation}

Execution of a $\mathrm{H}-\mathrm{C}\left(\mathrm{sp}^{3}\right)$ bond activation is regarded as difficult and is usually facilitated by the pre-coordination of a substrate donor function. This is also applicable to the internal $\mathrm{C}-\mathrm{H}$ cleavage mediated by transition metal complexes. ${ }^{[231]}$ Two scenarios are feasible to realize such a process which are (i) an oxidative addition of the substrate to the metal and (ii) the heterolytic cleavage of the $\mathrm{C}-\mathrm{H}$ bond supported by a Brønsted base able to abstract a proton as illustrated in equation 5.8. In the latter case, the oxidation

${ }^{\ddagger}$ A change in sign of the NOE to positive occured at around $-50^{\circ} \mathrm{C}$.

$\S$ Similar correlations were observed for the resonance at $-3.1 \mathrm{ppm}$. 
state of the metal center remains unchanged $\left(\mathrm{M}^{\mathrm{n}+}\right) . \underline{[231]}$

$$
\mathrm{H}^{+}+(\mathrm{M}-\mathrm{R})^{(\mathrm{n}-1)+} \longleftarrow \mathrm{M}^{\mathrm{n}+}+\mathrm{R}-\mathrm{H} \longrightarrow \mathrm{R}-\mathrm{M}^{\mathrm{n}+2}-\mathrm{H}
$$

The oxidative addition of the isopropyl substituent to one of the nickel centers is accompanied by a change of oxidation state from II to IV, which is not reasoable for the nickel complex $\mathbf{X}$. This mechanism would also lead to formation of a nickel-hydride, however, the only upfield shifted signal was observed at $\delta\left\{{ }^{1} \mathrm{H}\right\} \approx-3 \mathrm{ppm}$. Considering the usually observed metal hydride resonances from -5 to $-30 \mathrm{ppm}$ and comparing to the far upfield shift of the hydrides in the complex $\mathbf{K}\left[\mathbf{L}^{\text {bisnac }} \mathbf{N i}_{\mathbf{2}}(\mathbf{H})_{\mathbf{2}}\right]\left(\delta\left\{{ }^{1} \mathrm{H}\right\}=-24 \mathrm{ppm}\right)$, the presence of a nickel hydride complex is improbable, too. $\stackrel{[\underline{136}-143]}{\underline{14}}$

Therefore, a heterolytic cleavage process of the isopropyl $\mathrm{C}-\mathrm{H}$ bond was envisioned. In an internal $\mathrm{C}-\mathrm{H}$ activation process the abstraction of the proton can either be effected by one of the adjacent nitrogen atoms belonging to the ligand system ${ }^{[231.232]}$ or the donor function of a potential bridging unit. This process then leads to a pentacoordinate nickel center with a square pyramidal coordination sphere in which the alkyl unit is coordinated in the apical position. Since many pentacoordinate low spin $\mathrm{Ni}^{\mathrm{II}}$ systems are known and NMR based evidence suggests a diamagnetic compound, this option seems viable. $\stackrel{[233-239]}{\underline{[2}}$ Nonetheless, a situation in which the $\mathrm{CH}$ proton is abstracted by a ligand nitrogen was excluded by the fact that no NH correlation was observed in ${ }^{15} \mathrm{~N}-\mathrm{HMBC}$ or HSQC experiments. Furthermore, the NMR experiments imply a complex geometry similar to the compounds which were discussed in the earlier part of this work. A cleavage of a metal-nitrogen bond results in an entirely different geometry, which would be reflected by distinct NMR resonances.

The proton of the $\mu-\mathrm{OH}$ unit in $\left[\mathbf{L}^{\text {bisnac }} \mathbf{N i}_{\mathbf{2}}(\mu-\mathrm{OH})\right]$ is known to be highly electronically shielded $\left(\delta\left\{{ }^{1} \mathrm{H}\right\}=-7.26 \mathrm{ppm}\right)$, also indicating a high electron density on the oxygen atom. The facile abstraction of a proton can be furnished by such a highly basic hydroxide. Another favourable factor is the close spatial proximity of the DIPP isopropyl substituents to the bridging unit, which is observed in NOESY experiments.

$\mathrm{C}-\mathrm{H}$ bond activations mediated by hydroxide complexes are reported in literature and can be effected via several possible pathways as illustrated in Scheme 5.12, ${ }^{[240-245]}$ Thereby, the mechanism of activation is often described as a 1,2-CH-addition across the metal-heteroatom bond. ${ }^{[241]}$ However, these studies were based on mononuclear complexes.

Considering the preorganized dinuclear compounds of this work, another mechanism for intramolecular $\mathrm{C}-\mathrm{H}$ activation would be more appropriate, that is a mechanism involving $\sigma$-bond metathesis. As illustrated in Scheme 5.12, in a mononuclear system the $\mathrm{C}-\mathrm{H}$ cleavage by $\sigma$-bond metathesis yields a metalalkyl complex as outcome of an intramolecular reaction, which is accompanied by the release of water. In a dinuclear system like 


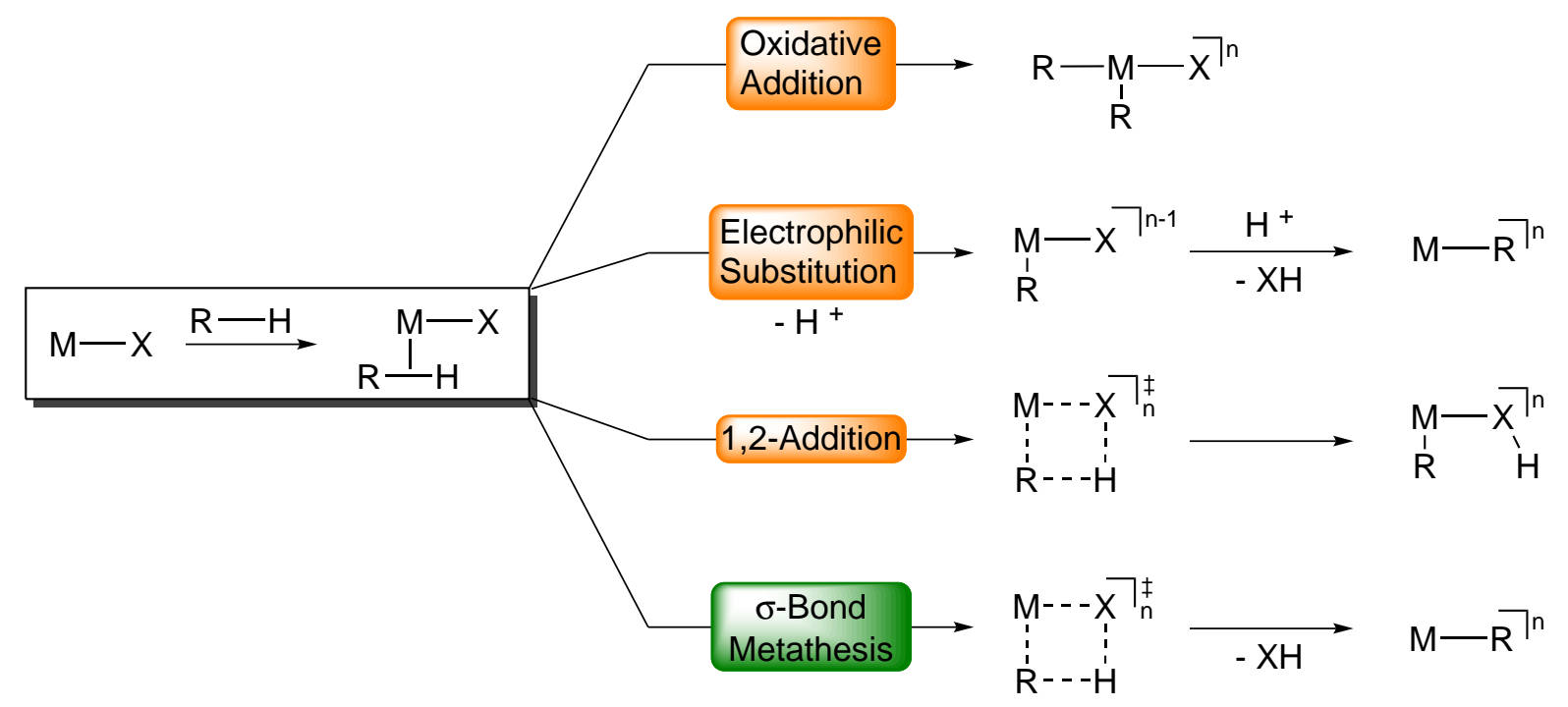

Scheme 5.12: Pathways for the metal-mediated $\mathrm{CH}$-activation $(X=O, O R, N R$, etc. $) .\left[\frac{[241]}{}\right.$

[L $\left.\mathbf{L}^{\text {bisnac }} \mathbf{N i}_{\mathbf{2}}(\mu-\mathrm{OH})\right]$, containing a bridging hydroxide, the $\sigma$-bond metathesis results in one alkyl coordinated nickel center while the other metal center most probably retains the formed water molecule.

This coordinated formal water molecule $\left(\mathrm{OH}^{-}, \mathrm{H}^{+}\right)$probably exhibits a fast exchange between its protons via rotation around the $\mathrm{Ni}-\mathrm{O}$ bond, which is in accordance with the observations made before. A possible stabilization of the proton $\left(\delta\left\{{ }^{1} \mathrm{H}\right\}_{223 \mathrm{~K}}=13.21 \mathrm{ppm}\right)$ can be effected by the $\pi$-system that belongs to the adjacent aryl sidearm. The already confirmed capability of a cation- $\pi$ interaction mediated by the DIPP sidearm substituents of the system (e.g. $\mathbf{K}\left[\mathbf{L}^{\text {bisnac }} \mathbf{N i}_{\mathbf{2}}(\mathbf{H})_{\mathbf{2}}\right]$ ) substantiates this possibility. Cation- $\pi$ interactions usually lead to a decrease of chemical shift of protons belonging to the cation $\left(\delta_{\mathrm{H}}\right.$ values are shifted upfield). Accordingly, chemical shift values of ligands increase (i.e. $\delta_{\mathrm{H}}$ values are shifted downfield) due to deshielding. $\underline{[246-249]}$

Although structurally distinct from $\left[\mathbf{L}^{\text {bisnac }} \mathbf{N i}_{\mathbf{2}}(\mu-\mathrm{OH})\right]$, downfield shifted signals of aryl hydrogen atoms are observed for $\mathbf{X}$. The difference between the most downfield shifted resonances $\left(\Delta \delta_{\mathrm{H}} \approx 0.2 \mathrm{ppm}\right)$ is comparable with ${ }^{1} \mathrm{H}$ NMR shift increases $\left(\Delta \delta_{\mathrm{H}} 0.1-0.3 \mathrm{ppm}\right)$ calculated for hydrogen halide $\cdots$ benzene complexes featuring $\mathrm{X}-\mathrm{H} \cdots \pi$ interactions $(\mathrm{X}$ $=\mathrm{F}, \mathrm{Cl}, \mathrm{Br}) .{ }^{[248]}$

The observed proton abstraction from the $\mathrm{CH}$ isopropyl-carbon atom is unusual since the expected result was that the $\mathrm{C}-\mathrm{H}$ activation would proceed at one of the methyl groups. ${ }^{[250,251]}$ A mechanistic proposal for the $\mathrm{C}-\mathrm{H}$ activation is depicted in Scheme 5.13. Cyclometalations are known to lead to highly stable products $\stackrel{[251]}{=}$ which is indicated by the formation of an additional five-ring chelate (- in Scheme 5.13). 


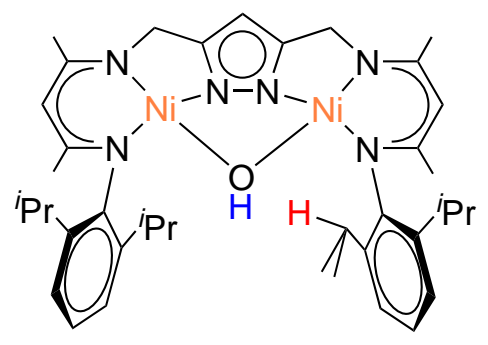

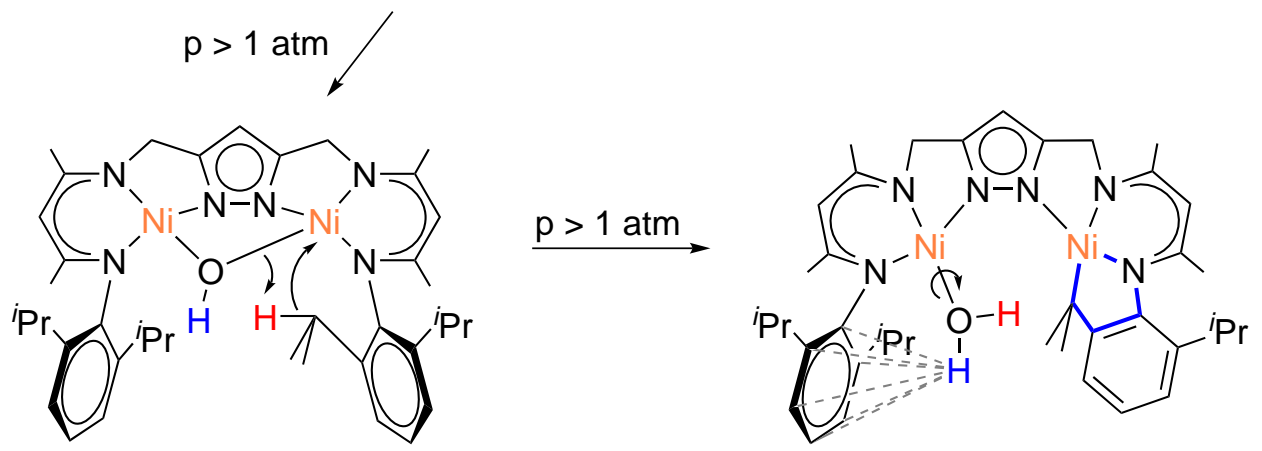

Scheme 5.13: Mechanistic hypothesis for the intramolecular $\mathrm{CH}$-activation mediated by [ $\mathrm{L}^{\text {bisnac }} \mathrm{Ni}_{2}(\mu-$ $\mathrm{OH})$ ] under high pressure.

\subsubsection{Conclusion}

In summary, it can be stated that the product of intramolecular $\mathrm{C}-\mathrm{H}$ activation did not initially originate from $\mathbf{K}\left[\mathbf{L}^{\text {bisnac }} \mathbf{N i}_{\mathbf{2}}\left(\mu-\eta^{\mathbf{1}}: \eta^{\mathbf{1}}-\mathbf{C H C P h}\right)\right]$ itself but was rather the result of a two step side reaction caused by traces of water in the sample mixture.

After the formation of $\left[\mathbf{L}^{\text {bisnac }} \mathbf{N i}_{\mathbf{2}}(\boldsymbol{\mu}-\mathbf{O H})\right]$ the intramolecular $\mathrm{C}-\mathrm{H}$ activation proceeded in a subsequent step, likely via $\sigma$-bond metathesis, to yield a new non-symmetric complex of neutral charge. The compound features a cyclometalated $\mathrm{Ni}^{\mathrm{II}}$ center and a formal water ligand coordinated to the other $\mathrm{Ni}^{\mathrm{II}}$. This new diamagnetic species can be considered as the more thermodynamically stable product. Effective mixing of the solution containing $\mathbf{K}\left[\mathbf{L}^{\text {bisnac }} \mathbf{N i}_{\mathbf{2}}\left(\mu-\eta^{\mathbf{1}}: \eta^{\mathbf{1}}-\mathbf{C H C P h}\right)\right]$ and high pressures of $\mathrm{H}_{2}$ led to immediate formation of $\mathbf{X}$. This conversion is dependent on the applied pressure since experiments performed at atmospheric conditions did not lead to $\mathrm{C}-\mathrm{H}$ activation. In a non-mixed solution an early equilibrium is reached, in which only a small part of $\left[\mathrm{L}^{\text {bisnac }} \mathbf{N i}_{2}(\mu-\mathrm{OH})\right]$ is converted. Heating of the solution promotes the $\mathrm{C}-\mathrm{H}$ activation process, indicating that the generation of the corresponding product is kinetically inhibited. 


\subsection{Summary and Outlook}

Treatment of $\mathbf{K}\left[\mathbf{L}^{\text {bisnac }} \mathbf{N i}_{\mathbf{2}}(\mathbf{H})_{\mathbf{2}}\right]$ with phenylacetylene led to the formation of a novel phenylvinyl-bridged complex which was characterized in detail. By means of isotopic labeling experiments with deuterium a proposal of the mechanistic pathway for the incorporation of the bridging unit was established. It was shown that the $\mathrm{Ni}-\mathrm{H}$ hydrogen atoms of $\mathbf{K}\left[\mathbf{L}^{\text {bisnac }} \mathbf{N i}_{\mathbf{2}}(\mathbf{H})_{\mathbf{2}}\right]$ are substituted by the substrate (PA) in a pairwise fashion yielding $\mathrm{H}_{2}$ in the process. In this regard the substitution of $\left.\mathbf{K}\left[\mathbf{L}^{\text {bisnac }} \mathbf{N i}_{\mathbf{2}} \mathbf{( H}\right)_{\mathbf{2}}\right]$ with PA is similar to the exchange/substitution with $\mathrm{D}_{2}$ and indicates an analogous mechanism. $\mathbf{K}\left[\mathbf{L}^{\text {bisnac }} \mathbf{N i}_{\mathbf{2}}\left(\mu-\eta^{\mathbf{1}}: \eta^{\mathbf{1}}-\mathbf{C H C P h}\right)\right]$ has shown to be capable of catalyzing the selective hydrogenation of phenylacetylene to styrene in an $\mathrm{H}_{2}$ atmosphere at room temperature, albeit with low rates. Additional isotopic labeling and hyperpolarization experiments using parahydrogen were conducted to investigate the stereochemical aspects for the hydrogenation. It was found that the hydrogenation process initially yields the cis-hydrogenated product styrene. By means of deuterium labeling it was further found that after incipient hydrogenation full deuteration of the olefin substituent occured, probably due to a H/D exchange reaction, mediated by the complex.

Full conversion of the substrate was reached when using an excess amount of phenylacetylene indicating a minimum turnover number of 15 according to the used amount of substrate. After consumption of phenylacetylene through this semihydrogenation process, full hydrogenation of styrene to ethylbenzene was promoted. However, this process did not lead to the entire consumption of styrene under the present conditions.

Although application of higher pressures of $\mathrm{H}_{2}$ resulted in greater initial conversion rates, side reactions mediated by traces of water were significantly enchanced leading to poisoning of the catalyst and accompanied formation of the $\mu$-hydroxo complex $\left[\mathbf{L}^{\text {bisnac }} \mathbf{N i}_{\mathbf{2}}(\mu-\right.$ $\mathbf{O H})]$. NMR spectroscopy showed that in $\left[\mathbf{L}^{\text {bisnac }} \mathbf{N i}_{2}(\mu-\mathbf{O H})\right]$ an intramolecular $\mathrm{C}-\mathrm{H}$ activation process occurs at the tertiary carbon of an isopropyl substituent belonging to one of the DIPP moieties. Elevated temperatures and effective mixing of the solution at high pressure contribute to the conversion. Similar to $\left[\mathbf{L}^{\text {bisnac }} \mathbf{N i}_{\mathbf{2}}(\mu-\mathbf{O H})\right]$ the stable product of intramolecular $\mathrm{C}-\mathrm{H}$ activation did not show any catalytic activity in regards of the semihydrogenation of phenylacetylene.

Nonetheless, with the overall information obtained by the conducted studies a mechanism for the catalytic semihydrogenation process was proposed. Future works could deal with a thorough establishment of the catalytic cycle through optimized experimental procedure (minimization of catalyst poisoning by water) and with the support of DFT calculations. Additional ligand modifications with particular respect to steric properties and sidearm alterations are thought to result in a more efficient semihydrogenation catalyst.

Alhough NMR spectroscopy indicates that the product of intramolecular $\mathrm{C}-\mathrm{H}$-activation $(\mathrm{X})$ originates from $\left[\mathbf{L}^{\text {bisnac }} \mathbf{N i}_{\mathbf{2}}(\mu-\mathrm{OH})\right]$, high pressure experiments including the isolated 
$\mu$-OH complex can confirm this. The pressure dependency can be further proven by use of $\mathrm{N}_{2}$ or argon instead of $\mathrm{H}_{2}$.

The insight, that the hydroxo-bridged compound $\left[\mathbf{L}^{\text {bisnac }} \mathbf{N i}_{\mathbf{2}}(\mu-\mathrm{OH})\right]$ is capable to perform an intramolecular $\mathrm{C}-\mathrm{H}$ activation reaction suggests further investigation in the direction of catalytic intermolecular $\mathrm{C}-\mathrm{H}$ activation. For this purpose the aryl sidearms can be modified to exert less steric shielding on the cavity. This offers the additional advantage of a minimized intramolecular $\mathrm{C}-\mathrm{H}$ activation processes due to the increased spatial distance. 



\section{New Model Complexes of Intermediates in the Process of Nitrogen Fixation}

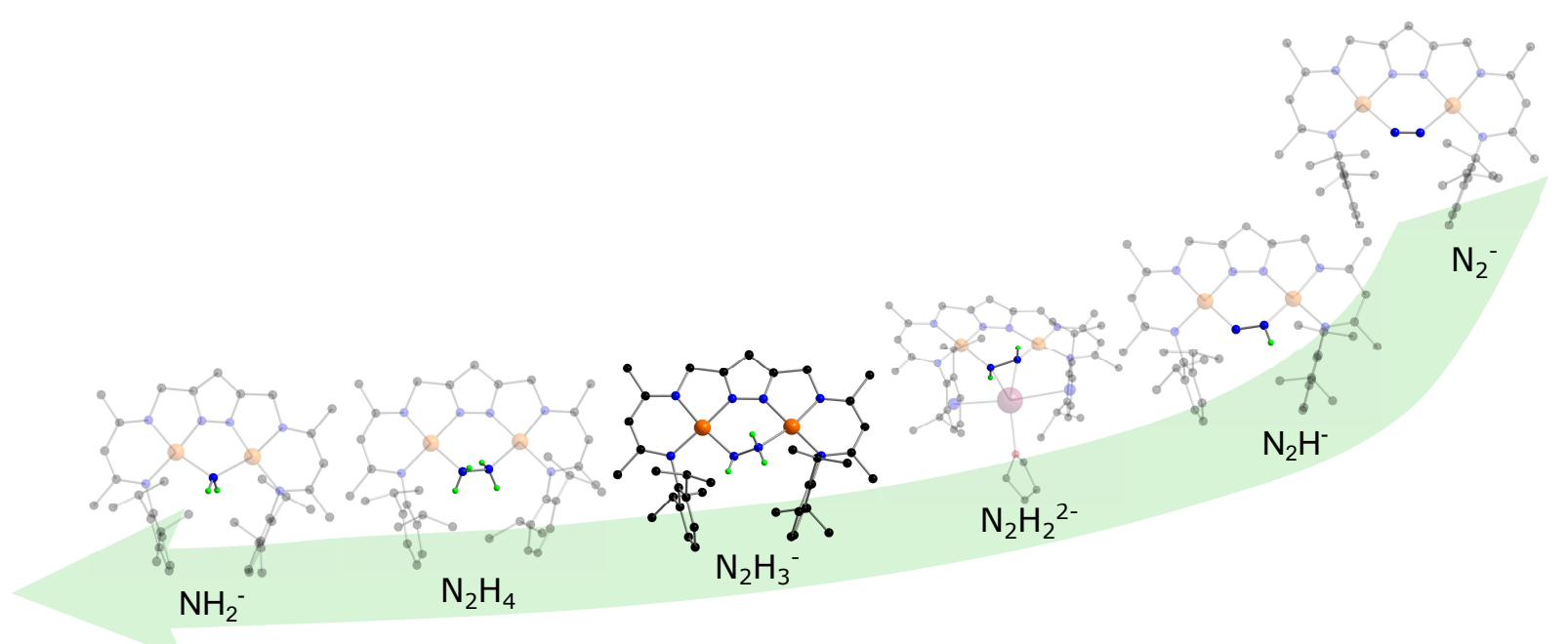

Abstract In the context of dinitrogen fixation the synthesis of new model complexes including the new "bisnac" system was achieved. The previously discussed $\left[\mathbf{L}^{\text {bisnac }} \mathbf{N i}_{\mathbf{2}}(\mu-\right.$ $\mathbf{B r})]$ and $\mathbf{K}\left[\mathbf{L}^{\text {bisnac }} \mathbf{N i}_{\mathbf{2}}(\mathbf{H})_{2}\right]$ served as precursors for the preparation. For instance, an end-on $\mathrm{N}_{2} \mathrm{H}_{3}{ }^{-}$hydrazido bridged dinickel system was established, which proved to be an excellent platform for the establishment of various related nitrogen based species. The scope of prepared complexes includes $\mu-\eta^{1}: \eta^{1}$-ligands like $\mathrm{N}_{2} \mathrm{H}_{4}, \mathrm{~N}_{2} \mathrm{H}_{3}{ }^{-}, \mathrm{N}_{2} \mathrm{H}_{2}{ }^{2-}, \mathrm{N}_{2} \mathrm{H}^{-}$, $\mathrm{N}_{2}{ }^{-}$and $\mu-\mathrm{NH}_{2}{ }^{-}$, which are supposed to represent important intermediates in the process of $\mathrm{N}_{2}$ fixation. 


\subsection{Introduction}

Approximately one third of the world's population relies on fertilizers for agricultural food production, which is constantly growing due to the increasing population on earth. $\stackrel{[252]}{[}$ Since these fertilizers are mainly based on ammonia, the need for this simple precursor is quite demanding - the annual production of ammonia amounts up to 160 million tonnes. $\stackrel{[252]}{ }$

While some microorganisms are able to generate ammonia from dinitrogen at ambient temperature and pressure via nitrogenases, mankind uses an artificial method to fix $\mathrm{N}_{2}$, also known as the Haber-Bosch process. In contrast to nature's ability to activate atmospheric $\mathrm{N}_{2}$ whose triple bond, nonpolarity and high ionization potential make it a very inert molecule, industrial generation of $\mathrm{NH}_{3}$ requires conditions of 200-400 atm and 400-800 ${ }^{\circ} \mathrm{C}$. Consequently, the Haber-Bosch process demands a very high amount of energy which sums up to over $1 \%$ of the world's energy produced every year. ${ }^{[252-254]}$

Therefore, one goal for industry and research over the last decades was to find a suitable method to catalytically produce ammonia from atmospheric nitrogen under ambient conditions. ${ }^{[252,255,256]}$ CHATT's pioneering work provided a mechanistic outline to approach this problem. ${ }^{[257]}$ It is now often referred to as the "Chatt"- or "distal"- mechanism. ${ }^{[253]}$ In this case, the hydrogenation takes place at the distal nitrogen atom of the coordinated $\mathrm{N}_{2}$, which eventually leads to the release of $\mathrm{NH}_{3}$ giving a nitrido complex..$^{[80]}$

So far only a few molybdenum based systems reported by NiSHIBAYASHI and SCHROCK and an iron based system by PETERs are capable to catalytically produce $\mathrm{NH}_{3}$ directly from $\mathrm{N}_{2}$, although in a limited fashion..$^{[253,258,259]}$

Molybdenum and iron are receiving a lot of attention since both metals are part of the Mo-dependent nitrogenase, whose active center is the iron-molybdenum cofactor (FeMoco).$\stackrel{[260]}{]}$ Moreover, iron is part of other kinds of nitrogenase enzymes and is, besides ruthenium, the base of the most effective catalysts in the Haber-Bosch process. $\stackrel{[256]}{\left[-{ }^{2}\right.}$ In further work by Holland et al. the reduction and hydrogenation of $\mathrm{N}_{2}$ to $\mathrm{NH}_{3}$ by a molecular iron potassium complex of a $\beta$-diketiminato ligand was achieved. ${ }^{[99]}$ Moreover, QU et al. have recently synthesized a diiron complex, featuring a $\left[\mathrm{Fe}_{2} \mathrm{~S}_{2}\right]$ scaffold, which is able to accomodate several nitrogenous donors. ${ }^{[252]}$ In particular, $\mu-\eta^{1}: \eta^{1}$ diazene, $\mu-\eta^{1}: \eta^{2}$ hydrazido $\left(\mathrm{N}_{2} \mathrm{H}_{3}{ }^{-}\right)$and amide bridged compounds were isolated. After stepwise reductive protonation, the diazene unit was transformed to $\mathrm{NH}_{2}{ }^{-}$via a $\mathrm{N}_{2} \mathrm{H}_{3}$ - intermediate, accompanied by release of ammonia. Theoretical calculations using DFT revealed, that $\mathrm{N}_{2} \mathrm{H}_{2}, \mathrm{~N}_{2} \mathrm{H}_{3}{ }^{-}$and $\mathrm{NH}_{2}{ }^{-}$are the most probable species which occur within natural enzymes during the reduction process of $\mathrm{N}_{2} \cdot{ }^{[252]}$

These particular nitrogen species are part of the so called "alternating" nitrogen reduction pathway. In contrast to the "distal" pathway, an alternating sequence of protonation and 
reduction of the $\mathrm{N}-\mathrm{N}$ bond at various different stages (Scheme 6.1) occurs. It is based on recent spectroscopic studies and is considered as a very likely pathway for dinitrogen reduction by nitrogenase. $\frac{[80,261,262]}{}$

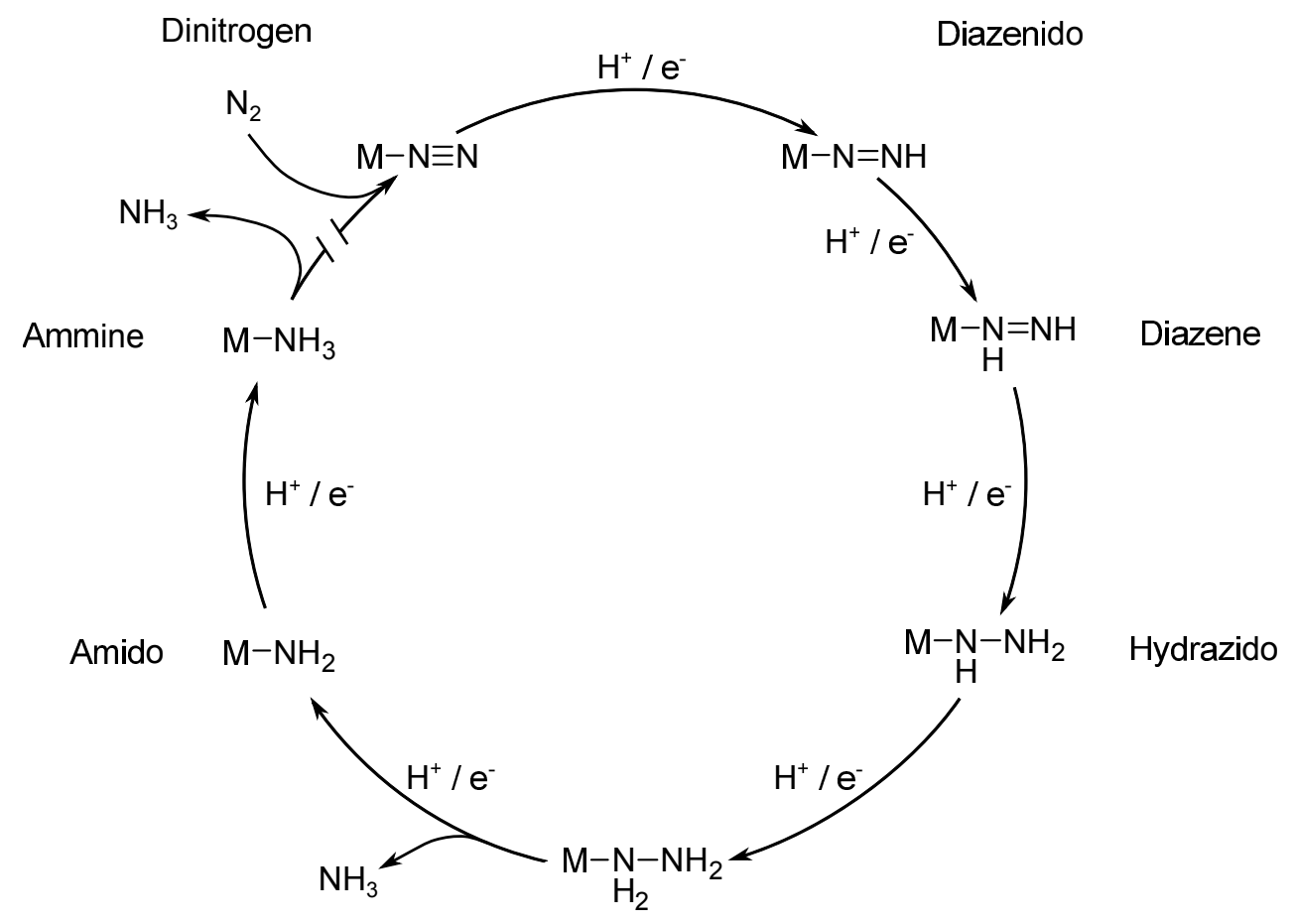

Hydrazine

Scheme 6.1: The alternating pathway as one possible reaction mechanism for nitrogenase. The different nitrogen species bound to the FeMo-cofactor (M) are indicated by names. ${ }^{[\underline{\underline{80}}]}$

Despite the high interest in Mo or Fe based systems, complexes of various other transition metals and even non-transition metals were investigated. ${ }^{[263]}$ Although the $\mathrm{N}_{2}$ molecule tends to be strongly activated and cleaved by early transition metal atoms, the resulting complexes often contain metal-nitrogen bonds, which prevent further conversions. ${ }^{[80]}$ For this reason, complexes of late transition metals are also receiving more and more interest. $\stackrel{[33,65,80,264-267]}{-2}$

For instance, Holland et al. also investigated a cobalt-complex of a $\beta$-diketiminato ligand, which is capable to accomodate $\mathrm{N}_{2}$ by formation of a dimer, which significantly weakens the $\mathrm{N}-\mathrm{N}$ bond. ${ }^{[65}$

Establishment of an end-on $\mathrm{N}_{2}$ bridged system $\left[\mathrm{L}^{t \mathrm{Bu}} \mathrm{Ni}\left(\mu-\eta^{1} ; \eta^{1}-\mathrm{N}_{2}\right) \mathrm{NiL}{ }^{t \mathrm{Bu}}\right]$ by LIMBERG et al., which was already mentioned in Chapter 2 of this work, allowed for comparison of a series of first-row late transition metal ( $\mathrm{Fe}, \mathrm{Co}, \mathrm{Ni}$ ) dinitrogen complexes with the same bulky $\beta$-diketiminate ligand. ${ }^{[67.80]}$ Based on derived $\mathrm{N}-\mathrm{N}$ bond lengths and $\mathrm{N}-\mathrm{N}$ stretching vibrations of these compounds, a nitrogen activation trend could be established. Although the shorter bond length and the associated higher stretching vibration wavenumber revealed that the nickel-based complex has the lowest activation potential in the series, it still showed a unique reduction chemistry, unlike the Fe and Co analogues. Additionally, 
using potassium graphite, it was possible to accomplish singly and doubly reduced forms of the $\mathrm{N}-\mathrm{N}$ bridged complex.

Besides other reported complexes of the same nickel $\beta$-diketiminato scaffold containing side-on bonded $\mathrm{N}_{2} \mathrm{H}_{2}, \mathrm{~N}_{2} \mathrm{H}_{3}{ }^{-}, \mathrm{N}_{2} \mathrm{H}_{4}$ and $\mathrm{NH}_{3}$ ligands, a $\mathrm{Ni}^{\mathrm{II}}$ hydrazido complex $\left[\mathrm{L}^{t \mathrm{Bu}} \mathrm{Ni}\left(\eta^{2}-\mathrm{N}_{2} \mathrm{H}_{3}\right)\right]$ was established by reacting a $\mathrm{Ni}^{\mathrm{II}}$ bromide precursor with an excess of hydrazine..$^{\left[\frac{58,79]}{3}\right.}$ The latter was deprotonated to yield a side-on bound diazene $\mathrm{Ni}^{\mathrm{II}}$ complex. ${ }^{[58]}$ Protonation led to reformation of the hydrazido species, and further addition of a weak acid resulted in a hydrazine complex, which was also reversible..$^{[58]}$

Furthermore, it was observed that at elevated temperatures the hydrazido complex decomposed to give an ammine complex as a result of hydrazine disproportionation. More interestingly, this ammine complex reacts with $\mathrm{KC}_{8}$ under atmospheric $\mathrm{N}_{2}$, which leads to release of $\mathrm{NH}_{3}$ and generation of the end-on bridged diazene compound $\mathrm{K}_{2}\left[\mathrm{~L}^{t \mathrm{Bu}} \mathrm{Ni}\right.$ $\left.\left(\mu-\eta^{1}: \eta^{1}-\mathrm{N}_{2}\right) \mathrm{NiL}{ }^{t \mathrm{Bu}}\right] .{ }^{\left[\frac{79]}{}\right.}$ In particular, regeneration of the $\mathrm{Ni}-\mathrm{N}_{2}-\mathrm{Ni}$ moiety represents an important step in any catalytical nitrogen fixation and conversion to $\mathrm{NH}_{3} \cdot{ }^{\left[{ }^{[80}\right]}$

\subsection{Motivation}

Despite the fact that in comparison to cobalt and iron based complexes of the bulky $\beta$-diketiminato ligand system the nickel analogue showed the lowest nitrogen activation potential, the promising results of LIMBERG significantly encouraged the investigation of the dinickel bis( $\beta$-diketiminate) system in the context of nitrogen activation.

In contrast to the previously reported systems, a highly preorganized dinuclear complex can provide better cooperation between both metal-centers, which could be favourable for potential nitrogen activation. ${ }^{\left[{ }^{54]}\right.}$ Establishment of the dinuclear bridged $\mathrm{Ni}^{\mathrm{II}}$ complexes [L $\left.\mathbf{L}^{\text {bisnac }} \mathbf{N i}_{2}(\mu-\mathrm{Br})\right], \mathbf{K}\left[\mathbf{L}^{\text {bisnac }} \mathbf{N i}_{2}(\mathbf{H})_{2}\right]$ and $\mathbf{K}\left[\mathbf{L}^{\text {bisnac }} \mathbf{N i}_{2}\left(\mu-\eta^{1}: \eta^{1}-\mathbf{C H C P h}\right)\right]$ revealed that the dinickel system is capable of accomodating bridging ligands of different steric demand by variation of the $\mathrm{Ni} \cdot \cdots \mathrm{Ni}$ distance.

In particular, the dihydride possesses the capability to coordinate alkali-cations via $\pi$-cation interactions with its aryl sidearms similar to the systems of LIMBERG or HOLLAND. Hence, a comparable redox chemistry was envisioned for the bisnac system. Moreover, steric demand of the bulky $\beta$-diketiminate sidearms can cause a strain of the cavity especially if a potassium cation is coordinated between the aryl sidearms as seen in $\mathbf{K}\left[\mathbf{L}^{\text {bisnac }} \mathbf{N i}_{\mathbf{2}}(\mathbf{H})_{\mathbf{2}}\right]\left(d_{\mathrm{Ni}-\mathrm{Ni}}=4.159 \AA\right)$. This strain can prove particularly advantageous in supporting further weakening of the strong bond of a potentially $\mu-\eta^{1}: \eta^{1}$ bridged $N_{2}$ molecule. 


\subsection{Preparation of an end-on Bridged Hydrazido Complex}

Since they have been postulated as intermediates in the conversion process of $\mathrm{N}_{2}$ to $\mathrm{NH}_{3}$, hydrazine transition metal complexes are of substantial interest in chemistry. ${ }^{[58,252,262]}$ Thus incorporation of hydrazine into the cavity of $\left[\mathbf{L}^{\mathbf{b i s n a c}} \mathbf{N i}_{\mathbf{2}}()\right]$ was envisioned to establish a fundament for further investigations of redox and acid/base reactivity of $\mathrm{N}_{\mathrm{x}} \mathrm{H}_{\mathrm{y}}$ species at the dinickel core.

According to Scheme 6.2 conversion of $\left[\mathbf{L}^{\text {bisnac }} \mathbf{N i}_{2}(\mu-\mathrm{Br})\right]$ with three equivalents of hy-

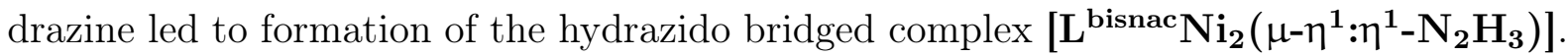
After filtration and subsequent crystallization, crystals were obtained, which were suitable for X-ray diffraction analysis.
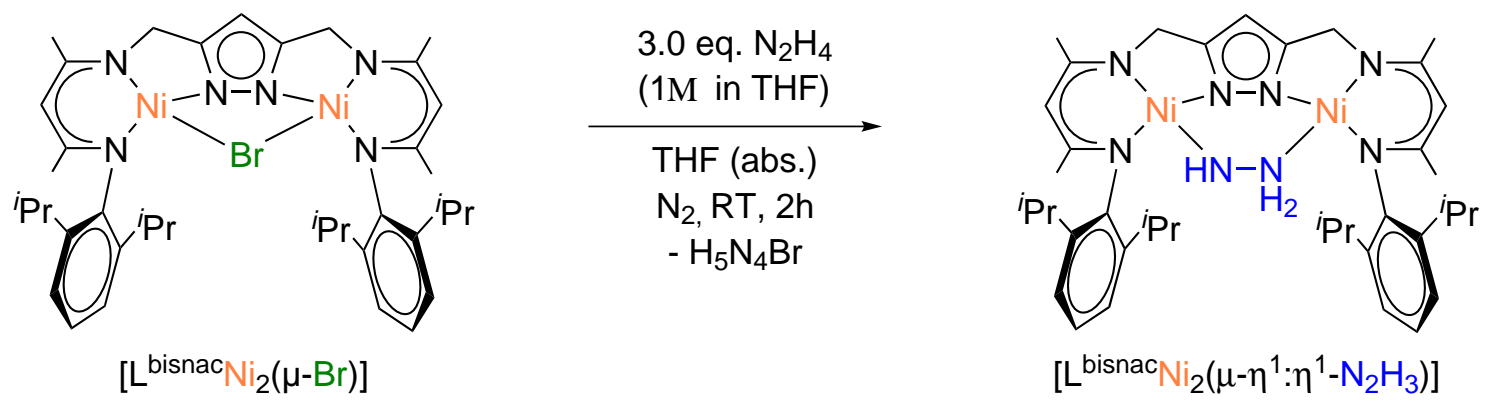

Scheme 6.2: Synthesis of [ $\left.L^{\text {bisnac }} \mathrm{Ni}_{2}\left(\mu-\eta \eta^{1}: \eta^{1} \mathrm{~N}_{2} \mathrm{H}_{3}\right)\right]$.

The ORTEP diagram of the molecular structure is depicted in Figure 6.1 and relevant bond lengths and distances are tabulated in Table 6.1. The two $\mathrm{Ni}^{\mathrm{II}}$ centers exhibit a distance of $3.94 \AA$, which corresponds well with known dinuclear nickel complexes of pyrazolate-based ligand systems. ${ }^{[10]}$

However, in comparison to other reported dinuclear nickel complexes of linked $\beta$-diketiminate ligand systems, the metal-metal distance is slightly larger $(3.597 \AA, 3.429 \AA) . \stackrel{[98]}{ }$ The distance $d_{\mathrm{N} 7-\mathrm{N} 8}$ in the $\mu-\eta^{1}: \eta^{1}$-hydrazido ligand is $1.45 \AA$ (Table 6.1). It is similar to the bond length found in crystalline $(1.47 \AA)$ or free $(1.45 \AA)$ hydrazine, confirming the presence of a $\mathrm{N}-\mathrm{N}$ single bond. $[\underline{[268,269]}$

Since a $\mu-\eta^{1}: \eta^{1}-\mathrm{N}_{2} \mathrm{H}_{3}{ }^{-}$binding motif is quite rare in literature, a direct comparison of the $\mathrm{N}-\mathrm{N}$ bond length in $\left[\mathbf{L}^{\text {bisnac }} \mathbf{N i}_{2}\left(\mu-\eta^{\mathbf{1}}: \eta^{\mathbf{1}}-\mathbf{N}_{\mathbf{2}} \mathbf{H}_{\mathbf{3}}\right)\right]$ with other systems, particularly containing nickel, was difficult. A $\mu-\eta^{1}: \eta^{2}-\mathrm{N}_{2} \mathrm{H}_{3}{ }^{-}$briging ligand was observed in a dinuclear iron complex featuring a N-N bond length of $1.40 \AA .{ }^{[252]}$ Although the nickel $\beta$-diketiminato system from LIMBERG contains a side-on hydrazido ligand, its reported $\mathrm{N}-\mathrm{N}$ bond length of $1.42-1.48 \AA$ is comparable with the bond length found in $\left[\mathbf{L}^{\text {bisnac }} \mathbf{N i}_{\mathbf{2}}\left(\mu-\eta^{1}: \eta^{\mathbf{1}}-\mathbf{N}_{\mathbf{2}} \mathbf{H}_{\mathbf{3}}\right)\right] .{ }^{[.58]}$ Moreover, the $\mathrm{Ni}-\mathrm{N}$ bond lengths $d(\mathrm{Ni}-\mathrm{N} 7)=1.891 \AA$ and 
$d(\mathrm{Ni}-\mathrm{N} 8)=1.914 \AA$ also correspond quite well with LIMBERG's side-on coordinated complex which features a $\mathrm{Ni}-\mathrm{N}\left(\mathrm{sp}^{3}\right)$ bond length of $1.871 \AA . \stackrel{[58]}{[.5}$

A slight distortion of the system is observed (right side of Figure 6.1, -) as a result of steric demand of the $\mu-\eta^{1}: \eta^{1}-\mathrm{N}_{2} \mathrm{H}_{3}{ }^{-}$ligand, reflected by the torsion angle $\Varangle(\mathrm{Ni1}-\mathrm{N} 7-\mathrm{N} 8-\mathrm{Ni} 2)=$ $89.71^{\circ}$.
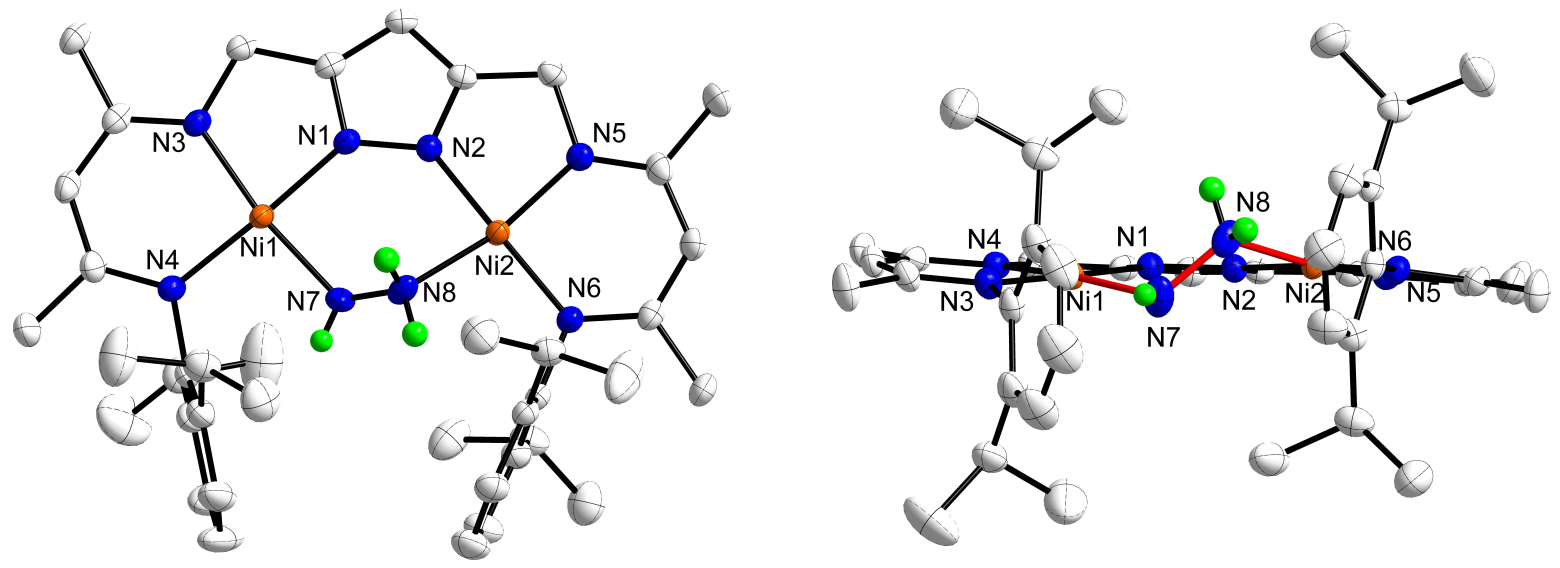

Figure 6.1.: Thermal displacement ellipsoids (shown at $50 \%$ probability) of the molecular structure of [ ${ }^{\text {bisnac }} \mathrm{Ni}_{2}\left(\mu-\eta^{1}: \eta^{1}-\mathbf{N}_{2} \mathbf{H}_{3}\right)$ ]. All hydrogen atoms except the nitrogen bound ones have been omitted for the sake of clarity (the hydrogen atoms are displayed with a fixed radius of $0.135 \AA$ ). Right: Front view of the molecular structure of $\left[\mathrm{L}^{\text {bisnac }} \mathrm{Ni}_{2}\left(\mu-\eta^{1}: \eta^{1}-\mathbf{N}_{2} \mathbf{H}_{3}\right)\right]$. The twist of the bond N7-N8 towards the ligand plane is indicated $(-)$.

Table 6.1.: Selected bond lengths and angles of [ $\left.L^{\text {bisnac }} \mathrm{Ni}_{2}\left(\mu-\eta^{1}: \eta^{1}-\mathrm{N}_{2} \mathrm{H}_{3}\right)\right]$.

\begin{tabular}{cccccc}
\hline \multicolumn{2}{c}{ Bond lengths / $\AA$} \\
\hline Ni1-N1 & $1.8695(2)$ & N1-Ni1-N7 & $88.13(9)$ & H7-N7-Ni1 & $112(2)$ \\
N1-N7 & $1.891(2)$ & N1-Ni1-N3 & $83.13(8)$ & N8-N7-Ni1 & $116.30(2)$ \\
Ni1-N3 & $1.8989(2)$ & N7-Ni1-N3 & $169.31(9)$ & H8B-N8-H8A & $104(3)$ \\
Ni1-N4 & $1.904(2)$ & N1-Ni1-N4 & $177.42(8)$ & H8B-N8-N7 & $111(2)$ \\
Ni2-N2 & $1.8621(2)$ & N7-Ni1-N4 & $94.45(9)$ & H8A-N8-N7 & $112(2)$ \\
Ni2-N5 & $1.8914(2)$ & N3-Ni1-N4 & $94.31(8)$ & H8B-N8-Ni2 & $111(2)$ \\
Ni2-N6 & $1.9015(2)$ & N2-Ni2-N5 & $83.26(8)$ & H8A-N8-Ni2 & $102(2)$ \\
Ni2-N8 & $1.914(2)$ & N2-Ni2-N6 & $175.90(8)$ & N7-N8-Ni2 & $115.36(2)$ \\
N7-H7 & $0.76(3)$ & N5-Ni2-N6 & $95.18(8)$ & & \\
N7-N8 & $1.451(3)$ & N2-Ni2-N8 & $87.37(9)$ & & \\
N8-H8B & $0.83(4)$ & N5-Ni2-N8 & $167.66(9)$ & & \\
N8-H8A & $0.88(4)$ & N6-Ni2-N8 & $94.70(9)$ & & \\
Ni1...Ni2 & $3.9366(5)$ & H7-N7-N8 & $103(2)$ & & \\
\hline
\end{tabular}

IR spectroscopy was performed illustrating the various $\mathrm{N}-\mathrm{H}$ stretching vibrations of the $\mathrm{N}_{2} \mathrm{H}_{3}{ }^{-}$bridging unit. The $\mathrm{N}-\mathrm{H}$ stretching vibration of the $\mathrm{NH}$ unit was observed at $\tilde{\nu}_{\mathrm{NH}}$ $=3253 \mathrm{~cm}^{-1}$. Two further bands at $\tilde{\nu}_{\mathrm{NH}}=3273 \mathrm{~cm}^{-1}$ and $\tilde{\nu}_{\mathrm{NH}}=3299 \mathrm{~cm}^{-1}$ were assigned 
to the symmetric and antisymmetric stretching vibration of the $\mathrm{NH}_{2}$ unit, respectively. Despite the fact that the ligand is end-on bridged, these vibrations are very similar to the literature known $\mathrm{N}-\mathrm{H}$ stretching vibration of the mononuclear nickel complex with a side-on bound hydrazido ligand, which showed an IR band at $\tilde{\nu}_{\mathrm{NH}}=3312 \mathrm{~cm}^{-1} .^{[58]}$

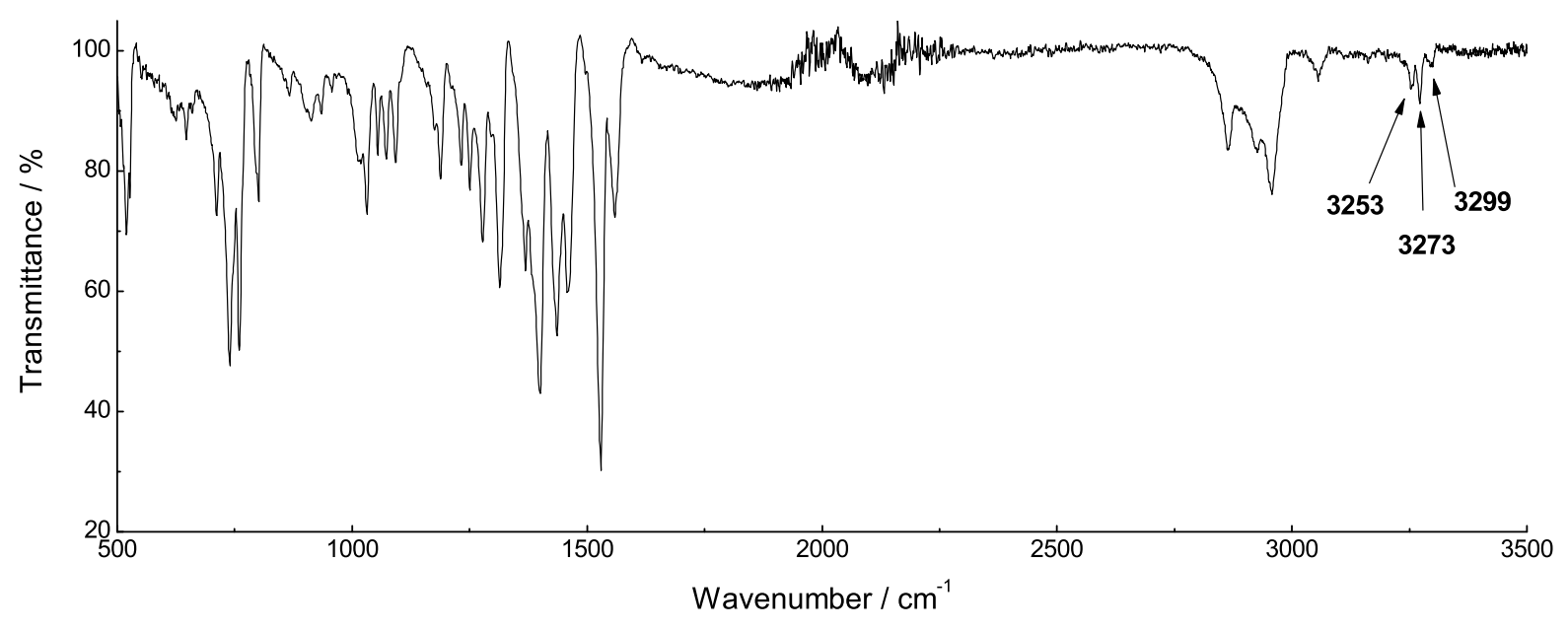

Figure 6.2.: IR spectrum [ $\left.\mathrm{L}^{\text {bisnac }} \mathrm{Ni}_{2}\left(\mu-\eta^{1}: \eta^{1}-\mathrm{N}_{2} \mathrm{H}_{3}\right)\right]$.

The ${ }^{1} \mathrm{H}$ NMR experiment at room temperature revealed a resonance pattern, which is associated with a non-symmetric complex (Figure 6.3). In fact the spectrum showed two distinct signals, which were assigned to the hydrazido protons: a doublet at $\delta\left\{{ }^{1} \mathrm{H}\right\}=$ $2.26 \mathrm{ppm}$ with a normalized integral of two corresponding to the $\mathrm{NH}_{2}$ and a triplet at $\delta\left\{{ }^{1} \mathrm{H}\right\}=-0.92 \mathrm{ppm}$ with an integral of one, which matches to NH. Both protons of the $\mathrm{NH}_{2}$ group yield a single signal indicating equivalence due to the dynamic process illustrated in Scheme 6.3.

The vicinal proton-proton correlation was proven by means of a ${ }^{1} \mathrm{H}_{-}{ }^{1} \mathrm{H}$ COSY experiment (Figure 6.4), showing a corresponding cross-signal for the two NH-resonances ( $\bullet \mathrm{NH}_{2}$ and $\boldsymbol{\square}$ for $\mathrm{NH}$ are indicated).

The ${ }^{15} \mathrm{~N}$ HMBC spectrum Figure 6.5 describes N-H coupling correlations ${ }^{\dagger}$ of the two proton resonances to nitrogen at $\delta\left\{{ }^{1} \mathrm{H}\right\}=2.26 \mathrm{ppm} / \delta\left\{{ }^{15} \mathrm{~N}\right\}=-343.3 \mathrm{ppm}\left({ }^{1} J_{\mathrm{NH}}\right.$ $=70 \mathrm{~Hz})$ and $\delta\left\{{ }^{1} \mathrm{H}\right\}=-0.92 \mathrm{ppm} / \delta\left\{{ }^{15} \mathrm{~N}\right\}=-313.8 \mathrm{ppm}\left({ }^{1} J_{\mathrm{NH}}=54 \mathrm{~Hz}\right)$. Until now, no ${ }^{15} \mathrm{~N}$ resonances for $\mu-\eta^{1}: \eta^{1} \mathrm{~N}_{2} \mathrm{H}_{3}{ }^{-}$briging ligands in nickel complexes were reported in the literature. Nonetheless, the proton and ${ }^{15} \mathrm{~N}$ NMR resonances found for $\left[\mathbf{L}^{\text {bisnac }} \mathbf{N i}_{\mathbf{2}}\left(\mu-\eta^{\mathbf{1}}: \eta^{\mathbf{1}}-\mathbf{N}_{\mathbf{2}} \mathbf{H}_{\mathbf{3}}\right)\right]$ are similar to reported resonances of a nickel complex with a side-on coordinated neutral $\mathrm{N}_{2} \mathrm{H}_{4}$ ligand $\left(\delta\left\{{ }^{1} \mathrm{H}\right\}=3.5 \mathrm{ppm} / \delta\left\{{ }^{15} \mathrm{~N}\right\}=-363.1 \mathrm{ppm}\right) . \stackrel{[79]}{.}$

${ }^{\dagger}$ Conditions for the non-decoupled ${ }^{1} \mathrm{H}-{ }^{15} \mathrm{~N}-\mathrm{HMBC}$ were optimized for a $\mathrm{N}-\mathrm{H}$ coupling of ${ }^{1} \mathrm{~J}_{\mathrm{NH}}=70 \mathrm{~Hz}$. In consequence, the other non-H-bound nitrogen atoms were not visible in the spectrum. 


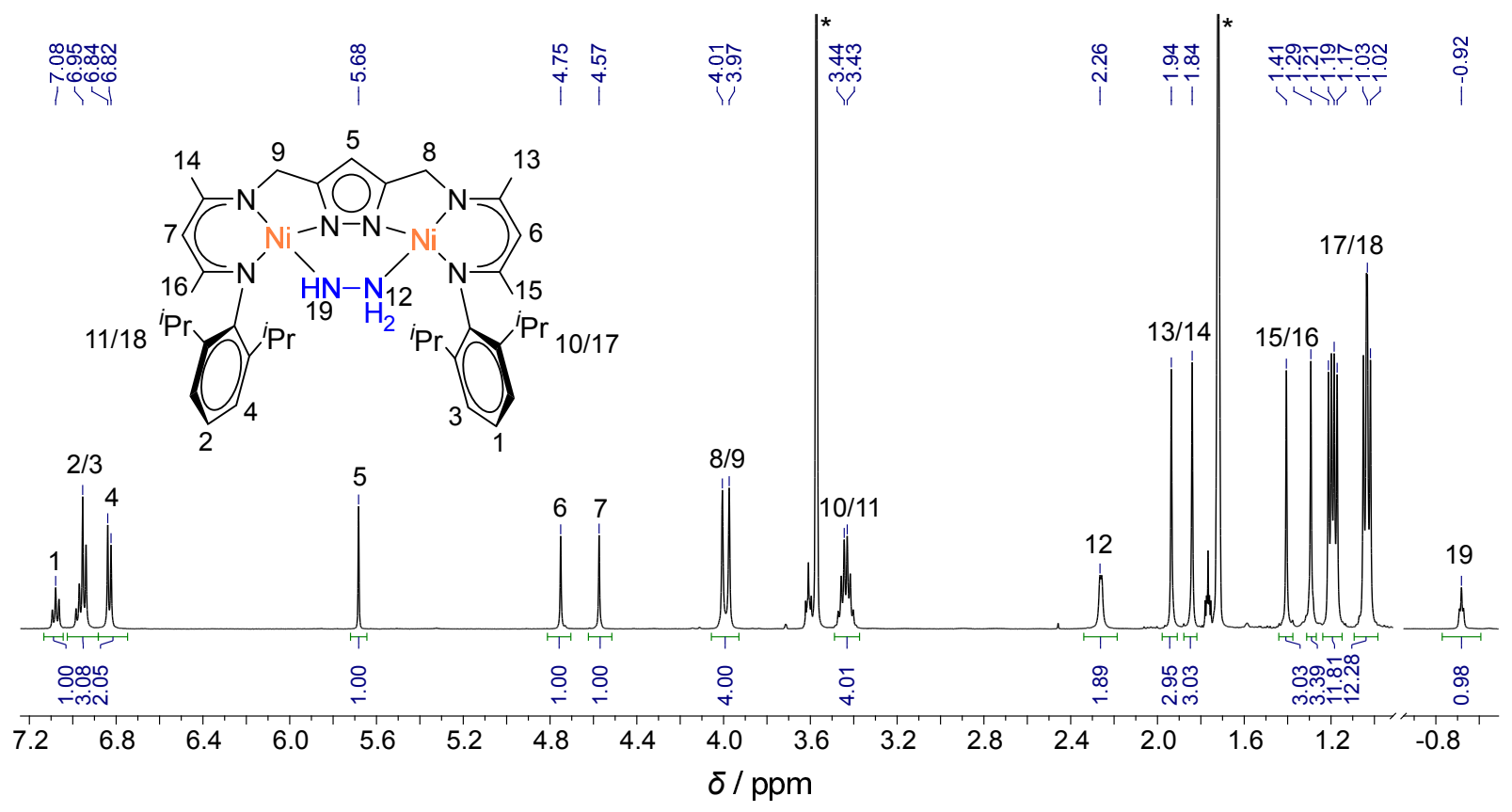

Figure 6.3.: ${ }^{1} \mathrm{H}$ NMR spectrum $(500 \mathrm{MHz})$ of $\left[\mathrm{L}^{\text {bisnac }} \mathrm{Ni}_{2}\left(\mu-\eta^{1}: \eta^{1}-\mathbf{N}_{2} \mathrm{H}_{3}\right)\right]$ measured in THF- $\mathrm{D}_{8}$ at room temperature. Residual solvents $\left(\right.$ THF- $\left._{7}\right)$ are marked $\left({ }^{*}\right)$.<smiles>CC=NNNN</smiles>

Scheme 6.3: Equivalence of the protons in the $\mathrm{NH}_{2}$ group of the bridging ligand caused by dynamic rearrangement.

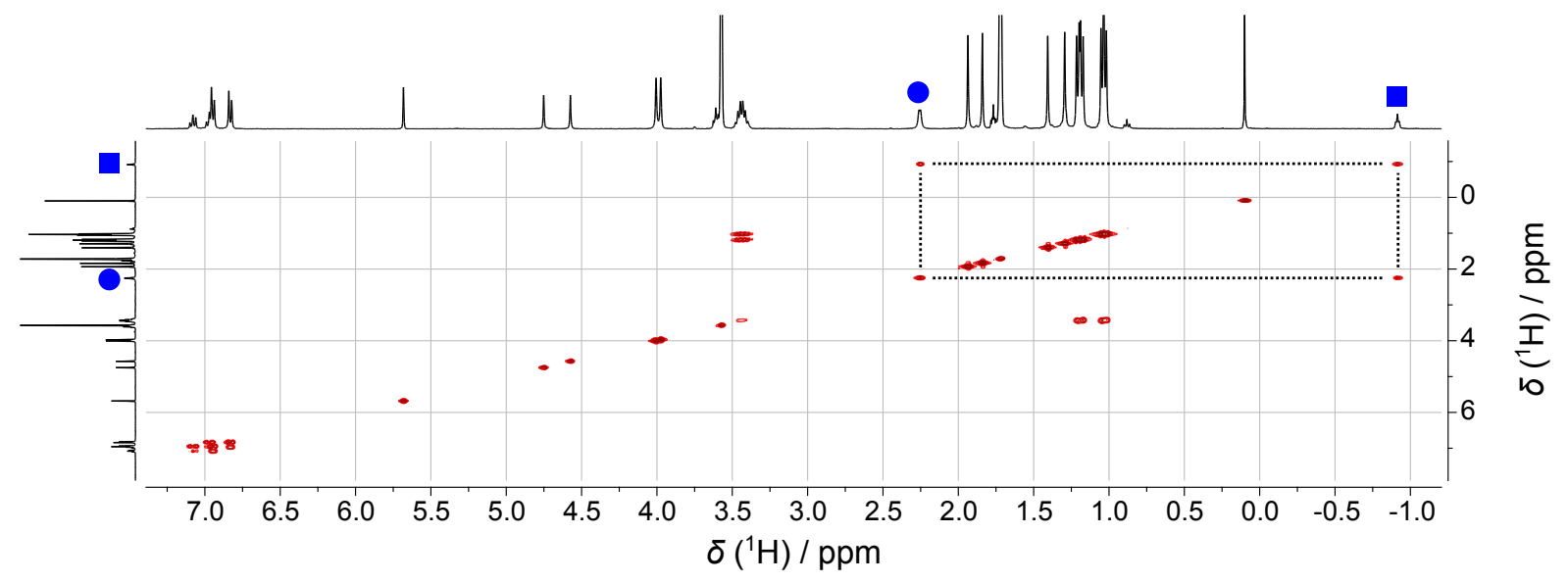

Figure 6.4.: ${ }^{1} \mathrm{H}-{ }^{1} \mathrm{H}$ COSY spectrum $(400 \mathrm{MHz})$ of $\left[\mathrm{L}^{\text {bisnac }} \mathrm{Ni}_{2}\left(\mu-\eta^{1}: \eta^{1}-\mathrm{N}_{2} \mathrm{H}_{3}\right)\right]$ measured in THF- $\mathrm{D}_{8}$ at room temperature. Resonances of the hydrazide ${ }^{-}$bridging unit are marked with $\bullet\left(\mathrm{NH}_{2}\right)$ and $\mathbf{\square}(\mathrm{NH})$. 


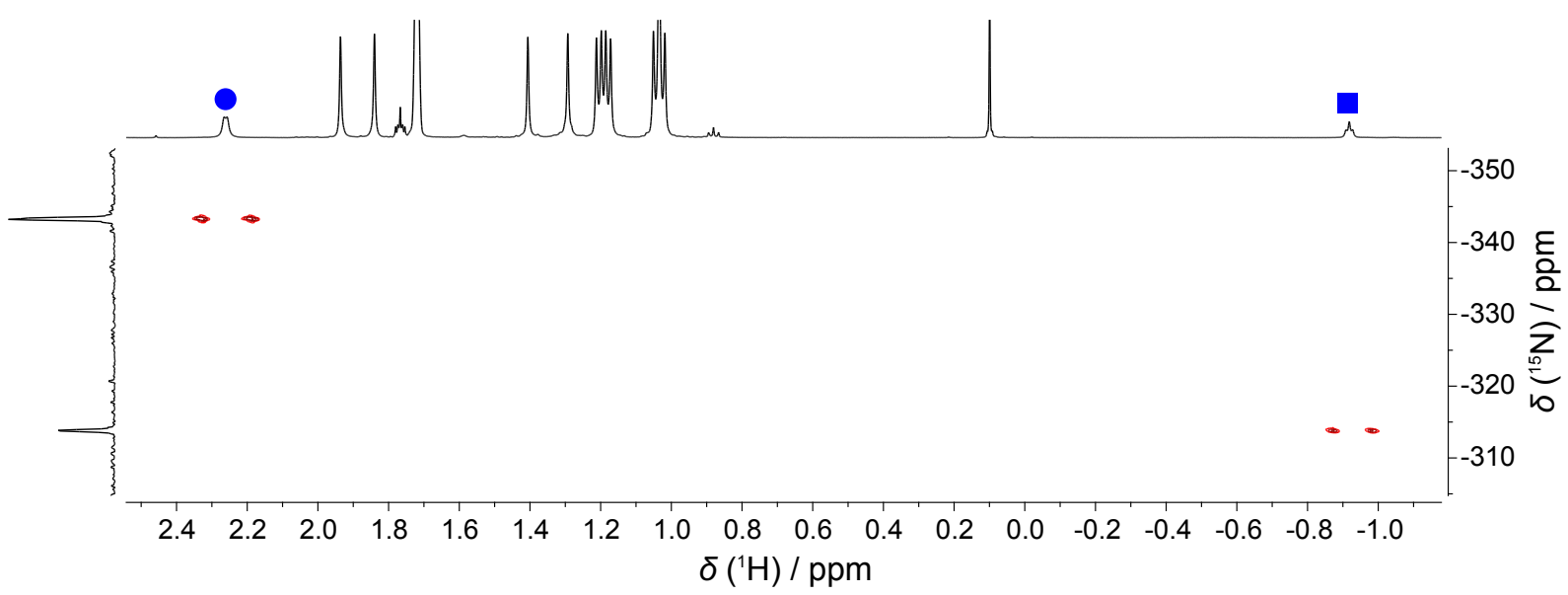

Figure 6.5.: ${ }^{1} \mathrm{H}^{-15} \mathrm{~N} \mathrm{HMBC}$ spectrum $(500 \mathrm{MHz})$ of $\left[\mathrm{L}^{\text {bisnac }} \mathrm{Ni}_{2}\left(\mu-\eta \eta^{1}: \eta^{1}-\mathrm{N}_{2} \mathrm{H}_{3}\right)\right]$ measured in THF- $\mathrm{D}_{8}$ at room temperature. The ${ }^{1} \mathrm{H}$ resonances of the hydrazido bridging unit are marked with $\bullet\left(\mathrm{NH}_{2}\right)$ and $(\mathrm{NH})$

\subsection{Deprotonation of the Hydrazido Bridge}

Starting from the derived $\mathrm{N}_{2} \mathrm{H}_{3}{ }^{-}$-bridged nickel complex, deprotonation was attempted to achieve a $\mathrm{N}_{2} \mathrm{H}_{2}{ }^{-}$bridging unit. Inspired by the dihydride complex $\mathbf{K}\left[\mathbf{L}^{\text {bisnac }} \mathbf{N i}_{\mathbf{2}}(\mathbf{H})_{\mathbf{2}}\right]$, which was discussed in chapter 4 , incorporation of a potassium cation into the complex was envisioned to stabilize the negative charge. Similar to the dihydride complex, the DIPP substituents of $\left[\mathrm{L}^{\text {bisnac }} \mathbf{N i}_{\mathbf{2}}\left(\mu-\eta^{\mathbf{1}}: \eta^{\mathbf{1}}-\mathbf{N}_{\mathbf{2}} \mathbf{H}_{\mathbf{3}}\right)\right]$ are also arranged orthogonally to the complex plane, creating conditions for a potential cation- $\pi$ interaction between the aryl units and potassium.

Deprotonation of $\left[\mathbf{L}^{\text {bisnac }} \mathbf{N i}_{\mathbf{2}}\left(\mu-\eta^{\mathbf{1}}: \eta^{\mathbf{1}}-\mathbf{N}_{\mathbf{2}} \mathbf{H}_{\mathbf{3}}\right)\right]$ was achieved with KH and dibenzo-18crown-6 (Scheme 6.4). The reaction led to an immediate color change from red to dark brown and visible but slow development of a gas, which was most probably $\mathrm{H}_{2}$.
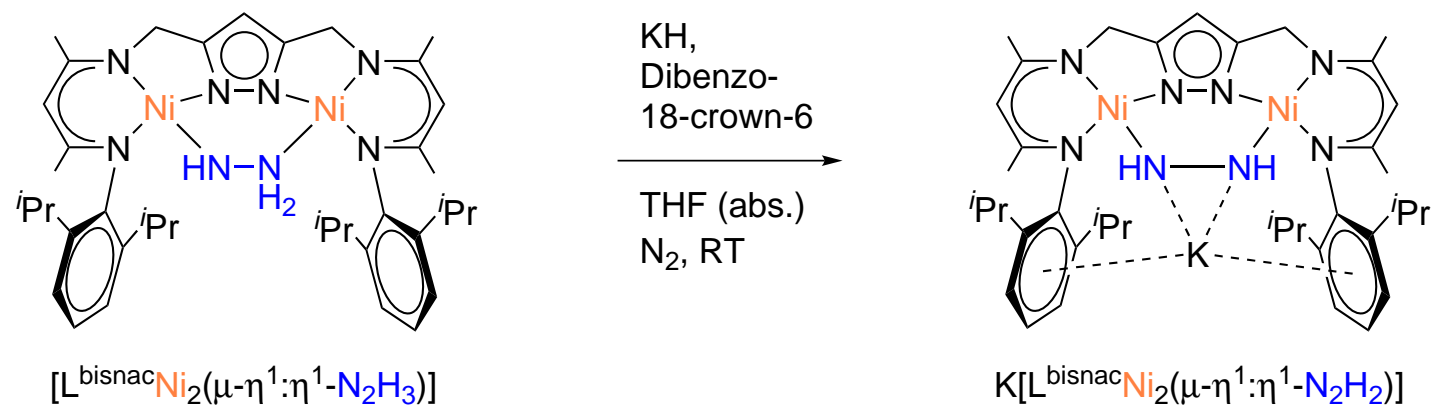

Scheme 6.4: Deprotonation of [ $\left.L^{\text {bisnac }} \mathbf{N i}_{2}\left(\mu-\eta^{1}: \eta^{1}-\mathbf{N}_{2} \mathbf{H}_{3}\right)\right]$ yielding $K\left[{ }^{\text {bisnac }} \mathbf{N i}_{2}\left(\mu-\eta^{1}: \eta^{1}-\mathbf{N}_{2} \mathbf{H}_{2}\right)\right]$.

After layering the reaction solution (THF) with hexanes and cooling to $-30^{\circ} \mathrm{C}$, crystalline material for X-ray analysis was obtained. The ORTEP diagram shown in Figure 6.6 illustrates the molecular structure of $\mathbf{K}\left[\mathbf{L}^{\text {bisnac }} \mathbf{N i}_{\mathbf{2}}\left(\mu-\eta^{\mathbf{1}}: \eta^{\mathbf{1}}-\mathbf{N}_{\mathbf{2}} \mathbf{H}_{\mathbf{2}}\right)\right]$. 

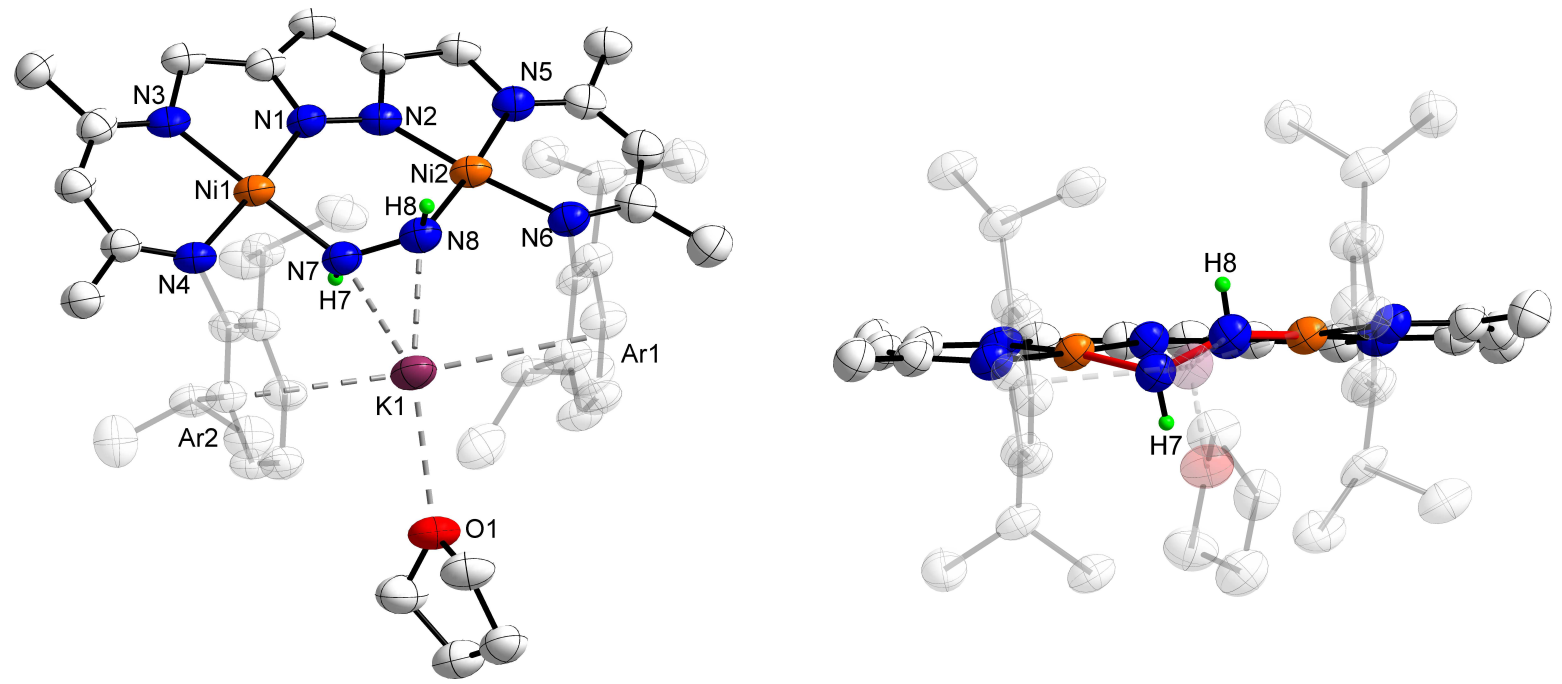

Figure 6.6.: Thermal displacement ellipsoids (shown at $50 \%$ probability) of the molecular structure of $K\left[L^{\text {bisnac }} \mathrm{Ni}_{2}\left(\mu-\eta^{1}: \eta^{1}-\mathbf{N}_{2} \mathbf{H}_{2}\right)\right]$. All hydrogen atoms except the nitrogen bound ones have been omitted for the sake of clarity (the hydrogen atom is displayed with a fixed radius of $0.135 \AA$. Left: Top view of the complex. Right: Front view of the molecular structure of $K\left[L^{\text {bisnac }} \mathbf{N i}_{2}\left(\mu-\eta^{1}: \eta^{1}-\mathbf{N}_{2} \mathbf{H}_{2}\right)\right]$. The twist of the bond N7-N8 with respect to the ligand plane is indicated (-).

Table 6.2.: Selected bond lengths and angles of $K\left[L^{\text {bisnac }} \mathrm{Ni}_{2}\left(\mu-\eta^{1}: \eta^{1}-\mathbf{N}_{2} \mathbf{H}_{2}\right)\right]$.

\begin{tabular}{|c|c|c|c|}
\hline \multicolumn{2}{|c|}{ Bond lengths / $\AA$} & \multicolumn{2}{|c|}{ Angle ${ }^{\circ}$} \\
\hline Ni1-N1 & $1.867(4)$ & N1-Ni1-N7 & $90.60(17)$ \\
\hline Ni1-N7 & $1.923(4)$ & N1-Ni1-N4 & $176.32(2)$ \\
\hline Ni1-N4 & $1.933(4)$ & N7-Ni1-N4 & $93.05(2)$ \\
\hline Ni1-N3 & $1.934(4)$ & N1-Ni1-N3 & $83.08(2)$ \\
\hline Ni2-N2 & $1.857(4)$ & N7-Ni1-N3 & $170.61(2)$ \\
\hline Ni2-N8 & $1.905(4)$ & N4-Ni1-N3 & $93.35(2)$ \\
\hline Ni2-N6 & $1.924(4)$ & N2-Ni2-N8 & $91.48(2)$ \\
\hline Ni2-N5 & $1.935(4)$ & N2-Ni2-N6 & $175.62(2)$ \\
\hline K1-N7 & $2.731(4)$ & N8-Ni2-N6 & $91.63(2)$ \\
\hline K1-N8 & $2.752(4)$ & N2-Ni2-N5 & $83.54(2)$ \\
\hline K1-O1 & $2.800(4)$ & N8-Ni2-N5 & $174.99(2)$ \\
\hline N7-N8 & $1.416(6)$ & N6-Ni2-N5 & $93.38(2)$ \\
\hline $\mathrm{Ni} \ldots \mathrm{Ni}$ & $3.9666(9)$ & & \\
\hline $\mathrm{K} \cdots \operatorname{Ar} 1^{\dagger}$ & $3.0761(1)$ & & \\
\hline $\mathrm{K} \cdots \mathrm{Ar}^{\dagger}$ & $2.9058(1)$ & & \\
\hline
\end{tabular}

†Measured distance of aryl centroids to the potassium cation. 
Indeed, it turned out that after deprotonation, the resulting negatively charged compound was stabilized by a potassium cation. Similar to $\left.\mathbf{K}\left[\mathbf{L}^{\text {bisnac }} \mathbf{N i}_{\mathbf{2}} \mathbf{( H}\right)_{\mathbf{2}}\right]$, the $\pi$-system of the diisopropyl-phenyl sidearms of the nacnac ligand are able to interact with the cation, which led to the stabilization of the entire compound, along with coordination of a THF molecule. The bound $\mathrm{N}_{2} \mathrm{H}_{2}{ }^{2-}$ bridge is positioned between the nickel centers in a similar fashion as observed for the precursor compound $\left[\mathbf{L}^{\text {bisnac }} \mathbf{N i}_{\mathbf{2}}\left(\mu-\eta^{\mathbf{1}}: \eta^{\mathbf{1}}-\mathbf{N}_{\mathbf{2}} \mathbf{H}_{\mathbf{3}}\right)\right]$, but exhibits a much smaller twist, which is illustrated by the red marked bonds at the right structure of Figure 6.6. The torsion angle of the bridging unit in $\mathbf{K}\left[\mathbf{L}^{\mathbf{b i s n a c}} \mathbf{N i}_{\mathbf{2}}\left(\mu-\eta^{\mathbf{1}}: \eta^{\mathbf{1}}-\mathbf{N}_{\mathbf{2}} \mathbf{H}_{\mathbf{2}}\right)\right]$ $\Varangle(\mathrm{Ni1}-\mathrm{N} 7-\mathrm{N} 8-\mathrm{Ni2})$ amounts to $60.09^{\circ}$ and is substantially smaller than the one observed for the $\mathrm{N}_{2} \mathrm{H}_{3}{ }^{-}$ligand of the precursor compound $\left(\Varangle(\mathrm{Ni1}-\mathrm{N} 7-\mathrm{N} 8-\mathrm{Ni} 2)=89.71^{\circ}\right)$.

This can be explained by contraction of the N-N bond from $1.451 \AA$ to $1.416 \AA$ after deprotonation of the hydrazido ligand. The N7-N8 bond distance found in $\mathbf{K}\left[\mathbf{L}^{\text {bisnac }} \mathbf{N i}_{\mathbf{2}}(\mu-\right.$ $\left.\eta^{1}: \eta^{1}-\mathbf{N}_{2} \mathbf{H}_{2}\right)$ ] is consistent with other reported bond lengths for $\mu-\eta^{1}: \eta^{1}$ $(1.413 \AA-1.420 \AA)^{\left[\frac{[270]}{\underline{n}}\right.}$ and $\mu-\eta^{2}: \eta^{2}$ bridged $(1.429 \AA)^{[271]} \mathrm{N}_{2} \mathrm{H}_{2}{ }^{2-}$ ligands, suggestive of a $\mathrm{N}-\mathrm{N}$ single bond.

$\mathbf{K}\left[\mathbf{L}^{\text {bisnac }} \mathbf{N i}_{\mathbf{2}}\left(\mu-\eta^{\mathbf{1}}: \eta^{\mathbf{1}}-\mathbf{N}_{\mathbf{2}} \mathbf{H}_{\mathbf{2}}\right)\right]$ features $\mathrm{Ni}-\mathrm{N}$ bond lengths of $1.923 \AA$ (Ni1-N7) and $1.905 \AA(\mathrm{Ni2}-\mathrm{N} 8)$ while in the precursor complex $\left[\mathbf{L}^{\text {bisnac }} \mathbf{N i}_{\mathbf{2}}\left(\mu-\eta^{\mathbf{1}}: \eta^{\mathbf{1}}-\mathbf{N}_{\mathbf{2}} \mathbf{H}_{\mathbf{3}}\right)\right]$ bond lengths are $1.891 \AA$ (Ni1-N7) and $1.914 \AA$ (Ni2-N8), which concur with $\mathrm{Ni}-\mathrm{N}\left(\mathrm{sp}^{3}\right)$ bond lengths, confirming $\mathrm{N}-\mathrm{N}$ single bond character in the $\mathrm{N}_{2} \mathrm{H}_{2}{ }^{2-}$ ligand. $[\underline{[58,78]}$

Despite the decrease in bond length of the bridging unit from $\mathrm{N}_{2} \mathrm{H}_{3}{ }^{-}$to $\mathrm{N}_{2} \mathrm{H}_{2}{ }^{2-}$, the $\mathrm{Ni}$...Ni distance exhibits an increase from $3.937 \AA$ to $3.967 \AA$. This is attributable to the potassium counterion residing between the two DIPP moieties, resulting in a strain on the system. A similar effect was also described for $\left.\mathbf{K}\left[\mathbf{L}^{\text {bisnac }} \mathbf{N i}_{\mathbf{2}} \mathbf{( H}\right)_{\mathbf{2}}\right]$, which features a $\mathrm{Ni} \cdots$ Ni distance of $4.159 \AA$. In $\mathbf{K}\left[\mathbf{L}^{\text {bisnac }} \mathbf{N i}_{\mathbf{2}}\left(\mu-\eta^{\mathbf{1}}: \eta^{\mathbf{1}}-\mathbf{N}_{\mathbf{2}} \mathbf{H}_{\mathbf{2}}\right)\right]$ the distance of the aryl centroids to the potassium amounts to $3.076 \AA$ and $2.906 \AA$, which is significantly longer than in the dihydride complex $\left(d_{\mathrm{K}-\mathrm{Ar}}=2.833 \AA, 2.850 \AA\right)$. This is in accordance with the longer $\mathrm{Ni} \cdots \mathrm{Ni}$ distance found in $\mathbf{K}\left[\mathbf{L}^{\text {bisnac }} \mathbf{N i}_{\mathbf{2}}(\mathbf{H})_{2}\right]$ since the hydrogen atoms are less sterically demanding than the $\mathrm{N}_{2} \mathrm{H}_{2}{ }^{2-}$ and the potassium is hosted deeper inside the cavity.

The $K \cdots N$ distances of $\mathbf{K}\left[\mathbf{L}^{\text {bisnac }} \mathbf{N i}_{\mathbf{2}}\left(\mu-\eta^{\mathbf{1}}: \eta^{\mathbf{1}}-\mathbf{N}_{\mathbf{2}} \mathbf{H}_{\mathbf{2}}\right)\right]$ are $2.731 \AA$ (K1-N7) and $2.752 \AA$ (K1-N8), which is marginally shorter than the $\mathrm{K} \cdots \mathrm{N}$ distance in the side-on coordinated nickel complex reported by LIMBERG et al. ${ }^{[\underline{[79]}}\left(d_{\mathrm{K}-\mathrm{N}}=2.760 \AA, 2.980 \AA\right)$. In the latter complex, the longer distance was attributed to steric hinderance that originates from a crown-ether coordinating to the cation.

The substitution of potassium with other cations like sodium was not attempted since literature reported no significant change in the activation potential of the $\mathrm{N}-\mathrm{N}$ bond. ${ }^{[81]}$ Nevertheless, incorporation of an alkali cation into the complex via cation- $\pi$ interactions with arylic substituents can be highly beneficial in terms of stabilization of the negative charge, which builds up during reduction of $\mathrm{N}_{2}$. It has been well established that the pres- 
ence of a positive charge in close proximity to the reduced core can increase stabilization of the complex..$^{[54,272]}$ Furthermore, coordinated alkali cations enhance overall activation of dinitrogen by pulling electron density from the system into the $\mathrm{N}_{2}$ bridging unit.

IR spectroscopic investigations revealed two bands at $\nu=3186 \mathrm{~cm}^{-1}$, which was assigned to the asymmetric stretching vibration and $\nu=3162 \mathrm{~cm}^{-1}$ assigned to the symmetric stretching vibration. Compared to literature reported systems with $\mu-\eta^{2}: \eta^{2} \mathrm{~N}_{2} \mathrm{H}_{2}{ }^{2-}$ ligands $\left(\nu=3297\right.$ and $\left.3582 \mathrm{~cm}^{-1}, \underline{[270]} \nu=3210-3398 \mathrm{~cm}^{-1[\underline{[271]}}\right)$, the stretching vibrations of $\mathbf{K}\left[\mathbf{L}^{\text {bisnac }} \mathbf{N i}_{\mathbf{2}}\left(\mu-\eta^{1}: \eta^{\mathbf{1}}-\mathbf{N}_{\mathbf{2}} \mathbf{H}_{\mathbf{2}}\right)\right]$ are shifted to lower wavenumbers. This finding may be attributed to the different binding motif $\left(\mu-\eta^{1}: \eta^{1}\right)$ of the ligand in $K\left[L^{\text {bisnac }} \mathrm{Ni}_{2}\left(\mu-\eta^{1}: \eta^{1}-\mathrm{N}_{2} \mathrm{H}_{2}\right)\right]$.

A ${ }^{1} \mathrm{H}$ NMR experiment (Figure 6.7 confirms the symmetric geometry of $\mathbf{K}\left[\mathbf{L}^{\text {bisnac }} \mathbf{N i}_{\mathbf{2}}\left(\mu-\eta^{\mathbf{1}}: \eta^{\mathbf{1}}-\mathbf{N}_{\mathbf{2}} \mathbf{H}_{2}\right)\right]$. The resonance associated with the isopropyl CH function (region marked by $\square$ ) was overlayed by the solvent residual resonance, but was determined by means of a ${ }^{1} \mathrm{H}-{ }^{1} \mathrm{H}$ COSY experiment (proton correlations are shown in the encased excerpt of the COSY spectrum in Figure 6.7). The ${ }^{3} J_{\mathrm{HH}}$ coupling between ispropyl CH- (6) and $\mathrm{CH}_{3}$ (7 and 9) protons yields a typical strong cross correlation in the spectrum. At $\delta\left\{{ }^{1} \mathrm{H}\right\}=1.03 \mathrm{ppm}$, the $\mu-\eta^{1}: \eta^{1}-\mathrm{N}_{2} \mathrm{H}_{2}{ }^{2-}$ ligand singlet proton resonance was detected.
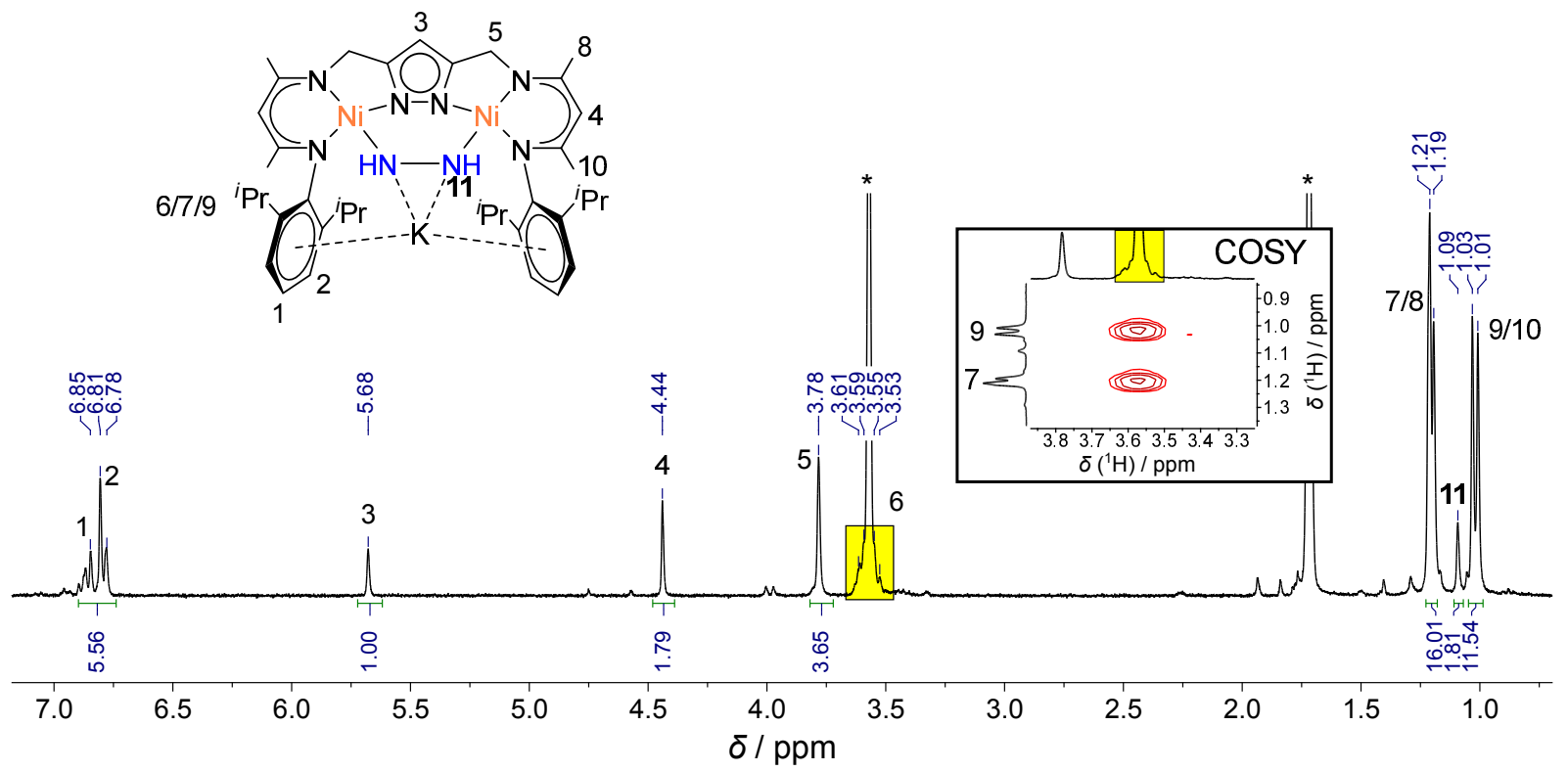

Figure 6.7.: ${ }^{1} \mathrm{H}$ NMR spectrum (300 MHz) of $K\left[L^{\text {bisnac }} \mathbf{N i}_{2}\left(\mu-\eta^{1}: \eta^{1}-\mathbf{N}_{2} \mathbf{H}_{2}\right)\right]$. The septet resonance of the isopropyl $\mathrm{CH}$ group is overlayed by the THF- $\mathrm{D}_{7}$ signal at $3.57 \mathrm{ppm} . \mathrm{A}^{1} \mathrm{H}^{-1} \mathrm{H}$ COSY experiment revealed the correlations for discrimination (encased spectrum). Residual solvents are marked $\left({ }^{*}\right)$.

The contour plot of the ${ }^{1} \mathrm{H}-{ }^{15} \mathrm{~N}$ HMBC experiment Figure 6.8 ${ }^{\dagger}$ confirms the presence of the nitrogen based bridge. Besides the observed ${ }^{1} J_{\mathrm{NH}}$ correlation with a ${ }^{15} \mathrm{~N}$ chem-

\footnotetext{
†The conditions of the ${ }^{1} \mathrm{H}-{ }^{15} \mathrm{~N}$ HMBC measurement were optimized for detection of ${ }^{1} \mathrm{~J}_{\mathrm{NH}}$ correlations (Topspin parameter CNST13 $=70 \mathrm{~Hz}$ ).
} 
ical shift at $-255.2 \mathrm{ppm}\left({ }^{1} J_{\mathrm{NH}}=59 \mathrm{~Hz}\right)$ the ${ }^{1} \mathrm{H}-{ }^{15} \mathrm{~N}$ long range coupling of ${ }^{15} \mathrm{~N}$ to the $\beta$-diketiminato $C H$-proton was detected. Similar to $\left[\mathbf{L}^{\mathbf{b i s n a c}} \mathbf{N i}_{\mathbf{2}}\left(\mu-\eta^{\mathbf{1}}: \eta^{\mathbf{1}}-\mathbf{N}_{\mathbf{2}} \mathbf{H}_{\mathbf{3}}\right)\right]$, a $\mu-\eta^{1}: \eta^{1}$ binding motif for $\mathrm{N}_{2} \mathrm{H}_{2}{ }^{2-}$ ligands in dinuclear metal complexes was not reported in the literature so far. Therefore, a comparison of NMR resonance chemical shifts was not possible.

Nonetheless, compared to the literature reported nickel-potassium complex from LIMBERG et al. featuring a side-on coordinated $\mu-\eta^{2}: \eta^{2} \mathrm{NPhNH}$ ligand with a $\mathrm{NH}$ proton resonance at $1.47 \mathrm{ppm}, \stackrel{[78]}{\underline{70}}$ the resonance of $\mathbf{K}\left[\mathbf{L}^{\text {bisnac }} \mathbf{N i}_{\mathbf{2}}\left(\mu-\eta^{\mathbf{1}}: \eta^{\mathbf{1}}-\mathbf{N}_{\mathbf{2}} \mathbf{H}_{\mathbf{2}}\right)\right]$ is quite similar. For a dinuclear iron based complex with side-on bridged $\mathrm{N}_{2} \mathrm{H}_{2}{ }^{2-}$ ligands a $\mathrm{NH}$ resonance at $2.59 \mathrm{ppm}$ was reported. $\underline{[271]}$

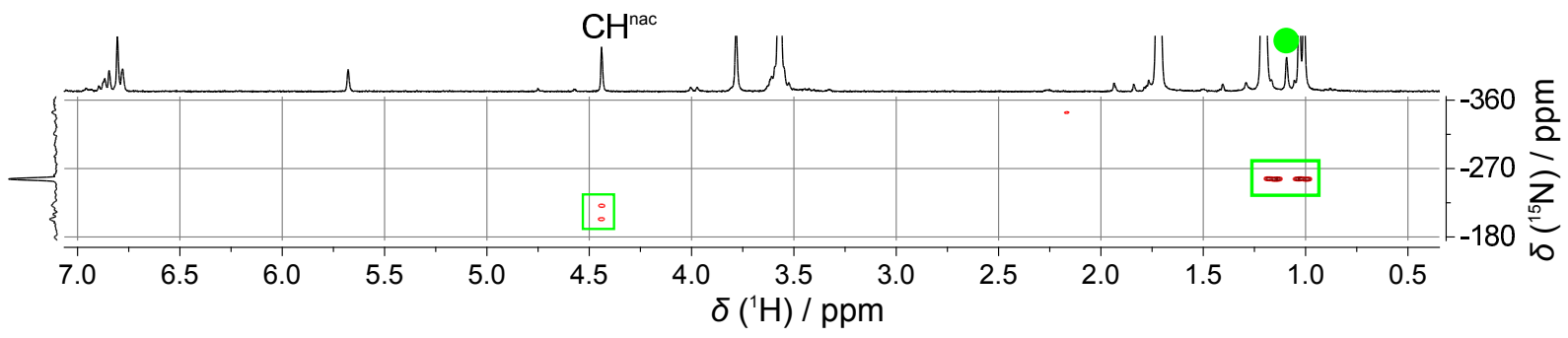

Figure 6.8.: ${ }^{1} \mathrm{H}-{ }^{15} \mathrm{~N}$ HMBC spectrum (400 MHz) of $\mathrm{K}\left[L^{\text {bisnac }} \mathrm{Ni}_{2}\left(\mu-\eta \eta^{1}: \eta^{1}-\mathbf{N}_{2} \mathrm{H}_{2}\right)\right]$. The $\mu-\eta^{1}: \eta^{1} \mathrm{~N}_{2} \mathrm{H}_{2}$ ligand shows a strong ${ }^{1} J_{\mathrm{NH}}$ correlation. Residual solvents are marked $\left(^{*}\right)$.

In order to gain insight into the spatial environment of the bridging $\mu-\eta^{1}: \eta^{1} \mathrm{~N}_{2} \mathrm{H}_{2}$ ligand, a ${ }^{1} \mathrm{H}$ NOESY experiment was conducted. The spectrum illustrated in Figure 6.9 revealed a weak NOE correlation of $\mathrm{NH}$ hydrogen atoms to the isopropyl $\mathrm{CH}$ functions ( $\square$ ). In contrast, a clear correlation to the isopropyl $\mathrm{CH}_{3}$ protons could not be determined from the spectrum. This finding concurs with the molecular structure of $\mathbf{K}\left[\mathbf{L}^{\text {bisnac }} \mathbf{N i}_{\mathbf{2}}(\mu-\right.$ $\left.\eta^{\mathbf{1}}: \eta^{\mathbf{1}}-\mathbf{N}_{\mathbf{2}} \mathbf{H}_{\mathbf{2}}\right)$ ] showing that the isopropyl methyl groups are pointing away from the $\mathrm{NH}$ protons thus increasing the distance between aliphatic and NH hydrogen atoms.

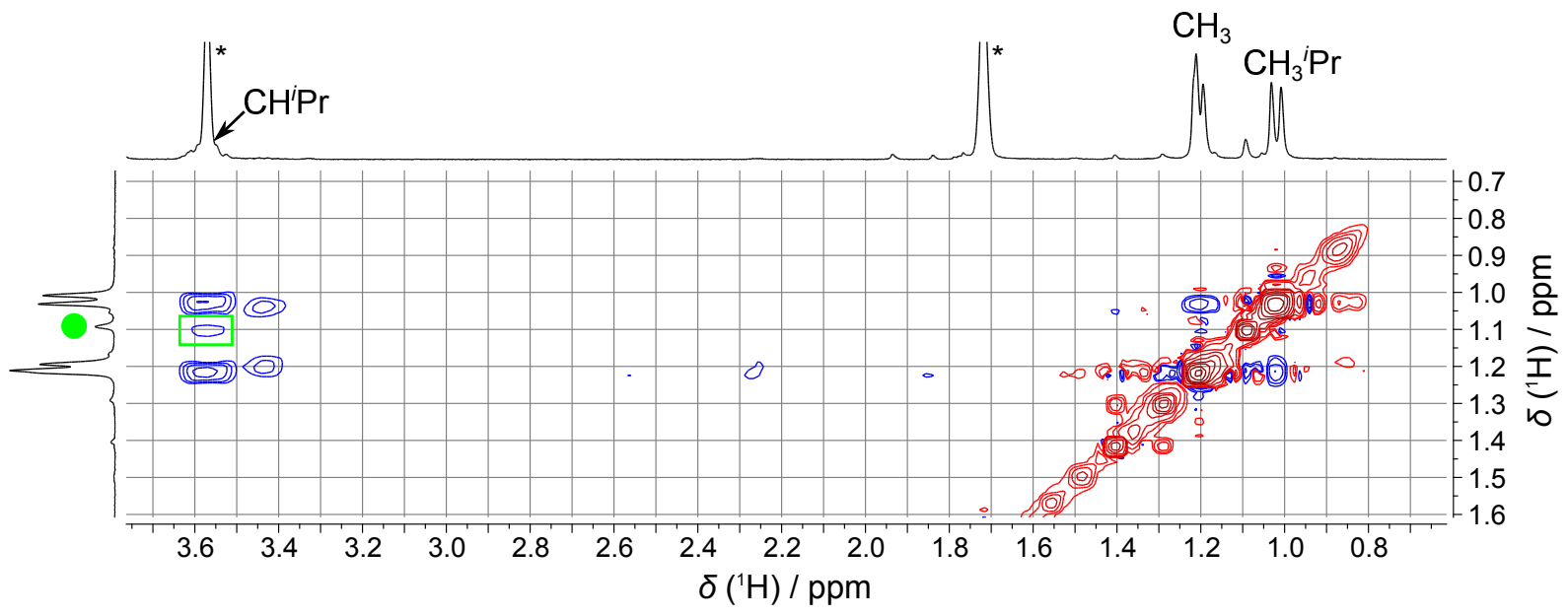

Figure 6.9.: ${ }^{1} \mathrm{H}$ NOESY spectrum (400 MHz) of $K\left[\mathrm{~L}^{\text {bisnac }} \mathrm{Ni}_{2}\left(\mu-\eta^{1}: \eta^{1}-\mathbf{N}_{2} \mathbf{H}_{2}\right)\right]$. Protons belonging to the $\mathrm{N}_{2} \mathrm{H}_{2}{ }^{2-}$ bridge show a NOE correlation to the $\mathrm{CH}$ proton of the ispropyl groups. Residual solvents are marked $\left({ }^{*}\right)$. 


\subsection{Protonation of the Hydrazido Bridge}

It was possible to protonate the $\mathrm{N}_{2} \mathrm{H}_{3}{ }^{-}$bridging ligand of $\left[\mathbf{L}^{\text {bisnac }} \mathrm{Ni}_{\mathbf{2}}\left(\mu-\eta^{\mathbf{1}}: \eta^{\mathbf{1}}-\mathbf{N}_{\mathbf{2}} \mathbf{H}_{\mathbf{3}}\right)\right]$ to yield [ $\left[\mathbf{L}^{\text {bisnac }} \mathbf{N i}_{2}\left(\mu-\eta^{1}: \eta^{1}-\mathbf{N}_{2} \mathbf{H}_{4}\right)\right]$ OTf (Scheme 6.5) $)$ Addition of [LutH]OTf (lutidinium triflate) to the THF solution of $\left[\mathbf{L}^{\text {bisnac }} \mathbf{N i}_{\mathbf{2}}\left(\mu-\eta^{\mathbf{1}}: \eta^{\mathbf{1}}-\mathbf{N}_{\mathbf{2}} \mathbf{H}_{\mathbf{3}}\right)\right]$ resulted in immediate color change from a deep red solution to a dark green, indicating facile protonation of the hydrazido ligand to hydrazine.
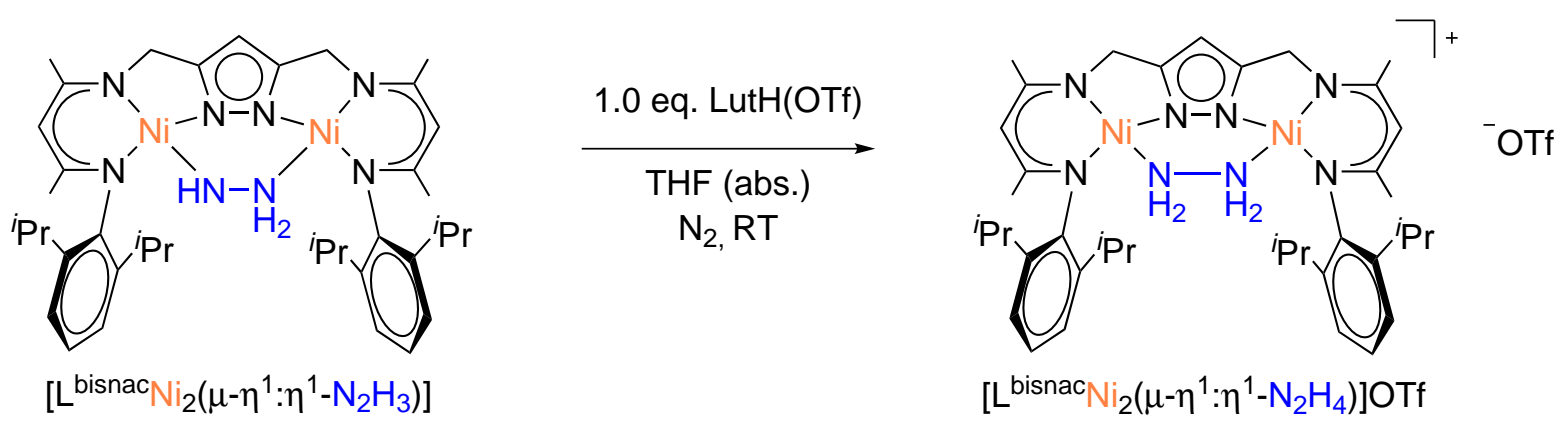

Scheme 6.5: Synthesis of [ $\left.{ }^{\text {bisnac }} \mathrm{Ni}_{2}\left(\mu-\eta^{1}: \eta^{1}-\mathbf{N}_{2} \mathrm{H}_{4}\right)\right] \mathrm{OTf}$ via protonation of [ $\left.L^{\text {bisnac }} \mathrm{Ni}_{2}\left(\mu-\eta^{1}: \eta^{1}-\mathrm{N}_{2} \mathrm{H}_{3}\right)\right]$.

Crystals of the protonated complex were obtained by slow diffusion of pentane vapours into the reaction solution or layering with hexanes. However, they were not optimal for X-ray analysis. The proposed molecular structure of $\left[\mathbf{L}^{\text {bisnac }} \mathbf{N i}_{2}\left(\mu-\eta^{1}: \eta^{1}-\mathbf{N}_{2} \mathbf{H}_{4}\right)\right] \mathbf{O T f}$ is shown in Figure 6.10. In the sideview of the complex, illustrated on the right side of Figure 6.10, a twist of the complex was observed. This finding fits the trend for the protonation state of the $\mu-\eta^{1}: \eta^{1}$ bridging unit and the associated steric demand which is reflected by an enlarged torsion angle for the hydrazine bridging ligand ( $\Varangle(\mathrm{Ni1}-\mathrm{N} 7-\mathrm{N} 8-\mathrm{Ni} 2)$ $\left.=92.42^{\circ}\right)$.
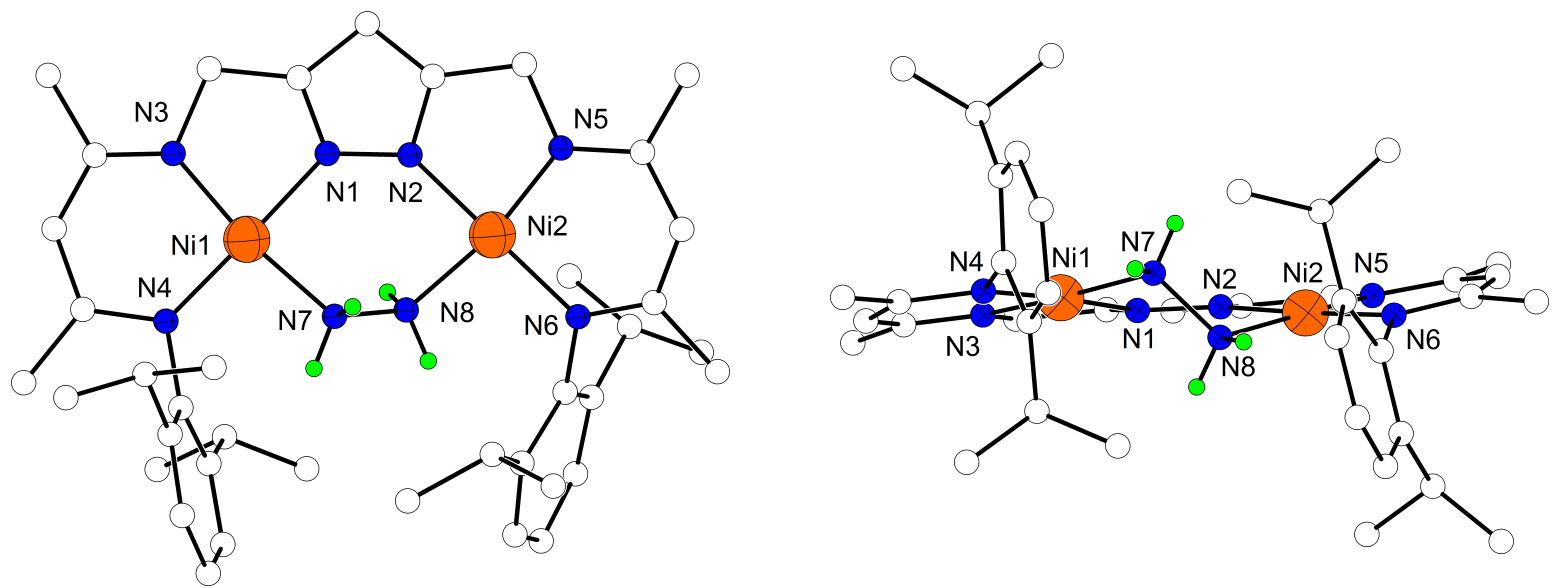

Figure 6.10.: The picture of $\left[\mathrm{Lisnac}^{\text {bisi }} \mathrm{Ni}_{2}\left(\mu-\eta^{1}: \eta^{1}-\mathrm{N}_{2} \mathrm{H}_{4}\right)\right] \mathrm{OTf}$ indicating the molecular structure. Left shows the topview and right the sideview of the complex. Hydrogen atoms except the nitrogen bound ones are omitted for clarity. 
The ${ }^{1} \mathrm{H}$ NMR spectrum of the reaction mixture (measured at $40{ }^{\circ} \mathrm{C}$ ) is depicted in Figure 6.11 and revealed the formation of a symmetric species as expected after protonation. A resonance at $3.74 \mathrm{ppm}$ corresponding to a normalized integral of four and a strong line broadening at room temperature is illustrated in the inset of Figure 6.11. This signal was assigned to the neutral hydrazine ligand $\mathrm{N}_{2} \mathrm{H}_{4}$. The found resonance concurs with other reported proton resonances of bridged $\mu^{2}-\eta^{1}: \eta^{1}$ hydrazine ligands in dimeric iron $\left(\delta\left\{{ }^{1} \mathrm{H}\right\}\right.$ $=2.54 \mathrm{ppm})^{[271]}$ and tungsten complexes $\left(\delta\left\{{ }^{1} \mathrm{H}\right\}=4.26 \mathrm{ppm}\right)^{[273]}$. A side-on $\eta^{2}-\mathrm{N}_{2} \mathrm{H}_{4}$ coordinated mononuclear $\mathrm{Ni}^{\mathrm{II}}$ complex showed a ${ }^{1} \mathrm{H}$ NMR shift of $3.50 \mathrm{ppm} .{ }^{[79]}$

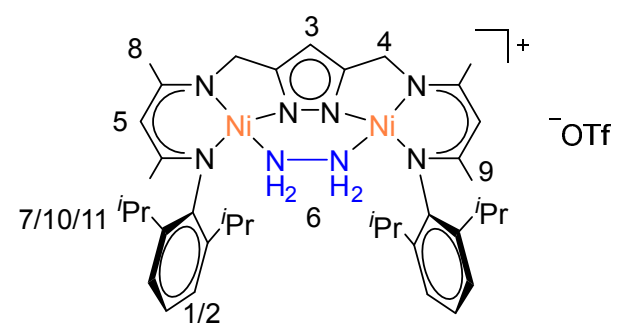

OTf

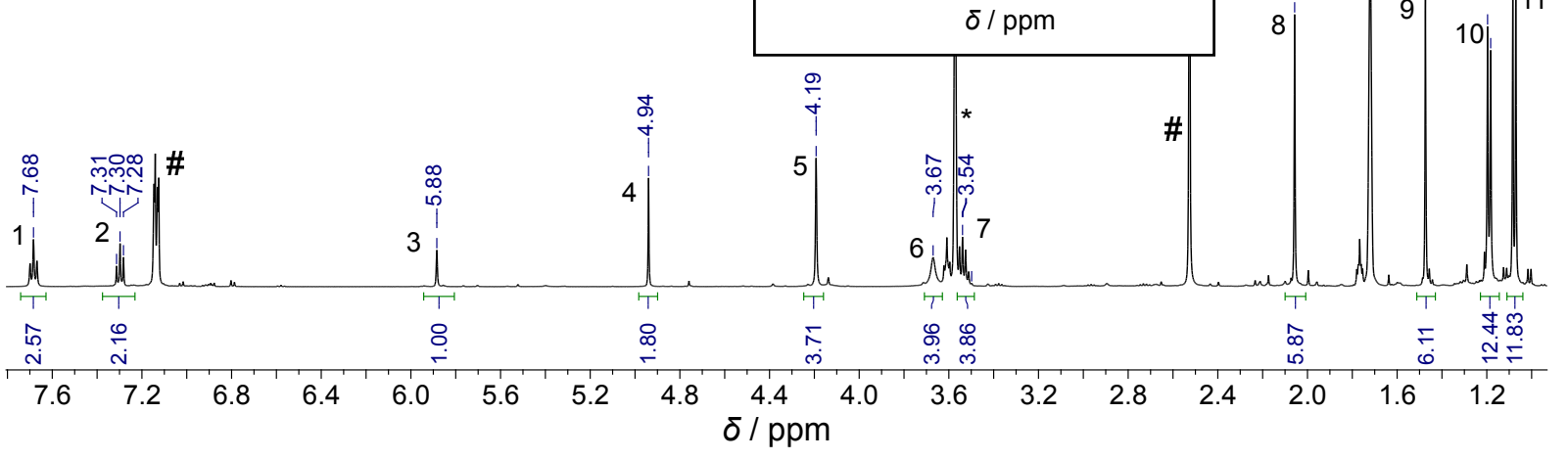

Figure 6.11.: ${ }^{1} \mathrm{H}$ NMR spectrum $(500 \mathrm{MHz})$ of $\left[\mathrm{L}^{\text {bisnac }} \mathrm{Ni}_{2}\left(\mu-\eta^{1}: \eta^{1}-\mathrm{N}_{2} \mathrm{H}_{4}\right)\right]$ OTf measured in THF- $\mathrm{D}_{8}$ at $40{ }^{\circ} \mathrm{C}$. The proton resonance of the $\mathrm{N}_{2} \mathrm{H}_{4}$ ligand (6) is observed at $3.67 \mathrm{ppm}$. Heating of the sample led to a decrease in line width and a shift to upfield (illustrated in the box). Resonances of lutidine (\#) and residual solvent $\left(^{*}\right)$ are indicated.

At elevated temperature the line width decreases due to a faster exchange between the ligand hydrogen atoms while the signal shifts to higher field, eventually leading to an overlay with solvent resonances at $60{ }^{\circ} \mathrm{C}$ (bottom spectrum shown in the inset).

The ${ }^{1} \mathrm{H}_{-}{ }^{15} \mathrm{~N}$ HMBC spectrum recorded at $40{ }^{\circ} \mathrm{C}$ is shown in Figure 6.12 (a). The ${ }^{1} J_{\mathrm{NH}}$ correlation occured at $-348.1 \mathrm{ppm}\left({ }^{1} J_{\mathrm{NH}}=71 \mathrm{~Hz}\right)$ and is similar to the chemical shift observed for the $\mathrm{NH}_{2}$ unit of the bridge in $\left[\mathbf{L}^{\text {bisnac }} \mathbf{N i}_{\mathbf{2}}\left(\mu-\eta^{\mathbf{1}}: \eta^{\mathbf{1}}-\mathbf{N}_{\mathbf{2}} \mathbf{H}_{\mathbf{3}}\right)\right]\left(\delta\left\{{ }^{1} \mathrm{H}\right\} 2.26 / \delta\left\{{ }^{15} \mathrm{~N}\right\}\right.$ $=-343.3 \mathrm{ppm})$. Accordingly, the same correlation is observed in the ${ }^{1} \mathrm{H}-{ }^{15} \mathrm{~N}-\mathrm{HSQC}$ experiment shown in Figure 6.12 (b).

The observed resonance agrees well with the reported mononuclear $\mu-\eta^{2}: \eta^{2}$ hydrazine coordinated $\mathrm{Ni}^{\mathrm{II}}$ complex from LIMBERG et al. $\left(\delta\left\{{ }^{15} \mathrm{~N}\right\}=-361.3 \mathrm{ppm}\right) . \stackrel{[79]}{[}^{2}$ 


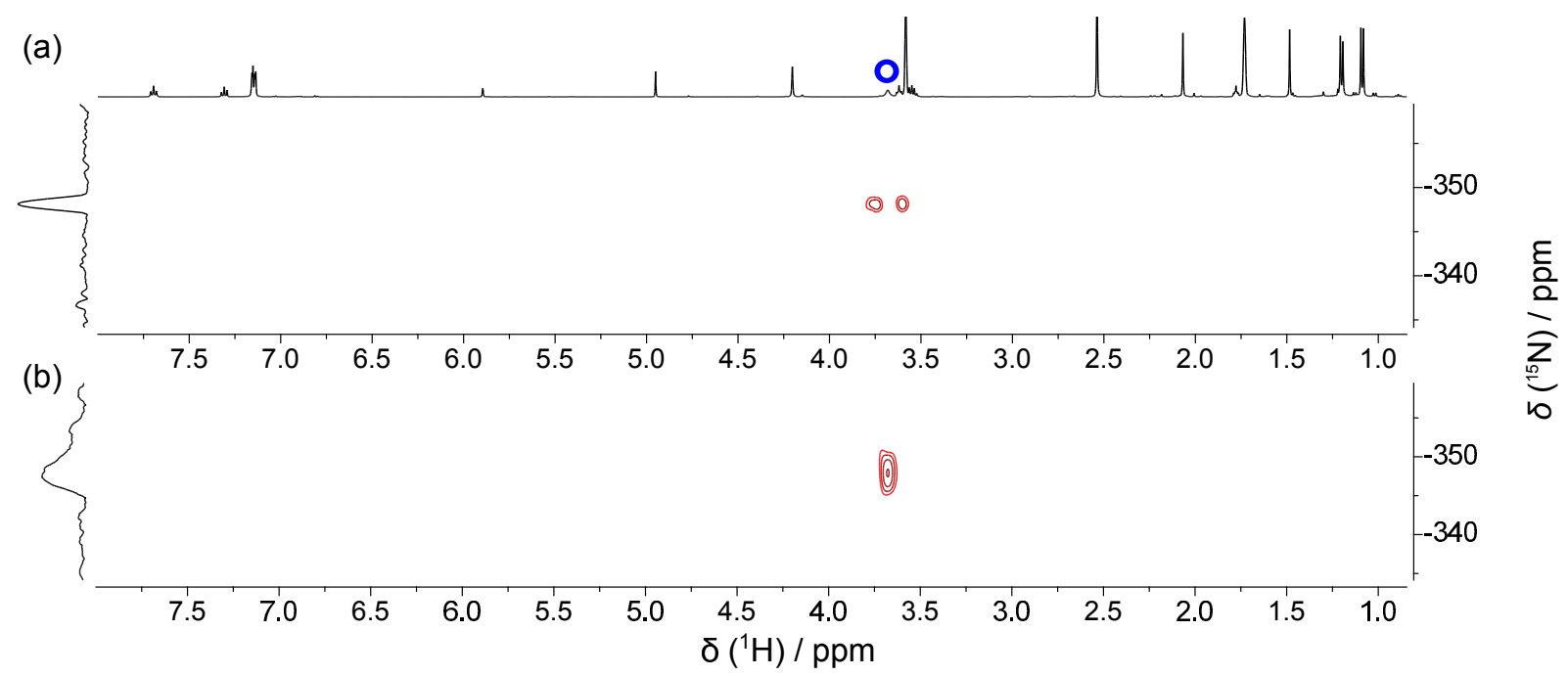

Figure 6.12.: ${ }^{1} \mathrm{H}^{-15} \mathrm{~N}$ correlation spectra $(500 \mathrm{MHz})$ of $\left[\mathrm{L}^{\text {bisnac }} \mathrm{Ni}_{2}\left(\mu-\eta^{1}: \eta^{1}-\mathrm{N}_{2} \mathrm{H}_{4}\right)\right] \mathrm{OTf}$. A correlation of the ${ }^{1} \mathrm{H}$ resonance (O) is observed for the $\mathrm{N}_{2} \mathrm{H}_{4}$ bridging ligand. (a) shows the ${ }^{1} \mathrm{H}^{-15} \mathrm{~N}-\mathrm{HMBC}$ spectrum. (b) shows the ${ }^{1} \mathrm{H}-{ }^{15} \mathrm{~N}-\mathrm{HSQC}$ spectrum. Both measurements have been conducted with samples of a THF- $\mathrm{D}_{8}$ solution at $40^{\circ} \mathrm{C}$.

Investigation by IR spectroscopy revealed bands at $\nu=3204 \mathrm{~cm}^{-1}, 3247 \mathrm{~cm}^{-1}, 3260 \mathrm{~cm}^{-1}$ and $3282 \mathrm{~cm}^{-1}$, which lie in the typical range of $\mathrm{NH}$ symmetric and asymmetric stretching vibrations of hydrazine ligands. ${ }^{[79,274,275]}$ Furthermore, the bands are well comparable to stretching vibrations reported for bridged $\mu^{2}-\eta^{1}: \eta^{1} \mathrm{~N}_{2} \mathrm{H}_{4}$ ligands. $\stackrel{[276]}{ }$ 


\subsection{Thermal Conversion to an Amido-Bridged Complex}

At elevated temperatures $\left(60-70{ }^{\circ} \mathrm{C}\right)\left[\mathbf{L}^{\text {bisnac }} \mathbf{N i}_{\mathbf{2}}\left(\mu-\eta^{\mathbf{1}}: \eta^{\mathbf{1}}-\mathbf{N}_{\mathbf{2}} \mathbf{H}_{\mathbf{3}}\right)\right]$ (dissolved in THF) was slowly transformed to the amido bridged $\left[\mathbf{L}^{\text {bisnac }} \mathbf{N i}_{\mathbf{2}}\left(\mu-\mathbf{N H}_{2}\right)\right]$ (Scheme 6.6) $)$.
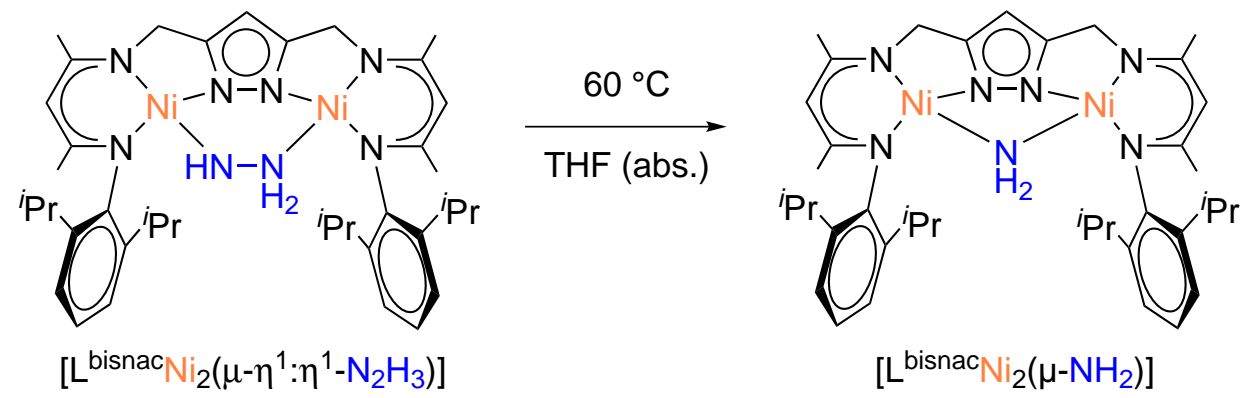

Scheme 6.6: The transformation of $\left[L^{\text {bisnac }} \mathrm{Ni}_{2}\left(\mu-\eta^{1}: \eta^{1}-\mathbf{N}_{2} \mathrm{H}_{3}\right)\right]$ to $\left[\mathrm{L}^{\text {bisnac }} \mathrm{Ni}_{2}\left(\mu-\mathrm{NH}_{2}\right)\right]$ at elevated temperature.

After full conversion to the amido bridged complex, slow diffusion of pentane vapours into the reaction solution led to formation of yellowish crystals, suitable for X-ray diffraction analysis. The ORTEP diagram of the molecular structure of $\left[\mathbf{L}^{\mathbf{b i s n a c}} \mathbf{N i}_{\mathbf{2}}\left(\mu-\mathbf{N H}_{\mathbf{2}}\right)\right]$ is shown in Figure 6.13 and selected bond lengths and angles of $\left[\mathbf{L}^{\mathbf{b i s n a c}} \mathbf{N i}_{\mathbf{2}}\left(\boldsymbol{\mu}-\mathbf{N H}_{\mathbf{2}}\right)\right]$ are listed in Table 6.3.

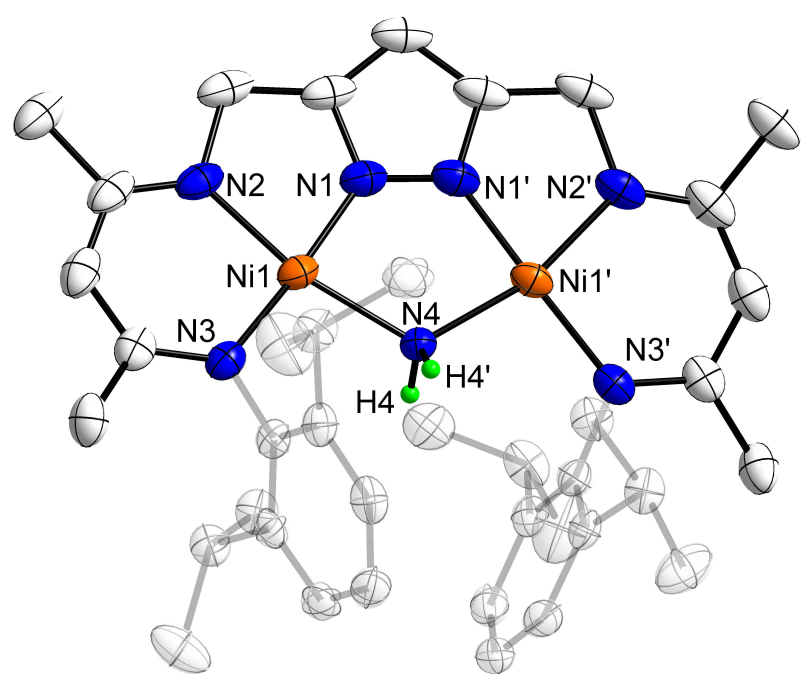

Figure 6.13.: Thermal displacement ellipsoids (shown at $50 \%$ probability) of the molecular structure of [ ${ }^{\text {bisnac }} \mathrm{Ni}_{2}\left(\mu-\mathrm{NH}_{2}\right)$ ]. All hydrogen atoms except the ones bound to $\mathrm{N} 4$ have been omitted for the sake of clarity (the hydrogen atoms are displayed with a fixed radius of $0.135 \AA$ ).

The Ni-N4 distance in $\left[\mathbf{L}^{\text {bisnac }} \mathbf{N i}_{\mathbf{2}}\left(\boldsymbol{\mu}-\mathbf{N H}_{\mathbf{2}}\right)\right]$ is $2.003 \AA$ which is consistent with other reported complexes containing a bridging $\mu-\mathrm{NH}_{2}{ }^{-}$ligand. $\stackrel{[277-281]}{ }$ Dinuclear nickel complexes of preorganized ligands including a $\mu-\mathrm{NH}_{2}{ }^{-}$bridging ligand were so far not reported in the literature. However, dimeric amido-bridged nickel complexes are known. ${ }^{[282.283]}$ For instance, a dimeric cyclopentadienylnickel amido complex $\left[\left(\eta-\mathrm{C}^{5} \mathrm{Me}_{4} \mathrm{R}^{\prime}\right) \mathrm{Ni}(\mu-\mathrm{NHR})\right]_{2}(\mathrm{R}$ 
Table 6.3.: Selected bond lengths and angles of $\left[\mathrm{L}^{\text {bisnac }} \mathrm{Ni}_{2}\left(\mu-\mathrm{NH}_{2}\right)\right]$.

\begin{tabular}{cccc}
\hline \multicolumn{2}{c}{ Bond lengths / } & \multicolumn{2}{c}{ Angle / } \\
\hline Ni1-N1 & $1.803(3)$ & N1-Ni1-N3 & $173.22(1)$ \\
Ni1-N3 & $1.895(2)$ & N1-Ni1-N2 & $81.29(1)$ \\
Ni1-N2 & $1.906(2)$ & N3-Ni1-N2 & $95.03(1)$ \\
Ni1-N4 & $2.0029(2)$ & N1-Ni1-N4 & $83.62(1)$ \\
Ni1...Ni1' & $3.4633(6)$ & N3-Ni1-N4 & $100.36(1)$ \\
& & N2-Ni1-N4 & $164.44(1)$ \\
& & Ni1-N4-Ni1' & $119.675(2)$ \\
\hline
\end{tabular}

$=\mathrm{Ph}, p$-tol, 2,6-xyl, ${ }^{t} \mathrm{Bu} ; \mathrm{R}^{\prime}=\mathrm{Me}$, Et) was characterized by Holland et al. ${ }^{[282]}$, which contains a secondary amide bridging unit with $\mathrm{Ni}-\mathrm{N}$ distances ranging between $1.942 \AA$ and $1.962 \AA$.

Compared to that and the precursor complex $\left[\mathbf{L}^{\text {bisnac }} \mathbf{N i}_{2}\left(\mu-\eta^{1}: \eta^{1}-\mathbf{N}_{\mathbf{2}} \mathbf{H}_{3}\right)\right]$, which features a Ni-N bond length of $d(\mathrm{Ni}-\mathrm{N} 7)=1.891(2) \AA$ and $d(\mathrm{Ni}-\mathrm{N} 8)=1.914(2) \AA$ (Table 6.1), the distance in $\left[\mathbf{L}^{\text {bisnac }} \mathbf{N i}_{\mathbf{2}}\left(\boldsymbol{\mu}-\mathbf{N H}_{\mathbf{2}}\right)\right]$ is significantly larger. This can be explained by the smaller size of the $\mu-\mathrm{NH}_{2}{ }^{-}$ligand, which in turn produces a strain on the entire system. Therefore, the Ni-N distance in $\left[\mathbf{L}^{\text {bisnac }} \mathbf{N i}_{\mathbf{2}}\left(\mu-\mathbf{N H}_{\mathbf{2}}\right)\right]$ is also very similar to bonds of other bridging ligands of comparable size like $\mathrm{OH}^{-}$in $\left[\mathbf{L}^{\text {bisnac }} \mathbf{N i}_{\mathbf{2}}(\mu-\mathrm{OH})\right]$, which amounts to $d_{\mathrm{Ni}-\mathrm{OH}}=1.994 \AA$. In contrast the distance of $\mathrm{Br}^{-}$in $\left[\mathbf{L}^{\text {bisnac }} \mathbf{N i}_{\mathbf{2}}(\mu-\mathbf{B r})\right]\left(d_{\mathrm{Ni}-\mathrm{Br}}=\right.$ $2.381 \AA$ and $2.398 \AA$ ) is significantly larger according to the greater size of the halide.

As a consequence of small size of the $\mu-\mathrm{NH}_{2}{ }^{-}$ligand, a decrease in the $\mathrm{Ni} \cdot \mathrm{Ni}$ distance from $3.936 \AA \quad\left(\left[\mathbf{L}^{\text {bisnac }} \mathbf{N i}_{\mathbf{2}}\left(\mu-\eta^{\mathbf{1}}: \eta^{\mathbf{1}}-\mathbf{N}_{\mathbf{2}} \mathbf{H}_{\mathbf{3}}\right)\right]\right)$ to $3.463 \AA$ is observed. The angle between the nitrogen of the bridging unit and adjacent nickel atoms is $\Varangle$ Ni1-N4-Ni1' $119.68^{\circ}$, which corresponds well with the observed angle for the hydroxo-bridged complex $\left[\mathbf{L}^{\text {bisnac }} \mathbf{N i}_{\mathbf{2}}(\mu-\mathrm{OH})\right]\left(\varangle \mathrm{Ni}-\mathrm{O}-\mathrm{Ni}=120.91^{\circ}\right)$. It is much larger than the bromido-bridged analogue $\left[\mathbf{L}^{\text {bisnac }} \mathbf{N i}_{2}(\mu-\mathrm{Br})\right]$, featuring an angle of $\Varangle \mathrm{Ni}-\mathrm{Br}-\mathrm{Ni}=105.60^{\circ}$. The significant difference between the angles can be attributed to the greater size of $\mathrm{Br}^{-}$.

Figure 6.14 shows the ${ }^{1} \mathrm{H}$ NMR spectrum of a sample containing the starting material $\left[\mathbf{L}^{\text {bisnac }} \mathbf{N i}_{2}\left(\mu-\eta^{1}: \eta^{1}-\mathbf{N}_{2} \mathbf{H}_{3}\right)\right]$ dissolved in THF-D $\left[\mathbf{L}^{\text {bisnac }} \mathbf{N i}_{2}\left(\mu-\mathbf{N H}_{2}\right)\right]$ (top spectrum) and the spectrum after thermal conversion to $\left[\mathbf{L}^{\text {bisnac }} \mathbf{N i}_{\mathbf{2}}\left(\mu-\mathbf{N H}_{2}\right)\right]$ (bottom spectrum). Quantitative transformation was observed (Figure 6.14). 

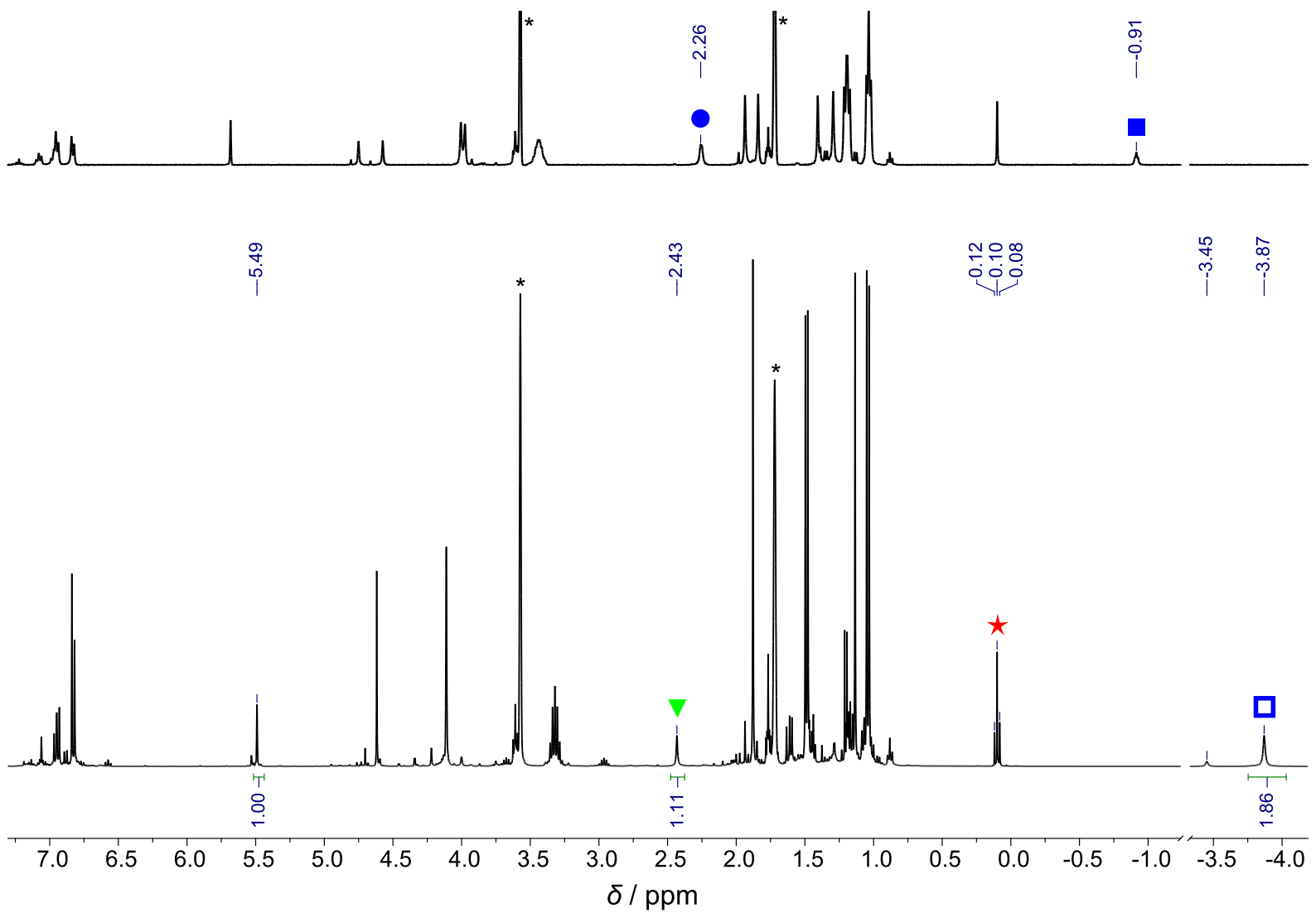

Figure 6.14.: Top: ${ }^{1} \mathrm{H}$ NMR spectrum $(400 \mathrm{MHz})$ of a sample containing [ $\mathrm{L}^{\text {bisnac }} \mathrm{Ni}_{2}\left(\mu-\eta^{1}: \eta^{1}-\mathbf{N}_{\mathbf{2}} \mathbf{H}_{3}\right)$ ]. Bottom: ${ }^{1} \mathrm{H}$ NMR spectrum (400 MHz) after the complete transformation to [ $\mathrm{L}^{\text {bisnac }} \mathbf{N i}_{2}\left(\mu-\mathrm{NH}_{2}\right)$ ]. Besides the development of a new upfield shifted resonance at $-3.87 \mathrm{ppm}(\square)$ and a signal at $2.43 \mathrm{ppm}(\nabla$, water) another pattern at $0.1 \mathrm{ppm}$ ( $\star$, decomposed silicon grease) arose. Residual solvents are marked $\left({ }^{*}\right)$. 


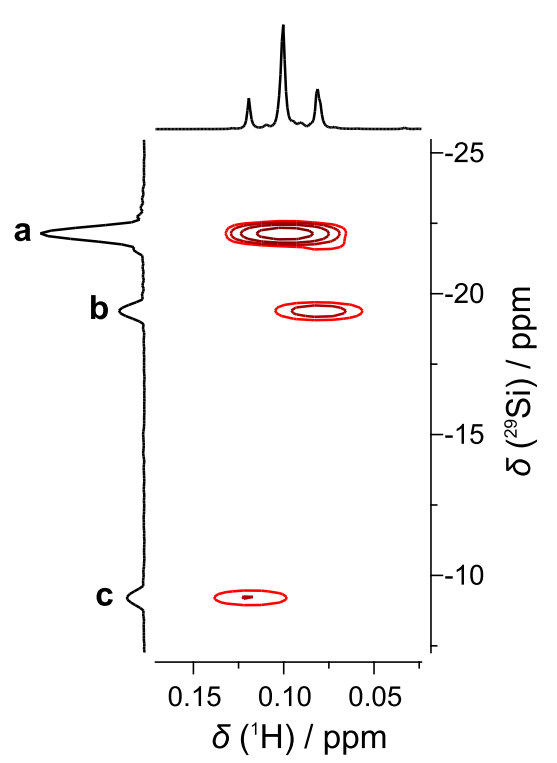

Figure 6.15.: Excerpt of the ${ }^{29} \mathrm{Si}$ HMBC spectrum $(500 \mathrm{MHz})$ of [L bisnac $\mathrm{Ni}_{2}\left(\mu-\mathrm{NH}_{2}\right)$ ] in $\mathrm{THF}-\mathrm{D}_{8}$.
Newly developed signals at $0.08 \mathrm{ppm}$ and $0.12 \mathrm{ppm}$ are associated with fragments originating from the cleavage of polydimethylsiloxane, a component of silicon grease. A ${ }^{29} \mathrm{Si}^{-}{ }^{1} \mathrm{H} \mathrm{HMBC}$ experiment proved the presence of silicon based species by associated correlations (Figure 6.15). It is reported that particular hydroxyl-terminated polymers are susceptible towards thermolysis, initially leading to the condensation at the chain ends followed by depolymerization. ${ }^{[293]}$ Consequently, degradation of hydroxy polydimethylsiloxane led to an increase of water $(2.43 \mathrm{ppm}$ in Figure 6.14).

The fact that at no point of the reaction $\left[\mathbf{L}^{\text {bisnac }} \mathbf{N i}_{2}(\mu-\mathrm{OH})\right]$ was produced, indicates that neither the precursor $\left[\mathbf{L}^{\text {bisnac }} \mathbf{N i}_{\mathbf{2}}\left(\mu-\eta^{\mathbf{1}}: \eta^{\mathbf{1}}-\mathbf{N}_{\mathbf{2}} \mathbf{H}_{\mathbf{3}}\right)\right.$ ] nor the transformation product $\left[\mathbf{L}^{\text {bisnac }} \mathbf{N i}_{\mathbf{2}}\left(\mu-\mathbf{N H}_{2}\right)\right]$ are susceptible towards water. This can be explained by the higher basicity ${ }^{\dagger}$ of the nitrogen based ligands in comparison to $\mathrm{OH}^{-}$, which results in preferred binding to the metal centers.

Furthermore, according to the HSAB concept, $\mathrm{OH}^{-}$is considered as a harder Lewis base than $\mathrm{NH}_{2}{ }^{-}$and $\mathrm{N}_{2} \mathrm{H}_{3}{ }^{-} \cdot \underline{[300-302]}$ The $\mathrm{Ni}^{2+}$ ions are considered as softer Lewis-acids. $\stackrel{[303,304]}{ }$ The effect is further promoted by the threefold anionic nacnac-ligand system. Therefore, softer Lewis-bases than $\mathrm{OH}^{-}$are preferentially bound to $\mathrm{Ni}^{2+}$, in particular $\mathrm{NH}_{2}{ }^{-}$and $\mathrm{N}_{2} \mathrm{H}_{3}^{-}$.

Time dependent ${ }^{1} \mathrm{H}$ NMR spectra illustrate the very slow process of conversion to $\left[\mathbf{L}^{\text {bisnac }} \mathbf{N i}_{\mathbf{2}}\left(\mu-\mathbf{N H}_{2}\right)\right.$ ] Figure 6.16). After heating the sample to $60{ }^{\circ} \mathrm{C}$ for about two weeks, characteristic proton resonances ( $\mathbf{\square}$ and $\bullet$ ) of the $\mu-\eta^{1}: \eta^{1}-\mathrm{N}_{2} \mathrm{H}_{3}$ - ligand gradually vanished. At the same time a new resonance at higher field emerged at $-3.87 \mathrm{ppm}(\boldsymbol{\square})$, which was attributed to the $\mathrm{NH}_{2}{ }^{-}$ligand. When increasing the temperature by ten degrees conversion was accelerated. Eventually, $\left[\mathbf{L}^{\text {bisnac }} \mathbf{N i}_{\mathbf{2}}\left(\mu-\eta^{\mathbf{1}}: \eta^{\mathbf{1}}-\mathbf{N}_{\mathbf{2}} \mathbf{H}_{\mathbf{3}}\right)\right]$ was completely consumed after around 13 weeks (top spectrum of Figure 6.16). Besides the main signal set of $\left[\mathbf{L}^{\text {bisnac }} \mathbf{N i}_{\mathbf{2}}\left(\mu-\mathbf{N H}_{2}\right)\right.$, a second set of peaks of much smaller intensity was determined. It includes another upfield shifted singlet resonance at $-3.45 \mathrm{ppm}$, also corresponding to two protons (not marked in Figure 6.16).

\footnotetext{
${ }^{\dagger} \mathrm{p} K_{\mathrm{a}\left[\mathrm{H}_{2} \mathrm{O}\right]}\left(\mathrm{H}_{2} \mathrm{O}\right)=14^{\left[\frac{294,295,304]}{4}\right.}, \mathrm{p} K_{\mathrm{a}[\mathrm{DMSO}]}\left(\mathrm{H}_{2} \mathrm{O}\right)=31.4^{\left[\frac{297]}{2}\right.} ; \mathrm{p} K_{\mathrm{a}\left[\mathrm{H}_{2} \mathrm{O}\right]}\left(\mathrm{NH}_{3}\right)=23^{\left[\frac{[304]}{}\right.}, \mathrm{p} K_{\mathrm{a}[\mathrm{DMSO}]}\left(\mathrm{NH}_{3}\right)=$ $41^{[298]} ; \mathrm{p} K_{\mathrm{a}[\mathrm{DMSO}]}\left(\mathrm{N}_{2} \mathrm{H}_{4}\right)=38^{[299]}$.
} 
Slow thermal degradation of hydroxy polydimethylsiloxane is indicated by increasing resonances of corresponding fragments $0.1 \mathrm{ppm}$ ( $\star$ in Figure 6.16). As a consequence of the hydroxy polydimethylsiloxane depolimerization the amount of water in the sample increased $(2.47 \mathrm{ppm}, \boldsymbol{\nabla})$.

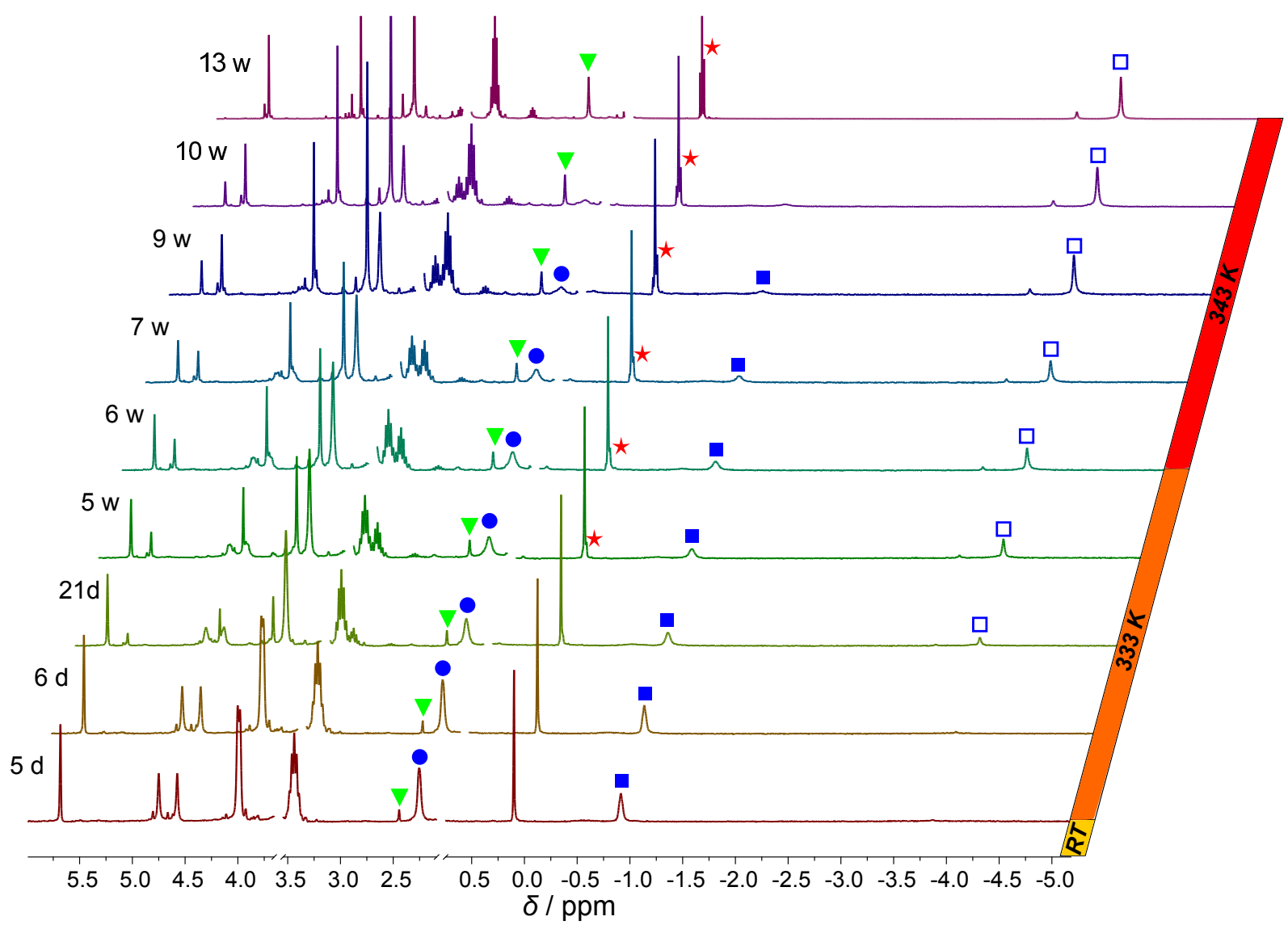

Figure 6.16.: Slow transformation of [ $\left[L^{\text {bisnac }} \mathbf{N i}_{2}\left(\mu-\eta^{1}: \eta^{1}-\mathbf{N}_{2} \mathbf{H}_{3}\right)\right]$ to [ $\left.\mathrm{L}^{\text {bisnac }} \mathbf{N i}_{2}\left(\mu-\mathrm{NH}_{2}\right)\right]$ at elevated temperatures $(298 \mathrm{~K}, 333 \mathrm{~K}$ and $343 \mathrm{~K})$ over 13 weeks illustrated by stacked ${ }^{1} \mathrm{H}$ NMR spectra $(300 \mathrm{MHz})$.

Compared to other reported systems of bridged $\mathrm{NH}_{2}{ }^{-}$ligands showing ${ }^{1} \mathrm{H}$ NMR chemical shifts ranging from -1.40 to $3.74 \mathrm{ppm}, \stackrel{[278,283-285]}{2}$ the $\mathrm{NH}$ proton resonance of $\left[\mathbf{L}^{\text {bisnac }} \mathbf{N i}_{\mathbf{2}}\left(\mu-\mathbf{N H}_{\mathbf{2}}\right]\right.$ is shifted to higher field $\left(\delta\left\{{ }^{1} \mathrm{H}\right\}=-3.87 \mathrm{ppm}\right)$. However, the resonance is comparable to the unusual upfield chemical shift of the hydroxo-bridged analogue $\left[\mathbf{L}^{\text {bisnac }} \mathbf{N i}_{2}(\mu-\mathrm{OH}]\right.$, featuring a hydroxo proton resonance at $-7.26 \mathrm{ppm}$. This further substantiates the tendency of the bis( $\beta$-diketiminato)-complex system to exert an enhanced electronic shielding effect on anionic bridging ligands.

Abscence of proton-proton correlations for the $\mathrm{N}_{2} \mathrm{H}_{3}{ }^{-}$ligand in the ${ }^{1} \mathrm{H}-{ }^{1} \mathrm{H}$ COSY experiment confirmed the complete conversion of $\left[\mathbf{L}^{\text {bisnac }} \mathbf{N i}_{\mathbf{2}}\left(\mu-\eta^{\mathbf{1}}: \eta^{\mathbf{1}}-\mathbf{N}_{\mathbf{2}} \mathbf{H}_{\mathbf{3}}\right)\right]$ (the spectrum is shown in the appendix, Figure D.11).

Similar to other complexes of this ligand type, which contain hydrogen atoms in a bridging unit, proton NOE correlations of the $\mathrm{NH}_{2}{ }^{-}$ligand ( $\mathbf{\square}$ ) to the aryl-isopropyl groups were also observed for $\left[\mathbf{L}^{\text {bisnac }} \mathbf{N i}_{\mathbf{2}}\left(\boldsymbol{\mu}-\mathbf{N H}_{\mathbf{2}}\right)\right](\mathbf{\square})$ in the NOESY experiment (Figure 6.17). 
Interestingly, a very small ${ }^{1} \mathrm{H}$ signal in the same upfield region, possibly corresponding to another amido-based species (•) also exhibits NOE correlations in the spectrum. The ${ }^{1} \mathrm{H}_{-}{ }^{15} \mathrm{~N}$ HMBC spectrum (Figure 6.18) confirmed that this signal originates from a $\mathrm{NH}$ based ligand.

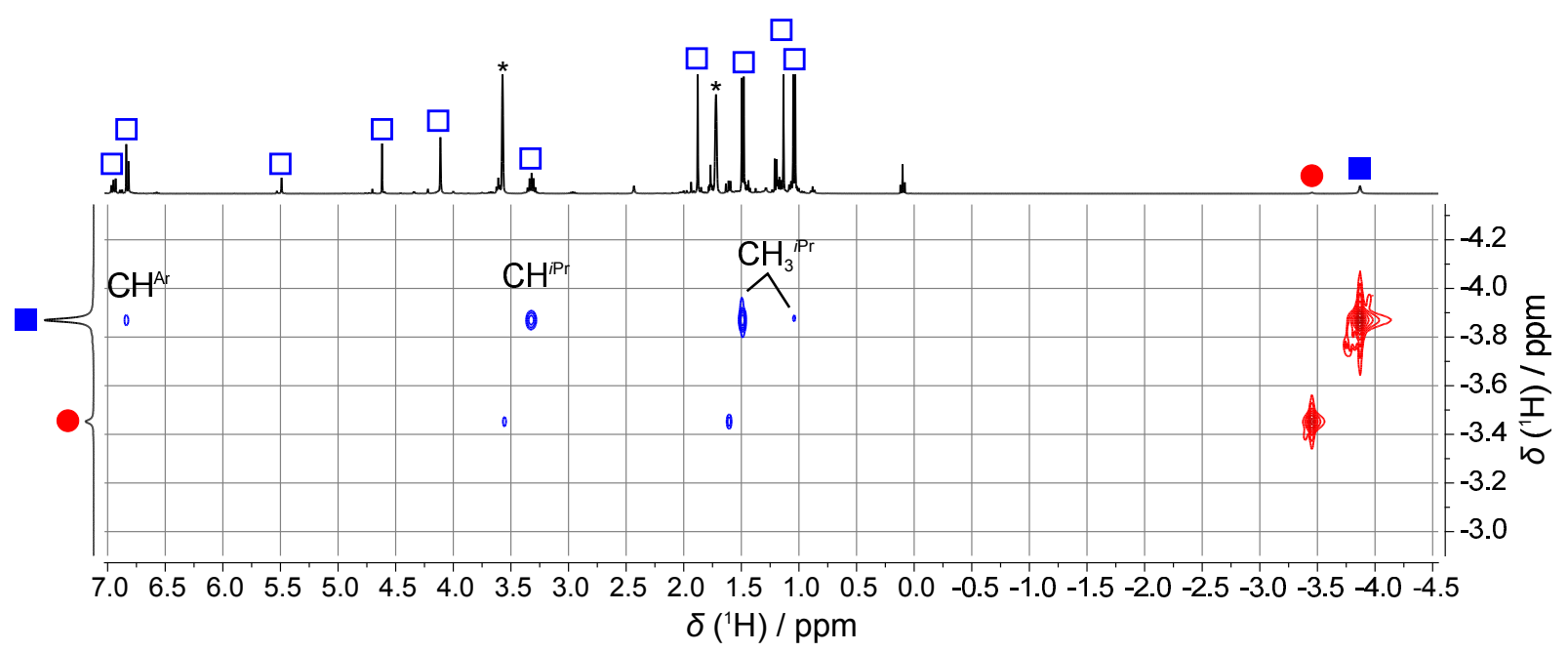

Figure 6.17.: Detail of the ${ }^{1} \mathrm{H}$ NOESY spectrum $(400 \mathrm{MHz})$ of the reaction mixture containing [ ${ }^{\text {bisnac }} \mathbf{N i}_{2}\left(\mu-\mathrm{NH}_{2}\right)$ ] in THF-D $\mathrm{D}_{8}$. Residual solvents are marked $\left(^{*}\right)$. While the $\mathrm{NH}_{2}{ }^{-}$ligand $(\mathbf{\square})$ of the main species ( $\square$ ) shows expected NOE correlations to the isopropyl substituents of the DIPP sidearms correlations associated to another compound were observed $(\bullet)$.

The contour plot of the ${ }^{1} \mathrm{H}^{-15} \mathrm{~N}$ HMBC experiment (Figure 6.18) yielded a strong $\mathrm{N}-\mathrm{H}$ correlation cross peak for the $\mu-\mathrm{NH}_{2}{ }^{-}$ligand $\left({ }^{1} J_{\mathrm{NH}}=59 \mathrm{~Hz}\right)$.

Moreover, further ${ }^{1} \mathrm{H}-{ }^{15} \mathrm{~N}$ HMBC experiments (optimized to long range $\mathrm{NH}$ couplings) did not reveal any ${ }^{15} \mathrm{~N}$ correlations of the ${ }^{1} \mathrm{H}$ signals at $2.43 \mathrm{ppm}(\nabla$, Figure 6.16) and $0.1 \mathrm{ppm}(\star$, Figure 6.16). This further confirms the association to water and silicon based fragments, rather than nitrogen based species.

The ${ }^{15} \mathrm{~N}$ resonance of the $\mathrm{NH}_{2}{ }^{-}$bridging ligand $(\boldsymbol{\square})$ occurs at $-452.6 \mathrm{ppm}\left({ }^{1} J_{\mathrm{NH}}=\right.$ $59 \mathrm{~Hz}$ ) and is shifted to higher fields than the resonances of literature known $\mu-\mathrm{NH}_{2}{ }^{-}$ ligands $(-45-67.7 \mathrm{ppm}) . \stackrel{[278,280]}{{ }^{2}}$ However, compared to the ${ }^{15} \mathrm{~N}$ shift of the precursor $\left[\mathbf{L}^{\text {bisnac }} \mathbf{N i}_{\mathbf{2}}\left(\mu-\eta^{\mathbf{1}}: \eta^{\mathbf{1}}-\mathbf{N}_{\mathbf{2}} \mathbf{H}_{\mathbf{3}}\right)\right]$, which was observed at $\delta\left\{{ }^{15} \mathrm{~N}\right\}=-313.8$ and $-343.3 \mathrm{ppm}$, the signal of the $\mathrm{NH}_{2}{ }^{-}$ligand is also shifted significantly to higher field as a result of higher electronic shielding of the $\mathrm{NH}_{2}{ }^{-}$nitrogen atom.

Furthermore, the spectrum revealed additional NH correlations $(\square)$ at $\delta\left\{{ }^{1} \mathrm{H}\right\}=1.37 \mathrm{ppm}$ $/ \delta\left\{{ }^{15} \mathrm{~N}\right\}=-398.15 \mathrm{ppm}, \delta\left\{{ }^{1} \mathrm{H}\right\}=3.55 \mathrm{ppm} / \delta\left\{{ }^{15} \mathrm{~N}\right\}=-249.48 \mathrm{ppm}$ and $\delta\left\{{ }^{1} \mathrm{H}\right\}=$ $-3.45 \mathrm{ppm} / \delta\left\{{ }^{15} \mathrm{~N}\right\}=-452.63 \mathrm{ppm}$, which are not associated with $\left[\mathbf{L}^{\text {bisnac }} \mathbf{N i}_{\mathbf{2}}\left(\mu-\mathbf{N H}_{\mathbf{2}}\right)\right]$ $(\bullet)$ or its precursor. In particular, the latter correlation exhibits a NH coupling constant of ${ }^{1} J_{\mathrm{NH}}=59 \mathrm{~Hz}$ which is consistent with the one observed for the bridging ligand in $\left[\mathbf{L}^{\text {bisnac }} \mathbf{N i}_{2}\left(\mu-\mathbf{N H}_{2}\right)\right]$. This observation along with similar chemical shifts suggests the presence of another amido based species.

The IR spectrum of $\left[\mathbf{L}^{\text {bisnac }} \mathbf{N i}_{\mathbf{2}}\left(\boldsymbol{\mu}-\mathbf{N H}_{\mathbf{2}}\right)\right]$ revealed bands at $3333 \mathrm{~cm}^{-1}$ and $3348 \mathrm{~cm}^{-1}$ 
corresponding to symmetric and asymmetric $\mathrm{N}-\mathrm{H}$ streches of the $\mathrm{NH}_{2}{ }^{-}$bridging ligand. They fall well within the expected range of the reported stretching vibrations for bridging amido ligands $\left(\tilde{\nu}_{\mathrm{NH}}=3258-3393 \mathrm{~cm}^{-1}\right) . \stackrel{[278,283-286]}{ }$ Since transition metal complexes with coordinated hydrazine are known to decompose to ammonia and nitrogen by disproportionation, $\stackrel{[79.80]}{\underline{ }}$ it was assumed that the same may be true for the decomposition of $\left[\mathbf{L}^{\text {bisnac }} \mathbf{N i}_{2}\left(\mu-\eta^{1}: \eta^{1}-\mathbf{N}_{2} \mathbf{H}_{3}\right)\right]$. In this case a disproportionation of three $\mathrm{N}_{2} \mathrm{H}_{3}{ }^{-}$ligands would yield one molecule of $\mathrm{NH}_{3}$ and one molecule of $\mathrm{N}_{2}$.

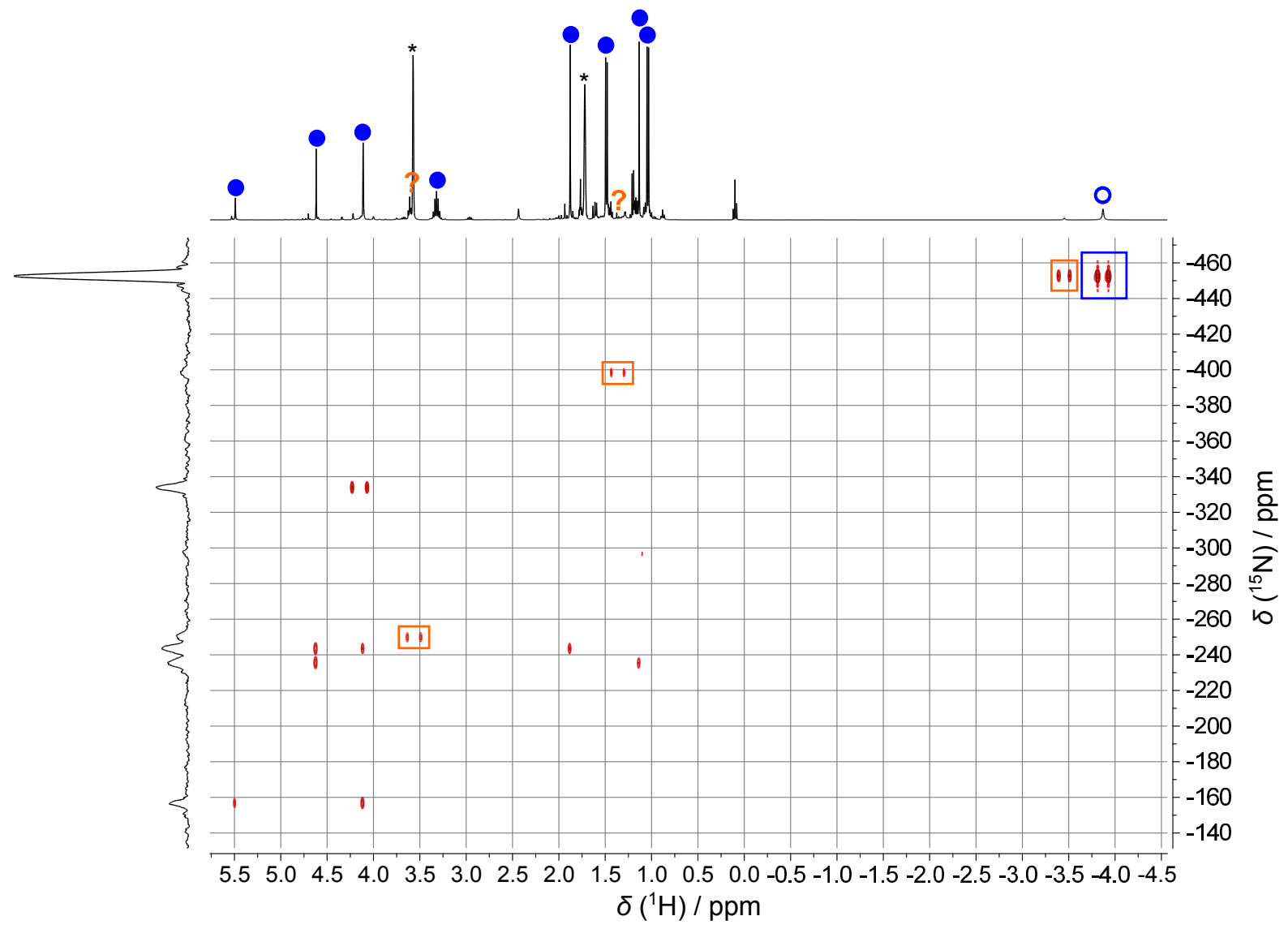

Figure 6.18.: ${ }^{1} \mathrm{H}-{ }^{15} \mathrm{~N}-\mathrm{HMBC}$ spectrum of $\left[\mathrm{L}^{\text {bisnac }} \mathrm{Ni}_{2}\left(\mu-\mathrm{NH}_{2}\right)\right]$. ${ }^{1} \mathrm{H}$ resonances associated with the main complex are indicated $\left(\bullet\right.$ and 0 ). In addition to ${ }^{15} \mathrm{~N}$ correlations of $\left[\mathrm{L}^{\text {bisnac }} \mathrm{Ni}_{2}\left(\mu-\mathrm{NH}_{2}\right)\right]$ (the ${ }^{1} \mathrm{~J}_{\mathrm{NH}^{-}}$ correlation of the bridging ligand is encased by a blue box), further weaker correlations are observed, which are encased by orange boxes. Residual solvents are marked $\left({ }^{*}\right)$.

Consequently, a $\mathrm{NH}_{3}$ proton resonance was expected around $0 \mathrm{ppm}$ in the ${ }^{1} \mathrm{H}$ NMR spectrum. ${ }^{[287-290]}$ During the course of decomposition of $\left[\mathbf{L}^{\text {bisnac }} \mathbf{N i}_{\mathbf{2}}\left(\mu-\eta^{\mathbf{1}}: \eta^{\mathbf{1}}-\mathbf{N}_{\mathbf{2}} \mathbf{H}_{\mathbf{3}}\right)\right]$, besides the new signals at $0.12-0.08 \mathrm{ppm}$, which were assigned to silicon based fragments of silicon oil degradation, no other resonances were observed in the ${ }^{1} \mathrm{H}$ NMR (bottom spectrum of Figure 6.14). A superimposed spectrum of a ${ }^{1} \mathrm{H}$ NMR reference data set of $\mathrm{NH}_{3}$ (atmospheric pressure) in THF- $\mathrm{D}_{8}$ and the reaction mixture is illustrated in Figure 6.19. Furthermore, no ${ }^{15} \mathrm{~N}-{ }^{1} \mathrm{H}$ correlation for $\mathrm{NH}_{3}\left({ }^{1} J_{\mathrm{NH}}=61.2 \mathrm{~Hz}\right)^{\left[\frac{[291]}{2}\right.}$ was found in the ${ }^{1} \mathrm{H}_{-}{ }^{15} \mathrm{~N}$ HMBC spectrum (Figure 6.18).

Due to the presence of water in the sample the development of $\mathrm{NH}_{4}{ }^{+}$was further con- 


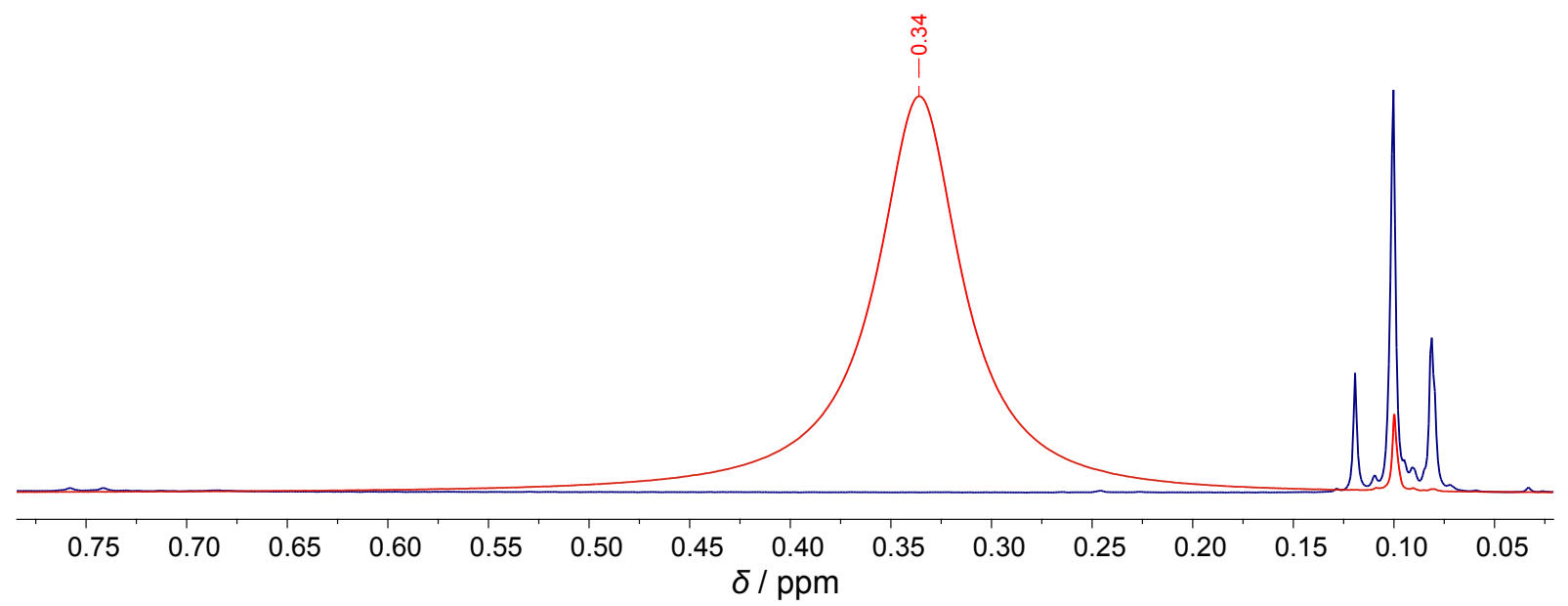

Figure 6.19.: ${ }^{1} \mathrm{H}$ NMR spectrum $(400 \mathrm{MHz})$ of $\left[\mathrm{L}^{\text {bisnac }} \mathrm{Ni}_{2}\left(\mu-\mathrm{NH}_{2}\right)\right](-)$ with a superimposed spectrum $(400 \mathrm{MHz})$ of ammonia in THF-D 8 (-). No signs of $\mathrm{NH}_{3}$ development were observed.

sidered. However, a doublet $\left({ }^{15} \mathrm{~N}\right)$ or triplet $\left({ }^{14} \mathrm{~N}\right)$ resonance at around $7.3 \mathrm{ppm}$ with a coupling constant of ${ }^{1} J_{15} \mathrm{NH}=71 \mathrm{~Hz}$ or ${ }^{1} J^{14} \mathrm{NH}=51 \mathrm{~Hz} \stackrel{[253]}{ }$ was not observed.

As illustrated in the ${ }^{15} \mathrm{~N}-{ }^{1} \mathrm{H}$ HMBC spectrum of the reaction mixture at its final state (Figure 6.18), other proton-nitrogen correlations (marked by orange boxes) cannot be assigned. These signals may be associated with NH containing (small) molecules or the formation of diazene as described in a) of Scheme 6.7. However, formed diazene readily decomposes to hydrazine and dinitrogen b), which would impede its detection. ${ }^{[304]}$ Nonetheless, ${ }^{1} \mathrm{H}_{-}{ }^{15} \mathrm{~N}$ correlations at $\delta\left\{{ }^{1} \mathrm{H}\right\}=3.55 \mathrm{ppm} / \delta\left\{{ }^{15} \mathrm{~N}\right\}=-249.48 \mathrm{ppm}$ found in the ${ }^{15} \mathrm{~N}-{ }^{1} \mathrm{H}$ HMBC spectrum (bottom left orange box in Figure 6.18) indicate the potential formation of $\mathrm{N}_{2} \mathrm{H}_{4} \cdot{ }^{[305.306]}$ Furthermore, the IR spectrum of the reaction mixture (illustrated in the appendix, Figure D.12) shows bands, which are indicative for the

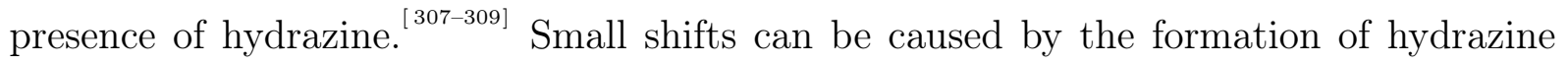
monohydrates, which are formed with water due to silicon grease decomposition.

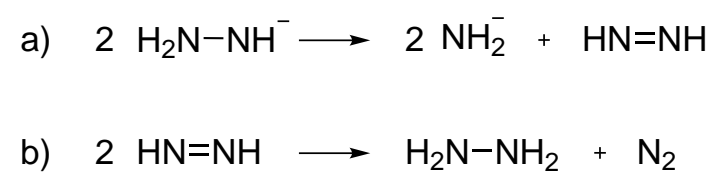

Scheme 6.7: Alternative possible pathway for the decompositon of the hydrazido ligand in [ $\mathrm{L}^{\text {bisnac }} \mathbf{N i}_{2}(\mu-$ $\left.\eta^{1}: \eta^{1}-\mathbf{N}_{2} \mathbf{H}_{3}\right)$ ]. In a first step a) the hydrazido ligand disproportionates to an amido ${ }^{-}$ligand and diazene. b) Due to its instability, formed diazene further disproportionates to hydrazine and dinitrogen.

To confirm the formation of hydrazine during the conversion of $\left[\mathbf{L}^{\text {bisnac }} \mathbf{N i}_{2}\left(\mu-\eta^{1}: \eta^{1}-\mathbf{N}_{2} \mathbf{H}_{3}\right)\right]$ to $\left[\mathbf{L}^{\text {bisnac }} \mathbf{N i}_{2}\left(\mu-\mathbf{N H}_{2}\right)\right]$, further experiments in larger scales and under exclusion of silicon grease are planned. 


\subsection{Oxidation of the Hydrazido Ligand}

Since a diazenido complex is usually regarded as first intermediate in the reduction of dinitrogen, $\stackrel{[312]}{[}$ the establishment of a $\mu-\eta^{1}: \eta^{1}-\mathrm{N}_{2} \mathrm{H}^{-}$bridged dinuclear nickel complex was envisioned. Indeed, it was possible to oxidize $\left[\mathbf{L}^{\text {bisnac }} \mathbf{N i}_{\mathbf{2}}\left(\mu-\eta^{\mathbf{1}}: \eta^{\mathbf{1}}-\mathbf{N}_{\mathbf{2}} \mathbf{H}_{\mathbf{3}}\right)\right]$ with (2,2,6,6-tetramethylpiperidin-1-yl)oxyl (TEMPO) to yield the diazenido-bridged complex $\left[\mathbf{L}^{\text {bisnac }} \mathbf{N i}_{\mathbf{2}}\left(\mu-\eta^{1}: \eta^{1}-\mathbf{N}_{2} \mathbf{H}\right)\right.$ ] (Scheme 6.8) . Upon addition of the oxidant to the clear deep red THF solution of $\left[\mathbf{L}^{\text {bisnac }} \mathbf{N i}_{\mathbf{2}}\left(\mu-\eta^{\mathbf{1}}: \eta^{\mathbf{1}}-\mathbf{N}_{\mathbf{2}} \mathbf{H}_{\mathbf{3}}\right)\right]$ an immediate color change to dark green occured. In solution, the generated compound was not stable at room temperature, where the complex decomposed accompanied by a color change from green-brownish to yellow-orange. Nevertheless, oxidation at lower temperatures $\left(-30^{\circ} \mathrm{C}\right)$, followed by layering of the solution with hexanes and subsequent cooling yielded crystalline material suitable for X-ray defraction measurements. The resulting ORTEP diagram (Figure 6.20) shows the molecular structure of $\left[\mathbf{L}^{\text {bisnac }} \mathbf{N i}_{2}\left(\mu-\eta^{1}: \eta^{1}-\mathbf{N}_{\mathbf{2}} \mathbf{H}\right)\right]$ and Table 6.4 lists selected bond lengths and angles.
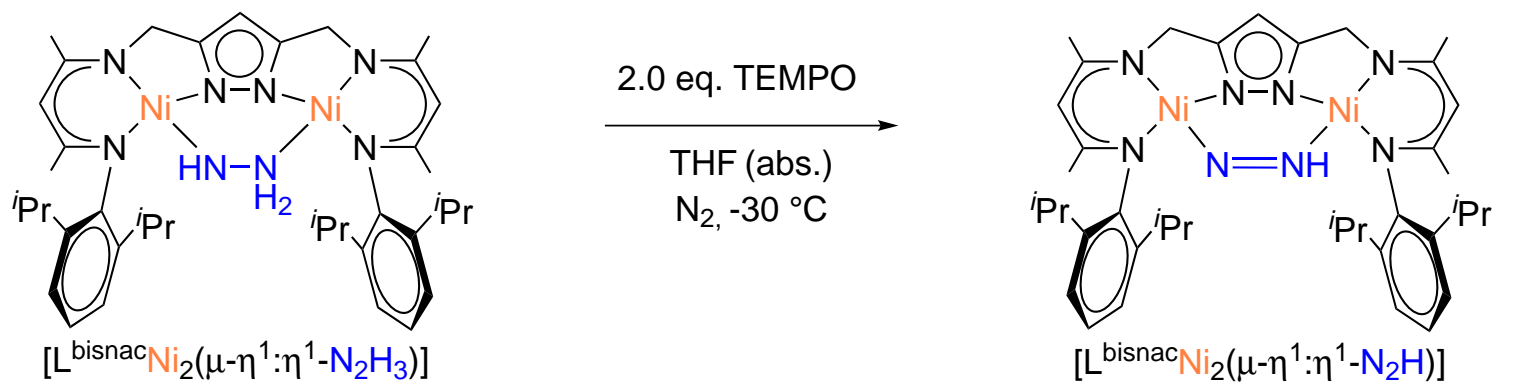

Scheme 6.8: The synthesis of [ $\left.L^{\text {bisnac }} \mathrm{Ni}_{2}\left(\mu-\eta^{1}: \eta^{1}-\mathrm{N}_{2} \mathrm{H}\right)\right]$.
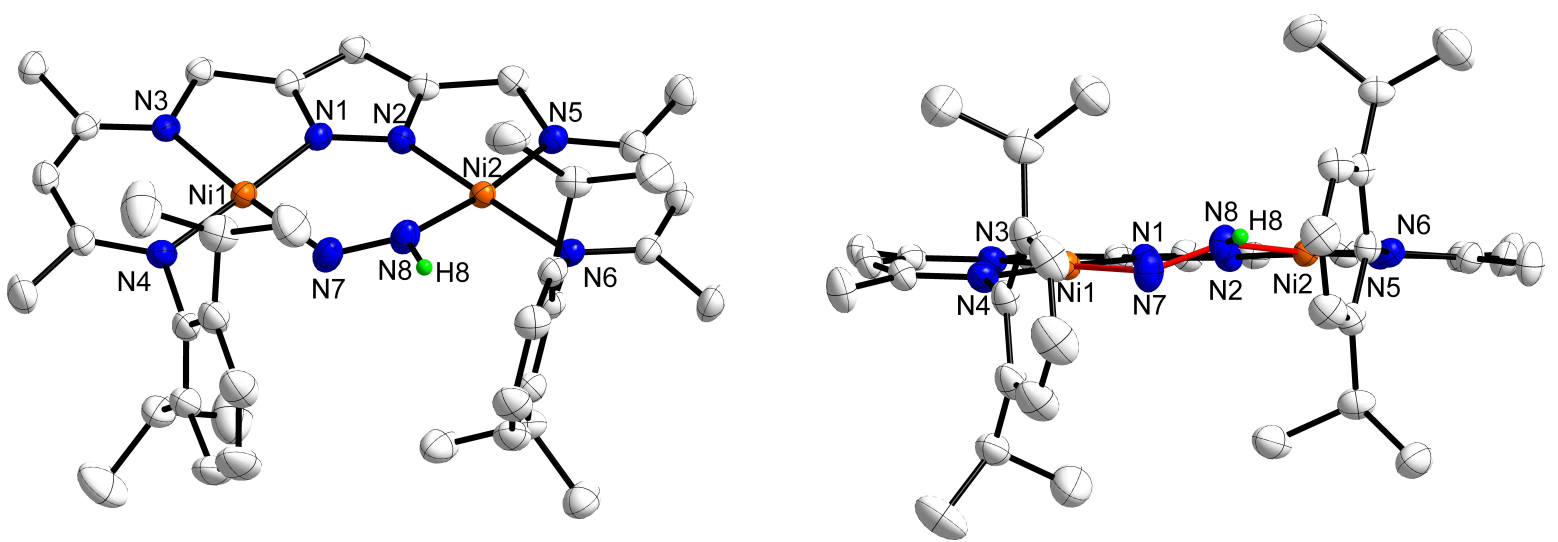

Figure 6.20.: Thermal displacement ellipsoids (shown at $50 \%$ probability) of the molecular structure of [ $L^{\text {bisnac }} \mathrm{Ni}_{2}\left(\mu-\eta^{1}: \eta^{1}-\mathrm{N}_{2} \mathrm{H}\right)$ ]. All hydrogen atoms except the nitrogen bound ones have been omitted for the sake of clarity (the hydrogen atom is displayed with a fixed radius of $0.135 \AA$ ). Right: Front view of the molecular structure of [ $\mathrm{L}^{\text {bisnac }} \mathrm{Ni}_{2}\left(\mu-\eta^{1}: \eta^{1}-\mathrm{N}_{2} \mathrm{H}\right)$ ]. The twist of the bond N7-N8 towards the ligand plane is indicated. The corresponding bonds are marked $(-)$. 
Table 6.4.: Selected bond lengths and angles of $\left[L^{\text {bisnac }} \mathrm{Ni}_{2}\left(\mu-\eta^{1}: \eta^{1}-\mathrm{N}_{2} \mathrm{H}\right)\right]$.

\begin{tabular}{cccc}
\hline \multicolumn{2}{c}{ Bond lengths / ̊ } & \multicolumn{2}{c}{ Angle / } \\
\hline Ni1-N1 & $1.8558(17)$ & N1-Ni1-N7 & $90.85(8)$ \\
Ni1-N7 & $1.8604(19)$ & N1-Ni1-N3 & $83.48(7)$ \\
Ni1-N3 & $1.8987(16)$ & N7-Ni1-N3 & $174.33(8)$ \\
Ni1-N4 & $1.9192(17)$ & N1-Ni1-N4 & $176.59(7)$ \\
Ni2-N8 & $1.8565(18)$ & N7-Ni1-N4 & $91.97(8)$ \\
Ni2-N2 & $1.8583(16)$ & N3-Ni1-N4 & $93.69(7)$ \\
Ni2-N5 & $1.9049(17)$ & N8-Ni2-N2 & $90.70(8)$ \\
Ni2-N6 & $1.9206(17)$ & N8-Ni2-N5 & $172.69(8)$ \\
N7-N8 & $1.311(3)$ & N2-Ni2-N5 & $83.13(7)$ \\
Ni1...N2 & $3.9078(5)$ & N8-Ni2-N6 & $92.16(7)$ \\
N8-H8 & $0.8933(317)$ & N2-Ni2-N6 & $176.44(7)$ \\
& & N5-Ni2-N6 & $94.16(7)$ \\
& & N8-N7-Ni1 & $130.92(15)$ \\
& & N7-N8-Ni2 & $130.50(15)$ \\
\hline
\end{tabular}

The torsion angle $\Varangle(\mathrm{Ni1}-\mathrm{N} 7-\mathrm{N} 8-\mathrm{Ni2})=48.19^{\circ}$ suggests a smaller distortion of the entire system caused by the bridging unit. This angle agrees well with the trend of the torsion angles observed for the bridged $\mathrm{N}_{2} \mathrm{H}_{4}, \mathrm{~N}_{2} \mathrm{H}_{3}{ }^{-}$and $\mathrm{N}_{2} \mathrm{H}_{2}{ }^{2-}$ ligands.

Furthermore, it concurs with a shorter $\mathrm{N}-\mathrm{N}$ bond length of the diazendio unit $(1.31 \AA)$ compared to the one observed for the $\mathrm{N}_{2} \mathrm{H}_{2}{ }^{2-}$ ligand $(1.42 \AA)$, which consequently requires less space and thus fits better into the cavity between the two metal centers. In comparison to free diazene (1.249-1.252 $\AA$ ), this bond appears slightly elongated but still

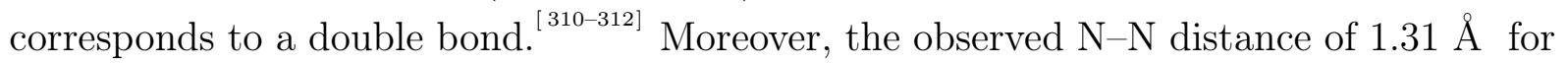
the $\mathrm{N}_{2} \mathrm{H}^{-}$bridge is also slightly elongated compared to other systems including end-on bridged diazenido $\left(\mathrm{N}_{2}\right)$ complexes $(1.19-1.24 \AA)^{\left[\frac{65,81,313]}{6}\right.}$ or complexes with substituted $\mu-\eta^{1}: \eta^{1}$ diazenido ligands $(1.20-1.29 \AA) . .^{[312.314-316]}$ Since dinuclear complexes containing $\mu-\eta^{1}: \eta^{1}-\mathrm{N}_{2} \mathrm{H}^{-}$ligands are rare, comparison of other properties is more difficult.

The IR spectrum shows a band at $3025 \mathrm{~cm}^{-1}$, which was assigned to the NH stretching vibration of the bridging ligand. Compared to free diazene $\left(\tilde{\nu}_{\mathrm{NH}}=3128 \mathrm{~cm}^{-1}\right)^{[310]}, \mu-\eta^{1}: \eta^{1}$ $\mathrm{N}_{2} \mathrm{H}_{2}\left(\tilde{\nu}_{\mathrm{NH}}=3242 \mathrm{~cm}^{-1}\right)^{\underline{[252]}}$ and $\mu-\eta^{1}: \eta^{1} \mathrm{~N}_{2} \mathrm{HAr}\left(\tilde{\nu}_{\mathrm{NH}}=3220 \mathrm{~cm}^{-1}\right)^{\underline{[312]}}$ ligands the wavenumber for the NH vibration of $\left[\mathbf{L}^{\text {bisnac }} \mathbf{N i}_{\mathbf{2}}\left(\mu-\eta^{1}: \eta^{1}-\mathbf{N}_{2} \mathbf{H}\right)\right]$ is significantly smaller. This may be attributed to the negative charge of the diazenido ligand.

Figure 6.21 shows an overlay of measured IR spectra of $\left[\mathbf{L}^{\text {bisnac }} \mathbf{N i}_{\mathbf{2}}\left(\mu-\eta^{\mathbf{1}}: \eta^{\mathbf{1}}-\mathbf{N}_{\mathbf{2}} \mathbf{H}\right)\right]$ () and $\left[\mathbf{L}^{\text {bisnac }} \mathbf{N i}_{\mathbf{2}}\left(\mu-\eta^{\mathbf{1}}: \eta^{\mathbf{1}}-\mathbf{N}_{\mathbf{2}} \mathbf{H}_{\mathbf{3}}\right)\right]$ (-) in the range from $500-1700 \mathrm{~cm}^{-1}$. No significant changes occured after oxidation except development of a new band at $1263 \mathrm{~cm}^{-1}$. This band was assigned to the $\mathrm{N}=\mathrm{N}$ stretching vibration of the $\mathrm{N}_{2} \mathrm{H}^{-}$ligand. In comparison to free diazene $\left(\tilde{\nu}_{\mathrm{NN}}=1529 \mathrm{~cm}^{-1}\right)^{[\underline{[310]}}$, bridged diazenido $(\mathrm{N}=\mathrm{N})$ complexes 
$\left(\tilde{\nu}_{\mathrm{NN}}=1583-1696\right)^{\left[\frac{[65,81,313]}{}\right.}$ or mononuclear complexes with end-on coordinated $\mathrm{N}_{2} \mathrm{H}\left(\tilde{\nu}_{\mathrm{NN}}\right.$ $\left.=1457 \mathrm{~cm}^{-1}\right)^{\left[\frac{[317]}{\underline{ }}\right.}$ the N-H stretching frequency for $\left[\mathbf{L}^{\text {bisnac }} \mathbf{N i}_{\mathbf{2}}\left(\mu-\eta^{\mathbf{1}}: \eta^{\mathbf{1}}-\mathbf{N}_{\mathbf{2}} \mathbf{H}\right)\right]$ is low. Nonetheless, the observed $N-N$ stretching vibration concurs well with a $\mu-\eta^{1}: \eta^{1}$-bridged substituted diazene $\left(\mathrm{N}_{2} \mathrm{HAr}\right) \mathrm{N}-\mathrm{N}$ band found at $1260 \mathrm{~cm}^{-1}$. In this case, the $\mathrm{N}-\mathrm{N}$ bond length $\left(d_{\mathrm{NN}}=1.29 \AA\right)^{\underline{[318]}}$ of the reported compound is very similar to the one of $\left[\mathbf{L}^{\text {bisnac }} \mathbf{N i}_{\mathbf{2}}\left(\mu-\eta^{1}: \eta^{1}-\mathbf{N}_{\mathbf{2}} \mathbf{H}\right)\right]\left(d_{\mathrm{NN}}=1.31 \AA\right)$.

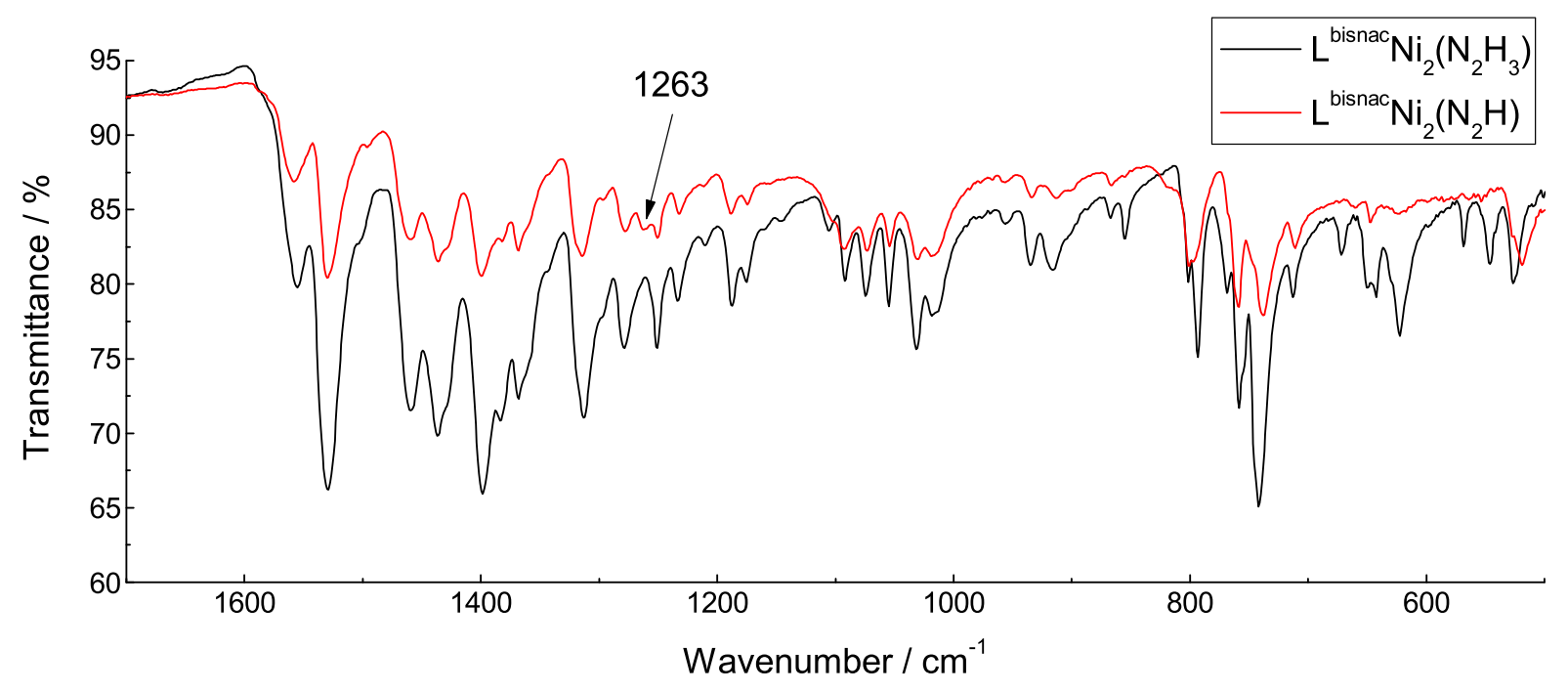

Figure 6.21.: Overlay of IR spectra of [ $\left.L^{\text {bisnac }} \mathrm{Ni}_{2}\left(\mu-\eta^{1}: \eta^{1}-\mathrm{N}_{2} \mathrm{H}\right)\right](-)$ and [ $\left[\mathrm{L}^{\text {bisnac }} \mathrm{Ni}_{2}\left(\mu-\eta^{1}: \eta^{1}-\mathbf{N}_{2} \mathbf{H}_{3}\right)\right](-)$. A new band occured at $1263 \mathrm{~cm}^{-1}$, which was assigned to the $\mathrm{N}=\mathrm{N}$ stretching vibration of the diazenido ligand.

NMR experiments with samples of crystalline material also showed that temperatures above $0{ }^{\circ} \mathrm{C}$ led to decomposition of the compound in solution. This may be attributed to residual TEMPOH or even unreacted traces of TEMPO, which could not be removed by crystallization. For this reason, the sample had to be measured at a temperature of $-35^{\circ} \mathrm{C}$ to minimize the progress of side reactions.

However, further work ${ }^{\left[\frac{[135]}{\underline{1}}\right.}$ showed that a milder form of oxidant (galvinoxyl free radical) ${ }^{[\underline{\underline{319}]}}$ furnished a much cleaner reaction, which eventually yielded crystalline material free of reactive impurities.

It was shown that clean material of $\left[\mathbf{L}^{\text {bisnac }} \mathbf{N i}_{\mathbf{2}}\left(\mu-\eta^{\mathbf{1}}: \eta^{\mathbf{1}}-\mathbf{N}_{\mathbf{2}} \mathbf{H}\right)\right]$ is stable in solution at room temperature. The ${ }^{1} \mathrm{H}$ NMR spectrum is shown in Figure 6.22.

The asymmetry of the compound is reflected by the ${ }^{1} \mathrm{H}$ NMR experiment, in particular by distinct resonances associated to the methylene groups and the nacnac imine moieties. Therefore, this experiment substantiates a similar molecular structure in solution and in the solid state.

In the spectrum a new resonance at low field $(9.5 \mathrm{ppm})$ was observed which was assigned to the $\mathrm{N}_{2} \mathrm{H}^{-}$proton. For reported complexes with $\mu-\eta^{1}: \eta^{1}$-bridged subsituted diazene $\left(\mathrm{N}_{2} \mathrm{ArH}\right)$ ligands, proton resonances at $12.6 \mathrm{ppm}$ and $15.6 \mathrm{ppm}$ were observed. ${ }^{[312]} \mathrm{A}$ reported non-bridged end-on coordinated $\mathrm{N}_{2} \mathrm{H}^{-}$complex features a proton chemical shift 
of $8.6 \mathrm{ppm} .{ }^{[292.320]}$ The shift of $\left[\mathbf{L}^{\text {bisnac }} \mathbf{N i}_{\mathbf{2}}\left(\mu-\eta^{\mathbf{1}}: \eta^{\mathbf{1}}-\mathbf{N}_{\mathbf{2}} \mathbf{H}\right)\right]$ fits into the range of these compounds.

The ${ }^{15} \mathrm{~N}$ shift of the $\mathrm{NH}$ group was determined by means of ${ }^{1} \mathrm{H}-{ }^{15} \mathrm{~N}$ HMBC (appendix, Figure D.13) to give a resonance at $2.21 \mathrm{ppm}\left(J_{\mathrm{NH}}=74 \mathrm{~Hz}\right)$. Compared to the chemical shift of the $\mathrm{N}_{2} \mathrm{H}_{3}{ }^{-}$-bridged complex, featuring signals at $\delta\left\{{ }^{15} \mathrm{~N}\right\}=-313 \mathrm{ppm}$ and $-343 \mathrm{ppm}$, this resonance is significantly shifted to lower fields, which is reasonable for an oxidized bridging unit.

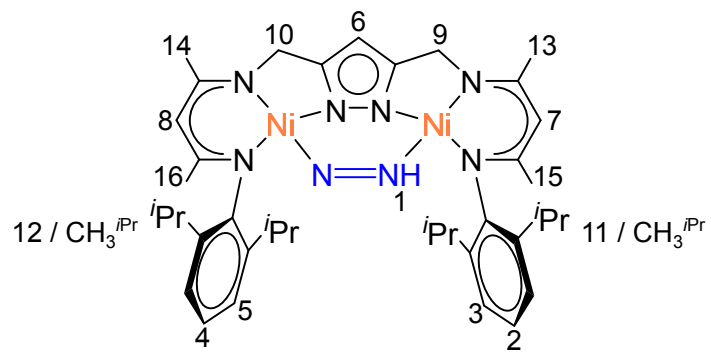

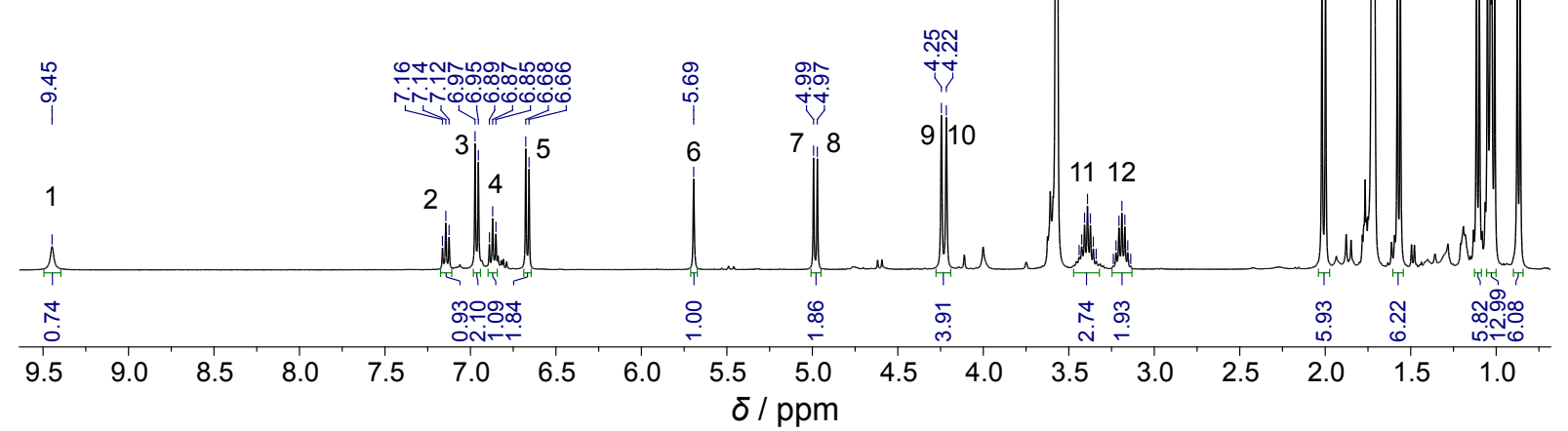

Figure 6.22.: ${ }^{1} \mathrm{H}$ NMR spectrum $(400 \mathrm{MHz})$ of $\left[\mathrm{L}^{\text {bisnac }} \mathrm{Ni}_{2}\left(\mu-\eta^{1}: \eta^{1}-\mathrm{N}_{2} \mathrm{H}\right)\right]$ measured at room temperature. Residual solvents $\left(\right.$ THF- $\left.D_{7}\right)$ are marked $\left(^{*}\right)$. 


\section{8. $\mathrm{K}\left[\mathrm{L}^{\text {bisnac }} \mathrm{Ni}_{2}(\mathrm{H})_{2}\right]$ - a Potential Scaffold for $\mathrm{N}_{2}$ Fixation}

Since dinitrogen fixation demands binding of $\mathrm{N}_{2}$ to the active center of the complex, a dinickel bis-( $\beta$-diketiminato) compound containing a coordinated dinitrogen molecule was envisioned.

In the context of this work, the dihydride complex $\mathbf{K}\left[\mathbf{L}^{\text {bisnac }} \mathbf{N i}_{\mathbf{2}}(\mathbf{H})_{\mathbf{2}}\right]$ has already proven to be very versatile. The fact that hydrides readily react with acids, leading to subsequent release of $\mathrm{H}_{2}$, prompted a corresponding reaction with $\mathbf{K}\left[\mathbf{L}^{\text {bisnac }} \mathbf{N i}_{\mathbf{2}}(\mathbf{H})_{\mathbf{2}}\right]$.

According to Scheme 6.9, a precooled solution $\left(-30{ }^{\circ} \mathrm{C}\right)$ of the dissolved dihydride compound in $\mathrm{THF}_{-} \mathrm{D}_{8}$ was added to a small vessel containing one equivalent of [LutH]OTf. The reaction was performed under an atmosphere of $\mathrm{N}_{2}$.
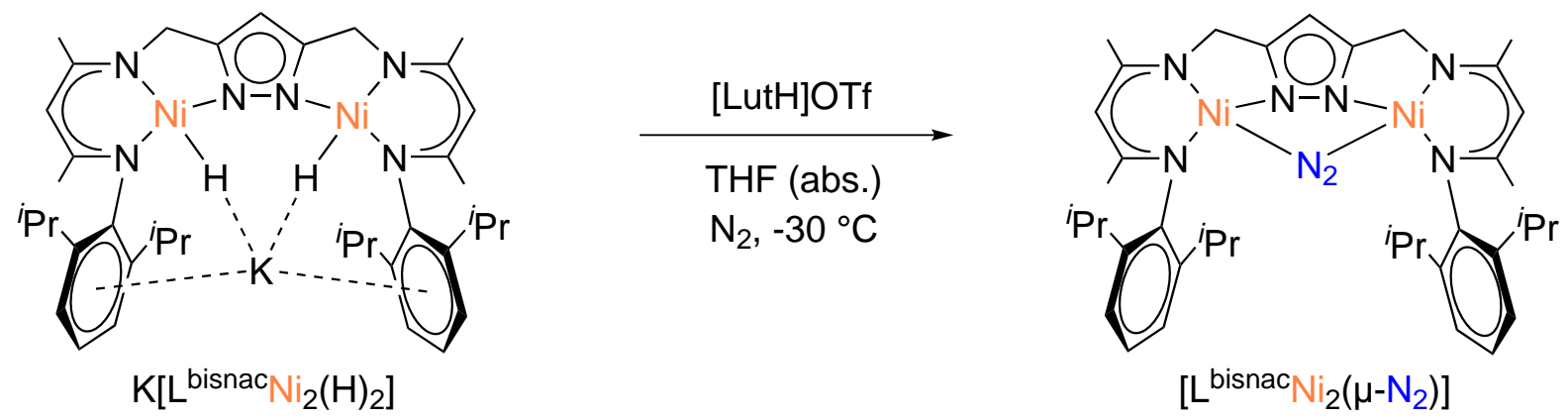

Scheme 6.9: Preparation of [ $\left.{ }^{\text {bisnac }} \mathrm{Ni}_{2}\left(\mu-\mathrm{N}_{2}\right)\right]$ from $\mathrm{K}\left[\mathrm{L}^{\text {bisnac }} \mathrm{Ni}_{2}(\mathrm{H})_{2}\right]$.

An immediate reaction occured, accompanied by a color change from an orange clear solution to deep brownish. Moreover, a significant release of gas from the solution could be observed. This gas development may be associated with the release of $\mathrm{H}_{2}$ generating a free binding pocket between both nickel ions. It was considered to facilitate the accomodation of a small molecule such as $\mathrm{N}_{2}$. However, exclusion of other small molecules was absolutely crucial for a succesful reaction. For instance, as a common side product $\mathbf{K}\left[\mathbf{L}^{\text {bisnac }} \mathbf{N i}_{\mathbf{2}}(\mu-\mathbf{O H})\right]$ was easily generated from the dihydride precursor, if the experiment was not conducted in a moisture free environment (Chapter 4.5). According to the HSAB concept this can be due to the larger chemical hardness of the $\mathrm{N}_{2}$ molecule in comparison to $\mathrm{H}_{2} \mathrm{O}$ or the $\mathrm{OH}^{-}$ion. ${ }^{[303]}$ Considering the $\mathrm{Ni}^{2+}$ cation as softer Lewis-acid (mentioned in 6.6), $\mathrm{OH}^{-}$is preferentially bound to the metal centers over $\mathrm{N}_{2}$.

After reaction under strictly inert conditions ( $\mathrm{N}_{2}$ atmosphere) $\left[\mathbf{L}^{\text {bisnac }} \mathbf{N i}_{\mathbf{2}}\left(\mu-\mathbf{N}_{\mathbf{2}}\right)\right]$ was crystallized at low temperature and isolated. The isolated brownish crystals were suitable for X-ray diffraction analysis, which revealed the structure of the complex (Figure 6.23). $\left[\mathbf{L}^{\text {bisnac }} \mathbf{N i}_{\mathbf{2}}\left(\mu-\mathbf{N}_{2}\right)\right]$ was reasonably stable as a solid in contrast to solution. A selection of bond lengths, distances and angles is shown in Table 6.5. 

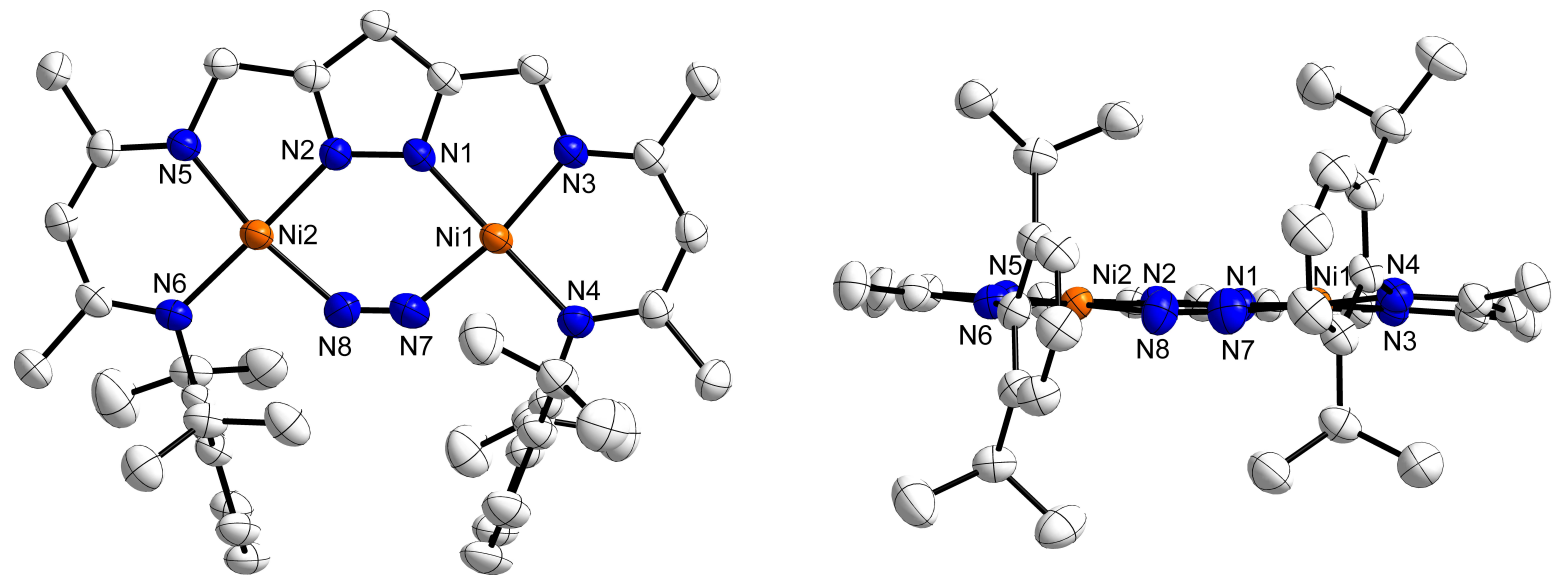

Figure 6.23.: Thermal displacement ellipsoids (shown at $50 \%$ probability) of the molecular structure of [ ${ }^{\text {bisnac }} \mathrm{Ni}_{2}\left(\mu-\eta^{1}: \eta^{1}-\mathbf{N}_{2}\right)$ ] from the top (left) and the front side (right).

[ $\mathbf{L}^{\text {bisnac }} \mathbf{N i}_{\mathbf{2}}\left(\mu-\mathbf{N}_{\mathbf{2}}\right)$ ] crystallizes in the monoclinic space-group $P 2_{1} / c$ with four molecules in the unit cell. Both metal centers are coordinated in the expected square-planar fashion similar to the previously mentioned complexes containing nitrogen based bridging ligands. For instance the two largest angles between Ni1 and the coordinated nitrogen atoms $\left(\Varangle \mathrm{N} 7-\mathrm{Ni1}-\mathrm{N} 3=169.29^{\circ}\right.$ and $\left.\Varangle \mathrm{N} 17-\mathrm{Ni1}-\mathrm{N} 4=175.43^{\circ}\right)$ are close to $180^{\circ}$ while the smaller angles are nearly $90^{\circ}$ (e.g. $\Varangle \mathrm{N} 1-\mathrm{Ni} 1-\mathrm{N} 3=83.60^{\circ}$ ). The same is found for Ni2 and shows a near ideal square-planar metal coordination environment. In the front view of the side-projection of the structure (right side of Figure 6.23), the planarity is demonstrated.

Table 6.5.: Selected bond lengths and angles of $\left[\mathrm{L}^{\text {bisnac }} \mathrm{Ni}_{2}\left(\mu-\mathrm{N}_{2}\right)\right]$.

\begin{tabular}{cccc}
\hline \multicolumn{2}{c}{ Bond lengths / } & \multicolumn{2}{c}{ Angle / } \\
\hline Ni1-N7 & $1.884(3)$ & N1-Ni1-N3 & $83.60(9)$ \\
Ni1-N3 & $1.889(2)$ & N7-Ni1-N3 & $169.29(1)$ \\
Ni1-N4 & $1.901(2)$ & N1-Ni1-N4 & $175.43(1)$ \\
Ni2-N2 & $1.871(2)$ & N7-Ni1-N4 & $94.56(1)$ \\
Ni2-N8 & $1.875(2)$ & N3-Ni1-N4 & $95.11(1)$ \\
Ni2-N5 & $1.904(2)$ & N2-Ni2-N8 & $87.27(1)$ \\
Ni2-N6 & $1.905(2)$ & N2-Ni2-N5 & $83.02(9)$ \\
N7-N8 & $1.113(3)$ & N8-Ni2-N5 & $170.17(1)$ \\
Ni1...Ni2 & $3.950(9)$ & N2-Ni2-N6 & $178.13(9)$ \\
& & N8-Ni2-N6 & $94.33(1)$ \\
& & N5-Ni2-N6 & $95.40(9)$ \\
\hline
\end{tabular}


The bond between the nitrogen atoms of the bridging unit is significantly shorter $\left(d_{\mathrm{N} 7-\mathrm{N} 8}\right.$ $=1.113 \AA$, Table 6.5) than the $\mathrm{N}-\mathrm{N}$ bond lengths of other reported $\mu-\eta^{1}: \eta^{1} \mathrm{~N}_{2}$ ligands $\left(d_{\mathrm{N}-\mathrm{N}}=1.120-1.208 \AA\right) \cdot \stackrel{[65,67,264,266,322,326]}{ }^{-}$In fact the $\mathrm{N}-\mathrm{N}$ bond length of $\left[\mathbf{L}^{\text {bisnac }} \mathbf{N i}_{\mathbf{2}}\left(\mu-\mathbf{N}_{\mathbf{2}}\right)\right]$ is close to the one found for free dinitrogen $(1.0976 \AA) .{ }^{[323]}$ This indicates a very weak activation of the triple bond in the $\mu-\mathrm{N}_{2}{ }^{-}$ligand.

Due to the shorter N-N bond length of the bridging ligand the torsion angle $\Varangle($ Ni1-N7-N8$\mathrm{Ni2})=4.02^{\circ}$ is substantially lower than the one observed for the hydrogen containing analogues $\mathrm{N}_{2}{ }^{-}, \mathrm{N}_{2} \mathrm{H}_{2}{ }^{2-}, \mathrm{N}_{2} \mathrm{H}_{3}{ }^{-}$and $\mathrm{N}_{2} \mathrm{H}_{4}\left(\Varangle(\mathrm{Ni1}-\mathrm{N} 7-\mathrm{N} 8-\mathrm{Ni} 2)=48.19-92^{\circ}\right)$. The central core structure with the six-membered $\mathrm{Ni}_{2} \mathrm{~N}_{4}$ ring is essentially planar.

IR- and Raman- spectroscopic investigations of crystalline substance yielded spectra showing a sharp isolated absorption at $1896 \mathrm{~cm}^{-1}$ (Figure 6.24). While substantially lower than the wavenumber of free $\mathrm{N}_{2}\left(\tilde{\nu}_{\mathrm{NN}}=2331 \mathrm{~cm}^{-1}\right)^{[323]}$ the band observed at $1896 \mathrm{~cm}^{-1}$ fits into the range of $\left(\mu-\eta^{1}: \eta^{1}\right)$ bridged dinitrogen ligands of other reported complexes $\left(\tilde{\nu}_{\mathrm{NN}}\right.$ $\left.=1685-2164 \mathrm{~cm}^{-1}\right) . \underline{[67,266,322,326]}$
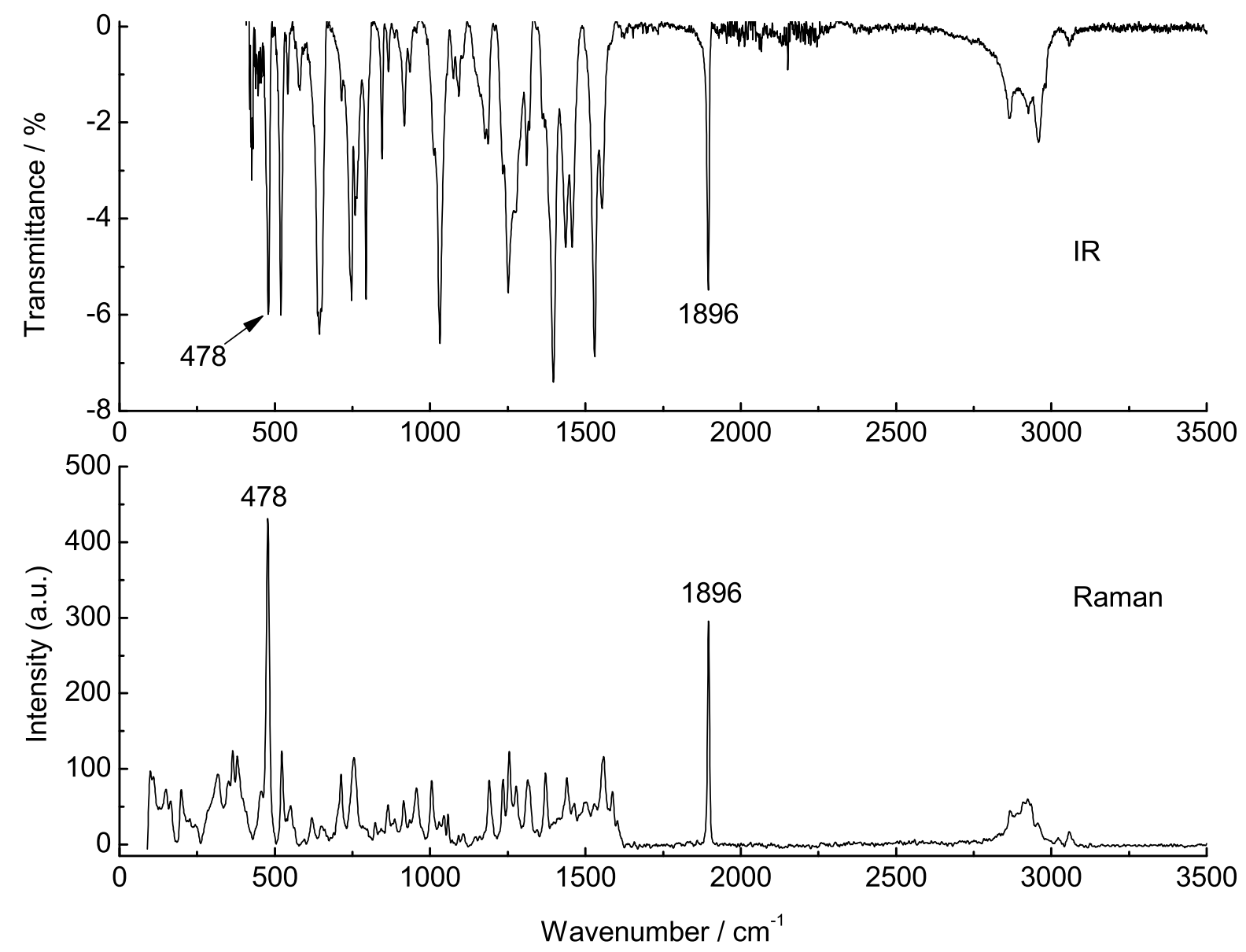

Figure 6.24.: The IR (top) and Raman spectrum (bottom, $\lambda_{\mathrm{ex}}=633 \mathrm{~nm}$ ) of [ ${ }^{\text {bisnac }} \mathbf{N i}_{2}\left(\mu-\mathbf{N}_{2}\right]$. Both spectra show strong bands at $1896 \mathrm{~cm}^{-1}$ and $478 \mathrm{~cm}^{-1}$ associated with the $\mathrm{N}-\mathrm{N}$ - and Ni-N- stretching vibration.

Additionally, a sharp band at $478 \mathrm{~cm}^{-1}$ was observed. It was attributed to the $\mathrm{Ni}-\mathrm{N}$ 
stretching vibration and is within the range of metal nitrogen stretching vibrations of reported mononuclear complexes with terminal coordinated $\mathrm{N}_{2}$ ligands $\left(\tilde{\nu}_{\mathrm{NN}}=1880-2315 \mathrm{~cm}^{-1}, \tilde{\nu}_{\mathrm{MN}}=393-564 \mathrm{~cm}^{-1}\right) . \underline{[\underline{317,320,324,325,327} \underline{\underline{331}}]}$

Conversion of $\mathbf{K}\left[\mathbf{L}^{\text {bisnac }} \mathbf{N i}_{\mathbf{2}}(\mathbf{H})_{\mathbf{2}}\right.$ ] with [LutH]OTf in a dry ${ }^{15} \mathrm{~N}_{2}$ atmosphere led to the labeled ${ }^{15} \mathrm{~N}_{2}$ ligand. In the IR-spectrum of the isolated crystalline material (bottom spectrum in Figure 6.25), a band shifted by $-65 \mathrm{~cm}^{-1}$ compared to the unlabeled complex was observed, which was attributed to the ${ }^{15} \mathrm{~N}-{ }^{15} \mathrm{~N}$ - stretching vibration.

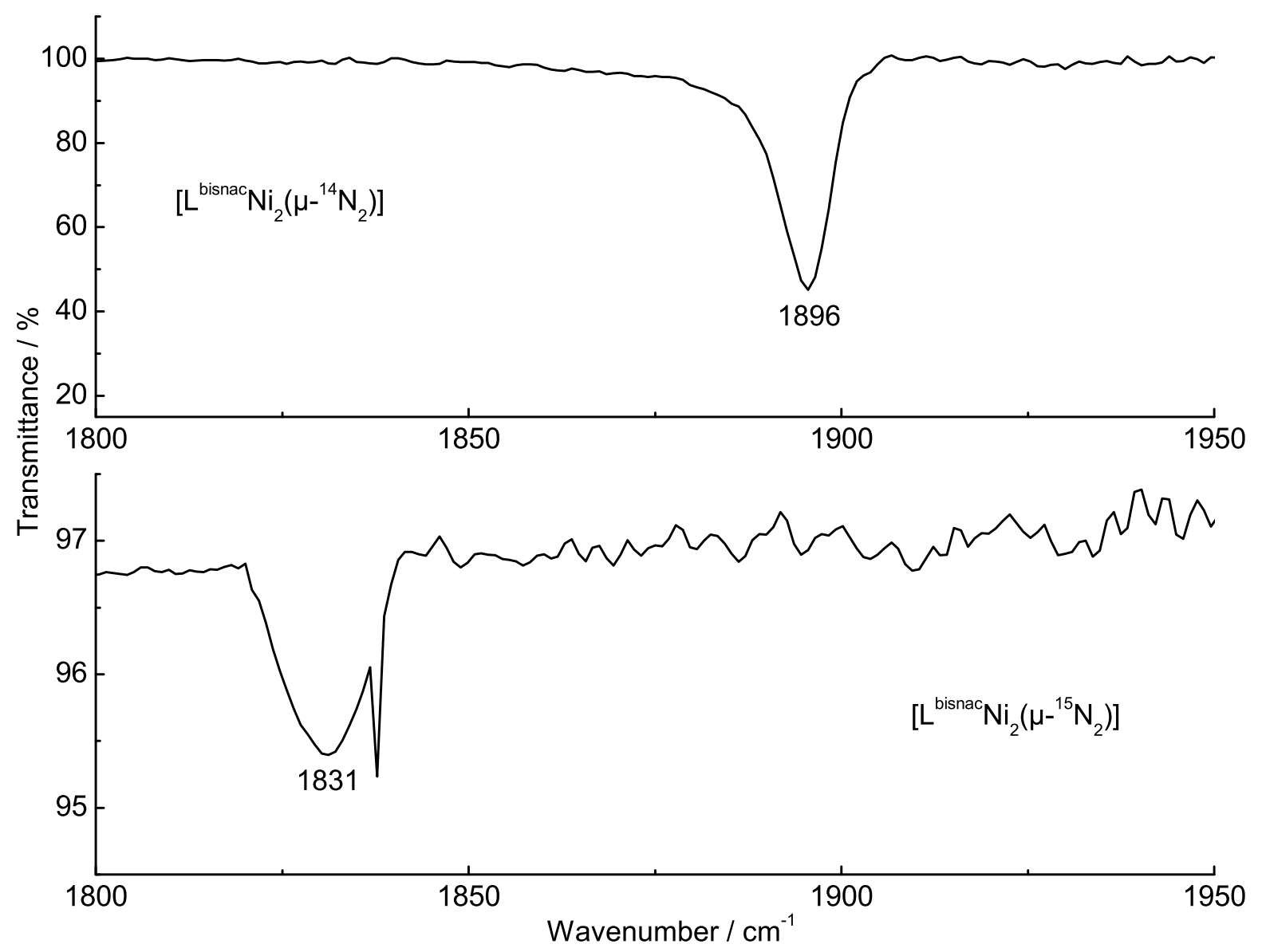

Figure 6.25.: Exerpt of the IR spectra collected from [ $\left.\mathrm{L}^{\text {bisnac }} \mathrm{Ni}_{2}\left(\mu-\mathrm{N}_{2}\right)\right]$ (top) showing the $\mathrm{N}-\mathrm{N}$ - stretching vibration. The ${ }^{15} \mathrm{~N}$ labeled complex exhibits a signal shift of $-65 \mathrm{~cm}^{-1}$ to $1831 \mathrm{~cm}^{-1}$ (bottom).

\section{Mechanism of Formation}

It was surprising that $\left[\mathbf{L}^{\text {bisnac }} \mathbf{N i}_{\mathbf{2}}\left(\mu-\mathbf{N}_{\mathbf{2}}\right]\right.$ formed by conversion of $\mathbf{K}\left[\mathbf{L}^{\text {bisnac }} \mathbf{N i}_{\mathbf{2}}(\mathbf{H})_{\mathbf{2}}\right]$ with only one equivalent of [LutH]OTf since elimination of one equivalent of $\mathrm{H}_{2}$ was expected. However, it was already shown that $\mathbf{K}\left[\mathbf{L}^{\text {bisnac }} \mathbf{N i}_{\mathbf{2}}(\mathbf{H})_{\mathbf{2}}\right]$ readily reacts with $\mathrm{H}_{2} \mathrm{O}$, which inter alia leads to formation of $\mathrm{H}_{2}$ by means of an acid-base reaction (discussed in Chapter 44). Full elimination of hydrides from $\mathbf{K}\left[\mathbf{L}^{\text {bisnac }} \mathbf{N i}_{\mathbf{2}}(\mathbf{H})_{2}\right]$ can thus be facilitated by traces of moisture in addition to the used [LutH]OTf. An undesired outcome of this process was 
production of a significant amount of the $\left[\mathbf{L}^{\text {bisnac }} \mathbf{N i}_{2}(\mu-\mathrm{OH})\right]$.

After hydride elimination from $\mathbf{K}\left[\mathbf{L}^{\text {bisnac }} \mathbf{N i}_{\mathbf{2}}(\mathbf{H})_{2}\right]$ and subsequent incorporation of $\mathrm{N}_{2}$ the resulting complex is expected to contain a postive charge provided that the oxidation state of the metal centers $\left(\mathrm{Ni}^{\mathrm{II}}\right)$ is retained. X-ray analyis revealed that the formed compound is of neutral charge. Therefore and according to IR and Raman spectra the bridging ligand in $\left[\mathbf{L}^{\text {bisnac }} \mathbf{N i}_{\mathbf{2}}\left(\mu-\mathbf{N}_{\mathbf{2}}\right)\right]$ is best described as $\mathrm{N}_{2}{ }^{-}$yielding the following equation:

$2\left[\mathrm{~L}^{\text {bisnac }} \mathrm{Ni}_{2}(\mathrm{H})_{2}\right] \underset{2 \mathrm{H}^{+}}{\stackrel{2 \mathrm{~N}_{2}}{\longrightarrow}} 2\left[\mathrm{~L}^{\text {bisnac }} \mathrm{Ni}_{2}\left(\mathrm{~N}_{2}\right)\right]+3 \mathrm{H}_{2}$.

In the context of nitrogen fixation binding of $\mathrm{N}_{2}$ to other model complexes is usually accomplished in a reductive fashion via use of $\mathrm{Na}, \mathrm{K}, \mathrm{Na} / \mathrm{Hg}$ or $\mathrm{KC}_{8} .{ }^{[67,99,253,258,260,332]}$

However, it is supposed that in natural nitrogen fixation by nitrogenase multiple reducing equivalents are accumulated as hydrides rather than as reduced metal ions. Reductive elimination of $\mathrm{H}_{2}$ from the FeMo-co then generates a state in which highly reduced FeMo-co binds $\mathrm{N}_{2}(\underline{\text { Scheme 6.10 }}) \cdot{ }^{[333]}$ After elimination of $\mathrm{H}_{2}$ from $\left.\mathbf{K}\left[\mathbf{L}^{\text {bisnac }} \mathbf{N i}_{\mathbf{2}} \mathbf{(} \mathbf{H}\right)_{\mathbf{2}}\right]$ and formal reduction a similar reduced state may be generated which facilitates the binding of $\mathrm{N}_{2}$.

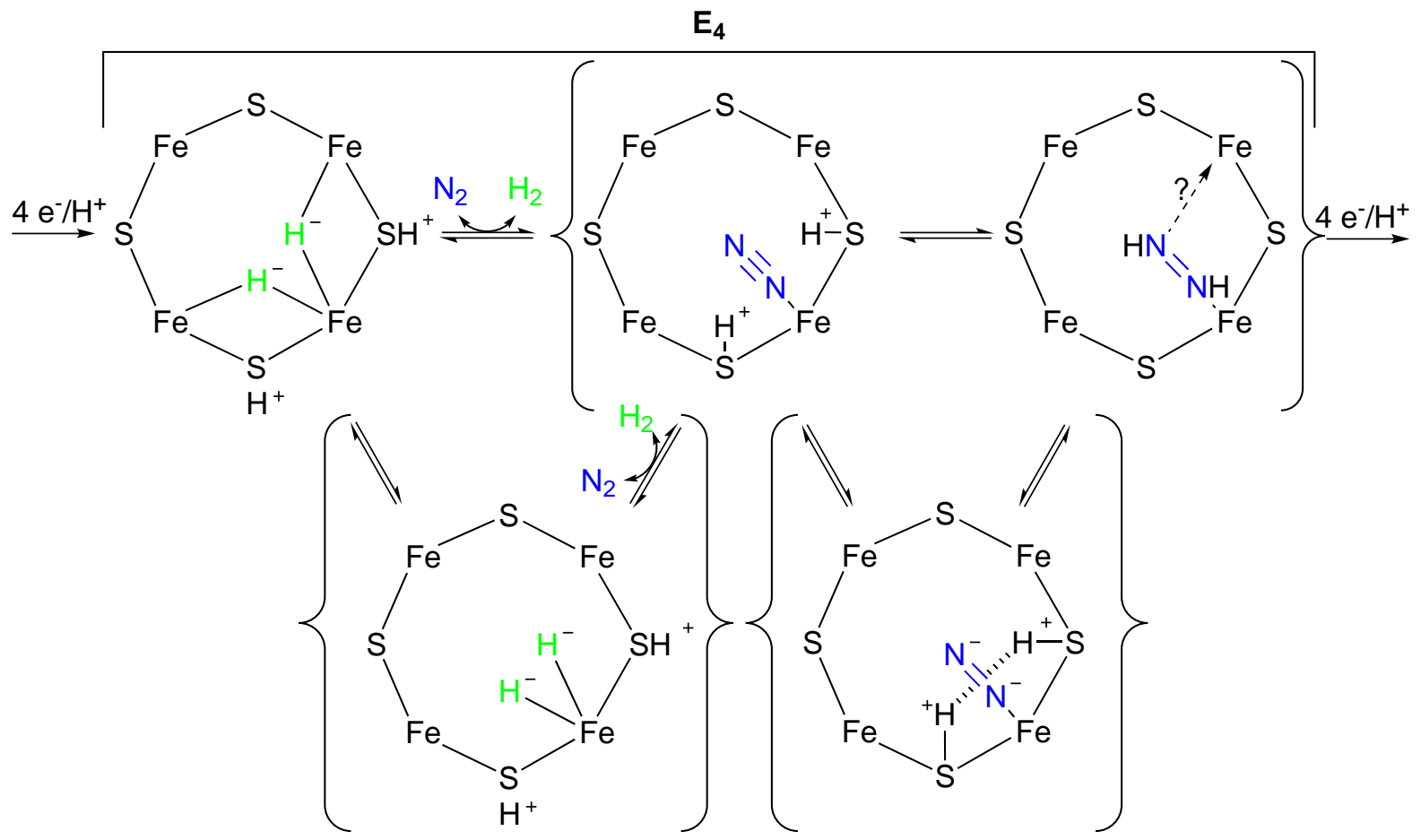

Scheme 6.10: Proposed mechanism of reductive elimination of $\mathrm{H}_{2}$ and subsequent binding of $\mathrm{N}_{2}$ to the $\mathrm{E}_{4}$ state of FeMo-co. ${ }^{\left[\frac{[333]}{}\right.}$ 


\subsection{Summary and Outlook}

The established nickel based pyrazolate-bridged bis( $\beta$-diketiminato) system has remarkably demonstrated its potential to accomodate a series of $\mathrm{N}_{\mathrm{x}} \mathrm{H}_{\mathrm{y}}{ }^{\mathrm{z}}(\mathrm{x}=1,2 ; \mathrm{y}=0,1,2$, $3,4 ; \mathrm{z}=0,1-, 2-)$ ligands, which are supposed to play an important role in nature's nitrogenase mediated dinitrogen fixation process. Moreover, the preorganized dinuclear $\mathrm{Ni}^{\mathrm{II}}$ system gave rise to a rare $\mu^{2}-\eta^{1}: \eta^{1}$ binding state of the nitrogen based ligands.

A hydrazido $\left(\mathrm{N}_{2} \mathrm{H}_{3}{ }^{-}\right)$bridged complex $\left[\mathbf{L}^{\text {bisnac }} \mathbf{N i}_{2}\left(\mu-\eta^{1}: \eta^{2}-\mathbf{N}_{\mathbf{2}} \mathbf{H}_{3}\right)\right]$, which was produced by reaction of the bromido-bridged precursor $\left[\mathbf{L}^{\text {bisnac }} \mathbf{N i}_{2}(\mu-\mathbf{B r})\right]$ with an excess of hydrazine (Scheme 6.11), served as scaffold to accomplish various other bridged $\mathrm{N}_{\mathrm{x}} \mathrm{H}_{\mathrm{y}}{ }^{\mathrm{z}}$ ligands.

$\left[\mathbf{L}^{\text {bisnac }} \mathbf{N i}_{\mathbf{2}}\left(\mu-\eta^{1}: \eta^{2}-\mathbf{N}_{\mathbf{2}} \mathbf{H}_{\mathbf{3}}\right)\right]$ was deprotonated with potassium hydride and dibenzo-18-crown-6 to yield a heterometallic $\mathrm{N}_{2} \mathrm{H}_{2}{ }^{2-}$-bridged complex $\mathbf{K}\left[\mathbf{L}^{\text {bisnac }} \mathbf{N i}_{\mathbf{2}}\left(\mu-\eta^{\mathbf{1}}: \eta^{\mathbf{2}}\right.\right.$ $\mathbf{N}_{2} \mathbf{H}_{2}$ )], featuring a potassium cation residing between the two DIPP moietes of the system comparable to the dihydride complex $\mathbf{K}\left[\mathbf{L}^{\text {bisnac }} \mathbf{N i}_{\mathbf{2}}(\mathbf{H})_{\mathbf{2}}\right]$. An inclusion of alkali metal cations into the system by cation- $\pi$ interactions with the DIPP moieties of the ligand system induces a stabilizing effect on the anionic complex. In particular, it can be highly beneficial for other related compounds containing $\mathrm{N}_{\mathrm{x}} \mathrm{H}_{\mathrm{y}}$ ligands in a reduced state. Moreover, the presence of a positive charge in close proximity to a potential $\mathrm{N}_{2}$ bridging unit can greatly enhance the activation potential of the dinitrogen core by pulling electron density of the coordination environment into it.

Protonation of $\left[\mathbf{L}^{\text {bisnac }} \mathbf{N i}_{\mathbf{2}}\left(\mu-\eta^{\mathbf{1}}: \eta^{\mathbf{2}}-\mathbf{N}_{\mathbf{2}} \mathbf{H}_{3}\right)\right.$ ] was accomplished by using the mild acid [LutH]OTf affording the cationic complex $\left[\mathbf{L}^{\text {bisnac }} \mathbf{N i}_{\mathbf{2}}\left(\mu-\eta^{\mathbf{1}}: \eta^{\mathbf{2}}-\mathbf{N}_{\mathbf{2}} \mathbf{H}_{\mathbf{4}}\right)\right] \mathrm{OTf}$. Both deprotonation and protonation of the hydrazido ligand in $\left[\mathrm{L}^{\text {bisnac }} \mathbf{N i}_{\mathbf{2}}\left(\mu-\eta^{\mathbf{1}}: \eta^{\mathbf{2}}-\mathbf{N}_{\mathbf{2}} \mathbf{H}_{\mathbf{3}}\right)\right]$ are reversible.

$\left[\mathbf{L}^{\text {bisnac }} \mathbf{N i}_{\mathbf{2}}\left(\mu-\eta^{\mathbf{1}}: \eta^{\mathbf{2}}-\mathbf{N}_{\mathbf{2}} \mathbf{H}_{\mathbf{3}}\right)\right]$ decomposes at elevated temperatures $\left(>30{ }^{\circ} \mathrm{C}\right)$ resulting in near quantitative formation of an amido-bridged complex $\left[\mathbf{L}^{\text {bisnac }} \mathbf{N i}_{\mathbf{2}}\left(\mu-\mathbf{N H}_{\mathbf{2}}\right)\right]$. No formation of $\mathrm{NH}_{3}$ was observed. Instead, NMR- and IR- spectroscopic investigations indicate the presence of hydrazine. It was likely formed from diazene, which in turn can be the result of the disproportionation of the $\mathrm{N}_{2} \mathrm{H}_{3}{ }^{-}$ligand.

Furthermore, oxidation of $\left[\mathbf{L}^{\text {bisnac }} \mathbf{N i}_{2}\left(\mu-\eta^{1}: \eta^{2}-\mathbf{N}_{2} \mathbf{H}_{3}\right)\right]$ led to formation of a $\mu-\eta^{1}: \eta^{1}$ diazenido bridged complex $\left[\mathbf{L}^{\text {bisnac }} \mathbf{N i}_{2}\left(\mu-\eta^{1}: \eta^{2}-\mathbf{N}_{2} \mathbf{H}\right)\right]$.

The conversion of $\left.\left[\mathbf{L}^{\text {bisnac }} \mathbf{N i}_{\mathbf{2}} \mathbf{( H}\right)_{\mathbf{2}}\right]$ with [LutH]OTf under a nitrogen atmosphere led to the end-on-bridged $\mathrm{N}_{2}$ complex $\left[\mathbf{L}^{\text {bisnac }} \mathbf{N i}_{\mathbf{2}}\left(\mu-\mathbf{N}_{\mathbf{2}}\right]\right.$ upon release of $\mathrm{H}_{2}$, confirming the nickel-nacnac scaffold to be capable of accomodating the small molecule. Remarkably, the process shows similarities to the proposed mechanism of $\mathrm{N}_{2}$ binding to the $\mathrm{E}_{4}$ state of FeMo-co, which involves the reductive elimination of $\mathrm{H}_{2}$. 
The derived compounds were characterized by means of NMR- and IR- spectroscopy and X-ray diffraction analysis ${ }^{\dagger}$. A compilation of acquired information is shown in Table 6.6.

${ }^{\dagger}$ The quality of the obtained crystals of $\left[L^{\text {bisnac }} \mathrm{Ni}_{2}\left(\mu-\eta^{1}: \eta^{2}-\mathrm{N}_{2} \mathrm{H}_{4}\right)\right] \mathrm{OTf}$ was not sufficient for a reasonable refinement. 


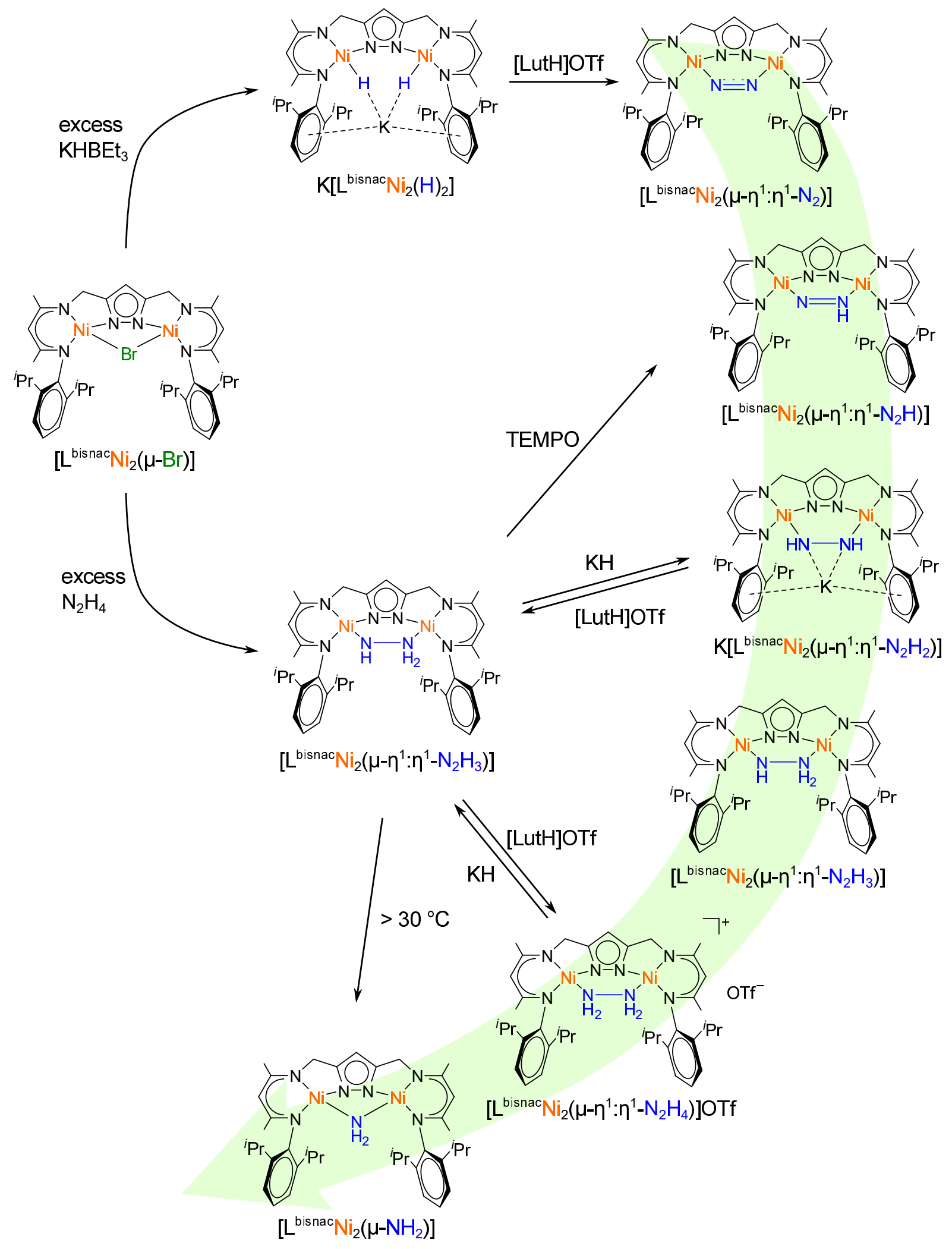

Scheme 6.11: Overview of synthesized Nill complexes with $\mathrm{N}_{\mathrm{x}} \mathrm{H}_{\mathrm{y}}{ }^{ }$bridging ligands. All compounds were prepared under a $\mathrm{N}_{2}$ atmosphere. 
Table 6.6.: Comparison of parameters derived for the bridging ligands of the dinickel- $L^{\text {bisnac }}$ system.

\begin{tabular}{|c|c|c|c|c|c|c|c|c|}
\hline$\mu$-ligand & $d_{\mathrm{N}-\mathrm{N}} / \AA$ & $\Varangle(\mathrm{Ni}-\mathrm{N}-\mathrm{N}-\mathrm{Ni}) /^{\circ}$ & $\tilde{\nu}_{\mathrm{NN}} / \mathrm{cm}^{-1}$ & $\tilde{\nu}_{15} \mathrm{~N}^{15} \mathrm{~N} / \mathrm{cm}^{-1}$ & $\tilde{\nu}_{\mathrm{NH}} / \mathrm{cm}^{-1}$ & $\delta\left\{{ }^{1} \mathrm{H}\right\} / \mathrm{ppm}$ & $\delta\left\{{ }^{15} \mathrm{~N}\right\} / \mathrm{ppm}$ & ${ }^{1} J_{\mathrm{NH}} / \mathrm{Hz}$ \\
\hline \multirow[t]{3}{*}{$\mathrm{N}_{2} \mathrm{H}_{4}$} & $1.5^{\ddagger}$ & $92^{\ddagger}$ & - & - & 3204 & 3.74 & -348.09 & 71 \\
\hline & & & & & 3247 & & & \\
\hline & & & & & 3260 & & & \\
\hline \multirow[t]{3}{*}{$\mathrm{N}_{2} \mathrm{H}_{3}^{-}$} & 1.451 & 89.71 & - & - & 3253 & -0.92 & -313.83 & 54 \\
\hline & & & & & 3273 & 2.26 & -343.26 & 70 \\
\hline & & & & & 3299 & & & \\
\hline \multirow[t]{2}{*}{$\mathrm{N}_{2} \mathrm{H}_{2}{ }^{2-}$} & 1.416 & 60.09 & - & - & 3186 & 1.03 & -255.16 & 59 \\
\hline & & & & & 3162 & & & \\
\hline $\mathrm{N}_{2} \mathrm{H}^{-}$ & 1.311 & 48.19 & 1263 & - & 3025 & 9.50 & 2.21 & 74 \\
\hline $\mathrm{N}_{2}^{-}$ & 1.113 & 4.02 & 1896 & 1831 & - & - & - & - \\
\hline \multirow[t]{2}{*}{$\mathrm{NH}_{2}{ }^{-}$} & - & - & - & - & 3333 & -3.87 & -452.63 & 59 \\
\hline & & & & & 3348 & & & \\
\hline
\end{tabular}

${ }^{\ddagger}$ Due to insufficient refinement of the $\mathrm{X}$-ray derived data the shown numbers only represent approximate values. 


\section{Outlook}

In the context of this work, new model complexes of intermediates, which are considered as important steps in the fixation of $N_{2}$ were accomplished. The $\mu-\eta^{1}: \eta^{1}$ binding state of $\mathrm{N}_{\mathrm{x}} \mathrm{H}_{\mathrm{y}}$ ligands in the preorganized dinuclear nickel scaffold is rare. Therefore, these complexes can serve as valuable models in the context of nitrogen fixation and can provide new insights for the mechanistic pathway. In particular, the process of $\mathrm{N}_{2}$ binding to $\left.\left[\mathbf{L}^{\text {bisnac }} \mathbf{N i}_{\mathbf{2}} \mathbf{( H}\right)_{\mathbf{2}}\right]$ via release of $\mathrm{H}_{2}$ is of interest, since it is similar to the proposed mechanism of $\mathrm{N}_{2}$ binding to FeMo-co.

Future work can focus on interconversion of obtained intermediates to establish the full cycle of nitrogen fixation. Final release of $\mathrm{NH}_{3}$ from the hydrazine compound $\left[\mathbf{L}^{\mathbf{b i s n a c}} \mathbf{N i}_{\mathbf{2}}(\mu-\right.$ $\left.\eta^{1}: \eta^{1}-\mathbf{N}_{2} \mathbf{H}_{4}\right)$ ]OTf by means of reductive protonation and eventual reestablishment of $\left[\mathbf{L}^{\text {bisnac }} \mathbf{N i}_{2}\left(\mu-\mathbf{N}_{2}\right)\right]$ is another goal en route to a catalytic nitrogen fixation.

In particular, due to the rare $\mu-\eta^{1}: \eta^{1} \mathrm{~N}_{\mathrm{x}} \mathrm{H}_{\mathrm{y}}{ }^{\mathrm{z}}(\mathrm{x}=1,2 ; \mathrm{y}=0,1,2,3,4 ; \mathrm{z}=0,1-$, $2-)$ binding motif, future studies based on other late transition metals may provide new insights into a potential nitrogen activation process. The versatile bis( $\beta$-diketiminato) system introduced in this work can undoubtedly aid this purpose. For instance, an iron based fundament ([ $\left.\mathbf{L}^{\text {bisnac }} \mathbf{F e}_{2}\left(\mu-\eta^{1}: \eta^{1}-\mathbf{O T f}\right)\right]$ ) for a potential accomodation of nitrogen

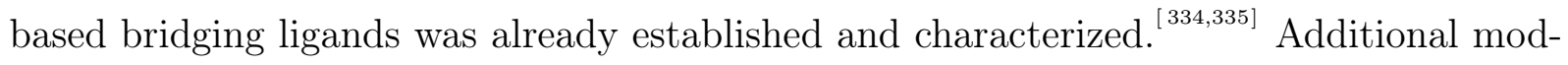
ifications on the $\left[\mathbf{L}^{\text {bisnac }}\right]^{3-}$ system can further promote the nitrogen cleavage capability and are certainly included in future work of the MEYER group. ${ }^{[135]}$ 


\section{Experimental Section}

\subsection{General Considerations}

\section{Materials and Methods}

Manipulations involving air- and moisture sensititve compounds were conducted under an atmosphere of dried (phosphorous pentoxide on solid support [Sicapent ${ }^{\circledR}$, Merck]) nitrogen or argon using standard Schlenk techniques or in a glovebox (MBRAUN LABmaster) and nitrogen atmosphere. Glasware was dried at $120{ }^{\circ} \mathrm{C}$ before use. Solvents were dried over sodium benzophenone ketyl (THF, toluene, $n$-hexane, $n$-pentane), $\mathrm{CaH}_{2}$ or $\mathrm{P}_{4} \mathrm{O}_{10}\left(\mathrm{DCM}, \mathrm{CHCl}_{3}, \mathrm{Et}_{3} \mathrm{~N}\right)$ and distilled and degassed before use. Deuterated solvents were dried analogously. Alternatively, toluene, $n$-hexane were dried using a solvent purification system (MB-SPS) and subsequent storage over molecular sieves (3 $\AA$ ). Hydrogen gas was purchased from Messer and deuterium gas from Linde. Freshly prepared parahydrogen gas was provided by the working group of Prof. Griesinger. Commercially available chemicals were purchased from Acros, Aldrich, Fluka, Deutero, Euriso-Top, Merck, ABCR and used without further purification unless stated otherwise. 3,5-bis(hydroxymethyl)-1H-pyrazole hydrochloride $\underline{[\underline{[21}}^{\underline{23}]}, 3$,4-diethylpyrrole ${ }^{[24,25]}$, pyrazole-3,5-dicarboxylic acid $\stackrel{[100]}{\underline{2}}$, pyrazole-3,5-carboxy-methyl ester $\stackrel{[101,102]}{ }$ and 2-[(2,6-diisopropylphenyl)imido]-pent-2-en-4-one ${ }^{\left[\frac{56]}{6}\right.}$ were synthesized according to published procedures. Column chromatographic purifications $(63-20 \mu \mathrm{m}$ particle size) were performed on silica. TLC was performed on silica gel (Macherey-Nagel, Polygram SIL G/UV254). NMR samples were prepared in a glovebox (MBRAUN LABmaster) under $\mathrm{N}_{2}$ atmosphere. Addition of water in exchange reactions with $\left[\mathbf{L}^{\mathbf{p y r}} \mathbf{N i}_{\mathbf{2}}(\mu-\mathbf{O H})\right]$ or $\left[\mathbf{L}^{\text {bisnac }} \mathbf{N i}_{\mathbf{2}}(\mathbf{H})_{\mathbf{2}}\right]$ was conducted in an argon stream using a Schlenk line. Experiments involving the addition of $\mathrm{D}_{2}, \mathrm{H}_{2}$ or para-hydrogen gas were conducted either in J. Young NMR tubes (for pressures up to 1.5 bar) or in Wilmad-LabGlass high pressure NMR tubes (for higher pressures up to 6 bar). The sample solution was degassed by means of 
pump-freeze-thaw procedure with liquid $\mathrm{N}_{2}$. The process was repeated three times. $\mathrm{D}_{2}$ and $\mathrm{H}_{2}$ were dried by transfer through cooling traps (liquid $\mathrm{N}_{2}$ ) or over $\mathrm{H}_{2} \mathrm{SO}_{4}$ followed by transfer over phosphorus pentoxide (Sicapent ${ }^{\circledR}$ ) in order to remove moisture.

\section{Instrumentation}

${ }^{1} \mathrm{H}$ NMR, ${ }^{2} \mathrm{H}$ NMR and ${ }^{13} \mathrm{C}$ NMR spectra were recorded on Bruker Avance spectrometers $\left(200 \mathrm{MHz}, 300 \mathrm{MHz}, 400 \mathrm{MHz}, 500 \mathrm{MHz}, 600 \mathrm{MHz}\right.$ for ${ }^{1} \mathrm{H}$ ) at $25{ }^{\circ} \mathrm{C}$ unless otherwise noted. Residual ${ }^{1} \mathrm{H}$ signals of the deuterated solvent in ${ }^{1} \mathrm{H}$ NMR or residual ${ }^{2} \mathrm{H}$ signals in ${ }^{2} \mathrm{H}$ NMR spectroscopy were used as internal references.

Electro ionization (EI) and Electrospray ionization (ESI) mass spectra were collected on an Applied Biosystems API 2000 device or on a Bruker HCTultra instrument. Moisture or oxygen sensitive samples were prepared in a glovebox (MBRAUN UNIlab) under an argon atmosphere and injected into the Bruker HTCultra instrument via a direct Peek ${ }^{\top \mathrm{M}}$ tubing connection.

Elemental analyses (standard CHN) were carried out on an Elementar 4.1 vario EL 3 element analyzer.

IR spectra were measured with either an Excalibur FTS 3000, a Bruker VERTEX 70 or a Cary 630 FTIR spectrometer equipped with a DialPath and Diamond ATR accessory (Agilent) placed in a glovebox (MBRAUN UNIlab, argon atmosphere). IR bands were labeled according to their relative intensities with vs (very strong), s (strong), m (medium), $\mathrm{w}$ (weak) and very weak (vw).

Melting points were determined using glas capillary tubes on a Stanford Research Systems OptiMelt MPA 100 device (given values are uncorrected).

X-ray data were collected on a STOE IPDS II diffractometer with an area detector (graphite monochromated Mo- $\mathrm{K}_{\alpha}$ radiation, $\Lambda=0.71073 \AA$ ) by use of $\omega$ scans at 133 $\mathrm{K}$. The structures were solved by direct methods with $S H E L X S-97^{[\underline{336]}}$ and refined on $F^{2}$ using all reflections with $\left(S H E L X L-97^{[337]} \text { or } S H E L X-2013 / 14\right)^{[338]}$. Most non-hydrogen atoms were refined anisotropically. Most hydrogen atoms were placed in calculated positions and assigned to an isotropic displacement parameter of $1.2 / 1.5 U_{\text {eq }}(\mathrm{C})$. The empirical formula and derived values are in accordance with the calculated cell content. Face-indexed absorption corrections were performed numerically with the programm $X$ $R E D . \stackrel{[339]}{ }$ 


\subsection{Synthesis of Ligands and Ligand Precursors}

\section{3,5-Bis(chloromethyl)-1H-pyrazole (2)}

To a suspension of 3,5-bis(cloromethyl)- $1 H$-pyrazole hydrochloride (9.39 g, $46.7 \mathrm{mmol}$, 1.0 eq.) in THF sodium carbonate (5 g) was added and the suspension stirred over night at room temperature. Afterwards the THF solution was slowly decanted, filtrated, evaporated to dryness and kept under an atmosphere of dinitrogen. To maximize the yield, THF was added to the remaining suspension and the above mentioned procedure repeated. The product was isolated as a white solid (7.27 g, $44.0 \mathrm{mmol}, 94 \%)$.

elemental formula: $\mathrm{C}_{5} \mathrm{H}_{7} \mathrm{Cl}_{3} \mathrm{~N}_{2}$.

molecular weight: $201.48 \mathrm{~g} \cdot \mathrm{mol}^{-1}$.

${ }^{1}$ H-NMR $\left(300 \mathrm{MHz}, \mathrm{CDCl}_{3}\right): \delta / \mathrm{ppm}=10.14\left(\mathrm{~s}_{b r}, 1 \mathrm{H}, \mathrm{NH}\right), 6.40\left(\mathrm{~s}, 1 \mathrm{H}, \mathrm{CH}^{\mathrm{pz}-4}\right)$, $4.65\left(\mathrm{~s}, 4 \mathrm{H}, 2 \mathrm{CH}_{2}\right)$.

\section{3,5-Bis-(3,4-dimethyl-1 $H$-pyrrol-2-yl-methyl)-1H-pyrazole (4)}

Freshly distilled 3,4-diethylpyrrole (5.2 g, $42 \mathrm{mmol}, 3.2$ eq.) was dissolved in dichloromethane $(300 \mathrm{~mL})$ under an atmosphere of dinitrogen and cooled to $-78{ }^{\circ} \mathrm{C}$. At this temperature a $2.5 \mathrm{M}$ soultion of n-butyllithium in hexane $(16.8 \mathrm{~mL}, 42 \mathrm{mmol}, 3.2 \mathrm{eq}$.) was slowly added and the solution stirred for $2 \mathrm{~h}$. Subsequently, a solution of 3,5-bis(chloromethyl)- $1 H$-pyrazole $(2.15 \mathrm{~g}, 13 \mathrm{mmol}, 1$ eq.) in dry dichloromethane $(70 \mathrm{~mL})$ was slowly added to the reaction mixture at $-78{ }^{\circ} \mathrm{C}$ which was stirred for $4 \mathrm{~h}$ at this temperature and afterwards warmed to room temperature over night. The orange-brown solution was neutralized by addition of a saturated aequous solution of ammonium chloride $(250 \mathrm{~mL})$ and the organic phase was separated. After extraction of the aequous phase with dichloromethane $(5 \times 120 \mathrm{~mL})$ all organic phases were combined, dried over magnesium sulfate and the solvent removed under reduced pressure. The crude, wine red, viscous product was purified by column chromatography (silica, dichloromethane/methanol 45:1), dried in vacuum and kept under an atmosphere of dinitrogen. The product was isolated as a brownish, readily polymerizing, hygroscopic solid (2.55 g, $7.5 \mathrm{mmol}, 58 \%$ ).

elemental formula: $\mathrm{C}_{21} \mathrm{H}_{30} \mathrm{~N}_{4}$.

molecular weight: $338.49 \mathrm{~g} \cdot \mathrm{mol}^{-1}$.

TLC: $\mathrm{R}_{\mathrm{f}}=0.18(\mathrm{DCM} / \mathrm{MeOH}, 20: 1)$.

${ }^{1}$ H-NMR $\left(300 \mathrm{MHz}, \mathrm{CDCl}_{3}\right): \delta / \mathrm{ppm}=8.00\left(\mathrm{sbr}, 2 \mathrm{H}, 2 \mathrm{NH} H^{\mathrm{py}}\right) 6.43-6.36(\mathrm{~m}, 2 \mathrm{H}$, $\left.\mathrm{CH}^{\mathrm{py}}\right), 5.92\left(\mathrm{~s}, 1 \mathrm{H}, \mathrm{C} H^{\mathrm{pz}}\right), 3.88\left(\mathrm{~s}, 4 \mathrm{H}, 2 \mathrm{CH}_{2}{ }^{\mathrm{py} / \mathrm{pz}}\right), 2.48\left(\mathrm{qd},{ }^{3} J_{H H}=7.5 \mathrm{~Hz},{ }^{4} J_{H H}=\right.$ $\left.0.8 \mathrm{~Hz}, 4 \mathrm{H}, 2 \mathrm{CH}_{2} \mathrm{CH}_{3}{ }^{\mathrm{Et}}\right), 2.47\left(\mathrm{q},{ }^{3} J_{H H}=7.5 \mathrm{~Hz}, 4 \mathrm{H}, 2 \mathrm{CH}_{2} \mathrm{CH}_{3}{ }^{\mathrm{Et}}\right), 1.22\left(\mathrm{t},{ }^{3} J_{H H}=\right.$ $\left.7.5 \mathrm{~Hz}, 6 \mathrm{H}, 2 \mathrm{CH}_{3}{ }^{\mathrm{Et}}\right), 1.12\left(\mathrm{t},{ }^{3} J_{\mathrm{HH}}=7.5 \mathrm{~Hz}, 6 \mathrm{H}, 2 \mathrm{CH}_{3}{ }^{\mathrm{Et}}\right)$. 


\section{3,5-Bis-(3,4-dimethyl-5-formylpyrrol-2-yl-methyl)-1 $H$-pyrazole (5)}

Oxalylchloride (0.94 mL, $10.8 \mathrm{mmol}, 2.1$ eq.) was slowly added to dimethylformamide (0.78 mL, $10.8 \mathrm{mmol}, 2.1$ eq.) under stirring at $10{ }^{\circ} \mathrm{C}$ (waterbath) to give a white solid which was afterwards warmed to room temperature for $10 \mathrm{~min}$ and dissolved in dichloromethane $(10 \mathrm{~mL})$. Thereupon a solution of 3,5-bis-(3,4-dimethyl-1H-pyrrol-2-yl-methyl)-1H-pyrazole $(1.74 \mathrm{~g}, 5.1 \mathrm{mmol}, 1.0 \mathrm{eq})$ in dichloromethane $(10 \mathrm{~mL})$ was slowly added to that solution over $10 \mathrm{~min}$. and stirred at room temperature for $2 \mathrm{~h}$. Afterwards hydrolysis was initiated by addition of an aequous solution of sodium acetate $(4.1 \mathrm{~g}$, $50 \mathrm{mmol}, 9.8 \mathrm{eq}$. in $45 \mathrm{ml}$ water) and the resulting reaction mixture was stirred at room temperature over night. The aequous phase was separated and extracted with dichloromethane $(8 \times 40 \mathrm{~mL})$. All organic phases were washed with a saturated, aequous solution of sodium hydrogen carbonate $(3 \times 70 \mathrm{~mL})$ and water $(3 \times 70 \mathrm{~mL})$. Subsequently, the organic phase was dried over magnesium sulfate, the solvent removed under reduced pressure and the crude, brownish, viscous product purified by column chromatography (silica, dichloromethane/methanole, 15:1). The isolated product was dissolved in ethanol and recrystallized at low temperature to yield a beige, fine powder (1.18 g, $3.0 \mathrm{mmol}$, $59 \%)$.

elemental formula: $\mathrm{C}_{23} \mathrm{H}_{30} \mathrm{~N}_{4} \mathrm{O}_{2}$.

molecular weight: $394.51 \mathrm{~g} \cdot \mathrm{mol}^{-1}$.

TLC: $\mathrm{R}_{\mathrm{f}}=0.54(\mathrm{DCM} / \mathrm{MeOH}, 15: 1)$.

${ }^{1} \mathrm{H}-\mathrm{NMR}\left(300 \mathrm{MHz}, \mathrm{CDCl}_{3}\right): \delta / \mathrm{ppm}=10.51$ (s, $\left.2 \mathrm{H}, 2 \mathrm{NH}{ }^{\mathrm{py}}\right), 9.51$ (s, $\left.2 \mathrm{H}, 2 \mathrm{CHO}\right)$, $5.99\left(\mathrm{~s}, 1 \mathrm{H}, \mathrm{CH}^{\mathrm{pz}}\right), 4.05\left(\mathrm{~s}, 4 \mathrm{H}, 2 \mathrm{CH}_{2}{ }^{\mathrm{py} / \mathrm{pz}}\right), 2.72\left(\mathrm{q},{ }^{3} J_{H H}=7.6 \mathrm{~Hz}, 4 \mathrm{H}, 2 \mathrm{CH}_{2} \mathrm{CH}_{3}{ }^{\mathrm{Et}}\right)$, $2.47\left(\mathrm{q},{ }^{3} J_{H H}=7.6 \mathrm{~Hz}, 4 \mathrm{H}, 2 \mathrm{CH}_{2} \mathrm{CH}_{3}{ }^{\mathrm{Et}}\right), 1.24\left(\mathrm{t},{ }^{3} J_{H H}=7.6 \mathrm{~Hz}, 6 \mathrm{H}, 2 \mathrm{CH}_{3}{ }^{\mathrm{Et}}\right), 1.10$ $\left(\mathrm{t},{ }^{3} J_{\mathrm{HH}}=7.5 \mathrm{~Hz}, 6 \mathrm{H}, 2 \mathrm{CH}_{3}{ }^{\mathrm{Et}}\right)$.

\section{$\mathrm{H}_{3} \mathrm{~L}^{\mathrm{pyr}}$}

The dialdehyde 5 (600 mg, $1.52 \mathrm{mmol}, 1.0$ eq.) was dissolved in dry methanol (360 mL) in an atmosphere of dinitrogen and heated to $60{ }^{\circ} \mathrm{C}$ generating a clear brown solution. In the next step freshly distilled 2,6-diisopropylaniline (1.72 mL, $9.13 \mathrm{mmol}, 6.0$ eq.) was added and the resulting solution stirred for $10 \mathrm{~min}$. Afterwards trifluoroacetic acid (1.13 mL, $15.2 \mathrm{mmol}, 10.0$ eq.) was carefully added and the reaction mixture heated to reflux for 3 days. Thereafter the solution was cooled to room temperature, the solvent removed under reduced pressure and the crude, brown, viscous product purified by column chromatography (silica, ethyl acetate/dichloromethane 10:1). The isolated product was dissolved in ethyl acetate and recrystallized at low temperature to yield a white fine powder (607 mg, $0.9 \mathrm{mmol}, 56 \%$ ). 
elemental formula: $\mathrm{C}_{47} \mathrm{H}_{64} \mathrm{~N}_{6}$.

molecular weight: $713.05 \mathrm{~g} \cdot \mathrm{mol}^{-1}$.

TLC: $R_{\mathrm{f}}=0.85(\mathrm{EtOAc} / \mathrm{DCM}, 10: 1)$.

${ }^{1} \mathbf{H}-\mathbf{N M R}\left(300 \mathrm{MHz}, \mathrm{CDCl}_{3}\right): \delta / \mathrm{ppm}=7.91(\mathrm{~s}, 2 \mathrm{H}, 2 \mathrm{HC}=\mathrm{N}), 7.21-7.07(\mathrm{~m}, 6 \mathrm{H}$, $\left.\mathrm{C} H^{\mathrm{Ph}}\right), 6.08\left(\mathrm{~s}, 1 \mathrm{H}, \mathrm{CH} H^{\mathrm{pz}}\right), 4.15\left(\mathrm{~s}_{\mathrm{br}}, 4 \mathrm{H}, 2 \mathrm{CH}_{2}{ }^{\mathrm{py} / \mathrm{pz}}\right), 3.07\left(\mathrm{sept},{ }^{3} J_{H H}=6.9 \mathrm{~Hz}, 4 \mathrm{H}\right.$, $\left.4 \mathrm{C} H^{i \operatorname{Pr}}\right), 2.57\left(\mathrm{q}^{3}{ }^{3} \mathrm{HH}_{\mathrm{H}}=7.6 \mathrm{~Hz}, 4 \mathrm{H}, 2 \mathrm{CH}_{2} \mathrm{CH}_{3}{ }^{\mathrm{Et}}\right), 2.44\left(\mathrm{q}_{\mathrm{br}}, 4 \mathrm{H}, 2 \mathrm{CH}_{2} \mathrm{CH}_{3}{ }^{\mathrm{Et}}\right), 1.16$ $\left(\mathrm{d},{ }^{3} J_{H H}=6.8 \mathrm{~Hz}, 24 \mathrm{H}, 8 \mathrm{CH}_{3}{ }^{i \operatorname{Pr}}\right), 1.14-1.00\left(\mathrm{~m}, 12 \mathrm{H}, 4 \mathrm{CH}_{3}{ }^{\mathrm{Et}}\right)$.

\section{$1 H$-pyrazole-3,5-dicarboxamide (a)}

Dimethyl-1H-pyrazole-3,5-dicarboxylate $(6.90 \mathrm{~g}, 20 \mathrm{mmol}, 1$ eq.) was dissolved in $7 \mathrm{M}$ $\mathrm{NH}_{3}$ in $\mathrm{MeOH}$ and the mixture was heated to $70{ }^{\circ} \mathrm{C}$ in a sealed tube for 48 hours. The solvent was then evaporated and washed with water to give the product as a solid (3.0 g, 19 mmol, $97 \%$ ).

elemental formula: $\mathrm{C}_{5} \mathrm{H}_{6} \mathrm{~N}_{4} \mathrm{O}_{2}$.

molecular weight: $154.13 \mathrm{~g} \cdot \mathrm{mol}^{-1}$.

elemental analysis: elemental analysis: Calcd. (\%) for $\mathrm{C}_{5} \mathrm{H}_{6} \mathrm{~N}_{4} \mathrm{O}_{2}$ : C $38.96 \mathrm{H} 3.92$ N 36.35. Found: C 38.53 H 4.06 N 35.35.

${ }^{1} \mathbf{H}$ NMR $\left(300 \mathrm{MHz}, \mathrm{DMSO}_{6}\right): \delta / \mathrm{ppm}=7.20\left(\mathrm{~s}, 1 \mathrm{H}, \mathrm{C} H^{\mathrm{Pz}}\right), 7.25(\mathrm{br}, 1 \mathrm{H}), 7.54$ (br, $2 \mathrm{H}), 13.8$ (br, $1 \mathrm{H})$.

${ }^{13} \mathbf{C}\left\{{ }^{1} \mathbf{H}\right\} \mathbf{N M R}\left(75 \mathrm{MHz}, \mathrm{DMSO}_{-}\right): \delta / \mathrm{ppm}=105.86$.

\section{$1 H$-pyrazole-3,5-dicarbonitrile (b)}

$1 H$-pyrazole-3,5-dicarboxamide (3.0 g, 19 mmol, 1 eq.) was suspended in MeCN (70 mL). The suspension was cooled to $0{ }^{\circ} \mathrm{C}$ and $\mathrm{POCl}_{3}(10 \mathrm{~mL})$ added dropwise. The mixture was heated $\left(120^{\circ} \mathrm{C}\right)$ under stirring in a sealed tube for 5 hours until all solid material was dissolved. Subsequently, the resulting black solution was transferred into a mixture of ice and water and extracted with EtOAc. The organic layer was separated, dried $\left(\mathrm{MgSO}_{4}\right)$, filtrated and evaporated in vacuo to yield the product as a solid $(1.7 \mathrm{~g}, 14 \mathrm{mmol}, 75 \%)$.

elemental formula: $\mathrm{C}_{5} \mathrm{H}_{2} \mathrm{~N}_{4}$.

molecular weight: $118.10 \mathrm{~g} \cdot \mathrm{mol}^{-1}$.

elemental analysis: Calcd. (\%) for $\mathrm{C}_{5} \mathrm{H}_{2} \mathrm{~N}_{4}$ : C $50.85 \mathrm{H} 1.71 \mathrm{~N} 47.44$. Found: $\mathrm{C} 50.62$ H 1.94 N 46.25 .

${ }^{\mathbf{1}} \mathbf{H}$ NMR $\left(300 \mathrm{MHz}, \mathrm{DMSO}-\mathrm{D}_{6}\right): \delta / \mathrm{ppm}=7.88\left(\mathrm{~s}, 1 \mathrm{H}, \mathrm{CH}{ }^{\mathrm{Pz}}\right)$.

${ }^{13} \mathbf{C}\left\{{ }^{1} \mathbf{H}\right\} \mathbf{N M R}\left(75 \mathrm{MHz}, \mathrm{DMSO}_{6}\right): \delta / \mathrm{ppm}=114.7(\mathrm{~s}), 110.5(\mathrm{~s})$.

MS (EI): $\left(\frac{m}{z} / \%\right)=118(100)$. 


\section{(1H-pyrazole-3,5-diyl)dimethanamine (c)}

A solution of $1 H$-pyrazole-3,5-dicarbonitrile (2.1 g, $18 \mathrm{mmol}, 1$ eq.) in THF (50 mL) was slowly added to a solution of $\mathrm{LiAlH}_{4}(36 \mathrm{mmol})$ in THF $(50 \mathrm{~mL})$. The resulting suspension was heated to reflux for 90 minutes and afterwards cooled in an ice bath. A mixture of water $(5 \mathrm{~mL})$ and THF $(20 \mathrm{~mL})$ was slowly added. Afterwards the solvent was removed under reduced pressure and the solid extracted with hot MeCN. The solvent from the extract was removed under reduced pressure. Kugelrohrdistillation $\left(10^{-5}\right.$ mbar, $\left.100{ }^{\circ} \mathrm{C}\right)$ yielded the product as a white solid (1.3 g, $10.3 \mathrm{mmol}, 57 \%$ ).

elemental formula: $\mathrm{C}_{5} \mathrm{H}_{10} \mathrm{~N}_{4}$.

molecular weight: $126.16 \mathrm{~g} \cdot \mathrm{mol}^{-1}$.

elemental analysis: Calcd. (\%) for $\mathrm{C}_{5} \mathrm{H}_{10} \mathrm{~N}_{4}$ : C $47.60 \mathrm{H} 7.99 \mathrm{~N} 44.51$. Found: C 46.83 H 7.99 N 44.51.

${ }^{1} \mathbf{H}$ NMR $\left(300 \mathrm{MHz}, \mathrm{D}_{2} \mathrm{O}\right): \delta / \mathrm{ppm}=3.79\left(\mathrm{~d}, 4 \mathrm{H}, \mathrm{CH} H_{2}\right), 6.26\left(\mathrm{~s}, 1 \mathrm{H}, \mathrm{CH}{ }^{\mathrm{Pz}}\right)$. ${ }^{13} \mathbf{C}\left\{{ }^{1} \mathbf{H}\right\} \mathbf{N M R}\left(75 \mathrm{MHz}, \mathrm{D}_{2} \mathrm{O}\right): \delta / \mathrm{ppm}=150.1(\mathrm{~s}), 101.1(\mathrm{~s}), 36.8(\mathrm{~s})$.

\section{$\mathrm{H}_{3} \mathrm{~L}^{\text {bisnac }}$}

2-[(2,6-diisopropylphenyl)imido]-pent-2-en-4-one (16.4 g, $63 \mathrm{mmol}, 2$ eq.) was dissolved in dry dichloromethane $(100 \mathrm{~mL})$ to give a colorless solution. To this solution a solution of triethyloxonium tetrafluoroborate $(12.7 \mathrm{~g}, 67 \mathrm{mmol}, 2.1 \mathrm{eq}$.) in dry dichloromethane $(20 \mathrm{~mL})$ was added slowly and the mixture stirred under argon over night at room temperature. The next day triethylamine $(9.3 \mathrm{~mL})$ was added to the reaction mixture which turned to red after 20 minutes. This solution was then slowly added to a suspension of bis(3,5-aminomethyl)pyrazole (4.0 g, $32 \mathrm{mmol}, 1 \mathrm{eq}$.) in dry triethylamine (20 mL). The red mixture was stirred for 3 days under argon at room tepmerature. After removal of the solvents under reduced pressure the resulting orange residue was extracted with toluene (yellow solution). The solvent was removed under reduced pressure and the remaining solid material was purified by Kugelrohrdistillation $\left(10^{-5}\right.$ mbar, $\left.110{ }^{\circ} \mathrm{C}\right)$. Recrystallization from ethanol yielded the ligand as a yellowish solid material (9.7 g, $16 \mathrm{mmol}, 43 \%$ ). $\mathbf{H}_{3} \mathbf{L}^{\text {bisnac }}$ is sensitive towards hydrolysis and should be kept under inert atmosphere.

elemental formula: $\mathrm{C}_{39} \mathrm{H}_{56} \mathrm{~N}_{6}$.

molecular weight: $608.92 \mathrm{~g} \cdot \mathrm{mol}^{-1}$.

TLC: $\mathrm{R}_{\mathrm{f}}=0.39\left(\mathrm{EE} / 5 \% \mathrm{Et}_{3} \mathrm{~N}\right)$.

${ }^{1} \mathrm{H}$ NMR $\left(300 \mathrm{MHz}, \mathrm{CDCl}_{3}\right): \delta / \mathrm{ppm}=7.10-7.02\left(\mathrm{~m}, 6 \mathrm{H}, \mathrm{C} H^{\mathrm{Ar}}\right), 6.02\left(\mathrm{~s}, 1 \mathrm{H}, \mathrm{CH}{ }^{\mathrm{Pz}}\right)$, $4.72(\mathrm{~s}, 2 \mathrm{H}, \mathrm{CH}), 4.40$ (s, $4 \mathrm{H}, \mathrm{CH}_{2}$ ), 2.91-2.78 (sept, $\left.4 \mathrm{H}, \mathrm{C} H^{i \operatorname{Pr}}\right), 1.95$ (s, $6 \mathrm{H}, \mathrm{CH}_{3}$ ), $1.64\left(\mathrm{~s}, 6 \mathrm{H}, \mathrm{CH}_{3}\right), 1.15$ (d, $\left.12 \mathrm{H}, \mathrm{CH}_{3}{ }^{i \operatorname{Pr}}\right), 1.06$ (d, $12 \mathrm{H}, \mathrm{CH}_{3}{ }^{i \operatorname{Pr}}$ ).

${ }^{13} \mathrm{C}$ NMR $\left(75 \mathrm{MHz}, \mathrm{CDCl}_{3}\right): \delta / \mathrm{ppm}=122.83\left(C \mathrm{H}^{\mathrm{Ar}}\right), 122.74\left(C \mathrm{H}^{\mathrm{Ar}}\right), 101.09\left(\left(C \mathrm{H}^{\mathrm{Pz}}\right)\right.$, $94.60(\mathrm{CH}), 40.11\left(\mathrm{CH}_{2}\right), 28.08\left(C \mathrm{H}^{i \operatorname{Pr}}\right), 23.75\left(\mathrm{CH}_{3}{ }^{i \operatorname{Pr}}\right), 22.73\left(\mathrm{CH}_{3}{ }^{i \operatorname{Pr}}\right), 21.58\left(\mathrm{CH}_{3}\right)$, 
$19.15\left(\mathrm{CH}_{3}\right)$.

MS $(\operatorname{ESI}(+), \operatorname{MeCN}):\left(\frac{m}{z} / \%\right)=609.36(40)\left[\mathrm{L}^{\text {bisnac }} \mathrm{Ni}_{2}(\mathrm{OH})+\mathrm{H}^{+}\right]^{+}$,

\subsection{Synthesis of Complexes}

\section{$\left[\mathrm{L}^{\text {pyr }} \mathrm{Ni}_{2}(\mu-\mathrm{OH})\right]$}

To a solution of $\mathbf{H}_{\mathbf{3}} \mathbf{L}^{\mathbf{1}}$ (200 mg, $0.28 \mathrm{mmol}, 1.0$ eq.) in THF (16 mL) triethylamine (0.4 mL, $2.8 \mathrm{mmol}, 10$ eq.) was added and the resulting solution stirred for $10 \mathrm{~min}$. at room temperature. Thereafter a solution of nickel(II) acetate tetrahydrate $(140 \mathrm{mg}$, $0.56 \mathrm{mmol}, 2.0$ eq.) was added and the resulting deep red soltution stirred at room temperature for 3 days. The solvent was removed under reduced pressure and the crude brownish, solid product dissolved in a solvent mixture of THF/DCM/MeCN (2:1:1). Slow evaporation of the solvents yielded red crystals in the shape of needles or rods $(150 \mathrm{mg}$, $0.18 \mathrm{mmol}, 64 \%$ ) suitable for X-ray structure analysis.

elemental formula: $\mathrm{C}_{47} \mathrm{H}_{62} \mathrm{~N}_{6} \mathrm{Ni}_{2} \mathrm{O}$.

molecular weight: $844.42 \mathrm{~g} \cdot \mathrm{mol}^{-1}$.

${ }^{1}$ H-NMR (200 MHz, THF-d $): \delta / \mathrm{ppm}=7.11-6.91\left(\mathrm{~m}, 2 \mathrm{H}, 2 \mathrm{CH}{ }^{\mathrm{Ar}}\right), 6.83\left(\mathrm{~d},{ }^{3} J_{H H}=\right.$ $\left.8.2 \mathrm{~Hz}, 4 \mathrm{H}, 4 \mathrm{CH}{ }^{\mathrm{Ar}}\right), 6.81(\mathrm{~s}, 2 \mathrm{H}, 2 H \mathrm{C}=\mathrm{N}), 5.96\left(\mathrm{~s}, 1 \mathrm{H}, \mathrm{CH}^{\mathrm{pz}}\right), 3.91\left(\mathrm{~s}, 4 \mathrm{H}, 2 \mathrm{CH}_{2}{ }^{\mathrm{py} / \mathrm{pz}}\right)$, 3.70 (sept, $\left.{ }^{3} J_{H H}=6.8 \mathrm{~Hz}, 4 \mathrm{CH}{ }^{i \operatorname{Pr}}\right), 2.44\left(\mathrm{q},{ }^{3} J_{H H}=7.6 \mathrm{~Hz}, 4 \mathrm{H}, 2 \mathrm{CH}_{2} \mathrm{CH}_{3}{ }^{\mathrm{Et}}\right), 2.33$ $\left(\mathrm{q},{ }^{3} J_{H H}=7.6 \mathrm{~Hz}, 4 \mathrm{H}, 2 \mathrm{CH}_{2} \mathrm{CH}_{3}{ }^{\mathrm{Et}}\right), 1.34\left(\mathrm{~d},{ }^{3} J_{H H}=6.9 \mathrm{~Hz}, 12 \mathrm{H}, 4 \mathrm{CH}_{3}{ }^{i \mathrm{Pr}}\right), 1.03$ $\left(\mathrm{t},{ }^{3} J_{H H}=7.2 \mathrm{~Hz}, 12 \mathrm{H}, 4 \mathrm{CH}_{3}{ }^{\mathrm{Et}}\right), 1.03\left(\mathrm{~d},{ }^{3} J_{H H}=6.7 \mathrm{~Hz}, 12 \mathrm{H}, 4 \mathrm{CH}_{3}{ }^{\mathrm{PPr}}\right),-6.71(\mathrm{~s}$, $1 \mathrm{H}, \mathrm{OH})$.

MS $\left(\operatorname{ESI}(+), \mathrm{CH}_{2} \mathrm{Cl}_{2}\right):\left(\frac{m}{z} / \%\right)=843(100)\left[\mathrm{L}^{1} \mathrm{Ni}_{2}(\mathrm{OH})+\mathrm{H}^{+}\right]^{+}$.

\section{$\left[\mathrm{L}^{\text {bisnac }} \mathrm{Ni}_{2}(\mu-\mathrm{Br})\right]$}

$\mathbf{H}_{3} \mathbf{L}^{\text {bisnac }}$ (505 mg, $0.83 \mathrm{mmol}, 1.0$ eq.) was dissolved in dry THF (4 mL) and cooled to $-78^{\circ} \mathrm{C}$. To the clear yellow solution $n$-BuLi (2.6 M in Toluene) was added slowly to give a clear orange solution. Under stirring the solution was warmed to room temperature over 15 min. to yield a dark red, clear solution. $\mathrm{NiBr}_{2}(\mathrm{DME})$ was then slowly added. The resulting dark-brown mixture was stirred over night at room temperature. The suspension was separated by centrifuge and the dark brown THF solution discarded while the solid material was washed with acetone for several times until the solvent remained colorless. After removing residual solvent in the vacuum, a beige colored fine powderous material was isolated (641 mg, $0.8 \mathrm{~mol}, 96 \%$ ). Evaporation from a concentrated solution in dichloromethane or chloroform yielded small green-brown, cube-shaped crystals suitable for X-ray structural analysis. 
elemental formula: $\mathrm{C}_{39} \mathrm{H}_{53} \mathrm{~N}_{6} \mathrm{BrNi}_{2}$.

molecular weight: $803.19 \mathrm{~g} \cdot \mathrm{mol}^{-1}$.

${ }^{1} \mathrm{H}$ NMR $\left(300 \mathrm{MHz}, \mathrm{CDCl}_{3}\right): \delta / \mathrm{ppm}=6.98-6.80\left(\mathrm{~m}, 6 \mathrm{H}, \mathrm{C} H^{\mathrm{Ar}}\right), 5.52\left(\mathrm{~s}, 1 \mathrm{H}, \mathrm{CH}{ }^{\mathrm{Pz}}\right)$, 4.75 (s, $2 \mathrm{H}, \mathrm{CH}$ ), 4.14 (s, $4 \mathrm{H}, \mathrm{CH}_{2}$ ), 3.39-3.25 (sept, $\left.{ }^{3} J_{\mathrm{HH}}=6.9 \mathrm{~Hz}, 4 \mathrm{H}, \mathrm{CH}{ }^{i \operatorname{Pr}}\right), 2.02$ $\left(\mathrm{s}, 6 \mathrm{H}, \mathrm{CH}_{3}\right), 1.46\left(\mathrm{~d},{ }^{3} J_{\mathrm{HH}}=7.0 \mathrm{~Hz}, 12 \mathrm{H}, \mathrm{CH}_{3}{ }^{i \mathrm{Pr}}\right), 1.33\left(\mathrm{~s}, 6 \mathrm{H}, \mathrm{CH}_{3}\right), 1.03\left(\mathrm{~d},{ }^{3} J_{\mathrm{HH}}\right.$ $\left.=7.0 \mathrm{~Hz}, 12 \mathrm{H}, \mathrm{CH}_{3}{ }^{i \mathrm{Pr}}\right)$.

${ }^{13} \mathrm{C}$ NMR $\left(75 \mathrm{MHz}, \mathrm{CDCl}_{3}\right): \delta / \mathrm{ppm}=160.01\left(C^{\mathrm{q}}-\mathrm{Me}\right), 153.41\left(C^{\mathrm{q}}-\mathrm{Me}\right), 147.83\left(C^{\mathrm{pz}}\right)$, $\left.\left.141.67\left(C^{\mathrm{Ar}}\right), 125.60 C \mathrm{H}^{\mathrm{Ar}}\right), 123.46 C \mathrm{H}^{\mathrm{Ar}}\right), 97.60(C \mathrm{H}), 91.67\left(C \mathrm{H}^{\mathrm{Pz}}\right), 54.59\left(C \mathrm{H}_{2}\right), 28.28$ $\left(C \mathrm{H}^{i \operatorname{Pr}}\right), 24.98\left(\mathrm{CH}_{3}{ }^{i \operatorname{Pr}}\right), 24.27\left(\mathrm{CH}_{3}{ }^{i \operatorname{Pr}}\right), 23.51\left(\mathrm{CH}_{3}\right), 21.67\left(\mathrm{CH}_{3}\right)$.

MS $(\mathrm{ESI}(+), \mathrm{MeOH}):\left(\frac{m}{z} / \%\right)=803.2(9)\left[\mathrm{L}^{\text {bisnac }} \mathrm{Ni}_{2} \mathrm{Br}+\mathrm{H}^{+}\right]^{+}, 721.3(28)\left[\mathrm{L}^{\text {bisnac }} \mathrm{Ni}_{2}\right.$ $\left.+\mathrm{H}^{+}\right]^{+}, 665.4(100)\left[\mathrm{L}^{\text {bisnac }} \mathrm{Ni}+\mathrm{H}^{+}\right]^{+}$.

HRMS $(\operatorname{ESI}(+), \mathrm{MeOH} / \mathrm{DCM}) \frac{m}{z}$ : calculated: $[\mathrm{M}+\mathrm{H}]^{+}$803.2273; experimental: $[\mathrm{M}+$ $\mathrm{H}]^{+} 803.2258$.

IR (KBr): $\tilde{\nu} / \mathrm{cm}^{-1}=3057(\mathrm{vw}), 2959(\mathrm{~m}), 2926(\mathrm{~m}), 2867(\mathrm{~m}), 1554(\mathrm{~m}), 1533(\mathrm{vs})$, 1464 (s), 1436 (s), 1399 (vs), 1381 (s), 1369 (s), 1314 (s), 1281 (m), 1265 (w), 1252 (m), $1234(\mathrm{w}) 1187(\mathrm{~m}), 1176(\mathrm{w}), 1108(\mathrm{vw}), 1091(\mathrm{~m}), 1056(\mathrm{~m}), 1033(\mathrm{~m}), 1012(\mathrm{~m}), 957$ (w), $937(\mathrm{w}) 898(\mathrm{vw}), 869(\mathrm{vw}), 860(\mathrm{vw}), 795(\mathrm{~m}), 766(\mathrm{~m}), 760(\mathrm{vs}), 745(\mathrm{vs}), 714(\mathrm{~m})$, $704(\mathrm{~m}), 650(\mathrm{vw}), 616(\mathrm{vw}), 544(\mathrm{w}), 528(\mathrm{w}), 478(\mathrm{w}), 431(\mathrm{vs})$.

\section{$\left[\mathrm{L}^{\text {bisnac }} \mathrm{Ni}_{2}(\mu-\mathrm{OH})\right]$}

[ $\mathbf{L}^{\text {bisnac }} \mathbf{N i}_{\mathbf{2}} \mathbf{B r}$ ] (300 mg, $0.37 \mathrm{mmol}, 1.0$ eq.) was suspended in THF (5 mL). An aqueous solution of $\mathrm{KOH}$ (21 mg. $0.37 \mathrm{mmol}, 1.0 \mathrm{eq}$, dissolved in $0.1 \mathrm{ml}$ of water) was added to the beige suspension. Stirring the mixture for several days at room temperature yielded a dark green solution which was filtered. A slow diffusion of pentane vapours into the filtrate yielded dark green rod shaped crystals suitable for X-ray structural analysis.

elemental formula: $\mathrm{C}_{39} \mathrm{H}_{54} \mathrm{~N}_{6} \mathrm{Ni}_{2} \mathrm{O}$.

molecular weight: $740.29 \mathrm{~g} \cdot \mathrm{mol}^{-1}$.

${ }^{1} \mathbf{H}$ NMR (400 MHz, THF-D $): \delta / \mathrm{ppm}=6.97-6.93\left(\mathrm{~m}, 2 \mathrm{H}, \mathrm{CH}{ }^{\mathrm{Ar}}\right), 6.81-6.79(\mathrm{~m}$, $\left.4 \mathrm{H}, \mathrm{C} H^{\mathrm{Ar}}\right), 5.46\left(\mathrm{~s}, 1 \mathrm{H}, \mathrm{C} H^{\mathrm{Pz}}\right), 4.59$ (s, $\left.2 \mathrm{H}, \mathrm{CH}\right), 4.00$ (s, $\left.4 \mathrm{H}, \mathrm{CH} H_{2}\right), 3.40-3.29$ (sept, $\left.{ }^{3} J_{\mathrm{HH}}=6.9 \mathrm{~Hz}, 4 \mathrm{H}, \mathrm{C} H^{i \operatorname{Pr}}\right), 1.85\left(\mathrm{~s}, 6 \mathrm{H}, \mathrm{C} H_{3}\right), 1.60\left(\mathrm{~d},{ }^{3} J_{\mathrm{HH}}=6.9 \mathrm{~Hz}, 12 \mathrm{H}, \mathrm{CH}_{3}{ }^{i \operatorname{Pr}}\right)$, $1.08\left(\mathrm{~d},{ }^{3} J_{\mathrm{HH}}=6.9 \mathrm{~Hz}, 12 \mathrm{H}, \mathrm{CH}_{3}{ }^{i \operatorname{Pr}}\right), 1.07\left(\mathrm{~s}, 6 \mathrm{H}, \mathrm{CH}_{3}\right)$.

${ }^{13} \mathrm{C}$ NMR (100 MHz, THF-D 8$): \delta / \mathrm{ppm}=160.28\left(C^{\mathrm{q}}-\mathrm{Me}\right), 158.43\left(C^{\mathrm{q}}-\mathrm{Me}\right), 148.85$ $\left.\left.\left(C^{\mathrm{pz}}\right), 144.52\left(C^{\mathrm{Ar}}\right), 141.31\left(C^{\mathrm{Ar}}\right), 125.07 C^{\mathrm{Ar}}\right), 124.42 C^{\mathrm{Ar}}\right), 97.23(C \mathrm{H}), 90.56$ $\left(C \mathrm{H}^{\mathrm{Pz}}\right), 54.47\left(C \mathrm{H}_{2}\right), 54.44\left(C \mathrm{H}_{2}\right), 28.24\left(C \mathrm{H}^{i \operatorname{Pr}}\right), 22.56\left(C_{3}{ }^{i \operatorname{Pr}}\right), 22.17\left(C \mathrm{H}_{3}\right), 20.31$ $\left(\mathrm{CH}_{3}\right)$.

${ }^{17} \mathrm{O}$ NMR $\left(54 \mathrm{MHz}, \mathrm{THF}-\mathrm{D}_{8}\right): \delta / \mathrm{ppm}=-168.71\left(\mathrm{~s}_{\mathrm{br}}, 1 \mathrm{O}, \mathrm{OH}\right.$.

$\operatorname{MS}(\operatorname{ESI}(+), \operatorname{THF} / \mathrm{MeCN}):\left(\frac{m}{z} / \%\right)=739.43(40)\left[\mathrm{L}^{\text {bisnac }} \mathrm{Ni}_{2}(\mathrm{OH})+\mathrm{H}^{+}\right]^{+}, 761.41(60)$ $\left[\mathrm{L}^{\text {bisnac }} \mathrm{Ni}_{2}(\mathrm{OH})+\mathrm{Na}^{+}\right]^{+}$. 
IR (KBr): $\tilde{\nu} / \mathrm{cm}^{-1}=3608(\mathrm{vw}), 3280(\mathrm{vw}), 3058(\mathrm{vw}), 2954(\mathrm{~m}), 2923(\mathrm{w}), 2864(\mathrm{w})$, 1553 (m), 1529 (s), 1462 (m), 1436 (m), 1394 (s), 1362 (m), 1323 (m), 1314 (m), 1271 (w), 1251 (w), 1234 (vw), 1197 (vw), 1179 (vw), 1159 (vw), 1139 (vw), 1104 (vw), 1082 (vw), 1060 (vw), $1033(\mathrm{w}), 1018(\mathrm{w}), 945(\mathrm{w}), 923(\mathrm{vw}), 873(\mathrm{vw}), 800(\mathrm{~m}), 757(\mathrm{~s}), 732$ (vs), $709(\mathrm{~m}), 641(\mathrm{w}), 621(\mathrm{vw}), 592(\mathrm{vw}), 544(\mathrm{vw}), 525(\mathrm{vw}), 492(\mathrm{vw}), 472(\mathrm{vw}), 430$ $(\mathrm{m})$.

\section{$\mathrm{K}\left[\mathrm{L}^{\text {bisnac }} \mathrm{Ni}_{2}(\mathrm{H})_{2}\right]$}

[ $\mathbf{L}^{\text {bisnac }} \mathbf{N i}_{\mathbf{2}} \mathbf{B r}$ ] (300 mg, $0.37 \mathrm{mmol}, 1.0$ eq.) was suspended in dry THF (4 mL). After slow addition of a $1 \mathrm{M}$ solution of $\mathrm{KHBEt}_{3}$ in THF $(0.93 \mathrm{ml}, 0.93 \mathrm{mmol}, 2.5 \mathrm{eq}$.) to the beige suspension the mixture was stirred at room temperature under inert conditions for $30 \mathrm{~min}$. The formed dark brownish suspension was filtered over a Whatman ${ }^{\circledR}$ glasfibre filter medium to yield a clear dark yellow-brownish solutuion. A slow diffusion of pentane vapours into the filtrate yielded the product as large, cube- rod- or needle shaped crystals (191 mg, $0.25 \mathrm{mmol}, 67 \%$ ) that were washed with pentane. The obtained crystals were suitable for X-ray structural analysis.

elemental formula: $\mathrm{C}_{39} \mathrm{H}_{55} \mathrm{KN}_{6} \mathrm{Ni}_{2}$.

molecular weight: $764.40 \mathrm{~g} \cdot \mathrm{mol}^{-1}$.

elemental analysis: Calcd. (\%) for $\mathrm{C}_{39} \mathrm{H}_{55} \mathrm{KN}_{6} \mathrm{Ni}_{2}$ : C 61.28 H $7.25 \mathrm{~N}$ 10.99. Found: C $60.73 \mathrm{H} 6.95 \mathrm{~N} 10.84$.

${ }^{1} \mathbf{H}$ NMR (400 MHz, THF-D $): \delta / \mathrm{ppm}=6.87-6.83\left(\mathrm{~m}, 2 \mathrm{H}, \mathrm{CH}{ }^{\mathrm{Ar}}\right), 6.87-6-76(\mathrm{~m}$, $\left.4 \mathrm{H}, \mathrm{C} H^{\mathrm{Ar}}\right), 5.55\left(\mathrm{~s}, 1 \mathrm{H}, \mathrm{C} H^{\mathrm{Pz}}\right), 4.56(\mathrm{~s}, 2 \mathrm{H}, \mathrm{CH}), 4.22$ (s, $\left.4 \mathrm{H}, \mathrm{CH} H_{2}\right), 3.49-3.39$ (sept, $\left.{ }^{3} J_{\mathrm{HH}}=7.0 \mathrm{~Hz}, 4 \mathrm{H}, \mathrm{CH}{ }^{i \operatorname{Pr}}\right), 1.82\left(\mathrm{~s}, 6 \mathrm{H}, \mathrm{CH} H_{3}\right), 1.21\left(\mathrm{~s}, 6 \mathrm{H}, \mathrm{CH} H_{3}\right), 1.12\left(\mathrm{~d},{ }^{3} J_{\mathrm{HH}}=\right.$ $\left.6.6 \mathrm{~Hz}, 12 \mathrm{H}, \mathrm{CH}_{3}{ }^{i \operatorname{Pr}}\right), 1.03\left(\mathrm{~d},{ }^{3} J_{\mathrm{HH}}=6.8 \mathrm{~Hz}, 12 \mathrm{H}, \mathrm{CH}_{3}{ }^{i \operatorname{Pr}}\right), 24.17$ (s, $\left.2 \mathrm{H}, \mathrm{Ni} H\right)$.

${ }^{13} \mathrm{C}$ NMR (100 MHz, THF-D 8$): \delta / \mathrm{ppm}=157.61$ ( $\left.C^{\mathrm{q}}-\mathrm{Me}\right), 156.54\left(C^{\mathrm{q}}-\mathrm{Me}\right), 156.10$ $\left(C^{\mathrm{q}}-\mathrm{Me}\right), 155.58\left(C^{\mathrm{q}}-\mathrm{Me}\right), 139.32\left(C^{\mathrm{pz}}\right), 122.87\left(C^{\mathrm{Ar}}\right), 122.15\left(C^{\mathrm{Ar}}\right), 95.99\left(C \mathrm{H}^{\mathrm{Pz}}\right), 91.20$ $(\mathrm{CH}), 52.30\left(\mathrm{CH}_{2}\right), 27.29\left(\mathrm{CH}^{i \mathrm{Pr}}\right), 23.42\left(\mathrm{CH}_{3}{ }^{i \mathrm{Pr}}\right), 22.84\left(\mathrm{CH}_{3}{ }^{i \mathrm{Pr}}\right), 21.61\left(\mathrm{CH}_{3}\right), 19.65$ $\left(\mathrm{CH}_{3}\right)$.

MS $(\mathrm{ESI}(+), \mathrm{THF} / \mathrm{MeCN}):\left(\frac{m}{z} / \%\right)=764.41(100)\left[\mathrm{L}^{\text {bisnac }} \mathrm{Ni}_{2}(\mathrm{H})_{2}+\mathrm{H}^{+}\right]^{+}$.

IR (KBr): $\tilde{\nu} / \mathrm{cm}^{-1}=3116(\mathrm{vw}), 3056(\mathrm{vw}), 2956(\mathrm{~m}), 2921(\mathrm{w}), 2860(\mathrm{w}), 1562(\mathrm{~m})$, 1519 (s), 1462 (m), 1431 (s), 1398 (vs), 1316 (m), 1273 (m), 1196 (w), 1098 (m), 1058 $(\mathrm{w}), 1029(\mathrm{~m}), 935(\mathrm{w}), 862(\mathrm{w}), 805(\mathrm{~m}), 773(\mathrm{~m}), 732(\mathrm{~s}), 720(\mathrm{~s}), 644(\mathrm{w}), 549(\mathrm{w}), 518$ (w), $456(\mathrm{w})$. 


\section{$\mathrm{K}\left[\mathrm{L}^{\text {bisnac }} \mathrm{Ni}_{2}(\mathrm{D})_{2}\right]$}

$\left.\mathbf{K}\left[\mathbf{L}^{\text {bisnac }} \mathbf{N i}_{\mathbf{2}} \mathbf{( H}\right)_{\mathbf{2}}\right](85 \mathrm{mg}, 100 \mu \mathrm{mol}, 1.0$ eq.) was dissolved in dry THF (3 mL) to give a clear, orange solution. The solution was degassed by three pump-freeze-thaw cycles before addition of dried $\mathrm{D}_{2}$ gas (atmospheric pressure) to the vessel. The solution was stirred over night at room temperature and the atmosphere removed as mentioned above. This procedure was repeated two more times. Afterwards the clear solution was cooled $\left(-30{ }^{\circ} \mathrm{C}\right)$ and layered with hexanes. A slow diffusion of the solvents yielded the crystaline product (65 mg, $85 \mu \mathrm{mol}, 85 \%$ ) in the shape of orange needles which were washed with pentane or hexane.

elemental formula: $\mathrm{C}_{39} \mathrm{H}_{53} \mathrm{D}_{2} \mathrm{KN}_{6} \mathrm{Ni}_{2}$.

molecular weight: $766.41 \mathrm{~g} \cdot \mathrm{mol}^{-1}$.

${ }^{\mathbf{2}} \mathbf{H} \mathbf{N M R}(77 \mathrm{MHz}, \mathrm{THF}): \delta / \mathrm{ppm}=24.11(\mathrm{~s}, 2 \mathrm{D}, \mathrm{Ni} D)$.

\section{$\mathrm{K}\left[\mathrm{L}^{\text {bisnac }} \mathrm{Ni}_{2}(\mathrm{OH})(\mathrm{H})\right]$}

$\left.\mathbf{K}\left[\mathbf{L}^{\text {bisnac }} \mathbf{N i}_{\mathbf{2}} \mathbf{( H )}\right)_{\mathbf{2}}\right](10 \mathrm{mg}, 13 \mu \mathrm{mol}, 1$ eq.) was dissolved in THF (0.5 mL) to give a clear, orange solution. Water $(2.4 \mu \mathrm{L}, 1$ eq.) was added resulting in a dark yellow solution. The reaction mixture was cooled $\left(-30{ }^{\circ} \mathrm{C}\right)$ and layered with hexanes. Slow solvent diffusion at $-30{ }^{\circ} \mathrm{C}$ yielded fine orange crystalline material.

elemental formula: $\mathrm{C}_{39} \mathrm{H}_{55} \mathrm{~N}_{6} \mathrm{ONi}_{2}$.

molecular weight: $741.30 \mathrm{~g} \cdot \mathrm{mol}^{-1}$.

${ }^{1} \mathbf{H}$ NMR (500 MHz, THF-D $): \delta / \mathrm{ppm}=6.97-6.94\left(\mathrm{~m}, 1 \mathrm{H}, \mathrm{CH}{ }^{\mathrm{Ar}}\right), 6.85-6.83((\mathrm{~m}$, $\left.2 \mathrm{H}, \mathrm{C} H^{\mathrm{Ar}}\right), 5.52\left(\mathrm{~s}, 1 \mathrm{H}, \mathrm{C} H^{\mathrm{Pz}}\right), 4.60(\mathrm{~s}, 1 \mathrm{H}, \mathrm{CH}), 4.51$ (s, $\left.1 \mathrm{H}, \mathrm{CH}\right), 4.09\left(\mathrm{~s}, 2 \mathrm{H}, \mathrm{CH}_{2}\right)$, $3.91-3.83$ (sept, $\left.{ }^{3} J_{\mathrm{HH}}=6.8 \mathrm{~Hz}, 4 \mathrm{H}, \mathrm{CH}{ }^{i \mathrm{Pr}}\right), 3.79\left(\mathrm{~s}, 2 \mathrm{H}, \mathrm{CH}{ }_{2}\right), 3.65-3.57$ (sept, ${ }^{3} J_{\mathrm{HH}}$ $\left.=6.8 \mathrm{~Hz}, 4 \mathrm{H}, \mathrm{C} H^{i \mathrm{Pr}}\right), 1.87\left(\mathrm{~s}, 3 \mathrm{H}, \mathrm{C} H_{3}\right), 1.74\left(\mathrm{~s}, 3 \mathrm{H}, \mathrm{CH}_{3}\right), 1.51\left(\mathrm{~d},{ }^{3} J_{\mathrm{HH}}=6.8 \mathrm{~Hz}\right.$ $\left.6 \mathrm{H}, \mathrm{CH}_{3}{ }^{i \mathrm{Pr}}\right), 1.42\left(\mathrm{~d},{ }^{3} J_{\mathrm{HH}}=6.8 \mathrm{~Hz}, 6 \mathrm{H}, \mathrm{CH}_{3}{ }^{i \mathrm{Pr}}\right), 1.15\left(\mathrm{~s}, 3 \mathrm{H}, \mathrm{CH} H_{3}\right), 1.12\left(\mathrm{~d},{ }^{3} J_{\mathrm{HH}}=\right.$ $\left.7.0 \mathrm{~Hz}, 6 \mathrm{H}, \mathrm{CH}_{3}{ }^{i \mathrm{Pr}}\right), 1.10\left(\mathrm{~d},{ }^{3} J_{\mathrm{HH}}=7.1 \mathrm{~Hz}, 6 \mathrm{H}, \mathrm{CH}_{3}{ }^{i \mathrm{Pr}}\right), 1.08\left(\mathrm{~s}, 3 \mathrm{H}, \mathrm{CH}_{3}\right),-2.06$ $\left(\mathrm{d},{ }^{3} J_{\mathrm{HH}}=5.6 \mathrm{~Hz}, 1 \mathrm{H}, \mathrm{Ni}-\mathrm{O} H\right),-26.0\left(\mathrm{~d},{ }^{3} J_{\mathrm{HH}}=5.6 \mathrm{~Hz}, 1 \mathrm{H}, \mathrm{Ni}-H\right)$.

${ }^{17} \mathrm{O}$ NMR $\left(54 \mathrm{MHz}, \mathrm{THF}_{-} \mathrm{D}_{8}\right): \delta / \mathrm{ppm}=-88.58\left(\mathrm{~s}_{\mathrm{br}}, 1 \mathrm{O}, \mathrm{OH}\right)$.

IR (KBr): $\tilde{\nu} / \mathrm{cm}^{-1}=3621(\mathrm{w}), 3059(\mathrm{vw}), 2960(\mathrm{~m}), 2922(\mathrm{w}), 2865(\mathrm{w}), 1555(\mathrm{~m})$, 1524 (s), 1459 (w), 1435 (m), 1400 (vs), 1321 (m), 1260 (vs), 1196 (w), 1096 (s), 1015 (vs), 800 (vs), 732 (vs). 


\section{$\mathrm{K}\left[\mathrm{L}^{\text {bisnac }} \mathrm{Ni}_{2}\left(\mu-\eta^{1}: \eta^{1}-\mathrm{CHCPh}\right)\right]$}

$\left.\mathbf{K}\left[\mathbf{L}^{\text {bisnac }} \mathbf{N i}_{\mathbf{2}} \mathbf{( H )}\right)_{\mathbf{2}}\right](70 \mathrm{mg}, 92 \mu \mathrm{mol}, 1.0$ eq. $)$ was dissolved in dry THF (3 mL) to give a clear, dark yellow solution. To this solution phenylacetylene $(12 \mu \mathrm{L}, 110 \mu \mathrm{mol}, 1.2 \mathrm{eq}$. was added, resulting in a dark green, clear solution which was cooled $\left(-30{ }^{\circ} \mathrm{C}\right)$ and layered with hexanes. A slow diffusion of the solvents at $-30{ }^{\circ} \mathrm{C}$ yielded the product as dark green, rod shaped crystals suitable for X-ray structural analysis.

elemental formula: $\mathrm{C}_{47} \mathrm{H}_{59} \mathrm{KN}_{6} \mathrm{Ni}_{2}$.

molecular weight: $864.52 \mathrm{~g} \cdot \mathrm{mol}^{-1}$.

elemental analysis: Calcd. (\%)

${ }^{1} \mathrm{H}$ NMR $\left(500 \mathrm{MHz}, \mathrm{THF}-\mathrm{D}_{8},-35^{\circ} \mathrm{C}\right): \delta / \mathrm{ppm}=10.14\left(\mathrm{~d},{ }^{3} J_{\mathrm{HH}}=7.8 \mathrm{~Hz}, 1 \mathrm{H}, \mathrm{CH}{ }^{\mathrm{Ph}}\right)$, $7.01-6.99\left(\mathrm{dd},{ }^{3} J_{\mathrm{HH}}=7.7 \mathrm{~Hz},{ }^{4} J_{\mathrm{HH}}=1.2 \mathrm{~Hz}, 2 \mathrm{H}, \mathrm{CH}{ }^{\mathrm{Ph}}\right), 6.97-6.95\left(\mathrm{dd},{ }^{3} J_{\mathrm{HH}}=7.7 \mathrm{~Hz}\right.$, $\left.{ }^{4} J_{\mathrm{HH}}=1.2 \mathrm{~Hz}, 1 \mathrm{H}, \mathrm{C} H^{\mathrm{Ph}}\right), 6.88\left(\mathrm{t},{ }^{3} J_{\mathrm{HH}}=7.7 \mathrm{~Hz}, 1 \mathrm{H} \mathrm{CH}{ }^{\mathrm{Ph}}\right), 6.87\left(\mathrm{t},{ }^{3} J_{\mathrm{HH}}=7.5 \mathrm{~Hz}\right.$, $\left.1 \mathrm{H} \mathrm{CH}{ }^{\mathrm{Ph}}\right), 6.75\left(\mathrm{dd},{ }^{3} J_{\mathrm{HH}}=7.6 \mathrm{~Hz},{ }^{4} J_{\mathrm{HH}}=1.3 \mathrm{~Hz}, 1 \mathrm{H}, \mathrm{CH}{ }^{\mathrm{Ar}}\right), 6.73\left(\mathrm{dd},{ }^{3} J_{\mathrm{HH}}=\right.$ $\left.7.7 \mathrm{~Hz},{ }^{4} J_{\mathrm{HH}}=1.4 \mathrm{~Hz}, 1 \mathrm{H}, \mathrm{C} H^{\mathrm{Ar}}\right), 6.68-6.62\left(\mathrm{~m}, 2 \mathrm{H} \mathrm{CH}{ }^{\mathrm{Ar}}\right), 6.24\left(\mathrm{~d},{ }^{3} J_{\mathrm{HH}}=7.5 \mathrm{~Hz}\right.$, $\left.1 \mathrm{H}, \mathrm{C} H^{\mathrm{Ph}}\right), 5.63\left(\mathrm{~s}, 1 \mathrm{H}, \mathrm{C} H^{\mathrm{Pz}}\right), 4.87\left(\mathrm{~s}, 1 \mathrm{H}, \mathrm{C} H^{\text {vinyl }}\right), 4.62(\mathrm{~s}, 1 \mathrm{H}, \mathrm{CH}), 4.63(\mathrm{~s}, 1 \mathrm{H}$, $\mathrm{CH}), 4.32\left(\mathrm{~d},{ }^{2} J_{\mathrm{HH}}=17.5 \mathrm{~Hz}, 1 \mathrm{H}, \mathrm{CH} H_{2}\right), 4.24\left(\mathrm{~d},{ }^{2} J_{\mathrm{HH}}=17.9 \mathrm{~Hz}, 1 \mathrm{H}, \mathrm{CH} H_{2}\right), 4.09(\mathrm{~d}$, $\left.{ }^{2} J_{\mathrm{HH}}=17.7 \mathrm{~Hz}, 1 \mathrm{H}, \mathrm{CH} H_{2}\right), 3.92\left(\mathrm{~d},{ }^{2} J_{\mathrm{HH}}=17.9 \mathrm{~Hz}, 1 \mathrm{H}, \mathrm{CH} H_{2}\right), 3.76-3.65\left(\mathrm{sept},{ }^{3} J_{\mathrm{HH}}=\right.$ $7.1 \mathrm{~Hz}, 1 \mathrm{H}, \mathrm{C} H^{i \mathrm{Pr}}$ ) , 3.58-3.48 (sept, ${ }^{3} J_{\mathrm{HH}}=7.1 \mathrm{~Hz}, 1 \mathrm{H}, \mathrm{C} H^{i \mathrm{Pr}}$ ), 3.29-3.19 (sept, ${ }^{3} J_{\mathrm{HH}}$ $\left.=7.0 \mathrm{~Hz}, 1 \mathrm{H}, \mathrm{C} H^{i \mathrm{Pr}}\right), 1.92\left(\mathrm{~s}, 3 \mathrm{H}, \mathrm{CH}_{3}\right), 1.84\left(\mathrm{~s}, 3 \mathrm{H}, \mathrm{C} H_{3}\right), 1.79\left(\mathrm{~d},{ }^{3} J_{\mathrm{HH}}=6.5 \mathrm{~Hz}\right.$, $3 \mathrm{H}, \mathrm{CH}_{3}{ }^{i \operatorname{Pr}}$ ), $1.39-1.29$ (sept, $\left.{ }^{3} J_{\mathrm{HH}}=7.1 \mathrm{~Hz}, 1 \mathrm{H}, \mathrm{C} H^{i \operatorname{Pr}}\right), 1.18\left(\mathrm{~d},{ }^{3} J_{\mathrm{HH}}=6.8 \mathrm{~Hz}, 3 \mathrm{H}\right.$, $\left.\mathrm{CH}_{3}{ }^{i \operatorname{Pr}}\right), 1.02\left(\mathrm{~d},{ }^{3} J_{\mathrm{HH}}=6.5 \mathrm{~Hz}, 3 \mathrm{H}, \mathrm{CH}_{3}{ }^{i \operatorname{Pr}}\right), 0.93\left(\mathrm{~d},{ }^{3} J_{\mathrm{HH}}=6.8 \mathrm{~Hz}, 3 \mathrm{H}, \mathrm{CH}_{3}{ }^{i \operatorname{Pr}}\right)$, $0.91\left(\mathrm{~d},{ }^{3} J_{\mathrm{HH}}=6.8 \mathrm{~Hz}, 3 \mathrm{H}, \mathrm{CH}_{3}{ }^{i \operatorname{Pr}}\right), 0.79\left(\mathrm{~d},{ }^{3} J_{\mathrm{HH}}=6.8 \mathrm{~Hz}, 3 \mathrm{H}, \mathrm{CH}_{3}{ }^{i \mathrm{Pr}}\right), 0.51(\mathrm{~d}$, $\left.{ }^{3} J_{\mathrm{HH}}=7.0 \mathrm{~Hz}, 3 \mathrm{H}, \mathrm{CH}{ }_{3}{ }^{i \mathrm{Pr}}\right), 0.23\left(\mathrm{~d},{ }^{3} J_{\mathrm{HH}}=6.5 \mathrm{~Hz}, 3 \mathrm{H}, \mathrm{CH}_{3}{ }^{i \operatorname{Pr}}\right)$.

${ }^{13} \mathrm{C}$ NMR (126 MHz, THF-D $\left.8,-35^{\circ} \mathrm{C}\right): \delta / \mathrm{ppm}=160.95\left(C^{\mathrm{q}}-\mathrm{Me}\right), 159.19\left(C^{\mathrm{q}}-\mathrm{Me}\right)$, $158.46\left(C \mathrm{H}^{\text {vinyl }}, 158.36\left(C^{\mathrm{q}}-\mathrm{Me}\right), 157.40\left(C^{\mathrm{q}}-\mathrm{Me}\right), 155.16\left(C^{\mathrm{pz}}\right), 155.14\left(C^{\mathrm{pz}}\right), 152.19\right.$ $\left(C^{\mathrm{Ar}}\right), 150.56\left(C^{\mathrm{Ar}}\right), 150.17\left(C^{\mathrm{Ar}}\right), 144.43\left(C^{\mathrm{Ar}}\right), 143.34\left(C^{\mathrm{Ar}}\right), 143.14\left(C^{\mathrm{Ar}}\right), 142.47$ $\left(C^{\text {vinyl }}\right), 140.14\left(C^{\mathrm{Ph}}\right), 128.34\left(C \mathrm{H}^{\mathrm{Ph}}\right), 127.18\left(C \mathrm{H}^{\mathrm{Ph}}\right), 126.15\left(C \mathrm{H}^{\mathrm{Ph}}\right), 125.89\left(C \mathrm{H}^{\mathrm{Ar}}\right)$, $124.92\left(C \mathrm{H}^{\mathrm{Ar}}\right), 124.38\left(C \mathrm{H}^{\mathrm{Ar}}\right), 124.10\left(C \mathrm{H}^{\mathrm{Ar}}\right), 123.81\left(C \mathrm{H}^{\mathrm{Ar}}\right), 123.35\left(C \mathrm{H}^{\mathrm{Ph}}\right), 121.63$ $\left(C \mathrm{H}^{\mathrm{Ph}}, C \mathrm{H}^{\mathrm{Ar}}\right), 98.55(C \mathrm{H}), 96.43(C \mathrm{H}), 92.73\left(C \mathrm{H}^{\mathrm{Pz}}\right), 52.76\left(C \mathrm{H}_{2}\right), 51.58\left(C \mathrm{H}_{2}\right), 29.71$ $\left(C \mathrm{H}_{3}{ }^{i \operatorname{Pr}}\right), 28.50\left(C \mathrm{H}^{i \operatorname{Pr}}\right), 28.14\left(C \mathrm{H}^{i \operatorname{Pr}}\right), 27.96\left(C \mathrm{H}^{i \operatorname{Pr}}\right), 26.76\left(C \mathrm{H}^{i \operatorname{Pr}}\right), 26.59\left(C_{3}{ }^{i \operatorname{Pr}}\right)$, $25.93\left(\mathrm{CH}_{3}\right), 24.78\left(\mathrm{CH}_{3}{ }^{i \mathrm{Pr}}\right), 24.76\left(\mathrm{CH}_{3}{ }^{i \mathrm{Pr}}\right), 24.33\left(\mathrm{CH}_{3}{ }^{i \operatorname{Pr}}\right), 24.27\left(\mathrm{CH}_{3}{ }^{i \mathrm{Pr}}\right), 24.14$ $\left(\mathrm{CH}_{3}{ }^{i \operatorname{Pr}}\right), 23.72\left(\mathrm{CH}_{3}\right), 23.03\left(\mathrm{CH}_{3}{ }^{i \operatorname{Pr}}\right), 21.43\left(\mathrm{CH}_{3}\right), 20.55\left(\mathrm{CH}_{3}\right)$.

MS $(\mathrm{ESI}(+), \operatorname{THF} / \mathrm{MeCN}):\left(\frac{m}{z} / \%\right)=823.42(100)\left[\mathrm{L}^{\text {bisnac }} \mathrm{Ni}_{2}(\mathrm{CHCPh})+\mathrm{H}^{+}\right]^{+}$.

IR (KBr): $\tilde{\nu} / \mathrm{cm}^{-1}=3278(\mathrm{vw}), 3119(\mathrm{vw}), 3051(\mathrm{w}), 2955(\mathrm{~m}), 2923(\mathrm{~m}), 2863(\mathrm{~m})$, 1553 (m), 1521 (s), 1503 (m), 1430 (s), 1397 (vs), 1314 (m), 1306 (m), 1271 (m), 1250 (m), 1229 (w), 1190 (w), $1175(\mathrm{~m}), 1091(\mathrm{~m}), 1054(\mathrm{~s}), 1028(\mathrm{~m}), 1017(\mathrm{~m}), 892(\mathrm{~m}), 799$ $(\mathrm{m}), 752(\mathrm{~s}), 726(\mathrm{~m}), 704(\mathrm{~s}), 645(\mathrm{w}), 624(\mathrm{w}), 595(\mathrm{w}), 554(\mathrm{w}), 545(\mathrm{w}), 523(\mathrm{w}), 447$ $(\mathrm{w}), 417(\mathrm{~m})$. 


\section{$\left[\mathrm{L}^{\text {bisnac }} \mathrm{Ni}_{2}(\mathrm{OH})\right](\mathrm{X})$}

$\mathbf{K}\left[\mathbf{L}^{\text {bisnac }} \mathbf{N i}_{\mathbf{2}}\left(\mu-\eta^{1}: \eta^{1}-\mathbf{C H C P h}\right)\right](10 \mathrm{mg}, 12 \mu \mathrm{mol}, 1.0$ eq.) was dissolved in THF $(0.5 \mathrm{~mL})$. The solution was degassed via repeated pump-freeze-thaw cycles. Subsequently, $\mathrm{H}_{2}$ (6 atm) was added and the soltution stirred vigorously, which led to a color change from green to brown. Slow solvent diffusion of the $n$-hexane-layered solution at $-30{ }^{\circ} \mathrm{C}$ resulted in the formation of fine crystalline material.

elemental formula: $\mathrm{C}_{39} \mathrm{H}_{54} \mathrm{~N}_{6} \mathrm{ONi}_{2}$.

molecular weight: $740.29 \mathrm{~g} \cdot \mathrm{mol}^{-1}$.

${ }^{1} \mathbf{H}$ NMR (400 MHz, THF-D $): \delta / \mathrm{ppm}=7.19-7.15\left(\mathrm{~m}, 1 \mathrm{H}, \mathrm{CH}{ }^{\mathrm{Ar}}\right), 7.10-7.08(\mathrm{~m}, 1 \mathrm{H}$, $\left.\mathrm{C} H^{\mathrm{Ar}}\right), 7.03-7.01\left(\mathrm{~m}, 2 \mathrm{H}, \mathrm{C} H^{\mathrm{Ar}}\right), 6.87-6.83\left(\mathrm{t},{ }^{3} J_{\mathrm{HH}}=7.8 \mathrm{~Hz}, 1 \mathrm{H}, \mathrm{C} H^{\mathrm{Ar}}\right), 6.53-6.50$ $\left(\mathrm{d},{ }^{3} J_{\mathrm{HH}}=7.8 \mathrm{~Hz}, 1 \mathrm{H}, \mathrm{C} H^{\mathrm{Ar}}\right), 5.51\left(\mathrm{~s}, 1 \mathrm{H}, \mathrm{C} H^{\mathrm{Pz}}\right), 4.92(\mathrm{~s}, 1 \mathrm{H}, \mathrm{CH}), 4.70(\mathrm{~s}, 1 \mathrm{H}$, $\mathrm{CH}), 3.95\left(\mathrm{~d},{ }^{2} J_{\mathrm{HH}}=17.1 \mathrm{~Hz}, 1 \mathrm{H}, \mathrm{CH}_{2}\right), 3.93\left(\mathrm{~d},{ }^{2} J_{\mathrm{HH}}=21.0 \mathrm{~Hz}, 2 \mathrm{H}, \mathrm{CH}_{2}\right), 3.84$ $\left(\mathrm{d},{ }^{2} J_{\mathrm{HH}}=17.1 \mathrm{~Hz}, 1 \mathrm{H}, \mathrm{CH} H_{2}\right), 3.79-3.68\left(\mathrm{sept},{ }^{3} J_{\mathrm{HH}}=6.9 \mathrm{~Hz}, 1 \mathrm{H}, \mathrm{CH}{ }^{i \operatorname{Pr}}\right), 3.56-3.45$ (sept, $\left.{ }^{3} J_{\mathrm{HH}}=6.9 \mathrm{~Hz}, 1 \mathrm{H}, \mathrm{CH}{ }^{i \operatorname{Pr}}\right), 2.63-2.53$ (sept, $\left.{ }^{3} J_{\mathrm{HH}}=6.9 \mathrm{~Hz}, 1 \mathrm{H}, \mathrm{CH}{ }^{i \operatorname{Pr}}\right), 1.90(\mathrm{~s}$, $\left.3 \mathrm{H}, \mathrm{CH}_{3}\right), 1.87\left(\mathrm{~s}, 3 \mathrm{H}, \mathrm{CH} H_{3}\right), 1.86\left(\mathrm{~d},{ }^{3} J_{\mathrm{HH}}=6.9 \mathrm{~Hz}, 3 \mathrm{H}, \mathrm{CH}_{3}{ }^{i \operatorname{Pr}}\right), 1.67\left(\mathrm{~d},{ }^{3} J_{\mathrm{HH}}=\right.$ $\left.6.9 \mathrm{~Hz}, 3 \mathrm{H}, \mathrm{CH}_{3}{ }^{i \operatorname{Pr}}\right), 1.45\left(\mathrm{~d},{ }^{3} J_{\mathrm{HH}}=6.9 \mathrm{~Hz}, 3 \mathrm{H}, \mathrm{CH}_{3}{ }^{i \operatorname{Pr}}\right), 1.43\left(\mathrm{~s}, 3 \mathrm{H}, \mathrm{CH}_{3}\right), 1.37(\mathrm{~s}$, $\left.3 \mathrm{H}, \mathrm{CH}_{3}\right), 1.37\left(\mathrm{~d},{ }^{3} J_{\mathrm{HH}}=6.9 \mathrm{~Hz}, 3 \mathrm{H}, \mathrm{CH}_{3}{ }^{i \mathrm{Pr}}\right), 1.27\left(\mathrm{~s}, 3 \mathrm{H}, \mathrm{CH}{ }_{3}{ }^{i \operatorname{Pr}}\right), 1.20\left(\mathrm{~d},{ }^{3} J_{\mathrm{HH}}\right.$ $\left.=6.9 \mathrm{~Hz}, 3 \mathrm{H}, \mathrm{CH}_{3}{ }^{i \mathrm{Pr}}\right), 1.15\left(\mathrm{~d},{ }^{3} J_{\mathrm{HH}}=6.9 \mathrm{~Hz}, 3 \mathrm{H}, \mathrm{CH}_{3}{ }^{i \mathrm{Pr}}\right), 1.13\left(\mathrm{~d},{ }^{3} J_{\mathrm{HH}}=6.9 \mathrm{~Hz}\right.$, $\left.3 \mathrm{H}, \mathrm{CH}_{3}{ }^{i \mathrm{Pr}}\right), 0.6\left(\mathrm{~s}, 3 \mathrm{H}, \mathrm{CH}_{3}{ }^{i \mathrm{Pr}}\right)$.

${ }^{13}$ C NMR (101 MHz, THF-D $): \delta / p p m=160.51$ ( $\left.C^{\mathrm{q}}-\mathrm{Me}\right), 160.09\left(C^{\mathrm{q}}-\mathrm{Me}\right), 158.92$ $\left(C^{\mathrm{q}}-\mathrm{Me}\right), 158.88\left(C^{\mathrm{q}}-\mathrm{Me}\right), 155.97\left(C^{\mathrm{pz}}\right), 154.48\left(C^{\mathrm{pz}}\right), 144.59\left(C^{\mathrm{Ar}}\right), 144.57\left(C^{\mathrm{Ar}}\right), 143.74$ $\left(C^{\mathrm{Ar}}\right), 143.52\left(C^{\mathrm{Ar}}\right), 143.34\left(C^{\mathrm{Ar}}\right), 141.23\left(C^{\mathrm{Ar}}\right), 126.42\left(C \mathrm{H}^{\mathrm{Ar}}\right), 124.83\left(C \mathrm{H}^{\mathrm{Ar}}\right), 124.49$ $\left(C \mathrm{H}^{\mathrm{Ar}}\right), 124.15\left(C \mathrm{H}^{\mathrm{Ar}}\right), 123.40\left(C \mathrm{H}^{\mathrm{Ar}}\right), 121.40\left(C \mathrm{H}^{\mathrm{Ar}}\right), 103.00(C \mathrm{H}), 97.84(C \mathrm{H}), 92.24$ $\left(\mathrm{CH}^{\mathrm{Pz}}\right), 52.68\left(\mathrm{CH}_{2}\right), 51.96\left(\mathrm{CH}_{2}\right), 29.56\left(\mathrm{CH}_{3}{ }^{i \operatorname{Pr}}\right), 29.50\left(\mathrm{CH}_{3}{ }^{i \operatorname{Pr}}\right), 25.46\left(\mathrm{CH}_{3}{ }^{i \operatorname{Pr}}\right), 25.34$ $\left(\mathrm{CH}_{3}{ }^{i \operatorname{Pr}}\right), 25.26\left(\mathrm{CH}_{3}{ }^{i \operatorname{Pr}}\right), 25.14\left(\mathrm{CH}_{3}{ }^{i \operatorname{Pr}}\right), 23.77\left(\mathrm{CH}_{3}{ }^{i \operatorname{Pr}}\right), 23.75\left(C_{3}{ }^{i \operatorname{Pr}}\right), 21.33\left(\mathrm{CH}_{3}\right)$, $21.09\left(\mathrm{CH}_{3}\right), 20.91\left(\mathrm{CH}_{3}{ }^{i \operatorname{Pr}}\right), 19.74\left(\mathrm{CH}_{3}\right)$.

\section{$\left[L^{\text {bisnac }} \mathrm{Ni}_{2}\left(\mu-\eta^{1}: \eta^{1}-\mathrm{N}_{2} \mathrm{H}_{3}\right)\right]$}

[L $\left.\mathbf{L}^{\text {bisnac }} \mathbf{N i}_{\mathbf{2}}(\boldsymbol{\mu}-\mathbf{B r})\right]$ (200 mg, $0.25 \mathrm{mmol}, 1.0$ eq.) was suspended in dry THF (3 mL). To the yellow-greenish suspension a $1 \mathrm{M}$ hydrazine solution $(0.75 \mathrm{~mL}, 0.75 \mathrm{mmol}, 3.0$ eq.) was slowly added. An immediate color change to dark red occured and the resulting mixture was stirred for 2 hours at room temperature. The mixture was then filtrated to yield a clear, dark red solution. A slow diffusion of pentane vapours into the THF-solution resulted in the formation of deep red, rod shaped crystals, suitable for X-ray structural analysis (58 $\mathrm{mg}, 0.08 \mathrm{mmol}, 31 \%$ ).

elemental formula: $\mathrm{C}_{39} \mathrm{H}_{56} \mathrm{~N}_{8} \mathrm{Ni}_{2}$.

molecular weight: $754.32 \mathrm{~g} \cdot \mathrm{mol}^{-1}$. 
elemental analysis: Calcd. (\%) for $\mathrm{C}_{39} \mathrm{H}_{56} \mathrm{~N}_{8} \mathrm{Ni}_{2}$ : C $62.10 \mathrm{H} 7.48 \mathrm{~N}$ 14.86. Found: C 62.18 H 7.66 N 14.04.

${ }^{1}$ H NMR $\left(500 \mathrm{MHz}, \mathrm{THF}-\mathrm{d}_{8}\right): \delta / \mathrm{ppm}=7.10-6.80\left(\mathrm{~m}, 6 \mathrm{H}, \mathrm{CH} H^{\mathrm{Ar}}\right), 5.68\left(\mathrm{~s}, 1 \mathrm{H}, \mathrm{CH}{ }^{\mathrm{Pz}}\right)$, 4.75 (s, $1 \mathrm{H}, \mathrm{CH}), 4.57$ (s, $1 \mathrm{H}, \mathrm{CH}), 4.01$ (s, $2 \mathrm{H}, \mathrm{CH}_{2}$ ), 3.97 (s, $2 \mathrm{H}, \mathrm{CH}_{2}$ ), 3.49-3.40 (sept, $\left.{ }^{3} J_{\mathrm{HH}}=6.9 \mathrm{~Hz}, 2 \mathrm{H}, \mathrm{CH}{ }^{i \operatorname{Pr}}\right), 3.47-3.39\left(\mathrm{sept},{ }^{3} J_{\mathrm{HH}}=6.9 \mathrm{~Hz}, 2 \mathrm{H}, \mathrm{C} H^{i \operatorname{Pr}}\right), 2.26$ $\left(\mathrm{d}, 2 \mathrm{H}, \mathrm{NH}_{2}\right), 1.94\left(\mathrm{~s}, 3 \mathrm{H}, \mathrm{CH}_{3}\right), 1.84\left(\mathrm{~s}, 3 \mathrm{H}, \mathrm{CH}_{3}\right), 1.41$ (s, $\left.3 \mathrm{H}, \mathrm{CH}_{3}\right), 1.29$ (s, $3 \mathrm{H}$, $\left.\mathrm{CH}_{3}\right), 1.20\left(\mathrm{~d},{ }^{3} J_{\mathrm{HH}}=7.0 \mathrm{~Hz}, 6 \mathrm{H}, \mathrm{CH}_{3}{ }^{i \mathrm{Pr}}\right), 1.18\left(\mathrm{~d},{ }^{3} J_{\mathrm{HH}}=7.0 \mathrm{~Hz}, 6 \mathrm{H}, \mathrm{CH}_{3}{ }^{i \operatorname{Pr}}\right), 1.04$ $\left(\mathrm{d},{ }^{3} J_{\mathrm{HH}}=7.0 \mathrm{~Hz}, 6 \mathrm{H}, \mathrm{CH}_{3}{ }^{i \operatorname{Pr}}\right), 1.02\left(\mathrm{~d},{ }^{3} J_{\mathrm{HH}}=7.0 \mathrm{~Hz}, 6 \mathrm{H}, \mathrm{CH}_{3}{ }^{i \operatorname{Pr}}\right),-0.92(\mathrm{t}, 1 \mathrm{H}$, $\mathrm{N} H)$.

${ }^{13} \mathrm{C}$ NMR $\left(126 \mathrm{MHz}, \mathrm{THF}-\mathrm{d}_{8}\right): \delta / \mathrm{ppm}=160.59\left(C^{\mathrm{q}}\right), 159.27\left(C^{\mathrm{q}}\right), 158.32\left(C^{\mathrm{q}}\right)$, $157.87\left(C^{\mathrm{q}}\right), 155.04\left(C^{\mathrm{q}}\right), 153.52\left(C^{\mathrm{q}}\right), 145.52\left(C^{\mathrm{q}}\right), 144.33\left(C^{\mathrm{q}}\right), 142.84\left(C^{\mathrm{q}}\right), 142.44$ $\left(C^{\mathrm{q}}\right), 125.84\left(C \mathrm{H}^{\mathrm{Ar}}\right), 124.15\left(C \mathrm{H}^{\mathrm{Ar}}\right), 123.70\left(C \mathrm{H}^{\mathrm{Ar}}\right), 122.36\left(C \mathrm{H}^{\mathrm{Ar}}\right), 96.76(C \mathrm{H}), 95.85$ $(C \mathrm{H}), 91.29\left(\left(\mathrm{CH}^{\mathrm{Pz}}\right), 51.14\left(\mathrm{CH}_{2}\right), 50.34\left(\mathrm{CH}_{2}\right), 27.62\left(C \mathrm{H}^{i \mathrm{Pr}}\right), 27.48\left(C \mathrm{H}^{i \operatorname{Pr}}\right), 24.65\right.$ $\left(\mathrm{CH}_{3}{ }^{i \operatorname{Pr}}\right), 24.25\left(\mathrm{CH}_{3}{ }^{i \operatorname{Pr}}\right), 23.63\left(\mathrm{CH}_{3}\right), 23.23\left(\mathrm{CH}_{3}\right), 23.19\left(\mathrm{CH}_{3}{ }^{i \operatorname{Pr}}\right), 23.09\left(\mathrm{CH}_{3}{ }^{i \operatorname{Pr}}\right)$, $20.81\left(\mathrm{CH}_{3}\right), 20.61\left(\mathrm{CH}_{3}\right)$.

${ }^{15} \mathrm{~N}$ NMR $\left(52 \mathrm{MHz}, \mathrm{THF}-\mathrm{d}_{8}\right): \delta / \mathrm{ppm}=-170.41\left(N^{\mathrm{pz}}\right),-172.43\left(N^{\mathrm{pz}}\right),-230.60\left(N^{\mathrm{nac}}\right)$, $-232.92\left(N^{\mathrm{nac}}\right),-234.44\left(N^{\mathrm{nac}}\right),-240.29\left(N^{\mathrm{nac}}\right),-313.81\left({ }^{1} J_{\mathrm{NH}}=70 \mathrm{~Hz}, N \mathrm{H}_{2}\right),-343.24$ $\left({ }^{1} J_{\mathrm{NH}}=54 \mathrm{~Hz}, \mathrm{NH}\right)$.

MS $\left(\operatorname{ESI}(+)\right.$, THF / MeCN): $\left(\frac{m}{z} / \%\right)=753.32(100)\left[\mathrm{L}^{\text {bisnac }} \mathrm{Ni}_{2}\left(\mu-\eta 1-\eta 1-\mathrm{N}_{2} \mathrm{H}_{3}\right)+\mathrm{H}\right]^{+}$. IR (KBr): $\tilde{\nu} / \mathrm{cm}^{-1}=3299(\mathrm{vw}), 2373(\mathrm{w}), 3253(\mathrm{w}), 3055(\mathrm{vw}), 2929(\mathrm{w}), 2864(\mathrm{w})$, 1559 (m), 1529 (vs), 1458 (m), 1436 (s), 1401 (s), 1369 (m), 1314 (m), 1278 (m), 1251 (m), $1233(\mathrm{w}), 1188(\mathrm{w}), 1092(\mathrm{w}), 1073(\mathrm{w}), 1055(\mathrm{w}), 1032(\mathrm{~m}), 1015(\mathrm{w}), 957(\mathrm{vw}), 935$ $(\mathrm{w}), 913(\mathrm{w}), 867(\mathrm{vw}), 801(\mathrm{~m}), 760(\mathrm{~s}), 740(\mathrm{~s}), 711(\mathrm{~m}), 647(\mathrm{w}), 625(\mathrm{vw}), 519(\mathrm{~m})$, $486(\mathrm{w}), 460(\mathrm{w}), 448(\mathrm{w}), 423(\mathrm{w})$.

\section{$\mathrm{K}\left[\mathrm{L}^{\text {bisnac }} \mathrm{Ni}_{2}\left(\mu-\eta^{1}: \eta^{1}-\mathrm{N}_{2} \mathrm{H}_{2}\right)\right]$}

[ $\mathbf{L}^{\text {bisnac }} \mathbf{N i}_{\mathbf{2}}\left(\mu-\eta^{\mathbf{1}}: \eta^{\mathbf{1}}-\mathbf{N}_{\mathbf{2}} \mathbf{H}_{\mathbf{3}}\right)$ ] (10 mg, $13.3 \mu \mathrm{mol}, 1.0$ eq.) was dissolved in dry THF $(0.5 \mathrm{~mL})$. To the dark red, clear solution $\mathrm{KH}(0.53 \mathrm{mg}, 13.3 \mu \mathrm{mol}, 1.0$ eq. $)$ and dibenzo-18-crown-6 (4.8 mg, $13.3 \mu \mathrm{mol}, 1.0$ eq.) was added to deprotonate the hydrazido bridging ligand. The resulting solution containing the potassium coordinated $\mathbf{K}\left[\mathbf{L}^{\mathbf{b i s n a c}} \mathbf{N i}_{\mathbf{2}}(\mu-\right.$ $\mathbf{N}_{\mathbf{2}} \mathbf{H}_{\mathbf{2}}$ )] was cooled and layered with hexanes. At $-30{ }^{\circ} \mathrm{C}$ red, rod shaped crystals grew, which were suitable for X-ray analysis. $\left[\mathbf{L}^{\text {bisnac }} \mathbf{N i}_{\mathbf{2}}\left(\mu-\eta^{\mathbf{1}}: \eta^{\mathbf{1}}-\mathbf{N}_{\mathbf{2}} \mathbf{H}_{\mathbf{3}}\right)\right]$ was reacquired by means of a conversion with [LutH]OTf (1.0 eq.) in a THF solution.

elemental formula: $\mathrm{C}_{39} \mathrm{H}_{55} \mathrm{KN}_{8} \mathrm{Ni}_{2}$.

molecular weight: $792.41 \mathrm{~g} \cdot \mathrm{mol}^{-1}$.

${ }^{1} \mathbf{H}$ NMR (300 MHz, THF-d $\left.8,40{ }^{\circ} \mathrm{C}\right): \delta / \mathrm{ppm}=6.9-6.85\left(\mathrm{~m}, 2 \mathrm{H}, \mathrm{CH}{ }^{\mathrm{Ar}}\right), 6.81-6.78$ $\left(\mathrm{m}, 4 \mathrm{H}, \mathrm{C} H^{\mathrm{Ar}}\right), 5.68\left(\mathrm{~s}, 1 \mathrm{H}, \mathrm{C} H^{\mathrm{Pz}}\right), 4.44(\mathrm{~s}, 2 \mathrm{H}, \mathrm{CH}), 3.78\left(\mathrm{~s}, 4 \mathrm{H}, \mathrm{CH} H_{2}\right), 3.63-3.53$ (sept, $\left.{ }^{3} J_{\mathrm{HH}}=7.0 \mathrm{~Hz}, 4 \mathrm{H}, \mathrm{CH}{ }^{i \operatorname{Pr}}\right), 1.21-1.19\left(\mathrm{~d},{ }^{3} J_{\mathrm{HH}}=7.0 \mathrm{~Hz}, 12 \mathrm{H}, \mathrm{CH}_{3}{ }^{i \operatorname{Pr}}\right), 1.09$ (s, 
$\left.1 \mathrm{H}, \mathrm{N}_{2} H_{2}\right), 1.03-1.01\left(\mathrm{~d},{ }^{3} J_{\mathrm{HH}}=7.0 \mathrm{~Hz}, 12 \mathrm{H}, \mathrm{CH}_{3}{ }^{i \mathrm{Pr}}\right)$.

${ }^{13}$ C NMR (101 MHz, THF-D 8$): \delta / p p m=158.66\left(C^{\mathrm{q}}-\mathrm{Me}\right), 157.13\left(C^{\mathrm{q}}-\mathrm{Me}\right), 154.23$ $\left(C^{\mathrm{pz}}\right), 148.31\left(C^{\mathrm{Ar}}\right), 142.68\left(C^{\mathrm{Ar}}\right), 123.84\left(C \mathrm{H}^{\mathrm{Ar}}\right), 123.05\left(C \mathrm{H}^{\mathrm{Ar}}\right), 96.05(C \mathrm{H}), 92.81$ $\left(C \mathrm{H}^{\mathrm{Pz}}\right), 48.66\left(C \mathrm{H}_{2}\right), 28.03\left(C \mathrm{H}^{i \mathrm{Pr}}\right), 23.84\left(\mathrm{CH}_{3}{ }^{i \mathrm{Pr}}\right), 22.01\left(C_{3}\right)$.

${ }^{15} \mathbf{N}$ NMR (41 MHz, THF-d $): \delta / p p m=-255.16\left({ }^{1} J_{\mathrm{NH}}=59 \mathrm{~Hz}, N_{2} \mathrm{H}_{2}\right)$.

IR (ATR): $\tilde{\nu} / \mathrm{cm}^{-1}=3282(\mathrm{vw}), 3260(\mathrm{w}), 3247(\mathrm{w}), 3204(\mathrm{w}), 3105(\mathrm{vw}), 3060(\mathrm{vw})$, $2962(\mathrm{~m}), 2927$ (w), 2868 (w), 1563 (m), 1531 (s), 1464 (m), 1433 (m), 1394 (m), 1318 (m), $1270(\mathrm{~m}), 1232$ (s), $1187(\mathrm{w}), 1147$ (s), $1094(\mathrm{w}), 1028(\mathrm{vs}), 982(\mathrm{w}), 803$ (w), 767 (m), $746(\mathrm{w}), 710(\mathrm{w}), 636(\mathrm{vs}), 571(\mathrm{~m}), 516(\mathrm{~s})$.

\section{$\left[L^{\text {bisnac }} \mathrm{Ni}_{2}\left(\mu-\eta^{1}: \eta^{1}-\mathrm{N}_{2} \mathrm{H}_{4}\right)\right] \mathrm{OTf}$}

$\left[\mathbf{L}^{\text {bisnac }} \mathbf{N i}_{\mathbf{2}}\left(\mu-\eta^{\mathbf{1}}: \eta^{\mathbf{1}}-\mathbf{N}_{\mathbf{2}} \mathbf{H}_{\mathbf{3}}\right)\right](10 \mathrm{mg}, 13.3 \mu \mathrm{mol}, 1.0$ eq.) was dissolved in dry THF $(0.5 \mathrm{~mL})$. To the dark red, clear solution [LutH]OTf (3.8 mg, $14.8 \mu \mathrm{mol}, 1.1$ eq.) was added, immediately forming a dark green, clear solution. The cooled solution was then layered with hexanes under inert conditions. A slow diffusion of the solvents yielded the product as dark green crystals. [ $\left.\mathbf{L}^{\text {bisnac }} \mathbf{N i}_{2}\left(\mu-\eta^{1}: \eta^{1}-\mathbf{N}_{2} \mathbf{H}_{3}\right)\right]$ was reacquired by means of a conversion with $\mathrm{KH}$ (1.1 eq.) in a THF solution.

elemental formula: $\mathrm{C}_{40} \mathrm{H}_{57} \mathrm{~F}_{3} \mathrm{~N}_{8} \mathrm{Ni}_{2} \mathrm{O}_{3} \mathrm{~S}$.

molecular weight: $904.39 \mathrm{~g} \cdot \mathrm{mol}^{-1}$.

${ }^{1} \mathbf{H}$ NMR $\left(500 \mathrm{MHz}, \mathrm{THF}-\mathrm{d}_{8}, 40{ }^{\circ} \mathrm{C}\right): \delta / \mathrm{ppm}=7.31-7.28\left(\mathrm{~m}, 2 \mathrm{H}, \mathrm{C} H^{\mathrm{Ar}}\right), 7.15-7.13$ $\left(\mathrm{m}, 4 \mathrm{H}, \mathrm{C} H^{\mathrm{Ar}}\right), 5.88\left(\mathrm{~s}, 1 \mathrm{H}, \mathrm{C} H^{\mathrm{Pz}}\right), 4.94(\mathrm{~s}, 2 \mathrm{H}, \mathrm{CH}), 4.19\left(\mathrm{~s}, 4 \mathrm{H}, \mathrm{C} H_{2}\right), 3.67\left(\mathrm{~s}_{\mathrm{br}}\right.$, $\left.4 \mathrm{H}, \mathrm{NH}_{2}\right), 3.58-3.50\left(\mathrm{sept},{ }^{3} J_{\mathrm{HH}}=7.0 \mathrm{~Hz}, 4 \mathrm{H}, \mathrm{CH}{ }^{i \mathrm{Pr}}\right), 2.06\left(\mathrm{~s}, 3 \mathrm{H}, \mathrm{CH}_{3}\right), 1.47(\mathrm{~s}, 3 \mathrm{H}$, $\left.\mathrm{CH}_{3}\right), 1.19\left(\mathrm{~d},{ }^{3} J_{\mathrm{HH}}=6.7 \mathrm{~Hz}, 12 \mathrm{H}, \mathrm{CH}_{3}{ }^{i \operatorname{Pr}}\right), 1.08\left(\mathrm{~d},{ }^{3} J_{\mathrm{HH}}=6.7 \mathrm{~Hz}, 12 \mathrm{H}, \mathrm{CH}_{3}{ }^{i \operatorname{Pr}}\right)$.

${ }^{15} \mathrm{~N}$ NMR $\left(52 \mathrm{MHz}, \mathrm{THF}-\mathrm{d}_{8}\right): \delta / \mathrm{ppm}=-347.97\left({ }^{1} J_{\mathrm{NH}}=71 \mathrm{~Hz}, \mathrm{NH}_{2}\right)$.

IR (ATR): $\tilde{\nu} / \mathrm{cm}^{-1}=3282(\mathrm{vw}), 3260(\mathrm{w}), 3247(\mathrm{w}), 3204(\mathrm{w}), 3105(\mathrm{vw}), 3060(\mathrm{vw})$, $2962(\mathrm{~m}), 2927$ (w), 2868 (w), 1563 (m), 1531 (s), 1464 (m), 1433 (m), 1394 (m), 1318 (m), $1270(\mathrm{~m}), 1232(\mathrm{~s}), 1187(\mathrm{w}), 1147(\mathrm{~s}), 1094(\mathrm{w}), 1028(\mathrm{vs}), 982(\mathrm{w}), 803(\mathrm{w}), 767$ $(\mathrm{m}), 746(\mathrm{w}), 710(\mathrm{w}), 636(\mathrm{vs}), 571(\mathrm{~m}), 516(\mathrm{~s})$.

\section{[ $\left.\mathrm{L}^{\text {bisnac }} \mathrm{Ni}_{2}\left(\mu-\mathrm{NH}_{2}\right)\right]$}

[ $\mathbf{L}^{\text {bisnac }} \mathbf{N i}_{\mathbf{2}}\left(\mu-\eta^{1}: \eta^{\mathbf{1}}-\mathbf{N}_{\mathbf{2}} \mathbf{H}_{\mathbf{3}}\right)$ ] (142 mg, $0.17 \mathrm{mmol}, 1.0$ eq.) was dissolved in dry THF $(4 \mathrm{~mL})$. The dark red clear solution was heated to $100{ }^{\circ} \mathrm{C}$ in an overpressure tube for $3 \mathrm{w}$ to give an orange clear solution. The cooled solution was then layered with hexanes under inert conditions. A slow diffusion of the solvents yielded the product as rod shaped yellow crystals. 
elemental formula: $\mathrm{C}_{39} \mathrm{H}_{55} \mathrm{~N}_{7} \mathrm{Ni}_{2}$.

molecular weight: $739.30 \mathrm{~g} \cdot \mathrm{mol}^{-1}$.

${ }^{\mathbf{1}} \mathbf{H}$ NMR (300 MHz, THF-d N $): \delta / \mathrm{ppm}=6.97-6.92\left(\mathrm{~m}, 2 \mathrm{H}, \mathrm{CH}{ }^{\mathrm{Ar}}\right), 6.84-6.81(\mathrm{~m}, 4 \mathrm{H}$, $\left.\mathrm{CH}^{\mathrm{Ar}}\right), 5.49\left(\mathrm{~s}, 1 \mathrm{H}, \mathrm{C} H^{\mathrm{Pz}}\right), 4.62(\mathrm{~s}, 2 \mathrm{H}, \mathrm{CH}), 4.11\left(\mathrm{~s}, 4 \mathrm{H}, \mathrm{CH}_{2}\right), 3.39-3.25$ (sept, ${ }^{3} J_{\mathrm{HH}}$ $\left.=7.0 \mathrm{~Hz}, 4 \mathrm{H}, \mathrm{C} H^{i \mathrm{Pr}}\right), 1.88\left(\mathrm{~s}, 3 \mathrm{H}, \mathrm{CH}{ }_{3}\right), 1.49\left(\mathrm{~d},{ }^{3} J_{\mathrm{HH}}=6.9 \mathrm{~Hz}, 12 \mathrm{H}, \mathrm{CH}_{3}{ }^{i \mathrm{Pr}}\right), 1.13$ $\left(\mathrm{s}, 3 \mathrm{H}, \mathrm{CH}_{3}\right), 1.04\left(\mathrm{~d},{ }^{3} J_{\mathrm{HH}}=6.9 \mathrm{~Hz}, 12 \mathrm{H}, \mathrm{CH}_{3}{ }^{i \mathrm{Pr}}\right),-3.87\left(\mathrm{~s}, 2 \mathrm{H}, \mathrm{NH}_{2}\right)$.

${ }^{13} \mathrm{C}$ NMR (101 MHz, THF-D 8$): \delta / \mathrm{ppm}=161.28$ ( $\left.C^{\mathrm{q}}-\mathrm{Me}\right), 159.49$ ( $\left.C^{\mathrm{q}}-\mathrm{Me}\right), 150.83$ $\left.\left.\left(C^{\mathrm{pz}}\right), 147.93\left(C^{\mathrm{Ar}}\right), 141.23\left(C^{\mathrm{Ar}}\right), 125.93 C \mathrm{H}^{\mathrm{Ar}}\right), 125.36 C \mathrm{H}^{\mathrm{Ar}}\right), 97.53(C \mathrm{H}), 91.60$ $\left(C \mathrm{H}^{\mathrm{Pz}}\right), 55.55\left(C \mathrm{H}_{2}\right), 28.87\left(C \mathrm{H}_{3}{ }^{i \operatorname{Pr}}\right), 25.84\left(\mathrm{CH}_{3}{ }^{i \mathrm{Pr}}\right), 23.98\left(C \mathrm{H}_{3}\right), 21.46\left(C \mathrm{H}_{3}\right)$.

${ }^{15} \mathrm{~N}$ NMR $\left(52 \mathrm{MHz}, \mathrm{THF}-\mathrm{d}_{8}\right): \delta / \mathrm{ppm}=-243.49\left(N^{\mathrm{nac}}\right),-235.39\left(N^{\mathrm{nac}}\right),-156.43\left(N^{\mathrm{pz}}\right)$, $-51.34\left({ }^{1} J_{\mathrm{NH}}=59 \mathrm{~Hz}, \mathrm{NH}_{2}\right)$.

IR (ATR): $\tilde{\nu} / \mathrm{cm}^{-1}=3348(\mathrm{w}), 3333(\mathrm{w}), 3311(\mathrm{vw}), 3059(\mathrm{w}), 2960(\mathrm{vs}), 2927(\mathrm{~s}), 2867$ (s), $2235(\mathrm{w}), 2082(\mathrm{w}), 1680(\mathrm{~m}), 1629(\mathrm{~m}), 1557$ (s), 1531 (vs), 1463 (s), 1439 (vs), 1404 (vs), $1322(\mathrm{~m}), 1260(\mathrm{~m}), 1195(\mathrm{w}), 1098(\mathrm{~m}), 1048(\mathrm{~m}), 956(\mathrm{w}), 936(\mathrm{w}), 802(\mathrm{~m}), 764$ $(\mathrm{m}), 735(\mathrm{~m})$.

\section{$\left[\mathrm{L}^{\text {bisnac }} \mathrm{Ni}_{2}\left(\mu-\eta^{1}: \eta^{1}-\mathrm{N}_{2} \mathrm{H}\right)\right]$}

$\left[\mathbf{L}^{\text {bisnac }} \mathbf{N i}_{\mathbf{2}}\left(\mu-\eta^{\mathbf{1}}: \eta^{\mathbf{1}}-\mathbf{N}_{\mathbf{2}} \mathbf{H}_{\mathbf{3}}\right)\right](10 \mathrm{mg}, 13.3 \mu \mathrm{mol}, 1.0$ eq. $)$ was dissolved in dry THF $(0.5 \mathrm{~mL})$. The dark red, clear solution was cooled to $-30{ }^{\circ} \mathrm{C}$ and added to TEMPO (4.2 mg, $26.6 \mu \mathrm{mol}, 2.0$ eq.) immediately forming a dark green, clear solution. The cooled solution was then layered with hexanes under inert conditions. A slow diffusion of the solvents yielded the product as rod shaped dark green crystals, suitable for X-ray structural analysis.

elemental formula: $\mathrm{C}_{39} \mathrm{H}_{54} \mathrm{~N}_{8} \mathrm{Ni}_{2}$.

molecular weight: $752.30 \mathrm{~g} \cdot \mathrm{mol}^{-1}$.

${ }^{1} \mathbf{H}$ NMR $\left(400 \mathrm{MHz}, \mathrm{THF}_{-} \mathrm{d}_{8},-35{ }^{\circ} \mathrm{C}\right): \delta / \mathrm{ppm}=9.43(\mathrm{~s}, 1 \mathrm{H}, \mathrm{N}=\mathrm{NH}), 7.19-7.15(\mathrm{~m}$, $\left.1 \mathrm{H}, \mathrm{C} H^{\mathrm{Ar}}\right), 6.99\left(\mathrm{~d},{ }^{3} J_{\mathrm{HH}}=7.8 \mathrm{~Hz}, 2 \mathrm{H}, \mathrm{C} H^{\mathrm{Ar}}\right), 6.91-6.87\left(\mathrm{~m}, 1 \mathrm{H}, \mathrm{C} H^{\mathrm{Ar}}\right), 6.67(\mathrm{~d}$, $\left.{ }^{3} J_{\mathrm{HH}}=7.5 \mathrm{~Hz}, 2 \mathrm{H}, \mathrm{C} H^{\mathrm{Ar}}\right), 5.73\left(\mathrm{~s}, 1 \mathrm{H}, \mathrm{CH}{ }^{\mathrm{Pz}}\right), 5.02(\mathrm{~s}, 1 \mathrm{H}, \mathrm{CH}), 4.99(\mathrm{~s}, 1 \mathrm{H}, \mathrm{CH})$, $4.26\left(\mathrm{~s}, 2 \mathrm{H}, \mathrm{CH} H_{2}\right), 4.23\left(\mathrm{~s}, 2 \mathrm{H}, \mathrm{C} H_{2}\right), 3.36-3.27$ (sept, ${ }^{3} J_{\mathrm{HH}}=6.9 \mathrm{~Hz}, 2 \mathrm{H}, \mathrm{C} H^{i \operatorname{Pr}}$ ), $3.17-3.06$ (sept, $\left.{ }^{3} J_{\mathrm{HH}}=6.9 \mathrm{~Hz}, 2 \mathrm{H}, \mathrm{CH}{ }^{i \operatorname{Pr}}\right), 2.02\left(\mathrm{~d},{ }^{3} J_{\mathrm{HH}}=8.4 \mathrm{~Hz}, 6 \mathrm{H}, \mathrm{CH}_{3}{ }^{i \operatorname{Pr}}\right), 1.55$ $\left(\mathrm{s}, 3 \mathrm{H}, \mathrm{CH}_{3}\right), 1.26\left(\mathrm{~s}, 3 \mathrm{H}, \mathrm{CH}_{3}\right), 1.10\left(\mathrm{~d},{ }^{3} J_{\mathrm{HH}}=7.2 \mathrm{~Hz}, 6 \mathrm{H}, \mathrm{CH}_{3}{ }^{i \operatorname{Pr}}\right), 1.00\left(\mathrm{~d},{ }^{3} J_{\mathrm{HH}}\right.$ $\left.=6.9 \mathrm{~Hz}, 6 \mathrm{H}, \mathrm{CH}_{3}{ }^{i \mathrm{Pr}}\right), 0.84\left(\mathrm{~d},{ }^{3} J_{\mathrm{HH}}=7.1 \mathrm{~Hz}, 6 \mathrm{H}, \mathrm{CH}_{3}{ }^{i \operatorname{Pr}}\right)$.

${ }^{15} \mathrm{~N}$ NMR $\left(51 \mathrm{MHz}, \mathrm{THF}-\mathrm{d}_{8}\right): \delta / \mathrm{ppm}=-400.16,-354.80,-323.35,-231.42\left(N^{\mathrm{nac}}\right)$, $3.86\left({ }^{1} J_{\mathrm{NH}}=74 \mathrm{~Hz}, \mathrm{~N}=\mathrm{NH}\right)$.

MS $(\mathrm{ESI}(+), \mathrm{THF} / \mathrm{MeCN}):\left(\frac{m}{z} / \%\right)=751.45(20)\left[\mathrm{L}^{\text {bisnac }} \mathrm{Ni}_{2}\left(\mathrm{~N}_{2} \mathrm{H}\right)+\mathrm{H}^{+}\right]^{+}, 753.47$ (100) $\left[\mathrm{L}^{\text {bisnac }} \mathrm{Ni}_{2}\left(\mu-\eta 1-\eta 1-\mathrm{N}_{2} \mathrm{H}_{3}\right)+\mathrm{H}\right]^{+}$.

IR (KBr): $\tilde{\nu} / \mathrm{cm}^{-1}=3282(\mathrm{w}), 3264(\mathrm{w}), 3056(\mathrm{vw}), 2958(\mathrm{~m}), 2926(\mathrm{w}), 2866(\mathrm{w}), 1560$ (m), 1530 (vs), 1461 (s), 1437 (s), 1398 (s), 1369 (m), 1314 (m), 1278 (m), 1251 (m), 1233 
(w), $1188(\mathrm{w}), 1175(\mathrm{w}), 1092(\mathrm{w}), 1074(\mathrm{w}), 1055(\mathrm{w}), 1032(\mathrm{~m}), 1019(\mathrm{w}), 957(\mathrm{vw}), 935$ $(\mathrm{w}), 910(\mathrm{w}), 867(\mathrm{w}), 798(\mathrm{~m}), 762(\mathrm{~s}), 712(\mathrm{~m}), 647(\mathrm{w}), 620(\mathrm{w}), 739(\mathrm{~s}), 520(\mathrm{~m}), 461$ (m), $446(\mathrm{~m}), 423(\mathrm{w})$.

\section{$\left[\mathrm{L}^{\text {bisnac }} \mathrm{Ni}_{2}\left(\mu-\eta^{1}: \eta^{1}-\mathrm{N}_{2}\right)\right]$}

$\left.\mathbf{K}\left[\mathbf{L}^{\text {bisnac }} \mathbf{N i}_{\mathbf{2}} \mathbf{( H}\right)_{\mathbf{2}}\right](100 \mathrm{mg}, 0.13 \mathrm{mmol}, 1 \mathrm{eq})$ was dissolved in dry THF (3 mL) under inert $\left(\mathrm{N}_{2}\right)$ atmosphere. This solution was added to solid lutidinium triflate $(34 \mathrm{mg}$, $0.13 \mathrm{mmol}, 1 \mathrm{eq})$ and the resulting mixture stirred for 5 minutes. The solution was cooled $\left(-30{ }^{\circ} \mathrm{C}\right)$ and layered with hexanes. Slow diffusion of the solvents at $-30{ }^{\circ} \mathrm{C}$ yielded crystalline material of the product, which was suitable for X-ray analysis.

elemental formula: $\mathrm{C}_{39} \mathrm{H}_{53} \mathrm{~N}_{8} \mathrm{Ni}_{2}$.

molecular weight: $751.30 \mathrm{~g} \cdot \mathrm{mol}^{-1}$.

IR (KBr): $\tilde{\nu} / \mathrm{cm}^{-1}=3057(\mathrm{vw}), 2958(\mathrm{~m}), 2926(\mathrm{w}), 2867(\mathrm{w}), 1896(\mathrm{~s})=\tilde{\nu}_{\mathrm{N}-\mathrm{N}}, 1554$ (m), 1530 (vs), 1458 (m), 1437 (m), 1397 (vs), 1369 (w), 1320 (w), 1311 (m), 1276 (m), $1252(\mathrm{~s}), 1234(\mathrm{~m}), 1186(\mathrm{~m}), 1177(\mathrm{~m}), 1102(\mathrm{w}), 1092(\mathrm{w}), 1075(\mathrm{w}), 1032(\mathrm{~s}), 1012(\mathrm{~m})$ $956(\mathrm{vw}), 935(\mathrm{w}), 917(\mathrm{~m}), 885(\mathrm{vw}), 866(\mathrm{w}), 845(\mathrm{~m}), 794(\mathrm{~s}), 764(\mathrm{~m}), 759(\mathrm{~m}), 747$ $(\mathrm{s}), 714(\mathrm{w}), 650(\mathrm{~s}), 644(\mathrm{~s}), 580(\mathrm{w}), 541(\mathrm{w}), 520(\mathrm{~s}), 478(\mathrm{~s}), 426(\mathrm{~m})$.

\subsection{DFT Calculations}

Calculations concerning the mechanisms for $\mathrm{H} / \mathrm{D}$ or $\mathrm{OH}$ exchange in $\left[\mathbf{L}^{\mathbf{p y r}} \mathbf{N i}_{\mathbf{2}}(\mu-\mathrm{OH})\right]$, $\left[\mathbf{L}^{\text {bisnac }} \mathbf{N i}_{\mathbf{2}}(\mu-\mathrm{OH})\right],\left[\mathbf{L}^{\text {bisnac }} \mathbf{N i}_{2}(\mathbf{H})_{2}\right]$ were carried out in cooperation with the group of Prof. Dr. Ricardo Mata at the Institute of Physical Chemistry of the Georg-August-University Göttingen. The BP86 functional was used with the def2-SVP basis for optimizations, frequency calculations and thermodynamic corrections, whereas the TZVP basis set was applied for energy calculations. Resolution of the identity approximations were used in all BP86 calculations (RI-J). $\stackrel{[340]}{ }^{[}$For the thermodynamic parameters the electronic energies were refined with B3LYP-D3/TZVP and LMP2/TZVP. In the case of B3LYP-D3 and LMP2, the RIJCOSX and density fitting methods were applied respectively. ${ }^{[341]}$ All density functional calculations were performed with the Orca program package. ${ }^{[342]}$ In the case of DF-LMP2, Pipek-Mezey orbitals were used, together with the NPA domain criterium TNPA $=0.03$ and default pair distance criteria. ${ }^{[343.344]}$ The default density fitting

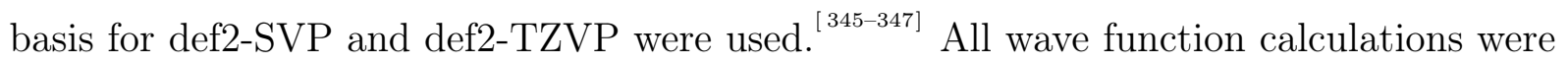
carried out with Molpro2012.1. $\stackrel{[348]}{ }$ 
Appendices 



\section{Structures of Important Compounds}

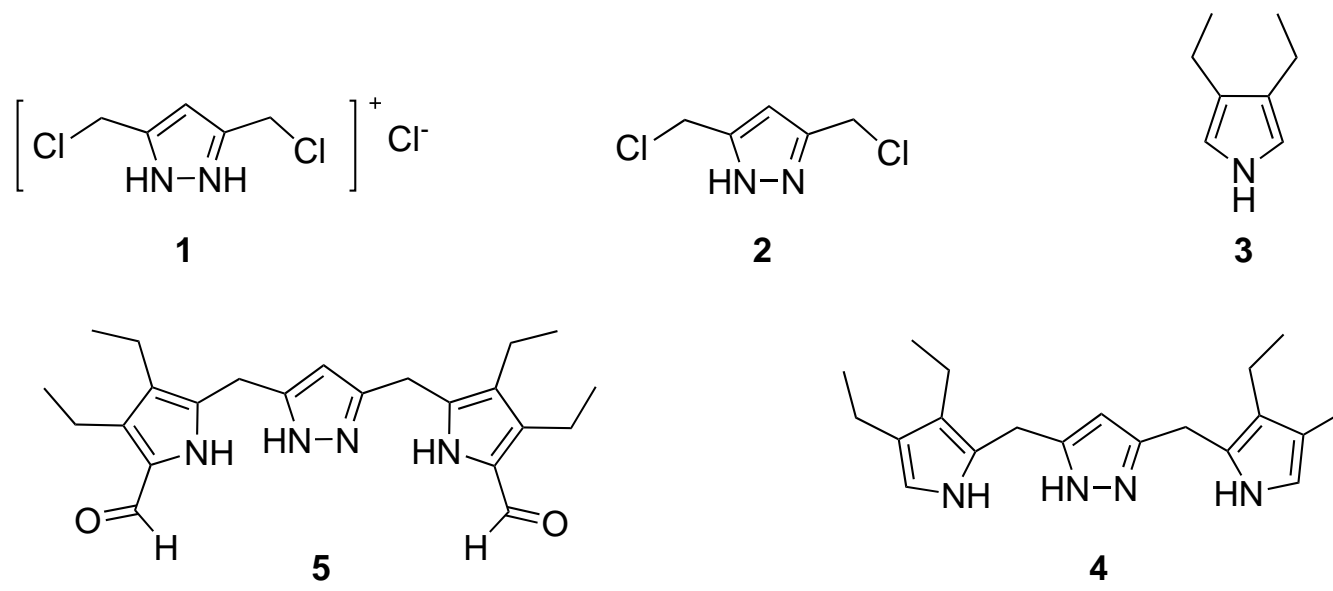<smiles>NC(=O)c1cc(C(N)=O)[nH]n1</smiles><smiles>N#Cc1cc(C#N)[nH]n1</smiles><smiles>NCc1cc(CN)[nH]n1</smiles>

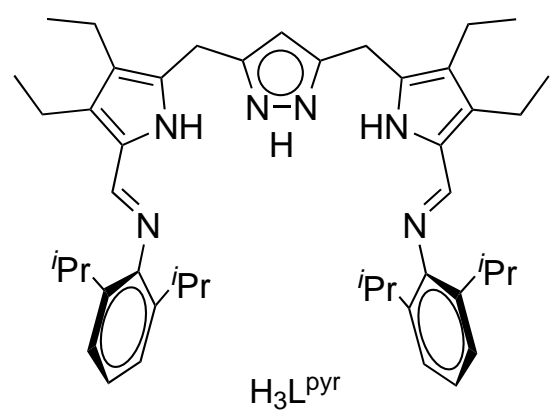

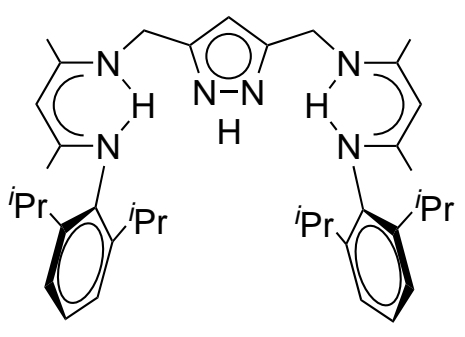

$\mathrm{H}_{3} \mathrm{~L}^{\text {bisnac }}$

Figure A.1.: Ligands and precursors. 


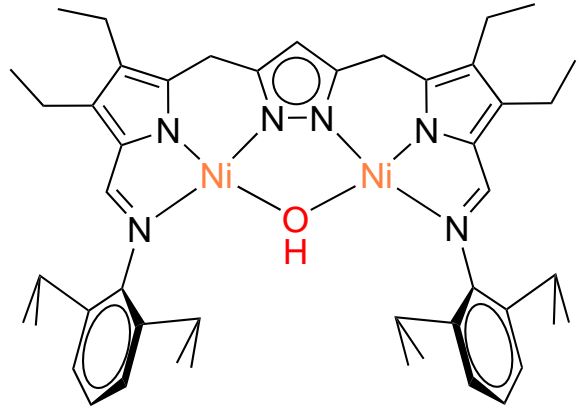

$\left[\mathrm{L}^{\mathrm{pyr}} \mathrm{Ni}_{2}(\mu-\mathrm{OH})\right]$

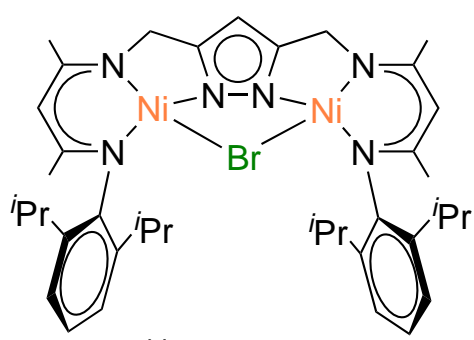

[ $\left.\mathrm{L}^{\text {bisnac }} \mathrm{Ni}_{2}(\mu-\mathrm{Br})\right]$

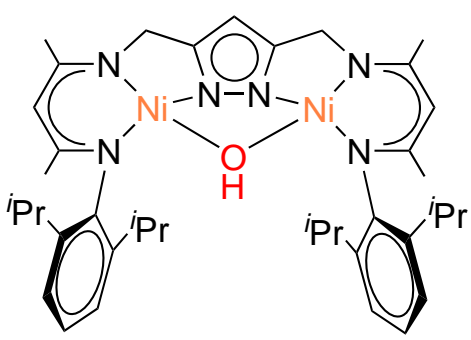

$\left[\mathrm{L}^{\text {bisnac }} \mathrm{Ni}_{2}(\mu-\mathrm{OH}]\right.$

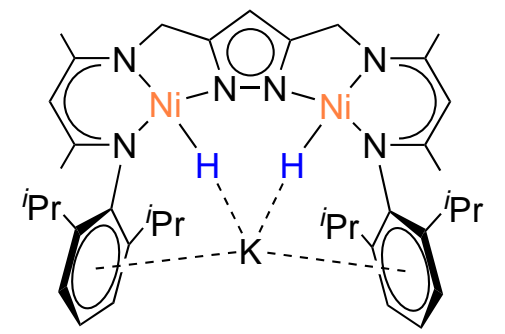

$\mathrm{K}\left[\mathrm{L}^{\text {bisnac }} \mathrm{Ni}_{2}(\mathrm{H})_{2}\right]$

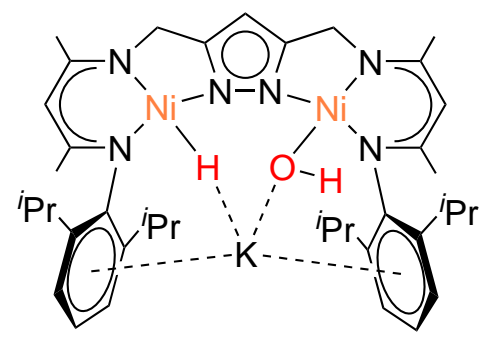

$\mathrm{K}\left[\mathrm{L}^{\text {bisnac }} \mathrm{Ni}_{2}(\mathrm{OH}) \mathrm{H}\right]$

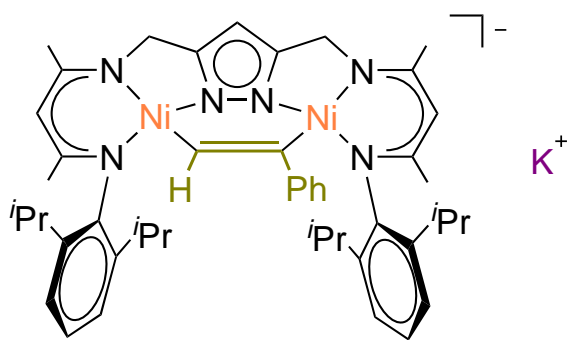

$\mathrm{K}\left[\mathrm{L}^{\text {bisnac }} \mathrm{Ni}_{2}\left(\mu-\eta^{1}: \eta^{1}-\mathrm{CHCPh}\right)\right]$

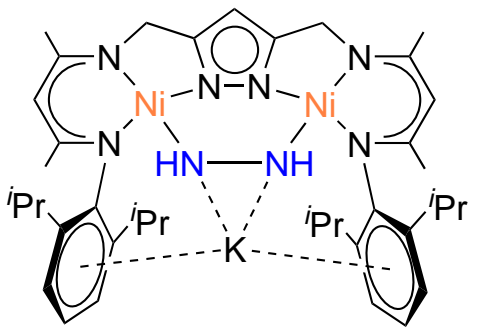

$\mathrm{K}\left[\mathrm{L}^{\text {bisnac }} \mathrm{Ni}_{2}\left(\mu-\eta^{1}: \eta^{1}-\mathrm{N}_{2} \mathrm{H}_{2}\right)\right]$

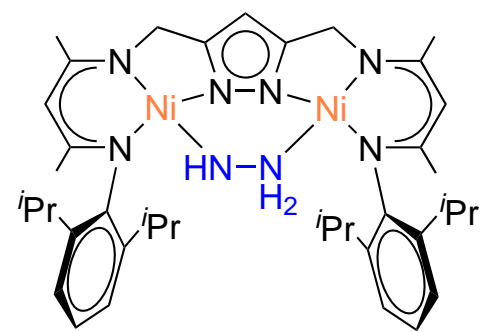

$\left[\mathrm{L}^{\text {bisnac }} \mathrm{Ni}_{2}\left(\mu-\eta^{1}: \eta^{1}-\mathrm{N}_{2} \mathrm{H}_{3}\right)\right]$

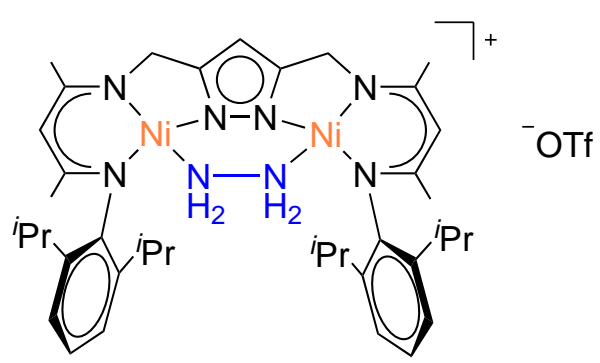

$\left[\mathrm{L}^{\text {bisnac }} \mathrm{Ni}_{2}\left(\mu-\eta^{1}: \eta^{1}-\mathrm{N}_{2} \mathrm{H}_{4}\right)\right] \mathrm{OTf}$

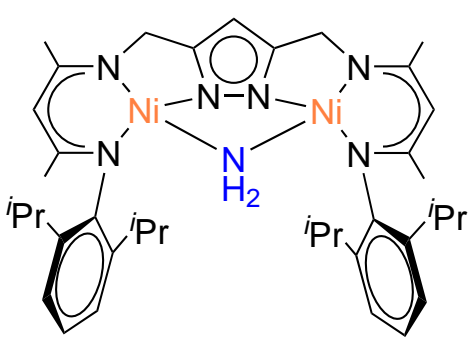

[ $\left.\mathrm{L}^{\text {bisnac }} \mathrm{Ni}_{2}\left(\mu-\mathrm{NH}_{2}\right)\right]$

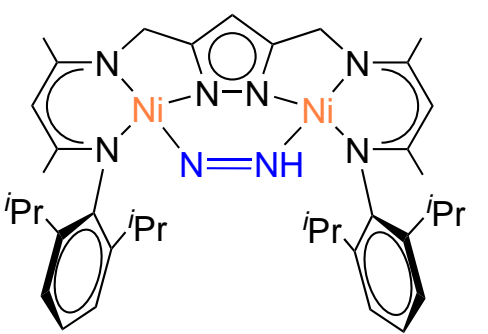

$\left[\mathrm{L}^{\text {bisnac }} \mathrm{Ni}_{2}\left(\mu-\eta^{1}: \eta^{1}-\mathrm{N}_{2} \mathrm{H}\right)\right]$

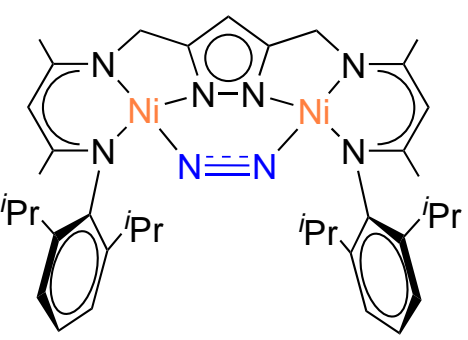

$\left[\mathrm{L}^{\text {bisnac }} \mathrm{Ni}_{2}\left(\mu-\eta^{1}: \eta^{1}-\mathrm{N}_{2}\right)\right]$

Figure A.2.: Complexes. 


\section{Crystallographic Data}


Table B.1.: Crystal data and refinement details.

\begin{tabular}{|c|c|c|c|}
\hline compound & {$\left[\mathrm{L}^{\mathrm{pyr}} \mathrm{Ni}_{2}(\mu-\mathrm{OH})\right]$} & [ $\left.L^{\text {bisnac }} \mathrm{Ni}_{2}(\mu-\mathrm{Br})\right]$ & {$\left[\mathrm{L}^{\text {bisnac }} \mathrm{Ni}_{2}(\mu-\mathrm{OH})\right]$} \\
\hline empirical formula & $\mathrm{C}_{47} \mathrm{H}_{62} \mathrm{~N}_{6} \mathrm{Ni}_{2} \mathrm{O}$ & $\mathrm{C}_{39.50} \mathrm{H}_{54} \mathrm{BrClN}_{6} \mathrm{Ni}_{2}$ & $\mathrm{C}_{39} \mathrm{H}_{54} \mathrm{~N}_{6} \mathrm{Ni}_{2} \mathrm{O}$ \\
\hline formula weight & 844.45 & 845.66 & 740.30 \\
\hline$T / \mathrm{K}$ & $133(2)$ & $133(2)$ & $133(2)$ \\
\hline $\begin{array}{l}\text { crystal size } / \mathrm{mm}^{3} \\
\text { crystal system }\end{array}$ & $\begin{array}{l}0.50 \times 0.49 \times 0.45 \\
\text { triclinic }\end{array}$ & $\begin{array}{l}0.26 \times 0.21 \times 0.18 \\
\text { monoclinic }\end{array}$ & $\begin{array}{l}0.50 \times 0.17 \times 0.15 \\
\text { monoclinic }\end{array}$ \\
\hline space group & $P \overline{1}$ & $P 2_{1} / c$ & $C 2 / c$ \\
\hline$a / \AA$ & $12.8084(6)$ & $17.1871(4)$ & $18.5319(9)$ \\
\hline$b / \AA$ & $14.0477(7)$ & $13.8483(3)$ & $16.4482(9)$ \\
\hline$c / \AA$ & $14.6069(7)$ & $17.3512(4)$ & $13.6531(7)$ \\
\hline$\alpha /^{\circ}$ & $62.148(3)$ & 90 & 90 \\
\hline$\beta / \circ$ & $89.579(4)$ & $111.488(2)$ & $118.285(3)$ \\
\hline$\gamma /{ }^{\circ}$ & $68.588(3)$ & 90 & 90 \\
\hline$V / \AA^{3}$ & $2120.40(18)$ & $3842.75(16)$ & $3664.8(3)$ \\
\hline$Z$ & 2 & 4 & 4 \\
\hline$\rho / \mathrm{g} \mathrm{cm}^{-3}$ & 1.323 & 1.462 & 1.342 \\
\hline$F(000)$ & 900 & 1764 & 1576 \\
\hline$\mu / \mathrm{mm}^{-1}$ & 0.931 & 2.126 & 1.067 \\
\hline$T_{\min } \mid T_{\max }$ & $0.7314 \mid 0.5462$ & $0.7252 \mid 0.5963$ & $0.8261 \mid 0.5304$ \\
\hline$\Theta$-range $/^{\circ}$ & $1.61-26.78$ & $1.273-26.732$ & $1.758-26.779$ \\
\hline \multirow[t]{3}{*}{ hkl-range } & $-16 \leq h \leq 16$ & $-21 \leq h \leq 21$ & $-23 \leq h \leq 23$ \\
\hline & $-17 \leq \mathrm{k} \leq 17$ & $-17 \leq \mathrm{h} \leq 17$ & $-20 \leq \mathrm{h} \leq 20$ \\
\hline & $-18 \leq \mathrm{I} \leq 18$ & $-21 \leq \mathrm{h} \leq 21$ & $-17 \leq \mathrm{h} \leq 14$ \\
\hline measured refl. & 31022 & 52042 & 24641 \\
\hline unique refl. / $R_{\text {int }}$ & $9024[0.0610]$ & 8142 [0.0643] & $3884[0.0558]$ \\
\hline completeness to $\Theta / \%$ & 99.6 & 100.0 & 99.8 \\
\hline data | res. | param. & $9024|1| 529$ & $8142|0| 472$ & $3884|1| 225$ \\
\hline goodness-of-fit $\left(F^{2}\right)$ & 1.043 & 1.031 & 1.050 \\
\hline$R 1, \omega R 2(I>2 \sigma(I))$ & $0.0339,0.0916$ & $.0355,0.0870$ & $0.0376,0.0841$ \\
\hline$R 1, \omega R 2$ (all data) & $0.0405,0.0937$ & $0.0467,0.0912$ & $0.0481,0.0885$ \\
\hline resid. el. dens. / e $\AA^{-3}$ & $0.656 \mid-0.435$ & $0.631 \mid-0.732$ & $0.596 \mid-0.327$ \\
\hline
\end{tabular}


Table B.2.: Crystal data and refinement details.

\begin{tabular}{|c|c|c|}
\hline compound & $\mathrm{K}\left[\mathrm{L}^{\text {bisnac }} \mathrm{Ni}_{2}(\mathrm{H})_{2}\right]$ & $\mathrm{K}\left[\mathrm{L}^{\text {bisnac }} \mathrm{Ni}_{2}\left(\mu-\eta^{1}: \eta^{1}-\mathrm{CHCPh}\right)\right]$ \\
\hline empirical formula & $\mathrm{C}_{39} \mathrm{H}_{55} \mathrm{KN}_{6} \mathrm{Ni}_{2}$ & $\mathrm{C}_{59} \mathrm{H}_{83} \mathrm{KN}_{6} \mathrm{Ni}_{2} \mathrm{O}_{3}$ \\
\hline formula weight & 764.41 & 1080.83 \\
\hline$T / \mathrm{K}$ & $133(2)$ & $133(2)$ \\
\hline crystal size / mm³ & $0.39 \times 0.26 \times 0.22$ & $0.34 \times 0.20 \times 0.14$ \\
\hline crystal system & monoclinic & triclinic \\
\hline space group & $P 2_{1} / n$ & $P \overline{1}$ \\
\hline$a / \AA$ & $15.7726(3)$ & $12.1168(3)$ \\
\hline$b / \AA$ & $17.7079(3)$ & $12.6383(3)$ \\
\hline$c / \AA$ & $27.8404(5)$ & $40.8254(10)$ \\
\hline$\alpha /^{\circ}$ & 90 & $92.634(2)$ \\
\hline$\beta /{ }^{\circ}$ & $98.8790(10)$ & $97.043(2)$ \\
\hline$\gamma /{ }^{\circ}$ & 90 & $113.936(2)$ \\
\hline$V / \AA^{3}$ & $7682.6(2)$ & $5639.4(3)$ \\
\hline$Z$ & 8 & 4 \\
\hline$\rho / \mathrm{g} \mathrm{cm}^{-3}$ & 1.322 & 1.273 \\
\hline$F(000)$ & 3248 & 2312 \\
\hline$\mu / \mathrm{mm}^{-1}$ & 1.124 & 0.790 \\
\hline$T_{\min } \mid T_{\max }$ & $0.5954 \mid 0.7919$ & $0.8876 \mid 0.7368$ \\
\hline$\Theta$-range / ${ }^{\circ}$ & $1.368-26.735$ & $1.516-25.616$ \\
\hline \multirow[t]{3}{*}{$h k l$-range } & $-19 \leq \mathrm{h} \leq 17$ & $-14 \leq h \leq 14$ \\
\hline & $-22 \leq \mathrm{k} \leq 22$ & $-15 \leq \mathrm{h} \leq 15$ \\
\hline & $-35 \leq \mathrm{I} \leq 35$ & $-49 \leq \mathrm{h} \leq 49$ \\
\hline measured refl. & 82995 & 68586 \\
\hline unique refl. / $R_{\text {int }}$ & $16258[0.0595]$ & 21235 [0.0439] \\
\hline completeness to $\Theta / \%$ & 99.8 & 99.9 \\
\hline data | res. | param. & 16258 | 67 | 925 & 21235 | 3 | 1315 \\
\hline goodness-of-fit $\left(F^{2}\right)$ & 1.003 & 1.021 \\
\hline$R 1, \omega R 2(I>2 \sigma(I))$ & $0.0352,0.0832$ & $0.0406,0.0954$ \\
\hline$R 1, \omega R 2$ (all data) & $0.0471,0.0872$ & $0.0590,0.1011$ \\
\hline resid. el. dens. / e $\AA^{-3}$ & $0.706 \mid-0.348$ & $0.537 \mid-0.406$ \\
\hline
\end{tabular}


Table B.3.: Crystal data and refinement details.

\begin{tabular}{|c|c|c|}
\hline compound & {$\left[L^{\text {bisnac }} \mathrm{Ni}_{2}\left(\mu-\eta^{1}: \eta^{1}-\mathbf{N}_{2} \mathbf{H}_{3}\right)\right]$} & $\mathrm{K}\left[\mathrm{L}^{\text {bisnac }} \mathrm{Ni}_{2}\left(\mu-\eta^{1}: \eta^{1}-\mathrm{N}_{2} \mathrm{H}_{2}\right)\right]$ \\
\hline empirical formula & $\mathrm{C}_{41} \mathrm{H}_{60} \mathrm{~N}_{8} \mathrm{Ni}_{2} \mathrm{O}_{0.50}$ & $\mathrm{C}_{47} \mathrm{H}_{77} \mathrm{KN}_{8} \mathrm{Ni}_{2} \mathrm{O}$ \\
\hline formula weight & 790.39 & 950.70 \\
\hline$T / \mathrm{K}$ & 133(2) & $133(2)$ \\
\hline crystal size $/ \mathrm{mm}^{3}$ & $0.50 \times 0.50 \times 0.32$ & $0.50 \times 0.50 \times 0.15$ \\
\hline crystal system & monoclinic & monoclinic \\
\hline space group & $P 2_{1} / c$ & $P 2_{1} / n$ \\
\hline$a / \AA$ & $17.2013(9)$ & $15.5613(8)$ \\
\hline$b / \AA$ & $14.2468(7)$ & $15.3945(11)$ \\
\hline$c / \AA$ & $17.4667(10)$ & $21.2119(11)$ \\
\hline$\alpha /^{\circ}$ & 90 & 90 \\
\hline$\beta /{ }^{\circ}$ & $112.975(4)$ & $106.260(4)$ \\
\hline$\gamma /{ }^{\circ}$ & 90 & 90 \\
\hline$V / \AA^{3}$ & $3940.9(4)$ & $4878.2(5)$ \\
\hline$Z$ & 4 & 4 \\
\hline$\rho / \mathrm{g} \mathrm{cm}^{-3}$ & 1.332 & 1.294 \\
\hline$F(000)$ & 1688 & 2040 \\
\hline$\mu / \mathrm{mm}^{-1}$ & 0.997 & 0.901 \\
\hline$T_{\min } \mid T_{\max }$ & $0.7449 \mid 0.5259$ & $0.8362 \mid 0.6552$ \\
\hline$\Theta$-range $/^{\circ}$ & $1.910-26.810$ & $1.447-25.768$ \\
\hline \multirow[t]{3}{*}{ hkl-range } & $-21 \leq \mathrm{h} \leq 21$ & $-18 \leq h \leq 18$ \\
\hline & $-18 \leq k \leq 18$ & $-18 \leq h \leq 18$ \\
\hline & $-22 \leq \mathrm{I} \leq 22$ & $-25 \leq \mathrm{h} \leq 25$ \\
\hline measured refl. & 54479 & 52165 \\
\hline unique refl. / $R_{\mathrm{int}}$ & 8355 [0.0799] & $9235[0.1402]$ \\
\hline completeness to $\Theta / \%$ & 100.0 & 100.0 \\
\hline data | res. | param. & $8355|77| 511$ & $9235|87| 606$ \\
\hline goodness-of-fit $\left(F^{2}\right)$ & 0.958 & 1.057 \\
\hline$R 1, \omega R 2(I>2 \sigma(I))$ & $0.0397,0.0929$ & $0.0707,0.1409$ \\
\hline$R 1, \omega R 2$ (all data) & $0.0564,0.0982$ & $0.1161,0.1594$ \\
\hline resid. el. dens. / e $\AA^{-3}$ & $0.715 \mid-0.442$ & $1.042 \mid-0.361$ \\
\hline
\end{tabular}


Table B.4.: Crystal data and refinement details.

\begin{tabular}{|c|c|c|}
\hline compound & {$\left[\mathrm{L}^{\text {bisnac }} \mathrm{Ni}_{2}\left(\mu-\eta^{1}: \eta^{1}-\mathrm{N}_{2} \mathrm{H}_{4}\right)\right] \mathrm{OTf}$} & {$\left[\mathrm{L}^{\text {bisnac }} \mathrm{Ni}_{2}\left(\mu-\mathrm{NH}_{2}\right)\right]$} \\
\hline empirical formula & $\mathrm{C}_{48} \mathrm{H}_{73} \mathrm{~F}_{3} \mathrm{~N}_{8} \mathrm{Ni}_{2} \mathrm{O}_{5} \mathrm{~S}$ & $\mathrm{C}_{39} \mathrm{H}_{55} \mathrm{~N}_{7} \mathrm{Ni}_{2}$ \\
\hline formula weight & 1048.62 & 739.32 \\
\hline$T / \mathrm{K}$ & $133(2)$ & $133(2)$ \\
\hline crystal size $/ \mathrm{mm}^{3}$ & - & $0.25 \times 0.14 \times 0.12$ \\
\hline crystal system & triclinic & monoclinic \\
\hline space group & $P \overline{1}$ & $C 2 / c$ \\
\hline$a / \AA$ & $11.590(2)$ & $18.4847(13)$ \\
\hline$b / \AA$ & $15.133(3)$ & $16.4274(9)$ \\
\hline$c / \AA$ & $15.647(3)$ & $13.6837(9)$ \\
\hline$\alpha /{ }^{\circ}$ & $87.71(3)$ & 90 \\
\hline$\beta / \circ$ & $69.42(3)$ & $117.818(5)$ \\
\hline$\gamma /{ }^{\circ}$ & 77.99(3) & 90 \\
\hline$V / \AA^{3}$ & $2511.3(10)$ & $3674.9(4)$ \\
\hline$Z$ & 2 & 4 \\
\hline$\rho / \mathrm{g} \mathrm{cm}^{-3}$ & 1.387 & 1.336 \\
\hline$F(000)$ & 1112 & 1576 \\
\hline$\mu / \mathrm{mm}^{-1}$ & 0.856 & 1.062 \\
\hline$T_{\min } \mid T_{\max }$ & - & $0.8962 \mid 0.7541$ \\
\hline$\Theta$-range $/{ }^{\circ}$ & $1.919-26.882$ & $1.757-26.859$ \\
\hline \multirow[t]{3}{*}{$h k l$-range } & $-14 \leq \mathrm{h} \leq 14$ & $-23 \leq h \leq 23$ \\
\hline & $-19 \leq k \leq 19$ & $-20 \leq h \leq 20$ \\
\hline & $-8 \leq 1 \leq 19$ & $-17 \leq h \leq 14$ \\
\hline measured refl. & 8859 & 23469 \\
\hline unique refl. / $R_{\text {int }}$ & $8859[-]$ & $3908[0.0660]$ \\
\hline completeness to $\Theta / \%$ & 83.4 & 99.9 \\
\hline data | res. | param. & 8859 | 558 | 617 & $3908|1| 228$ \\
\hline goodness-of-fit $\left(F^{2}\right)$ & 1.188 & 1.134 \\
\hline$R 1, \omega R 2(I>2 \sigma(I))$ & $0.1246,0.3849$ & $0.0516,0.0971$ \\
\hline$R 1, \omega R 2$ (all data) & $0.2151,0.4219$ & $0.0727,0.1035$ \\
\hline resid. el. dens. / e $\AA^{-3}$ & $1.655 \mid-0.707$ & $0.401 \mid-0.401$ \\
\hline
\end{tabular}


Table B.5.: Crystal data and refinement details.

\begin{tabular}{|c|c|c|}
\hline compound & {$\left[L^{\text {bisnac }} \mathrm{Ni}_{2}\left(\mu-\eta^{1}: \eta^{1}-\mathrm{N}_{2} \mathrm{H}\right)\right]$} & {$\left[\mathrm{L}^{\text {bisnac }} \mathrm{Ni}_{2}\left(\mu-\eta^{1}: \eta^{1}-\mathbf{N}_{2}\right)\right]$} \\
\hline empirical formula & $\mathrm{C}_{41} \mathrm{H}_{58} \mathrm{~N}_{8} \mathrm{Ni}_{2} \mathrm{O}_{0.50}$ & $\mathrm{C}_{41} \mathrm{H}_{57} \mathrm{~N}_{8} \mathrm{Ni}_{2} \mathrm{O}_{0.50}$ \\
\hline formula weight & 788.37 & 787.36 \\
\hline$T / \mathrm{K}$ & 133(2) & $133(2)$ \\
\hline crystal size $/ \mathrm{mm}^{3}$ & $0.35 \times 0.33 \times 0.28$ & $0.32 \times 0.30 \times 0.19$ \\
\hline crystal system & monoclinic & monoclinic \\
\hline space group & $P 2_{1} / c$ & $P 2_{1} / c$ \\
\hline$a / \AA$ & $17.2482(5)$ & $17.2064(7)$ \\
\hline$b / \AA$ & $14.1827(4)$ & $14.1966(4)$ \\
\hline$c / \AA$ & $17.4589(5)$ & $17.2998(7)$ \\
\hline$\alpha /^{\circ}$ & 90 & 90 \\
\hline$\beta /{ }^{\circ}$ & $112.742(2)$ & $112.736(3)$ \\
\hline$\gamma /{ }^{\circ}$ & 90 & 90 \\
\hline$V / \AA^{3}$ & $3938.9(2)$ & $3897.5(3)$ \\
\hline$Z$ & 4 & 4 \\
\hline$\rho / \mathrm{g} \mathrm{cm}^{-3}$ & 1.329 & 1.342 \\
\hline$F(000)$ & 1680 & 1676 \\
\hline$\mu / \mathrm{mm}^{-1}$ & 0.997 & 1.008 \\
\hline$T_{\min } \mid T_{\max }$ & $0.7978 \mid 0.7139$ & $0.8572 \mid 0.7129$ \\
\hline$\Theta$-range $/^{\circ}$ & $1.913-26.789$ & $1.283-26.786$ \\
\hline \multirow[t]{3}{*}{ hkl-range } & $-21 \leq \mathrm{h} \leq 21$ & $-20 \leq h \leq 21$ \\
\hline & $-17 \leq \mathrm{k} \leq 17$ & $-17 \leq h \leq 17$ \\
\hline & $-22 \leq \mathrm{I} \leq 22$ & $-21 \leq \mathrm{h} \leq 21$ \\
\hline measured refl. & 52420 & 49138 \\
\hline unique refl. / $R_{\text {int }}$ & $8345[0.0471]$ & $8281[0.0511]$ \\
\hline completeness to $\Theta / \%$ & 100.0 & 100.0 \\
\hline data | res. | param. & $8345 \mid 152$ | 519 & $8281|7| 508$ \\
\hline goodness-of-fit $\left(F^{2}\right)$ & 1.031 & 1.023 \\
\hline$R 1, \omega R 2(I>2 \sigma(I))$ & $0.0356,0.0783$ & $0.0461,0.1069$ \\
\hline$R 1, \omega R 2$ (all data) & $0.0497,0.0827$ & $0.0644,0.1143$ \\
\hline resid. el. dens. / e $\AA^{-3}$ & $0.567 \mid-0.262$ & $1.164 \mid-0.293$ \\
\hline
\end{tabular}




\section{C}

\section{Part One}

\section{C.1. Analysis of Isotopically Labeled [ $\left.\mathrm{L}^{\mathrm{pyr}} \mathrm{Ni}_{2}(\mu-\mathrm{OH})\right]$}

The $\mathrm{H} / \mathrm{D}$ exchange can be well observed via IR spectroscopy. Upon exchange with $\mathrm{D}_{2} \mathrm{O}$ the stretching vibration of the bridging $\mathrm{OH}$ group $\left(3603 \mathrm{~cm}^{-1}\right)$ slowly disappears while a new signal emerges at $2664 \mathrm{~cm}^{-1}$ (Figure C.1). The corresponding wavenumbers can be calculated using reduced masses $(\mu)$ of the OH and OD group respectively. The vibration equation for two connected masses $\mathrm{A}$ and $\mathrm{B}$ is shown in C.1 with $f_{A B}$ as force constant.

$$
\tilde{\nu}_{A B}=\frac{1}{2 \pi} \sqrt{\frac{f_{A B}}{\mu_{A B}}}
$$

Using C.1 for both $\mathrm{OH}$ and $\mathrm{OD}$ and forming the quotient of resulting wavenumbers $\frac{\tilde{\nu}_{O H}}{\tilde{\nu}_{O D}}$ yields an equation shown in C.2

$$
\frac{\tilde{\nu}_{O H}}{\tilde{\nu}_{O D}}=\sqrt{\frac{f_{O H} \mu_{O D}}{f_{O D} \mu_{O H}}}
$$

By assuming the force constants $f_{\mathrm{OH}}$ and $f_{\mathrm{OD}}$ to be of same value, C.2 is simplified to give C.3.

$$
\frac{\tilde{\nu}_{O H}}{\tilde{\nu}_{O D}}=\sqrt{\frac{\mu_{O D}}{\mu_{O H}}}
$$


After conversion of $\mathrm{C} .3 \tilde{\nu}_{O D}$ can be determined:

$$
\tilde{\nu}_{O D}=\tilde{\nu}_{O H} \sqrt{\frac{\mu_{O H}}{\mu_{O D}}}
$$

With the reduced masses $\mu_{O H}=0.948$ and $\mu_{O D}=1.789$ equation C.4 yields an theoretical OD wavenumber of about $\tilde{\nu}_{O D}=2625 \mathrm{~cm}^{-1}$. This is in accordance with the experimental value. For the ${ }^{17} \mathrm{O}$-labeled hydroxo group a wavenumber of $\tilde{\nu}_{17} \mathrm{OH}=3596 \mathrm{~cm}^{-1}$ (with $\left.\mu_{17} \mathrm{OH}=0.948\right)$ was calculated. Because of the very little mass difference of the ${ }^{16} \mathrm{O}$ and ${ }^{17} \mathrm{O}$ nucleus no distinct peak was observed for the ${ }^{17} \mathrm{OH}$ vibration rather than a superposition with the ${ }^{16} \mathrm{OH}$ band (Figure C.1).
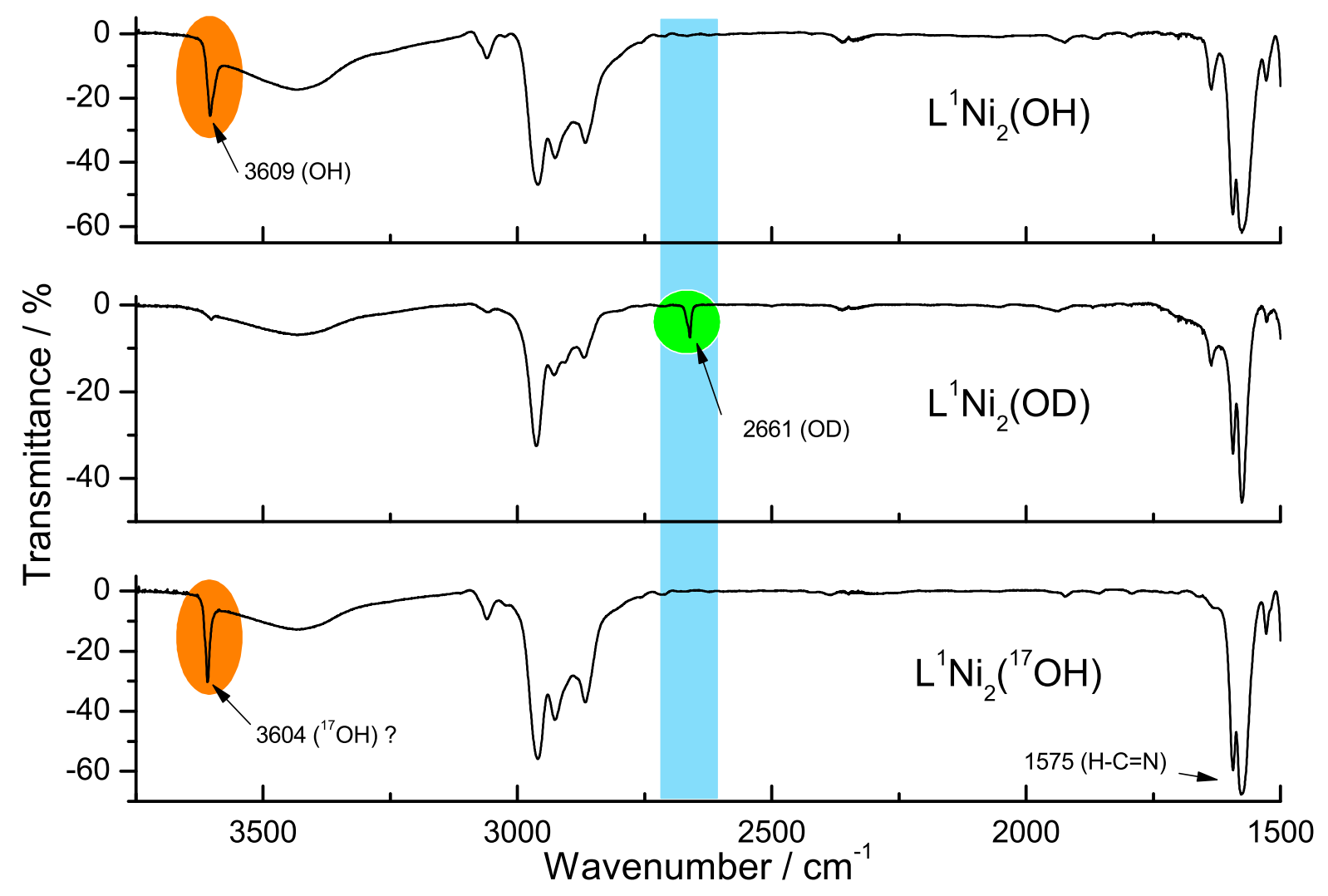

Figure C.1.: IR spectra $(\mathrm{KBr})$ of [ $\left.\mathrm{L}^{\mathrm{pyr}} \mathrm{Ni}_{2}(\mu-\mathrm{OH})\right]($ top $)$, [ $\left.\mathrm{L}^{\text {pyr }} \mathrm{Ni}_{2}(\mu-\mathrm{OD})\right]$ (middle) and [ $\left.\mathrm{L}^{\mathrm{pyr}} \mathrm{Ni}_{2}\left(\mu-{ }^{17} \mathrm{OH}\right)\right]$ (bottom). 

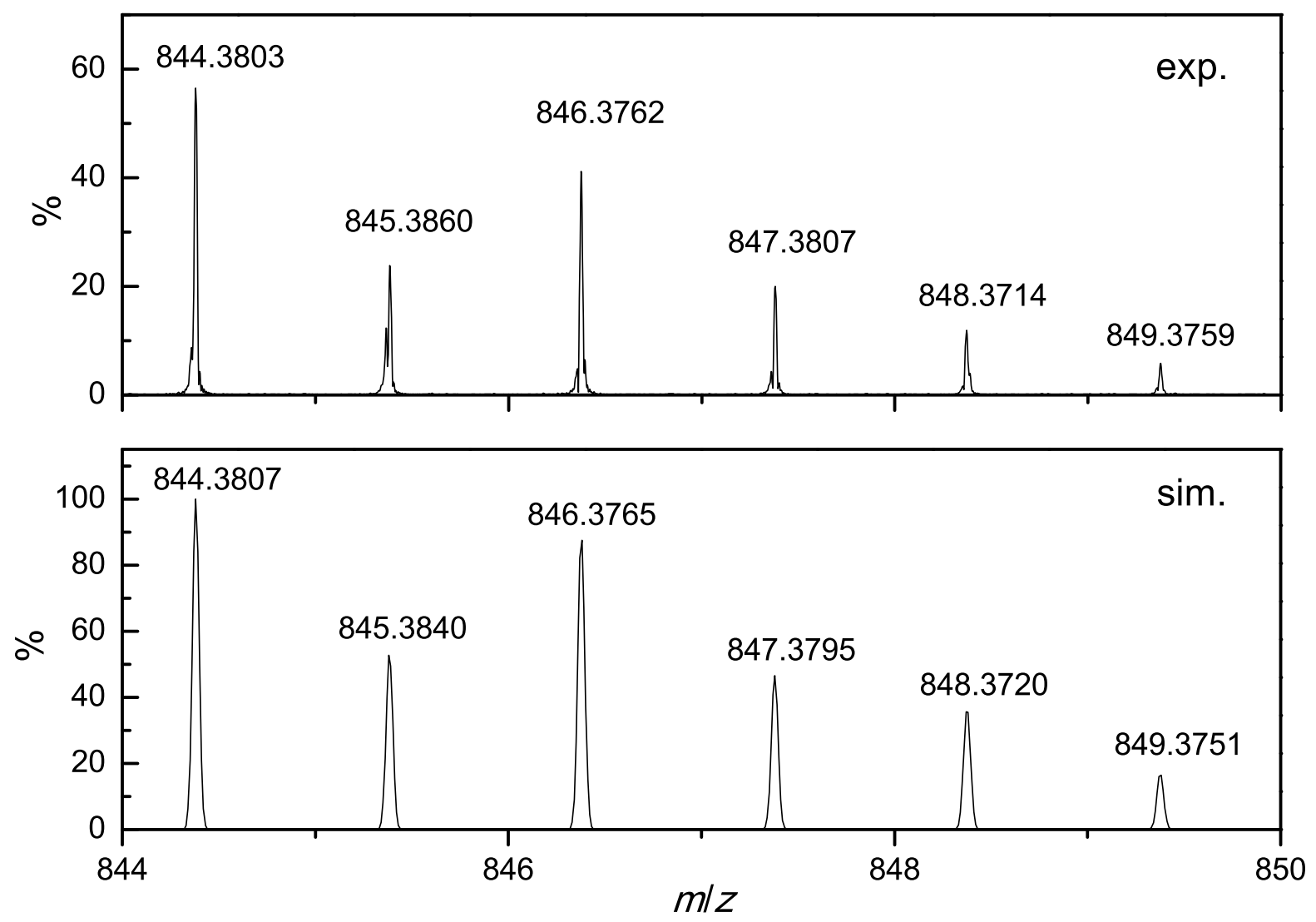

Figure C.2.: HRMS (ESI) spectrum of [ $\left.{ }^{\text {pyr }} \mathrm{Ni}_{2}\left(\mu-{ }^{17} \mathrm{OH}\right)\right]$. 


\section{C.2. Additional Plots}
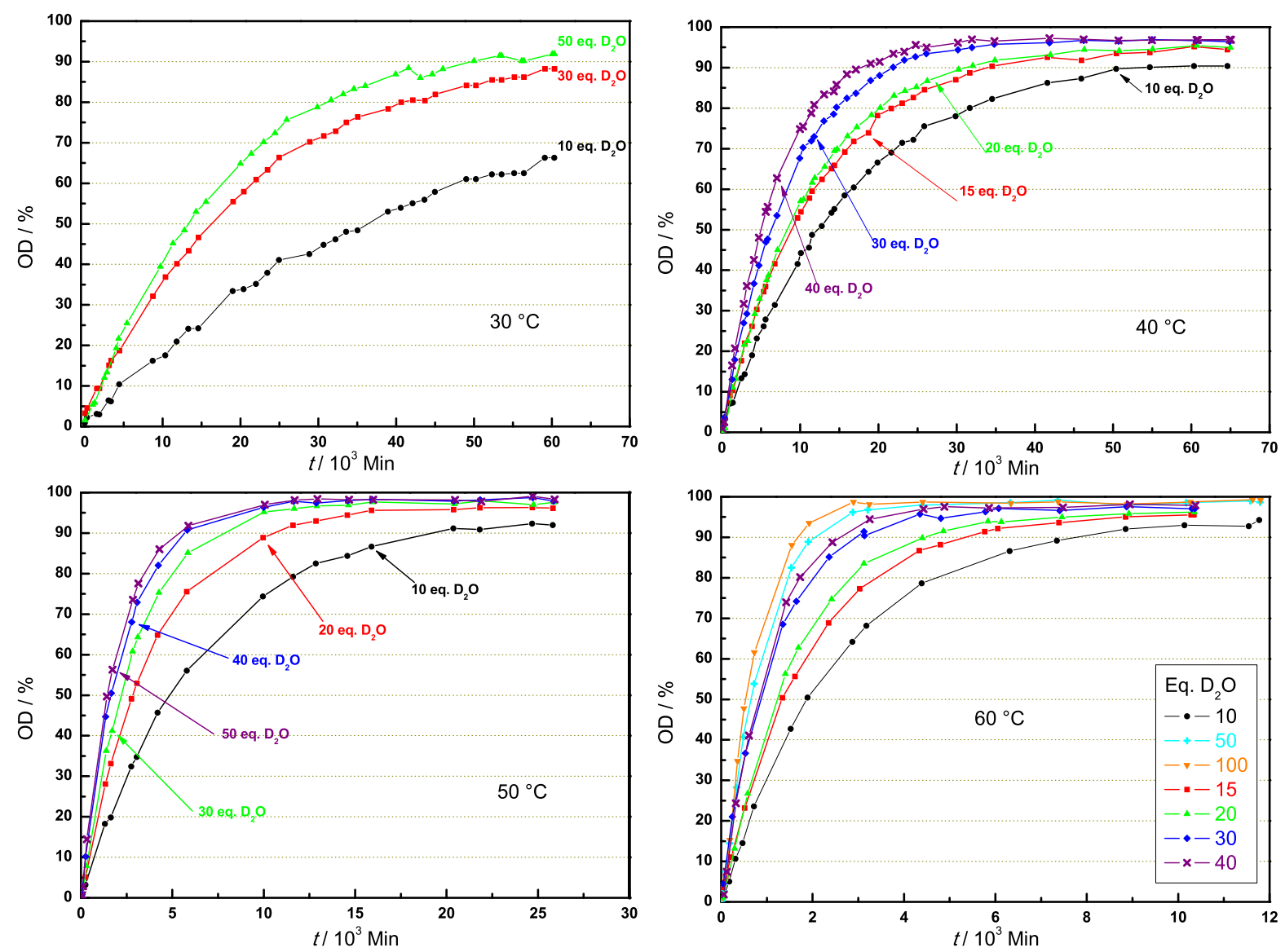

Figure C.3.: $\mathrm{H} / \mathrm{D}$ exchange of $\left[\mathrm{L}^{\text {pyr }} \mathrm{Ni}_{2}(\mu-\mathrm{OH})\right]$ with excess $\mathrm{D}_{2} \mathrm{O}$ in $\mathrm{THF}-\mathrm{d}_{8}$ measured at $30{ }^{\circ} \mathrm{C}$ (upper left), $40^{\circ} \mathrm{C}$ (upper right), $50^{\circ} \mathrm{C}$ (bottom left) and $60^{\circ} \mathrm{C}$ (bottom right) over time. 

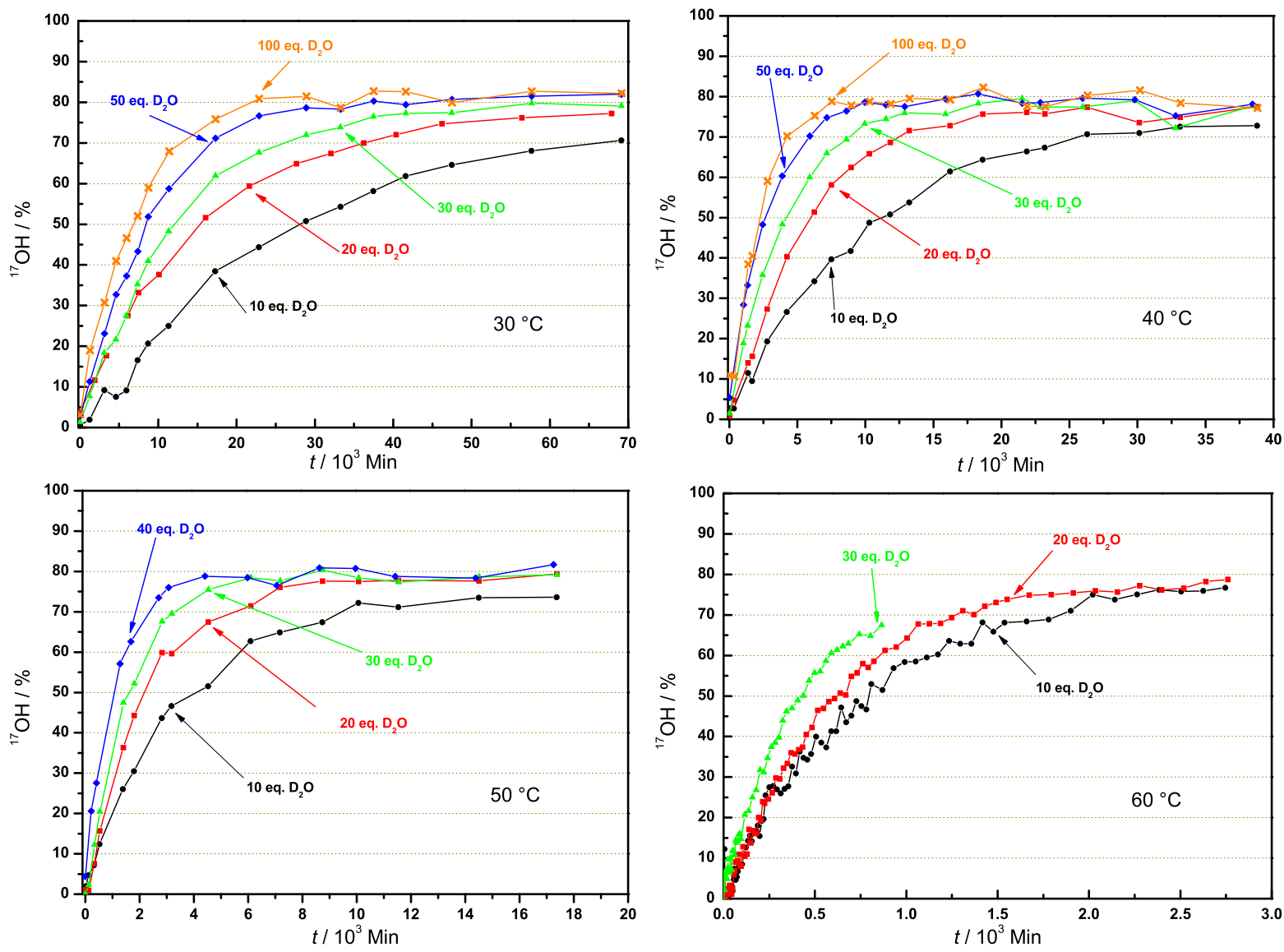

Figure C.4.: $\mathrm{OH}$ exchange of [ $\left.\mathrm{L}^{\text {pyr }} \mathrm{Ni}_{2}(\mu-\mathrm{OH})\right]$ with excess $\mathrm{H}_{2}{ }^{17} \mathrm{O}$ in THF- $\mathrm{d}_{8}$ measured at $30{ }^{\circ} \mathrm{C}$ (upper left), $40^{\circ} \mathrm{C}$ (upper right), $50^{\circ} \mathrm{C}$ (bottom left) and $60^{\circ} \mathrm{C}$ (bottom right) over time. 


\section{Determination of $k$}
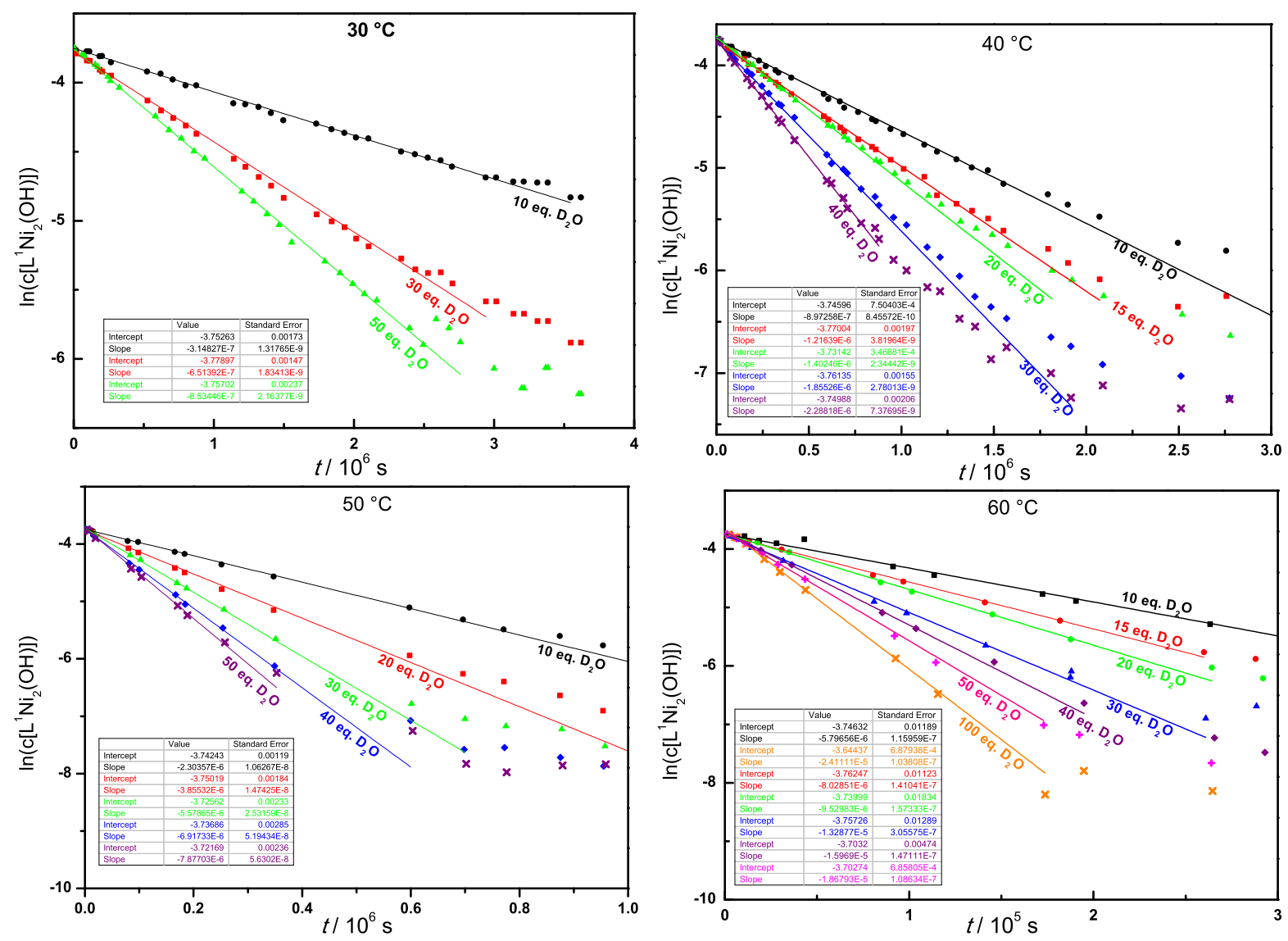

Figure C.5.: Halflogarithmic plot of decreasing concentration $\left[\mathrm{L}^{\mathrm{pyr}} \mathrm{Ni}_{2}(\mu-\mathrm{OH})\right]$ vs time for $30{ }^{\circ} \mathrm{C}$ (upper left), $40^{\circ} \mathrm{C}$ (upper right), $50^{\circ} \mathrm{C}$ (bottom left) and $60^{\circ} \mathrm{C}$ (bottom right) for $\mathrm{H} / \mathrm{D}$ exchange with $\mathrm{D}_{2} \mathrm{O}$. 

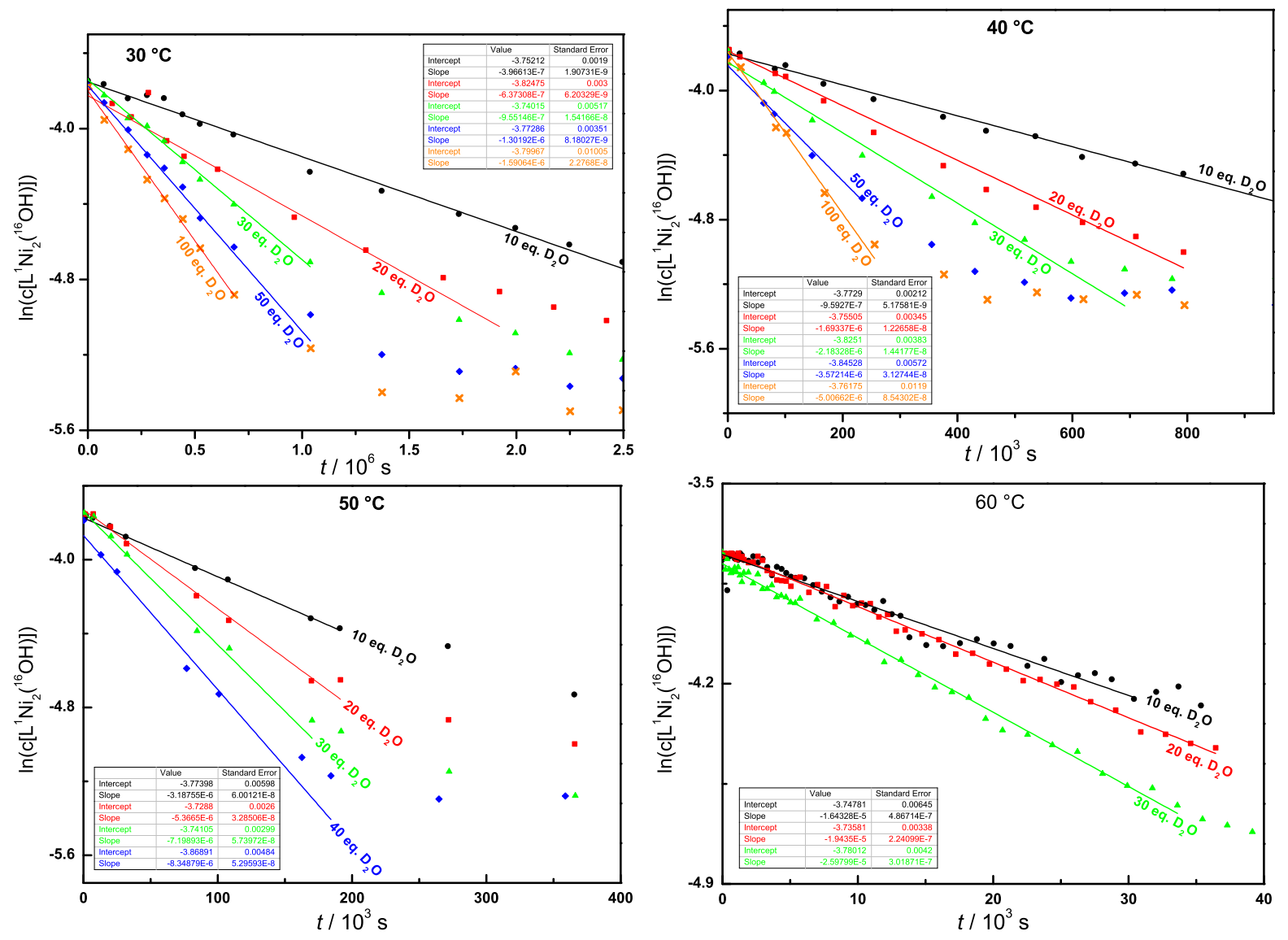

Figure C.6.: Halflogarithmic plot of decreasing concentration $\left[\mathrm{L}^{\text {pyr }} \mathrm{Ni}_{2}(\mu-\mathrm{OH})\right]$ vs time for $30{ }^{\circ} \mathrm{C}$ (upper left), $40^{\circ} \mathrm{C}$ (upper right), $50{ }^{\circ} \mathrm{C}$ (bottom left) and $60^{\circ} \mathrm{C}$ (bottom right) for $\mathrm{OH}$ exchange with $\mathrm{H}_{2}{ }^{17} \mathrm{O}$. 


\section{Determination of $k_{1}$ and $k_{2}$}
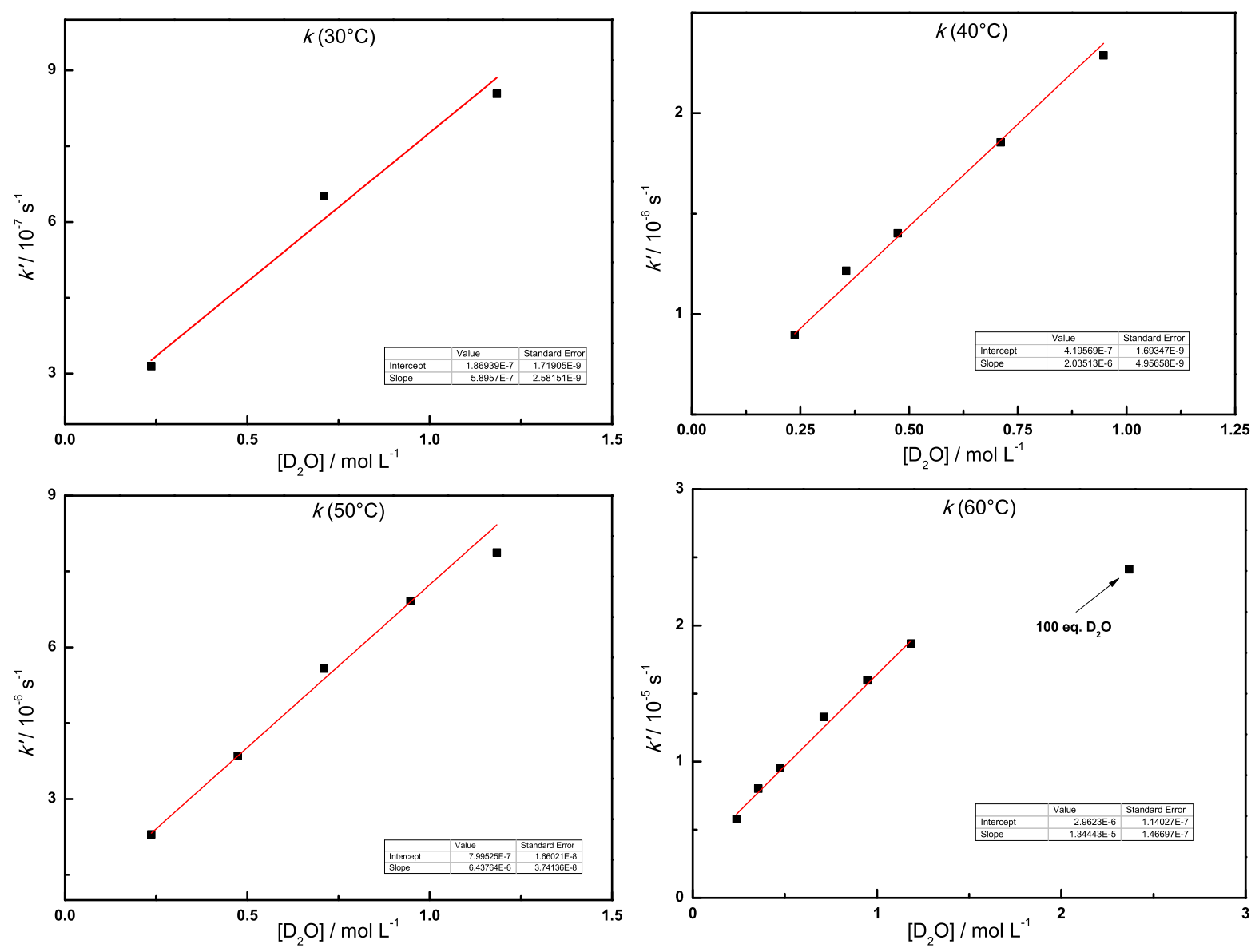

Figure C.7.: Plot of the experimental rate constant $k$ versus the excess concentration of $\mathrm{D}_{2} \mathrm{O}$ at $30{ }^{\circ} \mathrm{C}$ (upper left), $40{ }^{\circ} \mathrm{C}$ (upper right), $50{ }^{\circ} \mathrm{C}$ (bottom left) and $60^{\circ} \mathrm{C}$ (bottom right) for the H/D exchange. The data point representing 100 equivalents of $\mathrm{D}_{2} \mathrm{O}$ at $60^{\circ} \mathrm{C}$ was excluded because the saturation was already reached. 

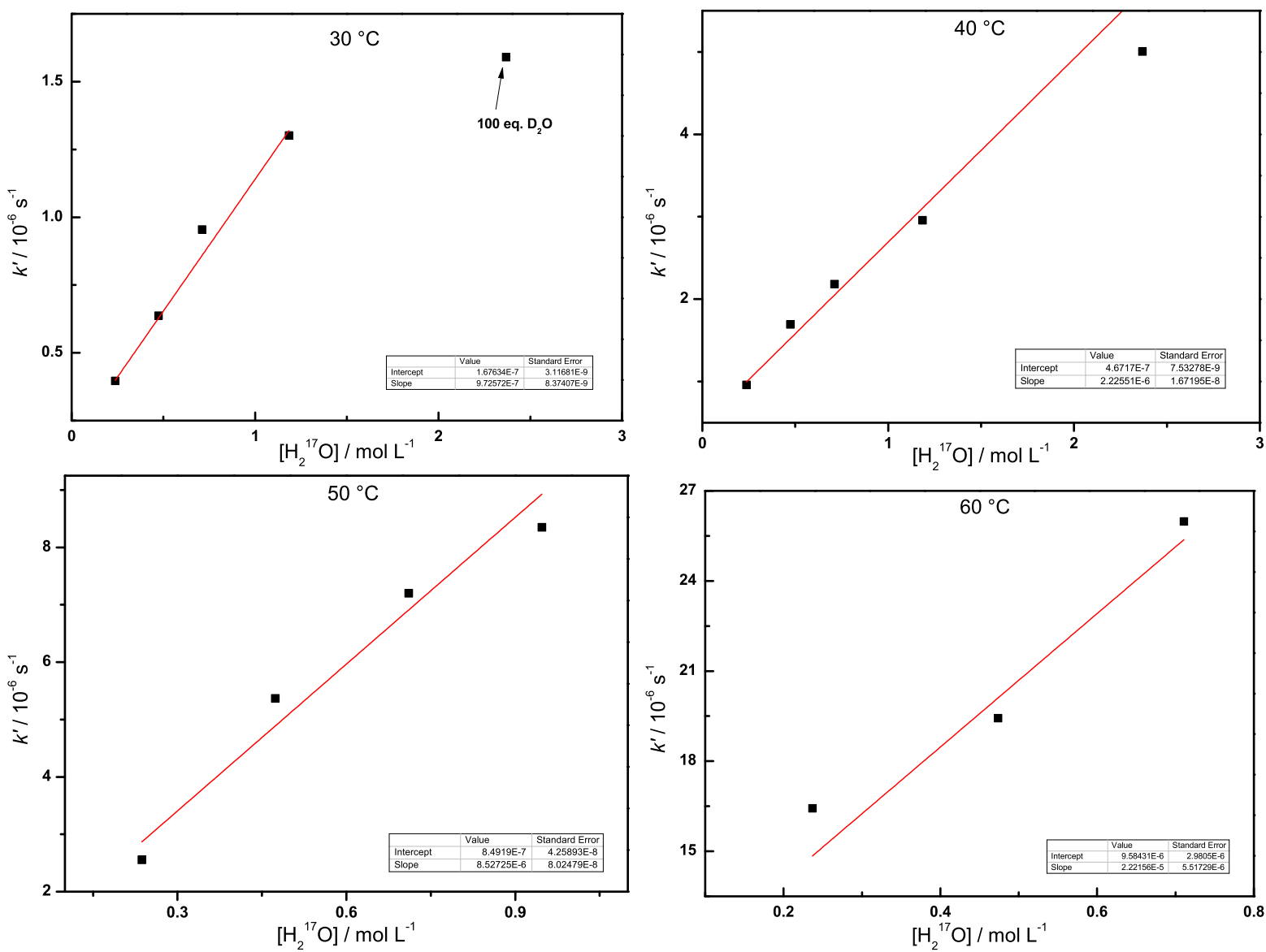

Figure C.8.: Plot of the experimental rate constant $k$ versus the excess concentration of $\mathrm{H}_{2}{ }^{17} \mathrm{O}$ at $30{ }^{\circ} \mathrm{C}$ (upper left), $40{ }^{\circ} \mathrm{C}$ (upper right), $50{ }^{\circ} \mathrm{C}$ (bottom left) and $60^{\circ} \mathrm{C}$ (bottom right) for the $\mathrm{OH}$ exchange. The data point representing 100 equivalents of $\mathrm{H}_{2}{ }^{17} \mathrm{O}$ at $30{ }^{\circ} \mathrm{C}$ was excluded because the saturation was already reached. 

Part Two 


\section{D.1. Gas Drying Procedure}

Exchange experiments involving $\mathrm{D}_{2}$ and $\mathrm{H}_{2}$ required removal of moisture. A cooling trap device with liquid $\mathrm{N}_{2}$ as a cooling medium was used Scheme D.1. The gas was transferred through the two cooling traps to freeze the moisture, ${ }^{\ddagger}$ before passing it over the Sicapent ${ }^{\circledR}$ drying column.

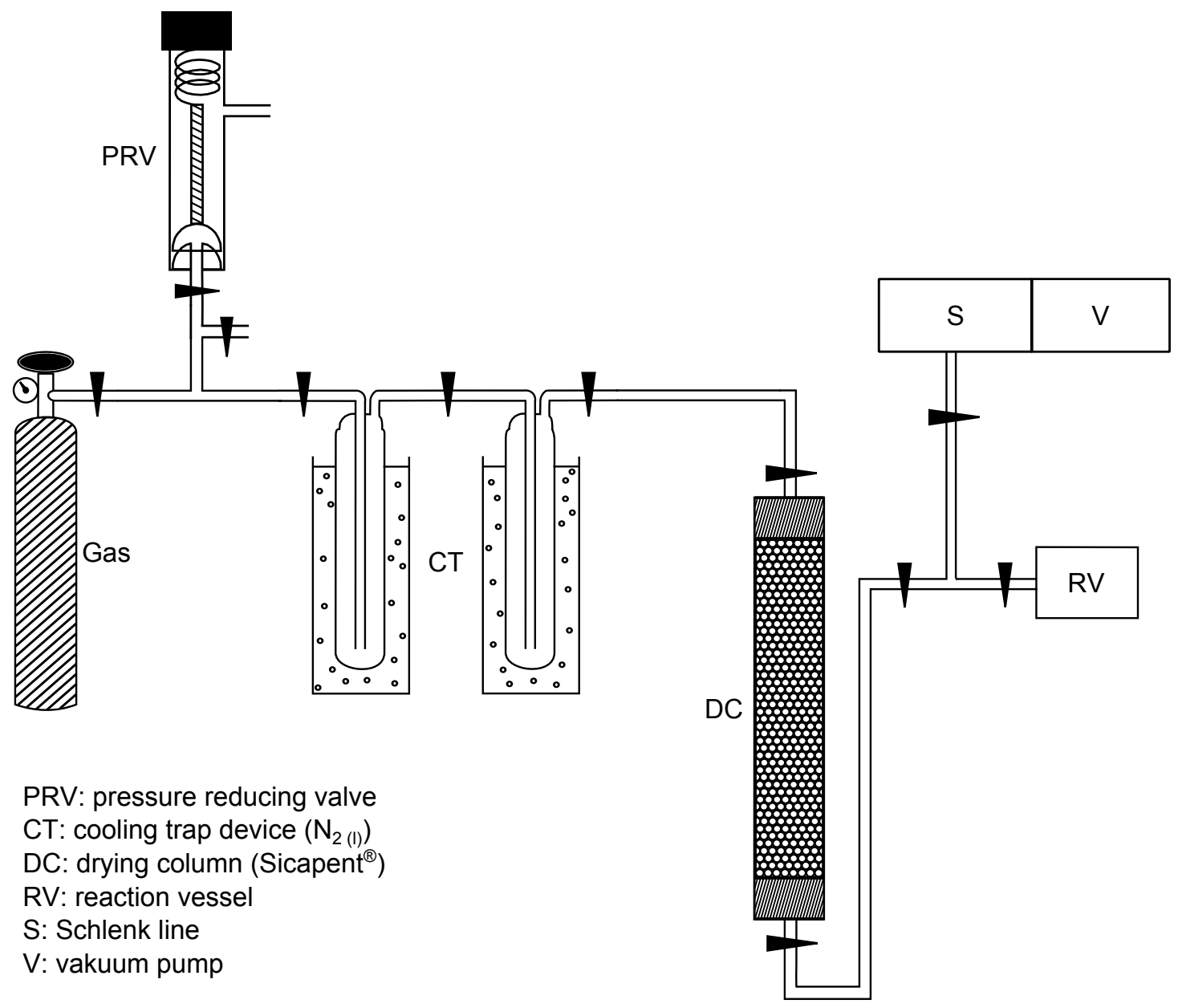

Scheme D.1: Experimental setup for the gas drying procedure.

$\ddagger$ Particular caution is recommended when the cooling traps are removed after the experiment. The residual amount of cooled gas expands quickly after warming! 


\section{D.2. Investigation of the Exchange of $\mathrm{K}\left[\mathrm{L}^{\text {bisnac }} \mathrm{Ni}_{2}(\mathrm{H})_{2}\right]$ with Parahydrogen}

\section{Motivation}

The previously obtained results for the exchange of $\mathbf{K}\left[\mathbf{L}^{\text {bisnac }} \mathbf{N i}_{\mathbf{2}}(\mathbf{H})_{\mathbf{2}}\right]$ with $\mathrm{D}_{2}$ and $\left[\mathbf{L}^{\text {bisnac }} \mathbf{N i}_{\mathbf{2}}(\mathbf{D})_{2}\right]$ with $\mathrm{H}_{2}$ respectively, indicated the direct pairwise exchange of dihydrogen with the $\mathrm{Ni}-\mathrm{H}$ hydrogen atoms (s. section 4.4). In order to confirm the mechanistic pathway an exchange with parahydrogen can be potentially useful (5.4.3).

However, substitution of the two Ni-H hydrogen atoms with one molecule of parahydrogen should not yield a PHIP in the ${ }^{1} \mathrm{H}$ NMR experiment for several reasons: firstly, the singlet symmetry of transferred parahydrogen is not broken because of the complex symmetry. As a consequence, the hydride resonance of $\left.\mathbf{K}\left[\mathbf{L}^{\text {bisnac }} \mathbf{N i}_{\mathbf{2}}(\mathbf{H})_{2}\right)\right]$ (i.e. the resonance at $\left.\delta\left\{{ }^{1} \mathrm{H}\right\}=-24.17 \mathrm{ppm}\right)$ is retained in the ${ }^{1} \mathrm{H}$ NMR experiment.

Despite the fact that the exchange process cannot be investigated by PHIP an indirect method was considered which is described in Scheme D.2. Since parahydrogen is not observable in the NMR due to its singlet nuclear spin state only the residual amount of orthohydrogen yields a signal. A pairwise exchange of Ni-H hydrogen atoms with parahydrogen generates a hydride complex containing both parahydrogen atoms $\left(\mathbf{K}\left[\mathbf{L}^{\text {bisnac }} \mathbf{N i}_{\mathbf{2}}\left(\mathbf{H}^{*}\right)_{\mathbf{2}}\right]\right)$ while dihydrogen in the thermal state is released. In the next step, an exchange can likely occur with another parahydrogen molecule. This time, the $\mathrm{Ni}-\mathrm{H}^{*}$ which originated from the formerly incorporated parahydrogen are released from the system, which then results in the reestablishment of the singlet nuclear spin alignment $\left(p-\mathrm{H}_{2}\right)$. As a consequence, during the ${ }^{1} \mathrm{H}$ NMR experiment the $\mathrm{H}_{2}$ resonance, which is correlated to orthohydrogen, is not expected to raise further during this process, provided that parahydrogen remains reasonably stable under the experimental conditions.

On the contrary a two step exchange process with parahydrogen is associated with a scrambling of the hydrogen molecule $\left(\mathrm{H}^{*} \mathrm{H}\right)$. Therefore, its singlet nuclear spin alignment is broken and the state of thermal equilibrium (3:1 ratio between ortho- and parahydrogen) in the dihydrogen gas would be eventually restored. This leads to a substantial increase of orthohydrogen and the associated growth of the $\mathrm{H}_{2}$ resonance in the spectrum.

\section{Experimental preparations}

Since the exchange of $\mathbf{K}\left[\mathbf{L}^{\text {bisnac }} \mathbf{N i}_{\mathbf{2}}(\mathbf{H})_{\mathbf{2}}\right]$ with para $-\mathrm{H}_{2}$ is dynamic, both the PASADENA and the ALTADENA protocols were suitable for the investigations. To determine the rate of conversion of the used parahydrogen back to orthohydrogen in solution, a blank degassed NMR sample of THF-D 8 was loaded with two atmospheres of freshly synthesized 


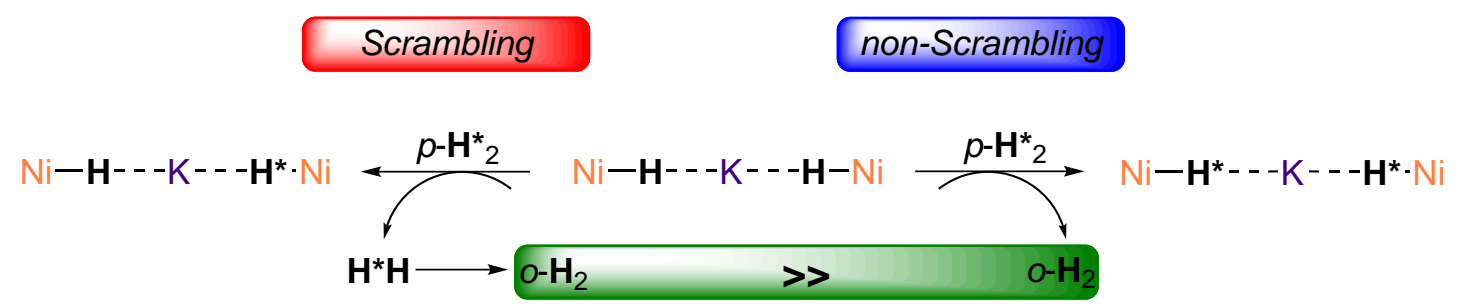

Scheme D.2: Illustration of the hydride exchange in $K\left[L^{\text {bisnac }} \mathbf{N i}_{2}(\mathbf{H})_{2}\right]$ with parahydrogen $\left(\mathrm{H}_{2}^{*}\right)$. During a scrambling process (left pathway) large amounts of parahydrogen are rapidly converted to orthohydrogen due to a dynamic exchange process, while a non-scrambling process only yields one equivalent of orthohydrogen according to the incipient release of $\mathrm{H}_{2}$ from the complex.

para $-\mathrm{H}_{2}$ gas $\left(\right.$ para $\mathrm{H}_{2} \geqslant 92 \%$ at $\left.36 \mathrm{~K}\right)$. The sample tube was shaken, transferred into the NMR spectrometer and monitored by ${ }^{1} \mathrm{H}$ NMR spectroscopy for 18 hours. Figure D.1 illustrates the progression of the orthohydrogen signal intensity at $4.53 \mathrm{ppm}$ over the course of time. As a benchmark for total ortho- $\mathrm{H}_{2}$ gas saturation of the solvent another degassed sample of $\mathrm{THF}_{-} \mathrm{D}_{8}$ loaded with two atmospheres of dihydrogen (in the thermal equilibrium state) was prepared and a ${ }^{1} \mathrm{H}$ NMR spectrum measured. In order to determine the conversion percentage, the absolute integral for the ortho-dihydrogen singlet resonance was measured and all integrals set into ratio to the benchmark integral which was set to $100 \%$. As can be seen the integral increases slowly over time according to the expected slow conversion of para $-\mathrm{H}_{2}$ back to ortho- $\mathrm{H}_{2}$. Furthermore, the plot shows that the starting amount of $o-\mathrm{H}_{2}$ in the sample corresponds to around $7 \%$. This finding well confirms the estimated amount of the freshly generated para- $\mathrm{H}_{2}(\geq 92 \%)$. The very slow loss of hyperpolarization proved a suitable stability of $p a r a-\mathrm{H}_{2}$ in $\mathrm{THF}-\mathrm{D}_{8}$, thus allowing for further experiments.

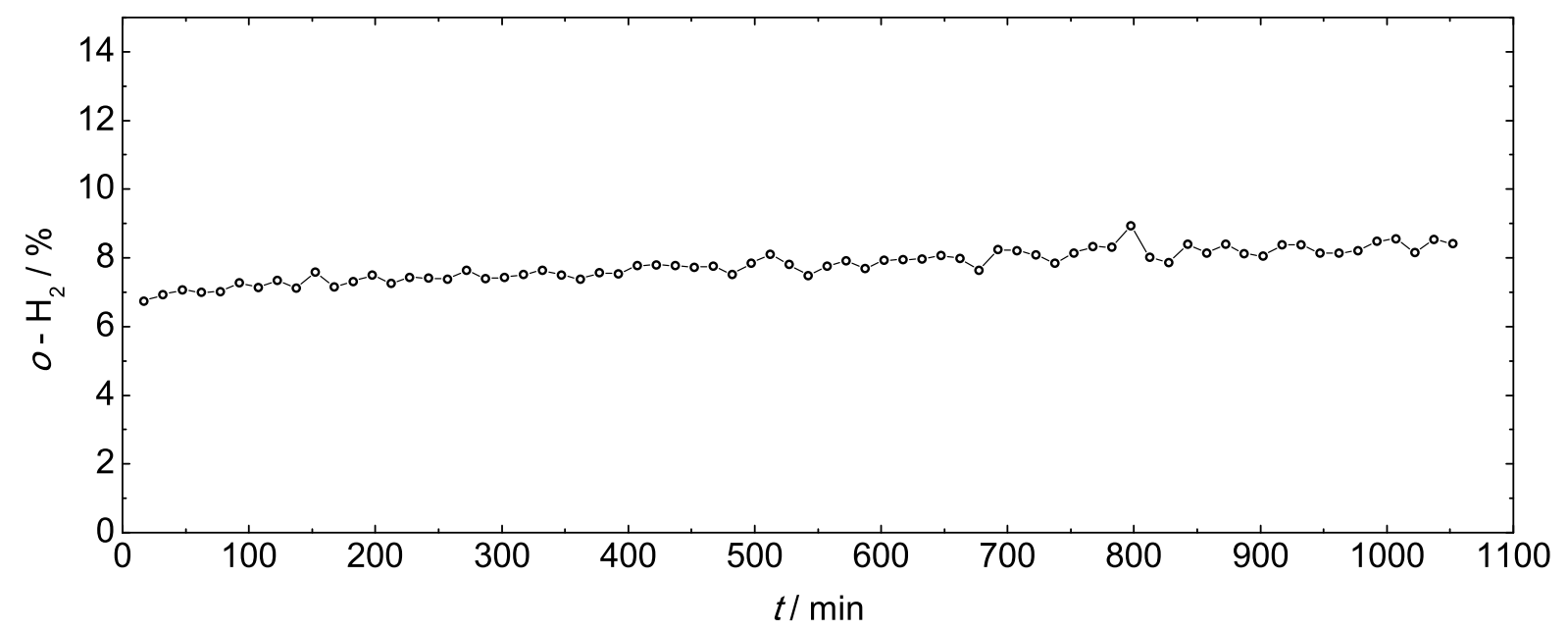

Figure D.1.: Increase of ortho- $\mathrm{H}_{2}$ in a sample of THF- $\mathrm{D}_{8}$ over 18 hours. The data was aquired by ${ }^{1} \mathrm{H}$ NMR spectroscopic monitoring. The depicted ratio was determined by measurement of absolute integrals of the dihydrogen signal at $4.53 \mathrm{ppm}$ adjusted to the absolute integral of the benchmark (determined by a seperate experiment with a THF- $\mathrm{D}_{8}$ solvent sample loaded with 2 atmospheres of dihydrogen gas, set to $100 \%)$. 


\section{Exchange of $\mathrm{K}\left[\mathrm{L}^{\text {bisnac }} \mathrm{Ni}_{2}(\mathrm{H})_{2}\right]$ with parahydrogen}

An experiment was conducted with a threefold pump-freeze-thaw degassed NMR sample of $\mathbf{K}\left[\mathbf{L}^{\text {bisnac }} \mathbf{N i}_{\mathbf{2}}(\mathbf{H})_{2}\right]$ dissolved in $\mathrm{THF}-\mathrm{D}_{8}$, which was loaded with para $-\mathrm{H}_{2}$ (2 atm.). After addition of parahydrogen the sample was vigorously shaken and transferred into the spectrometer. Single fid's were then collected in an intervall of 15 minutes (at the initial phase a shorter intervall was used). Three selected NMR spectra from the screening are illustrated in Figure D.2. The top spectrum (a) is associated with the degassed sample before the addition of the gas. After parahydrogen was added to the sample the hydride resonance at $-24.17 \mathrm{ppm}$ (O) slightly decreased, which is observed in spectrum (b). Furthermore, the addition of parahydrogen led to an immediate color change from orange to yellow. The third spectrum represents the last measurement after a total time of 18 hours (c). In addition to the formation of small amounts of $\left[\mathbf{L}^{\text {bisnac }} \mathbf{N i}_{2}(\mu-\mathrm{OH})\right](廿)$ and marginal amounts of a hydroxo-hydride intermediate $\left(\mathbf{K}\left[\mathbf{L}^{\text {bisnac }} \mathbf{N i}_{\mathbf{2}}(\mathbf{O H})(\mathbf{H})\right], \delta\left\{{ }^{1} \mathrm{H}_{\mathrm{H}}\right\}\right.$ $=-26 \mathrm{ppm}$ and $\left.\delta\left\{{ }^{1} \mathrm{H}_{\mathrm{OH}}\right\}=-2 \mathrm{ppm}\right)^{\dagger}$ no major changes were observed.

In order to determine the particular fraction of complexes formed during the cause of the reaction the characteristic resonances of the pyrazolate-backbone protons were used and the corresponding singlet signals for all pyrazolate based complex species merged to one absolute integral. This absolute integral equals the integral which was determined from the untreated sample as shown in spectrum (a) of Figure D.2. It remained reasonably stable as well during the whole course of the reaction and thus was suitable as a benchmark. By setting the absolute integrals of the characteristic resonances of each complex into ratio to this benchmark integral, the fraction of the specific compound present in the sample at a given time was calculated.

The amount of orthohydrogen in the sample was determined using a benchmark resonance derived from a measurement of a $\mathrm{THF}-\mathrm{D}_{8}$ sample, which was loaded with two atmospheres of $\mathrm{H}_{2}$. By using the absolute integral of both signals the percentage of orthohydrogen was calculated. An excerpt of Figure D.2 (Figure D.3) shows the development of the dihydrogen resonance $(\boldsymbol{\square})$ after addition of prepared parahydrogen according to the residual amounts of orthohydrogen (spectrum (b) of Figure D.3). The grey line in spectrum (c) represents the benchmark of the $\mathrm{H}_{2}$ saturated sample.

The plot shown in Figure D.4 describes the temporal course of performed measurements. It can be seen that the untreated NMR sample contained $95 \%$ of the dihydride compound and trace amounts of the hydroxo bridged complex. After addition of one atmosphere of parahydrogen, followed by mixing, rapid transfer into the spectrometer and repeated one scan measurements, this amount dropped to a value of $87 \%$. After a short time a stable

†The observed resonance corresponds to a hydroxo-hydride complex, which forms with the dihydride complex in the presence of water. Detailed studies associated with this compound are described in chapter 4 . 
value for $\mathbf{K}\left[\mathbf{L}^{\text {bisnac }} \mathbf{N i}_{\mathbf{2}}(\mathbf{H})_{\mathbf{2}}\right]$ of $\approx 80 \%$ was reached. This is explainable by the formation of sideproducts, which are mainly correlated to reactions with moisture $\left(\left[\mathbf{L}^{\text {bisnac }} \mathbf{N i}_{2}(\mu-\right.\right.$ $\left.\mathrm{OH})], \mathbf{K}\left[\mathrm{L}^{\text {bisnac }} \mathrm{Ni}_{2}(\mathrm{OH})(\mathrm{H})\right]\right)$. The slow increase of $\left[\mathrm{L}^{\text {bisnac }} \mathrm{Ni}_{2}(\mu-\mathrm{OH})\right]$ is indicated in the plot ( + in Figure D.4).

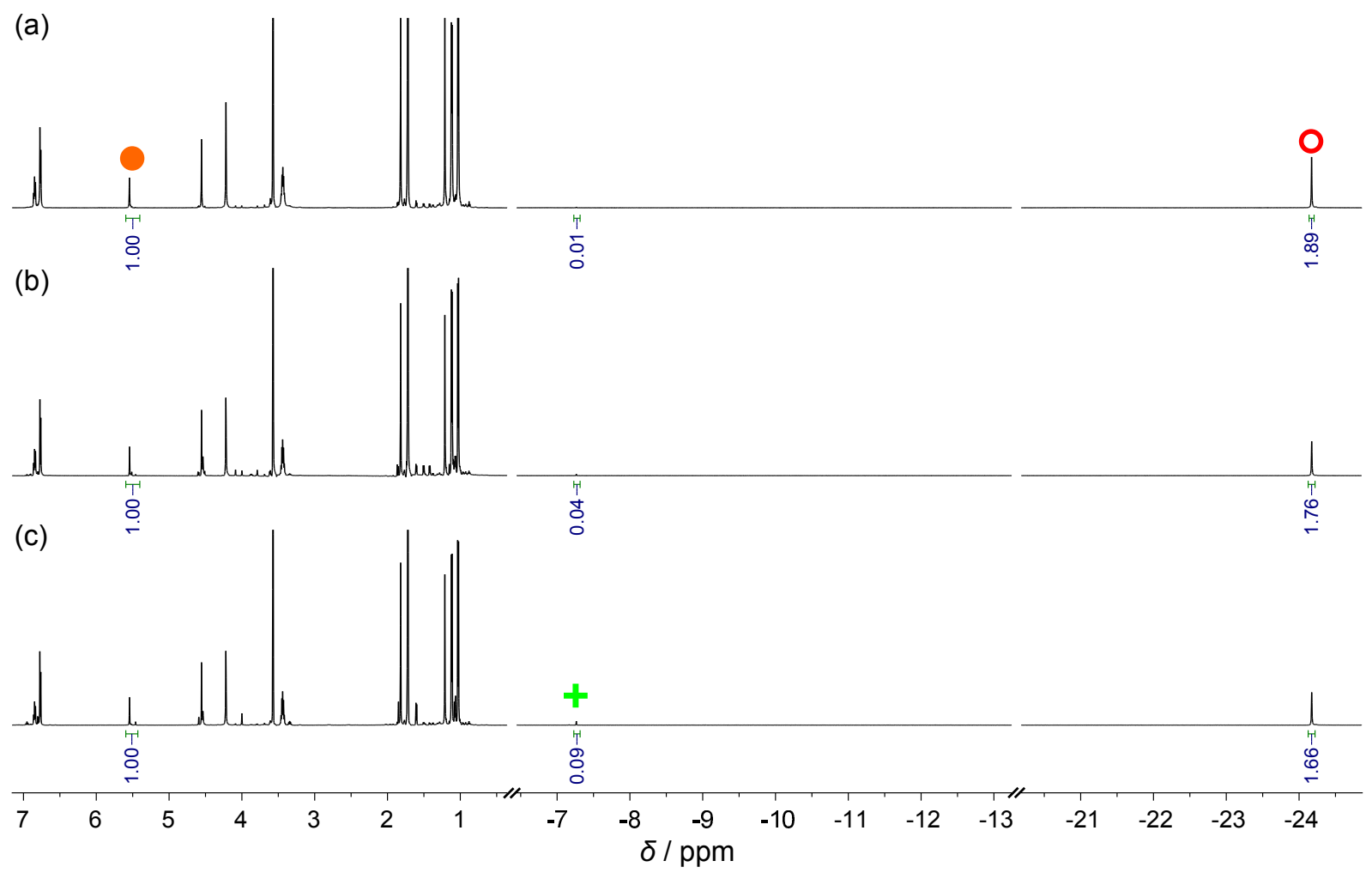

Figure D.2.: Selected ${ }^{1} \mathrm{H}$ NMR spectra of the monitored exchange experiment of $K\left[\mathrm{~L}^{\text {bisnac }} \mathrm{Ni}_{\mathbf{2}}(\mathbf{H})_{2}\right]$ with parahydrogen (2 atm.). (a) The untreated, degassed sample of $K\left[L^{\text {bisnac }} \mathrm{Ni}_{2}(\mathbf{H})_{2}\right]$. (b) The spectrum recorded 30 seconds after the initiation of the exchange with added parahydrogen. (c) The last recorded spectrum after 18 hours. The pyrazolate backbone hydrogen resonances of complex associated species $(\bullet)$, the hydride resonance $(0)$ and the characteristic resonance of the main side product $\left[\mathrm{L}^{\text {bisnac }} \mathrm{Ni}_{2}(\mathrm{OH})\right](+)$ are indicated.

Moreover, the fraction of orthohydrogen ( $\boldsymbol{\square}$ ) rapidly raised to a maximum of $120 \%$ (according to the reference) and afterwards stabilized at $110 \%$.

Surprisingly, this indicates the rapid loss of hyperpolarization in parahydrogen. The increase of $\mathrm{H}_{2}$ beyond $100 \%$, which represents the full conversion of parahydrogen to the thermal equilibrium state is explainable by formation of non-hydride side-products. In particular, reaction with water leads to production of $\mathrm{H}_{2}$ (discussed in detail in 4.5). 

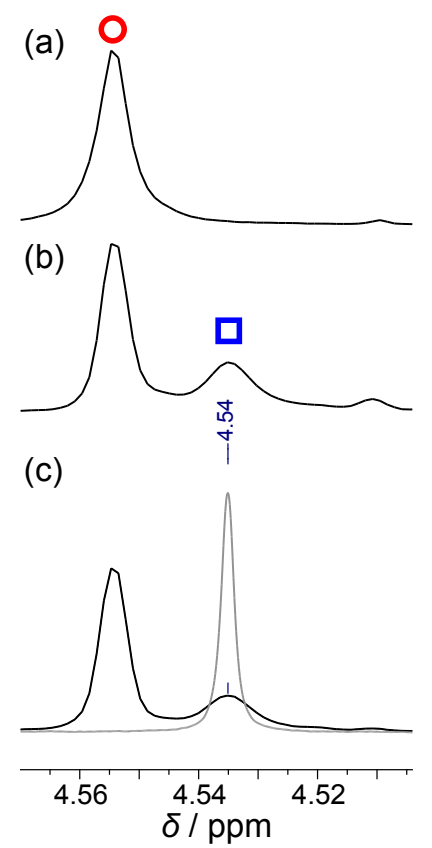

Figure D.3.: Detail of the selected spectra shown in Figure D.2. After the addition of parahydrogen (b), a resonance according to the residual amount of orthohydrogen emerged ( $\square$ ). The grey line in spectrum (c) represents the $\mathrm{H}_{2}$ signal of a THF-D $\mathrm{D}_{8}$ solution saturated with $2 \mathrm{~atm}$. of thermal hydrogen. The resonance at $4.56 \mathrm{ppm}$ corresponds to the $\beta$-diketiminate $\mathrm{CH}(\mathrm{O})$ from $\mathrm{K}\left[\mathrm{L}^{\text {bisnac }} \mathrm{Ni}_{2}(\mathrm{H})_{2}\right]$.

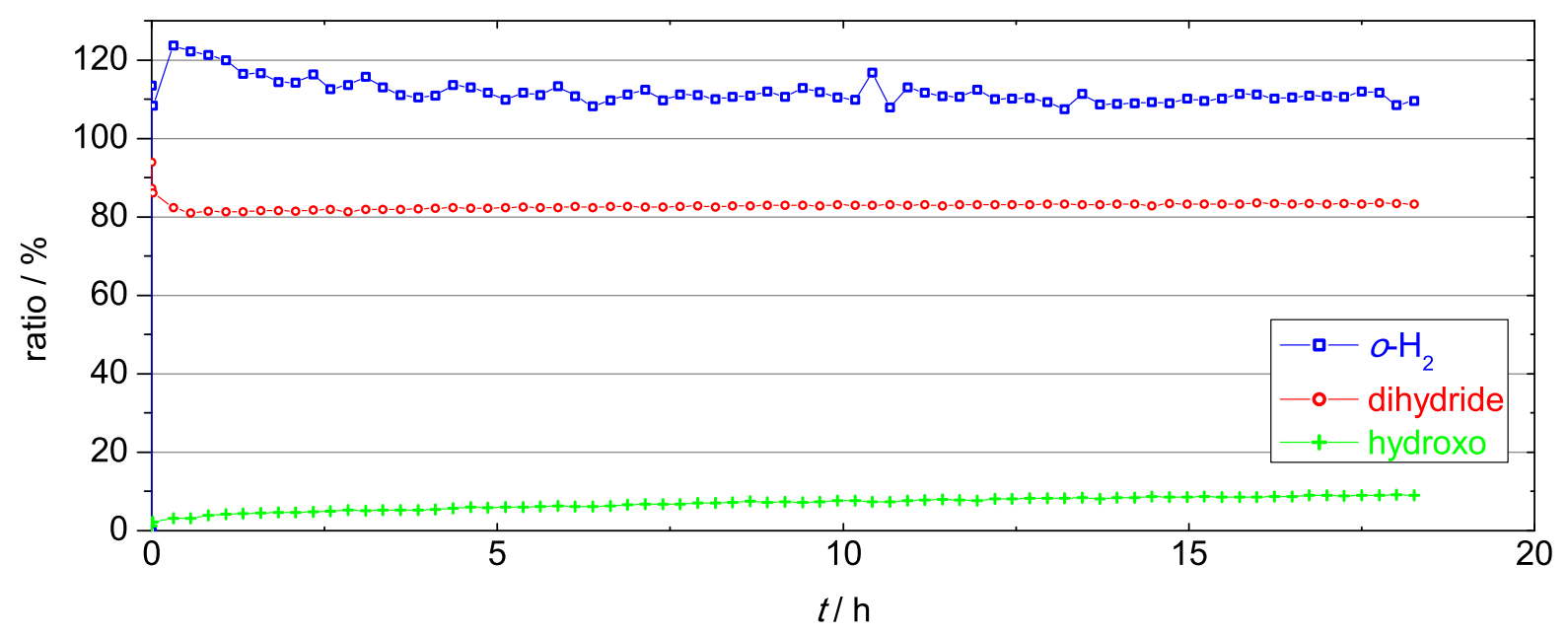

Figure D.4.: Development of signal intensity during the exchange experiment of $K\left[L^{\text {bisnac }} \mathrm{Ni}_{2}(\mathbf{H})_{2}\right]$ with para- $\mathrm{H}_{2}$ (2 atm.) over a 18 hour time frame. After the first three collected single fid's, the progress of the experiment was monitored in a defined interval of 900 seconds. The plot describes a strong change during the incipient phase of the experiment and the rapid development of an equilibrium of the observed species $(\mathbf{O}, \mathbf{\square})$, except the amount of $\left[\mathrm{L}^{\text {bisnac }} \mathrm{Ni}_{2}(\mu-\mathrm{OH})\right](+)$, which slowly increased in consquence of a reaction with moisture present in the sample. 


\section{Exchange of $\mathrm{K}\left[\mathrm{L}^{\text {bisnac }} \mathrm{Ni}_{2}(\mathrm{D})_{2}\right]$ with Parahydrogen}

The exchange reaction was repeated with the deuterium labeled dihydride complex $\left(\mathbf{K}\left[\mathbf{L}^{\text {bisnac }} \mathbf{N i}_{\mathbf{2}}(\mathbf{D})_{\mathbf{2}}\right]\right)$ and monitored by ${ }^{1} \mathrm{H}$ NMR spectroscopy. Figure D.5 represents the result. Exchange with parahydrogen led to formation of $\mathbf{K}\left[\mathbf{L}^{\text {bisnac }} \mathbf{N i}_{\mathbf{2}}(\mathbf{H})_{\mathbf{2}}\right]$ up to a value of $70 \%$ (in Figure D.5). This is again is associated with the conversion of $\mathbf{K}\left[\mathbf{L}^{\text {bisnac }} \mathbf{N i}_{2}(\mathrm{D})_{2}\right]$ and $\mathbf{K}\left[\mathbf{L}^{\text {bisnac }} \mathbf{N i}_{2}(\mathbf{H})_{2}\right]$ to hydroxide based side-products. In contrast to the previous experiment with $\mathbf{K}\left[\mathbf{L}^{\text {bisnac }} \mathbf{N i}_{\mathbf{2}}(\mathbf{H})_{\mathbf{2}}\right]$ the amount of orthohydrogen ( $\mathbf{\text { in }}$ Figure D.5) stabilizes at a value of $\approx 95 \%$. The lower amount of dihydrogen is explainable by the use of the deuterated form of $\mathbf{K}\left[\mathbf{L}^{\text {bisnac }} \mathbf{N i}_{\mathbf{2}}(\mathbf{H})_{\mathbf{2}}\right]$, which yielded $\mathrm{D}_{2}$ instead of $\mathrm{H}_{2}$ upon exchange (section 4.5). No formation of HD was observed in the ${ }^{1} \mathrm{H}$ NMR spectrum.

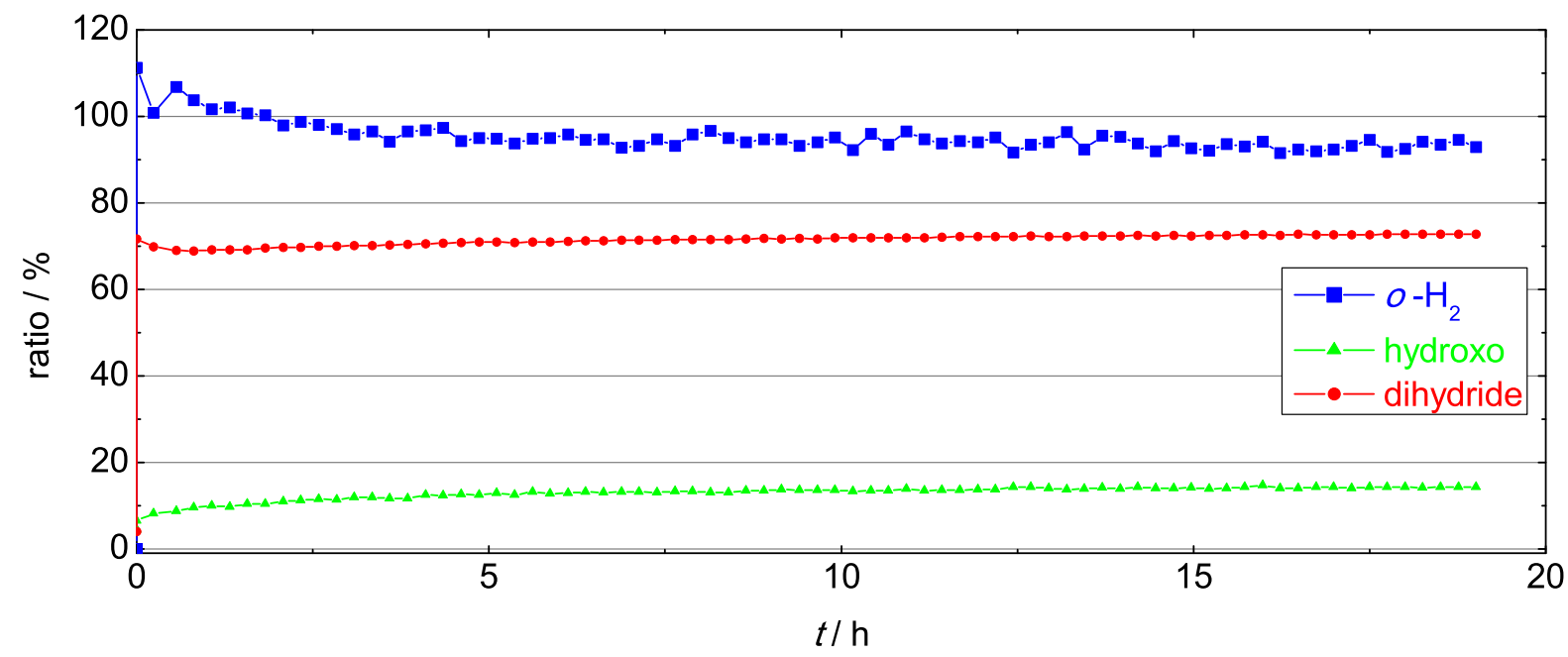

Figure D.5.: Development of respective characteristic signal intensities during the exchange experiment of $\mathbf{K}\left[\mathrm{L}^{\text {bisnac }} \mathrm{Ni}_{2}(\mathbf{D})_{2}\right]$ with para- $\mathrm{H}_{2}$ (2 atm.) over a 20 hour time frame. 


\section{Discussion}

The result of both experiments show that the hyperpolarization of parahydrogen is rapidly lost leading to the reestablishment of the thermal equlibrium in $\mathrm{H}_{2}\left(3\right.$ ortho $_{2}: 1$ para $\left.\mathrm{H}_{2}\right)$. A scrambling of $\mathrm{H}_{2}$ mediated by an exchange with $\mathbf{K}\left[\mathbf{L}^{\text {bisnac }} \mathbf{N i}_{\mathbf{2}}(\mathbf{H})_{2}\right]$ or $\mathbf{K}\left[\mathbf{L}^{\text {bisnac }} \mathbf{N i}_{\mathbf{2}}(\mathbf{D})_{2}\right]$ can explain the conversion to thermal state but was not proven due to absence of HD in experiments conducted with deuterium labeled complex. Therefore, other reasons have to be considered (Scheme D.3). An important factor is the low spin-lattice relaxation, which is short in the large molecule. It can significantly contribute to a potential loss of hyperpolarization. Furthermore, impurities, which are capable to catalyze the conversion of parahydrogen back to thermal state could not be clearly excluded. Since both a scrambling and non-scrambling mediated exchange of parahydrogen with $\mathbf{K}\left[\mathbf{L}^{\text {bisnac }} \mathbf{N i}_{\mathbf{2}}(\mathbf{H})_{2}\right]$ lead to the same observed outcome, no statement in direction of a scrambling or non-scrambling mediated exchange process can be made.

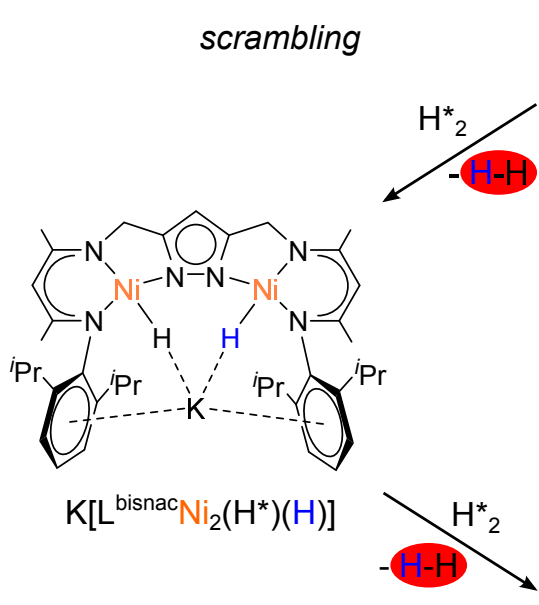

scrambling

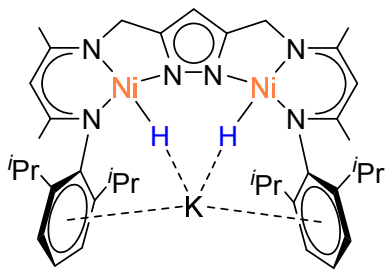

$\mathrm{K}\left[\mathrm{L}^{\text {bisnac }} \mathrm{Ni}_{2}(\mathrm{H})_{2}\right]$ non-scrambling
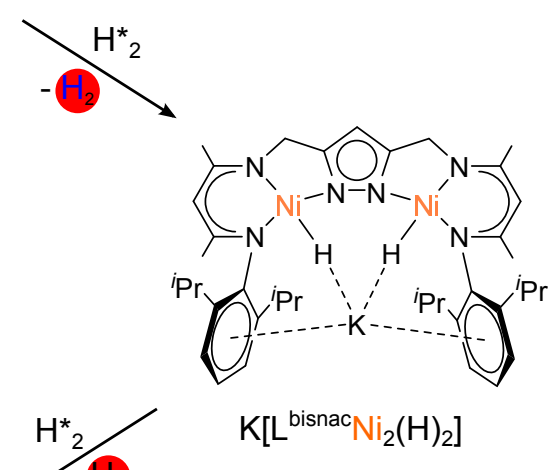

non-scrambling

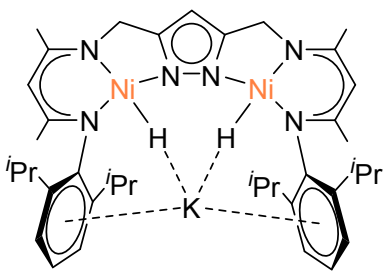

$\mathrm{K}\left[\mathrm{L}^{\text {bisnac }} \mathrm{Ni}_{2}(\mathrm{H})_{2}\right]$

(thermal equilibrium state)

Scheme D.3: Illustration of the reformation of parahydrogen to thermal state. Both scrambling and nonscrambling mediated exchanges lead to the same outcome. Produced $\mathrm{H}_{2}$ in thermal state is indicated $(\bullet)$. 


\section{D.3. $K\left[L^{\text {bisnac }} \mathrm{Ni}_{2}\left(\mu-\eta^{1}: \eta^{1}-\mathrm{CHCPh}\right)\right]$}

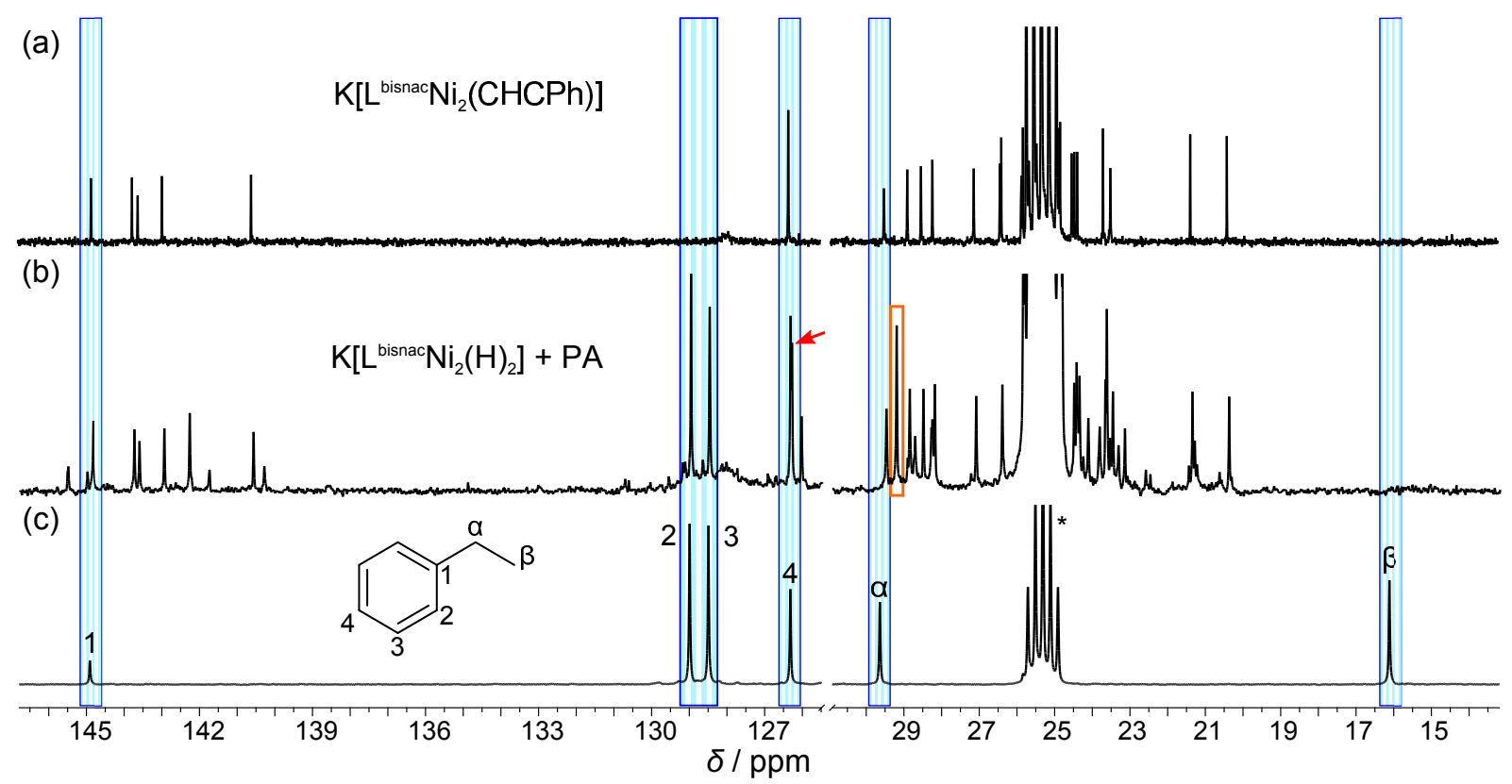

Figure D.6.: Comparison of ${ }^{13} \mathrm{C}\left\{{ }^{1} \mathrm{H}\right\}$ NMR spectra $(101 \mathrm{MHz})$ measured in THF- $\mathrm{D}_{8}\left(^{*}\right)$. (a) reference spectrum of the pure complex of $\mathrm{K}\left[\mathrm{L}^{\text {bisnac }} \mathrm{Ni}_{2}\left(\mu-\eta^{1}: \eta^{1}-\mathrm{CHCPh}\right)\right]$. (b) reaction mixture days after conclusion of the reaction of $\mathrm{K}\left[\mathrm{L}^{\text {bisnac }} \mathrm{Ni}_{2}(\mathrm{D})_{2}\right]$ with two equivalents of phenylacetylene. Spectrum (c) describes the ${ }^{13} \mathrm{C}$ resonances of an ethylbenzene sample. The characteristic resonances are encased $(\square)$. The numbering (1-4) of the ethylbenzene resonances indicates the position of the aromatic carbon atoms relative to the substituted carbon atom (ortho, meta, para) while the symbols $\alpha$ and $\beta$ refer to the aliphatic carbon positions relative to the substituted carbon of the aromatic ring. The marked carbon resonance $(\searrow)$ was difficult to distinguish since overlayed and could be correlated to the possible para-carbon of ethylbenzene. 


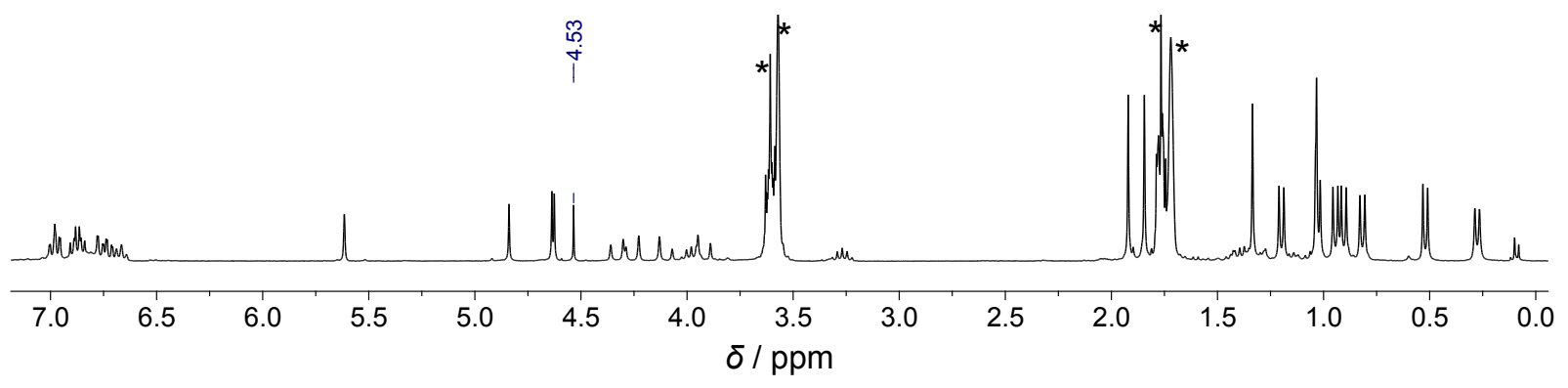

Figure D.7.: ${ }^{1} \mathrm{H}$ NMR spectrum (300 MHz) of a degassed sample of $K\left[L^{\text {bisnac }} \mathrm{Ni}_{2}\left(\mu-\eta^{1}: \eta^{1}-\mathrm{CHCPh}\right)\right]$ dissolved in THF- $\mathrm{D}_{8}$ treated with one atmosphere of dried dihydrogen (4.53 ppm). Residual solvents are marked $\left(^{*}\right)$.

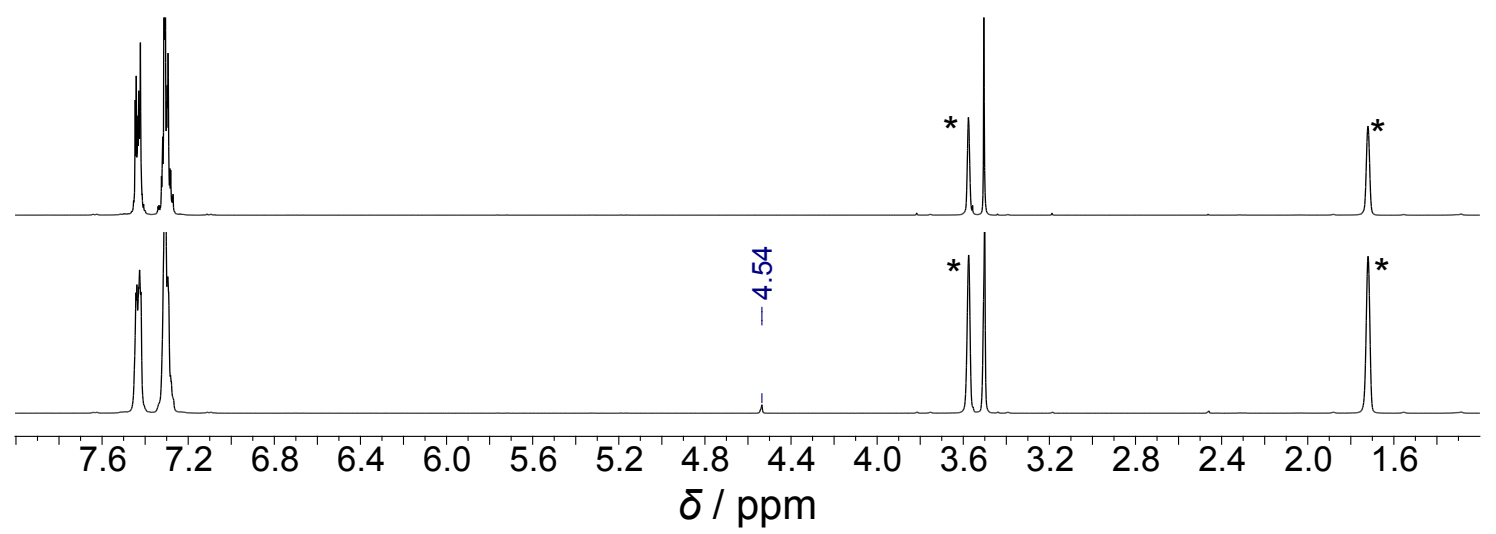

Figure D.8.: ${ }^{1} \mathrm{H}$ NMR spectra $(400 \mathrm{MHz})$ of a blind experiment with phenylacetylene in the presence of $\mathrm{H}_{2}$ without $\mathrm{K}\left[\mathrm{L}^{\text {bisnac }} \mathrm{Ni}_{2}\left(\mu-\eta \eta^{1}: \eta^{1}-\mathrm{CHCPh}\right)\right]$. Top: starting point without dihydrogen. Bottom: dihydrogen loaded (1 atm.) sample after one week. Residual solvents are marked $\left(^{*}\right)$.

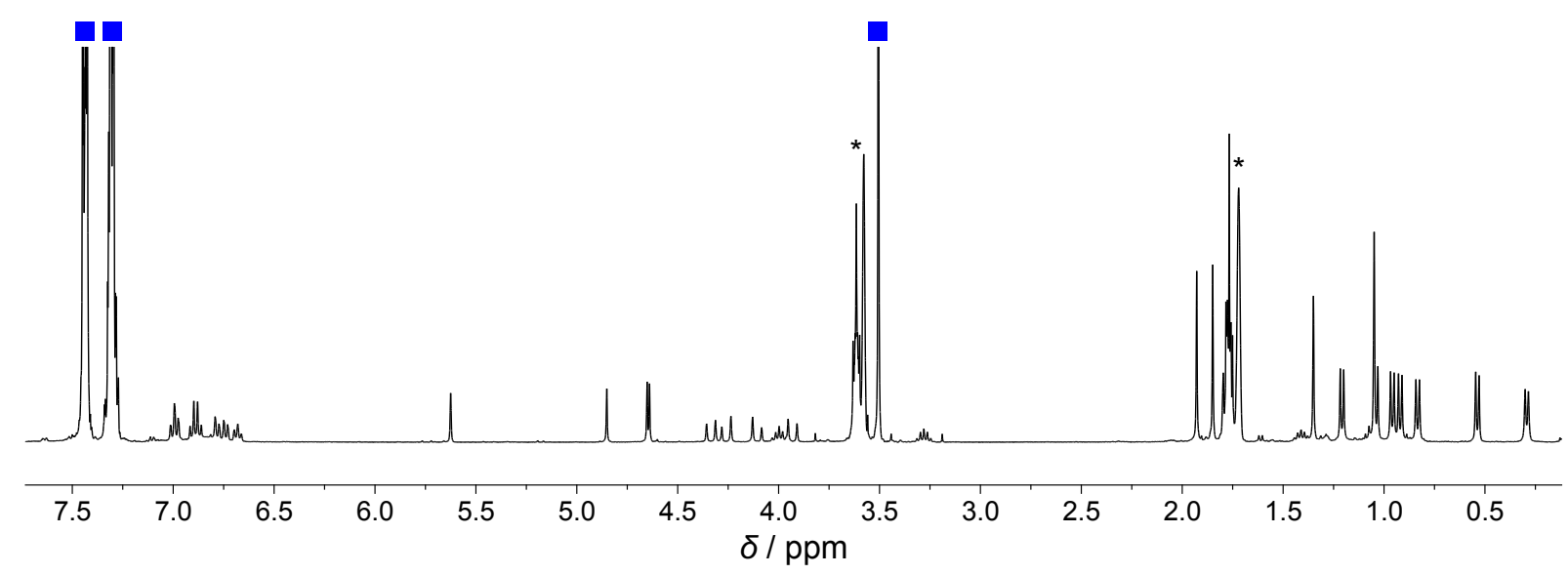

Figure D.9.: ${ }^{1} \mathrm{H}$ NMR spectrum $(400 \mathrm{MHz})$ of $\mathrm{K}\left[\mathrm{L}^{\text {bisnac }} \mathrm{Ni}_{2}\left(\mu-\eta^{1}: \eta^{1}-\mathrm{CHCPh}\right)\right]$ (crystalline material dissolved in THF- $\mathrm{D}_{8}$ ) treated with an excess (15 eq.) of phenylacetylene ( $\square$ ). Residual solvents are marked $\left({ }^{*}\right)$. 


\section{D.4. [ [ $\left.{ }^{\text {bisnac }} \mathrm{Ni}_{2}\left(\mu-\eta^{1}: \eta^{1}-\mathrm{N}_{2} \mathrm{H}_{3}\right)\right]$}

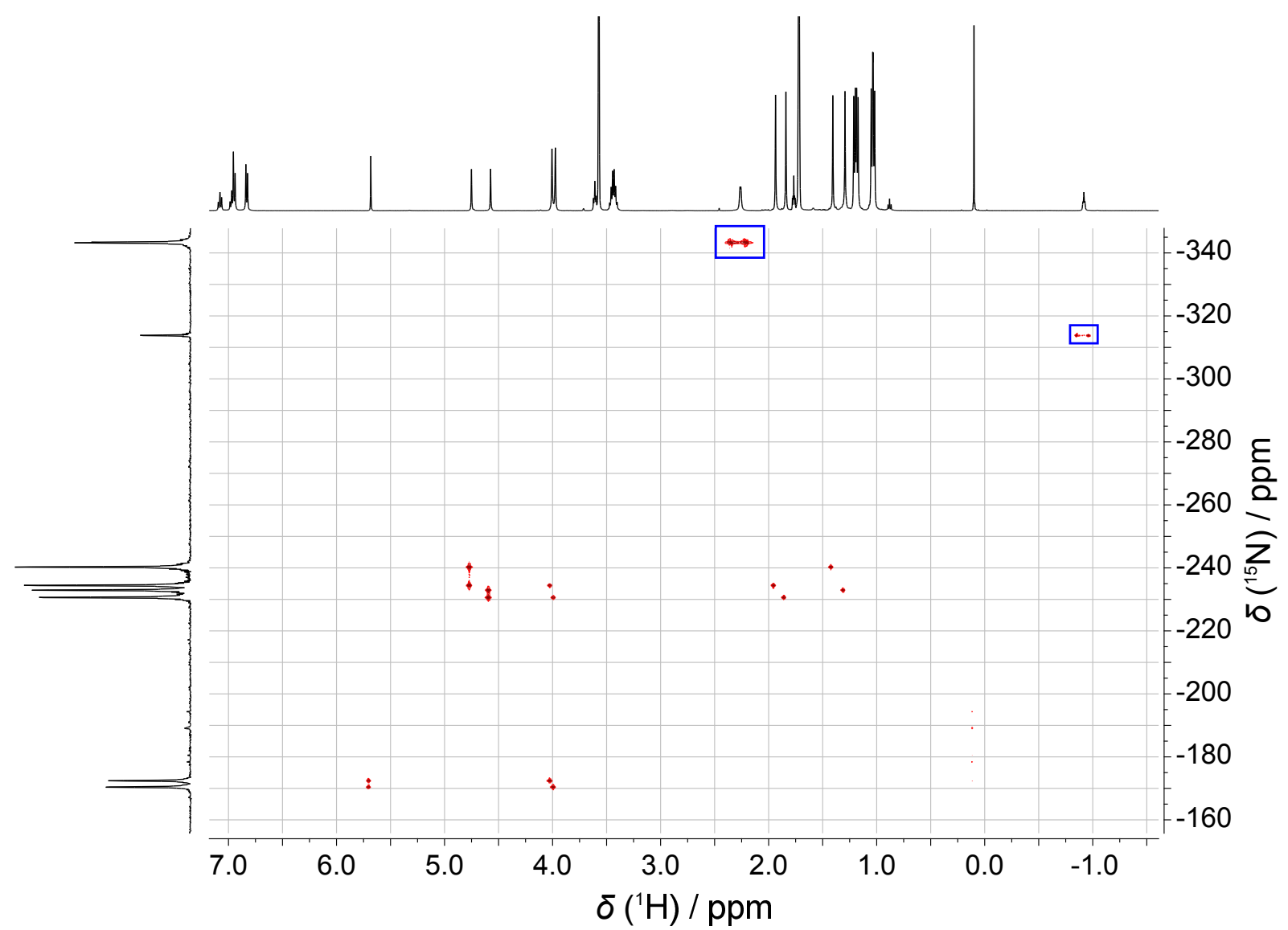

Figure D.10.: ${ }^{15} \mathrm{~N}$ HMBC NMR spectrum $(500 \mathrm{MHz})$ of [ $\left.{ }^{\text {bisnac }} \mathbf{N i}_{2}\left(\mu-\eta^{1}: \eta^{1}-\mathbf{N}_{2} \mathbf{H}_{3}\right)\right]$ measured in THF-D 8 at room temperature $(\mathrm{CNST} 13=10 \mathrm{~Hz}) .{ }^{1} \mathrm{~J}_{\mathrm{NH}}$ correlations of the compound are marked $(\square)$. 


\section{D.5. [ [ $\left.{ }^{\text {bisnac }} \mathrm{Ni}_{2} \mu-\left(\mathrm{NH}_{2}\right)\right]$}

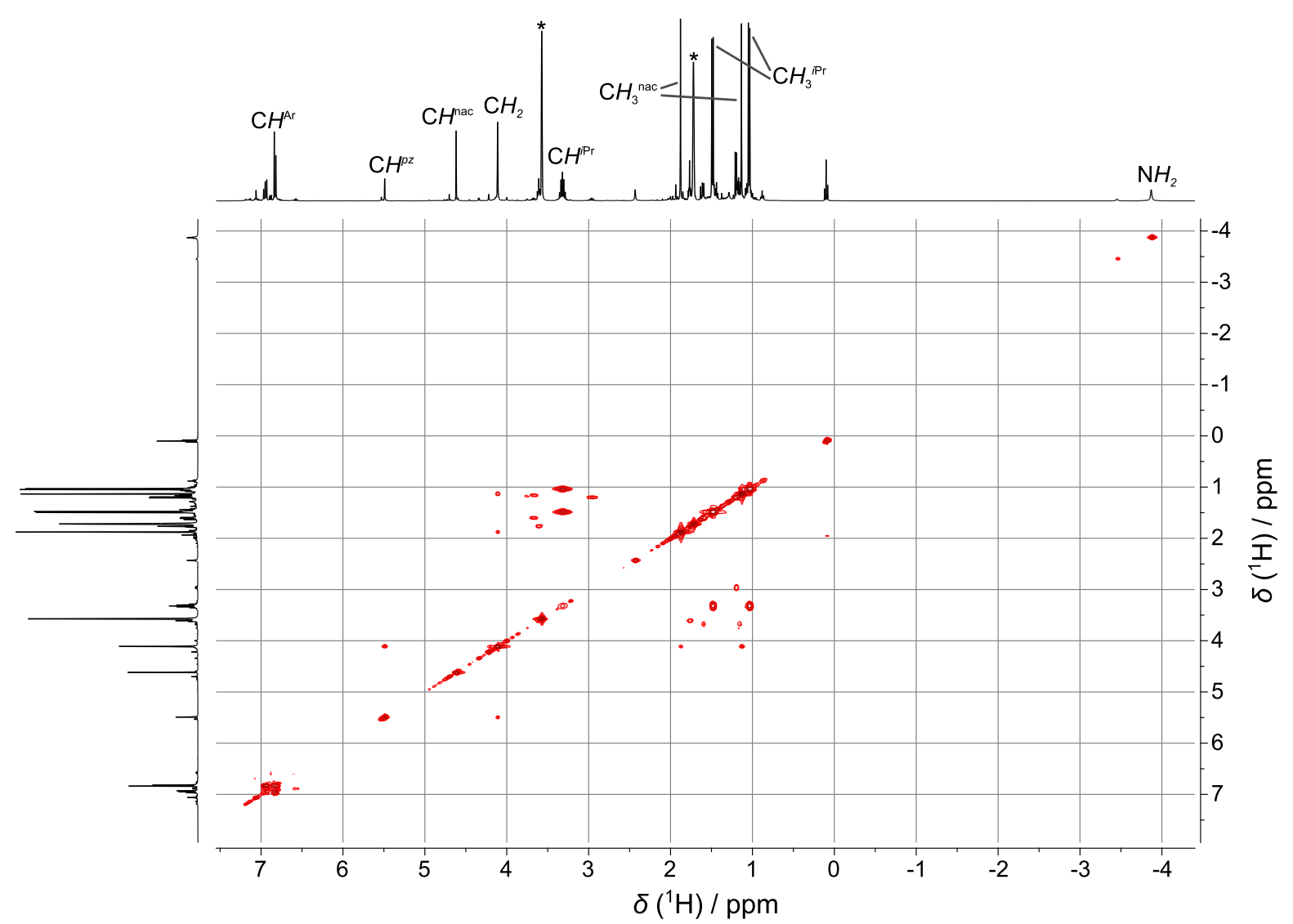

Figure D.11.: ${ }^{1} \mathrm{H}-{ }^{1} \mathrm{H}$ COSY spectrum $(400 \mathrm{MHz})$ of [ $\left.\mathrm{L}^{\text {bisnac }} \mathbf{N i}_{2}\left(\mu-\mathrm{NH}_{2}\right)\right]$. The spectrum was measured in THF-D ${ }_{8}$. Residual solvents are marked ( $\left.{ }^{*}\right)$. 


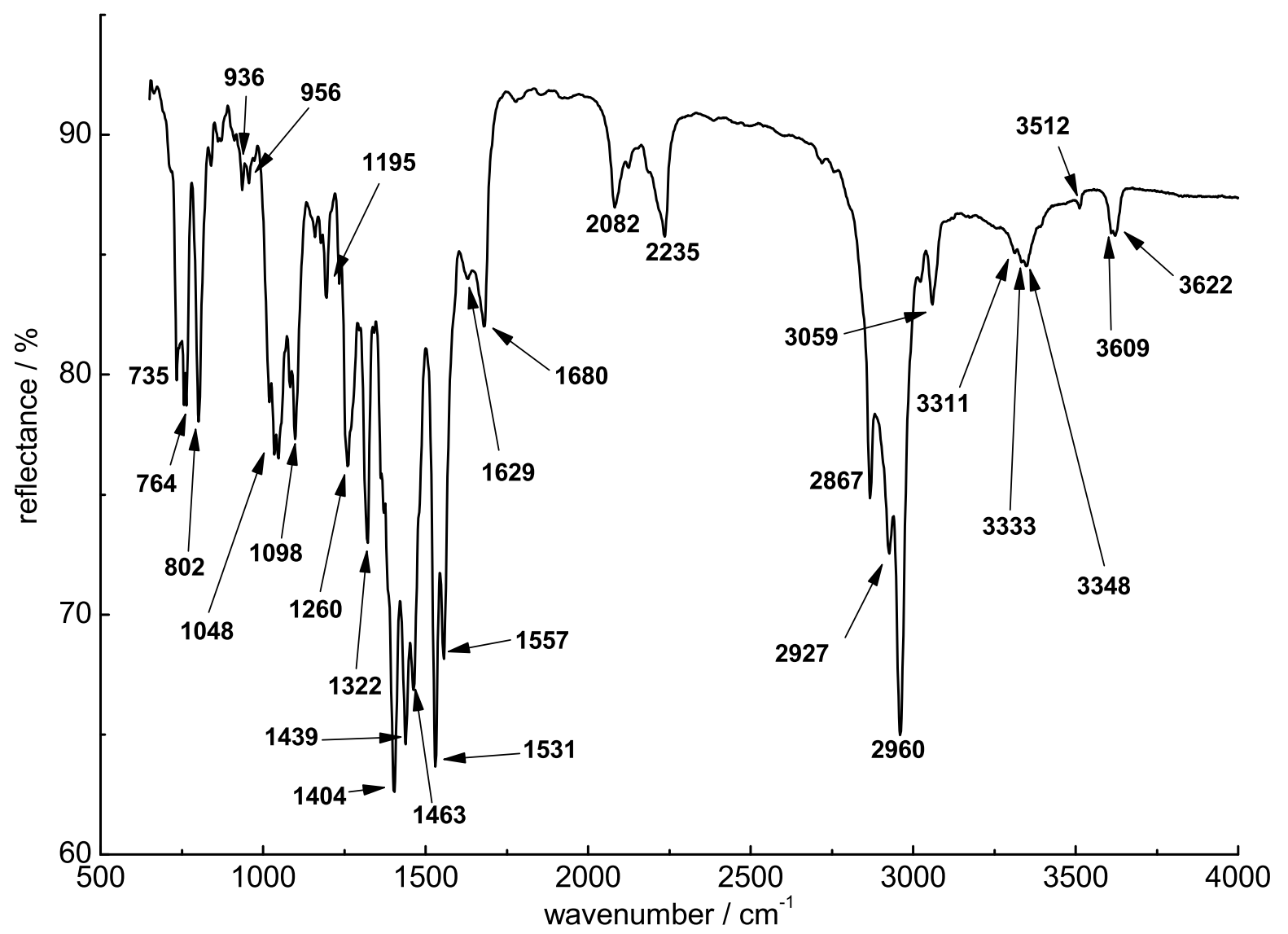

Figure D.12.: IR (ATR) spectrum of [ $\left.{ }^{\text {bisnac }} \mathrm{Ni}_{2}\left(\mu-\mathrm{NH}_{2}\right)\right]$. 


\section{D.6. $\left[\mathrm{L}^{\text {bisnac }} \mathrm{Ni}_{2}\left(\mu-\eta^{1}: \eta^{1}-\mathrm{N}_{2} \mathrm{H}\right)\right]$}

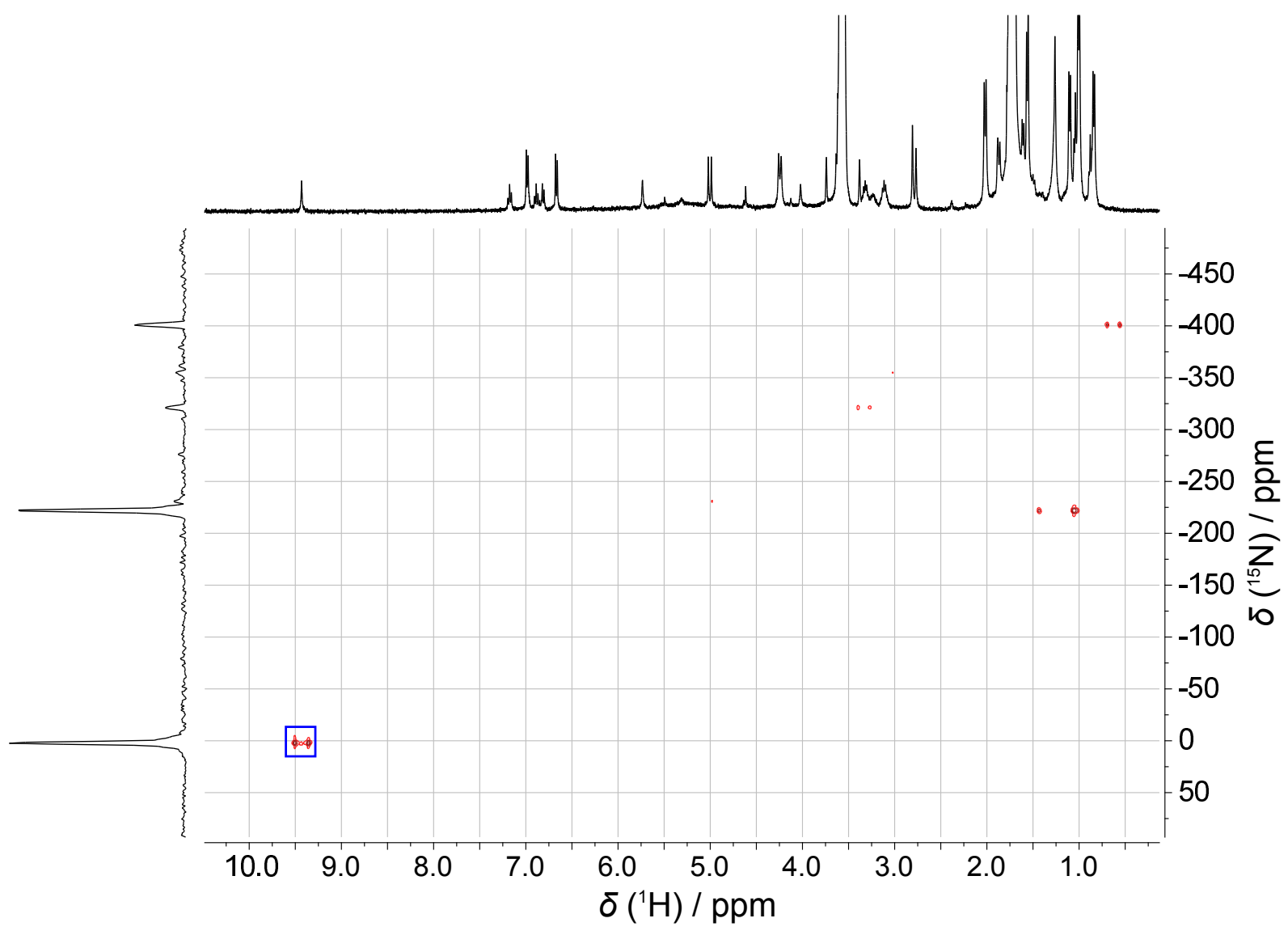

Figure D.13.: ${ }^{15} \mathrm{~N}-\mathrm{H}$ HMBC spectrum $(500 \mathrm{MHz})$ of $\left[\mathrm{L}^{\text {bisnac }} \mathrm{Ni}_{2}\left(\mu-\eta^{1}: \eta^{1}-\mathrm{N}_{2} \mathbf{H}\right)\right]$. The spectrum was measured in THF-D $8 .{ }^{1} J_{\mathrm{NH}}$ correlations of the compound are marked $(\square)$. 


\section{D.7. [ $\left.\mathrm{L}^{\text {bisnac }} \mathrm{Ni}_{2}\left(\mu-\eta^{1}: \eta^{1}-\mathrm{N}_{2}\right)\right]$}

\section{Experimental procedure for the isotopic labeling with ${ }^{15} \mathrm{~N}_{2}$}

In order to accomplish a ${ }^{15} \mathrm{~N}$ labeling of the bridging ligand, the generation of $\left[\mathbf{L}^{\text {bisnac }} \mathbf{N i}_{\mathbf{2}}\left(\mu-\eta^{\mathbf{1}}: \eta^{\mathbf{1}}-\mathbf{N}_{\mathbf{2}}\right)\right]$ under an atmosphere of ${ }^{15} \mathrm{~N}_{2}$ was attempted.

The solid lutidinium triflate was transferred into an improvised container which was made of a long glas pipette stuffed with a piece of glasfibre filter medium under inert conditions. This vessel was then inserted into the J. Young valve sample tube, followed by careful addition of already dissolved measured amount of the starting dihydride complex $\left[\mathbf{L}^{\text {bisnac }} \mathbf{N i}_{\mathbf{2}}(\mathbf{H})_{2}\right]$ to the bottom of the tube making sure that it did not came into contact with the acid as indicated in the picture (a) of Figure D.14. The next step required attachment of the sample to a dried and evacuated Schlenk apparatus (picture b of Figure D.14) and conduction of pump freeze thaw cycles to degas the complex solution. Afterwards the ${ }^{15} \mathrm{~N}_{2}$ gas which was dried in an improvised cooling trap device (liquid $\mathrm{N}_{2}$ ) was transferred to the degassed sample. By turning over and carefully shaking the sample tube, the complex solution came into contact with the acid, which initiated the reaction and led to the formation of gas (picture $\mathrm{c}$ and $\mathrm{d}$ of Figure D.14). The sample was immediately transferred into the precooled NMR spectrometer $\left(-35{ }^{\circ} \mathrm{C}\right)$ to collect a ${ }^{1} \mathrm{H}$ NMR spectrum. Unfortunately, various side products formed, which is indicated by occurence of multiple pyrazolate-backbone hydrogen resonances. Besides the strong resonance for ${ }^{15} \mathrm{~N}_{2}$ at $70 \mathrm{ppm}$, the ${ }^{15} \mathrm{~N}$ NMR experiment yielded no further resonances over a spectral width of $2000 \mathrm{ppm}$.

Conversion of $\left[\mathbf{L}^{\text {bisnac }} \mathbf{N i}_{2}(\mathbf{H})_{2}\right]$ to $\left[\mathbf{L}^{\text {bisnac }} \mathbf{N i}_{2}\left(\mu-\eta^{1}: \eta^{1}-\mathbf{N}_{2}\right)\right]$ is extremely sensitive towards moisture and mostly yields $\left[\mathbf{L}^{\text {bisnac }} \mathbf{N i}_{\mathbf{2}}(\mu-\mathbf{O H})\right]$. However, conduction of the experiment in larger scale (100 mg complex) followed by swift layering with hexane and cooling to $-30{ }^{\circ} \mathrm{C}$ led to formation of $\left[\mathbf{L}^{\text {bisnac }} \mathbf{N i}_{2}\left(\mu-\eta^{\mathbf{1}}: \eta^{1} \mathbf{1}^{15} \mathbf{N}_{2}\right)\right]$, although in very low yields (the amount of isolated crystalline material was to low for ${ }^{15} \mathrm{~N} N M R$ ). 

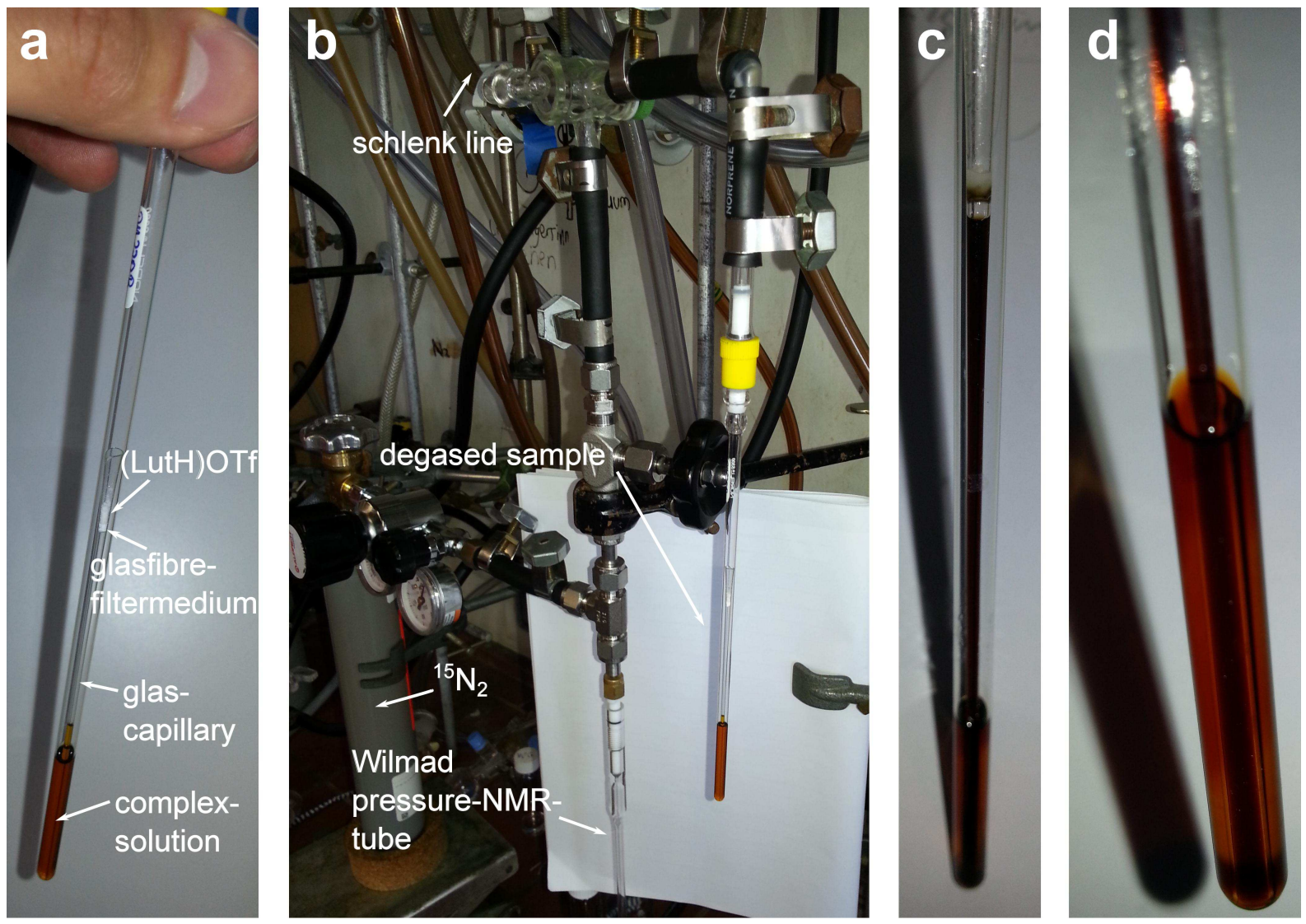

a: untreated sample; b: experimental setup; c / d: ${ }^{15} \mathrm{~N}_{2}$ loaded sample

Figure D.14.: Experimental setup for the ${ }^{15} \mathrm{~N}$ labeling experiment. 



\section{References}

[1] M. Lundberg, M. R. A. Blomberg, P. E. M. Siegbahn, Theor. Chem. Acc. 2003, 110, 130-143.

[2] M. M. Najafpour, M. A. Isaloo, J. J. Eaton-Rye, T. Tomo, H. Nishihara, K. Satoh, R. Carpentier, J. R. Shen, S. I. Allakhverdiev, Biochim. Biophys. Acta-Bioenerg. 2014, 1837, 1395-1410.

[3] W. Hillier, T. Wydrzynski, Coord. Chem. Rev. 2008, 252, 306-317.

[4] J. H. Weber, Inorg. Chem. 1967, 6, 258-262.

[5] E. A. Katayev, K. Severin, R. Scopelliti, Y. A Ustynyuk, Inorg. Chem. 2007, 46, $5465-5467$.

[6] H. Du, A. H. Velders, P. J. Dijkstra, Z. Zhong, X. Chen, and J. Feijen, Macromolecules 2009, 42, 1058-1066.

[7] G. Noël, J. C. Röder, S. Dechert, H. Pritzkow, L. Bolk, S. Mecking, and F. Meyer, Adv. Synth. Catal. 2006, 348, 887-897.

[8] D. J. de Geest, A. Noble, B. Moubaraki, K. S. Murray, D. S. Larsen, and S. Brooker, Dalton Trans. 2007, 16, 467-475.

[9] S. Katsiaouni, Dissertation, Georg-August-University Göttingen 2007.

[10] J. Klingele, S. Dechert, F. Meyer, Coord. Chem. Rev. 2009, 253, 2698-2741.

[11] D.-H. Manz, Diploma Thesis, Georg-August-University Göttingen 2010.

[12] J. Cámpora, I. Matas, P. Palma, C. Graiff, A. Tiripicchio, Organometallics 2005, 24, 2827-2830.

[13] D. Huang, R. H. Holm, J. Am. Chem. Soc. 2010, 132, 4693-4701.

[14] B. R. Dible, M. S. Sigman, J. Am. Chem. Soc. 2003, 125, 872-873.

[15] M. K. Samantaray, M. M. Shaikh, P. Ghosh, Organometallics 2009, 28, 2267-2275.

[16] G. T. Venkanna, H. D. Arman, Z. J. Tonzetich, ACS Catal. 2014, 4, 2941-2950. 
[17] R. C. Poulten, I. López, A. Llobet, M. F. Mahon, M. K. Whittlesey, Inorg. Chem. 2014, 53, 7160-7169.

[18] D. Huang, O. V Makhlynets, L. L. Tan, S. C. Lee, E. V Rybak-akimova, R. H. Holm, Inorg. Chem. 2011, 50, 10070-10081.

[19] J. Cámpora, P. Palma, D. Del Río, Á. Eleuterio, Organometallics 2004, 23, $1652-1655$.

[20] A. Castonguay, L. Beauchamp, D. Zargarian, Inorg. Chem. 2009, 48, 3177-3184.

[21] J. C. Röder, F. Meyer, H. Pritzkow, Organometallics 2001, 20, 811-817.

[22] J. S. Bradshaw, R. B. Nielsen, P. Tse, G. Arena, B. E. Wilson, N. K. Dalley, J. D. Lamb, J. C. Christensen, R. M. Izatt, J. Heterocyclic Chem. 1986, 23, 361.

[23] T. G. Schenck, J. M. Downes, C. R. C. Milne, P. B. Mackenzie, J. W. H. Boucher, B. Bosnich, Inorg. Chem. 1985, 24, 2334-2337.

[24] G. D. Hartman, L. M. Weinstock, Org. Synth. 1988, 6, 620.

[25] J. L. Sessler, A. Mazaffari, M. R. Johnson, Org. Synth. 1998, 9, 242.

[26] A. Mikhaleva, A. Ivanov, E. Skital'tseva, I. Ushakov, A. Vasil'tsov, and B. Trofimov, Synthesis 2009, 587-590.

[27] H. M. Hultman, K. Djanashvili, J. A. Peters, Magn. Reson. Chem. 2003, 41, 959-961.

[28] J. M. Robert, R. F. Evilia, J. Am. Chem. Soc, 1985, 10\%, 3733-3735.

[29] I. P. Gerothanassis, C. G. Tsanaktsidis, Concepts in Magnetic Resonance 1996, 8, $63-74$.

[30] M. L. Tobe, Inorganic Reaction Mechanisms, Thomas Nelson \& Sons Limited, London 1972.

[31] R. G. Wilkins, Kinetics and Mechanism of Reactions of Transition Metal Complexes, VCH, Weinheim, 1991.

[32] S. Buchler, F. Meyer, E. Kaifer, H. Pritzkow, Inorganica Chim. Acta 2002, 337, 371-386.

[33] D. J. Mindiola, Angew. Chem. Int. Ed. 2009, 48, 6198-200.

[34] L. Bourget-Merle, P. B. Hitchcock, M. F. Lappert, J. Organomet. Chem. 2004, 689, 4357-4365.

[35] L. Bourget-Merle, M. F. Lappert, J. R. Severn, Chem. Rev. 2002, 102, 3031-3066.

[36] Y. Tsai, Coord. Chem. Rev. 2012, 256, 722-758. 
[37] J. Spielmann, S. Harder, Dalton Trans. 2011, 40, 8314-8319.

[38] X. Wei, J. D. Farwell, B. Hitchcock, M. F. Lappert, Dalton Trans. 2008, 4, 1073-1080.

[39] D. F. Piesik, S. Range, S. Harder, Organometalics 2008, 27, 6178-6187.

[40] S. P. Sarish, S. Nembenna, S. Nagendran, H. W. Roesky, Acc. Chem. Res. 2011, 44, 157-170.

[41] M. R. Crimmin, M. S. Hill, B. Hitchcock, M. F. Mahon, New J. Chem. 2010, 34, $1572-1578$.

[42] S. E. Baillie, V. L. Blair, T. D. Bradley, W. Clegg, J. Cowan, R. W. Harrington, A. Hernán-Gómez, A. R. Kennedy, Z. Livingstone, E. Hevia, Chem. Sci. 2013, 4, 1895-1905.

[43] S. P. Green, C. Jones, A. Stasch, Science 2007, 318, 1754-1757.

[44] H. Zhu, J. Chai, V. Chandrasekhar, H. W. Roesky, J. Magull, D. Vidovic, H.-G. Schmidt, M. Noltemeyer, P. P. Power, W. A. Merrill, J. Am. Chem. Soc. 2004, 126, 9472-9473.

[45] D. Vidovic, M. Findlater, A. H. Cowley, J. Am. Chem. Soc. 2007, 129, 8436-8437.

[46] W. D. Woodul, E. Carter, M. Robert, A. F. Richards, A. Stasch, M. Kaupp, D. M. Murphy, M. Driess, C. Jones, J. Am. Chem. Soc. 2011, 133, 10074-10077.

[47] C. E. Radzewich, M. P. Coles, R. F. Jordan, J. Am. Chem. Soc. 1998, 120, 9384-9385.

[48] N. Burford, P. J. Ragogna, K. N. Robertson, T. S. Cameron, N. J. Hardman, P. P. Power, J. Am. Chem. Soc. 2002, 124, 382-383.

[49] H. Andres, E. L. Bominaar, J. M. Smith, N. a Eckert, P. L. Holland, E. Münck, J. Am. Chem. Soc. 2002, 124, 3012-3025.

[50] G. Bai, P. Wei, D. W. Stephan, Organometallics 2005, 24, 5901-5908.

[51] S. M. Bellows, T. R. Cundari, P. L. Holland, Organometallics 2013, 32, 4741-4751.

[52] P. H. M. Budzelaar, A. B. van Oort, A. G. Orpen, Eur. J. Inorg. Chem. 1998, 1485-1494.

[53] C. Chen, T. R. Dugan, W. W. Brennessel, D. J. Weix, P. L. Holland, J. Am. Chem. Soc. 2014, 136, 945-955.

[54] K. P. Chiang, S. M. Bellows, W. W. Brennessel, P. L. Holland, Chem. Sci. 2014, $5,267-274$. 
[55] X. Dai, P. Kapoor, T. H. Warren, J. Am. Chem. Soc. 2004, 126, 4798-4799.

[56] X. He, Y. Yao, X. Luo, J. Zhang, Y. Liu, L. Zhang, Q. Wu, Organometallics 2003, 22, 4952-4957.

[57] B. Horn, C. Limberg, C. Herwig, B. Braun, Chem. Commun. 2013, 49, 10923-10925.

[58] C. Köthe, R. Metzinger, C. Limberg, Eur. J. Inorg. Chem. 2013, 3937-3942.

[59] S. Yao, M. Driess, Acc. Chem. Res. 2012, 45, 276-287.

[60] R. Jiao, X. Shen, M. Xue, Y. Zhang, Y. Yao, Q. Shen, Chem. Commun. 2010, 46, 4118-4120.

[61] M. J. Monreal, R. J. Wright, D. E. Morris, B. L. Scott, T. Golden, P. P. Power, J. L. Kiplinger, Organometallics 2013, 32, 1423-1434.

[62] L. Chatelain, V. Mougel, P. Jacques, M. Mazzanti, Chem. Sci. 2012, 3, 1075-1079.

[63] J. M. Smith, A. R. Sadique, T. R. Cundari, K. R. Rodgers, G. Lukat-Rodgers, R. J. Lachicotte, C. J. Flaschenriem, J. Vela, P. L. Holland, J. Am. Chem. Soc. 2006, 128, 756-769.

[64] S. Yao, C. Herwig, Y. Xiong, A. Company, E. Bill, C. Limberg, M. Driess, Angew. Chem. Int. Ed. 2010, 49, 7054-7058.

[65] K. Ding, A. W. Pierpont, W. W. Brennessel, G. Lukat-Rodgers, K. R. Rodgers, T. R. Cundari, E. Bill, P. L. Holland, J. Am. Chem. Soc. 2009, 131, 9471-9472.

[66] T. R. Dugan, K. C. Macleod, W. W. Brennessel, P. L. Holland, Eur. J. Inorg. Chem. 2013, 3891-3897.

[67] S. Pfirrmann, C. Limberg, C. Herwig, R. Stösser, B. Ziemer, Angew. Chem. Int. Ed. 2009, 48, 3357-3361.

[68] S. Pfirrmann, S. Yao, B. Ziemer, R. Stösser, M. Driess, C. Limberg, Organometallics 2009, 28, 6855-6860.

[69] P. L. Holland, T. R. Cundari, L. L. Perez, N. A. Eckert, R. J. Lachicotte, J. Am. Chem. Soc. 2002, 124, 14416-14424.

[70] N. A Eckert, E. M. Bones, R. J. Lachicotte, P. L. Holland, Inorg. Chem. 2003, 42, 1720-1725.

[71] N. A. Eckert, A. Dinescu, T. R. Cundari, P. L. Holland, Inorg. Chem. 2005, 44, 7702-7704.

[72] E. Kogut, A. Zeller, T. H. Warren, T. Strassner, J. Am. Chem. Soc. 2004, 126, 11984-11994. 
[73] S. C. Puiu, T. H. Warren, Organometallics 2003, 22, 3974-3976.

[74] M. M. Melzer, S. Jarchow-Choy, E. Kogut, T. H. Warren, Inorg. Chem. 2008, 47, $10187-10189$.

[75] S. Wiese, M. J. B. Aguila, E. Kogut, T. H. Warren, Organometallics 2013, 32, $2300-2308$.

[76] G. Bai, P. Wei, A. K. Das, D. W. Stephan, Dalton Trans. 2006, 1141-1146.

[77] G. Bai, P. Wei, A. Das, D. Stephan, Organometallics 2006, 25, 5870-5878.

[78] C. Köthe, R. Metzinger, C. Herwig, C. Limberg, Inorg. Chem. 2012, 51, 9740-9747.

[79] C. Köthe, B. Braun, C. Herwig, C. Limberg, Eur. J. Inorg. Chem. 2014, 5296-5303.

[80] C. Köthe, C. Limberg, Z. Anorg. und Allg. Chem. 2015, 641, 18-30.

[81] B. Horn, S. Pfirrmann, C. Limberg, C. Herwig, B. Braun, S. Mebs, R. Metzinger, Z. Anorg. Allg. Chem. 2011, 637, 1169-1174.

[82] B. Horn, C. Limberg, C. Herwig, B. Braun, Inorg. Chem. 2014, 53, 6867-6874.

[83] S. Pfirrmann, C. Limberg, B. Ziemer, Dalton Trans. 2008, 6689-6691.

[84] S. Pfirrmann, C. Limberg, C. Herwig, C. Knispel, B. Braun, E. Bill, R. Stösser, J. Am. Chem. Soc. 2010, 132, 13684-13691.

[85] D. V Vitanova, F. Hampel, K. C. Hultzsch, Dalton Trans. 2005, 1565-1566.

[86] D. V Vitanova, F. Hampel, K. C. Hultzsch, J. Organomet. Chem. 2005, 690, 5182-5197.

[87] D. F.-J. Piesik, R. Stadler, S. Range, S. Harder, Eur. J. Inorg. Chem. 2009, $3569-3576$.

[88] S. Harder, J. Spielmann, J. Intemann, H. Bandmann, Angew. Chem. Int. Ed. 2011, 50, 4156-4160.

[89] J. Intemann, J. Spielmann, P. Sirsch, S. Harder, Chem. Eur. J. 2013, 19, 8478-8489.

[90] S. Harder, J. Spielmann, J. Intemann, Dalton Trans. 2014, 43, 14284-14290.

[91] J. Intemann, P. Sirsch, S. Harder, Chem. Eur. J. 2014, 20, 11204-11213.

[92] S. Gong, H. Ma, J. Huang, Dalton Trans. 2009, 8237-8247.

[93] S. Sun, H. Ouyang, Y. Luo, Y. Zhang, Q. Shen, Y. Yao, Dalton Trans. 2013, 42, 16355-16364.

[94] S. Sun, Q. Sun, B. Zhao, Y. Zhang, Q. Shen, Y. Yao, Organometallics 2013, 32, 1876-1881. 
[95] L. Li, C. Wu, D. Liu, S. Li, D. Cui, Organometallics 2013, 32, 3203-3209.

[96] J. Spielmann, D. E. J. Piesik, S. Harder, Chem. - A Eur. J. 2010, 16, 8307-8318.

[97] J. Spielmann, J. Intemann, S. Harder, Dalton Trans. 2014, 43, 14284-14290.

[98] H. Gehring, R. Metzinger, C. Herwig, J. Intemann, S. Harder, C. Limberg, Chem. Eur. J. 2013, 19, 1629-1636.

[99] M. M. Rodriguez, E. Bill, W. W. Brennessel, P. L. Holland, Science 2011, 334 , 780-783.

[100] H. H. Lee, B. F. Cain, W. A. Denny, J. Org. Chem. 1989, 54, 428-431.

[101] J. L. Chou, J. P. Chyn, F. L. Urbach, D. F. Gervasio, Polyhedron 2000, 19, 2215-2223.

[102] B. C. Askew, C. J. Mclntyre, C. A. Hunt, D. A. Claremon, R. J. Gould, R. J. Lynch, D. J. Armstrong, Bioorg. Med. Chem. Lett. 1995, 5, 475-480.

[103] N. A. Eberhardt, H. Guan, Chem. Rev. 2016, 116, 8373-8426.

[104] M. L. H. Green, T. Saito, Chem. Commun. 1969, 208.

[105] M. J. Tenorio, M. C. Puerta, P. Valgera, J. Am. Chem. Soc. Dalt. Trans. 1996, 1305-1308.

[106] P. Du, R. Eisenberg, Energy Environ. Sci. 2012, 5, 6012-6021.

[107] J. C. Fontecilla-Camps, A. Volbeda, C. Cavazza, Y. Nicolet, Chem. Rev. 2007, 107, 4273-4303.

[108] C. C. Tard, C. J. Pickett, Chem. Rev. 2009, 109, 2245-2274.

[109] B. E. Barton, C. M. Whaley, T. B. Rauchfuss, D. L. Gray, J. Am. Chem. Soc. 2009, 131, 6942-6943.

[110] G. M. Chambers, M. T. Huynh, Y. Li, S. Hammes-Schiffer, T. B. Rauchfuss, E. Reijerse, W. Lubitz, Inorg. Chem. 2015, 55, 419-431.

[111] Z. Li, Y. Ohki, K. Tatsumi, J. Am. Chem. Soc. 2005, 127, 8950-8951.

[112] S. Canaguier, V. Fourmond, C. U. Perotto, J. Fize, J. Pécaut, M. Fontecave, M. J. Field, V. Artero, Chem. Commun. 2013, 49, 5004-5006.

[113] Z. Han, W. R. McNamara, M. S. Eum, P. L. Holland, R. Eisenberg, Angew. Chem. Int. Ed. 2012, 51, 1667-1670.

[114] M. L. Helm, M. P. Stewart, R. M. Bullock, M. R. DuBois, D. L. DuBois, Science 2011, 333, 863-866.

[115] K. S. Sandhya, C. H. Suresh, Dalton Trans. 2014, 43, 12279-12287. 
[116] N. Armaroli, V. Balzani, Angew. Chem. Int. Ed. 2007, 46, 52-66.

[117] W. Lubitz, W. Tumas, Chem. Rev. 2007, 10\%, 3900-3903.

[118] V. Balzani, A. Credi, M. Venturi, ChemSusChem 2008, 1, 26-58.

[119] K. S. Sandhya, C. H. Suresh, Organometallics 2011, 30, 3888-3891.

[120] A. J. Bard, M. A. Fox, Acc. Chem. Res. 1995, 28, 141-145.

[121] S. W. Kohl, L. Weiner, L. Schwartsburd, L. Konstantinovski, L. J. W. Shimon, Y. Ben-david, M. A. Iron, D. Milstein, Science 2009, 324, 74-77.

[122] D. M. Heinekey, H. Mellows, T. Pratum, J. Am. Chem. Soc. 2000, 122, 6498-6499.

[123] D. G. Gusev, D. Nietlispach, I. L. Eremenko, H. Berke, Inorg. Chem. 1993, 32, $3628-3636$.

[124] X. L. Luo, R. H. Crabtree, J. Am. Chem. Soc. 1990, 112, 6912-6918.

[125] C. A. Bayse, M. B. Hall, B. Pleune, R. Poli, Organometallics 1998, 17, 4309-4315.

[126] R. H. Crabtree, M. Lavin, J. Chem. Soc. Chem. Commun. 1985, 794-795.

[127] W. J. Oldham, A. S. Hinkle, D. M. Heinekey, J. Am. Chem. Soc. 1997, 119, 11028-11036.

[128] G. J. Kubas, Chem. Rev. 2007, 10\%, 4152-4205.

[129] W. B. Tolman, Activation of Small Molecules: Organometallic and Bioinorganic Perspectives, Wiley-VCH, Weinheim, 2006.

[130] S. A. Abraham, D. Jose, A. Datta, ChemPhysChem 2012, 13, 695-698.

[131] C. Ruan, M. T. Rodgers, J. Am. Chem. Soc. 2004, 126, 14600-14610.

[132] C. L. Pan, W. Chen, J. Song, Organometallics 2011, 30, 2252-2260.

[133] L. Pu, A. D. Phillips, A. F. Richards, M. Stender, R. S. Simons, M. M. Olmstead, P. P. Power, J. Am. Chem. Soc. 2003, 125, 11626-11636.

[134] L. Pu, M. O. Senge, M. M. Olmstead, P. P. Power, J. Am. Chem. Soc. 1998, 120, 48, 12682-12683.

[135] P. Duan, Manuscript in preparation, University of Göttingen.

[136] Q. Dong, Y. Zhao, Y. Su, J.-H. Su, B. Wu, X.-J. Yang, Inorg. Chem. 2012, 51, $13162-13170$.

[137] W. Chen, S. Shimada, M. Tanaka, Y. Kobayashi, K. Saigo, J. Am. Chem. Soc. 2004, 126, 8072-8073.

[138] S. Chakraborty, J. A. Krause, H. Guan, Organometallics 2009, 28, 582-586. 
[139] T. Steinke, C. Gemel, M. Cokoja, M. Winter, R. A. Fischer, Angew. Chem. Int. Ed. 2004, 43, 2299-2302.

[140] K. V. Vasudevan, B. L. Scott, S. K. Hanson, Eur. J. Inorg. Chem. 2012, 4898-4906.

[141] J. Breitenfeld, R. Scopelliti, X. Hu, Organometallics 2012, 31, 2128-2136.

[142] S. Lin, M. W. Day, T. Agapie, J. Am. Chem. Soc. 2011, 133, 3828-3831.

[143] N. Koen de Vries, The Handbook of Homogeneous Hydrogenation (Eds.: J. G. de Vries, C. J. Elsevier), Vol. 1, Wiley-VCH, Weinheim, 2007.

[144] S. Ramakrishnan, S. Chakraborty, W. W. Brennessel, C. E. D. Chidsey, W. D. Jones, Chem. Sci. 2016, \%, 117-127.

[145] R. A. Schunn, Inorg. Chem. 1970, 9, 394-395.

[146] H. Günther, NMR-Spektroskopie: Grundlagen, Konzepte und Anwendungen der Protonen- und Kohlenstoff-13-Kernresonanz-Spektroskopie in der Chemie, 3. neubearb. und erw. Aufl., Thieme, Stuttgart; New York, 1992.

[147] J. Y. Chen, A. A. Marti, N. J. Turro, K. Komatsu, Y. Murata, R. G. Lawler, J. Phys. Chem. B 2010, 114, 14689-14695.

[148] Y. I. Neronov, S. G. Karshenboim, Phys. Lett. A 2003, 318, 126-132.

[149] S. G. Karshenboim, V. G. Ivanov, Y. I. Neronov, B. P. Nikolaev, Y. N. Tolparov, Can. J. Phys. 2005, 83, 405-412.

[150] G. R. Fulmer, A. J. M. Miller, N. H. Sherden, H. E. Gottlieb, A. Nudelman, B. M. Stoltz, J. E. Bercaw, K. I. Goldberg, Organometallics 2010, 29, 2176-2179.

[151] B. R. Bender, G. J. Kubas, L. H. Jones, B. I. Swanson, J. Eckert, K. B. Capps, C. D. Hoff, J. Am. Chem. Soc. 1997, 119, 9179-9190.

[152] C.-F. Lin, J. Res. Inst. Catal. 1967, 15, 218-227.

[153] J. Halpern, B. R. James, Can. J. Chem. 1966, 44, 671-675.

[154] Walter J. Moore, Grundlagen der Physikalischen Chemie, Walter de Gruyter, Berlin / New Y 1990, 377.

[155] G. Pratt, D. Rogers, J. Chem. Soc., Faraday Trans. 1 1976, 72, 1589-1600.

[156] J. Casanueva, J. S. Fabián, E. Díez, A. L. Esteban, J. Mol. Struct. 2001, 565-566, $449-454$.

[157] A. J. Lough, S. Park, R. Ramachandran, R. H. Morris, J. Am. Chem. Soc. 1994, $116,8356-8357$. 
[158] J. C. Lee Jr., E. Peris, A. L. Rheingold, R. H. Crabtree, J. Am. Chem. Soc. 1994, 116, 11014-11019.

[159] D. Milstein, J. C. Calabrese, I. D. Williams, J. Am. Chem. Soc. 1986, 108, 6387-6389.

[160] R. C. Stevens, R. Bau, D. Milstein, O. Blum, T. F. Koetzle, J. Chem. Soc. Dalton Trans. 1990, 1429-1432.

[161] A. Adhikary, J. R. Schwartz, L. M. Meadows, J. A. Krause, H. Guan, Inorg. Chem. Front. 2014, 71-82.

[162] M. Bassetti, S. Marini, J. Díaz, M. P. Gamasa, J. Gimeno, Y. Rodríguez-Álvarez, S. Garcia-Granda, Organometalics 2002, 4815-4822.

[163] M. Ciclosi, F. Estevan, P. Lahuerta, V. Passarelli, J. Pérez-Prieto, M. Sanaú, Adv. Synth. Catal. 2008, 350, 234-236.

[164] M. Jiménez-Tenorio, M. C. Puerta, P. Valerga, Organometallics 2009, 28, $2787-2798$.

[165] C. Bianchini, P. Innocenti, A. Meli, M. Peruzzini, F. Zanobini, Organometalics 1990, 2514-2522.

[166] W. J. Evans, I. Bloom, W. E. Hunter, J. L. Atwood, J. Am. Chem. Soc. 1981, 103, 6507-6508.

[167] C. Kohrt, G. Wienhöfer, C. Pribbenow, M. Beller, D. Heller, ChemCatChem 2013, 5, 2818-2821.

[168] C. Belger, B. Plietker, Chem. Commun. 2012, 48, 5419-5421.

[169] R. Gao, D. R. Pahls, T. R. Cundari, C. S. Yi, Organometallics 2014, 33, 6937-6944.

[170] C. Gunanathan, M. Hölscher, F. Pan, W. Leitner, J. Am. Chem. Soc. 2012, 134 , 14349-14352.

[171] C. Bianchini, F. V. Andrea Mell, Maurizio Peruzzini, F. Zanobini, Organometallics 1989, 8, 2080-2082.

[172] C. Bianchini, C. Bohanna, M. A. Esteruelas, P. Frediani , A. Meli , L. A. Oro, Organometallics 1992, 11, 3837-3844.

[173] C. Bianchini, A. Meli, M. Peruzzini, P. Frediani, C. Bohanna, M. A. Esteruelas, L. A. Oro, Organometallics 1992, 11, 138-145.

[174] C. Kohrt, G. Wienhöfer, C. Pribbenow, M. Beller, D. Heller, ChemCatChem 2013, 5, 2818-2821. 
[175] Kirk-Othmer, Encyclopedia of Chemical Technology (Eds.: J. I. Kroschwitz, M. Howe-Grant), 4th ed., 22 vol., John Wiley \& Sons Inc., Chichester, 1997, pp. 956-994.

[176] W. S. Emerson, Chem. Rev. 1949, 45, 347-383.

[177] I. Serafin, A. Kotarba, M. Grzywa, Z. Sojka, H. Bińczycka, P. Kuśtrowski, J. Catal. 2006, 239, 137-144.

[178] T. Matsumoto, R. A. Periana, D. J. Taube, H. Yoshida, J. Catal. 2002, 206, 272-280.

[179] B. A. Vaughan, M. S. Webster-Gardiner, T. R. Cundari, T. B. Gunnoe, Science 2015, 348, 6233, 421-424.

[180] H. Cao, T. Chen, Y. Zhou, D. Han, S.-F. Yin, L.-B. Han, Adv. Synth. Catal. 2014, 356, 765-769.

[181] R. Waterman, G. L. Hillhouse, Organometallics 2003, 22, 5182-5184.

[182] A. Reyes-Sánchez, F. Cañavera-Buelvas, R. Barrios-Francisco, O. L. Cifuentes-Vaca, M. Flores-Alamo, J. J. García, Organometallics 2011, 30, 3340-3345.

[183] G. L. O. Wilson, M. Abraha, J. a. Krause, H. Guan, Dalton Trans. 2015, 44, 12128-12136.

[184] E. Bouwman, The Handbook of Homogenous Hydrogenation (Eds.: J. G. de Vries, C. J. Elsevier), Vol. 1, Wiley-VCH, Weinheim, 2007.

[185] I. M. Angulo, A. M. Kluwer, E. Bouwman, Chem. Comm. 1998, 2689-2690.

[186] I. L. Fedushkin, M. V Moskalev, A. N. Lukoyanov, A. N. Tishkina, E. V Baranov, G. a Abakumov, Chem. - A Eur. J. 2012, 18, 11264-11276.

[187] C. Bianchini, D. Masi, A. Meli, M. Peruzzini, A. Vacca, F. Laschi, P. Zanello, Organometallics 1991, 10, 636-645.

[188] M. J. Burn, G.-Y. Kiel, F. Seils, J. Takats, J. Washington, J. Am. Chem. Soc. 1989, 111, 6850-6852.

[189] S. I. Hommeltoft, D. H. Berry, R. Eisenberg, J. Am. Chem. Soc. 1986, 108, 5345-5347.

[190] D. Taher, H. Pritzkow, B. Walfort, H. Lang, J. Organomet. Chem. 2006, 691, 793-798.

[191] T. De Simone, R. S. Dickson, B. W. Skelton, A. H. White, Inorganica Chim. Acta 1995, 240, 323-333.

[192] J. Takats, Polyhedron 1988, 7, 931-941. 
[193] G. R. County, R. S. Dickson, S. M. Jenkins, J. Johnson, O. Paravagna, J. Organomet. Chem. 1997, 530, 49-57.

[194] B. D. Rowsell, R. Mcdonald, M. J. Ferguson, M. Cowie, Organometallics 2003, 22, 2944-2955.

[195] F. H. Allen, O. Kennard, D. G. Watson, L. Brammer, A. G. Orpen, R. Taylor, J. Chem. Soc. Perkins Trans. II 1987, 1-19.

[196] C. Cui, X. Li, C. Wang, J. Zhang, J. Cheng, X. Zhu, Angew. Chemie Int. Ed. 2006, 45, 2245-2247.

[197] K. Ohkita, H. Asano, H. Kurosawa, T. Hirao, Y. Miyaji, I. Ikeda, Can. J. Chem. 1996, 74, 1936-1944.

[198] F. P. Gasparro, N. H. Kolodny, J. Chem. Educ. 1977, 54, 258-261.

[199] H. Friebolin, Ein- und zweidimensionale NMR-Spektroskopie, 4. Aufl., Wiley-VCH GmbH \& Co. KGaA, Weinheim, 2006.

[200] J. E. Anderson, D. J. D. Barkel, C. J. Cooksey, Tetrahedron Lett. 1983, 24 , 1077-1080.

[201] G. B. Deacon, P. W. Felder, J. Am. Chem. Soc. 1968, 90, 495-497.

[202] P. C. Wailes, H. Weigold, P. Bell, J. Organomet. Chem. 1971, 27, 373-378.

[203] P. C. Wailes, H. Weigold, A. P. Bell, J. Organomet. Chem. 1972, 43, 32-34.

[204] S. B. Jones, J. L. Petersen, Organometallics 1985, 4, 966-971.

[205] C. Bianchini, A. Meli, M. Peruzzini, F. Vizza, Organometallics 1990, 9, 1146-1155.

[206] A. Di Giuseppe, R. Castarlenas, J. J. Perez-Torrente, F. J. Lahoz, V. Polo, L. A. Oro, Angew. Chem. Int. Ed. 2011, 50, 3938-3942.

[207] A. Harthun, PhD Thesis, University of Bonn, 1996.

[208] A. Harthun, R. Giernoth, C. J. Elsevier, J. Bargon, Chem. Commun. 1996, 2483-2484.

[209] J. Natterer, J. Bargon, Prog. Nucl. Magn. Reson. Spectrosc. 1997, 31, 293-315.

[210] C. R. Bowers, D. P. Weitekamp, J. Am. Chem. Soc. 1987, 109, 5541-5542.

[211] T. C. Eisenschmid, R. U. Kirss, P. P. Deutsch, S. I. Hommeltoft, R. Eisenberg, J. Am. Chem. Soc. 1987, 109, 8089-8091.

[212] L. Buljubasich, María Beleńe Franzoni, K. Münnemann, Duckett, R. E. Mewis, Hyperpolarization Methods in NMR Spectroscopy (Ed.: L. T. Kuhn), Springer-Verlag Berlin Heidelberg, 2013, 33-82. 
[213] R. W. Adams, J. A. Aguilar, K. D. Atkinson, M. J. Cowley, P. I. P. Elliott, S. B. Duckett, G. G. R. Green, I. G. Khazal, J. Lopez-Serrano, D. C. Williamson, Science 2009, 323, 1708-1711.

[214] S. B. Duckett, R. E. Mewis, Acc. Chem. Res. 2012, 45, 1247-1257.

[215] C. R. Bowers, D. P. Weitekamp, Phys. Rev. Lett. 1986, 57, 2645-2648.

[216] R. Eisenberg, Acc. Chem. Res. 1991, 24, 110-116.

[217] K. Münnemann, H. W. Spiess, Nat. Phys. 2011, 7, 522-523.

[218] K. L. Ivanov, A. V. Yurkovskaya, H.-M. Vieth, Z. Phys. Chem. 2012, 226, $1315-1342$.

[219] D. Schulman, R. Zhou, R. Bowers, J. Undergrad. Res. 2014, 15, 1-7.

[220] A. S. Kiryutin, K. L. Ivanov, A. V. Yurkovskaya, R. Kaptein, H.-M. Vieth, Z. Phys. Chem. 2012, 226, 1343-1362.

[221] M. Irfan, N. Eshuis, P. Spannring, M. Tessari, M. C. Feiters, F. P. J. T. Rutjes, J. Phys. Chem. C 2014, 118, 13313-13319.

[222] A. S. Kiryutin, A. V Yurkovskaya, R. Kaptein, H. Vieth, K. L. Ivanov, J. Phys. Chem. Lett. 2013, 4, 2514-2519.

[223] H. S. La Pierre, J. Arnold, F. D. Toste, Angew. Chemie - Int. Ed. 2011, 50, 3900-3903.

[224] R. Noyori, M. Ohta, Y. Hsiao, M. Kitamura, T. Ohta, H. Takaya, J. Am. Chem. Soc. 1986, 108, 7117-7119.

[225] Eisenschmid, T. C. Ph.D. Dissertation, University of Rochester, 1989.

[226] B. Feng, Z. Hou, X. Wang, Y. Hu, H. Li, Y. Qiao, Green Chem. 2009, 11, 1446-1452.

[227] N. Narkhede, A. Patel, S. Singh, Dalton Trans. 2014, 43, 2512-2520.

[228] M. J. Rak, M. Lerro, A. Moores, Chem. Commun. 2014, 50, 12482-2485.

[229] J.-Q. Wang, F. Cai, E. Wang, L.-N. He, Green Chem. 2007, 9, 882-887.

[230] C. J. Curtis, A. Miedaner, R. Ciancanelli, W. W. Ellis, B. C. Noll, M. Rakowski DuBois, D. L. DuBois, Inorg. Chem. 2003, 42, 216-227.

[231] A. Walstrom, M. Pink, N. P. Tsvetkov, H. Fan, M. Ingleson, K. G. Caulton, J. Am. Chem. Soc. 2014, 127, 16780-16781.

[232] N. P. Tsvetkov, M. F. Laird, H. Fan, M. Pink, K. G. Caulton, Chem. Commun. 2009, 4578-4580.

[233] L. Sacconi, I. Bertini, J. Am. Chem. Soc. 1968, 90, 5443-5446. 
[234] E. C. Alyea, D. W. Meek, J. Am. Chem. Soc. 1968, 91, 5761-5768.

[235] E. K. Barefield, D. H. Busch, Inorg. Chem. 1971, 10, 1216-1220.

[236] W. Levason, C. A. McAuliffe, D. G. Watson, J. Coord. Chem. 1975, 4, 173-179.

[237] E. Grimley, D. W. Meek, Inorg. Chem. 1986, 25, 2049-2053.

[238] D. A. Sweigart, Patricia Heidtmann, J. C. S. Chem. Comm. 1973, 556-557.

[239] S. P. Walg, A. D. Schmidt, M. Schmitz, S. Farsadpour, J. Lang, M. Niebergall, Y. Sun, P. W. Roesky, G. Niedner-Schatteburg, W. R. Thiel, Dalton Trans. 2015, 44, $1317-1322$.

[240] Y. Feng, K. A. Barakat, M. Lail, T. R. Cundari, T. B. Gunnoe, J. L. Petersen, J. Am. Chem. Soc. 2005, 127, 14174-14175.

[241] J. R. Webb, S. A. Burgess, T. R. Cundari, T. B. Gunnoe, Dalton Trans. 2013, 42, 16646-16665.

[242] T. L. Lohr, W. E. Piers, M. Parvez, Chem. Sci. 2013, 4, 770-775.

[243] J. Oxgaard, W. J. Tenn, R. J. Nielsen, R. a. Periana, W. a. Goddard, Organometallics 2007, 26, 1565-1567.

[244] W. J. Tenn, K. J. H. Young, J. Oxgaard, R. J. Nielsen, W. a. Goddard, R. a. Periana, Organometallics 2006, 25, 5173-5175.

[245] B. G. Hashiguchi, K. J. H. Young, M. Yousufuddin, W. a. Goddard, R. a. Periana, J. Am. Chem. Soc. 2010, 132, 12542-12545.

[246] X. Qu, X. Wang, D. Zhu, Environ. Sci. Technol. 2007, 41, 8321-8327.

[247] X.-L. Yuan, X.-L. Cheng, X.-F. Su, Int. J. Quantum Chem. 2009, 109, 972-981.

[248] A. Ebrahimi, M. Habibi, H. R. Masoodi, Chem. Phys. Lett. 2009, 478, 120-126.

[249] A. Ebrahimi, M. H. Khorassani, H. R. Masoodi, Chem. Phys. Lett. 2010, 493, $27-32$.

[250] A. C. Behrle, L. Castro, L. Maron, J. R. Walensky, J. Am. Chem. Soc. 2015, 137, 14846-14849.

[251] J. Carroll, J. P. Gagnier, A. W. Garner, J. G. Moots, R. D. Pike, Y. Li, S. Huo, Organometallics 2013, 32, 4828-4836.

[252] Y. Y. Li, B. Wang, Y. Luo, D. Yang, P. Tong, J. Zhao, L. Luo, Y. Zhou, S. Chen, F. Cheng, et al., Nat. Chem. 2013, 5, 320-326.

[253] J. S. Anderson, J. Rittle, J. C. Peters, Nature 2013, 501, 84-88.

[254] M. D. Fryzuk, Acc. Chem. Res. 2009, 42, 127-133. 
[255] J. a Pool, E. Lobkovsky, P. J. Chirik, Nature 2004, 427, 527-530.

[256] L. D. Field, Nat. Chem. 2010, 2, 520-521.

[257] J. Chatt, J. R. Dilworth, R. L. Richards, Chem. Rev. 1978, 78, 589-625.

[258] K. Arashiba, Y. Miyake, Y. Nishibayashi, Nat. Chem. 2011, 3, 120-125.

[259] D. V. Yandulov, R. R. Schrock, Science 2003, 301, 76-78.

[260] K. C. Macleod, P. L. Holland, Nat. Chem. 2013, 5, 559-565.

[261] C. C. Lee, J. A. Wiig, Y. Hu, M. W. Ribbe, Bioinspired Catalysis (Eds.: W. Weigand, P. Schollhammer), Wiley-VCH Verlag GmbH \& Co. KGaA, 2014.

[262] D. Lukoyanov, S. A. Dikanov, Z. Yang, B. M. Barney, R. I. Samoilova, K. V Narasimhulu, D. R. Dean, L. C. Seefeldt, B. M. Ho, J. Am. Chem. Soc. 2011, 133, 11655-11664.

[263] M. Hidai, Y. Mizobe, Chem. Rev. 1995, 95, 1115-1133.

[264] T. A. Betley, J. C. Peters, J. Am. Chem. Soc. 2003, 125, 10782-10783.

[265] S. Vogel, A. Barth, G. Huttner, T. Klein, L. Zsolnai, R. Kremer, Angew. Chem. Int. Ed. Engl. 1991, 30, 303-304.

[266] A. R. Fout, F. Basuli, H. Fan, J. Tomaszewski, J. C. Huffman, M. H. Baik, D. J. Mindiola, Angew. Chem. Int. Ed. Engl. 2006,45, 3291-3295.

[267] L. J. Murray, W. W. Weare, J. Shearer, A. D. Mitchell, K. A. Abboud, J. Am. Chem. Soc. 2014, 136, 13502-3505.

[268] R. L. Collin, W. N. Lipscomb, Acta Crystallogr. 1951, 4, 10-14.

[269] F. Cardulla, H. Beach, J. Chem. Educ. 1983, 60, 505-508.

[270] W. H. Bernskoetter, J. A. Pool, E. Lobkovsky, P. J. Chirik, J. Am. Chem. Soc. 2005, 127, 7901-7911.

[271] C. T. Saouma, P. Müller, J. C. Peters, J. Am. Chem. Soc. 2009, 131, 10358-10359.

[272] S. F. McWilliams, P. L. Holland, Acc. Chem. Res. 2015, 48, 2059-2065.

[273] L. Blum, I. D. Williams, R. R. Schrock, J. Am. Chem. Soc. 1984, 106, 8316-8317.

[274] J. L. Crossland, L. N. Zakharov, D. R. Tyler, Inorg. Chem. 2007, 46, 10476-10478.

[275] C. T. Saouma, C. C. Lu, J. C. Peters, Inorg. Chem. 2012, 51, 10043-10054.

[276] M. Jahncke, A. Neels, H. Stoeckli-Evans, G. Süss-Fink, J. Organomet. Chem. 2000, 565, 97-103.

[277] D. M. Roundhill, A. L. Rheingold, Inorg. Chem. 1987, 26, 3972-3974. 
[278] I. Mena, E. a Jaseer, M. a Casado, P. García-Orduña, F. J. Lahoz, L. a Oro, Chem. Eur. J. 2013, 19, 5665-5675.

[279] I. P. Parkin, D. J. Williams, Polyhedron 1989, 8, 1979-1981.

[280] I. Mena, M. a. Casado, P. García-Orduña, V. Polo, F. J. Lahoz, A. Fazal, L. a. Oro, Angew. Chemie Int. Ed. 2011, 50, 11735-11738.

[281] S. Park, A. L. Rheingold, D. M. Roundhill, Organometallics 1991, 10, 615-623.

[282] P. L. Holland, R. a. Andersen, R. G. Bergman, J. Am. Chem. Soc. 1996, 118, 1092-1104.

[283] H. Klein, H. H. Karsch, Chem. Ber. 1973, 106, 2438-2454.

[284] W. Henderson, A. G. Oliver, Inorg. Chim. Acta 2014, 416, 49-56.

[285] T. Kimura, H. Arita, K. Ishiwata, S. Kuwata, T. Ikariya, Dalton Trans. 2009, $2912-2914$.

[286] C. A. O’Mahoney, I. P. Parkin, D. J. Williams, D. Woollins, Polyhedron 1989, 8, 1979-1981.

[287] J. P. Chauvel, M. M. Folkendt, N. S. True, Magn. Reson. Chem. 1987, 25, 101-104.

[288] P. Garbacz, K. Jackowski, W. Makulski, R. E. Wasylishen, J. Phys. Chem. A 2012, 116, 11896-11904.

[289] F. Haase, J. Sauer, J. Phys. Chem. 1994, 98, 3083-3085.

[290] W. G. Schneider, H. J. Bernstein, J. A. Pople, J. Chem. Phys. 1958, 28, 601-607.

[291] R. A. Bernheim, H. Batiz-Hernandez, J. Chem. Phys. 1964, 40, 3446-3447.

[292] W. W. Weare, X. Dai, M. J. Byrnes, J. M. Chin, R. R. Schrock, P. Müller, Proc. Natl. Acad. Sci. 2006, 103, 17099-17106.

[293] M. Zeldin, B.-R. Qian, S. J. Choi, J. Polym. Sci. 1983, 21, 1361-1369.

[294] W. L. Marshall, E. U. Franck, J. Phys. Chem. Ref. Data 1981, 10, 295-304.

[295] A. V. Bandura, S. N. Lvov, J. Phys. Chem. Ref. Data 2006, 35, 15-30.

[296] E. C. Meister, M. Willeke, W. Angst, A. Togni, P. Walde, Helv. Chim. Acta 2014, $97,1-31$.

[297] W. N. Olmstead, Z. Margolin, F. G. Bordwell, J. Org. Chem. 1980, 45, 3295-3299.

[298] F. G. Bordwell, D. J. Algrim, J. Am. Chem. Soc. 1988, 110, 2964-2968.

[299] U. Ragnarsson, L. Grehn, J. Koppel, O. Loog, O. Tšubrik, A. Bredikhin, U. Mäeorg, I. Koppel, J. Org. Chem. 2005, 70, 5916-5921. 
[300] R. G. Pearson, J. Songstad, J. Am. Chem. Soc. 1967, 89, 1827-1836.

[301] R. G. Pearson, J. Am. Chem. Soc. 1988, 110, 7684-7690.

[302] R. G. Pearson, Coord. Chem. Rev. 1990, 100, 403-425.

[303] R. G. Pearson, Inorg. Chem. 1988, 27, 734-740.

[304] N. Wiberg, Lehrbuch der Anorganischen Chemie, 101. Aufl., Walter de Gruyter, Berlin/New York 1995, 763-775.

[305] G. V. Lagodzinskaya, N. G. Yunda, G. B. Manelis, Bull. Acad. Sci. USSR 1980, $89,532-539$.

[306] R. L. Lichter, J. D. Roberts, J. Am. Chem. Soc. 1972, 94, 4904-4906.

[307] E. Catalano, R. H. Sanborn, J. W. Frazer, J. Chem. Phys. 1963, 38, 2265-2272.

[308] J. R. Durig, S. F. Bush, E. E. Mercer, J. Chem. Phys. 1966, 44, 4238-4247.

[309] J. S. Ziomek, M. D. Zeidler, J. Mol. Spectrosc. 1963, 11, 163-184.

[310] J. Demaison, F. Hegelund, H. Bürger, J. Mol. Struct. 1997, 413-414, 447-456.

[311] H. J. a. Jensen, P. Joergensen, T. Helgaker, J. Am. Chem. Soc. 1987, 109, 2895-2901.

[312] P. Schollhammer, Er. Guénin, F. Y. Pétillon, J. Talarmin, Organometallics 1998, 2, 1922-1924.

[313] G. E. Greco, R. R. Schrock, Inorg. Chem. 2001, 40, 3861-3878.

[314] P. Schollhammer, B. Didier, N. Le Grand, F. Y. Pétillon, J. Talarmin, K. W. Muir, S. J. Teat, Eur. J. Inorg. Chem. 2002, 658-663.

[315] X. Yan, R. J. Batchelor, F. W. B. Einstein, D. Sutton, Inorg. Chem. 1996, 35, 7818-7828.

[316] F. W. B. Einstein, X. Yan, D. Sutton, J. Chem. Soc., Chem. Commun. 1990, 1466-1467.

[317] N. Lehnert, F. Tuczek, Inorg. Chem. 1999, 38, 1671-1682.

[318] S. S. Rozenel, J. Arnold, Inorg. Chem. 2012, 51, 9730-9739.

[319] G. M. Coppinger, J. Am. Chem. Soc. 1957, 79, 501-502.

[320] D. V. Yandulov, R. R. Schrock, J. Am. Chem. Soc. 2002, 124, 6252-6253.

[321] D. V. Yandulov, R. R. Schrock, A. L. Rheingold, C. Ceccarelli, W. M. Davis, Inorg. Chem. 2003, 42, 796-813. 
[322] J. M. Smith, R. J. Lachicotte, K. A. Pittard, T. R. Cundari, G. Lukat-Rodgers, K. R. Rodgers, P. L. Holland, J. Am. Chem. Soc. 2001, 123, 9222-9223.

[323] A. J. L. Pombeiro, Rev. Port. Quím. 1984, 26, 30-60.

[324] O. Franke, B. E. Wiesler, N. Lehnert, F. Tuczek, Z. anorg. allg. Chem. 2002, 628, 2395-2402.

[325] I. Čorić, B. Q. Mercado, E. Bill, D. J. Vinyard, P. L. Holland, Nature 2015, 526, 96-99.

[326] W. A. Chomitz, J. Arnold, Dalton Trans. 2009, 1714-1720.

[327] M. B. O’Donoghue, W. M. Davis, R. R. Schrock, Inorg. Chem. 1998, 37, 5149-5158.

[328] J. M. Blacquiere, C. S. Higman, S. I. Gorelsky, N. J. Beach, S. J. Dalgarno, D. E. Fogg, Angew. Chemie - Int. Ed. 2011, 123, 946-949.

[329] O. Hübner, L. Manceron, H.-J. Himmel, Chem. - A Eur. J. 2014, 20, 17025-17038.

[330] S. Schenk, B. Kirchner, M. Reiher, Chem. - A Eur. J. 2009, 15, 5073-5082.

[331] L. D. Field, H. L. Li, A. M. Magill, Inorg. Chem. 2009, 48, 5-7.

[332] S. Kuriyama, K. Arashiba, K. Nakajima, H. Tanaka, K. Yoshizawa, Y. Nishibayashi, Chem. Sci. 2015, 6, 3940-3951.

[333] B. M. Hoffman, D. Lukoyanov, Z. Y. Yang, D. R. Dean, L. C. Seefeldt, Chem. Rev. 2014, 114, 4041-4062.

[334] S. Neumann, Master Thesis, University of Göttingen, 2015.

[335] E. Ferretti, Manuscript in preparation, University of Göttingen.

[336] G. M. Sheldrick, SHELXS-97, Program for Crystal Structure Solution, Universität Göttingen 1997.

[337] G. M. Sheldrick, SHELXL-97, Program for Crystal Structure Refinement, Universität Göttingen 1997.

[338] G. M. Sheldrick, Acta Cryst. 2008, A64, 112-122.

[339] STOE \& CIE GmbH, Darmstadt, Germany, 2002.

[340] J. L. Whitten, J. Chem. Phys. 1973, 58, 4496.

[341] F. Neese, F. Wennmohs, A. Hansen and U. Becker, Chem. Phys. 2009, 356, 98-109.

[342] F. Neese, ORCA, An ab initio, DFT and semiempirical SCF-MO package 2.9.0 2012.

[343] J. Pipek and P. G. Mezey, J. Chem. Phys. 1989, 90, 4916-4926. 
[344] R. A. Mata and H.-J. Werner, Mol. Phys. 2007, 105, 2753-2761.

[345] F. Weigend, Phys. Chem. Chem. Phys. 2006, 8, 1057.

[346] F. Weigend, J. Comput. Chem. 2007, 29, 167.

[347] A. Hellweg, C. Hättig, S. Hoefener and W. Klopper, Theoretical Chemistry Accounts $2007,117,587$.

[348] H.-J. Werner, P. J. Knowles, G. Knizia, F. R. Manby and M. Schütz et al., MOLPRO, version 2012.1, a package of ab initio programs 2012, see http://www.molpro.net. 


\section{List of Abbreviations}

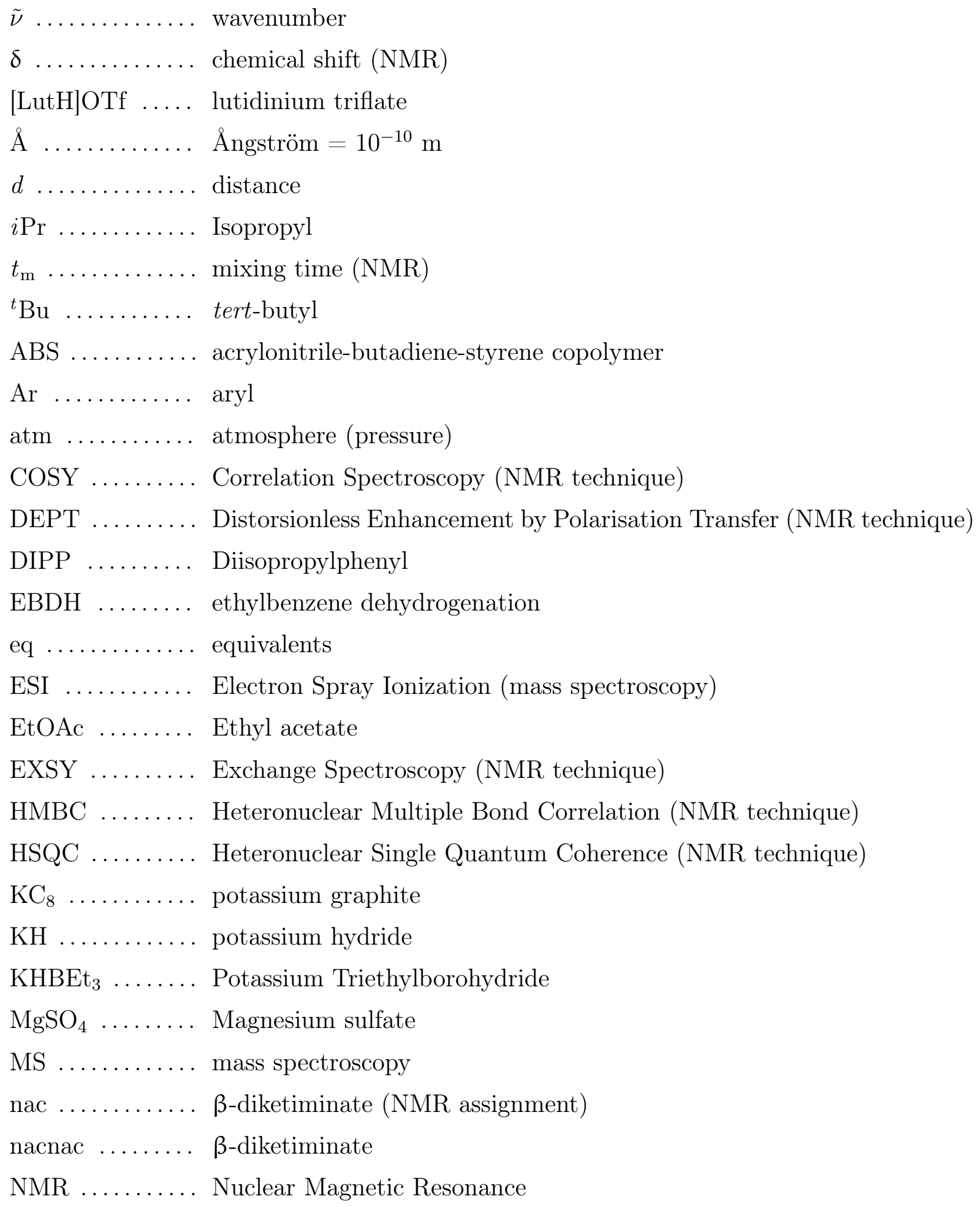


NOESY ...... Nuclear Overhauser Effect Spectroscopy (NMR technique)

ORTEP ....... Oak Ridge Thermal Ellipsoid Plot - program

PA .......... phenylacetylene

$\mathrm{Ph} \ldots \ldots \ldots$...... phenyl

PO $\ldots \ldots \ldots \ldots$ propylene oxide

POCOP ...... bis(phosphinite)- based pincer ligand

$\operatorname{ppm} \ldots \ldots \ldots$ parts per million

RT .......... room temperature

SAN ......... styrene-arylonitrile resins

SB $\ldots \ldots \ldots$ styrene-butadiene latex

SBR .......... styrene-butadiene-rubber

$\mathrm{SM} \ldots \ldots \ldots$....... styrene monomer

TACN ........ 1,4,7-triazacyclononane

TEMPO ..... (2,2,6,6-Tetramethylpiperidin-1-yl)oxyl

THF ........ tetrahydrofuran

$\mathrm{THF}-\mathrm{D}_{8} \ldots \ldots$ deuterated tetrahydrofuran

TOF ......... turnover frequency

UPR ........ unsaturated polyester resins 


\section{List of Scientific Contributions}

\section{Presentation at Conference}

D.-H. Manz, "A dinuclear pyrazolate-bridged bis( $\beta$-diketiminato) nickel hydride", Koordinationschemie-Tagung 2015, Paderborn, Germany, March 23 2015.

\section{Publications}

D.-H. Manz, T. Stolper, S. Katsiaouni, S. Dechert, M. John, R. Mata and F. Meyer, "Extremely Slow Proton and Water Exchange of a Metal-Bridging Hydroxide", manuscript in preparation.

D.-H. Manz, P.-C. Duan, S. Dechert, R. Mata, M. John and F. Meyer, "Synchronous $\mathrm{H}_{2} / \mathrm{D}_{2}$ Exchange and $\mathrm{H}_{2}$ Substitution at a Bimetallic Dinickel(II) Dihydride Complex", manuscript in preparation.

D.-H. Manz, P.-C. Duan, E.-B. Dücker, S. Dechert, M. John, C. Griesinger and F. Meyer, "Semihydrogenation of Phenylacetylene by a Dinuclear Phenylvinyl-Bridged Nickel (II) Complex", manuscript in preparation.

D.-H. Manz, P.-C. Duan, S. Dechert, M. John, R. Mata and F. Meyer, "New Model Complexes of Intermediates in the Process of Nitrogen Fixation", manuscript in preparation.

\section{Posters at Conferences and Workshops}

D.-H. Manz, S. Katsiaouni, S. Dechert, S. Demeshko, R. Mata, F. Meyer, "Extremely Slow Proton Exchange in Hydroxide-Bridged Bimetallic Complexes of Pyrrole/Pyrazole-HybridLigands", Göttinger Chemie-Forum 2012, Göttingen, Germany, July 11 ${ }^{\text {th }}, 2012$.

D.-H. Manz, S. Katsiaouni, S. Dechert, S. Demeshko, R. Mata, F. Meyer, "Extremely Slow Proton Exchange in Hydroxide-Bridged Bimetallic Complexes of Pyrrole/Pyrazole-HybridLigands", 40 International Conference on Coordination Chemistry (ICCC40), Valencia, Spain, September $9^{\text {th }}$ to $13^{\text {th }}, \mathbf{2 0 1 2}$.

D.-H. Manz, S. Katsiaouni, S. Dechert, S. Demeshko, R. Mata, F. Meyer, "Extremely Slow Proton Exchange in Hydroxide-Bridged Bimetallic Complexes of Pyrrole/Pyrazole-HybridLigands", 16. Vortragstagung der Wöhler-Vereinigung, Göttingen, Germany, September $26^{\text {th }}$ to $28^{\text {th }}, \mathbf{2 0 1 2}$. 
D.-H. Manz, S. Katsiaouni, S. Dechert, S. Demeshko, T. Stolper, R. Mata, F. Meyer, "Extremely Slow Proton Exchange in Hydroxide-Bridged Bimetallic Complexes of Pyrrole/Pyrazole-Hybrid-Ligands", Göttinger Chemie-Forum 2013, Göttingen, Germany, June $5^{\text {th }}, 2013$. 


\section{Acknowledgments}

At first, i would like to thank my supervisor Prof. Dr. Franc Meyer for his guidance and the opportunity to work on so many different exciting topics. I am grateful for his outstanding, constant optimism which motivated me to face and overcome experimental challenges. Thank you Franc for providing me with best equipment and your support for technical ideas. I would further like to address special thanks to my cosupervisor Prof. Dr. Sven Schneider, and the whole examination board.

I owe many thanks to my colleagues and external cooperation partners who contributed to this work: Prof. Dr. Ricardo Mata and Thorsten Stolper (Institute of Physical Chemistry, Georg-August-University Göttingen) for the DFT calculations concerning the mechansims of hydroxo exchange in $\left[\mathbf{L}^{\mathbf{p y r}} \mathbf{N i}_{2}(\mu-\mathrm{OH})\right]$ and hydrogen exchange in $\left[\mathbf{L}^{\text {bisnac }} \mathbf{N i}_{\mathbf{2}}(\mathbf{H})_{\mathbf{2}}\right]$; Prof. Dr. Christian Griesinger and Eibe Behrend Dücker (Department of NMR-based Structural Biology, Georg-August-University Göttingen) for the opportunity to conduct mechanistical investigations by use of parahydrogen which provided valuable information concerning the hydrogenation of phenylacetylene to styrene in presence of $\mathbf{K}\left[\mathbf{L}^{\text {bisnac }} \mathbf{N i}_{\mathbf{2}}\left(\mu-\eta^{\mathbf{1}}: \eta^{\mathbf{1}} \mathbf{- C H C P h}\right)\right]$; Dr. Michael John, Ralf Schöne and Wolfgang Zolke for teaching me numerous NMR techniques, and helping with problems concerning NMR spectroscopy; Dr. Sebastian Dechert for performing X-ray diffraction experiments and X-ray data refinements; Anne Schober and Nicole Kindermann for further X-ray diffraction experiments and corresponding structure refinements; Dr. Sebastian Dechert for Raman measurements; Jörg Teichgräber and Mattia Veronelli for cyclic voltammetry measurements; Angelika Wraage and the analytical laboratory for elemental analyses; Dr. Claudia Stückl for EPR measurements; Dr. Holm Frauendorf and Thomas Schuchardt for mass spectrometric measurements; Oliver Mitevski, Stefan Resch, Markus Steinert, Iris Klawitter, and Anastasia Vogel for further mass spectrometric measurements; Matthias Hesse, Andreas Schwarz and Jörg Teichgräber for the distribution of chemicals and laboratory equipment; Petra Unger, Britta Mueller, Dr. Claudia Stückl, Andreas Schwarz, Jörg Teichgräber, and the workshops for technical and administrative support. Thank you for all your help which was essential for the success of this work!

Furthermore, i would like to thank my research students Hauke Schmidt, Elena Polo, Susanne Löffler, Stefan Beulig, Helena Keil for supporting me with their experimental work. For effective cooperative experimental work i thank Pengcheng Duan who will succeed with studies on the "bisnac" system. Special thanks i address to the present and former labmates Fabian Schax, Tongxun Guo, Pierre Goursot, Pengcheng Duan, Dr. Subhas Samanta, Anastasia Vogel, and Andreas Schwarz for the wonderful working atmosphere in the lab which included scientific and non-scientific discussions, motivating music and funny conversations. For the great time during regular joint sports activities like Badminton and Flag Football i thank Markus Steinert, Oliver Mitevski and Kai Kalz. 
Moreover, i would like to acknowledge the many group activites with former and present members of the working group which significantly contributed to a great atmosphere. I also like to thank Ralf Schöne, Wolfgang Zolke and Eibe Behrend Dücker for all the technical tipps concerning NMR spectroscopy, their patience, and the long scientific and non-scientific discussions. For corrections, suggestions and linguistic improvements of this work i would like to thank Stefan Resch, Pierre Goursot, Dr. Michael John, Pengcheng Duan, Oliver Mitevski, Alexander Gers-Barlag, Eleonora Ferretti, Joanne Wong, Jana Lücken, Anna Koelpin, Andreas Schwarz, Dr. Sarina Dorazio and Dr. Torben Böhnisch. I thank all my friends, especially Halil, Manuel, Kai, Markus, Olli and Pierre for the great time in Göttingen.

Last but not the least, I would like to thank my parents and my sister for their unwavering support in every regard. 